Thesis submitted for the Doctor of Philosophy degree in Statistics

\title{
Semi-parametric copula sample selection models for count responses
}

Karol Wyszynski

Primary supervisor:

Dr Giampiero Marra

Secondary supervisor:

Dr Christian Hennig

Department of Statistical Science

University College London

January 2016 
I, Karol Wyszynski confirm that the work presented in this report is my own. Where information has been derived from other sources, I confirm that this has been indicated in the report. 


\begin{abstract}
Non-random sample selection arises when observations do not come from a random sample. Instead, individuals select themselves into (or out of) the sample on the basis of observed and unobserved characteristics. In this case, estimates obtained using standard methods such as linear or logistic regression will be biased and inconsistent. This problem can be addressed using sample selection models. In the methodological literature a lot of attention has been given to sample selection models with continuous response. At the same time, not much work has been attributed to sample selection models with count response. The aim of this project is to develop a copula-based sample selection model for count data with flexible covariate effects. First, the literature on sample selection models will be reviewed. Second, two motivating data sets originating from the German Socio-Economic Panel (SOEP) and the United States Veterans' Administration (VA) will be described and explored. Third, the parametric count sample selection model will be depicted. Fourth, flexible covariate effects will be introduced together with inferential and model selection methods. Fifth, the model will be illustrated on the previously mentioned data sets. Finally, potential extensions for future research will be discussed.
\end{abstract}

Keywords: unmeasured confounding, count data, sample selection bias, Heckman model, missingness not at random. 


\section{Acknowledgements}

I would like to express my sincere appreciation to my supervisor, Dr Giampiero Marra, for his guidence and support over the past three years. Giampiero exposed to me a new fascinating area of statistics and econometrics. His invaluable knowledge and experience allowed me to pursue my own research.

I would like to thank my second supervisor, Dr Christian Hennig, for providing me constructive feedback and advice on improving my thesis. I also thank Dr Rosalba Radice and Dr Małgorzata Wojtyś for support in developing new ideas and implementing the elements of my thesis into the software package. 


\section{Contents}

$\begin{array}{ll}\text { Abstract } & 1\end{array}$

$\begin{array}{lr}\text { Acknowledgements } & 2\end{array}$

$\begin{array}{lr}\text { List of Figures } & 7\end{array}$

List of Tables $\quad 22$

1 Introduction $\quad 25$

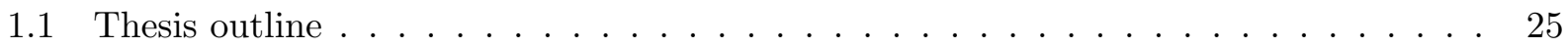

2 Non-random sample selection $\quad 27$

2.1 Brief overview of some imputation methods . . . . . . . . . . . . . . . . 29

2.2 Model definition of some sample selection models and their applications . . . . . . . 34

3 Variants of sample selection models $\quad 40$

3.1 Heckman model for continuous response . . . . . . . . . . . . . . . . . 40

3.2 Greene's count model . . . . . . . . . . . . . . . . . . . . . 43

3.2 .1 Model specification . . . . . . . . . . . . . . . . . 43

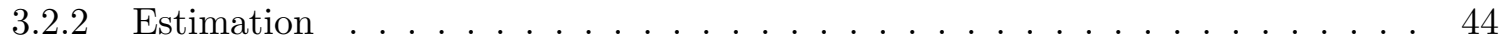

3.2 .3 Disadvantages . . . . . . . . . . . . . . . . . . . . . . 48

3.3 The copula approach . . . . . . . . . . . . . . . . . . . . 49

3.3 .1 Some copula theory . . . . . . . . . . . . . . . . . . . 49

3.3.2 Measures of association . . . . . . . . . . . . . . 54

3.3.3 Archimedean copulas . . . . . . . . . . . . . . . 56 
3.3.4 Copulas in sample selection models . . . . . . . . . . . . . 57

3.3.5 Benefits of copula approach . . . . . . . . . . . . . . 62

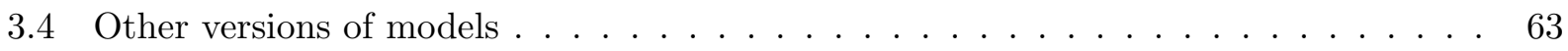

3.4.1 Why not a Bayesian approach? . . . . . . . . . . . . . 65

4 Data and preliminary analysis $\quad 67$

4.1 Veterans' Administration data . . . . . . . . . . . . . . . 67

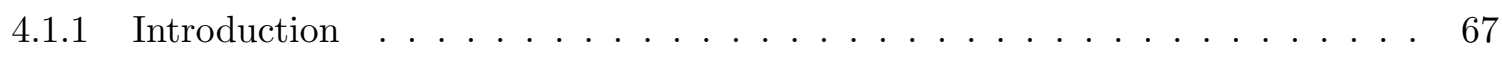

4.1 .2 Data . . . . . . . . . . . . . . . . . . . 69

4.2 German Socio-Economic Panel data . . . . . . . . . . . . . . . . 82

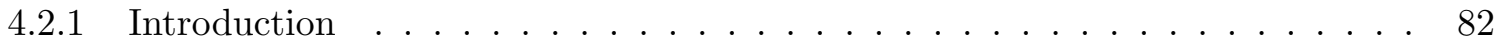

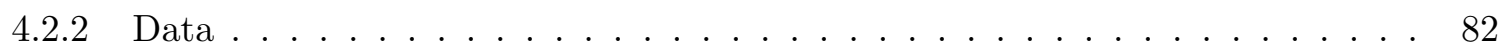

5 Count sample selection models with linear or pre-specified covariate effects 88

5.1 Model specification . . . . . . . . . . . . . . . . . . . 88

5.2 Likelihood . . . . . . . . . . . . . . . . . . . . . . 89

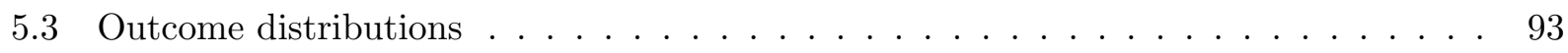

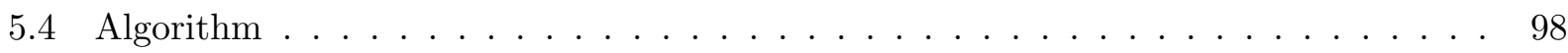

5.5 Starting values . . . . . . . . . . . . . . . . . . 100

5.6 Some numerical aspects $\ldots \ldots \ldots \ldots 1 \ldots \ldots \ldots$

5.7 Simulations . . . . . . . . . . . . . . . . . . . . . 104

5.8 Analytical gradients . . . . . . . . . . . . . . . . . 106

5.8 .1 Analytical expressions of first derivatives f . . . . . . . . 106

5.8 .2 Numerical checks . . . . . . . . . . . . . . . . . . 108

5.8 .3 Simulations . . . . . . . . . . . . . . . . . 112

5.9 Hessian and trust algorithm . . . . . . . . . . . . . . . . . 112

5.9 .1 General form of second derivatives . . . . . . . . . . . . . 112

5.9 .2 Trust algorithm . . . . . . . . . . . . . . . . . . . 113

5.9 .3 Hessian checks . . . . . . . . . . . . . . . . . . . 117

5.9 .4 Simulations . . . . . . . . . . . . . . . . . 120

5.9 .5 Exclusion restriction . . . . . . . . . . . . . . . . . 120 
5.10 Copula rotations . . . . . . . . . . . . . . . . . . . 124

5.11 Remarks on using Kendall's $\tau$ for a discrete and continuous margin . . . . . . . . 126

6 Introducing splines $\quad 127$

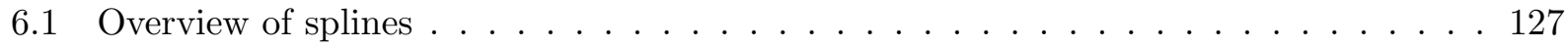

6.2 Introducing non-parametric terms in sample selection models . . . . . . . . . . 135

6.2 .1 Model specification . . . . . . . . . . . . . . . . 135

6.2 .2 Deriving penalized linear working model . . . . . . . . . . . . . . 136

6.2.3 Smoothing parameter selection . . . . . . . . . . . . . . 140

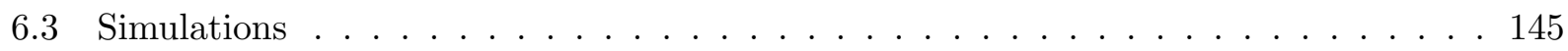

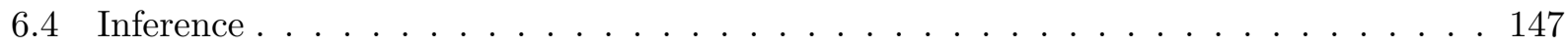

6.4 Frequentist confidence intervals . . . . . . . . . . . . 147

6.4 .2 Bayesian confidence intervals (credible intervals) . . . . . . . . . . . 148

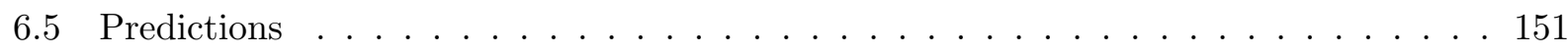

6.6 Model selection . . . . . . . . . . . . . . . . . . . . . . 152

6.7 Asymptotic considerations . . . . . . . . . . . . . . . 153

7 Data illustration using the SemiParSampleSel package $\quad 159$

7.1 Veterans Administration data . . . . . . . . . . . . . . . . 160

7.2 German Socio-Economic Panel data . . . . . . . . . . . . . . . . 170

8 Discussion $\quad 177$

A Derivation of the conditional expected value for the sample selection model with $\begin{array}{lr}\text { continuous response } & 181\end{array}$

B Derivation of the likelihood function for the sample selection model with contin$\begin{array}{ll}\text { uous response and bivariate normal distribution } & 184\end{array}$

C Derivation of the conditional expected value for the Greene count model 186

$\begin{array}{lc}\text { D gamlss diagnostic plots for VA and SOEP data } & 188\end{array}$

$\begin{array}{ll}\text { E Starting value for } \theta^{*} \text { and data simulation } \mathrm{R} \text { code } & 199\end{array}$ 
F Derivation of the score vector components

G Simulations for likelihood function using optim for numerical and anaytical gradient and specification with and without instrumental variable

H General form of analytical Hessian

I Copula, pmf and cdf second derivatives

J Simulation for likelihood function using trust for analytical Hessian and specification with and without instrumental variable

K First and second derivatives of rotated copulas

L Simulation for rotated copulas

M Data simulation $\mathrm{R}$ code (splines)

N Spline simulation for unrotated copulas and same covariates

O Spline simulation for rotated copulas and same covariates

P Spline simulation for unrotated copulas and IV

Q Spline simulation for rotated copulas and IV

R Data illustration results for VA

Bibliography 


\section{List of Figures}

2.1 Selection and outcome process in HIV survey . . . . . . . . . . . . . . . . . 28

3.1 Conditional density plot domain of $f_{2 \mid 1}\left(y_{2} \mid y_{1}^{*}>0\right)$. The white area represents possible $y_{2}$ response values under sample selection. In the peach area, outcomes are not observed. 59

4.1 Histograms of responses for the VA data. The kernel density lines were obtained using density and lines functions. The smoothing kernel of density lines was chosen to be Gaussian, which is the default. The reponses indicate strong positive skewness and many extreme values. . . . . . . . . . . . . . . . . 71

4.2 Poisson Inverse Gaussian model diagnostics for the NUMVAOUT response. . . . . . . . 76

4.3 Sichel model diagnostics for the NUMOTH response. . . . . . . . . . . . . . 76

4.4 Sichel model diagnostics for the VANITES response. . . . . . . . . . . . . . . . 77

4.5 Poisson inverse Gaussian model diagnostics for the OTHNITES response. . . . . . . . 77 
4.6 Selection (left) and outcome (right) equation smooth terms (NUMVAOUT). The horizontal axis represents veterans' age and the vertical axis shows the smooth value on the linear predictor scale. The numbers in brackets on the vertical axis are the effective degrees of freedom of the smooth which will be described in Chapter 6 . The smooth from the selection equation suggests that the likelihood of having an outpatient visit increases with age up to approximately the age of 65 . Then it decreases as the patient may require an inpatient treatment. The shape of the outcome equation smooth may indicate that the patients first seek standard low-cost health care methods by visiting the VA administration and later require more specialised health care and hence address non-VA inpatient facilities. Alternatively, one can speculate that the 40-year old patients overutilise the VA health care system. . . . . . . . . . . . . 80

4.7 Naïve probit (left) and naïve GAM (right) smooth terms (NUMVAOUT). The horizontal axis represents veterans' age and the vertical axis shows the smooth value on the linear predictor scale. The numbers in brackets on the vertical axis are the effective degrees of freedom of the smooth which will be described in Chapter 6. The shape of the selection equation smooth is similar to the one in Figure 4.6 and the same interpretation follows. However, the GAM smooth differs from the outcome equation smooth - the individuals steadily more use VA outpatient facilities until they reach the age of 60; after that there is a sharp decline in their usage. Not accounting for non-random sample selection may change the interpretation of the smooth terms. . . 81

4.8 Delaporte model diagnostics for the SOEP response. . . . . . . . . . . . . . . . . . 84 
4.9 Selection (left) and outcome (right) equation smooth terms (direct job changes). The horizontal axis represents full time experience and length of education. The vertical axis shows the smooth value on the linear predictor scale. The numbers in brackets on the vertical axis are the effective degrees of freedom of the smooth which will be described in Chapter 6. The selection equation smooth suggests that the likelihood of being active on the labor market as a dependent worker increases when gaining full time work experience. Then, it gradually decreases as individuals start to receive pensions or enter into self-employment. The outcome smooth suggests that the longer the education of an individual lasts the less likely he is to change his job voluntarily. This is because the individual is more specialised in a particular area as he completes subsequent stages of education. Therefore, it is harder for him to find an alternative job or there are no financial incentives to search another one. An extreme example of that could be medical doctors. . . . . . . . . . . . . . . . . 86

4.10 Naïve probit (left) and naïve GAM (right) smooth terms (direct job changes). The horizontal axis represents full time experience and length of education. The vertical axis shows the smooth value on the linear predictor scale. The numbers in brackets on the vertical axis are the effective degrees of freedom of the smooth which will be described in Chapter 6 . The shape of the selection equation smooth is the same as in Figure 4.9. Unlike for the sample selection model, the job mobility first increases with the years of education until the 14th year and then decreases. Again, not accounting for non-random sample selection may change the interpretation of the smooth terms.

5.1 Graphical representation of likelihood of observed entries. Assume that $y_{2}=2$. The light blue area is the domain of $F\left(0, y_{2}-1\right)$ whereas the light blue and the light green area jointly constitute $F\left(0, y_{2}\right)$. The red area is our domain of interest i.e. $f\left(1, y_{2}\right)$. By conducting the operation $f_{2}\left(y_{2}\right)-F\left(0, y_{2}\right)+F\left(0, y_{2}-1\right)$ one obtains $f\left(1, y_{2}\right) \ldots$ 92

5.2 Poisson density plots (red: $\mu=1$; blue: $\mu=2$; orange: $\mu=5$ ). . . . . . . . 95

5.3 Negative binomial density plots (red: $\mu=1, \sigma=1$; blue: $\mu=5, \sigma=2$; orange:

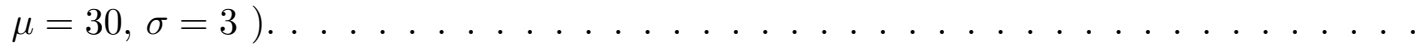


5.4 Delaporte density plots (red: $\mu=1, \sigma=1, \nu=0.1$; blue: $\mu=5, \sigma=2, \nu=0.3$;

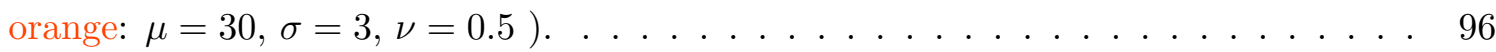

5.5 Poisson inverse Gaussian density plots (red: $\mu=1, \sigma=1$; blue: $\mu=5, \sigma=2$; orange: $\mu=30, \sigma=3) \ldots \ldots \ldots \ldots \ldots \ldots \ldots$

5.6 Sichel density plots (red: $\mu=1, \sigma=1, \nu=1$; blue: $\mu=5, \sigma=2, \nu=1$; orange:

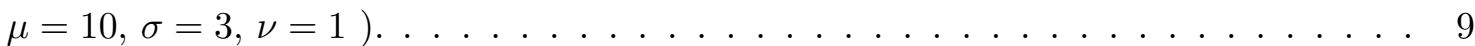

5.7 Comparison of the algorithm against line search methods based on a two parameter likelihood function (extracted from: Nocedal and Wright, 2006). The current point lies in upper end while the minimum point lies in the lower end of the valley of $f(\boldsymbol{x})$ contours. The quadratic model $m_{k}$, whose elliptical contours are shown by the dashed lines, is based on the information of $f(\boldsymbol{x})$ and its first and second derivatives. As shown by the longer arrow, a line search method based on this model searches along the step to the minimiser of $m_{k}$. This direction, however, allows only a small reduction in $f(\boldsymbol{x})$ even if an optimal step is taken. A trust-region method, as shown by the shorter arrow, shifts to the minimiser of $m_{k}$ within the dotted circle, which yields a more significant reduction in $f(\boldsymbol{x})$ and a better step. . . . . . . . . . .

5.8 Profile likelihood of Sichel Frank model with IV. Kendall's $\tau=0.5$ and $\theta=\theta^{*}=5.7$. The shape of the profile likelihood for the remaining parameters is the same for the case with and without instrumental variable. . . . . . . . . . . . . . 12

5.9 Profile likelihood of Sichel Frank model without IV. Kendall's $\tau=0.5$ and $\theta=\theta^{*}=$ 5.7. The shape of the profile likelihood for the remaining parameters is the same for the case with and without instrumental variable. The difference in shapes as compared to Figure 5.8 is likely to cause identification problems. . . . . . . . . . . . 122

5.10 Profile likelihood of Negative binomial Frank model with IV. Kendall's $\tau=0.5$ and $\theta=\theta^{*}=5.7$. The shape of the profile likelihood for the remaining parameters is the same for the case with and without instrumental variable. . . . . . . . . . . . 123

5.11 Profile likelihood of Negative binomial Frank model without IV. Kendall's $\tau=0.5$ and $\theta=\theta^{*}=5.7$. The shape of the profile likelihood for the remaining parameters is the same for the case with and without instrumental variable. The difference in shapes as compared to Figure 5.10 is likely to cause identification problems. . . . . . 123 
5.12 Contours of original Clayton and rotated copulae by 90, 180, 270 degrees with normal margins. Kendall's $\tau$ is equal to $0.5,-0.5,0.5$ and -0.5 respectively. The 90 and 270 degrees copulae show negative dependence. The 180 degrees (survival) copula still shows only positive dependence, but greater tail dependence now turns out to be in the upper right corner as compared to the unrotated copula. . . . . . . . . . . 125

6.1 Illustration of a B-spline. 7 equally spaced basis functions are depicted on the $[0,1]$ interval, where the knots are given by $[0,0.4,0.6,0.8,1]$ and $m=2$ (cubic B-spline). As one one can see, each B-spline basis function is non-zero between 5 adjacent knots (or on 4 intervals). The boundary knots are given by $[-0.6,1.6] \ldots \ldots \ldots$. . . . 131 131

6.2 Test functions, $f_{1}\left(x_{1}\right)=0.4\left[-4-\left(5.5 x_{1}-2.9\right)+3\left(4.5 x_{1}-2.3\right)^{2}-\left(4.5 x_{1}-2.3\right)^{3}\right]$ and $f_{2}\left(x_{1}\right)=x_{1} \sin \left(8 x_{1}\right)$, for the selection and outcome equation respectively. Both functions are neither monotonically increasing nor decreasing. . . . . . . . . . . 146

7.1 Selection (left) and outcome (right) smooth plots for Poisson inverse Gaussian Frank model for NUMVAOUT. The horizontal axis represents veterans' age and the vertical axis shows the smooth value on the linear predictor scale. The numbers in brackets on the vertical axis are the effective degrees of freedom of the smooth. For the selection smooth, the probability of having an outpatient VA visit increases up to the age of 65 ; then it decreases gradually. The shape of the outcome equation smooth is first decreasing, then increasing and then decreasing again. This suggests that the patients first seek standard low-cost health care methods by visiting the VA administration and later require more specialised health care and hence address non-VA inpatient facilities. Alternatively, one can speculate that the 45-year old patients overutilise the VA health care system. . . . . . . . . . . . . . . . . 167 
7.2 Selection (left) and outcome (right) smooth plots for Poisson inverse Gaussian Clayton 90 model for NUMVAOUT. The horizontal axis represents veterans' age and the vertical axis shows the smooth value on the linear predictor scale. The numbers in brackets on the vertical axis are the effective degrees of freedom of the smooth. For the selection smooth, the probability of having an outpatient VA visit increases up to the age of 65 ; then it decreases gradually. The shape of the outcome equation smooth is first decreasing, then increasing and then decreasing again. This suggests that the patients first seek standard low-cost health care methods by visiting the VA administration and later require more specialised health care and hence address nonVA inpatient facilities. Alternatively, one can speculate that the 45-year old patients overutilise the VA health care system. . . . . . . . . . . . . . . . 168

7.3 Selection (left) and outcome (right) smooth plots for Poisson inverse Gaussian Gumbel 270 model for NUMVAOUT. The horizontal axis represents veterans' age and the vertical axis shows the smooth value on the linear predictor scale. The numbers in brackets on the vertical axis are the effective degrees of freedom of the smooth. For the selection smooth, the probability of having an outpatient VA visit increases up to the age of 65 ; then it decreases gradually. The shape of the outcome equation smooth is first decreasing, then increasing and then decreasing again. This suggests that the patients first seek standard low-cost health care methods by visiting the VA administration and later require more specialised health care and hence address nonVA inpatient facilities. Alternatively, one can speculate that the 45-year old patients overutilize the VA health care system. . . . . . . . . . . . . . . . . . . 169 
7.4 Selection (left) and outcome (right) equation smooth for Delaporte outcome and Frank copula. The horizontal axis represents full time experience and length of education. The vertical axis shows the smooth value on the linear predictor scale. The numbers in brackets on the vertical axis are the effective degrees of freedom of the smooth. The plots do not differ substantially in shape as compared to Figure 4.9. The selection equation smooth suggests that the likelihood of being active on the labor market as a dependent worker increases when gaining full time work experience. Then, the likelihood of being active gradually decreases as individuals start to receive pensions or enter into self-employment. The outcome smooth suggests that the longer the education of an individual lasts the less likely he is to change his job. This is due to increasing specialisation in his professional area as he becomes more qualified. Therefore, it is harder for him to find an alternative occupation or there are no financial incentives for changing jobs. . . . . . . . . . . . . . . . . . . 174

7.5 Selection (left) and outcome (right) equation smooth for Delaporte outcome and Joe 90 copula. The horizontal axis represents full time experience and length of education. The vertical axis shows the smooth value on the linear predictor scale. The numbers in brackets on the vertical axis are the effective degrees of freedom of the smooth. The plots do not differ substantially in shape as compared to Figure 4.9. The selection equation smooth suggests that the likelihood of being active on the labor market as a dependent worker increases when gaining full time work experience. Then, the likelihood of being active gradually decreases as individuals start to receive pensions or enter into self-employment. The outcome smooth suggests that the longer the education of an individual lasts the less likely he is to change his job. This is due to increasing specialisation in his professional area as he becomes more qualified. Therefore, it is harder for him to find an alternative occupation or there are no financial incentives for changing jobs. . . . . . . . . . . . . . . . . 175 
7.6 Selection (left) and outcome (right) equation smooth for Delaporte outcome and Clayton 90 copula. The horizontal axis represents full time experience and length of education. The vertical axis shows the smooth value on the linear predictor scale. The numbers in brackets on the vertical axis are the effective degrees of freedom of the smooth. The plots do not differ substantially in shape as compared to Figure 4.9. The selection equation smooth suggests that the likelihood of being active on the labor market as a dependent worker increases when gaining full time work experience. Then, the likelihood of being active gradually decreases as individuals start to receive pensions or enter into self-employment. The outcome smooth suggests that the longer the education of an individual lasts the less likely he is to change his job. This is due to increasing specialisation in his professional area as he becomes more qualified. Therefore, it is harder for him to find an alternative occupation or there are no financial incentives for changing jobs. . . . . . . . . . . 176

D.1 Poisson model diagnostics for the NUMVAOUT response. . . . . . . . . . . . . 189

D.2 Negative binomial model diagnostics for the NUMVAOUT response. . . . . . . . . 189

D.3 Delaporte model diagnostics for the NUMVAOUT response. . . . . . . . . . . . . 190

D.4 Sichel model diagnostics for the NUMVAOUT response. . . . . . . . . . . . . . 190

D.5 Poisson model diagnostics for the NUMOTH response. . . . . . . . . . . . . . . . 191

D.6 Negative binomial model diagnostics for the NUMOTH response. . . . . . . . . . . . 191

D.7 Delaporte model diagnostics for the NUMOTH response. . . . . . . . . . . . . . 192

D.8 Poisson Inverse Gaussian model diagnostics for the NUMOTH response. . . . . . . . . . 192

D.9 Poisson model diagnostics for the VANITES response. . . . . . . . . . . . . 193

D.10 Negative binomial model diagnostics for the VANITES response. . . . . . . . . . 193

D.11 Delaporte model diagnostics for the VANITES response. . . . . . . . . . . . . . . 194

D.12 Poisson Inverse Gaussian model diagnostics for the VANITES response. . . . . . . . 194

D.13 Poisson model diagnostics for the OTHNITES response. . . . . . . . . . . . . . 195

D.14 Negative binomial model diagnostics for the OTHNITES response. . . . . . . . . 195

D.15 Delaporte model diagnostics for the OTHNITES response. . . . . . . . . . . . . 196

D.16 Sichel model diagnostics for the OTHNITES response. . . . . . . . . . . . . 196 
D.17 Poisson model diagnostics for the SOEP response. . . . . . . . . . . . . . 197

D.18 Negative binomial model diagnostics for the SOEP response. . . . . . . . . . . . 197

D.19 Poisson Inverse Gaussian model diagnostics for the SOEP response. . . . . . . . . . 198

D.20 Sichel model diagnostics for the SOEP response. . . . . . . . . . . . . . . . 198

G.1 Parameter estimate boxplots for the same set of covariates in both equations and Poisson margin. . . . . . . . . . . . . . . . . . . . . . 208

G.2 Parameter estimate boxplots for the same set of covariates in both equations and negative binomial margin. . . . . . . . . . . . . . . . . . . 209

G.3 Parameter estimate boxplots for the same set of covariates in both equations and Delaporte margin. . . . . . . . . . . . . . . . . . . . . 210

G.4 Parameter estimate boxplots for the same set of covariates in both equations and Poisson inverse Gaussian margin. . . . . . . . . . . . . . . . . . . . . . 211

G.5 Parameter estimate boxplots for the same set of covariates in both equations and Sichel margin. . . . . . . . . . . . . . . . . . . . . . . . . 212

G.6 Parameter estimate boxplots for the case with instrumental variable and Poisson

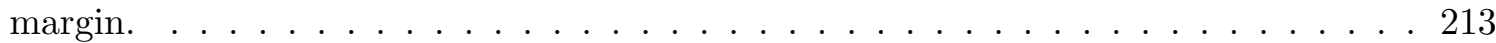

G.7 Parameter estimate boxplots for the case with instrumental variable and negative binomial margin. . . . . . . . . . . . . . . . . . . . . . . 214

G.8 Parameter estimate boxplots for the case with instrumental variable and Delaporte

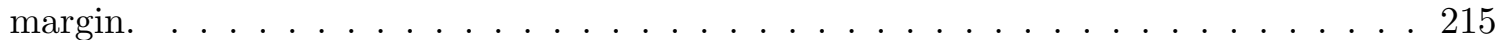

G.9 Parameter estimate boxplots for the case with instrumental variable and Poisson inverse Gaussian margin. . . . . . . . . . . . . . . . . . . . . 216

G.10 Parameter estimate boxplots for the case with instrumental variable and Sichel margin.217

G.11 Parameter estimate boxplots for the same set of covariates in both equations and Poisson margin (analytical gradient) . . . . . . . . . . . . . . . . 218

G.12 Parameter estimate boxplots for the same set of covariates in both equations and negative binomial margin (analytical gradient) . . . . . . . . . . . . . . . 219

G.13 Parameter estimate boxplots for the same set of covariates in both equations and Delaporte margin (analytical gradient). 
G.14 Parameter estimate boxplots for the same set of covariates in both equations and Poisson inverse Gaussian margin (analytical gradient) . . . . . . . . . . . . . . 221

G.15 Parameter estimate boxplots for the same set of covariates in both equations and Sichel margin (analytical gradient) . . . . . . . . . . . . . . . . . . 222

G.16 Parameter estimate boxplots for the case with instrumental variable and Poisson margin (analytical gradient). . . . . . . . . . . . . . . . . 223

G.17 Parameter estimate boxplots for the case with instrumental variable and negative binomial margin (analytical gradient). . . . . . . . . . . . . . . . . . . 224

G.18 Parameter estimate boxplots for the case with instrumental variable and Delaporte margin (analytical gradient). . . . . . . . . . . . . . . . . 225

G.19 Parameter estimate boxplots for the case with instrumental variable and Poisson inverse Gaussian margin (analytical gradient) . . . . . . . . . . . . . . . . 226

G.20 Parameter estimate boxplots for the case with instrumental variable and Sichel margin (analytical gradient) . . . . . . . . . . . . . . . . . . 227

J.1 Parameter estimate boxplots for the same set of covariates in both equations and Poisson margin (analytical Hessian) . . . . . . . . . . . . . . . . . 245

J.2 Parameter estimate boxplots for the same set of covariates in both equations and negative binomial margin (analytical Hessian) . . . . . . . . . . . . . . 246

J.3 Parameter estimate boxplots for the same set of covariates in both equations and Delaporte margin (analytical Hessian) . . . . . . . . . . . . . . . 247

J.4 Parameter estimate boxplots for the same set of covariates in both equations and Poisson inverse Gaussian margin (analytical Hessian). . . . . . . . . . . . . . . . 248

J.5 Parameter estimate boxplots for the same set of covariates in both equations and Sichel margin (analytical Hessian) . . . . . . . . . . . . . . . . . . 249

J.6 Parameter estimate boxplots for the case with instrumental variable and Poisson margin (analytical Hessian) . . . . . . . . . . . . . . . . . 250

J.7 Parameter estimate boxplots for the case with instrumental variable and negative binomial margin (analytical Hessian) . . . . . . . . . . . . . . . . 251 
J.8 Parameter estimate boxplots for the case with instrumental variable and Delaporte margin (analytical Hessian) . . . . . . . . . . . . . . . . . . 252

J.9 Parameter estimate boxplots for the case with instrumental variable and Poisson inverse Gaussian margin (analytical Hessian) . . . . . . . . . . . . . . . . . 253

J.10 Parameter estimate boxplots for the case with instrumental variable and Sichel margin (analytical Hessian).

L.1 Parameter estimate boxplots for the same set of covariates in both equations and Poisson margin. . . . . . . . . . . . . . . . . . . . . . 258

L.2 Parameter estimate boxplots for the same set of covariates in both equations and negative binomial margin. . . . . . . . . . . . . . . . . . . 259

L.3 Parameter estimate boxplots for the same set of covariates in both equations and Delaporte margin. . . . . . . . . . . . . . . . . . . . 260

L.4 Parameter estimate boxplots for the same set of covariates in both equations and Poisson inverse Gaussian margin. . . . . . . . . . . . . . . . . . . . . . 261

L.5 Parameter estimate boxplots for the same set of covariates in both equations and Sichel margin. . . . . . . . . . . . . . . . . . . . . . . . 262

L.6 Parameter estimate boxplots for the case with instrumental variable and Poisson

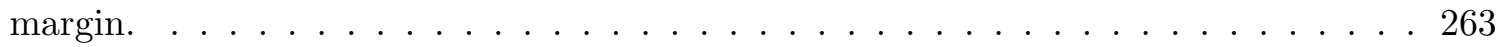

L.7 Parameter estimate boxplots for the case with instrumental variable and negative binomial margin. . . . . . . . . . . . . . . . . . . . . . 264

L.8 Parameter estimate boxplots for the case with instrumental variable and Delaporte

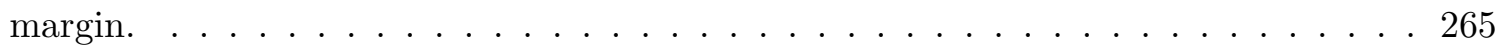

L.9 Parameter estimate boxplots for the case with instrumental variable and Poisson inverse Gaussian margin. . . . . . . . . . . . . . . . . . . 266

L.10 Parameter estimate boxplots for the case with instrumental variable and Sichel margin.267

N.1 Spline simulations for Poisson models with FGM, normal, AMH, Clayton and Frank copulas (same covariates) . . . . . . . . . . . . . . . . . 272

N.2 Spline simulations for Poisson models with Joe and Gumbel copulas (same covariates).273 
N.3 Spline simulations for negative binomial models with FGM, normal, AMH, Clayton and Frank copulas (same covariates) . . . . . . . . . . . . . . . . 274

N.4 Spline simulations for negative binomial models with Joe and Gumbel copulas (same

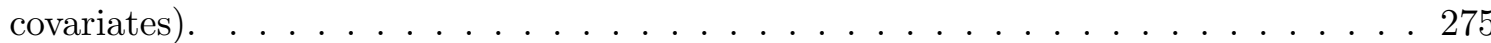

N.5 Spline simulations for Delaporte models with FGM, normal, AMH, Clayton and Frank copulas (same covariates) . . . . . . . . . . . . . . . 276

N.6 Spline simulations for Delaporte models with Joe and Gumbel copulas (same covari-

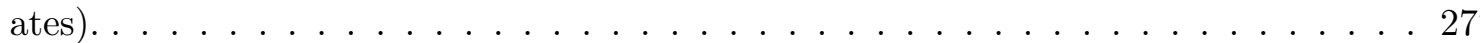

N.7 Spline simulations for Poisson inverse Gaussian models with FGM, normal, AMH, Clayton and Frank copulas (same covariates) . . . . . . . . . . . . . . . . 278

N.8 Spline simulations for Poisson inverse Gaussian models with Joe and Gumbel copulas (same covariates). . . . . . . . . . . . . . . . . . . 279

N.9 Spline simulations for Sichel models with FGM, normal, AMH, Clayton and Frank copulas (same covariates) . . . . . . . . . . . . . . . . . . 280

N.10 Spline simulations for Sichel models with Joe and Gumbel copulas (same covariates). 281

O.1 Spline simulations for Poisson models with Clayton 90, Clayton 180, Clayton 270, Joe 90 and Joe 180 copulas (same covariates) . . . . . . . . . . . . . . . 283

O.2 Spline simulations for Poisson models with Joe 270, Gumbel 90, Gumbel 180 and Gumbel 270 copulas (same covariates) . . . . . . . . . . . . . . . . . . 284

O.3 Spline simulations for negative binomial models with Clayton 90, Clayton 180, Clayton 270 , Joe 90 and Joe 180 copulas (same covariates) . . . . . . . . . . . . 285

O.4 Spline simulations for negative binomial models with Joe 270, Gumbel 90, Gumbel 180 and Gumbel 270 copulas (same covariates). . . . . . . . . . . . . . . 286

O.5 Spline simulations for Delaporte models Clayton 90, Clayton 180, Clayton 270, Joe 90 and Joe 180 copulas (same covariates) . . . . . . . . . . . . . . . . 287

O.6 Spline simulations for Delaporte models with Joe 270, Gumbel 90, Gumbel 180 and Gumbel 270 copulas (same covariates) . . . . . . . . . . . . . . . . . 288

O.7 Spline simulations for Poisson inverse Gaussian models Clayton 90, Clayton 180, Clayton 270, Joe 90 and Joe 180 copulas (same covariates). . . . . . . . . . . . . 289 
O.8 Spline simulations for Poisson inverse Gaussian models with Joe 270, Gumbel 90, Gumbel 180 and Gumbel 270 copulas (same covariates). . . . . . . . . . . . . . . 290

O.9 Spline simulations for Sichel models Clayton 90, Clayton 180, Clayton 270, Joe 90 and Joe 180 copulas (same covariates) . . . . . . . . . . . . . . . . . . 291

O.10 Spline simulations for Sichel models with Joe 270, Gumbel 90, Gumbel 180 and Gumbel 270 copulas (same covariates) . . . . . . . . . . . . . . . . . 292

P.1 Spline simulations for Poisson models with FGM, normal, AMH, Clayton and Frank copulas $(\mathrm{IV}) \ldots \ldots \ldots \ldots \ldots \ldots \ldots \ldots \ldots \ldots \ldots$

P.2 Spline simulations for Poisson models with Joe and Gumbel copulas (IV). . . . . . 295

P.3 Spline simulations for negative binomial models with FGM, normal, AMH, Clayton and Frank copulas $(\mathrm{IV}) \ldots \ldots \ldots \ldots \ldots \ldots \ldots$

P.4 Spline simulations for negative binomial models with Joe and Gumbel copulas (IV). 297

P.5 Spline simulations for Delaporte models with FGM, normal, AMH, Clayton and

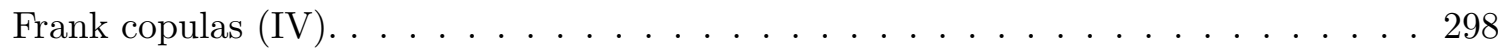

P.6 Spline simulations for Delaporte models with Joe and Gumbel copulas (IV). . . . . . 299

P.7 Spline simulations for Poisson inverse Gaussian models with FGM, normal, AMH, Clayton and Frank copulas (IV) . . . . . . . . . . . . . . . . . . 300

P.8 Spline simulations for Poisson inverse Gaussian models with Joe and Gumbel copulas (IV)

P.9 Spline simulations for Sichel models with FGM, normal, AMH, Clayton and Frank copulas (IV)

P.10 Spline simulations for Sichel models with Joe and Gumbel copulas (IV). . . . . . . . 303

Q.1 Spline simulations for Poisson models with Clayton 90, Clayton 180, Clayton 270, Joe 90 and Joe 180 copulas (IV)

Q.2 Spline simulations for Poisson models with Joe 270, Gumbel 90, Gumbel 180 and

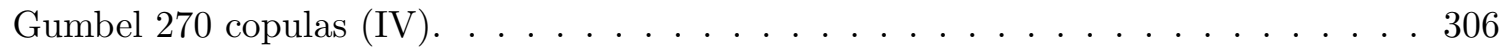

Q.3 Spline simulations for negative binomial models with Clayton 90, Clayton 180, Clayton 270 , Joe 90 and Joe 180 copulas (IV). 
Q.4 Spline simulations for negative binomial models with Joe 270, Gumbel 90, Gumbel 180 and Gumbel 270 copulas (IV) . . . . . . . . . . . . . . . 308

Q.5 Spline simulations for Delaporte models Clayton 90, Clayton 180, Clayton 270, Joe

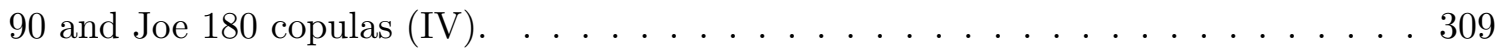

Q.6 Spline simulations for Delaporte models with Joe 270, Gumbel 90, Gumbel 180 and Gumbel 270 copulas $(\mathrm{IV}) \ldots \ldots \ldots$. . . . . . . . . . . . . . 310

Q.7 Spline simulations for Poisson inverse Gaussian models Clayton 90, Clayton 180, Clayton 270, Joe 90 and Joe 180 copulas (IV). . . . . . . . . . . . . . . 311

Q.8 Spline simulations for Poisson inverse Gaussian models with Joe 270, Gumbel 90, Gumbel 180 and Gumbel 270 copulas (IV). . . . . . . . . . . . . . . . . 312

Q.9 Spline simulations for Sichel models Clayton 90, Clayton 180, Clayton 270, Joe 90

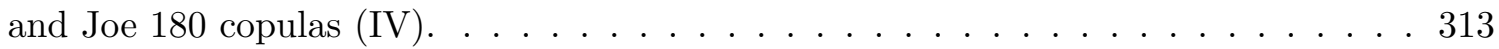

Q.10 Spline simulations for Sichel models with Joe 270, Gumbel 90, Gumbel 180 and Gumbel 270 copulas $(\mathrm{IV}) \ldots \ldots \ldots$. . . . . . . . . . . . . . 314

R.1 Selection (left) and outcome (right) smooth plots for Sichel Frank model for NUMOTH. 316

R.2 Selection (left) and outcome (right) smooth plots for Sichel Clayton 90 model for NUMOTH.

R.3 Selection (left) and outcome (right) smooth plots for Sichel Gumbel 270 model for NUMOTH.

R.4 Selection (left) and outcome (right) smooth plots for Sichel Frank model for VANITES. 317

R.5 Selection (left) and outcome (right) smooth plots for Sichel Clayton 90 model for

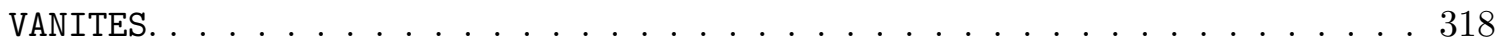

R.6 Selection (left) and outcome (right) smooth plots for Sichel Gumbel 270 model for

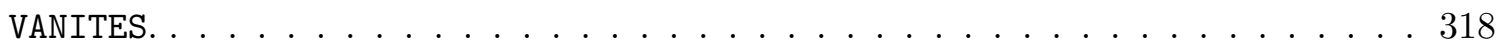

R.7 Selection (left) and outcome (right) smooth plots for Poisson inverse Gaussian Frank model for OTHNITES. . . . . . . . . . . . . . . . . . . . . . 319

R.8 Selection (left) and outcome (right) smooth plots for Poisson inverse Gaussian Clayton 90 model for OTHNITES. . . . . . . . . . . . . . . . . . . . . 319 
R.9 Selection (left) and outcome (right) smooth plots for Poisson inverse Gaussian Gumbel 270 model for OTHNITES. . . . . . . . . . . . . . . . . . . . . . 320 


\section{List of Tables}

3.1 Examples of ranks for Spearman's $\rho \ldots \ldots \ldots \ldots \ldots$

3.2 Examples of families of (bivariate) Archimedean copulas. . . . . . . . . . . . . . 57

3.3 Expressions for the Archimedean copula factor in the likelihood function (Smith, 2003) 62

4.1 Responses in the VA data. . . . . . . . . . . . . . . . . . . . 70

4.2 Descriptive statistics of VA data responses. . . . . . . . . . . . . . . 70

4.3 P-values for the chi-squared tests applied to the count responses described in Table

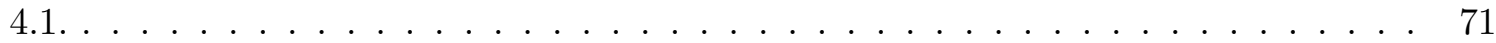

4.4 VA covariate description. The two continuous variables are VETSAGE and DEBTS. All other variables are factors. Covariates indicating a medical condition are binary. . . 74

4.5 Kendall's $\tau$ for the four VA data responses. For three out of four responses nonrandom sample selection is likely to be present. Kendall's $\tau$ for VANITES is small and negative, but non-random sample selection is unlikely to be present. . . . . . . . . 79

4.6 Variables used for gamlss and sample selection model for SOEP data . . . . . . . . 83

5.1 Discrete distribution summary. The distributions listed above are parametrised in terms of $\mu, \sigma$ and $\nu$. These are parameters representing location, scale and shape respectively. For all distributions, $E(Y)=\mu=\exp (\eta)$, where $\eta$ is the linear predictor. For Poisson inverse Gaussian and Sichel distributions, $K_{\lambda}(t)=$ $\frac{1}{2} \int_{0}^{\infty} x^{\lambda-1} \exp \left(-\frac{1}{2} t\left(x+x^{-1}\right)\right) d x$ is the modified Bessel function of the third kind. Note that $c=K_{\nu+1}\left(\frac{1}{\sigma}\right)\left[K_{\nu}\left(\frac{1}{\sigma}\right)\right]^{-1} \ldots \ldots \ldots \ldots \ldots \ldots \ldots \ldots$ 
$5.2 \theta^{*}$ defined in terms of $\theta$. The values of $\theta^{*}$ are corrected with $\epsilon$ for Clayton, Frank and Joe to prevent the optimisation algorithm from reaching boundaries of $\theta$ which are not included in the parameter space. $\epsilon$ is set to $10^{-8} \ldots \ldots \ldots$. . . . . . . 101

5.3 Copula-specific steps for obtaining starting values for $\theta^{*}$. The "inverse" $\rho$ and $\tau$ mean that the quantity is inserted into a function which treats it as if it was Spearman's $\rho$ or Kendall's $\tau$. The value returned by the inverse function is the initial value of $\theta$. This value is then transformed into a guesstimate of $\theta^{*}$ corresponding to the copula. 102

5.4 Parameter summary for selection and outcome equations. . . . . . . . . . . . . . 104

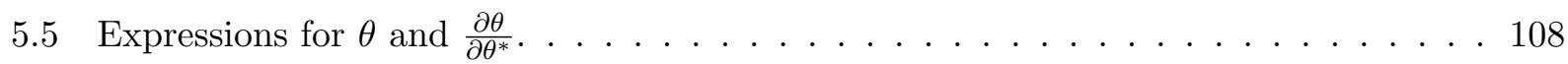

5.6 Numerical versus analytical gradient check: percentage of times gradient norms were close enough. In this case, "closeness" meant that either the percentage difference did not exceed 1 per cent or the analytical gradient components did not differ by more than one nominal unit. For instance, if the the analytical gradient component was 14.5 and the numerical gradient component was 14.4, this would suggest that the analytical gradient was derived correctly. . . . . . . . . . . . 110

5.7 Numerical versus analytical Hessian check: percentage of times all Hessian elements were close enough. . . . . . . . . . . . . . . . . . . . . . 119

7.1 Responses in the VA data. . . . . . . . . . . . . . . . . . . . . 161

7.2 Average predictions and association parameters for Frank, Clayton 90 and Gumbel 270 models. The values in brackets indicate $95 \%$ confidence intervals. For outpatient responses, the predicted values do not vary substantially. However, the confidence intervals for Frank and Gumbel 270 in NUMOTH do not overlap. For inpatient responses, there are considerable differences in average predicted values between Frank and the remaining models. Note that the average predictions of the univariate model for NUMOTH and OTHNITES are smaller than the ones of the sample selection models. As we can see, the choice of copula can have an impact on the predicted values. . . . . . 164 
7.3 Akaike and Bayesian information criteria for Frank, Clayton 90 and Gumbel 270 models. For example, the lowest AIC and BIC values for NUMVAOUT can be found for the Poisson inverse Gaussian Clayton 90 model. Hence, Clayton 90 would be preferred over the Frank and Gumbel 270 models. For none of the responses Frank is the favoured model. . . . . . . . . . . . . . . . . . . 165

7.4 Variables used for sample selection model for SOEP data . . . . . . . . . . . . . 171

7.5 Akaike and Bayesian information criteria for the Delaporte models for SOEP data. The lowest AIC and BIC scores can be obtained with Delaporte Frank. Hence, if the modeller is interested in making predictions of the number of job changes, he may consider Delaporte Frank. . . . . . . . . . . . . . . . . . . . . . . . . 172

7.6 Average predictions of job changes and estimates of association parameters for Delaporte model and Frank, Joe 90 and Clayton 270. The values in brackets indicate 95\% confidence interval bounds. For all three models, the confidence intervals of $\theta$ do not reach their bound, which implies that non-random sample selection is likely to be present. The average predictions do not differ substantially from another. In fact, their confidence intervals of all three models overlap. Thus, the copula assumption does not seem to have a major impact on the predictions. Note that the confidence interval of the univariate model ignoring non-random sample selection does not overlap in predictions with the sample selection models. This suggests that non-random sample selection needs to be accounted for. . . . . . . . . . . . . . . . . . 173

8.1 Additional discrete distributions implemented in SemiParSampleSel R package. The brackets underneath the link function indicate the parameter range. For binomialtype distributions, the binomial denominator is determined by the modeller. Note that the support of the logarithmic distribution does not include zero. . . . . . . . 179 


\section{Chapter 1}

\section{Introduction}

Non-random sample selection arises in observational studies when observations do not come from a random sample and were selected or selected themselves into (or out of) the sample based on a combination of unobservable and observable variables. In this situation the coefficient estimators for models like linear regression or generalized linear models will not be consistent. To rectify this situation sample selection models can be used. Since the work by Heckman (1979), many variants of sample selection models have been developed for continuous and binary responses. However, not much attention was attributed to count data. In this thesis, a copula-based sample selection modelling approach for count responses is proposed. The model will be enhanced with splines commonly known in Generalized Additive Models. This will allow for a flexible specification of continuous covariate effects when the true specification is unknown.

\subsection{Thesis outline}

The thesis aims at establishing a new methodology for count sample selection modelling. The steps to achieve this are described below.

Chapter two will introduce the problem of non-random sample selection or missingness not at random on the response. In chapter three, variants of sample selection models will be presented. This will include the classic Heckman model (Heckman, 1979), Greene count model (Greene, 1997), the copula approach (Smith, 2003) as well some other sample selection models.

In chapter four, two motivating case studies will be presented and explored. In both cases, the 
count response suffers from non-random sample selection. The former originates from the United States Veterans Administration and the latter from the German Socio-economic Panel (SOEP v28, 2012).

Chapter five will describe the proposed copula sample selection model. The rationale behind the likelihood will be explained mathematically and graphically. Examples of outcome distributions will be presented. The computational aspects including starting values and optimisation algorithm will be discussed. In chapter six, the proposed model will be augmented by flexible covariate effects. Some examples of splines will be described. The model will be respecified, the unbiased risk estimator (UBRE) derived, inferential methods and asymptotic arguments presented.

In chapter seven, the proposed model will be illustrated using the SemiParSampleSel R package. For this purpose, we will use the motivating data sets presented in Chapter four. Chapter eight will summarise all findings of the thesis.

The outputs of this thesis are:

1. Marra, G., Radice, R., Wojtyś, M., Wyszynski, K., 2015. SemiParSampleSel: semiparametric sample selection modelling. $\mathrm{R}$ package version 1.3 .

2. Marra, G., Wyszynski, K. (submitted). Semi-parametric copula sample selection models for count responses

3. Marra, G., Wyszynski, K. (submitted). Sample selection model for count data: a tutorial in $R$ using the package SemiParSampleSel 


\section{Chapter 2}

\section{Non-random sample selection}

Non-random sample selection arises in observational studies when observations do not come from a random sample and were selected or selected themselves into (or out of) the sample based on a combination of unobservable and observable variables (Gronau, 1974; Lewis, 1974; Heckman, 1979). In this situation the coefficient estimators for models such as linear regression or generalized linear models will not be consistent.

Let us consider an example where non-random sample selection can have an impact on public policy recommendations. Consider Figure 2.1. Suppose, the aim is to estimate HIV prevalence of an African country. Individuals are asked whether they agree to participate in a blood test. If they do, the blood test is conducted and their HIV status will be either positive or negative. If they do not, their HIV status will be unknown. Also, suppose there are observed and unobserved variables influencing the survey participation and HIV status such as age and fear of facing the truth. Thus, those who are more fearless or older are likely to respond and their HIV status is more likely to be negative. On the other hand, individuals who are younger and fearful are likely not to participate. In this case, the sample of participants and non-participants will differ in HIV prevalence rates and non-random sample selection will be present. 


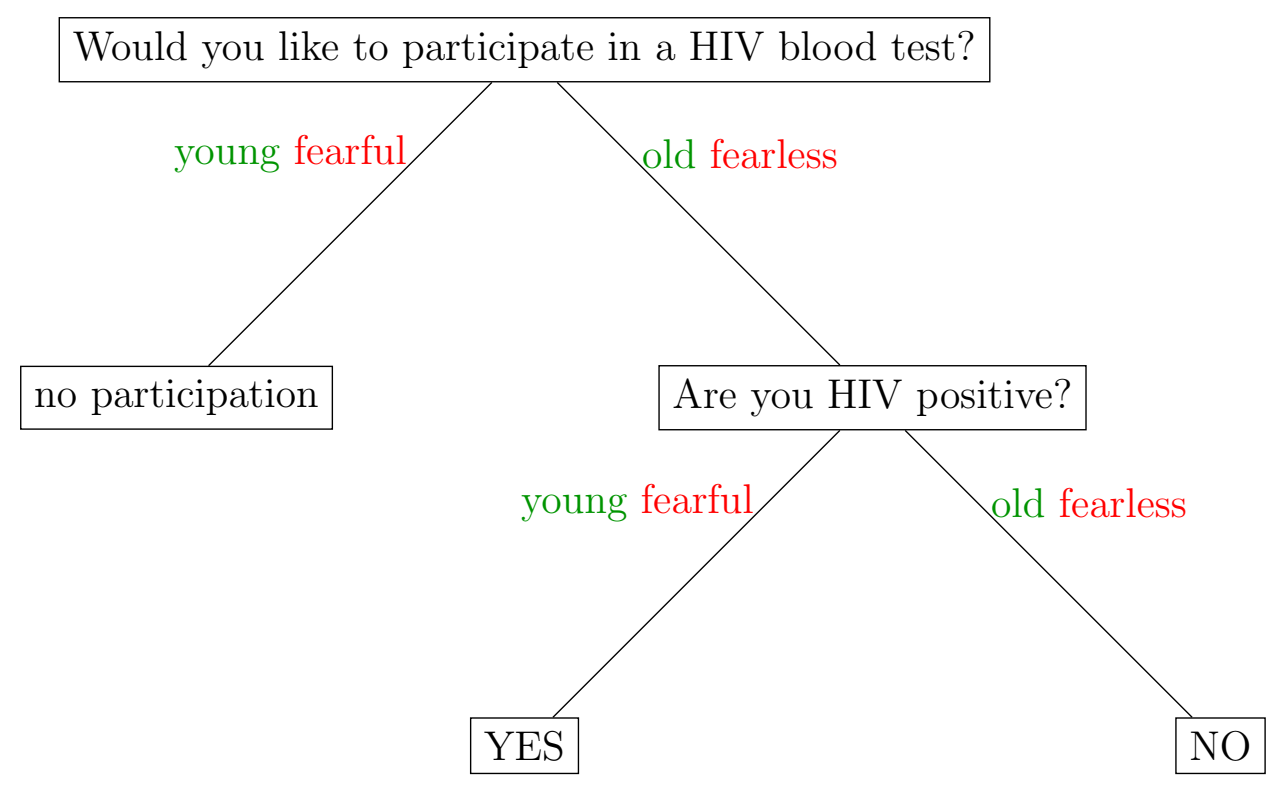

Figure 2.1: Selection and outcome process in HIV survey

Failing to correct for non-random sample selection may lead policymakers to draw erroneous conclusions, which may have a detrimental effect on targeted groups in the society, for instance. Bärnighausen et al. (2011) and McGovern et al. (2015) correct for non-random sample selection of HIV prevalence estimated from data of the 2007 Zambia Demographic and Health Survey. Here, the aim was to estimate a model which predicts the probability of being HIV positive. The results for men showed that sample selection was existent. The HIV prevalence is expected to be higher among men who did not consent to HIV testing than among those who consented. The results indicate that the correction for non-random sample selection changes the identification of groups at high risk of HIV infection and therefore the need of treatment and prevention. For example, the prevalence for men increases from 12 to 21 per cent when comparing the results of a classic imputation model and sample selection model. Given this, country's authorities may consider introducing stricter prevention measures such as more detailed blood checks for blood donors or improving early-treatment access for infected individuals (World Health Organisation, 2009).

In the remainder of this chapter we will first describe some imputation methods for response. We will then provide a general overview of existing sample selection models and their applications. 


\subsection{Brief overview of some imputation methods}

As we have seen in the HIV case study, non-random sample selection can be viewed as a problem of missing data on the response. This section aims at briefly describing types of missing data as defined by Rubin (1976). Then, we will introduce some imputation techniques for responses and explain why they fail to correct for non-random sample selection. Hence, we will outline pattern mixture and sample selection models as a means of correcting for non-random sample selection.

Let us first define the notation that will be used for the rest of this section. Consider a set of three variables $\boldsymbol{X}_{\mathbf{1}}, \boldsymbol{X}_{\mathbf{2}}$ and $\boldsymbol{X}_{\mathbf{3}}$. Also, suppose that $\boldsymbol{X}_{\mathbf{1}}$ and $\boldsymbol{X}_{\mathbf{2}}$ are fully observed and that $\boldsymbol{X}_{\mathbf{3}}$ has missing entries. If those entries were Missing at Random (MAR) the probability of missingness of $\boldsymbol{X}_{\mathbf{3}}$ would depend on $\boldsymbol{X}_{\mathbf{1}}$ and $\boldsymbol{X}_{\mathbf{2}}$ but not on the value of $\boldsymbol{X}_{\mathbf{3}}$ (Rubin, 1976). Suppose, that $\boldsymbol{R}$ is an indicator variable for a missing observation in $\boldsymbol{X}_{\mathbf{3}}$. Formally, the probability of MAR can be written as (e.g. Kenward and Carpenter, 2007)

$$
P\left(\boldsymbol{R} \mid \boldsymbol{X}_{\mathbf{1}}, \boldsymbol{X}_{\mathbf{2}}, \boldsymbol{X}_{\mathbf{3}}\right)=P\left(\boldsymbol{R} \mid \boldsymbol{X}_{\mathbf{1}}, \boldsymbol{X}_{\mathbf{2}}\right) .
$$

Note that $\boldsymbol{X}_{\mathbf{1}}$ and $\boldsymbol{X}_{\mathbf{2}}$ may be correlated with $\boldsymbol{X}_{\mathbf{3}}$ but $\boldsymbol{X}_{\mathbf{3}}$ will not be correlated with $\boldsymbol{R}$. For example, suppose we have missingness on the income variable, because some individuals have not reported it. The probability of reporting may depend on whether an individual has depression or not. Also, depression status may be correlated with income as those who have depression are likely to have low income (Howell, 2012). Still, in case of MAR income will not be associated with the probability of reporting income.

If the data were Missing Completely at Random (MCAR), then $\boldsymbol{X}_{\mathbf{3}}$ would not depend on other covariates and would be a random sample of all data values (Yuan, 2010). Thus,

$$
P\left(\boldsymbol{R} \mid \boldsymbol{X}_{\mathbf{1}}, \boldsymbol{X}_{\mathbf{2}}, \boldsymbol{X}_{\mathbf{3}}\right)=P(\boldsymbol{R}) .
$$

If the data are neither MAR nor MCAR they are said to be Missing not at Random (MNAR; e.g. Rubin, 1976; Kenward and Carpenter, 2007). Note that this is the case of non-random sample selection as the probability of missingness may depend on observed and/or unobserved variables.

Before we consider imputation techniques on the response it is essential to discuss the multiple 
imputation by Rubin (1987). It is of paramount importance to note that the aim of multiple imputation is to obtain valid inferences rather than to "fill in" the data. In the first stage, multiple imputation (MI) consists of creating a given number of data sets with imputed values. This is done to represent the uncertainty about the right value to impute. Second, each data set is analysed using a standard procedure such as linear regression. Finally, the results are combined together and "averaged". The literature on imputation algorithms is wide and we will not discuss it in this section; some examples are given in Yuan (2010).

When it comes to missing responses we can distinguish between three types of imputation methods (Young and Johnson, 2010):

1. excluding the dependent variable from the imputation model;

2. including the dependent variable in the imputation model and retaining it for the following analysis;

3. multiple imputation and then deletion (MID).

The algorithm for the first method can be summarised as follows:

1. Exclude the dependent variable from the data set.

2. Create multiple copies of incomplete data sets.

3. Using the desired algorithm, in each copy replace the missing values with plausible random values. The values are drawn conditionally upon the observed values of other variables.

4. Exclude observations where the response was missing.

5. Analyse imputed data sets separately using standard methods such as linear regression.

6. Combine the results of the analyses using formulas accounting for variation between data sets.

It can be shown that if the data are MCAR, imputation of the response cannot improve upon the complete case analysis which is performed in steps 5 and 6 of the method above. This is because our missing cases are a simple random sample from the sample of missing and complete cases. Since simple random sampling does not induce any bias, imputation would not make any bias corrections. However, in practice complete case analysis may result in a substantial reduction of sample size 
and data loss. Most importantly, if the MCAR assumption is not valid and MNAR holds, then considering only complete cases may lead to bias.

The second method differs from the first through the fact that the dependant variable is not excluded and the observations which are initially missing are included in the imputation model. Unlike in the first method, the imputation model will take into account the potential MAR and correlations between all variables. Nevertheless, again, this imputation technique cannot account for non-random sample selection.

The third method, MID, proposed by Von Hippel (2007) differs from the first in that the dependent variable is not excluded. The advantage of MID in comparison to the second technique is efficiency. Von Hippel (2007) showed analytically and numerically that MID yields less variable point estimates and smaller confidence intervals. In terms of hypothesis testing, this implies greater power for the same significance levels. All of this applies especially if there are a lot of missing values or few imputed data sets. Unfortunately, there are problems with this approach. For instance, nonlinear dependencies between the response and the explanatory variables may be removed from the imputation model. Also, depending on the software, discrete, non-negative continuous and factor variables may be modelled as if they were normally distributed. Finally, unobserved confounders are ignored in the MID approach.

Von Hippel (2007) provides examples of MID. One of them concerns sexual harassment at work. The cases are firms and the response can take four possible values. Regardless of practical problems that the researchers had with imputing data it seems that sample selection bias was not addressed even though such data are likely to suffer from it. Ilies et al. (2003) claim that sample selection bias may arise as complete-case samples originate from women who filed grievances or sought help. Thus, it may be argued they selected themselves into the sample on the basis of courage.

Even though the imputation techniques described above can address the issue of MAR and MCAR, they are not able to cope with the problem of non-random sample selection. It may be pointed out that they have not indeed been constructed for that purpose. We will now briefly present the idea behind pattern mixture models which account for MNAR.

Recall $\boldsymbol{R}$, which indicates missing observations. Let $\boldsymbol{Y}=\left(Y_{1}, Y_{2}, \ldots, Y_{n}\right)$ be the response. The 
likelihood function will then be the product of joint densities,

$$
L=\prod_{i=1}^{n} f\left(Y_{i}, R_{i}\right)
$$

As we will see in the next chapter, sample selection models factorise the likelihood components as

$$
f\left(Y_{i}, R_{i}\right)=f\left(Y_{i}\right) f\left(R_{i} \mid Y_{i}\right)
$$

The first factor characterises the distribution of $Y_{i}$ in the population and the second characterises the incidence of missing data as a function of $Y_{i}$ (Little and Rubin, 2002). On the other hand, a pattern mixture model factorises the components as

$$
f\left(Y_{i}, R_{i}\right)=f\left(R_{i}\right) f\left(Y_{i} \mid R_{i}\right)
$$

The first distribution models the incidence of missing data and the second distribution depicts $Y_{i}$ in the strata defined by $R_{i}$. Hence, the term "pattern-mixture" implies that the distribution $f\left(Y_{i}\right)$ is a mixture of distributions $f\left(Y_{i} \mid R_{i}\right)$ (Little, 1993).

Many applications of pattern-mixture models can be found in longitudinal studies dealing with MNAR on the response. For example, Fairclough et al. (1998) assess the quality of life of lung cancer patients treated with chemotherapy, where 30 per cent of data is missing. On the other hand, only a few cross-sectional studies mention pattern-mixture models. Little and Rubin (2002) examine the household net worth based on assets and liabilities. It can be speculated that the reason for not using pattern mixture models in cross-sectional studies is that most of them originate from social sciences, where sample selection models are more common.

The advantage of using pattern mixture models is that they do not require to set a bivariate distribution to relate the missingness mechanism with $Y$. For sample selection models it may be troublesome to combine the two mechanisms, especially when the outcome is not continuous. In the pattern mixture approach, $f\left(Y_{i} \mid R_{i}\right)$ is a univariate distribution whose parameters of the chosen distribution differ depending on the stratum. In some applications it is reasonable to have a separate distribution for responders and non-responders. For instance, suppose we want to measure quality of life of subjects under treatment in a longitudinal study. Pattern mixture models will allow to 
model survivors and non-survivors using two different distributions. Hence, we will have two sets of parameters for survivors and non-survivors. Since it is more reasonable to consider quality of life of survivors than non-survivors, the parameters of non-survivor stratum will be of little interest. Similarly, if the two outcome strata would be analysed as one, as it is done in sample selection modelling approach, the marginal distribution of survivors and non-survivors and its associated parameters would be of little relevance (Little, 2008).

Pattern mixture models do not require instrumental variables, which are usually needed in sample selection models. An instrumental variable (or exclusion restriction) is an explanatory variable which is correlated with the selection but not with the outcome process. Typically, exclusion restrictions are used to ensure identifiability of the model. Such variables may be difficult to find in empirical studies.

On the other hand, distribution mixtures can be regarded as a disadvantage. It is more natural to model the full population $Y$ in the absence of missingness and $f\left(R_{i} \mid Y_{i}\right)$ determining which $Y$ is observed. It is also more intuitive to model $Y$ conditioned on covariates rather than $Y$ given covariates in a stratum. There are no restrictions set on parameters related to $f\left(Y_{i}\right)$ and hence they are directly obtained from maximum likelihood estimation (Little, 2008).

The other disadvantage of pattern-mixture models is that their parameters are often not identified. For example, in equation (2.1.3) it is not possible to obtain parameters for $f\left(Y_{i} \mid R_{i}\right)$ when $Y_{i}$ is missing. This can be mitigated by imposing Bayesian priors which would relate these parameters to the stratum where $Y_{i}$ is complete. Nevertheless, this suggests computationally intensive estimation, in particular when many parameters are involved. It is worth noticing that, according to the author's best knowledge, the most common implementation can be found in SAS (SAS Institute, 2013). Finally, pattern mixture models do not offer any ways of checking the MNAR assumption. For example, sample selection models encompass association parameter showing the presence of non-random sample selection. Inferences can be made since maximum likelihood estimation is used. Pattern mixture models do not offer equivalent methods.

We will describe sample selection models whose primary aim is to correct for the selection bias arising due to the existence of unobserved confounders. 


\subsection{Model definition of some sample selection models and their applications}

Consider the classic case of a continuous response. The model consists of two equations

$$
\begin{gathered}
Y_{1}^{*}=\boldsymbol{\gamma}^{\top} \boldsymbol{z}+\epsilon_{1}, \\
Y_{2}=\boldsymbol{\beta}^{\top} \boldsymbol{x}+\epsilon_{2} .
\end{gathered}
$$

The first is the selection equation and the second is the outcome equation. $Y_{1}^{*}$ is a latent variable whereas $Y_{2}$ is the outcome of interest. Also, the first equation is assumed to be a probit regression, hence $Y_{1}^{*} \sim N\left(\boldsymbol{\gamma}^{\top} \boldsymbol{z}, 1\right)$. Furthermore, $Y_{2} \sim N\left(\boldsymbol{\beta}^{\top} \boldsymbol{x}, \sigma_{2}^{2}\right) . \boldsymbol{z}$ and $\boldsymbol{x}$ are the covariate vectors of the selection and outcome equations with corresponding parameter vectors $\boldsymbol{\gamma}$ and $\boldsymbol{\beta}$. $\epsilon_{1}$ and $\epsilon_{2}$ denote the error terms of the equations. The following decision rules exist

$$
\begin{gathered}
Y_{1}= \begin{cases}1 & \text { if } Y_{1}^{*}>0 \\
0 & \text { if } Y_{1}^{*} \leq 0\end{cases} \\
Y_{2}=\left\{\begin{array}{ll}
Y_{2} & \text { if } Y_{1}^{*}>0 \\
\text { not observed } & \text { if } y_{1}^{*} \leq 0
\end{array} .\right.
\end{gathered}
$$

To elaborate, consider a vector of six observations $y_{2}=[5.6,8.5,6,-,-,-]^{\top}$, where - indicates missing entries. In the model framework above, we would write $y_{1}=[1,1,1,0,0,0]^{\top}$. Also, the latent $y_{1}^{*}$, which we do not observe, could have taken values such that $y_{1}^{*}=[1.2,0.3,0.9,-2.1,-1.1,-0.8]^{\top}$. Hence, by the decision rules above, the first three entries are observed and the remaining three are not.

The error components are typically assumed to follow the distribution

$$
\left(\begin{array}{c}
\epsilon_{1} \\
\epsilon_{2}
\end{array}\right) \sim \mathcal{N}\left(\left[\begin{array}{l}
0 \\
0
\end{array}\right],\left[\begin{array}{cc}
1 & \rho \sigma_{2} \\
\rho \sigma_{2} & \sigma_{2}^{2}
\end{array}\right]\right) .
$$

Note that $\rho$ represents the correlation between the unobserved confounders in the two equations. We set the variance of $Y_{1}^{*}$ to unity for convenience, but other specifications are possible. 
When the outcome is binary, we use $Y_{2}^{*}$ instead of $Y_{2}$ and $\sigma_{2}^{2}=1$. In this case, both equations will be probit regressions. The following decision rule will then apply

$$
\begin{gathered}
Y_{1}=\left\{\begin{array}{ll}
1 & \text { if } Y_{1}^{*}>0 \\
0 & \text { if } Y_{1}^{*} \leq 0
\end{array} Y_{2}= \begin{cases}1 & \text { if } Y_{1}^{*}>0 \text { and } Y_{2}^{*}>0 \\
0 & \text { if } Y_{1}^{*}>0 \text { and } Y_{2}^{*} \leq 0 \\
\text { not observed } & \text { if } Y_{1}^{*} \leq 0\end{cases} \right.
\end{gathered}
$$

and

$$
\left(\begin{array}{c}
\epsilon_{1} \\
\epsilon_{2}
\end{array}\right) \sim \mathcal{N}\left(\left[\begin{array}{l}
0 \\
0
\end{array}\right],\left[\begin{array}{ll}
1 & \rho \\
\rho & 1
\end{array}\right]\right) .
$$

When typing Heckman sample selection model in the Google Scholar search engine we receive over 80,000 results, most of which are for applied papers. This shows the vast applied literature on sample selection models. However, we will only discuss some applications. The first possible application of sample selection models is mentioned in one of the seminal works by Heckman and concerns women's wages (Heckman, 1976). Suppose, that the data of interest includes women who are active on the labour market and women who are not. Active women will report a positive wage and those who are inactive will report a zero wage. It can be argued, that more ambitious women select themselves into the sample of working women. If this is the case, the market wage needs to be corrected for this non-random sample selection.

In health economics, Jung and Liu (2012) examine the impact of health insurance on individual out-of-pocket (OOP) health expenditure in China using sample selection models. Here, individuals might select themselves into the sample based on unobserved characteristics such as lifestyle or habits. Three sample selection models for continuous response were estimated. The first model was estimated using the two-step method which will be presented in the next section. This model did not incorporate exclusion restrictions or instrumental variables. The second model was based on the two-step estimation with exclusion restrictions. The third model was estimated using maximum likelihood with exclusion restrictions. It was found that for two out of the three models, non-random sample selection was existent. The most important finding was that the probability of having any 
OOP health expenditure rises with the availability of health insurance whereas the actual level of health expenditures decreases. Furthermore, people who work, live in rural areas or have larger families tend to spend less on health care. On the other hand, people who are older, female or married tend to spend more on health care.

Yuan and Yen (2012) investigate the alcohol consumption of individuals in the United States using data drawn from the 2003-2004 National Health and Nutrition Examination Survey. The importance of this research is justified by the fact that drinking is the third leading cause of preventable death in the United States and is a risk of many societal and health problems. The authors apply a sample selection model for continuous data. Explicit reasons for the application of sample selection models are not provided although one can speculate that individuals select themselves into the subsample of non-drinkers on the basis of health conditions or personal convictions. The results showed that the sample selection was existent. The probability of drinking decreases with age, income and education. Men are more likely to drink than women and marriage decreases drinking. When applying a model not accounting for non-random sample selection some of the results change. For example, drinking increases with income for the two-part model.

Liu et al. (2012) model household expenditures on food depending on the facility used i.e. fullservice restaurants, fast-food restaurants and other facilities based on the 2008 and 2009 Consumer Expenditure Survey of the United States Bureau of Labor Statistics. They employ sample selection models with a three equation system for each of the food-away-from home facility. Also, three models were estimated for subsamples of single-person households, husband-wife households without children and husband-wife households with children. They argue that sample selection arises on unavailable variables such as prices, income or hours worked. For example, for single-person households sample selection turned out to be existent for fast-food chains but not for the remaining two facilities. No model ignoring non-random sample selection was estimated for comparison. The findings of the model analysis can help the state authorities to modify their policies and support the food service firms in adjusting their marketing campaigns. For instance, men in single-person households like to spend more than women at full-service restaurants. The authorities which are interested in promoting healthy nutrition could launch an educational campaign to encourage men to eat home-made food. On the other hand, the food service firms can target men with special promotions of expensive menus. 
Applications of sample selection models with binary response can be found in epidemiology and credit risk, for instance. An example of the former was provided in the beginning of Chapter 2 . An example of the latter is provided by Greene (1998). This involves estimating a sample selection probit model that would estimate the probability and consequently predict whether an individual would default on a credit card. The selection consists of an individual's credit card application being accepted before a default on a credit card can take place. However, it is not clear what unobservable factors might influence the acceptance decision. Moreover, non-random sample selection seems to be marginal. Still, the results for the sample selection model indicate greater marginal influence of particular covariates than it would be under a standard probit model.

Regarding the discrete (count) response case, the methodological literature on sample selection models is limited. This thesis aims at rectifying this situation. Many applications of sample selection models of counts can be found in health and labour economics.

For example, Sapelli and Vial (2003) argue that sample selection arises in the Chilean health insurance industry which can be subdivided into private and public health insurance. They explain that the beneficiaries of private health insurance make unauthorised sequential use of both types of insurance relative to price and quality. Also, private insurance beneficiaries are permitted to switch back to public insurance as a last-resort insurer. Thus, the public insurance beneficiaries should be exhibiting greater non-observable risk (i.e. confounding risk) than private insurance beneficiaries. To control for this sample selection problem, based on the two-step approach by Greene (1997), the authors create count data sample selection models for the dependent and independent workers who were either insured in the public or private sector. The response was number of hospitalisation days. The estimation results showed that non-random sample selection was present for the dependent workers with public insurance.

In labour economics, Winkelmann (1998) constructs two modifications of sample selection models for labour mobility defined as the number of job changes over the period of ten years. The two models are generalisations of the under-reporting and the zero-inflation models. Their first common component is the expected value for observation $i$

$$
E\left(Y_{i}^{*} \mid \boldsymbol{x}_{i}, u_{i}\right)=\exp \left(\boldsymbol{x}_{i}^{\top} \boldsymbol{\beta}+u_{i}\right)
$$


where $\boldsymbol{x}_{i}$ is a vector of covariates, $\boldsymbol{\beta}$ is the vector of parameters, $u_{i}$ is the error term and $Y_{i}^{*}$ is the latent variable associated with the outcome process in the zero-inflated and underreporting models (for details see Winkelmann, 1998). The selection equation follows a latent process

$$
C_{i}^{*}=\boldsymbol{z}_{i}^{\top} \gamma+\epsilon_{i}
$$

where $\boldsymbol{z}_{i}$ is a vector of covariates, $\boldsymbol{\gamma}$ is the vector of the parameters and $\epsilon_{i}$ is the error term. The decision rule is

$$
C_{i}= \begin{cases}1 & \text { if } C_{i}^{*} \geq 0 \\ 0 & \text { if } C_{i}^{*}<0\end{cases}
$$

When non-random sample selection arises, the unobservables will enter $u_{i}$ and $\epsilon_{i}$. Thus, the error terms will be correlated. If all relevant covariates could be observed then selection would be solely based on observables and the correlation between the two terms would be zero. $u_{i}$ and $\epsilon_{i}$ are assumed to follow the distribution

$$
\left(\begin{array}{l}
u_{i} \\
\epsilon_{i}
\end{array}\right) \sim \mathcal{N}\left(\left[\begin{array}{l}
0 \\
0
\end{array}\right],\left[\begin{array}{cc}
\sigma^{2} & \rho \sigma \\
\rho \sigma & 1
\end{array}\right]\right)
$$

where $\rho$ is the correlation coefficient and $\sigma^{2}$ is the variance of $u_{i}$. The variance of $\epsilon_{i}$ is equal to 1 since we are dealing with a probit. In the application to labour mobility data, it turned out that sample selection was present. As the correlation coefficient associated with sample selection was negative, its interpretation is that workers who are likely to be on the labour market are less likely to change jobs and vice versa. The author also finds a 10 per cent level statistically significant negative association between union membership and job mobility, and experience and job mobility. Mobility was found to be lower for more educated, white collar and married workers, but the associated covariates were not statistically significant.

We will provide more details on the specification and estimation of sample selection models in Chapter 3. Chapter 4 will explore Veterans Administration (VA) and German Socioeconomic Panel (SOEP) data. We will be using the already established sample selection models and models that do not account for non-random sample selection, with the aim of gaining insight whether non-random sample selection is existent and what distributions best model count data. The subsequent chapters 
will focus on the development of sample selection models for count response. Finally, the VA and SOEP data will be re-analysed. 


\section{Chapter 3}

\section{Variants of sample selection models}

The aim of this chapter is to review some sample selection models relevant in the context of count data. It will be common sense to start with the original Heckman-type model. Then, we will discuss Greene's $(1994 ; 1997)$ sample selection approaches to count data. We will also mention other approaches to sample selection that are available in the literature. Afterwards, the copula modelling approach will be discussed. Hence, we will compare the copula modelling approach with Greene's approach. This will provide a platform to build count sample selection models, which will be discussed in the next chapter.

\subsection{Heckman model for continuous response}

The classic Heckman-type sample selection model consists of two equations:

$$
\begin{gathered}
Y_{1}^{*}=\boldsymbol{\gamma}^{\top} \boldsymbol{z}+\epsilon_{1}, \\
Y_{2}=\boldsymbol{\beta}^{\top} \boldsymbol{x}+\epsilon_{2} .
\end{gathered}
$$

The first is the selection equation and the second is the outcome equation. $Y_{1}^{*}$ is a latent variable whereas $Y_{2}$ is the outcome of interest. The first equation is a probit regression with $\epsilon_{1} \sim N(0,1)$.

Furthermore, $\epsilon_{2} \sim N\left(0, \sigma^{2}\right) . \quad \boldsymbol{z}$ and $\boldsymbol{x}$ are the covariate vectors of the selection and outcome equations respectively with corresponding parameter vectors $\boldsymbol{\gamma}$ and $\boldsymbol{\beta}$. $\epsilon_{1}$ and $\epsilon_{2}$ denote the error terms for both equations. To avoid clutter we have discarded the usual observation index $i$ in the 
equations above. The following decision rules exist

$$
Y_{1}= \begin{cases}1 & \text { if } Y_{1}^{*}>0 \\ 0 & \text { if } Y_{1}^{*} \leq 0\end{cases}
$$

and

$$
Y_{2}=\left\{\begin{array}{ll}
Y_{2} & \text { if } Y_{1}^{*}>0 \\
\text { not observed } & \text { if } Y_{1}^{*} \leq 0
\end{array} .\right.
$$

The random components are typically assumed to follow the distribution

$$
\left(\begin{array}{l}
\epsilon_{1} \\
\epsilon_{2}
\end{array}\right) \sim \mathcal{N}\left(\left[\begin{array}{l}
0 \\
0
\end{array}\right],\left[\begin{array}{cc}
1 & \rho \sigma_{2} \\
\rho \sigma_{2} & \sigma_{2}^{2}
\end{array}\right]\right)
$$

where $\sigma_{2}^{2}$ is the variance of the outcome equation. $\rho$ is the Pearson's correlation coefficient and models the (linear) association between selection and outcome. Note that $\sigma_{1}=1$, because the first equation is a probit equation. The expected value of $Y_{2}$ that is observed is

$$
E\left(Y_{2} \mid Y_{1}^{*}>0\right)=\boldsymbol{\beta}^{\top} \boldsymbol{x}+\rho \sigma_{2} \lambda\left(-\boldsymbol{\gamma}^{\top} \boldsymbol{z}\right)
$$

where $\lambda\left(-\gamma^{\top} \boldsymbol{z}\right)$ is a function equal to $\frac{\phi\left(-\gamma^{\top} \boldsymbol{z}\right)}{1-\Phi\left(-\boldsymbol{\gamma}^{\top} \boldsymbol{z}\right)}$ (see Appendix A). Note that $\phi$ and $\Phi$ stand for the probability density function (pdf) and the cumulative distribution function (cdf) of the standard normal respectively.

There are two approaches for estimating the model: two-step estimation and maximum likelihood (ML). The former can be summarised as follows:

1. Estimate the selection equation using a probit model for the full sample.

2. Extract the coefficients from the probit model in step 1 and calculate $-\hat{\gamma}^{\top} \boldsymbol{z}$ for the observations which are observed (i.e. $\left.y_{1}=1\right)$. Hence, calculate $\lambda\left(-\hat{\boldsymbol{\gamma}}^{\top} \boldsymbol{z}\right)$.

3. Estimate equation (3.1.3) for the observed cases where $\lambda\left(-\hat{\gamma}^{\top} \boldsymbol{z}\right)$ is included as a regressor.

The estimates obtained using the two-step method will be consistent. Intuitively, the probit estimates are consistent and by the continuous mapping theorem, $\lambda\left(-\hat{\gamma}^{\top} \boldsymbol{z}\right)$ will converge in probability 
to its true value. Thus, the parameter estimators associated with $\lambda$ will also converge in probability to their true value. As a consequence $\hat{\boldsymbol{\beta}} \stackrel{p}{\rightarrow} \boldsymbol{\beta}$ (Heckman, 1976).

Alternatively, one can use ML estimation. The general likelihood function for the sample selection models is given by

$$
L=\prod_{i=1}^{n_{0}} \operatorname{Pr}\left(Y_{1}^{*} \leq 0\right) \prod_{j=1}^{n_{1}} f_{2 \mid 1}\left(y_{2} \mid y_{1}^{*}>0\right) \operatorname{Pr}\left(Y_{1}^{*}>0\right)
$$

The first term is the product of all cases when the outcome is not observed $\left(n_{0}\right)$ and the second term is the product of all cases when the outcome is observed $\left(n_{1}\right)$. Assuming a bivariate normal distribution, (3.1.4) will be (see Appendix B)

$$
L=\prod_{i=1}^{n_{0}} \Phi\left(-\boldsymbol{\gamma}^{\boldsymbol{\top}} \boldsymbol{z}\right) \prod_{j=1}^{n_{1}} \frac{1}{\sigma_{2}} \phi\left(\frac{y_{2}-\boldsymbol{\beta}^{\boldsymbol{\top}} \boldsymbol{x}}{\sigma_{2}}\right) \Phi\left(\frac{\boldsymbol{\gamma}^{\boldsymbol{\top}} \boldsymbol{z}+\frac{\rho}{\sigma_{2}}\left\{y_{2}-\boldsymbol{\beta}^{\boldsymbol{\top}} \boldsymbol{x}\right\}}{\sqrt{1-\rho^{2}}}\right) .
$$

Note that both the two-step and ML approaches will correct for non-random sample selection. In the two-step approach, the regression equation based on (3.1.3) accounts for non-random sample selection by introducing the omitted variable $\lambda\left(-\hat{\gamma}^{\top} \boldsymbol{z}\right)$ (compare: Chapter 2). In the ML approach the likelihood function in (3.1.4) recognises sample selection as the likelihood of the observations that were selected non-randomly into the sample.

The advantage of using the ML approach is that, unlike the two-step estimation, the standard errors are consistent. Also, the ML approach does not require to analytically derive the conditional expected value beforehand, which makes the model specification in terms of joint distribution and outcome margins more flexible. Therefore, the remainder of this work will be dealing with the ML estimation method for sample selection models.

We will now explore sample selection models beginning with the Greene's count model. This model will serve as a motivation for creating a new model for count data. 


\subsection{Greene's count model}

\subsubsection{Model specification}

In the model specification proposed by Greene (1994) the selection equation is the same as the one in the standard sample selection model:

$$
Y_{1}^{*}=\gamma^{\top} z+\epsilon_{1}
$$

where $\epsilon_{1} \sim N(0,1)$. On the other hand, the outcome process follows a Poisson distribution such that:

$$
P\left(Y_{2}=y_{2} \mid \epsilon_{2}\right)=\frac{\exp \left[-\omega\left(\epsilon_{2}\right)\right] \omega\left(\epsilon_{2}\right)^{y_{2}}}{y_{2} !}
$$

where $E\left(Y_{2} \mid \epsilon_{2}\right)=\omega\left(\epsilon_{2}\right)=\exp \left(\boldsymbol{\beta}^{\top} \boldsymbol{x}+\epsilon_{2}\right)$ and $\epsilon_{2} \sim N\left(0, \sigma^{2}\right)$. We will later see that non-random sample selection is taken into account in the expected value and likelihood function: both of them include the correlation coefficient linking the outcome and selection equations. In terms of notation, we will refer to $P(\cdot)$ as the probability mass function of a random variable and $\operatorname{Pr}(\cdot)$ will be used in the context of cumulative distribution functions.

Note that the distribution we are dealing with is conditional on the error term of the outcome. The exact distribution of the modelled response remains unknown (see: Greene, 2002). The idea behind specifying the model like in (3.2.2) starts with the premise that a Poisson model can suffer from overdispersion. However, if we manage to integrate out the error term in a feasible way, we will arrive at a distribution which will have a scale parameter and the unconditional mean will not have to be equal to the unconditional variance. To see this, let us derive the first two moments. The mean in this case would be defined as (see: Agresti, 2002)

$$
\begin{aligned}
E\left(Y_{2}\right) & =E_{\epsilon_{2}}\left[E\left(Y_{2} \mid \epsilon_{2}\right)\right] \\
& =e^{\boldsymbol{\beta}^{\top} \boldsymbol{x}} E_{\epsilon_{2}}\left(e^{\epsilon_{2}}\right) \\
& =e^{\boldsymbol{\beta}^{\top} \boldsymbol{x}+\frac{\sigma^{2}}{2}} .
\end{aligned}
$$


The variance will look as follows (Agresti, 2002)

$$
\begin{aligned}
\operatorname{Var}\left(Y_{2}\right) & =\operatorname{Var}\left[E\left(Y_{2} \mid \epsilon_{2}\right)\right]+E\left[\operatorname{Var}\left(Y_{2} \mid \epsilon_{2}\right)\right] \\
& =\operatorname{Var}\left[e^{\boldsymbol{\beta}^{\top} \boldsymbol{x}+\epsilon_{2}}\right]+E\left[e^{\boldsymbol{\beta}^{\boldsymbol{\top}} \boldsymbol{x}+\epsilon_{2}}\right] \\
& =\exp \left(\boldsymbol{\beta}^{\boldsymbol{\top}} \boldsymbol{x}\right)^{2}\left(e^{\sigma^{2}}-1\right) e^{\sigma^{2}}+\exp \left(\boldsymbol{\beta}^{\boldsymbol{\top}} \boldsymbol{x}\right) \exp \left(\frac{\sigma^{2}}{2}\right) \\
& =E\left(Y_{2}\right)\left[\left(e^{\sigma^{2}}-1\right) E\left(Y_{2}\right)+1\right] .
\end{aligned}
$$

As one can see, $E\left(Y_{2}\right)=\operatorname{Var}\left(Y_{2}\right)$ only in the special case when $\sigma^{2}=0$. Then, the unconditional distribution reduces itself to the Poisson distribution.

The decision rules are the same as in the Heckman model:

$$
\begin{gathered}
Y_{1}=\left\{\begin{array}{ll}
1 & \text { if } Y_{1}^{*}>0 \\
0 & \text { if } Y_{1}^{*} \leq 0
\end{array},\right. \\
Y_{2}= \begin{cases}Y_{2} & \text { if } Y_{1}^{*}>0 \\
\text { not observed } & \text { if } Y_{1}^{*} \leq 0\end{cases}
\end{gathered}
$$

The random components $\epsilon_{1}$ and $\epsilon_{2}$ also follow the bivariate normal distribution as described in Section 3.1.

\subsubsection{Estimation}

There are two ways of estimating the model: two-step estimation and full information maximum likelihood (FIML) estimation. We will first introduce the former:

1. Estimate the selection equation using a probit model for the full sample.

2. Calculate $-\hat{\gamma}^{\top} \boldsymbol{z}$ for the observed subsample and estimate the following regression via nonlinear least squares:

$$
E\left(Y_{2} \mid Y_{1}^{*}>0\right)=\exp \left(\boldsymbol{\beta}^{\top} \boldsymbol{x}+\frac{\sigma_{2}^{2}}{2}\right) \frac{\Phi\left(-\hat{\boldsymbol{\gamma}}^{\top} \boldsymbol{z}+\rho \sigma_{2}\right)}{\Phi\left(-\hat{\boldsymbol{\gamma}}^{\top} \boldsymbol{z}\right)} .
$$

For the derivation of the conditional expected value see Appendix C. Greene (1997) also provides details for obtaining the asymptotic covariance matrix based on White (1980) and Murphy and Topel (1985), which needs to be adjusted since an estimate of $\gamma$ is used. 
In the FIML estimation method the log-likelihood function to be maximised is

$$
\ell=\sum_{i=1}^{n_{0}} \log \operatorname{Pr}\left(Y_{1}=0\right)+\sum_{j=1}^{n_{1}} \log P\left(Y_{2}=y_{2}, Y_{1}=1\right)
$$

We will first need to obtain probabilities for both terms. Note that to avoid clutter we dropped conditioning on $\boldsymbol{z}$ and $\boldsymbol{x}$. Let us start with $P\left(Y_{2}=y_{2}, Y_{1}=1\right)$.

$$
\begin{aligned}
P\left(Y_{2}=y_{2}, Y_{1}=1\right) & =\int_{-\infty}^{\infty} P\left(Y_{2}=y_{2}, Y_{1}=1, \epsilon_{2}\right) d \epsilon_{2} \\
& =\int_{-\infty}^{\infty} P\left(Y_{2}=y_{2}, Y_{1}=1 \mid \epsilon_{2}\right) f\left(\epsilon_{2}\right) d \epsilon_{2} .
\end{aligned}
$$

We know that $Y_{1}^{*}$ depends on $\epsilon_{1}, Y_{2}$ depends on $\epsilon_{2}$, and $\epsilon_{1}$ and $\epsilon_{2}$ are correlated. Therefore, conditioned on $\epsilon_{2}, Y_{1}$ and $Y_{2}$ are independent (we pretend to "move" the unobservable from the error term into the deterministic part of the model - by doing so we remove the source of correlation between both equations). Thus,

$$
P\left(Y_{2}=y_{2}, Y_{1}=1 \mid \epsilon_{2}\right)=P\left(Y_{2}=y_{2} \mid \epsilon_{2}\right) \operatorname{Pr}\left(Y_{1}=1 \mid \epsilon_{2}\right)
$$

The first term, $P\left(Y_{2}=y_{2} \mid \epsilon_{2}\right)$, is the conditional Poisson distribution with an error term defined in (3.2.2). As by joint normality $f\left(\epsilon_{1} \mid \epsilon_{2}\right)=N\left(\frac{\rho}{\sigma_{2}} \epsilon_{2},\left(1-\rho^{2}\right)\right)$,

$$
\begin{aligned}
\operatorname{Pr}\left(Y_{1}=1 \mid \epsilon_{2}\right) & =\operatorname{Pr}\left(\epsilon_{1}>-\boldsymbol{\gamma}^{\top} \boldsymbol{z} \mid \epsilon_{2}\right) \\
& =\int_{-\boldsymbol{\gamma}^{\top} \boldsymbol{z}}^{\infty} f\left(\epsilon_{1} \mid \epsilon_{2}\right) d \epsilon_{1} \\
& =\int_{-\boldsymbol{\gamma}^{\top} \boldsymbol{z}}^{\infty} \frac{1}{\sqrt{1-\rho^{2}}} \frac{1}{\sqrt{2 \pi}} \exp \left[\left(-\frac{1}{2\left(1-\rho^{2}\right)}\right)\left(\epsilon_{1}-\frac{\rho}{\sigma_{2}} \epsilon_{2}\right)^{2}\right] d \epsilon_{1} .
\end{aligned}
$$

The standard normal conditional cdf will be

$$
\operatorname{Pr}\left(Y_{1}=1 \mid \epsilon_{2}\right)=\Phi\left(\frac{\boldsymbol{\gamma}^{\top} \boldsymbol{z}+\left(\frac{\rho}{\sigma} \epsilon_{2}\right)}{\sqrt{1-\rho^{2}}}\right)
$$


Combining (3.2.4), (3.2.5) and (3.2.7) yields

$$
P\left(Y_{2}=y_{2}, y_{1}=1\right)=\int_{-\infty}^{\infty} \frac{\exp \left[-\omega\left(\epsilon_{2}\right)\right] \omega\left(\epsilon_{2}\right)^{y_{2}}}{y_{2} !} \Phi\left(\frac{\boldsymbol{\gamma}^{\top} \boldsymbol{z}+\left(\frac{\rho}{\sigma} \epsilon_{2}\right)}{\sqrt{1-\rho^{2}}}\right) \frac{1}{\sigma_{2} \sqrt{2 \pi}} \exp \left[\frac{-\epsilon_{2}^{2}}{2 \sigma_{2}^{2}}\right] d \epsilon_{2} 3
$$

Now, let us proceed to some aspects related to numerical optimisation. As we will see in the next paragraphs, in order to maximise the log-likelihood function we will need to reparametrise (3.2.8). Thus, let $v=\frac{\epsilon_{2}}{\sigma_{2} \sqrt{2}}, \theta=\sigma_{2} \sqrt{2}, \tau=\sqrt{2} \frac{\rho}{\sqrt{1-\rho^{2}}}$ and $\boldsymbol{\alpha}=\frac{1}{\sqrt{1-\rho^{2}}} \gamma$. This will allow us to use Gauss-Hermite quadrature and evaluate $P\left(Y_{2}=y_{2}, Y_{1}=1\right)$. After changing the variables and reparametrising the probability one obtains

$$
P\left(Y_{2}=y_{2}, Y_{1}=1\right)=\frac{1}{\sqrt{\pi}} \int_{-\infty}^{\infty} \exp \left(-v^{2}\right) \frac{\exp [-\omega(v)] \omega(v)^{y_{2}}}{y_{2} !} \Phi\left(\boldsymbol{\alpha}^{\top} \boldsymbol{z}+v \tau\right) d v
$$

where $\omega(v)=\exp \left(\boldsymbol{\beta}^{\top} \boldsymbol{x}+v \theta\right)$. The integral in (3.2.9) can be evaluated using Hermite quadrature or simulation. In general, if an integral is supposed to demonstrate the area under the curve then a quadrature would create "rectangles" of equal width to approximate this area. Gauss-Hermite quadrature appproximates integrals of the kind (Abramowitz et al., 1964)

$$
\int_{-\infty}^{\infty} e^{-t^{2}} f(t) d t
$$

In this case the approximation would look as follows

$$
\int_{-\infty}^{\infty} e^{-t^{2}} f(t) d t \approx \sum_{h=1}^{H} w_{h} f\left(t_{h}\right)
$$

Analogically, Gauss-Hermite quadrature would be evaluated as follows

$$
\begin{aligned}
P\left(Y_{2}=y_{2}, Y_{1}=1\right) & \approx P^{*}\left(Y_{2}=y_{2}, Y_{1}=1\right) \\
& =\frac{1}{\sqrt{\pi}} \sum_{h=1}^{H} w_{h}\left[\frac{\exp [-\omega(v)] \omega(v)^{y_{2}}}{y_{2} !} \Phi\left(\boldsymbol{\alpha}^{\top} \boldsymbol{z}+v \tau\right)\right]
\end{aligned}
$$

where $H$ determines the number of sample points to be used. For the table of weights $w_{h}$ and $v$ see Abramowitz et al. (1964). Sufficiently accurate approximation will depend on the number of points chosen. Greene (1997) does not provide details on how to choose points. Also, the literature on how 
to do this in practice is broad and we will not further elaborate on that matter. For details on point selection see, for example, Rabe-Hesketh et al. (2005). Then, the second part of the log-likelihood function in (3.2.3) can be approximated by

$$
\sum_{j=1}^{n_{1}} \log P^{*}\left(Y_{2}=y_{2}, Y_{1}=1\right) .
$$

Assume that a simulation approach is chosen. Let us respecify the model such that $E\left(Y_{2} \mid \epsilon_{2}\right)=$ $\lambda\left(\epsilon_{2}\right)=\exp \left(\boldsymbol{\beta}^{\boldsymbol{\top}} \boldsymbol{x}+\sigma_{2} \epsilon_{2}\right)$ where $\epsilon_{2} \sim N(0,1)$. Then by the law of large numbers if the observations $\epsilon_{2_{r}}$ are iid,

$$
\begin{aligned}
\operatorname{plim}_{R \rightarrow \infty} \frac{1}{R} \sum_{r=1}^{R} P\left(Y_{2}=y_{2}, Y_{1}=1 \mid \epsilon_{2_{r}}\right) & =E_{\epsilon_{2}}\left[P\left(Y_{2}=y_{2}, Y_{1}=1 \mid \epsilon_{2}\right)\right] \\
& =P\left(Y_{2}=y_{2}, Y_{1}=1\right)
\end{aligned}
$$

It can be shown that this result can be used in maximum likelihood estimation. First, we sample $R$ realisations from the standard normal random number generator for each observation. Second, we approximate the true probability for each observation i.e. calculate $P^{\prime}\left(Y_{2}=y_{2}, Y_{1}=1\right)=$ $\frac{1}{R} \sum_{r=1}^{R} P\left(Y_{2}=y_{2}, Y_{1}=1 \mid \hat{\epsilon}_{2 r}\right)$ where $\hat{\epsilon}_{2 r}$ is the $r^{t h}$ draw from the standard normal random number generator. Finally, we plug the $P^{\prime}\left(Y_{2}=y_{2}, Y_{1}=1\right)$ values into the second part of the log-likelihood function in (3.2.3) and use the approximation

$$
\sum_{j=1}^{n_{1}} \log P^{\prime}\left(Y_{2}=y_{2}, Y_{1}=1\right) .
$$

There is no preference for one method or the other. The quadrature method will always contain an approximation error. On the other hand, Greene (1997) points out that the quadrature method will be more efficient as the usual value of draws in the simulations will amount to hundreds.

The only ingredient left for the evaluation of the log-likelihood function in (3.2.3) is the term 
with the probability of those observations that are not observed.

$$
\begin{aligned}
\operatorname{Pr}\left(Y_{1}=0\right) & =\int_{-\infty}^{\infty} P\left(Y_{1}=0, \epsilon_{2}\right) d \epsilon_{2} \\
& =\int_{-\infty}^{\infty} \operatorname{Pr}\left(\epsilon_{1} \leq-\boldsymbol{\gamma}^{\top} \boldsymbol{z} \mid \epsilon_{2}\right) f\left(\epsilon_{2}\right) d \epsilon_{2} \\
& =\int_{-\infty}^{\infty}\left[1-\operatorname{Pr}\left(\epsilon_{1}>-\boldsymbol{\gamma}^{\top} \boldsymbol{z} \mid \epsilon_{2}\right)\right] f\left(\epsilon_{2}\right) d \epsilon_{2}
\end{aligned}
$$

Note that

$$
\left[1-\operatorname{Pr}\left(\epsilon_{1}>\boldsymbol{\gamma}^{\top} \boldsymbol{z} \mid \epsilon_{2}\right)\right]=1-\Phi\left[\boldsymbol{\alpha}^{\top} \boldsymbol{z}+\frac{\tau \epsilon_{2}}{\sqrt{2} \sigma_{2}}\right]
$$

and therefore

$$
\operatorname{Pr}\left(Y_{1}=0\right)=\frac{1}{\sqrt{\pi}} \int_{-\infty}^{\infty} \exp \left(-v^{2}\right) \Phi\left[-\left(\boldsymbol{\alpha}^{\top} \boldsymbol{z}+v \tau\right)\right] d v
$$

Similarly, quadrature or simulation methods have to be used to evaluate the integral.

\subsubsection{Disadvantages}

As it is explicitly shown in Greene's work, the score vector components can be easily derived. However, his methodology has drawbacks.

First, quadrature or simulation methods have to be applied twice in order to obtain unconditional probabilities. This, of course, creates substantial computational burden. Also, as the integrals are approximated there will be an additional computational error involved.

Second, the model specifies that the outcome conditioned on the error term of the outcome equation follows a Poisson distribution. After integrating out the error term one would expect the unconditional distribution of the outcome to follow a negative binomial distribution. Unfortunately, the above reasoning holds if the error term follows a gamma distribution and not the normal distribution like we assumed in Section 3.2. Therefore, the unconditional distribution of the outcome is unknown.

We will now present the copula modelling technique and its advantages towards the Greene count model. 


\subsection{The copula approach}

In the standard sample selection model the classic assumption is that the errors follow a bivariate normal distribution (see Section 3.1). When this assumption fails the consistency of the maximum likelihood estimator of the standard Heckman sample selection model will not hold. For this reason the bivariate normality asssumption is frequently questioned. Bivariate normality may be viewed as a problem of a priori conjectures on the model specification rather than a problem of data (Pigini, 2012). Thus, the literature mainly offers two alternatives - the semi-parametric and the (parametric) copula approaches. The semi-parametric methods, unlike the copula models, tend to be quite computer-intensive. Additionally, there are identification constraints which do not allow the model user to fit the model with the desired specification (compare: Pigini, 2012, p. 31-33). Furthermore, convergence problems may occur for more complex models which include discrete covariates and interaction terms (Wojtyś et al., 2016). On the other hand, as we will see, the copula modelling approach uses well-known maximum likelihood techniques allowing for simultaneous estimation of the parameters from both equations (Smith, 2003). It can be argued, that maximum likelihood estimators are not consistent if the distributional assumption is not correct. In this case, the copula approach allows for piece-wise model specification. For instance, it is possible to use normal margins for different choices of copulas that will be discussed in this section. Genius and Strazzera (2008) argue that the copula approach allows for direct estimation of the dependence structure, while the semi-parametric methods do not. In this work we will only focus on the copula modelling techniques. The notation and explanation will mainly follow from Smith (2003). For the reader's interest, more on semiparametric estimation methods can be found in Gallant and Nychka (1987).

\subsubsection{Some copula theory}

Sklar's theorem asserts that by binding the margins using a copula function one can obtain a multivariate distribution (Sklar, 1959). Later we will see, it turns out that when the Archimedean class of copulas are used the resulting log-likelihood and the score function expressions facilitate maximum likelihood estimation (Smith, 2003).

Beforehand, let us define a two-place copula $C$. Consider a vector of continuous random variables $\left(X_{1}, X_{2}\right)$, where cdfs of the marginals are defined as $F_{1}\left(x_{1}\right)=\operatorname{Pr}\left(X_{1} \leq x_{1}\right)$ and $F_{2}\left(x_{2}\right)=\operatorname{Pr}\left(X_{2} \leq\right.$ 
$\left.x_{2}\right)$. Applying probability integral transform to each component yields vector (Nelsen, 1999)

$$
(U, V)=\left(F_{1}\left(X_{1}\right), F_{2}\left(X_{2}\right)\right)
$$

where the marginals $U$ and $V$ are both uniformly distributed on the interval $[0,1]$. The copula of $\left(X_{1}, X_{2}\right)$, is defined as the joint cumulative distribution function of $(U, V)$ (Nelsen, 1999):

$$
C(u, v)=\operatorname{Pr}[U \leq u, V \leq v]
$$

Note that the copula $C$ contains information of the dependance structure between $\left(X_{1}, X_{2}\right)$ (irrespective of margin forms) and the cumulative distribution functions $u=F_{1}\left(x_{1}\right)$ and $v=F_{2}\left(x_{2}\right)$ contain information on the marginal distributions.

Theorem 1 (Sklar's theorem; Sklar, 1959) Let $F_{1}\left(x_{1}\right)$ and $F_{2}\left(x_{2}\right)$ denote the cdf of two random variables $X_{1}$ and $X_{2}$ respectively i.e. $F_{i}\left(x_{i}\right)=\operatorname{Pr}\left(X_{i} \leq x_{i}\right)$, where $x_{i} \in \overline{\mathbb{R}}(i=1,2 ; \overline{\mathbb{R}}$ denotes the real line $\mathbb{R} \cup[-\infty,+\infty]$, and let $F\left(x_{1}, x_{2}\right)=\operatorname{Pr}\left(X_{1} \leq x_{1}, X_{2} \leq x_{2}\right)$ denote the joint cdf. Then, every joint cdf can be expressed only in terms of cdfs and copula $C$ :

$$
F\left(x_{1}, x_{2}\right)=C\left(F_{1}\left(x_{1}\right), F_{2}\left(x_{2}\right)\right) .
$$

Further in this section, we will see that equation (3.3.1) will help us in re-expressing the likelihood of the sample selection model. There are several remarks to be made:

1. Even though the copula function depends on $F_{1}$ and $F_{2}$ the joint cdf itself does not depend on $F_{1}$ and $F_{2}$. To see this, recall the bivariate distribution function $F\left(x_{1}, x_{2}\right)$ with continuous univariate marginal distributions $F_{1}\left(x_{1}\right)$ and $F_{2}\left(x_{2}\right)$. Since $u=F_{1}\left(x_{1}\right)$ and $v=F_{2}\left(x_{2}\right)$, it follows that $x_{1}=F_{1}^{-1}(u)$ and $x_{2}=F_{2}^{-1}(v)$. Thus,

$$
\begin{aligned}
F\left(x_{1}, x_{2}\right) & =F\left(F_{1}^{-1}(u), F_{2}^{-1}(v)\right) \\
& =\operatorname{Pr}\left[F_{1}^{-1}(U) \leq F_{1}^{-1}(u), F_{2}^{-1}(V) \leq F_{2}^{-1}(v)\right] \\
& =\operatorname{Pr}[U \leq u, V \leq v] \\
& =C(u, v)
\end{aligned}
$$


is a unique copula associated with the distribution function (compare: Trivedi and Zimmer, 2007). The step from line 2 to line 3 is explained by the fact that the inverse quantile functions are monotonic functions.

2. If $F_{1}$ and $F_{2}$ are continuous then (3.3.1) is unique for any $\left(x_{1}, x_{2}\right) \in \overline{\mathbb{R}}^{2}$. Otherwise, there are other many possible copulas, but all of them are unique only on the closure $\operatorname{Ran}\left(F_{1}\right) \times \operatorname{Ran}\left(F_{2}\right)$ where $\operatorname{Ran}(F)$ denotes the range of $F$ (Nikoloulopoulos and Karlis, 2009). However, the general lack of uniqueness for discrete-discrete or discrete-continuous margins is a theoretical aspect that needs to be confronted in analytical proofs and does not affect empirical applications. More intuitively, note that for marginals which are discrete, a unique $F^{-1}$ does not exist. However, practically if one knows the $F_{1}$, then the "corresponding $F_{1}^{-1}$ " would be the integer among the possible $F_{1}^{-1}$ values. For example, suppose that for an arbitrary discrete distribution $F_{1}(x), F_{1}(3)=0.5$ and $F_{1}(4)=0.6$. We would like to find " $F_{1}^{-1}=x$ " when $F_{1}=0.56$. Since $F_{1}$ is above 0.5 , but still below 0.6 it will be set to a lower threshold i.e. " $F_{1}^{-1}$ " will be equal to 3 . Also, in practice researchers are more interested in choosing the appropriate copula and its margins rather than knowing the exact mathematical form of the joint distribution which is a requirement for finding a unique copula (Trivedi and Zimmer, 2007).

3. Other interesting properties are:

$$
\begin{gathered}
C(0, v)=\lim _{x_{1} \rightarrow-\infty} F\left(x_{1}, x_{2}\right)=0, \\
C(u, 0)=\lim _{x_{2} \rightarrow-\infty} F\left(x_{1}, x_{2}\right)=0, \\
C(1, v)=\lim _{x_{1} \rightarrow \infty} F\left(x_{1}, x_{2}\right)=F\left(x_{2}\right)=v, \\
C(u, 1)=\lim _{x_{2} \rightarrow \infty} F\left(x_{1}, x_{2}\right)=F\left(x_{1}\right)=u,
\end{gathered}
$$

where $(u, v) \in \mathbb{I}^{2}$ and $\mathbb{I}$ denotes an interval $[0,1]$ of the real line (Trivedi and Zimmer, 2007).

Three copulas have to be mentioned: the product copula and the Fréchet lower and upper bound copulas. At this stage, we need to emphasise that the Fréchet bounds will have implications in establishing the association coverage of a given family of couplas. The product copula is

$$
\Pi=u v
$$


The Fréchet lower bound for copulas is (Smith, 2003)

$$
\begin{aligned}
W & =\frac{u+v-1+|u+v-1|}{2}, \\
& =\max (u+v-1,0) .
\end{aligned}
$$

The Fréchet upper bound for copulas takes the following form (Smith, 2003)

$$
\begin{aligned}
M & =\frac{u+v-|u-v|}{2}, \\
& =\min (u, v) .
\end{aligned}
$$

For all closed $C$ on $\mathbb{I}^{2}$ the interval $[W, M]$ has the following property:

$$
W \leq C \leq M
$$

These are the Fréchet bounds for copulas (for proof of the inequality and the bounds see: Nelsen, 1999, chapter 2). Rewriting (3.3.6) (in terms of bivariate copulas) yields:

$$
\max \left(F_{1}\left(x_{1}\right)+F_{2}\left(x_{2}\right)-1,0\right) \leq F\left(x_{1}, x_{2}\right) \leq \min \left(F_{1}\left(x_{1}\right), F_{2}\left(x_{2}\right)\right) .
$$

For our modelling purposes, we need an association parameter which would show the degree of association between the two variables. We will denote it as $\theta$. Thus,

$$
C_{\theta}(u, v)
$$

We assume that $F_{1}$ and $F_{2}$ do not depend on $\theta$ and consequently representation (3.3.1) will hold. Let us provide some examples of copulas.

Example 1 Family of normal copulas is given by (e.g., Smith, 2003):

$$
C_{\theta}(u, v)=\Phi_{2}\left(\Phi^{-1}(u), \Phi^{-1}(v) ; \theta\right)
$$

where $-1 \leq \theta \leq 1$ and $\Phi(\cdot)$ denotes the cdf of a standard normal variate and $\Phi_{2}(\cdot, \cdot ; \theta)$ the cdf of a bivariate standard normal variate with Pearson's product moment correlation coefficient $\theta$. 
Example 2 Family of Farlie-Gumbel-Morgenstern (FGM) copulas is given by (Smith, 2003):

$$
C_{\theta}(u, v)=u v(1+\theta(1-u)(1-v))
$$

where $-1 \leq \theta \leq 1$. It is worthwhile to note that this family might be useful to work with due to its mathematical simplicity.

Example 3 Family of Plackett copulas is given by (Smith, 2003):

$$
C_{\theta}(u, v)= \begin{cases}\frac{1}{2(\theta-1)}\left(s-\sqrt{s^{2}-4 u v \theta(\theta-1)}\right), & \text { if } \theta>0, \theta \neq 1 \text { and }, \\ u v, & s=1+(u+v)(\theta-1) .\end{cases}
$$

The ability to show association between variables can be verified using upper and lower Fréchet bounds. This is done by considering the extremes of the parameter space for $\theta$. For example, the Plackett family has full coverage i.e. is comprehensive. This is because (Smith, 2003)

$$
\begin{aligned}
\lim _{\theta \rightarrow+0} C_{\theta}(u, v) & =\lim _{\theta \rightarrow+0} \frac{1}{2(\theta-1)}\left[s-\sqrt{s^{2}-4 u v \theta(\theta-1)}\right] \\
& =\lim _{\theta \rightarrow+0} \frac{1}{2(\theta-1)}\left[1+(u+v)(\theta-1)-\sqrt{(1+(u+v)(\theta-1))^{2}-4 u v \theta(\theta-1)}\right] \\
& =\frac{1}{2(0-1)}\left[1+(u+v)(0-1)-\sqrt{(1+(u+v)(0-1))^{2}-0}\right] \\
& =\frac{1}{2}(u+v-1+|1-u-v|) \\
& =\frac{1}{2}(u+v-1+|u+v-1|) \\
& =W
\end{aligned}
$$


and

$$
\begin{aligned}
\lim _{\theta \rightarrow \infty} C_{\theta}(u, v)= & \lim _{\theta \rightarrow \infty} \frac{1}{2(\theta-1)}\left[s-\sqrt{s^{2}-4 u v \theta(\theta-1)}\right] \\
= & \lim _{\theta \rightarrow \infty} \frac{1}{2(\theta-1)}\left[1+(u+v)(\theta-1)-\sqrt{(1+(u+v)(\theta-1))^{2}-4 u v \theta(\theta-1)}\right] \\
= & \lim _{\theta \rightarrow \infty} \frac{\theta}{2 \theta\left(1-\frac{1}{\theta}\right)} \\
& {\left[\frac{1}{\theta}+(u+v)\left(1-\frac{1}{\theta}\right)-\sqrt{\left(\frac{1}{\theta}+(u+v)\left(1-\frac{1}{\theta}\right)\right)^{2}-4 u v\left(1-\frac{1}{\theta}\right)}\right] } \\
= & \lim _{\theta \rightarrow \infty} \frac{1}{2\left(1-\frac{1}{\theta}\right)} \\
& {\left[\frac{1}{\theta}+(u+v)\left(1-\frac{1}{\theta}\right)-\sqrt{\left(\frac{1}{\theta}+(u+v)\left(1-\frac{1}{\theta}\right)\right)^{2}-4 u v\left(1-\frac{1}{\theta}\right)}\right] } \\
= & \frac{1}{2}(u+v-|u-v|) \\
= & M .
\end{aligned}
$$

Also, the bivariate normal family is comprehensive as $C_{-1}(u, v)=W$ and $C_{1}(u, v)=M$. On the other hand, the FGM family is not comprehensive as it includes $\Pi$ but neither $W$ nor $M$. If a family is comprehensive then the family parametrises the full range of association irrespective of the margins. From the sample selection modelling point of view this feature may be important for a modeller who has some prior intuition about the degree and direction of correlation between the error terms. For example, if the modeller suspects that the correlation will be low and negative then the FGM family might be appropriate as it incorporates negative low degrees of association.

\subsubsection{Measures of association}

Another important question to be answered is: what association parameter is supposed to be used once $\theta$ is estimated? The most common one is the Pearson's product moment coefficient. However, it suffers from the lack of transformation invariance with respect to the margins. Thus, we will be using Kendall's $\tau$ and Spearman's $\rho$ which are both bounded by -1 and 1 ; both are equal -1 at $W$, 1 at $M$ and 0 at $\Pi$. Let us define both measures. For Kendall's $\tau$, let $\left(x_{1}, y_{2}\right), \ldots,\left(x_{n}, y_{n}\right)$ be a set of pairs of $X$ and $Y$ variable realisations respectively. Any pair of realisations $\left(x_{i}, y_{i}\right)$ and $\left(x_{j}, y_{j}\right)$ is said to be concordant if both $x_{i}<x_{j}$ and $y_{i}<y_{j}$ or if both $x_{i}>x_{j}$ and $y_{i}>y_{j}$. A pair is said 
to be discordant if $x_{i}<x_{j}$ and $y_{i}>y_{j}$ or if $x_{i}>x_{j}$ and $y_{i}<y_{j}$. If either $x_{i}=x_{j}$ or $y_{i}=y_{j}$ the pair is neither concordant nor discordant. The Kendall's $\tau$ is defined as:

$$
\tau=\frac{\text { (number of concordant pairs })-(\text { number of discordant pairs })}{\frac{1}{2} n(n-1)} .
$$

If tied pairs arise Kendall's $\tau$ may not hold in the range [-1,1]. Instead, Kendall's $\tau$-b may be used to correct for the existence of ties:

$$
\tau=\frac{(\mathrm{ncp})-(\mathrm{ndp})}{\left(\mathrm{ncp}+\mathrm{ndp}+X_{0}\right)\left(\mathrm{ncp}+\mathrm{ndp}+Y_{0}\right)}
$$

where ncp is the number of concordant pairs, ndp is the number of discordant pairs, $X_{0}$ is the number of pairs tied only on the $\mathrm{X}$ variable, $Y_{0}$ is the number of pairs tied only on the $\mathrm{Y}$ variable.

On the other hand, Spearman's $\rho$ is defined as the commonly known Pearson's correlation coefficient between ranked variables. If for an $n$-sized sample the realisations $X_{i}, Y_{i}$ are converted into ranks $x_{i}, y_{i}$, then in order to obtain Spearman's $\rho$ one has to calculate the Pearson's coefficient correlation

$$
\rho=\frac{\sum_{i}\left(x_{i}-\bar{x}\right)\left(y_{i}-\bar{y}\right)}{\sqrt{\sum_{i}\left(x_{i}-\bar{x}\right)^{2} \sum_{i}\left(y_{i}-\bar{y}\right)^{2}}} .
$$

Let us provide an example on how ranks can be obtained. Consider Table 3.1. The variables in the first two columns are converted into ranks in ascending order relatively to their position in the variable. If two realisations have the same value on either of the two variables, the two consecutive ranks are averaged. For instance, in $y$ there are two entries taking the value of 0.4 . If the two entries would take values, say, 0.39 and 0.41 then we would assign them ranks 2 and 3. However, because the entries are taking the same value we average those ranks i.e. $(2+3) / 2=2.5$.

\begin{tabular}{|c|c|c|c|}
\hline$x_{i}$ & $y_{i}$ & $x_{(i)}$ & $y_{(i)}$ \\
\hline 8 & 0.4 & 4 & $(2+3) / 2=2.5$ \\
\hline 9 & 0.4 & 5 & $(2+3) / 2=2.5$ \\
\hline 1 & 0.2 & 2 & 1 \\
\hline 0 & 0.7 & 1 & 4 \\
\hline 3 & 0.8 & 3 & 5 \\
\hline
\end{tabular}

Table 3.1: Examples of ranks for Spearman's $\rho$.

Most importantly, both measures are transformation invariant to strictly increasing transforma- 
tion of variables, implying that they depend only on the copula of the joint distribution and not the margins. For instance, for the FGM, which is not comprehensive, $\tau=2 \theta / 9$ and $\rho=\theta / 3$. So, $-2 / 9 \leq \tau \leq 2 / 9$ and $-1 / 3 \leq \tau \leq 1 / 3$ (see Nelsen, 1999, Section 5.1).

\subsubsection{Archimedean copulas}

Let us proceed with the discussion of Archimedean copulas. The mathematical properties of the Archimedean class of copulas are captured by an additive generator function $\varphi$. We will see that $\varphi$ is useful when deriving the likelihood function. The properties of $\varphi$ are as follows (Smith, 2003):

- $\varphi: \mathbb{I} \rightarrow[0, \infty]$

- continuity,

- convexity and decreasing $\left(\varphi^{\prime}(t)<0\right.$ and $\left.\varphi^{\prime \prime}(t)>0\right)$,

- terminal at $\varphi(1)=0$,

- can be indexed by $\theta$ i.e. the whole family of copulas can be Archimedean.

In the bivariate case, $\varphi$ generates the copula as follows

$$
\varphi(C(u, v))=\varphi(u)+\varphi(v) .
$$

For details on obtaining this expression see Nelsen (2006, p. 109). Equation (3.3.10) can be recovered by

$$
C(u, v)=\varphi^{-1}(\varphi(u)+\varphi(v)) .
$$

For example, $\varphi(t)=-\log t$ is a generator of the Product copula $\Pi$. Examples of Archimedean copulas are listed in table 3.2 .

If the terminal $\varphi(0)=\infty$ the generator is called strict. In this case the inverse function $\varphi^{-1}$ exists and the copulas can be recovered as shown in (3.3.11). Otherwise, when $\varphi(0)<\infty$ the generators are non-strict and a pseudo-inverse $\varphi^{[-1]}$ has to be defined. As stated by Smith (2003, 


\begin{tabular}{|c|c|c|c|c|}
\hline Name & Copula $C_{\theta}(u, v)$ & $\begin{array}{c}\text { Parameter } \\
\text { space }\end{array}$ & Generator $\varphi(t)$ & Kendall's $\tau$ \\
\hline AMH & $u v /(1-\theta(1-u)(1-v))$ & $-1 \leq \theta \leq 1$ & $\log \frac{1-\theta(1-t)}{t}$ & $-0.1817 \leq \tau<\frac{1}{3}$ \\
\hline AP & $\begin{array}{c}\frac{1}{2}\left(r+\sqrt{r^{2}+4 \theta}\right), \\
=u+v-1-\theta\left(\frac{1}{u}+\frac{1}{v}-1\right)\end{array}$ & $0 \leq \theta<\infty$ & $(1+\theta / t)(1-t)$ & $-1 \leq \tau<\frac{1}{3}$ \\
\hline Clayton & $\left(u^{-\theta}+v^{-\theta}-1\right)^{-1 / \theta}$ & $0 \leq \theta<\infty$ & $\frac{1}{\theta}\left(t^{-\theta}-1\right)$ & $0 \leq \tau<1$ \\
\hline Frank & $\begin{array}{c}-\theta^{-1} \log \left(1+\left(e^{-\theta u}-1\right)\left(e^{-\theta v}-1\right) /\right. \\
\left.\left(e^{-\theta}-1\right)\right)\end{array}$ & $-\infty<\theta<\infty$ & $-\log \frac{e^{-\theta t}-1}{e^{-\theta}-1}$ & $-1<\tau<1$ \\
& $\begin{array}{c}\exp \left(-\left((-\log u)^{\theta}+(-\log v)^{\theta}\right)^{1 / \theta}\right) \\
\text { Gumbel }\end{array}$ & $1 \leq \theta<\infty$ & $(-\log t)^{\theta}$ & $0 \leq \tau<1$ \\
\hline Joe & $1-\left((1-u)^{\theta}+(1-v)^{\theta}-(1-u)^{\theta}\right.$ & $1 \leq \theta<\infty$ & $-\log \left(1-(1-t)^{\theta}\right)$ & $0 \leq \tau<1$ \\
\hline
\end{tabular}

Table 3.2: Examples of families of (bivariate) Archimedean copulas.

p. 105), in the modelling context it is not clear what gains might be made by specifying non-strict generators. Thus, like in Smith (2003) we will only refer to copulas with strict generators.

Also, for the Archimedean copula with generator $\varphi$, we have that

$$
\tau=1+4 \int_{0}^{1} \frac{\varphi(t)}{\varphi^{\prime}(t)} d t
$$

where $\varphi^{\prime}(t)=\frac{\partial}{\partial t} \varphi(t)$ (see Nelsen, 1999, p.130). Finally,

$$
\frac{\partial}{\partial v} C_{\theta}(u, v)=\frac{\varphi^{\prime}(v)}{\varphi^{\prime}\left(C_{\theta}(u, v)\right)}
$$

This result follows from (3.3.10) by simply differentiating both sides with respect to $v$ and reordering.

The wide range of distributional shapes that Archimedean copulas can depict is an indicator that members of this class may be useful in modelling. The copula approach determines each component of the overall model, then engineers them together using copula function. Thus, the copula approach does not guarantee unique identification of parameters as even for the same margins two different copulas will produce different set of parameter estimates. This is because the copula components of the likelihood function are different and the maximum likelihood parameter estimates will be different. Additionally, identicality in marginal distributions in the copula approach is not necessary (Smith, 2003).

\subsubsection{Copulas in sample selection models}

We will now discuss copulas in the sample selection modelling framework. For the time being, we will assume that both margins are continuous. The discrete-continuous case relevant for modelling 
counts will be considered in the next chapters. Let us recall equation (3.1.4) from Section 3.1,

$$
L=\prod_{i=1}^{n_{0}} \operatorname{Pr}\left(Y_{1}^{*} \leq 0\right) \prod_{j=1}^{n_{1}} f_{2 \mid 1}\left(y_{2} \mid y_{1}^{*}>0\right) \operatorname{Pr}\left(Y_{1}^{*}>0\right) .
$$

The probability in the first product can be rewritten as follows:

$$
\begin{aligned}
F_{1}(0) & =\operatorname{Pr}\left(Y_{1}^{*} \leq 0\right) \\
& =1-\operatorname{Pr}\left(Y_{1}^{*}>0\right)
\end{aligned}
$$

For the second product we know that

$$
\begin{aligned}
P\left(Y_{2}=y_{2}, y_{1}^{*}>0\right) & =f\left(y_{2}, y_{1}^{*}>0\right) \\
& =f_{2 \mid 1}\left(y_{2} \mid y_{1}^{*}>0\right) \operatorname{Pr}\left(Y_{1}^{*}>0\right)
\end{aligned}
$$

and so by rearranging

$$
f_{2 \mid 1}\left(y_{2} \mid y_{1}^{*}>0\right)=\frac{f\left(y_{2}, y_{1}^{*}>0\right)}{\operatorname{Pr}\left(Y_{1}^{*}>0\right)}
$$

Simultaneously,

$$
\begin{aligned}
F_{2 \mid 1}\left(y_{2} \mid y_{1}^{*}>0\right) & =\operatorname{Pr}\left(Y_{2} \leq y_{2} \mid Y_{1}^{*}>0\right) \\
& =\frac{\operatorname{Pr}\left(Y_{2} \leq y_{2}, Y_{1}^{*}>0\right)}{\operatorname{Pr}\left(Y_{1}^{*}>0\right)} \\
& =\frac{F\left(1, y_{2}\right)}{\operatorname{Pr}\left(Y_{1}^{*}>0\right)} .
\end{aligned}
$$

To obtain $f_{2 \mid 1}$ we have to differentiate the conditional cdf $F_{2 \mid 1}$ w.r.t. $y_{2}$. Thus,

$$
\begin{aligned}
f_{2 \mid 1}\left(y_{2} \mid y_{1}^{*}>0\right) & =\frac{\partial}{\partial y_{2}} F_{2 \mid 1}\left(y_{2} \mid y_{1}^{*}>0\right) \\
& =\frac{\partial}{\partial y_{2}} \frac{F\left(1, y_{2}\right)}{\operatorname{Pr}\left(Y_{1}^{*}>0\right)}
\end{aligned}
$$

The domain of the conditional density $f_{2 \mid 1}\left(y_{2} \mid y_{1}^{*}>0\right)$ in the second term can be plotted as shown in Figure 3.1. The values of the $y_{2}$ response are chosen arbitrarily to distinguish between the outcome 
and the latent selection process. The non-coloured area depicts the possible outcomes of $y_{2}$ under sample selection. Of course, the coloured area outcomes are not available as the outcomes are not observed.

Figure 3.1: Conditional density plot domain of $f_{2 \mid 1}\left(y_{2} \mid y_{1}^{*}>0\right)$. The white area represents possible $y_{2}$ response values under sample selection. In the peach area, outcomes are not observed.

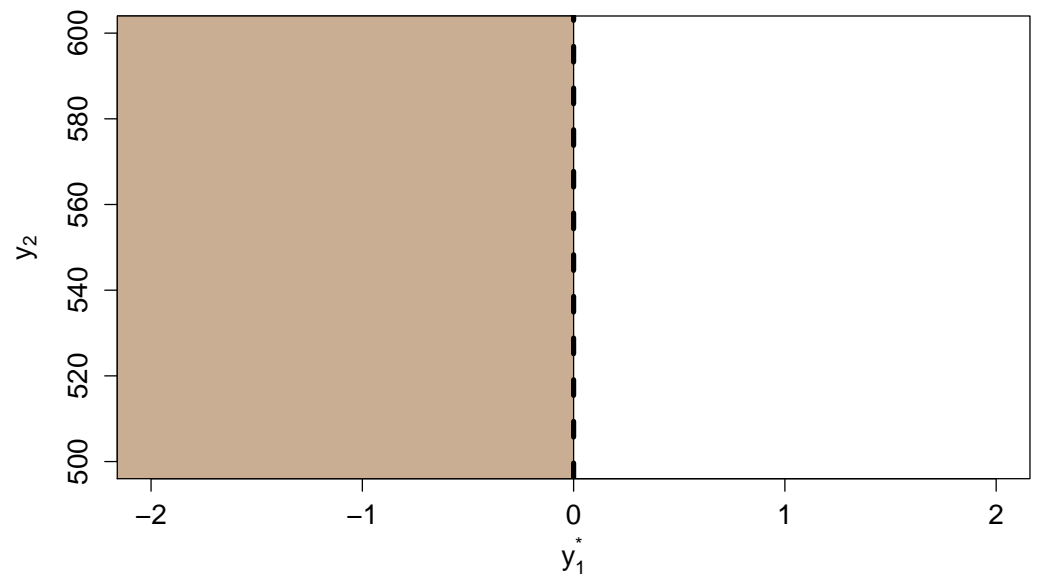

Specifically, the cdf for the coloured area is given as

$$
F\left(0, y_{2}\right)=F_{2}\left(y_{2}\right)-F\left(1, y_{2}\right)
$$

A rearrangement yields the intuitive result

$$
F_{2}\left(y_{2}\right)=F\left(0, y_{2}\right)+F\left(1, y_{2}\right)
$$

However, for our purposes we need to obtain an expression for $F\left(y_{2}, 1\right)$ i.e.

$$
F\left(1, y_{2}\right)=F_{2}\left(y_{2}\right)-F\left(0, y_{2}\right)
$$


Thus, the conditional density can be rewritten as follows:

$$
\begin{aligned}
f_{2 \mid 1}\left(y_{2} \mid y_{1}^{*}>0\right) & =\frac{\partial}{\partial y_{2}} \frac{F_{2}\left(y_{2}\right)-F\left(0, y_{2}\right)}{F_{1}(1)} \\
& =\frac{1}{1-F_{1}(0)} \frac{\partial}{\partial y_{2}}\left(F_{2}\left(y_{2}\right)-F\left(0, y_{2}\right)\right) \\
& =\frac{1}{1-F_{1}(0)}\left(f_{2}\left(y_{2}\right)-\frac{\partial}{\partial y_{2}} F\left(0, y_{2}\right)\right) .
\end{aligned}
$$

Substitution of (3.3.12) and (3.3.12) into (3.1.4) yields

$$
\begin{aligned}
L & =\prod_{i=1}^{n_{0}} F_{1}(0) \prod_{j=1}^{n_{1}}\left(f_{2}\left(y_{2}\right)-\frac{\partial}{\partial y} F\left(0, y_{2}\right)\right) \\
& =\prod_{i=1}^{n_{0}} F_{1} \prod_{j=1}^{n_{1}}\left(f_{2}-\frac{\partial}{\partial y_{2}} F\left(0, y_{2}\right)\right) .
\end{aligned}
$$

Example 4 The Lee's model with bivariate normal specification as a special case sets

$$
F\left(y_{1}, y_{2}\right)=\Phi_{2}\left(\Phi^{-1}\left(F_{1}\left(y_{1}^{*}\right)\right), \Phi^{-1}\left(F_{2}\left(y_{2}\right)\right) ; \theta\right)
$$

Assuming normality for both margins, i.e. $F_{1}\left(y_{1}^{*}\right)=\Phi\left(y_{1}^{*}-\boldsymbol{x}_{1}^{\top} \boldsymbol{\beta}_{1}\right)$ and $F_{2}\left(y_{2}\right)=\Phi\left(\frac{y_{2}-\boldsymbol{x}_{2}^{\top} \boldsymbol{\beta}_{2}}{\sigma}\right)$, equation (3.3.14) yields the standard sample selection model for the continuous response. The likelihood for the Lee model is:

$$
L=\prod_{i=1}^{n_{0}} F_{1} \prod_{j=1}^{n_{1}}\left(1-\Phi\left(\left(\Phi^{-1}\left(F_{1}\right)-\theta \Phi^{-1}\left(F_{2}\right)\right) / \sqrt{1-\theta^{2}}\right)\right) f_{2} .
$$

Example 5 The FGM model sets:

$$
F\left(y_{1}, y_{2}\right)=F_{1}\left(y_{1}^{*}\right) F_{2}\left(y_{2}\right)\left(1+\theta\left(1-F_{1}\left(y_{1}^{*}\right)\right)\left(1-F_{2}\left(y_{2}\right)\right)\right)
$$

Despite its mathematical simplicity the FGM copula is limited by its coverage $(-2 / 9 \leq \tau \leq 2 / 9)$. The likelihood for the FGM model is:

$$
L=\prod_{i=1}^{n_{0}} F_{1} \prod_{j=1}^{n_{1}}\left(1-F_{1}\right)\left(1-\theta F_{1}\left(1-2 F_{2}\right)\right) f_{2}
$$


We will derive the FGM likelihood function in the next lines. Recall (3.3.13). The first product of the likelihood in (3.3.13) is the same as above. Let us consider the second product i.e. $\left(f_{2}-\frac{\partial}{\partial y_{2}} F\left(0, y_{2}\right)\right)$. For FGM

$$
F\left(0, y_{2}\right)=F_{1}(0) F_{2}\left(y_{2}\right)\left(1+\theta\left(1-F_{1}(0)\right)\left(1-F_{2}\left(y_{2}\right)\right)\right)
$$

and its derivative w.r.t. $y_{2}$

$$
\frac{\partial}{\partial y_{2}} F\left(0, y_{2}\right)=F_{1}\left(1+\theta\left(1-F_{1}\right)\left(1-F_{2}\right)-F_{2} \theta\left(1-F_{1}\right)\right) f_{2}
$$

Thus,

$$
\begin{aligned}
f_{2}-\frac{\partial}{\partial y_{2}} F\left(0, y_{2}\right) & =f_{2}-F_{1}\left(1+\theta\left(1-F_{1}\right)\left(1-F_{2}\right)-F_{2} \theta\left(1-F_{1}\right)\right) f_{2} \\
& =\left(1-F_{1}-F_{1} \theta\left(1-F_{1}\right)\left(1-F_{2}\right)+F_{1} F_{2} \theta\left(1-F_{1}\right)\right) f_{2} \\
& =\left(1-\theta F_{1}\left(1-F_{2}\right)+F_{1} F_{2} \theta\right)\left(1-F_{1}\right) f_{2} \\
& =\left(1-\theta F_{1}\left(1-2 F_{2}\right)\right)\left(1-F_{1}\right) f_{2} .
\end{aligned}
$$

For more on Lee's and the FGM model see Lee (1983) and Prieger (2002). For the Archimedean class of copula models the term $\frac{\partial}{\partial y_{2}} F\left(0, y_{2}\right)$ in the likelihood function (3.3.13) simplifies to

$$
\begin{aligned}
\frac{\partial}{\partial y_{2}} F\left(0, y_{2}\right) & =\left.\frac{\partial}{\partial v} C_{\theta}\left(F_{1}, v\right)\right|_{v \rightarrow F_{2}} \times \frac{\partial F_{2}}{\partial y_{2}} \\
& =\frac{\varphi^{\prime}\left(F_{2}\right)}{\varphi\left(C_{\theta}\right)} \times f_{2},
\end{aligned}
$$

where $C_{\theta}$ denotes $C_{\theta}\left(F_{1}, F_{2}\right)=C_{\theta}\left(F_{1}(0), F_{2}\left(y_{2}\right)\right)$. Thus, by plugging into (3.3.13) one obtains

$$
L=\prod_{i=1}^{n_{0}} F_{1} \prod_{j=1}^{n_{1}}\left(1-\frac{\varphi^{\prime}\left(F_{2}\right)}{\varphi^{\prime}\left(C_{\theta}\right)}\right) f_{2}
$$

Table 3.3 shows expressions for the Archimedean copulas for the component $\left(1-\frac{\varphi^{\prime}\left(F_{2}\right)}{\varphi^{\prime}\left(C_{\theta}\right)}\right)$ :

Note that expressions of $\left(1-\frac{\varphi^{\prime}\left(F_{2}\right)}{\varphi^{\prime}\left(C_{\theta}\right)}\right)$ for the bivariate normal and the FGM copula are $(1-$ $\left.\Phi\left(\left(\Phi^{-1}\left(F_{1}\right)-\theta \Phi^{-1}\left(F_{2}\right)\right) / \sqrt{1-\theta^{2}}\right)\right)$ and $\left(1-F_{1}\right)\left(1-\theta F_{1}\left(1-2 F_{2}\right)\right)$, respectively, which have been used before. 


\begin{tabular}{|c|c|}
\hline Name & Expressions for $1-\frac{\varphi^{\prime}\left(F_{2}\right)}{\varphi^{\prime}\left(C_{\theta}\right)}$ \\
\hline AMH & $1-\frac{(1-\theta) F_{1}+\theta F_{1}^{2}}{\left(1-\theta\left(1-F_{1}\right)\left(1-F_{2}\right)\right)^{2}}$ \\
\hline Clayton & $1-F_{2}^{-(\theta+1)}\left(F_{1}^{-\theta}+F_{2}^{-\theta}-1\right)^{-\frac{1+\theta}{\theta}}$ \\
\hline Frank & $\frac{e^{\theta F_{2}}\left(e^{\theta F_{1}}-e^{\theta}\right)}{e^{\theta\left(F_{1}+F_{2}\right.}+e^{\theta}\left(1-e^{\theta F_{1}}-e^{\left.\theta F_{2}\right)}\right.}$ \\
\hline Gumbel & $1-C_{\theta}\left(F_{1}, F_{2}\right)\left(\left(-\log F_{1}\right)^{\theta}+\left(-\log F_{2}\right)^{\theta}\right)^{-1+1 / \theta}\left(-\log F_{2}\right)^{\theta-1} F_{2}^{-1}$ \\
\hline Joe & $\begin{array}{c}\left(1-\bar{F}_{1}^{\theta}\right) \bar{F}_{2}{ }^{\theta-1}\left(\bar{F}_{1}{ }^{\theta}+\bar{F}_{2}{ }^{\theta}-\bar{F}_{1}{ }^{\theta} \bar{F}_{2}{ }^{\theta}\right)^{-1+1 / \theta} \\
\text { where } \bar{F}_{1}=1-F_{1} \text { and } \bar{F}_{2}=1-F_{2}\end{array}$ \\
\hline
\end{tabular}

Table 3.3: Expressions for the Archimedean copula factor in the likelihood function (Smith, 2003)

\subsubsection{Benefits of copula approach}

The copula approach possesses highly desired features.

1. Unlike in the case of Greene the exact distribution of the response is known. Thus, we know what is being modelled, which will have its merits in practical applications.

2. The log-likelihood function can be determined for various marginal distributions with great simplicity. This suggests that the derivation of the gradient and the Hessian will be involved, but feasible.

3. Numerical integration and/or simulations are not necessary in fitting; except for the bivariate normal copula.

4. In principle, the optimisation can be conducted using commonly known techniques such as the quasi-Newton method (e.g. the BFGS algorithm).

5. As we are dealing with ML estimation the Akaike and Bayesian Information Criteria can be used to evaluate the competing models when performing sensitivity analysis for different copulas. Alternatively, we can apply the Vuong test (Vuong, 1989). In short, the purpose of the Vuong test is to infer about the closeness of two competing, nested or non-nested models to the true model. The null hypothesis states that both models are equally close to the true model. Formally

$$
H_{0}: E\left(\ln \frac{f(\boldsymbol{y} \mid \boldsymbol{x}, \boldsymbol{\alpha})}{g(\boldsymbol{y} \mid \boldsymbol{x}, \boldsymbol{\beta})}\right)=0
$$

where the expected value is defined with respect to the true data generating process, $f$ and 
$g$ are the competing distributions, $\boldsymbol{y}$ is the response data vector, $\boldsymbol{x}$ is the covariate vector, $\boldsymbol{\alpha}$ and $\boldsymbol{\beta}$ are the parameter vectors of the two competing models. The one-sided alternative hypothesis can be that either the model with distribution $f$ or the model with distribution $g$ is correct. The form of the test statistic will vary depending on whether the models are nested or non-nested (Cameron and Trivedi, 2005; Greene, 2002). For applied purposes it is important to note that the Vuong test can be used to compare models with the same covariates but different distributions or copulas.

6. ML properties such as the asymptotic normality, consistency and efficiency will hold if the usual (mild) regularity conditions are met.

The benefits of using copulas in a sample selection context seem to outnumber those of the approach by Greene. Thus, we will develop sample selection models for count data using the copula approach, with extensions to spline modelling. The reason for including splines of the continuous covariates is that the functional relation between covariates and response is typically not known a priori. In this situation employing splines reduces the risk of functional form misspecification.

\subsection{Other versions of models}

In this section we will outline other versions of sample selection models which may not be relevant in later sections but that are worth mentioning.

Terza (1998) introduces a count data endogenous switching model where both endogeneity of dummy variables (i.e. treatment effects) and sample selection effects are mitigated. Endogeneity arises if the model error is correlated with one or more covariates due to unobserved confounding. Ignoring endogeneity will lead to biased estimates. Two estimators are discussed: two-stage method of moments for the partially parametric model and non-linear weighted least squares for a fully parametric model. The two-stage method of moments consists of, first, estimating a probit equation like in Section 3.1. The second stage uses nonlinear least squares estimation for the outcome equation (for details see Terza, 1998, pp.133-135). On the other hand, the non-linear weighted least squares approach involves a two-stage method of moments estimation of the parameters in two equations as well as the correlation coefficient, whereas the variance of the outcome equation is estimated using a regression approach or conditional maximum likelihood. 
Chib et al. (2009) estimate a semiparametric model for data suffering from non-random sample selection and endogeneity based on continuous, binary, censored, or ordered regressors. We will now give an example of a one-equation semiparametric model without elaborating on its details. Consider

$$
g(\mu)=\boldsymbol{x}^{\top} \boldsymbol{\beta}+f_{1}\left(z_{1}\right)+f_{2}\left(z_{2}\right)+f_{3}\left(z_{3}, z_{4}\right)
$$

where $\boldsymbol{x}$ is a covariate vector assumed to have a parametric effect, $\boldsymbol{\beta}$ is the vector of parameters associated with the parametric component and $f_{1}, f_{2}$ and $f_{3}$ are the smooth (spline) functions of the continuous covariates $z_{1}, z_{2}, z_{3}$ and $z_{4}$ (Wood, 2006; Hastie and Tibshirani, 1986). $\mu=E(Y)$ and response $Y$ follows a distribution from the exponential family. Chib et al. (2009) argue that the introduction of non-parametric functions allows flexibility in modelling continuous covariate effects. Inference is based on Bayesian Markov Chain Monte Carlo (MCMC) methods. The algorithm does not require simulation of the missing outcomes which reduces the computational burden and improves the mixing properties of the proposed MCMC algorithm.

Kneib and Wiesenfarth (2010) introduce a geoadditive sample selection model to model postearthquake relief in Pakistan. They extend the Heckman model for continuous reponse by introducing spatial and temporal effects in the selection and outcome equations. The reason for this is that data were collected over time and the coefficients may vary with time. Also, to (at least partly) account for spatial correlation, spatial and temporal effects are modelled using Bayesian versions of smoothing splines. Inference is based on MCMC methods.

Marra and Radice (2013a) introduce a sample selection model for continuous data and model covariates flexibly using the regression spline approach. The specification is similar to the one used by Kneib and Wiesenfarth (2010) although the estimation approach is frequentist. Generally, in simulation the model by Marra and Radice (2013a) performs similar or better in reducing bias than that of Kneib and Wiesenfarth (2010). Also, the former approach is less time-consuming. The method is depicted in the RAND Health Insurance Experiment on annual health expenditures.

The same authors propose a sample selection modelling technique for binary data (Marra and Radice, 2013b). They propose two estimation procedures - the multi-stage and the bivariate probit estimation approaches. The two proposed approaches and a naïve probit are applied to the data 
from the American National Election Survey of 1992. Specifically, respondents were asked whether the government should intervene in ensuring that white and black children go to the same school. The individuals can be subdivided into two groups - those who responded and those who did not respond. Those who responded stated whether they support or do not support government intervention. The authors suggest that sample selection bias arises as those who did not respond felt that their opinion might have been socially unacceptable.

Marchenko and Genton (2012) develop the selection-t model in which the errors are modelled using a bivariate t-distribution. The model is applied to analyse ambulatory expenditures of individuals where the selection-t model indicated the presence of non-random sample selection as opposed to the classic Heckman model.

Hasebe and Vijverberg (2012) establish a sample selection model based on copulas and Generalized Tukey Lambda (GTL) distributions. The authors argue that GTL is a flexible univariate distribution allowing for skewness and thin- and heavy-tailed response behaviour. The models are tested in Monte Carlo simulations. Four illustrations are provided - wages of married women in Portugal, wages of school-aged children in Mexico, US Health Expenditures and speeding tickets in Massachusetts.

Miranda and Rabe-Hesketh (2006) discuss sample selection models for various types of responses including counts. The outcome equation of the count sample selection model is the linear predictor of the Poisson model. The selection equation is a probit including a random effect with a free parameter. The random effect is also the error term of the outcome equation. It can be shown that the model allows for overdispersion both when sample selection is existent and when it is not. No illustration specifically related to count sample selection modelling is provided.

\subsubsection{Why not a Bayesian approach?}

Before we proceed to the next chapter it is worth mentioning briefly the Bayesian approach. Note that despite the discussion below is not exhaustive, it provides some reasons why a frequentist approach is considered here. The advantage of Bayesian modelling is its flexibility as it decomposes

the model into smaller elements that are treated separately via the use of full conditionals. For example, if we believe that a parameter in the outcome equation has not a normal but gamma distribution, then we can easily replace the normal prior with a gamma prior. Thus, by changing 
the prior of one parameter the other parameter is not influenced. Moreover, the decomposition of MCMC into small parameter blocks has the advantage that estimation complexity grows linearly with the number of model terms added and not in a quadratic or cubic manner as it is the case of the frequentist approach. Also, MCMC does not require asymptotic results for inferences. On the other hand, if the sample size is small, then the choice of priors may lead to questioning of the results obtained using MCMC. Furthermore, MCMC methods may be less computationally efficient than maximum likelihood methods (Marra and Radice, 2013a).

The advantage of the frequentist approach is that it does not depend on the choice of priors or sampling performance. In addition, the estimates can be interpreted as the roots of the score function which can be useful for theoretical considerations. The disadvantage is that it is not in principle as modular as the Bayesian approach; introducing small changes in the model specification typically leads to the redevelopment of the numerical implementation. However, this issue can be mostly dealt with by identifying a general structure for the score vector and Hessian. Finally, inferences based on frequentist estimation rely on asymptotic proofs which may be difficult in some cases.

Many authors including Smith (2003) and Marra and Radice (2013a) have already used the frequentist approach for copula sample selection models. Here, we opt as well for the frequentist approach. We will now move on to the exploration of two data sets which will eventually illustrate the proposed count data sample selection models. Once this is done, we will introduce count sample selection models with linear or pre-specified covariate effects. 


\section{Chapter 4}

\section{Data and preliminary analysis}

The following two sections explain two data sets that can be used for sample selection modelling for count data. The first data set concerns hospital overutilisation whereas the second data set refers to labour mobility in labour economics. First, the practical significance of both data sets will be presented. Second, each data set will be explained in terms of its response and covariates. Third, an exploratory data analysis for the responses will be conducted together with formal distributional tests. Several gamlss-type models will be fitted using the gamlss R package (Stasinopoulos and Rigby, 2007) to find the distributions that suit the responses best. The reason for using gamlss is that it incorporates a wide variety of discrete distributions. Finally, we will apply the SemiParSampleSel R package (Marra et al., 2015) which can estimate semi-parametric sample selection models for continuous responses: the aim of this will be to approximately check whether non-random sample selection is likely to arise. To imitate continuous nature of data, the responses will be log-transformed. Non-transformed responses will be analysed in Chapter 7 using the proposed count data sample selection models.

\subsection{Veterans' Administration data}

\subsubsection{Introduction}

Since the end of World War II until the 1980s the health care costs in the United States have been rising approximately by 4 per cent per year in real terms (Levit et al., 1985). The most prominent explanation was the spread of health insurance which led to a higher quality and increased quantity 
of medical services. Simultaneously, it has been also argued that shifting part of the costs to the individual (i.e. introducing coinsurance) might lower total private health expenditure substantially. However, until the early 1980s no one has shown this (Manning et al., 1988).

From 1974 until 1982 the Research and Development (RAND) Institute conducted a social experiment whose aim was to explore whether and by how much varying coinsurance rates influence health care utilisation. The individuals were assigned to coinsurance groups by the experiment designers. Generally, the results showed a negative impact of co-insurance on health expenditure. The effects of the experiment followed immediately - Manning et al. (1988) claim that, despite the cost of 80 million US dollars between 1982 and 1984, there was a remarkable increase in cost sharing programmes. Hospital days among individuals under 65 decreased by 12.8 per cent and it is estimated that the cost saving amounted to 7.25 billion US dollars.

The link between the RAND experiment described above and sample selection models is that non-random sample selection can occur in such experiments (see: Manning et al., 1988, p. 17). The reason for this is that, as this is a social experiment and not a traditional confounder-controlled experiment, there exist unmeasured factors such as attitudes towards risks or life style which need to be controlled for (compare: Trivedi and Zimmer, 2007, p. 76). Based on those, less prudent individuals will select themselves into the sample of those who had positive health expenditures; hence, if this kind of sample selection on unobservables is not accounted for, then estimates obtained using standard modelling methods will be inconsistent. To cope with the problem of non-random sample selection, the authors of the RAND Institute experiment evaluation employ a four-equation model (Manning et al., 1988). As mentioned previously, the model indicated a negative impact of co-insurance on health expenditures. It can be speculated that a similar result can be obtained using sample selection models. If this is the case, then policymakers may consider introducing co-insurance schemes for public insurance.

More recent application of sample selection models in health care utilisation concerns hospital stay durations (Prieger, 2002). Prieger explains that hospitals have the tendency to release patients earlier than expected depending on whether the insurance company is willing to pay for the patient's stay. Also, he mentions that hospitals alter their care depending on the generosity of the payer. In this case, for example, if the public insurance patients stay shorter in hospitals than other patients, then it might be that the public system is underfinanced. Conversely, if the hospital stays are too 
long, then this would imply that the public system is overfinanced. Alternatively, a sample selection model can be used as a supportive tool in insurance pricing. Here, the interpretation will depend on the aim of the insuring institution. For instance, suppose that the insurance company aims at maximising client satisfaction. Also, in a modelling analysis it would turn out that their clients stay longer than other insurance holders. Then, it would imply that the product is well-recognised by hospitals and no actions have to be taken to modify it by the insurance company. On the other hand, let us assume that the company aims at short-term profit maximisation. In this case, the same modelling result would force the company to consider renegotiating contracts with the hospitals.

As we can observe, sample selection models can have a high utility in health economics as they can help answering questions from various angles that are essential to policymakers or insurers. Unlike in the previous applications where the responses were continuous, our responses will be counts.

\subsubsection{Data}

The data set on outpatient and inpatient visits originates from the 2001 National Survey of Veterans. Outpatients are individuals who do not stay overnight in the hospital (and attend doctor visits, medical tests etc.) whereas inpatients stay overnight in the hospital. Here, non-random sample selection arises due to the fact that individuals decide whether they should be treated or not. If they decide to be treated, then they choose between the Veterans' Administration (VA) or nonVeterans' Administration (non-VA) facilities and how many times they should be treated (Lahiri and Xing, 2004, p. 441). VA is a health care system run by the United States government for the war veterans. In this case the unmeasured confounders based on which non-random sample selection might occur are unidentified health shocks that might increase the number of hospital visits (e.g. car accidents), attitudes towards health risks (e.g. smoking or drinking) and life style (e.g. sport activity); see an analogical example in Trivedi and Zimmer (2007, p. 76).

The dataset contains 20,041 observations and 39 relevant variables including responses. Following the same methodology as Lahiri and Xing (2004) we will consider the inpatient and outpatient visits for VA and non-VA separately. The four responses are summarised in Tables 4.1 and 4.2. It is of interest to know the sample mean and variance for each response as one can intuitively model counts using the Poisson distribution for the outcome equation. Unfortunately, the respective sam- 
ple means and variances differ substantially for all responses, hence suggesting that a different distribution for the outcomes is needed.

\begin{tabular}{|c|c|}
\hline Response & Meaning \\
\hline NUMVAOUT & Number of VA outpatient visits \\
\hline NUMOTH & Number of non-VA outpatient visits \\
\hline VANITES & Nights hospitalised in VA facilities \\
\hline OTHNITES & Nights hospitalised in non-VA facilities \\
\hline
\end{tabular}

Table 4.1: Responses in the VA data.

\begin{tabular}{|c|c|c|}
\hline Response & Mean & Variance \\
\hline NUMVAOUT & 2.98 & 127.47 \\
\hline NUMOTH & 5.48 & 117.66 \\
\hline VANITES & 19.21 & 1108.075 \\
\hline OTHNITES & 9.99 & 228.38 \\
\hline
\end{tabular}

Table 4.2: Descriptive statistics of VA data responses.

Similarly, the histograms of the responses in Figure 4.1 indicate that there are extreme values which most likely contributed to the differences between the means and variances. At the same time, we cannot remove them as if they were outliers. Preliminary analysis, which consisted of determining whether the number of days during the year is realistic, did not reveal any problem with such observations. Hence they can be regarded as realisations of the true distribution. Again, it seems that for our modelling purposes we will have to consider an alternative distribution to the Poisson distribution.

The conclusions from the two previous paragraphs are confirmed by the Pearson's $\chi^{2}$ count data tests in which the null hypothesis was that the responses follow a Poisson distribution (for details see: Meyer et al., 2005). Clearly, as one can see in Table 4.3, the p-values of the tests are approximately zero indicating that the distributions are not Poisson. The same type of test was conducted for the negative binomial distribution and the null hypothesis was also rejected for all responses.

Still, the fact that the distributions are neither Poisson nor negative binomial does not discard them from future modelling. In fact, the previous analyses look at response distributions unconditional on covariates. Of course, as the final aim is to model the responses conditional on covariates, it seems reasonable to model the responses using various distributions including the Poisson and 
Figure 4.1: Histograms of responses for the VA data. The kernel density lines were obtained using density and lines functions. The smoothing kernel of density lines was chosen to be Gaussian, which is the default. The reponses indicate strong positive skewness and many extreme values.
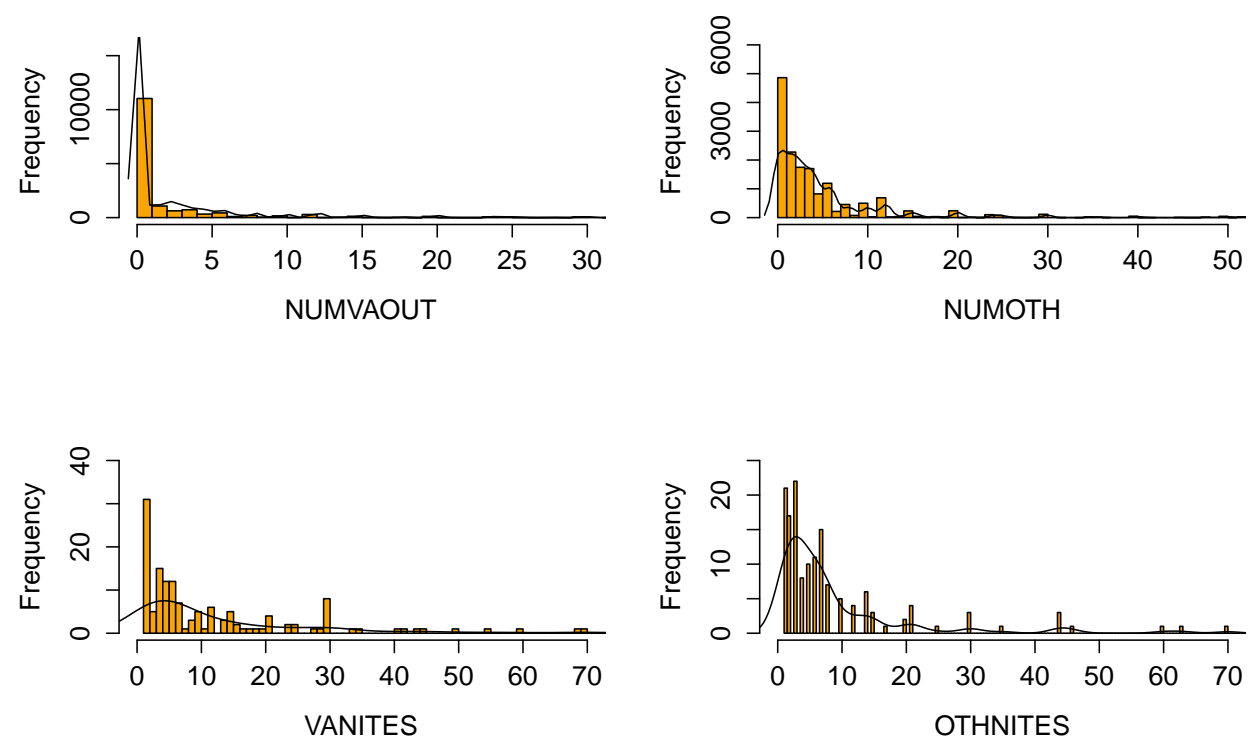

\begin{tabular}{|c|c|c|}
\hline Response & Poisson & Negative Binomial \\
\hline NUMVAOUT & 0 & 0 \\
\hline NUMOTH & 0 & 0 \\
\hline VANITES & 0 & $7.35 \times 10^{-28}$ \\
\hline OTHNITES & 0 & $1.17 \times 10^{-27}$ \\
\hline
\end{tabular}

Table 4.3: P-values for the chi-squared tests applied to the count responses described in Table 4.1.

the negative binomial as well. For the time being, we will ignore non-random sample selection and focus on finding which distributions are best suited for modelling the responses of interest.

We will now use the gamlss $R$ package to model each of the four responses (Stasinopoulos and Rigby, 2007). In brief, the gamlss R package is an implementation of GAMLSS models, which are aimed at univariate regression type statistical problems using new ways of dealing with overdispersion, skewness and kurtosis in the response variable (Stasinopoulos and Rigby, 2005). This is done by allowing for a wide variety of distributions (which can be different to the ones from the exponential family). Since many variants of discrete distributions are also included, gamlss seems to be a natural choice. Thus, in addition to the Poisson and the negative binomial we will consider other discrete distributions such as the Delaporte, Poisson Inverse Gaussian and Sichel distributions.

For each response we will use the same set of covariates except for the two inpatient responses 
where the variable indicating individuals' debts (DEBTS; see Table 4.4) was not included. The reason for this is that when estimating Delaporte gamlss for VA inpatients, which includes DEBTS, an error occurs. The error message stated that the global deviance was increasing and a different choice of specification is suggested. Possibly, the convergence problem may have been due to the fact that debt of individuals ranged from zero to tens of thousands of US dollars. Thus, for comparability of fit across models DEBTS was dropped for inpatients.

Only observations with recorded counts will be considered (i.e. those individuals who used a given medical service from any provider). The general equation of the outpatient models' linear predictor is

$$
\begin{aligned}
\eta= & \beta_{0}+f_{1}(\text { VETSAGE })+\beta_{1} \text { HRSWORK }+\beta_{2} \text { DEBTS }+\beta_{3} \text { HEATLH }+\beta_{4} \text { MARRY }+\beta_{5} \text { DISACOND } \\
& +\beta_{6} \text { GRADE }+\beta_{7} \text { WHITE }+\beta_{8} \text { MEDICAID }+\beta_{9} \text { MEDICARE }+\beta_{10} \text { EXTSEX }+\beta_{11} \text { HIGHBP } \\
& +\beta_{12} \text { LUNG }+\beta_{13} \text { HEAR }+\beta_{14} \text { ENT }+\beta_{15} \text { EYE }+\beta_{16} \text { CANCER }+\beta_{17} \text { HEART } \\
& +\beta_{18} \text { STROKE }+\beta_{19} \text { KIDNEY }+\beta_{20} \text { RHEUM }+\beta_{21} \text { LIVER }+\beta_{22} \text { HIV }+\beta_{23} \text { DIABETES } \\
& +\beta_{24} \text { STOMACH }+\beta_{25} \text { CHRONIC }+\beta_{26} \text { DRUGS }+\beta_{27} \text { PTSD }+\beta_{28} \text { MENTAL }+\beta_{29} \text { INJURY } \\
& +\beta_{30} \text { TXOTH },
\end{aligned}
$$

where $f_{1}$ is a smooth function which represents the non-parametric part of the equation. In our case it was a cubic smoothing spline. All variables mentioned in equation (4.1.1) are explained in Table 4.4. It is worthwhile to note that (4.1.1) is dominated by factor variables. These include the most frequent health problems individuals encountered. Also, we included Medicaid and Medicare insurance plans. Those variables might be interesting for a practitioner when, for instance, the relative performance of insurance plans needs to be assessed. As mentioned previously, by estimating parameters associated with Medicaid and Medicare, it may be concluded whether the public insurance system is under- or overfinanced.

Figures D.1, D.2, D.3, 4.2 and D.4 show the VA outpatient diagnostic plots for the Poisson, negative binomial, Delaporte, Poisson Inverse Gamma and Sichel distributions respectively. To avoid clutter, most figures were placed in Appendix D and only the "best" ones were kept. It is important to note that the residuals were normalised and randomised. Therefore, for the discrete case the 
normalised and randomised residuals will be given by $\hat{r}=\Phi^{-1}\left(u_{i}\right)$ where $\Phi^{-1}$ is the inverse cdf of a standard normal and $u_{i}$ is a random value from the uniform distribution on $\left[F\left(y_{i}-1 \mid \hat{\boldsymbol{\theta}}^{\boldsymbol{i}}\right), F\left(y_{i} \mid \hat{\boldsymbol{\theta}}^{\boldsymbol{i}}\right)\right]$ where $y_{i}$ is the observed response and $\hat{\boldsymbol{\theta}}^{i}$ is the vector of estimated parameters. Parameter estimates have the superscript $i$ to indicate that the estimated mean will be different for each observation since different covariate values will be used. Due to normalisation our interpretation of the graphs will be the same as if our reference distribution was standard normal. Randomisation consists in making the discrete distribution as if it was continuous (see Smyth and Dunn, 1996). For drawing conclusions on QQ-plots, Stasinopoulos et al. (2008) recommend to use multiple plots with many realisations of $u_{i}$. However, as we are dealing with a large data set and need to only have a distributional overview we will avoid this step.

The plots in the upper right corner of each figure are the default diagnostic option in gamlss which is supposed to be used for checking the independence assumption. However, since we do not have an ordering variable it is difficult to check this assumption. The quantile-residual-fitted-value plots in the upper left corner of the plots indicate a considerable spread for the lower values whereas as the fitted values increase the spread decreases. This is a sign of heteroskedasticity. However, of greater interest are the bottom plots in each figure, i.e. the residual density and the quantilequantile plots which evaluate the suitability of the chosen distribution to the data. For example, as in Figure D.1 the observations do not lie on the QQ-plot reference line it is unlikely that the data follow the Poisson distribution. The plots for the negative binomial, Delaporte and Sichel distributions indicate a considerable improvement. The best fit regarding the distribution can be obtained via the Poisson Inverse Gaussian distribution as almost all the points lie on the straight line. 


\begin{tabular}{|c|c|}
\hline Variable & Description \\
\hline VETSAGE & Age of the veteran in years \\
\hline HRSWORK & Usual number of hours worked each week \\
\hline DEBTS & Debt value in USD excluding mortgage loans \\
\hline HEATLH & Self-rated health condition 1-5 (factor; 1 - best, 5 worst) \\
\hline MARRY & $\begin{array}{l}\text { Marital status ( } 1 \text { - married and living with spouse, } \\
2 \text { - married and not living with spouse, } 3 \text { - widowed, } \\
4 \text { - divorced, } 5 \text { - legally separated, } 6 \text { - never married) }\end{array}$ \\
\hline DISACOND & Disability condition (binary) \\
\hline GRADE & $\begin{array}{c}\text { Highest educational grade } \\
\text { (0 - no formal schooling to } 14 \text { - Doctoral Degree) }\end{array}$ \\
\hline WHITE & White race (binary) \\
\hline MEDICAID & Covered by Medicaid/Medi-Cal (binary) \\
\hline MEDICARE & Covered by Medicare (binary) \\
\hline EXTSEX & Individual's gender (binary) \\
\hline & Last year treatment received for... (binary) \\
\hline HIGHP & high blood pressure \\
\hline LUNG & lung trouble \\
\hline HEAR & a hearing condition requiring a hearing aid \\
\hline ENT & other ear, throat, nose condition \\
\hline EYE & eye/vision problem including needing glasses \\
\hline CANCER & cancer \\
\hline HEART & heart trouble \\
\hline STROKE & a stroke \\
\hline KIDNEY & kidney or bladder trouble \\
\hline RHEUM & arthritis or rheumatism \\
\hline LIVER & hepatitis C or other liver disease \\
\hline HIV & an immune deficiency disease like HIV/AIDS \\
\hline DIABETES & diabetes requiring insulin or diet treatment \\
\hline STOMACH & stomach or digestive disorder \\
\hline CHRONIC & severe chronic pain \\
\hline DRUGS & drug abuse or alcoholism \\
\hline PTSD & post-traumatic stress disorder \\
\hline MENTAL & other mental or emotional problems \\
\hline INJURY & an accident-related injury \\
\hline TXOTH & any other serious condition \\
\hline
\end{tabular}

Table 4.4: VA covariate description. The two continuous variables are VETSAGE and DEBTS. All other variables are factors. Covariates indicating a medical condition are binary. 
Figures D.5, D.6, D.7, D.8 and 4.3 represent the non-VA outpatient diagnostic plots for the same set of distributions as before. Given the quantile-residual-versus-fitted-value plots we are dealing with heteroskedasticity, although this seems to be not as apparent as for the VA outpatient diagnostic plots. The Poisson distribution seems to be the worst distributional choice. The best choice would be the Sichel distribution. All other distributions show a reasonable fit. Similarly, Figures D.9, D.10, D.11, D.12 and 4.4 depict the VA inpatient diagnostic plots for the Poisson, negative binomial, Delaporte, Poisson Inverse Gamma and Sichel distributions. Unlike in the case of the outpatients, heteroskedasticity does not seem to be particularly troublesome. Generally, the fit of the distributions is not as good as that of the previous ones. Nevertheless, the Delaporte, Poisson Inverse Gaussian and Sichel distributions provide a satisfactory fit to the data. Finally, Figures D.13, D.14, D.15, 4.5 and D.16 show the non-VA inpatient diagnostic plots. In the upper left panel of Figure 4.5 observations are concentrated in the lower left corner of the plot, which may be a sign of heteroskedasticity. In other cases, it is hard to deduce from the plots whether heteroskedasticity is present. Similarly to the VA inpatients, the best fit is provided by the Delaporte, Poisson Inverse Gaussian and Sichel distribution based models. 
Figure 4.2: Poisson Inverse Gaussian model diagnostics for the NUMVAOUT response.

Against Fitted Values

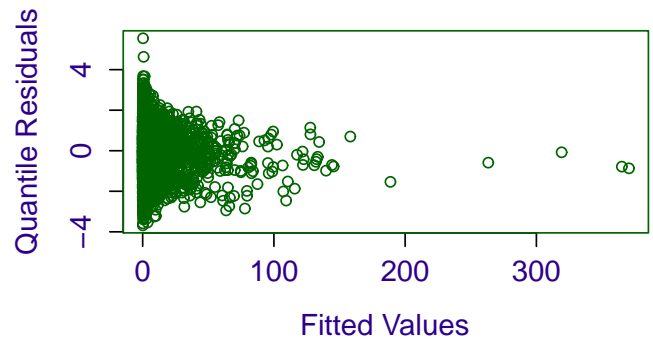

Density Estimate

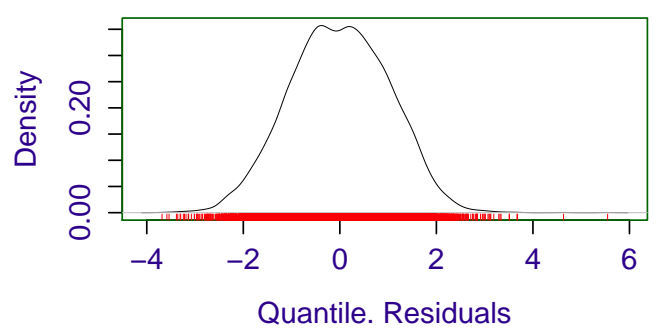

Against index

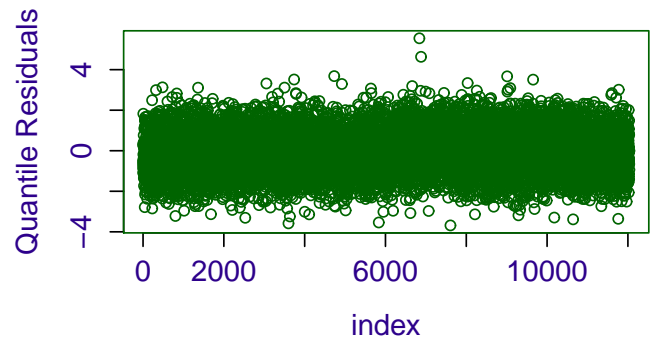

Normal Q-Q Plot

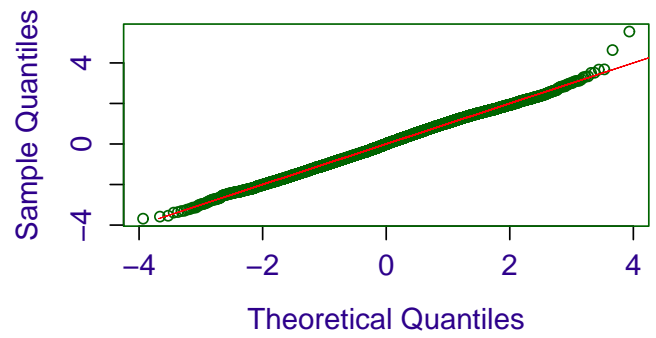

Figure 4.3: Sichel model diagnostics for the NUMOTH response.

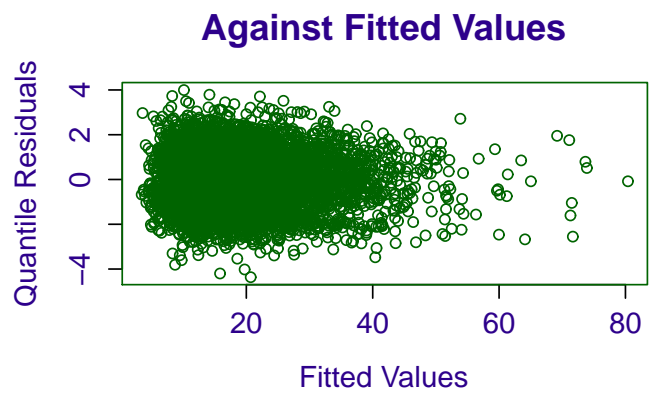

Density Estimate

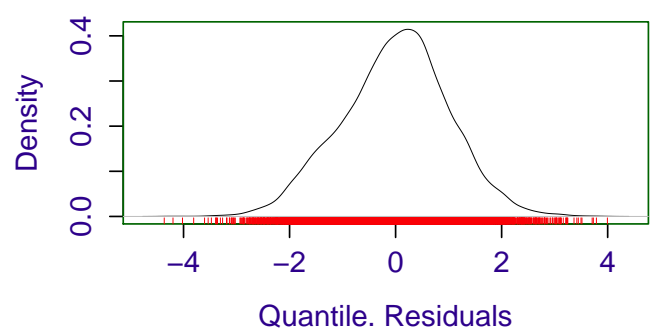

Against index

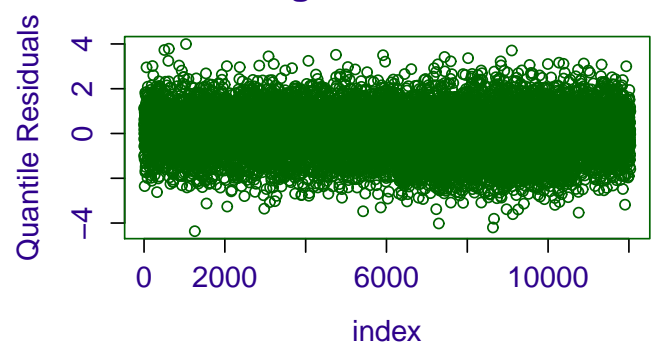

Normal Q-Q Plot

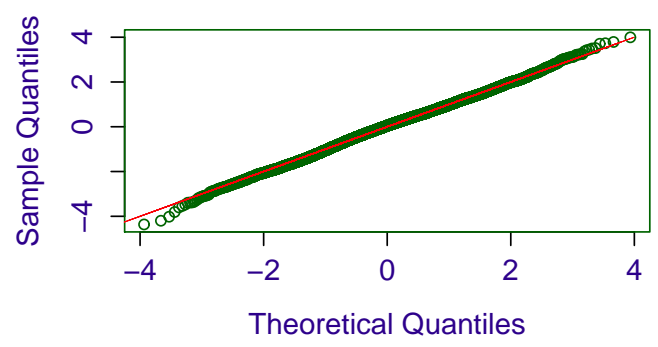


Figure 4.4: Sichel model diagnostics for the VANITES response.

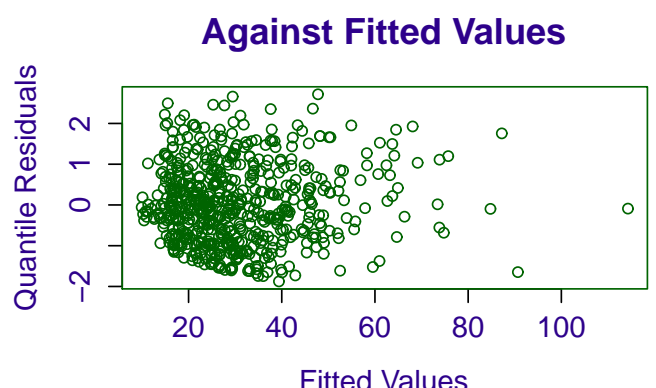

Density Estimate

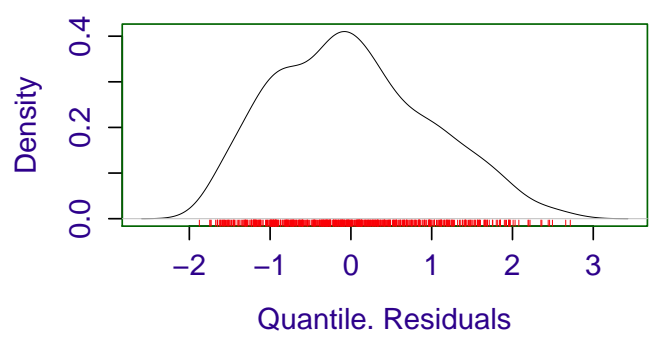

Against index

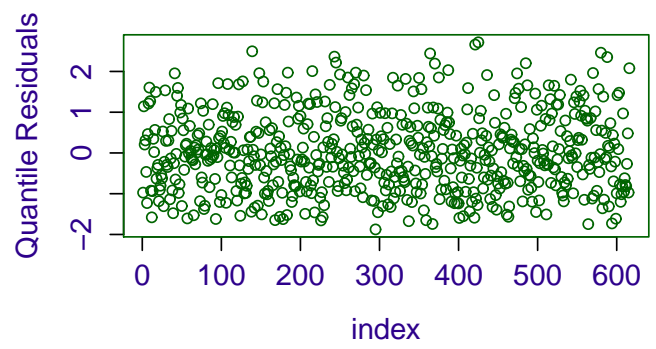

Normal Q-Q Plot

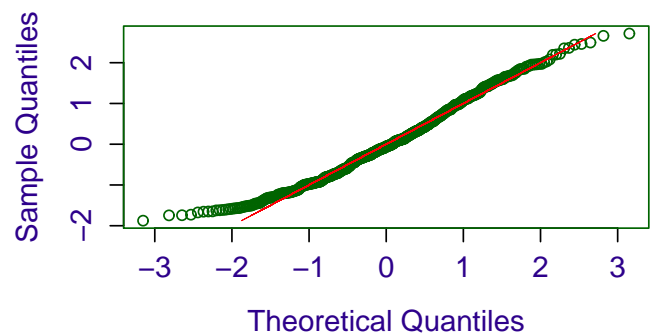

Figure 4.5: Poisson inverse Gaussian model diagnostics for the OTHNITES response.

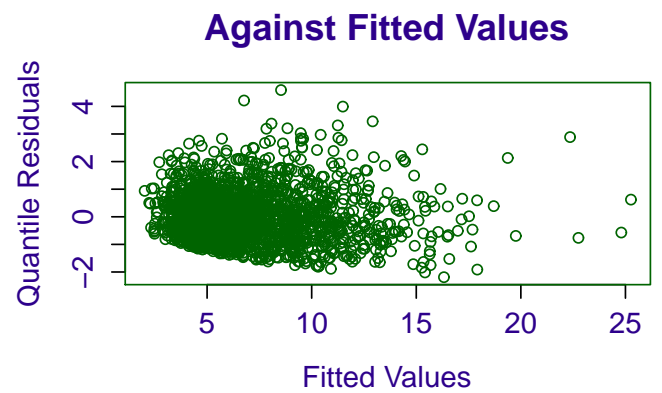

Density Estimate

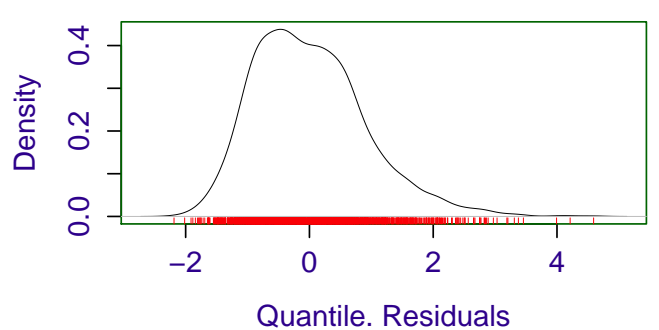

Against index

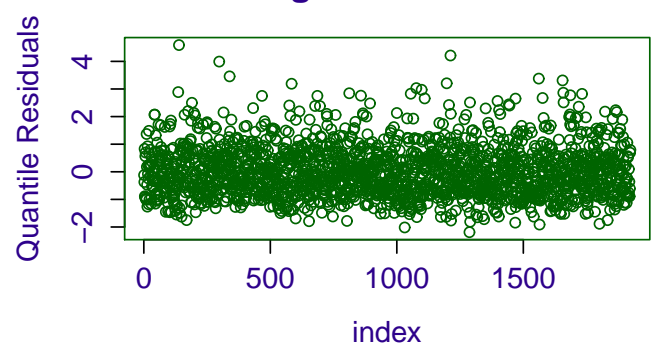

Normal Q-Q Plot

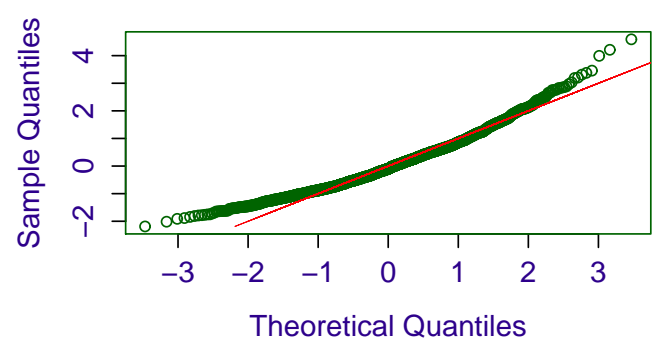


To preliminarily assess the extent of non-random sample selection issue we estimated a semiparametric sample selection model for normally distributed outcome. For simplicity, we will only focus on the response NUMVAOUT and the modelling remarks for other responses will be only stated. As NUMVAOUT is a count variable we will apply the log-transformation in order to model the skewness arising from the discrete nature of data. The selection and outcome equations are

$$
\begin{aligned}
Y_{1}^{*}= & \gamma_{0}+f_{11}(\text { VETSAGE })+\gamma_{1} \text { HRSWORK }+\gamma_{2} \text { EXTSEX }+\gamma_{3} \text { HEATLH }+ \\
& \gamma_{4} \text { DISACOND }+\gamma_{5} \text { HIGHBP }+\gamma_{6} \text { LUNG }+\gamma_{7} \text { HEAR }+ \\
& \gamma_{8} \text { ENT }+\gamma_{9} \text { EYE }+\gamma_{10} \text { CANCER }+\gamma_{11} \text { HEART }+ \\
& \gamma_{12} \text { STROKE }+\gamma_{13} \text { KIDNEY }+\gamma_{14} \text { RHEUM }+\gamma_{15} \text { LIVER }+ \\
& \gamma_{16} \text { HIV }+\gamma_{17} \text { DIABETES }+\gamma_{18} \text { STOMACH }+\gamma_{19} \text { CHRONIC }+ \\
& \gamma_{20} \text { DRUGS }+\gamma_{21} \text { PTSD }+\gamma_{22} \text { MENTAL }+\gamma_{23} \text { INJURY }+ \\
& \gamma_{24} \text { TXOTH }, \\
Y_{2}= & \beta_{0}+f_{21}(\text { VETSAGE })+\beta_{1} \text { HRSWORK }+\beta_{2} \text { DEBTS }+\beta_{3} \text { EXTSEX }+ \\
& \beta_{4} \text { HEATLH }+\beta_{5} \text { DISACOND }+\beta_{6} \text { GRADE }+\beta_{7} \text { WHITE }+ \\
& \beta_{8} \text { MEDICAID }+\beta_{9} \text { MEDICARE }+\beta_{10} \text { HIGHBP }+\beta_{11} \text { LUNG }+ \\
& \beta_{12} \text { HEAR }+\beta_{13} \text { ENT }+\beta_{14} \text { EYE }+\beta_{15} \text { CANCER }+ \\
& \beta_{16} \text { HEART }+\beta_{17} \text { STROKE }+\beta_{18} \text { KIDNEY }+\beta_{19} \text { RHEUM }+ \\
& \beta_{20} \text { LIVER }+\beta_{21} \text { HIV }+\beta_{22} \text { DIABETES }+\beta_{23} \text { STOMACH }+ \\
& \beta_{24} \text { CHRONIC }+\beta_{25} \text { DRUGS }+\beta_{26} \text { PTSD }+\beta_{27} \text { MENTAL }+ \\
& \beta_{28} \text { INJURY }+\beta_{29} \text { TXOTH },
\end{aligned}
$$

where $Y_{1}^{*}$ and $Y_{2}$ are the latent and (log-transformed) outcome variables respectively. Note that like in the original approach by Lahiri and Xing (2004) no instrumental variable was used. In our case, the number of variables in the outcome is greater than that in the selection equation. The estimation results showed that Kendall's $\tau$ amounted to -0.078 with the 95 per cent confidence interval (-0.129,0.025). This indicates that we are dealing with a weak, but probably existent non-random sample selection. Also the smooth terms in Figure 4.6 indicate shapes that agree with common sense. For 
example, the likelihood of having an outpatient visit increases with age up to approximately the age of 65 . Then it decreases as the patient may require an inpatient treatment. According to the author's best knowledge, no similar result was found in the literature. The interpretation of the smooth from the outcome equation could be that the patients first seek standard low-cost health care methods by visiting the VA administration and later require more specialised health care and hence address non-VA inpatient facilities. Alternatively, one can speculate that the 40-year old patients overutilise the VA health care system. In order to verify this more research is needed. Figure 4.7 shows the smooths of the standard generalized additive model (GAM) and probit model. As compared to the sample selection model, the probit smooth has the same shape as the selection equation smooth. However, the GAM smooth differs from the outcome equation smooth - the individuals steadily use more VA outpatient facilities until they reach the age of 60 ; after that there is a sharp decline in their usage. As we can observe, when not accounting for non-random sample selection the interpretation of the smooth terms may change.

For the three remaining responses the same set of covariates was used as for NUMVAOUT. The correlation coefficients for each response are presented in Table 4.5. As we can see all estimates are negative which implies a downward bias. Also, three out of four confidence intervals do not include zero indicating that the selection and outcome equations are correlated and hence nonrandom sample selection arises. The lowest value of Kendall's $\tau$ amounting to -0.45 is obtained for NUMOTH. A possible explanation of this result is that individuals who are more risk-prone try to avoid utilising costly non-VA facilities and search alternative treatment methods.

\begin{tabular}{|c|c|c|}
\hline Response & Kendall's $\tau$ & Confidence interval \\
\hline NUMVAOUT & -0.078 & $(-0.129,-0.025)$ \\
\hline NUMOTH & -0.45 & $(-0.486,-0.414)$ \\
\hline VANITES & -0.121 & $(-0.555,0.416)$ \\
\hline OTHNITES & -0.189 & $(-0.364,-0.003)$ \\
\hline
\end{tabular}

Table 4.5: Kendall's $\tau$ for the four VA data responses. For three out of four responses non-random sample selection is likely to be present. Kendall's $\tau$ for VANITES is small and negative, but nonrandom sample selection is unlikely to be present. 
Figure 4.6: Selection (left) and outcome (right) equation smooth terms (NUMVAOUT). The horizontal axis represents veterans' age and the vertical axis shows the smooth value on the linear predictor scale. The numbers in brackets on the vertical axis are the effective degrees of freedom of the smooth which will be described in Chapter 6 . The smooth from the selection equation suggests that the likelihood of having an outpatient visit increases with age up to approximately the age of 65 . Then it decreases as the patient may require an inpatient treatment. The shape of the outcome equation smooth may indicate that the patients first seek standard low-cost health care methods by visiting the VA administration and later require more specialised health care and hence address non-VA inpatient facilities. Alternatively, one can speculate that the 40-year old patients overutilise the VA health care system.
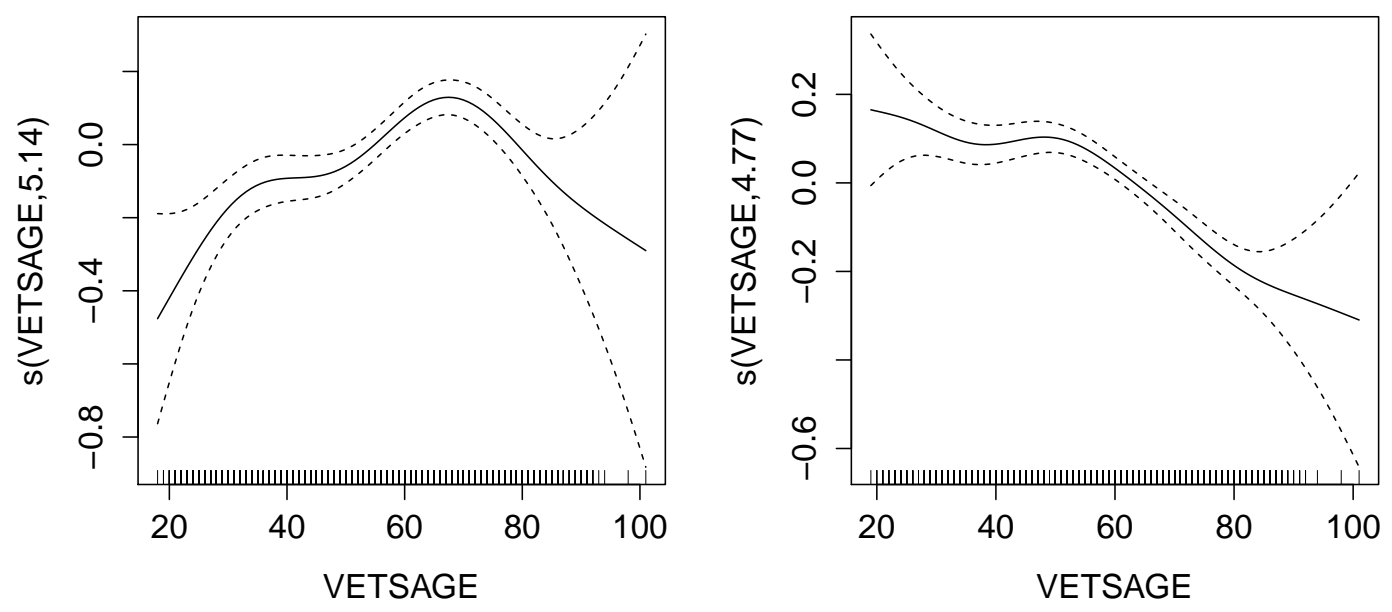
Figure 4.7: Naïve probit (left) and naïve GAM (right) smooth terms (NUMVAOUT). The horizontal axis represents veterans' age and the vertical axis shows the smooth value on the linear predictor scale. The numbers in brackets on the vertical axis are the effective degrees of freedom of the smooth which will be described in Chapter 6 . The shape of the selection equation smooth is similar to the one in Figure 4.6 and the same interpretation follows. However, the GAM smooth differs from the outcome equation smooth - the individuals steadily more use VA outpatient facilities until they reach the age of 60; after that there is a sharp decline in their usage. Not accounting for non-random sample selection may change the interpretation of the smooth terms.
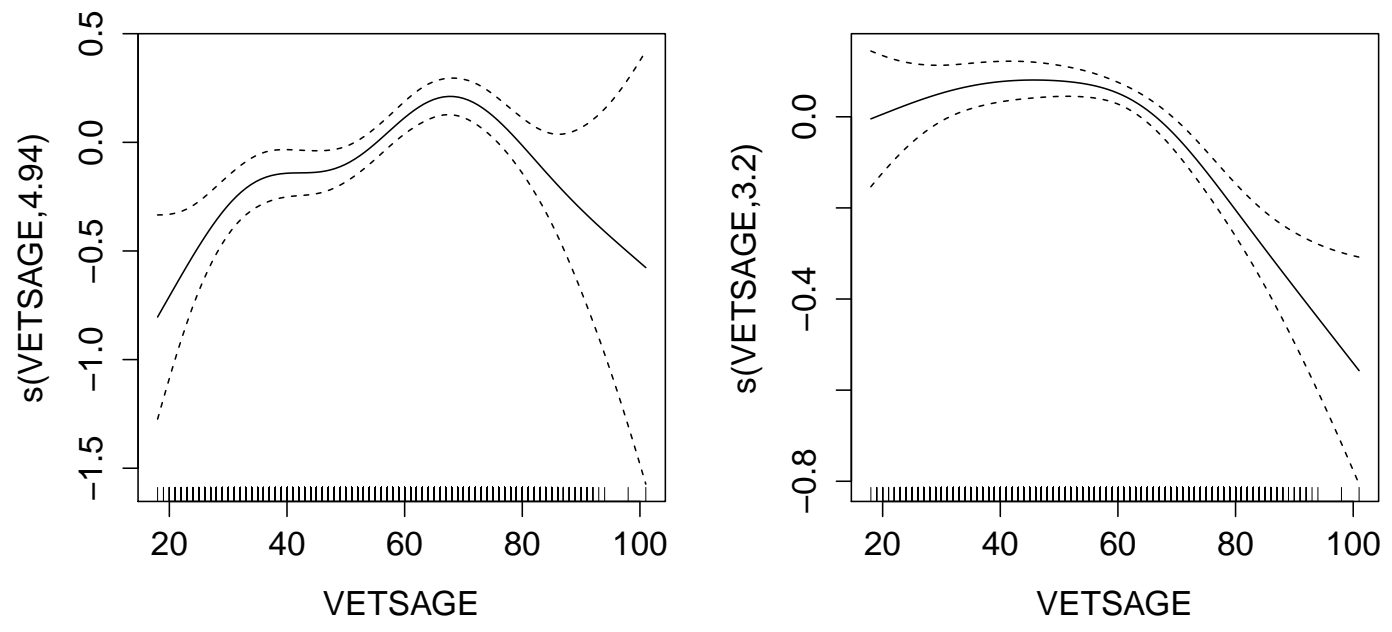


\subsection{German Socio-Economic Panel data}

\subsubsection{Introduction}

In labour economics labour mobility, defined as the number of job changes within a time period, plays an essential role for explaining unemployment. First, if labour mobility is high, then the labourers will be more flexible in changing jobs from declining industries (e.g. heavy industry) to rising industries (e.g. high-tech industry). If this is the case then the problem of mismatch between labour supply and demand, commonly known as structural unemployment, can be mitigated (Klau and Mittelstadt, 1986). Second, empirically there is a negative correlation between job creation/destruction rates and unemployment rates. For instance, countries such as the United States or the United Kingdom have higher job creation and job destruction rates, and lower unemployment rates than other countries. Therefore, intuitively, it is in the policymakers' interest to foster labour mobility (see also Boeri and van Ours, 2008, Chapter 10). In this part of the work we will explore a data set on labour mobility counts and try to fit gamlss-type models with different distributions.

\subsubsection{Data}

The data set is from the German Socio-Economic Panel survey of 1984 (SOEP v28). Following Winkelmann (1998) the response of interest will be 'direct job changes' of German male workers aged between 35 and 60 over ten years which is defined as the difference between job changes and the number of unemployment periods. This is supposed to stand for the 'voluntary' mobility (Winkelmann, 1998). In the following setup, individuals decide on whether they are engaged in search in the first place. If they are, then they may voluntarily change jobs a number of times. It can be the case that individuals who decided to be active on the labour market differ from the inactive ones on non-observed characteristics such as, for instance, labour market wage. Hence, the estimates obtained using standard models will be biased and inconsistent. On the other hand, if unobserved characteristics could be included in the standard modelling approach, then selection bias could be avoided.

The subsample consists of 2651 observations. The mean of the response is 0.53 and the variance 1.19. $66 \%$ of workers did not voluntarily change their jobs over ten years. These results are very similar to the ones obtained by Winkelmann except for the fact that our sample was twice as large. 
Similarly, to the VA data we conducted a chi-squared test for count data with the null hypothesis that the response follows a Poisson or negative binomial distribution. As the p-values were close to zero it is unlikely that the response unconditional on covariates follows either the Poisson or negative binomial distribution.

As for the VA data case, we will fit gamlss with different distributions. The explanatory variables used for gamlss and sample selection models are listed in Table 4.6. In the original work by Winkelmann (1998) one of the explanatory variables was union membership. However, due to the fact that we could not find it a 'proxy' variable was created. This was a binary variable indicating whether someone was a strong or a very strong supporter of the Socialdemocratic Party of Germany (SPD). To justify the choice of the proxy variable, according to Hartenstein and Liepelt (1962), in 1961 there were 6 million labour unionists among workers of which two thirds were repetitively casting SPD votes. As we can see there is association between labour union membership and political views. The linear predictor equation looks as follows

$$
\eta=\beta_{0}+f_{1}(\text { LEduc })+\beta_{1} \text { WhiteCollar }+\beta_{2} \text { SPDSup }
$$

where $f_{1}$ is the non-parametric component (defined in terms of cubic smoothing splines) and $\eta$ is the linear predictor.

\begin{tabular}{|c|c|}
\hline Variable & Description \\
\hline EXPFT & Full-time employment working experience \\
\hline Single & 1 if the individual is single; 0 otherwise \\
\hline WhiteCollar & 1 if the indivdual is a white collar worker; 0 otherwise \\
\hline LEduc & Length of education in years \\
\hline SPDSup & 1 if the indivdual is a strong or a very strong supporter of SPD; 0 otherwise \\
\hline
\end{tabular}

Table 4.6: Variables used for gamlss and sample selection model for SOEP data

Considering Figures D.17, D.18, 4.8, D.19 and D.20 we can state that all distributions provide a good fit to the data apart from the Poisson distribution. Surprisingly, unlike for the VA data heteroskedasticity does not seem to constitute a problem. 
Figure 4.8: Delaporte model diagnostics for the SOEP response.

Against Fitted Values

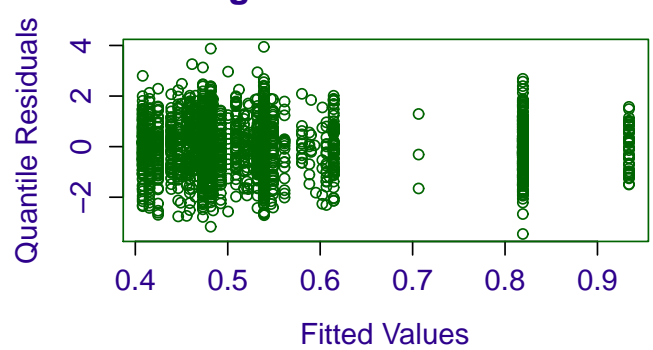

Density Estimate

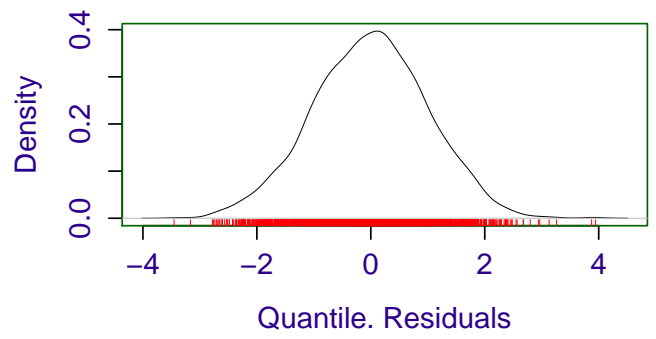

Against index

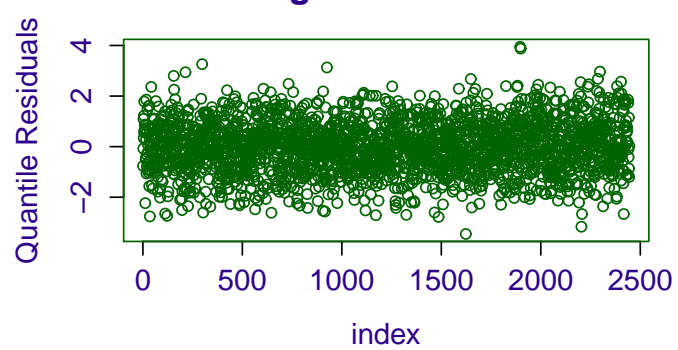

Normal Q-Q Plot

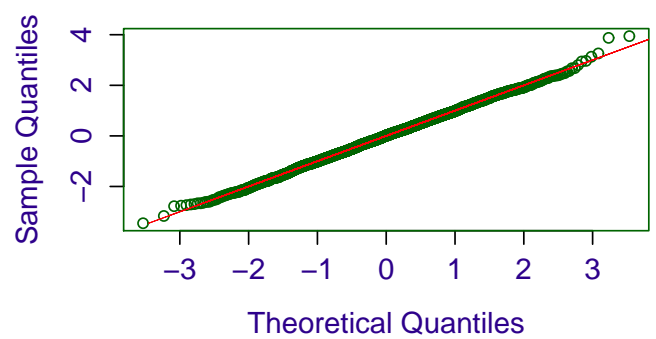

Replicating the original modelling approach by Winkelmann (1998) we selected two explanatory variables for the selection equation and three regressors for the outcome equation. According to Winkelmann (1998), unionised labour market participants are less likely to be mobile. The effect of education and employment status remains open. Despite the specification by Winkelmann (1998), it is not clear whether length of education should be modelled as a low degree polynomial or not. The same applies to full-time experience which is a regressor of the probability of labour market participation. For this reason, we will apply smooth functions to EXPFT and LEduc in the selection and outcome equations. Formally, the selection and outcome equations may be expressed as follows:

$$
\begin{aligned}
Y_{1}^{*} & =\gamma_{0}+f_{11}(\text { EXPFT })+\gamma_{1} \text { Single } \\
Y_{2} & \left.=\beta_{0}+f_{21} \text { (LEduc }\right)+\beta_{1} \text { WhiteCollar }+\beta_{2} \text { SPDSup }
\end{aligned}
$$

where $f_{11}$ and $f_{21}$ denote the non-parametric components (splines) and $Y_{2}$ and $Y_{1}^{*}$ are the (logtransformed) outcome and latent variables respectively.

The estimation results using the semi-parametric sample selection model for normally distributed outcome show that no parameter in both selection and outcome equation was statistically significant 
at 10 per cent level. However, Kendall's $\tau$ amounted to -0.106 (with the confidence interval from -0.202 to -0.011) implying that there is a weak non-random sample selection in the data. It is not difficult to find an interpretation for the smoothing terms in Figure 4.9. Apparently, the likelihood of being active on the labor market as a dependent worker increases when gaining full time work experience (see Figure 4.9, left). Then, it gradually decreases as individuals start to receive pensions or enter into self-employment. Furthermore, the longer the education of an individual lasts the less likely he is to change his job voluntarily (Figure 4.9, right). This is because the individual is more specialised in a particular area as he completes subsequent stages of education. Therefore, it is harder for him to find an alternative job or there are no financial incentives to search another one. An extreme example of that could be medical doctors. When comparing those results to the smooths of standard probit and GAM in Figure 4.10 it turns out that, like in the case of VA outpatient visits, the probit equation has the same shape. On the other hand, the outcome equation smooth differs substantially from the GAM. In the case of GAM, job mobility first increases with the years of education until the 14th year and then decreases. Again, the interpretation of smooths changes once non-random sample selection is accounted for. 
Figure 4.9: Selection (left) and outcome (right) equation smooth terms (direct job changes). The horizontal axis represents full time experience and length of education. The vertical axis shows the smooth value on the linear predictor scale. The numbers in brackets on the vertical axis are the effective degrees of freedom of the smooth which will be described in Chapter 6 . The selection equation smooth suggests that the likelihood of being active on the labor market as a dependent worker increases when gaining full time work experience. Then, it gradually decreases as individuals start to receive pensions or enter into self-employment. The outcome smooth suggests that the longer the education of an individual lasts the less likely he is to change his job voluntarily. This is because the individual is more specialised in a particular area as he completes subsequent stages of education. Therefore, it is harder for him to find an alternative job or there are no financial incentives to search another one. An extreme example of that could be medical doctors.
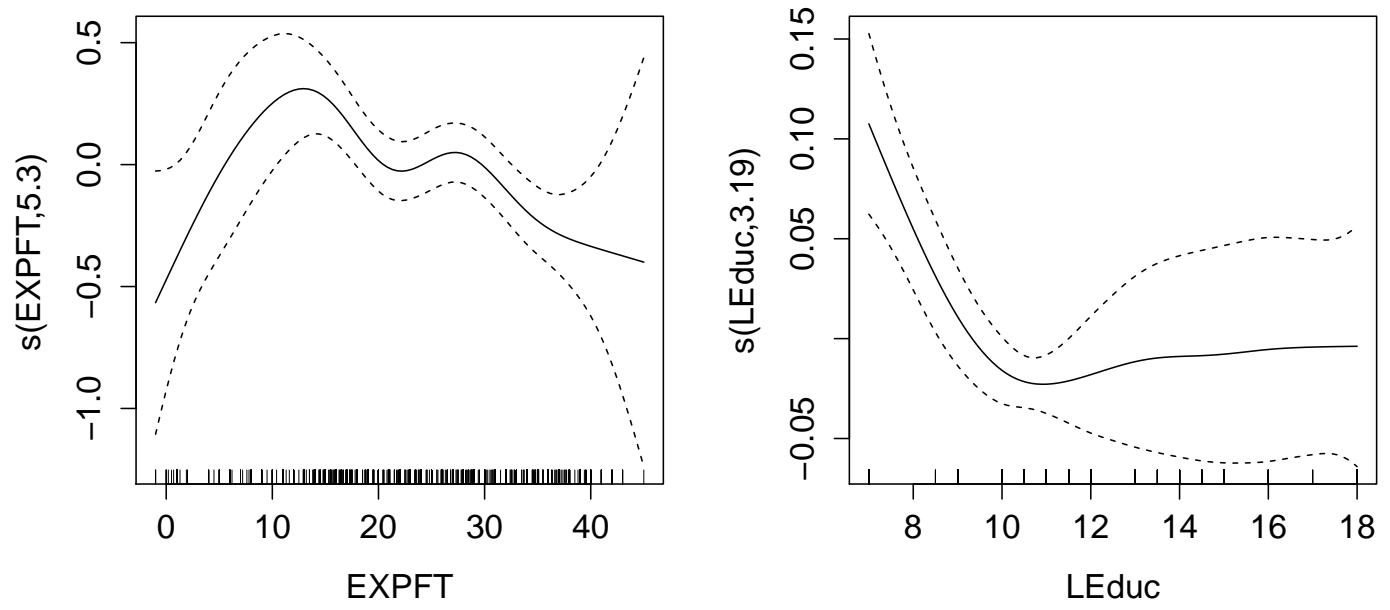
Figure 4.10: Naïve probit (left) and naïve GAM (right) smooth terms (direct job changes). The horizontal axis represents full time experience and length of education. The vertical axis shows the smooth value on the linear predictor scale. The numbers in brackets on the vertical axis are the effective degrees of freedom of the smooth which will be described in Chapter 6 . The shape of the selection equation smooth is the same as in Figure 4.9. Unlike for the sample selection model, the job mobility first increases with the years of education until the 14th year and then decreases. Again, not accounting for non-random sample selection may change the interpretation of the smooth terms.
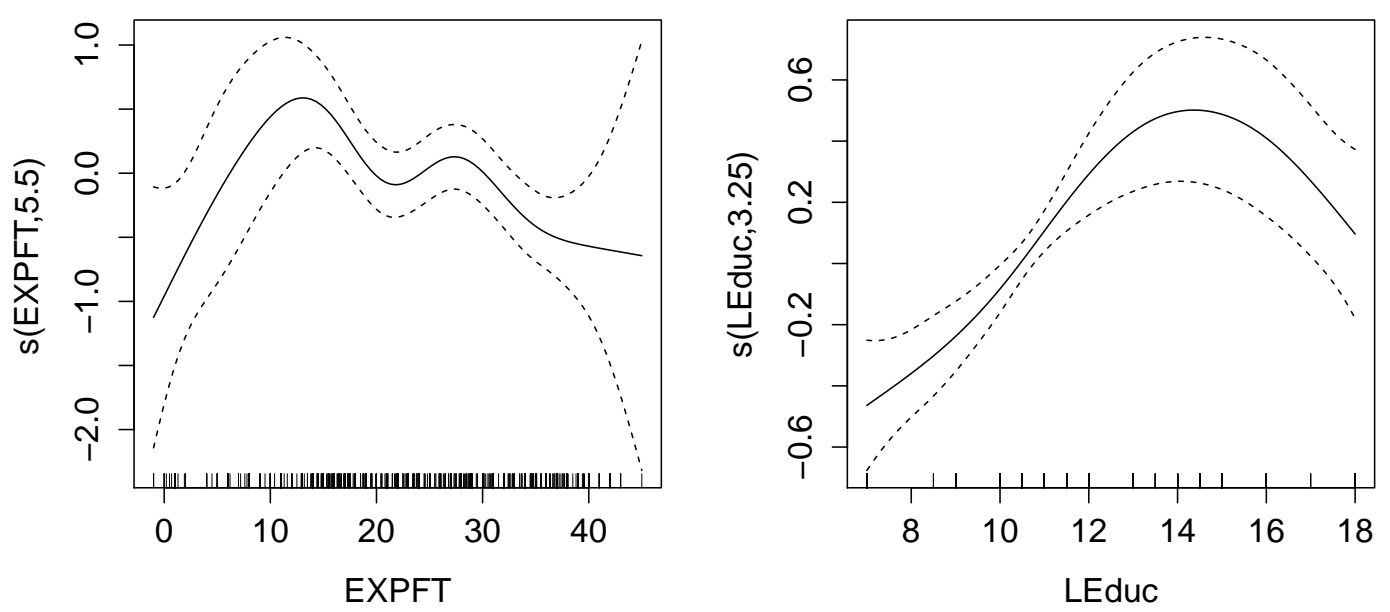


\section{Chapter 5}

\section{Count sample selection models with linear or pre-specified covariate effects}

As explained in Chapter 3 the copula modelling approach seems to be advantageous to build sample selection models for counts. The aim of this chapter is to introduce models that involve a combination of regressors both in the outcome and selection equations and account for non-random sample selection. First, a general model with its modified likelihood function will be introduced. Second, some discrete outcome margins will be discussed. Third, the algorithm used in parameter estimation will be briefly described. Fourth, numerical considerations related to the optimisation procedure will be provided. Fifth, the simulation procedure used to test the models will be described and the results will be presented for the case without analytical gradients. Finally, the analytical gradient and Hessian will be derived and checked against numerical one.

\subsection{Model specification}

As in the previous models, the selection process follows a probit regression

$$
Y_{1}^{*}=\eta_{1}+\epsilon_{1}=\boldsymbol{\gamma}^{\top} \boldsymbol{z}+\epsilon_{1}
$$


where $Y_{1}^{*} \sim N\left(\gamma^{\top} \boldsymbol{z}, 1\right)$. On the other hand, the outcome equation takes the following form

$$
Y_{2} \sim \mathcal{F}(\mu, \sigma, \nu)
$$

where $\mathcal{F}$ is a discrete distribution, $E\left(Y_{2}\right)=\mu=\exp \left(\eta_{2}\right)=\exp \left(\boldsymbol{\beta}^{\top} \boldsymbol{x}\right), \sigma$ and $\nu$ are the scale and shape parameters. The decision rules are the same as in the Heckman model:

$$
\begin{gathered}
Y_{1}=\left\{\begin{array}{ll}
1 & \text { if } Y_{1}^{*}>0 \\
0 & \text { if } Y_{1}^{*} \leq 0
\end{array},\right. \\
Y_{2}=\left\{\begin{array}{ll}
Y_{2} & \text { if } Y_{1}^{*}>0 \\
\text { not observed } & \text { if } Y_{1}^{*} \leq 0
\end{array} .\right.
\end{gathered}
$$

$Y_{1}^{*}$ and $Y_{2}$ follow a bivariate distribution defined by an arbitrary copula.

\subsection{Likelihood}

Let us recall the general likelihood function for the sample selection model first stated in the previous section

$$
L=\prod_{i=1}^{n_{0}} \operatorname{Pr}\left(Y_{1}^{*} \leq 0\right) \prod_{j=1}^{n_{1}} f_{2 \mid 1}\left(y_{2} \mid y_{1}^{*}>0\right) \operatorname{Pr}\left(Y_{1}^{*}>0\right)
$$

Recall from Section 3.3.4 that for the continuous outcome case the first component of the second product in $(3.1 .4)$ is

$$
\begin{aligned}
f_{2 \mid 1}\left(y_{2} \mid y_{1}^{*}>0\right) & =\frac{\partial}{\partial y_{2}} \frac{F_{2}\left(y_{2}\right)-F\left(0, y_{2}\right)}{F_{1}(1)} \\
& =\frac{1}{1-F_{1}(0)} \frac{\partial}{\partial y_{2}}\left(F_{2}\left(y_{2}\right)-F\left(0, y_{2}\right)\right) \\
& =\frac{1}{1-F_{1}(0)}\left(f_{2}\left(y_{2}\right)-\frac{\partial}{\partial y_{2}} F\left(0, y_{2}\right)\right) .
\end{aligned}
$$

This result cannot be used when dealing with discrete distributions. The reasoning follows from the properties of $F_{2}\left(y_{2}\right) . F_{2}\left(y_{2}\right)$ is a discrete distribution and therefore is discontinuous on the integers in its domain. Thus, $F_{2}\left(y_{2}\right)$ is not differentiable with respect to $y_{2}$. The same can be said about 
$F\left(0, y_{2}\right)$ as $F\left(0, y_{2}\right)$ includes $F_{2}\left(y_{2}\right)$. An alternative way for computing derivatives with respect to $y_{2}$ is to calculate finite differences. For the use of a discrete univariate cdf, in our case $F_{2}\left(y_{2}\right)$, the finite difference is the difference between the cumulative probability at $y_{2}$ and that at $y_{2}-1$. The result will be the probability mass function (pmf) of $y_{2}$ i.e. $f_{2}\left(y_{2}\right)$. Formally,

$$
f_{2}\left(y_{2}\right)=F_{2}\left(y_{2}\right)-F_{2}\left(y_{2}-1\right)
$$

Note that if $y_{2}=0$ then $f_{2}(0)=F_{2}(0)$. This is because $F_{2}(-1)$ is not in the support of a discrete distribution and therefore will have probability equal to 0.

If both margins of the bivariate cdf are discrete and $\theta$ is the association parameter of the copula then the joint pmf will have the following form (e.g. Nikoloulopoulos and Karlis, 2010; Karlis and Pedeli, 2013)

$$
\begin{aligned}
f\left(y_{1}, y_{2} ; \theta\right)= & C\left(F_{1}\left(y_{1}\right), F_{2}\left(y_{2}\right) ; \theta\right)-C\left(F_{1}\left(y_{1}-1\right), F_{2}\left(y_{2}\right) ; \theta\right) \\
& -C\left(F_{1}\left(y_{1}\right), F_{2}\left(y_{2}-1\right) ; \theta\right)+C\left(F_{1}\left(y_{1}-1\right), F_{2}\left(y_{2}-1\right) . \theta\right)
\end{aligned}
$$

Equation (5.2.3) can be proved as follows. By taking finite differences of $F\left(y_{1}, y_{2} ; \theta\right)=C\left(F_{1}\left(y_{1}\right)\right.$, $\left.F_{2}\left(y_{2}\right) ; \theta\right)$ with respect to $y_{1}$, we have $C\left(F_{1}\left(y_{1}\right), F_{2}\left(y_{2}\right) ; \theta\right)-C\left(F_{1}\left(y_{1}-1\right), F_{2}\left(y_{2}\right) ; \theta\right)$. Again, calculating finite differences of each of the elements with respect to $y_{2}$ will yield (5.2.3).

An analogical reasoning can be applied in our case where we have copulas with continuousdiscrete margins. Recall the second line of (3.3.12). Instead of calculating the derivative with respect to $y_{2}$ we will calculate the finite difference of the expression in brackets. Thus,

$$
\begin{aligned}
f_{2 \mid 1}\left(y_{2} \mid y_{1}^{*}>0\right) & =\frac{1}{1-F_{1}(0)}\left\{F_{2}\left(y_{2}\right)-F_{2}\left(y_{2}-1\right)\right\}-\frac{1}{1-F_{1}(0)}\left\{F\left(0, y_{2}\right)-F\left(0, y_{2}-1\right)\right\} \\
& =\frac{1}{1-F_{1}(0)}\left[\left\{F_{2}\left(y_{2}\right)-F_{2}\left(y_{2}-1\right)\right\}-\left\{F\left(0, y_{2}\right)-F\left(0, y_{2}-1\right)\right\}\right] \\
& =\frac{1}{1-F_{1}(0)}\left\{F_{2}\left(y_{2}\right)-F_{2}\left(y_{2}-1\right)-F\left(0, y_{2}\right)+F\left(0, y_{2}-1\right)\right\} \\
& =\frac{1}{1-F_{1}(0)}\left\{f_{2}\left(y_{2}\right)-F\left(0, y_{2}\right)+F\left(0, y_{2}-1\right)\right\}
\end{aligned}
$$


Given (5.2.4) the likelihood function will now assume the following form

$$
L=\prod_{0} F_{1}(0) \prod_{1}\left\{f_{2}\left(y_{2}\right)-F\left(0, y_{2}\right)+F\left(0, y_{2}-1\right)\right\}
$$

and the log-likelihood is given by

$$
\ell=\sum_{0} \log F_{1}(0)+\sum_{1} \log \left(f_{2}-F\left(0, y_{2}\right)+F\left(0, y_{2}-1\right)\right)
$$

The subscripts 0 and 1 under products and capital sigmas will from now on indicate whether an observation is missing or not. It is useful to show intuitively that $\left(f_{2}-F\left(0, y_{2}\right)+F\left(0, y_{2}-1\right)\right)$ is the joint likelihood of observations which are observed, i.e. $f\left(1, y_{2}\right)$. Let us consider Figure 5.1. The graph depicts the domain of $F\left(y_{1}^{*}, y_{2}\right)$. As one can see on the horizontal axis, if $y_{1}^{*}>0$, then a value will be observed; otherwise it will not. The vertical axis depicts the outcome values. Assume that $y_{2}=2$. The light blue area is the domain of $F\left(0, y_{2}-1\right)$ whereas the light blue and the light green area jointly constitute $F\left(0, y_{2}\right)$. The red area is our domain of interest i.e. $f\left(1, y_{2}\right)$. By conducting the operation $f_{2}\left(y_{2}\right)-F\left(0, y_{2}\right)+F\left(0, y_{2}-1\right)$ one obtains $f\left(1, y_{2}\right)$. This is because

$$
\begin{aligned}
f_{2}\left(y_{2}\right)-F\left(0, y_{2}\right)+F\left(0, y_{2}-1\right)= & \left\{f\left(1, y_{2}\right)+f\left(0, y_{2}\right)\right\}-F\left(0, y_{2}\right)+F\left(0, y_{2}-1\right) \\
= & \left\{f\left(1, y_{2}\right)+f\left(0, y_{2}\right)\right\}-\sum_{0}^{y_{2}} f\left(0, y_{2}\right)+\sum_{0}^{y_{2}-1} f\left(0, y_{2}-1\right) \\
= & \left\{f\left(1, y_{2}\right)+f\left(0, y_{2}\right)\right\}-\left\{\sum_{0}^{y_{2}-1} f\left(0, y_{2}-1\right)+f\left(0, y_{2}\right)\right\} \\
& +\sum_{0}^{y_{2}-1} f\left(0, y_{2}-1\right) \\
= & \left\{f\left(1, y_{2}\right)+f\left(0, y_{2}\right)\right\}-f\left(0, y_{2}\right) \\
= & f\left(1, y_{2}\right) .
\end{aligned}
$$

We will now describe the selected distributions for count sample selection modelling. Afterwards, we will move on to the algorithm and numerical issues associated with it. 
Figure 5.1: Graphical representation of likelihood of observed entries. Assume that $y_{2}=2$. The light blue area is the domain of $F\left(0, y_{2}-1\right)$ whereas the light blue and the light green area jointly constitute $F\left(0, y_{2}\right)$. The red area is our domain of interest i.e. $f\left(1, y_{2}\right)$. By conducting the operation $f_{2}\left(y_{2}\right)-F\left(0, y_{2}\right)+F\left(0, y_{2}-1\right)$ one obtains $f\left(1, y_{2}\right)$.

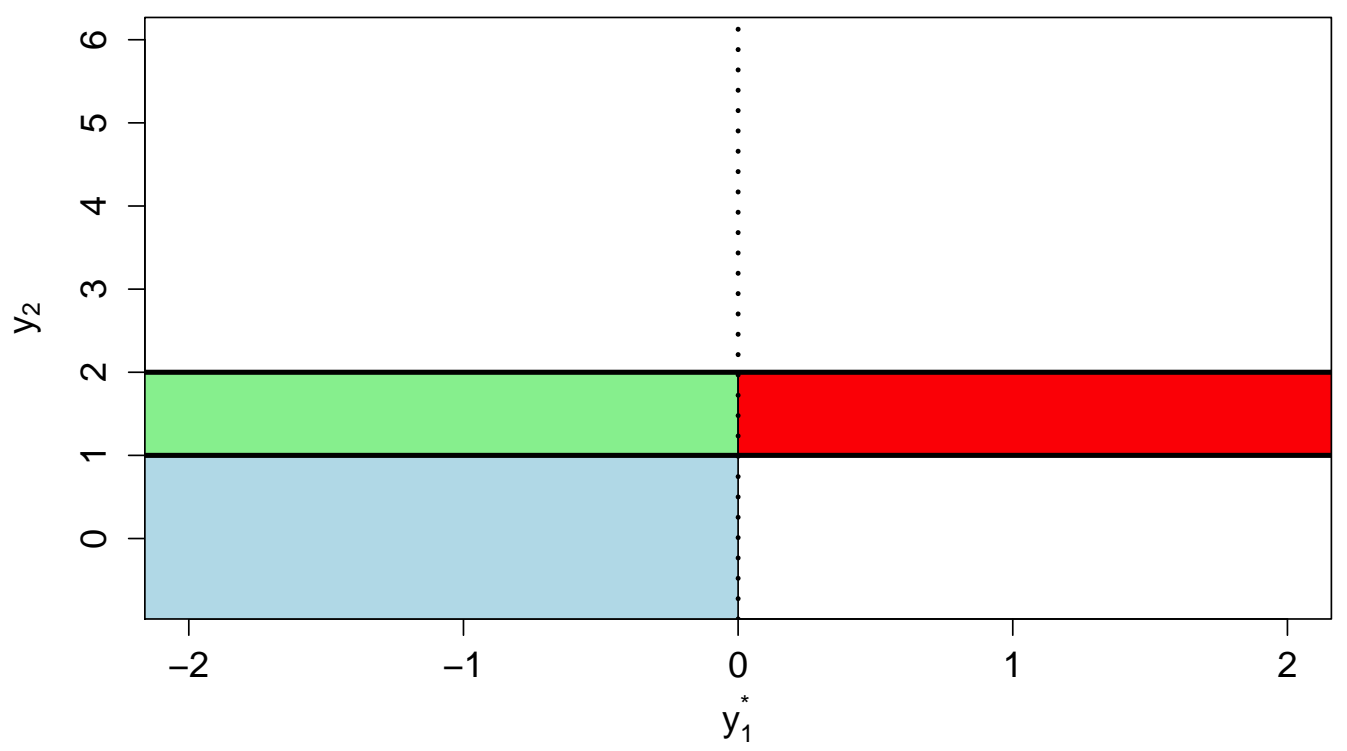




\subsection{Outcome distributions}

The likelihood derived in Section 5.2 can incorporate a wide range of discrete margins. The advantage of this approach is that it allows for greater flexibility in modelling data. For instance, if the modeller believes that the Poisson distribution is not suitable for the data at hand, then the negative binomial distribution may be chosen instead, for instance. This includes a scale parameter $\sigma$ which allows for greater flexibility in modelling.

Recall that in the data exploration section we ignored sample selection and investigated the suitability of models for which the distribution of the observed outcome was Poisson, negative binomial, Delaporte, Poisson Inverse Gaussian and Sichel. We concluded that it is worth using these distributions in sample selection modelling with discrete outcome. These distributions, implemented in the gamlss R package (Stasinopoulos and Rigby, 2007), will be used as our outcome margins. It is worth illustrating them.

Note that the distributions implemented in gamlss are parametrised with respect to location, scale and shape. We will denote them as $\mu, \sigma$ and $\nu$ respectively. The parametrisation with respect to $\mu$ allows us to specify the outcome equation in the desired way since $E(Y)=\mu=\exp (\eta)$, where $\eta$ is the linear predictor. Table 5.1 summarises the distributions used. All distributions apart from Poisson have a scale parameter and only Delaporte and Sichel have a shape parameter. Also, the ranges of the shape parameters differ for both distributions.

Figures 5.2-5.6 depict the pmf plots of the five distributions for some arbitrary parameter values (for more see Stasinopoulos and Rigby, 2007). The values chosen for the coefficients reported in the captions show the modelling flexibility of those distributions. In agreement with what was found in the data exploration, the Poisson distribution is worse at encompassing extreme values than other distributions. For example, when comparing the Poisson distribution with Delaporte or Poisson Inverse Gaussian we can see that the tail may be thicker for the two distributions than for Poisson, hence suggesting that these distributions are suitable for modelling data with extreme values. 


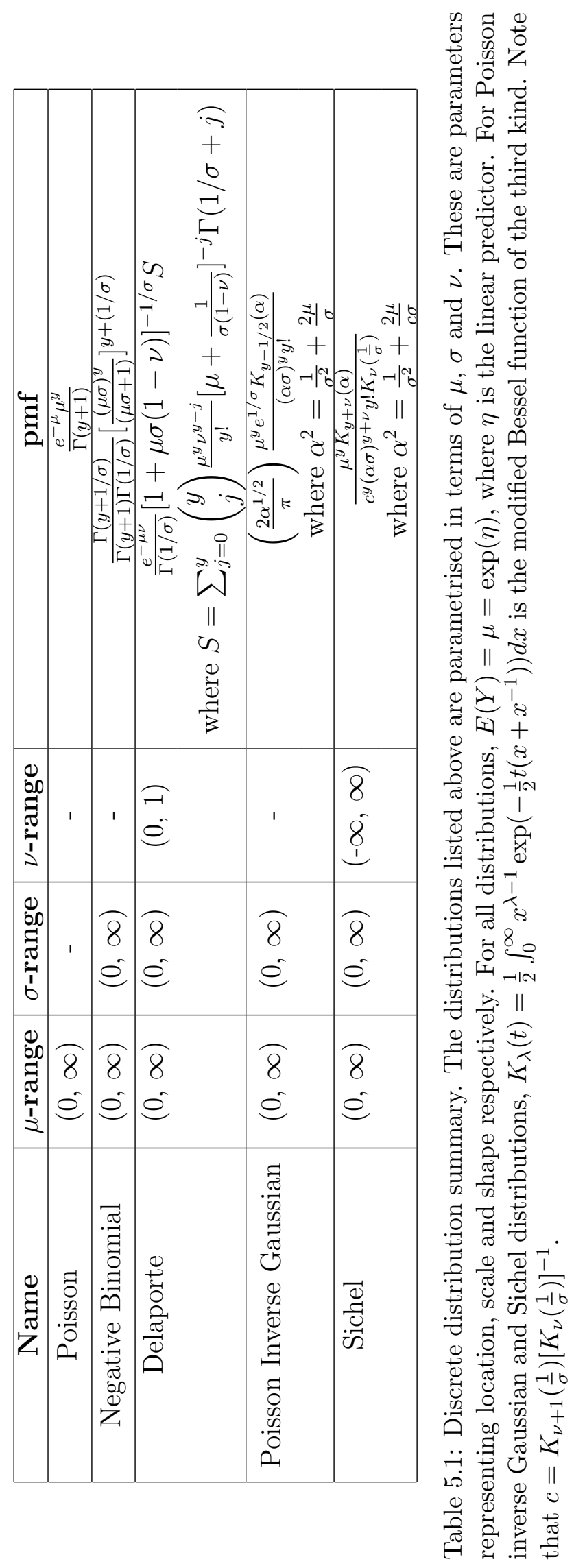


Figure 5.2: Poisson density plots (red: $\mu=1$; blue: $\mu=2$; orange: $\mu=5$ ).

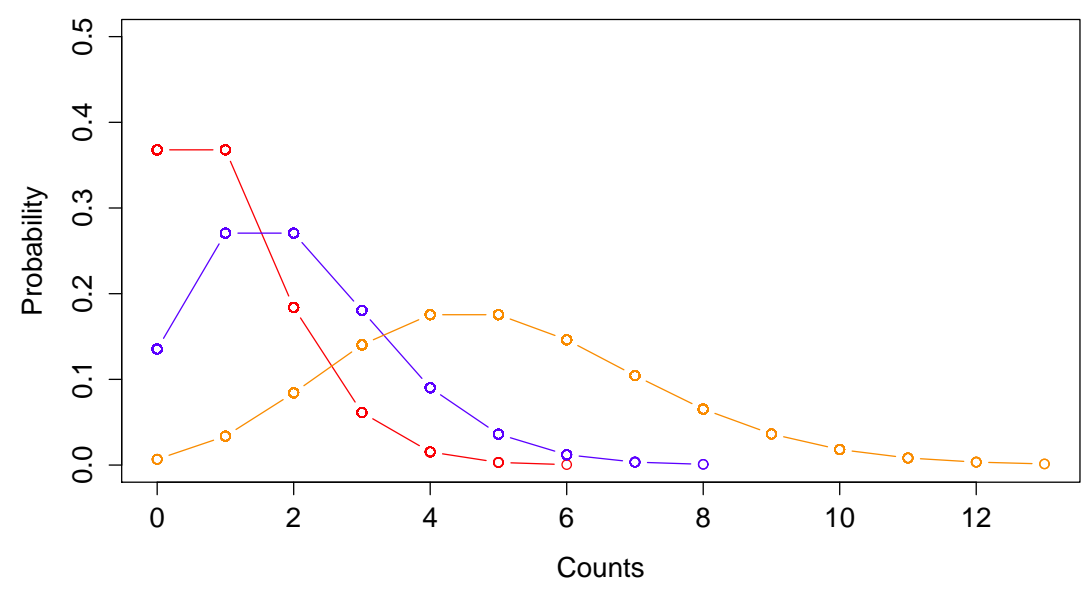

Figure 5.3: Negative binomial density plots (red: $\mu=1, \sigma=1$; blue: $\mu=5, \sigma=2$; orange: $\mu=30$, $\sigma=3$ ).

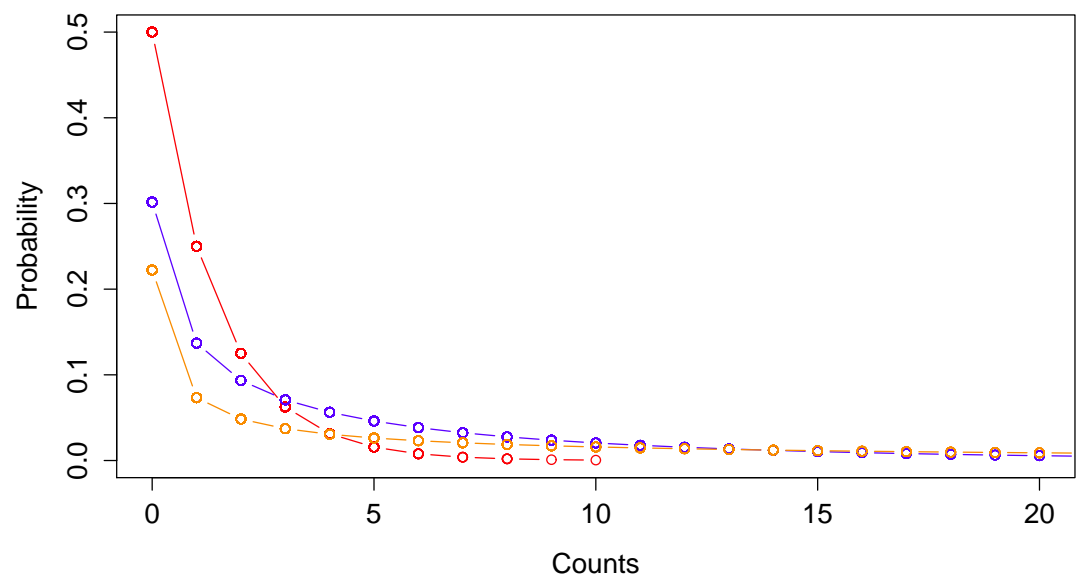


Figure 5.4: Delaporte density plots (red: $\mu=1, \sigma=1, \nu=0.1$; blue: $\mu=5, \sigma=2, \nu=0.3$; orange: $\mu=30, \sigma=3, \nu=0.5)$.

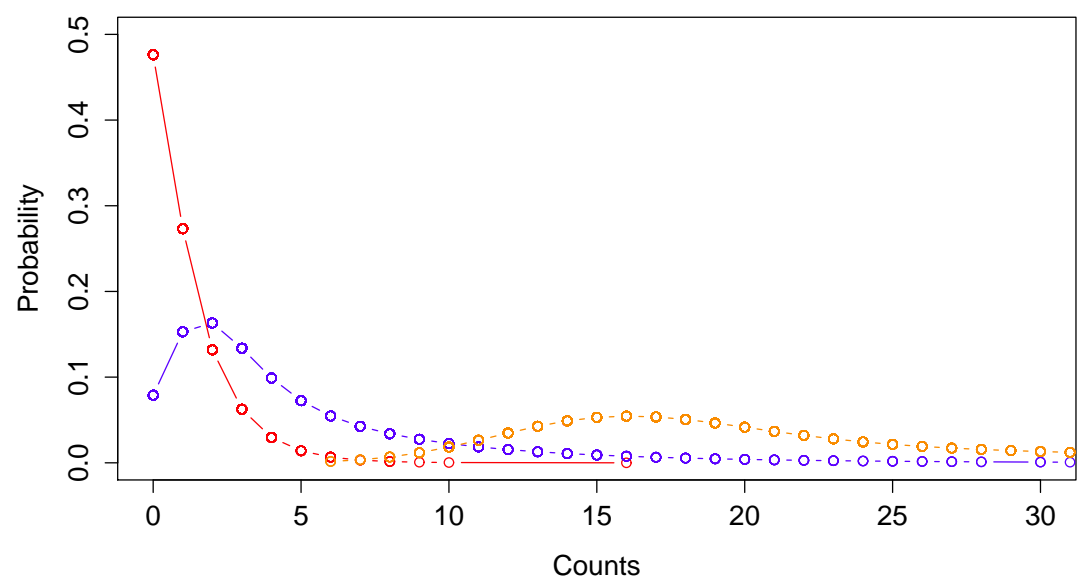

Figure 5.5: Poisson inverse Gaussian density plots (red: $\mu=1, \sigma=1$; blue: $\mu=5, \sigma=2$; orange: $\mu=30, \sigma=3$ ).

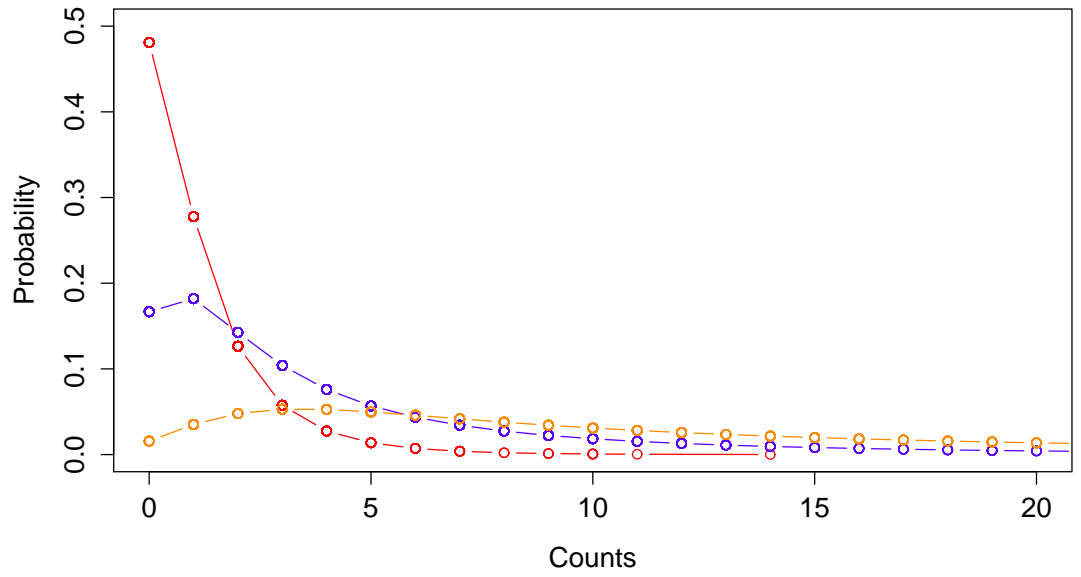


Figure 5.6: Sichel density plots (red: $\mu=1, \sigma=1, \nu=1$; blue: $\mu=5, \sigma=2, \nu=1$; orange: $\mu=10, \sigma=3, \nu=1)$.

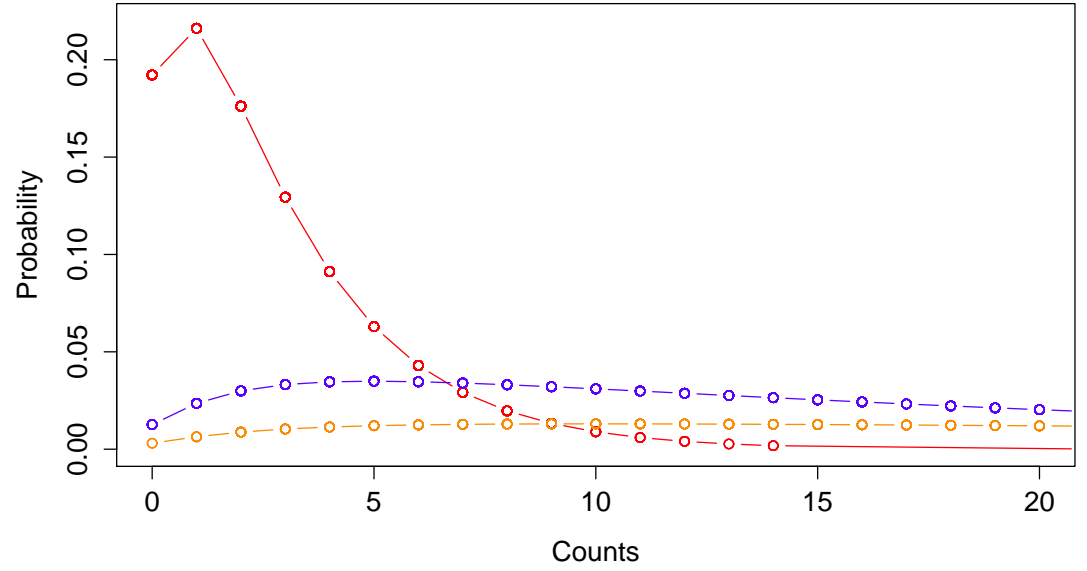




\subsection{Algorithm}

As we are dealing with a maximum likelihood problem which cannot be solved analytically the in-built R function optim can be used to perform numerical optimisation. We will use the BroydenFletcher-Goldfarb-Shanno (BFGS) algorithm. This section serves as a revision based on Nocedal and Wright (2006) and there will be no original contribution to the literature. Later, we will compare the function performance in terms of efficiency when a.) neither analytical gradient or Hessian is used, b.) only gradient is analytical c.) both gradient and Hessian are derived analytically.

Let us first briefly explain the BFGS algorithm (e.g., Broyden, 1970). BFGS is an example of quasi-Newton method, where the idea is to gather information about the objective function, gradient and Hessian from the previous steps without requiring the Hessian to be computed. As we will see the approximation of the Hessian will be more computationally efficient than its analytical version. We start by defining Taylor's approximation to the objective function $f(\boldsymbol{x})$ at iterate $k$ (Nocedal and Wright, 2006):

$$
\begin{aligned}
f(\boldsymbol{x}) & \approx m_{k}\left(\boldsymbol{p}_{\boldsymbol{k}}\right) \\
& =f_{k}+\nabla \boldsymbol{f}_{\boldsymbol{k}}^{\top} \boldsymbol{p}_{\boldsymbol{k}}+\frac{1}{2} \boldsymbol{p}_{\boldsymbol{k}}^{\top} \boldsymbol{B}_{\boldsymbol{k}} \boldsymbol{p}_{\boldsymbol{k}}
\end{aligned}
$$

where $\boldsymbol{p}_{\boldsymbol{k}}=\boldsymbol{x}-\boldsymbol{x}_{\boldsymbol{k}}, \boldsymbol{\nabla} \boldsymbol{f}_{\boldsymbol{k}}$ is the gradient vector and $\boldsymbol{B}_{\boldsymbol{k}}$ is the Hessian matrix at $k$-th iteration. By differentiating with respect to $\boldsymbol{p}_{\boldsymbol{k}}$ and setting the derivative to zero the first order condition is obtained (Nocedal and Wright, 2006):

$$
p_{k}=-B_{k}^{-1} \nabla f_{k}
$$

$\boldsymbol{p}_{\boldsymbol{k}}$ is then used as a search direction at iteration $k$. The new iterate is defined as

$$
x_{k+1}=x_{k}+\alpha p_{k}
$$

where the step length $\alpha$ is chosen via a line search procedure to satisfy Wolfe's condition (see Nocedal and Wright, 2006). The intuition behind Wolfe's condition is to conduct the line search

procedure efficiently without the need of repeatedly evaluating the line search objective function 
and its derivatives. It is possible to find an approximate Hessian matrix without using analytical expressions or finite differentiating at each iteration. To see this, consider $m(\cdot)$ at iteration $k+1$ (Nocedal and Wright, 2006):

$$
m_{k+1}\left(\boldsymbol{p}_{\boldsymbol{k}+\mathbf{1}}\right)=f_{k+1}+\nabla \boldsymbol{f}_{\boldsymbol{k}+\mathbf{1}}^{\top} \boldsymbol{p}_{\boldsymbol{k}+\mathbf{1}}+\frac{1}{2} \boldsymbol{p}_{\boldsymbol{k}+\mathbf{1}}^{\top} \boldsymbol{B}_{\boldsymbol{k}+\mathbf{1}} \boldsymbol{p}_{\boldsymbol{k}+\mathbf{1}}
$$

where $\boldsymbol{p}_{\boldsymbol{k}+\mathbf{1}}=\boldsymbol{x}-\boldsymbol{x}_{\boldsymbol{k}+\mathbf{1}}$. The gradient will then have the form

$$
\nabla m_{k+1}\left(p_{k+1}\right)=\nabla f_{k+1}+B_{k+1} p_{k+1}
$$

For quasi-Newton methods, the gradient of $m_{k+1}$ should be equal to the gradient of $f$ at $k$ and $k+1$ (Nocedal and Wright, 2006). The second equality is satisfied immediately since for $m_{k+1}$ at $k+1, \boldsymbol{p}_{\boldsymbol{k}+\mathbf{1}}=\boldsymbol{x}_{\boldsymbol{k}+\mathbf{1}}-\boldsymbol{x}_{\boldsymbol{k}+\mathbf{1}}=\mathbf{0}$. Hence, $\boldsymbol{\nabla} m_{k+1}(\mathbf{0})=\boldsymbol{\nabla} \boldsymbol{f}_{\boldsymbol{k}+\mathbf{1}}$. On the other hand, for $m_{k+1}$ at $k$, $\boldsymbol{p}_{\boldsymbol{k}+\mathbf{1}}=\boldsymbol{x}_{\boldsymbol{k}}-\boldsymbol{x}_{\boldsymbol{k}+\mathbf{1}}=-\alpha_{k} \boldsymbol{p}_{\boldsymbol{k}}$. Then,

$$
\begin{aligned}
\nabla m_{k+1}\left(-\alpha \boldsymbol{p}_{\boldsymbol{k}}\right) & =\boldsymbol{\nabla} \boldsymbol{f}_{\boldsymbol{k}+\mathbf{1}}-\alpha_{k} \boldsymbol{B}_{\boldsymbol{k}+\mathbf{1}} \boldsymbol{p}_{\boldsymbol{k}} \\
& =\boldsymbol{\nabla} \boldsymbol{f}_{\boldsymbol{k}}
\end{aligned}
$$

Rearranging yields (Nocedal and Wright, 2006)

$$
B_{k+1} s_{k}=y_{k}
$$

where $\boldsymbol{s}_{\boldsymbol{k}}=\boldsymbol{x}_{\boldsymbol{k}+\boldsymbol{1}}-\boldsymbol{x}_{\boldsymbol{k}}$ and $\boldsymbol{y}_{\boldsymbol{k}}=\boldsymbol{\nabla} \boldsymbol{f}_{\boldsymbol{k}+\boldsymbol{1}}-\boldsymbol{\nabla} \boldsymbol{f}_{\boldsymbol{k}}$. Equation (5.4.2) will be possible for a positive definite Hessian $\boldsymbol{B}_{\boldsymbol{k}+\mathbf{1}}$ under certain conditions imposed on $\boldsymbol{s}_{\boldsymbol{k}}$ and $\boldsymbol{y}_{\boldsymbol{k}}$. These can be satisfied by imposing appropriate restrictions on the selection of step length $\alpha$ (see Wolfe's conditions, Chapter 3, Nocedal and Wright, 2006).

Equation (5.4.2) is not sufficient to find a unique $\boldsymbol{B}_{\boldsymbol{k}+\mathbf{1}}$. Several additional conditions are needed to be fulfilled. Let us define the inverse of the Hessian i.e. $\boldsymbol{H}_{\boldsymbol{k}}=\left(\boldsymbol{B}_{\boldsymbol{k}}\right)^{-\mathbf{1}}$. This will make the algorithm more computationally efficient since (5.4.1) uses the inverse of the Hessian whereas no inversion will be required here. The desired $\boldsymbol{H}_{\boldsymbol{k}+\mathbf{1}}$

1. satisfies (5.4.2) i.e. $\boldsymbol{H}_{\boldsymbol{k}+\mathbf{1}} \boldsymbol{y}_{\boldsymbol{k}}=\boldsymbol{s}_{\boldsymbol{k}}$, 
2. is as close to $\boldsymbol{H}_{\boldsymbol{k}}$ as possible,

3. is positive definite (Nocedal and Wright, 2006).

"Closeness" in condition 2 is evaluated by the weighted Frobenius norm. The unique BFGS update for $\boldsymbol{H}_{\boldsymbol{k}+\mathbf{1}}$ is given by (Nocedal and Wright, 2006)

$$
\boldsymbol{H}_{\boldsymbol{k}+\boldsymbol{1}}=\left(\boldsymbol{I}-\rho_{k} \boldsymbol{s}_{\boldsymbol{k}} \boldsymbol{y}_{\boldsymbol{k}}^{\top}\right) \boldsymbol{H}_{\boldsymbol{k}}\left(\boldsymbol{I}-\rho_{k} \boldsymbol{y}_{\boldsymbol{k}} \boldsymbol{s}_{\boldsymbol{k}}^{\top}\right)+\rho_{k} \boldsymbol{s}_{\boldsymbol{k}} \boldsymbol{s}_{\boldsymbol{k}}^{\top}
$$

where $\rho=\frac{1}{\boldsymbol{y}_{\boldsymbol{k}}{ }^{\top} \boldsymbol{s}_{\boldsymbol{k}}}$.

To summarise, suppose that the starting value vector is $\boldsymbol{x}_{\mathbf{0}}$, the convergence tolerance $\epsilon$ and initial inverse Hessian approximation $\boldsymbol{H}_{\mathbf{0}}$. Also, let $k$ be the iteration index and hence initially $k=0$. Then, until the norm of the gradient $\left\|\boldsymbol{\nabla} \boldsymbol{f}_{\boldsymbol{k}}\right\|>\epsilon$

1. Compute search direction $\boldsymbol{p}_{\boldsymbol{k}}=-\boldsymbol{H}_{\boldsymbol{k}} \boldsymbol{\nabla} \boldsymbol{f}_{\boldsymbol{k}}$.

2. Set $\boldsymbol{x}_{\boldsymbol{k}+\mathbf{1}}=\boldsymbol{x}_{\boldsymbol{k}}+\alpha_{k} \boldsymbol{p}_{\boldsymbol{k}}$ where $\alpha_{k}$ is calculated from the line search procedure.

3. Define $s_{k}=x_{k+1}-x_{k}$ and $y_{k}=\nabla f_{k+1}-\nabla f_{k}$.

4. Compute $\boldsymbol{H}_{\boldsymbol{k}+\boldsymbol{1}}=\left(\boldsymbol{I}-\rho_{k} \boldsymbol{s}_{\boldsymbol{k}} \boldsymbol{y}_{\boldsymbol{k}}^{\top}\right) \boldsymbol{H}_{\boldsymbol{k}}\left(\boldsymbol{I}-\rho_{k} \boldsymbol{y}_{\boldsymbol{k}} \boldsymbol{s}_{\boldsymbol{k}}^{\top}\right)+\rho_{k} \boldsymbol{s}_{\boldsymbol{k}} \boldsymbol{s}_{\boldsymbol{k}}^{\top}$ where $\rho=\frac{1}{\boldsymbol{y}_{\boldsymbol{k}}^{\top} \boldsymbol{s}_{\boldsymbol{k}}}$.

5. Increase iteration by one i.e. $k \rightarrow k+1$ (Nocedal and Wright, 2006).

We will now proceed to numerical considerations regarding the starting values, objective function and optim.

\subsection{Starting values}

The starting values for the selection equation are based on the estimates obtained from a standard probit procedure. Additionally, we extract starting values for the outcome equation from the gamlss model which is estimated using observed values only.

We will describe the procedure of obtaining the starting value for the association parameter $\theta^{*}$.

$\theta^{*}$ is a monotonic mathematical transformation of $\theta$. Table 5.2 shows the $\theta^{*}$ parameters defined in terms of $\theta$ for each copula. 


\begin{tabular}{|c|c|}
\hline Copula & $\theta^{*}$ \\
\hline FGM & $\tanh ^{-1}(\theta)$ \\
\hline Normal & $\tanh ^{-1}(\theta)$ \\
\hline AMH & $\tanh ^{-1}(\theta)$ \\
\hline Clayton & $\log (\theta-\epsilon)$ \\
\hline Frank & $\theta-\epsilon$ \\
\hline Gumbel & $\log (\theta-1)$ \\
\hline Joe & $\log (\theta-1-\epsilon)$ \\
\hline
\end{tabular}

Table 5.2: $\theta^{*}$ defined in terms of $\theta$. The values of $\theta^{*}$ are corrected with $\epsilon$ for Clayton, Frank and Joe to prevent the optimisation algorithm from reaching boundaries of $\theta$ which are not included in the parameter space. $\epsilon$ is set to $10^{-8}$.

The rationale behind using $\theta^{*}$ instead of $\theta$ is that $\theta^{*}$ is not contrained by its parameter space. The starting value for $\theta^{*}$ is obtained by creating a new variable based on the probit equation and the inverse Mills ratio described in the previous sections. The new variable is included in the gamlss model and its parameter estimated together with the outcome equation parameters. The parameter estimate of the new variable is then divided by an estimate of $\sigma$. This is then inserted into a tanh function so that it is constrained by -1 and 1 . Finally, the new quantity is transformed depending on the copula. These transformations are depicted in Table 5.3. The copula-specific transformations were extracted and modified from the starting value function st.theta.star of the SemiParSampleSel package (Marra et al., 2015). The resulting quantity is the guesstimate of $\theta^{*}$. Intuitively, the transformations in Table 5.3 convert Kendall's $\tau$ or Spearman's $\rho$ first into $\theta$ and then into $\theta^{*}$. For Clayton, Gumbel and Joe the association parameter is set to an arbitrary number. The $\mathrm{R}$ code for obtaining $\theta^{*}$ can be found in Appendix E.

\subsection{Some numerical aspects}

Regarding the objective function several aspects are worth mentioning. First, expression $F\left(0, y_{2}-1\right)$ in (5.2.5) will include $F_{2}\left(y_{2}-1\right)$. If $y_{2}=0$ then $F_{2}\left(y_{2}-1\right)=F_{2}(-1)$ which is equal to zero as -1 lies outside the support of the distribution. The cdf functions implemented in gamlss will produce an error message when $F_{2}(-1)$. Thus, as $f_{2}\left(y_{2}\right)=F_{2}\left(y_{2}\right)-F_{2}\left(y_{2}-1\right)$ and hence $F_{2}\left(y_{2}\right)-f_{2}\left(y_{2}\right)=$ $F_{2}\left(y_{2}-1\right)$ we will use $F_{2}\left(y_{2}\right)-f_{2}\left(y_{2}\right)$ when $y_{2}=0$ (recall that $F_{2}\left(y_{2}\right)=f_{2}\left(y_{2}\right)$ ). To carry out the optimisation on $\mathbb{R}^{p}$, where $p$ is equal to the total number of parameters, we used the transformation $\sigma^{*}=\log (\sigma)$. For Delaporte margins, after each iteration $\nu$ was transformed using the logistic 


\begin{tabular}{|c|l|}
\hline Copula & Steps \\
\hline FGM & $\begin{array}{l}\text { 1. Bound by }-0.233 \text { and } 0.233 . \\
\text { 2. Find "inverse" Spearman's } \rho \text { for FGM copula. } \\
\text { 3. Use atanh. }\end{array}$ \\
\hline Normal & $\begin{array}{l}\text { 1. Bound by }-0.9 \text { and } 0.9 . \\
\text { 2. Use atanh. }\end{array}$ \\
\hline AMH & $\begin{array}{l}\text { 1. Bound by }-0.18 \text { and } 0.33 . \\
\text { 2. Find "inverse" Kendall's } \tau \text { for AMH copula. } \\
\text { 3. Use atanh. }\end{array}$ \\
\hline Clayton & Set to log(3). \\
\hline Frank & $\begin{array}{l}\text { 1. Find "inverse" Spearman's } \rho \text { for Frank copula. } \\
\text { 2. Bound by -15 and } 15 .\end{array}$ \\
\hline Gumbel & Set to log(3). \\
\hline Joe & Set to $\log (3)$. \\
\hline
\end{tabular}

Table 5.3: Copula-specific steps for obtaining starting values for $\theta^{*}$. The "inverse" $\rho$ and $\tau$ mean that the quantity is inserted into a function which treats it as if it was Spearman's $\rho$ or Kendall's $\tau$. The value returned by the inverse function is the initial value of $\theta$. This value is then transformed into a guesstimate of $\theta^{*}$ corresponding to the copula.

function in order to prevent it from taking values outside $(0,1)$. To prevent the mean parameter from taking negative values, $\mu$ was exponentiated i.e. $\mu=\exp \left(\eta_{2}\right)$, where $\eta_{2}$ is the linear predictor of the outcome. Third, it is necessary to set precision bounds on the individual cdfs and copulas. This is because if a cdf or a copula is exactly equal to 0 or to 1 NaNs (numerical under- and overflows) may be produced. For instance, let us recall the log-likelihood:

$$
\ell=\sum_{0} \log F_{1}(0)+\sum_{1} \log \left(f_{2}-F\left(0, y_{2}\right)+F\left(0, y_{2}-1\right)\right)
$$

If in a given iteration $F_{1}(0)$ is zero, then the logarithm would be equal to $-\infty$ and there would be an overflow. Similarly, if $F\left(y_{2}\right)=1$ and $f_{2}+F\left(0, y_{2}-1\right)$ is approximately equal to $F\left(0, y_{2}\right)$, then the logarithm would be equal to $-\infty$. The precision lower and upper bounds for the cdfs and copulas were $10^{-8}$ and $1-10^{-8}$ respectively. For example, if for an iteration the cdf value was evaluated to be $10^{-10}$ the final value of the cdf would be set to $10^{-8}$. Exactly the same reasoning applies to the final value of $\nu$ used in the Delaporte distribution. $\nu$ cannot take values equal to 1 or 0 . If this is the case then the cdfs and pmfs of Delaporte return a NaN. The same analogy applies to $\sigma$ where only the lower bound was set. For $\mu$ the lower bound was set to 0.0001 as it cannot take values which are close to 0 . For lower values of $\mu$ NaNs were produced.

Finally, as in simulation all non-Poisson margin models returned an error message whilst eval- 
uating the function, the scaling option available in optim was used. The error message stated that $\mathrm{NA} / \mathrm{NaN} / \mathrm{Inf}$ is passed into the parameter argument of a cdf or pmf. In unconstrained optimisation, if the objective function is poorly scaled, then the change of some parameters may affect the change of the objective function more than others (Nocedal and Wright, 2006). For instance, consider function $f\left(x_{1}, x_{2}\right)=100 x_{1}^{3}+2 x_{2}$. A small change in $x_{1}$ will change $f\left(x_{1}, x_{2}\right)$ more than a small change in $x_{2}$. A remedy for this is function scaling, which consists of dividing the objective function and gradient by an arbitrary number and performing optimisation on the new quantity. In the given example, if $f\left(x_{1}, x_{2}\right)$ is divided by 100 , the effect of $x_{1}$ on $f\left(x_{1}, x_{2}\right)$ will not be as large as initially. However, how does this relate to optimisation of models with non-Poisson margins? Arguably, the issue concerns finite differencing to obtain the gradient. This is justified by the fact that when tracing the optim's iterations it happened that it could evaluate the initial objective function but it could not evaluate it in consecutive iteration. Thus, an error within the optimising step must have occured. Simultaneously, when calculating the cdfs implemented in gamlss it turns out that for certain very large values of parameters, say, $\sigma$ the same error is returned as for the estimation. Therefore, it is likely that the gradient returns an $\mathrm{NaN}$ as its value is either too small or too large. By using function scaling and shrinking the objective function the estimation was feasible and as shown in the simulation section the resulting estimates were satisfactory. Apparently, the gradient or gradient components took up very high values and therefore returned NA/NaN/Inf message. Once scaling was used, the objective function was shrunk; hence numerical derivatives were shrunk as well. Thus, overflows were avoided and the optimisation procedure could terminate successfully.

The argument described in the previous paragraph can be verified when using analytical gradients which will be derived and implemented. As a final remark for this section the objective functions were shrunk by 10, 10, 10 and 500 for the models with negative binomial, Delaporte, Poisson Inverse Gaussian and Sichel outcome margins respectively. The scaling factor for each distribution was obtained via trial and error. Thus, the scaling factor will depend on the model specification. The Poisson margin models did not require any scaling at all. 


\subsection{Simulations}

Before we comment on the results we will present the simulation setup. 7 copula models for each of the 5 distributions mentioned in Section 5.3 were implemented. The copulas were FGM, bivariate normal, AMH, Clayton, Frank, Joe and Gumbel. In addition, we will consider two model specifications. Overall, a total of 70 model combinations were examined. The first specification will have an intercept and two explanatory variables, continuous $x_{1}$ and binary $x_{2}$, in both equations. The second specification will include an additional binary explanatory variable $x_{3}$ in the selection equation. We will refer to the selection equation parameters as $\alpha_{0}, \alpha_{1}, \alpha_{2}, \alpha_{3}$ respectively. The outcome equation parameters will be denoted as $\beta_{0}, \beta_{1}, \beta_{2}$ respectively. The scale and shape parameters will be referred to as $\sigma$ and $\nu$.

The reason for performing simulations on both specifications is that if both linear predictors have the same covariates, then the model may be poorly identified and the parameter estimates may be biased. According to the author's best knowledge, there is no formal proof of (lack of) identification, when the covariates are the same in both equations. However, it can be shown via simulations that the model may yield biased and inconsistent estimates. For instance, Leung and $\mathrm{Yu}$ (1996) perform simulations in which they show that under certain conditions maximum likelihood estimates are biased when the covariates are the same in both equations. To remedy this, many authors use instrumental variables. In our case, $x_{3}$ will serve as an instrumental variable. Thus, the simulation will show how sensitive the model is to the inclusion of $x_{3}$.

Table 5.4 summarises the parameter settings for both equations. Note that the values of $\alpha$ are close to the corresponding $\beta$ parameters. The reason for this was explained above. The values of $\sigma$ and $\nu$ were taken for simplicity, so that the same value of each parameter could be applied across all margins.

\begin{tabular}{|c|c|c|c|}
\hline Parameter & Value & Parameter & Value \\
\hline$\alpha_{0}$ & 1.0 & $\beta_{0}$ & 1.1 \\
\hline$\alpha_{1}$ & 2.0 & $\beta_{1}$ & 1.8 \\
\hline$\alpha_{2}$ & -2.0 & $\beta_{2}$ & -1.9 \\
\hline$\alpha_{3}$ & 0.3 & $\nu$ & 0.5 \\
\hline$\tau$ & $\begin{array}{c}\text { depending on copula } \\
\text { (see below) }\end{array}$ & $\sigma$ & 1.0 \\
\hline
\end{tabular}

Table 5.4: Parameter summary for selection and outcome equations. 
For each model combination 100 simulations were run with 1000 observations each. Note that in this setup we are interested in checking whether the function can (on average) recover the parameters, rather than investigating coverage properties. First, the two (three) covariates were generated from a trivariate normal distribution with the zero mean vector, covariance matrix with ones on the main diagonal and Pearson's correlation coefficient equal to 0.5. Then, the covariates were transformed using a standard normal cdf. The reason for this is that the cdf bounds the covariates by 0 and 1 . This implies that we can control for the outliers which can have an impact on the estimability of the model. For example, if for some observations one of the covariates has a value of -4 and its associated parameter is 2 , then the linear predictor is likely to have a negative value. Since the inverse Mills ratio is linear for negative values of the linear predictor, in the two-step approach the parameters may not be identified. Analogical problem may arise in the case of MLE. Hence, the unconditional expected mean values were calculated and the parameters $\mu, \sigma$ and $\nu$ were inserted into the $\operatorname{mvdc}()$ function which created random outcome responses from a previously defined copula. The R code for this can be found in Appendix E.

For copulas, Kendall's $\tau$ between the outcome and the selection equation was fixed at 0.2 for FGM, 0.3 for AMH and 0.5 for the remainder of the models. This is because the copulas used have different association coverages. Afterwards, the responses for which the corresponding latent $y_{1}^{*}$ were greater than zero were retained; otherwise responses were arbitrarily coded as -99 i.e. unobserved. To save time, in the starting value procedure the maximum number of iterations for gamlss was set to 100. The resulting starting parameter values were close to the ones that would be obtained if no iteration number constraints were imposed.

Figures G.1-G.5 in Appendix G show boxplot sets of each combination of outcome margin and copula used for the same set of covariates. In general, the boxplot medians are located close to the reference lines which represent true parameter values. The boxplots seem to be symmetric around the true parameters. All of this suggests that the likelihood maximisation algorithm works well. The same can be said about Figures G.6-G.10 which depict boxplot sets for the case where the instrumental variable is included in the selection equation. 


\subsection{Analytical gradients}

In this section we will focus on deriving and implementing gradients. The reason for this is that analytical gradients are more precise than their numerical approximations. First, we will provide a general framework for deriving score vector components. Second, we will check our gradients by comparing them to the numerical ones. This will consist of checking relative differences, convergence in the optimisation procedure and computational efficiency.

\subsubsection{Analytical expressions of first derivatives}

As we are dealing with a number of copula-outcome-margin combinations it is essential to derive a general derivative expression. Then, specific derivatives of copulas and univariate cdfs and pmfs can be calculated. Therefore, let us commence with the derivation of the general expression. Recall the log-likelihood stated in (5.2.5):

$$
\ell=\sum_{0} \log F_{1}(0)+\sum_{1} \log \left(f_{2}-F\left(0, y_{2}\right)+F\left(0, y_{2}-1\right)\right)
$$

To obtain the score vector we have to calculate derivatives with respect to first equation parameters $\boldsymbol{\delta}_{\mathbf{1}}$, second equation parameters $\boldsymbol{\delta}_{\mathbf{2}}, \sigma$ and $\nu$ and association parameter $\theta$. Thus,

$$
\begin{aligned}
\frac{\partial \ell}{\partial \boldsymbol{\delta}_{\mathbf{1}}} & =\sum_{0} \frac{1}{F_{1}} \frac{\partial F_{1}}{\partial \boldsymbol{\delta}_{\mathbf{1}}}+\sum_{1} \frac{1}{f_{2}-F\left(0, y_{2}\right)+F\left(0, y_{2}-1\right)} \frac{\partial}{\partial \boldsymbol{\delta}_{\mathbf{1}}}\left(f_{2}-F\left(0, y_{2}\right)+F\left(0, y_{2}-1\right)\right) \\
& =\sum_{0} \frac{1}{F_{1}} \frac{\partial F_{1}}{\partial \boldsymbol{\delta}_{\mathbf{1}}}+\sum_{1} \frac{1}{f_{2}-F\left(0, y_{2}\right)+F\left(0, y_{2}-1\right)}\left(-\frac{\partial F\left(0, y_{2}\right)}{\partial F_{1}} \frac{\partial F_{1}}{\partial \boldsymbol{\delta}_{\mathbf{1}}}+\frac{\partial F\left(0, y_{2}-1\right)}{\partial F_{1}} \frac{\partial F_{1}}{\partial \boldsymbol{\delta}_{\mathbf{1}}}\right) \\
& =\sum_{0} \frac{1}{F_{1}} \frac{\partial F_{1}}{\partial \boldsymbol{\delta}_{\mathbf{1}}}+\sum_{1} \frac{1}{f_{2}-F\left(0, y_{2}\right)+F\left(0, y_{2}-1\right)}\left(-\frac{\partial F\left(0, y_{2}\right)}{\partial F_{1}}+\frac{\partial F\left(0, y_{2}-1\right)}{\partial F_{1}}\right) \frac{\partial F_{1}}{\partial \boldsymbol{\delta}_{\mathbf{1}}}
\end{aligned}
$$

For notational convenience we will denote $F_{1}(0)$ as $F_{1}$. Note that in line 2 above we used the fact that $\frac{\partial}{\partial \boldsymbol{\delta}_{1}} F\left(0, y_{2}\right)=\frac{\partial F\left(0, y_{2}\right)}{\partial F_{1}} \frac{\partial F_{1}}{\partial \boldsymbol{\delta}_{1}}$. For the outcome equation parameters, we have that

$$
\frac{\partial \ell}{\partial \boldsymbol{\delta}_{\mathbf{2}}}=\sum_{1} \frac{1}{f_{2}-F\left(0, y_{2}\right)+F\left(0, y_{2}-1\right)}\left(\frac{\partial f_{2}}{\partial \boldsymbol{\delta}_{\mathbf{2}}}-\frac{\partial F\left(0, y_{2}\right)}{\partial F_{2}} \frac{\partial F_{2}}{\partial \boldsymbol{\delta}_{\mathbf{2}}}+\frac{\partial F\left(0, y_{2}-1\right)}{\partial\left(F_{2}-f_{2}\right)} \frac{\partial\left(F_{2}-f_{2}\right)}{\partial \boldsymbol{\delta}_{\mathbf{2}}}\right)
$$


It is worth noticing that $F\left(y_{2}-1\right)=F_{2}-f_{2}$ where $F_{2}=F\left(y_{2}\right)$ and $f_{2}=f\left(y_{2}\right)$. The reason for such notation is that for $y_{2}=0, F\left(y_{2}-1\right)$ and $f\left(y_{2}-1\right)$ would be undefined. Analogically,

$$
\begin{aligned}
\frac{\partial \ell}{\partial \sigma} & =\sum_{1} \frac{1}{f_{2}-F\left(0, y_{2}\right)+F\left(0, y_{2}-1\right)}\left(\frac{\partial f_{2}}{\partial \sigma}-\frac{\partial F\left(0, y_{2}\right)}{\partial F_{2}} \frac{\partial F_{2}}{\partial \sigma}+\frac{\partial F\left(0, y_{2}-1\right)}{\partial\left(F_{2}-f_{2}\right)} \frac{\partial\left(F_{2}-f_{2}\right)}{\partial \sigma}\right), \\
\frac{\partial \ell}{\partial \nu} & =\sum_{1} \frac{1}{f_{2}-F\left(0, y_{2}\right)+F\left(0, y_{2}-1\right)}\left(\frac{\partial f_{2}}{\partial \nu}-\frac{\partial F\left(0, y_{2}\right)}{\partial F_{2}} \frac{\partial F_{2}}{\partial \nu}+\frac{\partial F\left(0, y_{2}-1\right)}{\partial\left(F_{2}-f_{2}\right)} \frac{\partial\left(F_{2}-f_{2}\right)}{\partial \nu}\right)
\end{aligned}
$$

and

$$
\frac{\partial \ell}{\partial \theta}=\sum_{1} \frac{1}{f_{2}-F\left(0, y_{2}\right)+F\left(0, y_{2}-1\right)}\left(-\frac{\partial F\left(0, y_{2}\right)}{\partial \theta}+\frac{\partial F\left(0, y_{2}-1\right)}{\partial \theta}\right)
$$

Similarly to the case where numerical gradients were used, for all distributions parametrised with $\sigma$ we will maximise the likelihood for $\sigma^{*}$, where $\sigma=\exp \left(\sigma^{*}\right)$. Thus,

$$
\begin{aligned}
\frac{\partial \ell}{\partial \sigma^{*}} & =\frac{\partial \ell}{\partial \sigma} \frac{\partial \sigma}{\partial \sigma^{*}} \\
& =\frac{\partial \ell}{\partial \sigma} \sigma .
\end{aligned}
$$

Also, since for Delaporte $\nu$ is constrained by 0 and 1 we will use $\nu^{*}$, where $\nu=\frac{\exp \left(\nu^{*}\right)}{1+\exp \left(\nu^{*}\right)}$. Then,

$$
\begin{aligned}
\frac{\partial \ell}{\partial \nu^{*}} & =\frac{\partial \ell}{\partial \nu} \frac{\partial \nu}{\partial \nu^{*}} \\
& =\frac{\partial \ell}{\partial \nu} \frac{\frac{\nu}{1-\nu}\left(1+\frac{\nu}{1-\nu}\right)-\left(\frac{\nu}{1-\nu}\right)^{2}}{\left(1+\frac{\nu}{1-\nu}\right)^{2}}
\end{aligned}
$$

Finally, $\theta$ will be replaced by $\theta^{*}$ and therefore

$$
\frac{\partial \ell}{\partial \theta^{*}}=\frac{\partial \ell}{\partial \theta} \frac{\partial \theta}{\partial \theta^{*}}
$$

The expression of $\frac{\partial \theta}{\partial \theta^{*}}$ for each copula is given in Table 5.5. Derivatives of copulas with respect to margins and the association parameter can be obtained after some tedious calculations. Similarly, the derivatives of pmfs and cdfs with respect to their parameters can also be obtained. All derivations can be found in Appendix F. 


\begin{tabular}{|c|c|c|}
\hline Copula & $\theta$ & $\frac{\partial \theta}{\partial \theta^{*}}$ \\
\hline FGM & $\tanh \left(\theta^{*}\right)$ & $1-\theta^{2}$ \\
\hline Normal & $\tanh \left(\theta^{*}\right)$ & $1-\theta^{2}$ \\
\hline AMH & $\tanh \left(\theta^{*}\right)$ & $1-\theta^{2}$ \\
\hline Clayton & $\exp \left(\theta^{*}\right)+\epsilon$ & $\theta-\epsilon$ \\
\hline Frank & $\theta^{*}+\epsilon$ & 1 \\
\hline Gumbel & $1+\exp \left(\theta^{*}\right)$ & $\theta-1$ \\
\hline Joe & $1+\exp \left(\theta^{*}\right)+\epsilon$ & $\theta-1-\epsilon$ \\
\hline
\end{tabular}

Table 5.5: Expressions for $\theta$ and $\frac{\partial \theta}{\partial \theta^{*}}$.

\subsubsection{Numerical checks}

In this section we will check whether the analytical gradients were derived correctly. The specification we will consider is the one with the same covariates in both equations. First, the analytical gradients will be checked against numerical gradients for two different sample sizes i.e. $n=1000$ and 5000. For numerical gradients, we will use the function grad implemented in the numDeriv $\mathrm{R}$ package (Gilbert and Varadhan, 2015). Specifically, grad computes the first derivatives of the input function. Second, we will compare the likelihood value to which the optim function converged when both types of gradients are used. Third, the nlm function, which performs non-linear minimisation in $\mathrm{R}$, will be used to see whether the gradient was approximately zero for the final iteration. Fourth, we will compare computational times for optim to terminate the optimisation procedure for analytical and numerical gradients. This will be done using system.time function, which is an in-built $\mathrm{R}$ routine designed for this purpose. Finally, simulation results will be presented.

The gradients were compared by generating simulated data, creating starting values and submitting the line code for the analytical and numerical gradients. Once this was done the absolute percentage difference between each of the gradient components was calculated. The gradient functions assume the equation setup described in Section 5.7. Also, for all checks $\sigma$ of the simulated data was set to $1, \nu$ to 0.5 and Pearson's correlation coefficient between covariates to 0.5. The value of Pearson's correlation coefficient is arbitrary. Kendall's $\tau$ was set to 0.2 for simplicity as it is within coverage of all copulas used. Table 5.6 shows the percentage of simulated data gradients for each outcome distribution and copula combination where the analytical gradients were close enough to the numerical ones. In this case, "closeness" meant that either the percentage difference did not exceed 1 per cent or the analytical gradient components did not differ by more than one nominal unit. For instance, if the the analytical gradient component was 14.5 and the numerical gradient 
component was 14.4 , this would suggest that the analytical gradient was derived correctly.

From Table 5.6 one can see that for the negative binomial, Delaporte, Poisson inverse Gaussian and Sichel distributions for different sample sizes the bivariate normal copula has a proportion of analytical gradients which are far from the numerical ones. By "far" is meant up to 5 per cent of discrepancy. The reason for this turned out to be the precision bounds on copulas. Consider the following numerical derivative of a copula with respect to the second margin:

$$
\frac{\partial C}{\partial F_{2}}=\frac{C\left(F_{1}, F_{2}+\omega\right)-C\left(F_{1}, F_{2}\right)}{\omega} .
$$

Typically, $C\left(F_{1}, F_{2}+\omega\right) \neq C\left(F_{1}, F_{2}\right)$. However, by setting bounds on $C$, for small $C$ it may be that $C\left(F_{1}, F_{2}+\omega\right)=C\left(F_{1}, F_{2}\right)$. Hence, the numerator of (5.8.3) will be equal to zero and the numerical derivative will be zero. The same happens when the VGAM, pbivnorm, copula or the CDVine packages are used to evaluate bivariate copulas. Nevertheless, when using optim for analytical gradients which were far from the numerical ones, the final parameter estimate values were close to the ones obtained with numerical gradients. Furthermore, the terminal log-likelihood values did not differ at all. Also, since the check for the Poisson distribution and bivariate normal copula model did not reveal any problems, the structure of the copula derivatives is likely to be correct. 


\begin{tabular}{|c|c|c|c|}
\hline Outcome distribution & Copula & $n=1000$ & $n=5000$ \\
\hline \multirow{7}{*}{ Poisson } & FGM & 100 & 100 \\
\hline & Bivariate normal & 100 & 100 \\
\hline & $\mathrm{AMH}$ & 100 & 100 \\
\hline & Clayton & 100 & 100 \\
\hline & Frank & 100 & 100 \\
\hline & Joe & 100 & 100 \\
\hline & Gumbel & 100 & 100 \\
\hline \multirow[t]{7}{*}{ Negative binomial } & FGM & 100 & 100 \\
\hline & Bivariate normal & 50 & 10 \\
\hline & $\mathrm{AMH}$ & 100 & 100 \\
\hline & Clayton & 100 & 100 \\
\hline & Frank & 80 & 100 \\
\hline & Joe & 100 & 100 \\
\hline & Gumbel & 100 & 100 \\
\hline \multirow[t]{7}{*}{ Delaporte } & FGM & 100 & 100 \\
\hline & Bivariate normal & 50 & 20 \\
\hline & $\mathrm{AMH}$ & 100 & 100 \\
\hline & Clayton & 100 & 100 \\
\hline & Frank & 100 & 100 \\
\hline & Joe & 100 & 100 \\
\hline & Gumbel & 100 & 100 \\
\hline \multirow[t]{7}{*}{ Poisson inverse Gaussian } & FGM & 100 & 100 \\
\hline & Bivariate normal & 50 & 0 \\
\hline & $\mathrm{AMH}$ & 100 & 100 \\
\hline & Clayton & 100 & 100 \\
\hline & Frank & 100 & 100 \\
\hline & Joe & 100 & 100 \\
\hline & Gumbel & 100 & 100 \\
\hline \multirow[t]{7}{*}{ Sichel } & FGM & 100 & 100 \\
\hline & Bivariate normal & 50 & 20 \\
\hline & $\mathrm{AMH}$ & 100 & 100 \\
\hline & Clayton & 100 & 100 \\
\hline & Frank & 100 & 70 \\
\hline & Joe & 100 & 100 \\
\hline & Gumbel & 100 & 100 \\
\hline
\end{tabular}

Table 5.6: Numerical versus analytical gradient check: percentage of times gradient norms were close enough. In this case, "closeness" meant that either the percentage difference did not exceed 1 per cent or the analytical gradient components did not differ by more than one nominal unit. For instance, if the the analytical gradient component was 14.5 and the numerical gradient component was 14.4, this would suggest that the analytical gradient was derived correctly. 
Frank copula also shows imprecision for the negative binomial and Sichel distribution. The same explanation follows as for the bivariate normal case. The point estimates obtained in the optim procedure differed by more than three decimal places when comparing analytical to numerical gradients.

It is worth mentioning that for Sichel and Poisson Inverse Gaussian distributions when $n=5000$, NaNs for parameters associated with the outcome distribution occurred. This was first remedied by introducing upper bounds on the Bessel functions of third kind. Unfortunately, instead of NaNs high discrepancies started to occur. Ultimately, the derivatives of pmfs and cdfs had to be computed numerically with respect to linear predictors and scale and shape parameters (see: Appendix F). This solved the problem of NaNs and smaller discrepencies were observed.

In the next stage we will consider optimisation convergence issues. It turned out that for all outcome and copula combinations optim's terminal log-likelihood was the same regardless of whether the default in-built approximated gradient or analytical gradient were used. For the nlm optimising function, when using analytical gradients all Poisson and negative binomial distribution models converged arriving at a gradient norm approximately equal to zero. Delaporte and Poisson inverse Gaussian distribution also arrived at a zero gradient after introducing an upper bound of $10^{7}$ on $\mu$ and $\sigma$ parameters. However, for all copulas with Sichel margin the algorithm could not terminate since there was an overflow. To remedy this, the maximum allowable scaled step length was set to 5 . Once this was done, the algorithm could terminate at the iteration where the gradient norm was equal to zero.

The $\mathrm{R}$ in-built system.time function was used to compare the computational burden of optim when numerical and analytical gradients were used. For each model check, data were generated as if the outcome distribution and copula were known. $\sigma$ was set to $1, \nu$ to $0.5, \tau$ to 0.2 and the Pearson correlation coefficient between covariates was 0.5. For Sichel distribution functions function scaling of 200 was used. For the Poisson, Delaporte, Poisson inverse Gaussian and Sichel distribution the optimisation with analytical gradient was on average faster by $0.2,10,35$ and 150 seconds respectively. For the negative binomial distribution, the numerical gradient was on average 1.5 seconds faster then the analytical one. Moreover, when increasing the sample size from 1000 to 5000 numerical gradients for Poisson distribution were faster than the analytical ones. One may speculate that the cdf functions of Poisson and negative binomial do not require looping as opposed 
to the remaining distributions. Thus, calculating analytical derivatives which require looping may be more computationally intensive than the numerical ones.

\subsubsection{Simulations}

Figures G.11-G.15 in Appendix G show simulation results analogical to ones in Section 5.7. The setup is the same apart from the fact that analytical gradients are used. Similarly, all boxplots in all figures are overlapping with the dotted lines. Again, it seems that the maximum likelihood algorithm enhanced by analytical gradients works well.

\subsection{Hessian and trust algorithm}

In this section of the work we will introduce the analytical Hessian, which typically makes the estimates more precise and the algorithm more efficient. However, this may not always be true. For example, previously we have seen that the numerical derivative of the the Sichel margin may be more precise since for the analytical one the Bessel functions tend to overflow i.e. very large numbers cannot be evaluated. The trust region algorithm (Sorensen, 1982) will then be discussed. The analytical Hessian will be checked against the numerical one. Finally, simulations for the trust region algorithm and analytical Hessian will be conducted.

\subsubsection{General form of second derivatives}

Let us derive the general form of the Hessian components. For instance, the second derivative with respect to selection equation parameters is

$$
\begin{aligned}
\frac{\partial^{2} \ell}{\partial \boldsymbol{\delta}_{\mathbf{1}} \partial \boldsymbol{\delta}_{\mathbf{1}}} & =\sum_{0}-\frac{1}{F_{1}^{2}}\left(\frac{\partial F_{1}}{\partial \boldsymbol{\delta}_{\mathbf{1}}}\right)+\frac{1}{F_{1}} \frac{\partial^{2} F_{1}}{\partial \boldsymbol{\delta}_{\mathbf{1}} \partial \boldsymbol{\delta}_{\mathbf{1}}} \\
& +\sum_{1}-\frac{1}{\left(f_{2}-F\left(0, y_{2}\right)+F\left(0, y_{2}-1\right)\right)^{2}}\left[\frac{\partial}{\partial \boldsymbol{\delta}_{\mathbf{1}}}\left(-F\left(0, y_{2}\right)+F\left(0, y_{2}-1\right)\right)\right]^{2} \\
& +\frac{1}{f_{2}-F\left(0, y_{2}\right)+F\left(0, y_{2}-1\right)} \frac{\partial^{2}}{\partial \boldsymbol{\delta}_{\mathbf{1}} \partial \boldsymbol{\delta}_{\mathbf{1}}}\left(-F\left(0, y_{2}\right)+F\left(0, y_{2}-1\right)\right) .
\end{aligned}
$$

The remaining derivations can be found in Appendix H. The general form consists of copula and margin derivatives (see manual derivations in: Appendix I). Note that the general form, copula and 
selection margin derivatives are entirely analytical. The derivatives related to the outcome margin are numerical. The reason for calculating numerical second derivatives of the outcome margins is that it would be too complicated to derive them analytically. For instance, consider the first derivatives of the Delaporte pmf:

$$
\begin{aligned}
\frac{\partial f_{2}}{\partial \delta_{2}} & =f_{2}\left[(-\mu \nu)-\frac{1}{\sigma} \mu \sigma(1-\nu)(1+\mu \sigma(1-\nu))^{-1}+S_{\boldsymbol{\delta}_{\mathbf{2}}}^{\prime} / S\right] \mathbf{X}_{\mathbf{2}} \\
\frac{\partial f_{2}}{\partial \sigma} & =f_{2}\left[d^{\prime} / d+\psi\left(\frac{1}{\sigma}\right)\left(\frac{1}{\sigma^{2}}\right)+S_{\sigma}^{\prime} / S\right] \\
\frac{\partial f_{2}}{\partial \nu} & =f_{2}\left[-\mu+\mu(1+\mu \sigma(1-\nu))^{-1}+S_{\nu}^{\prime} / S\right]
\end{aligned}
$$

where $S=\sum_{j=0}^{y_{2}}\left(\begin{array}{c}y_{2} \\ j\end{array}\right) \frac{\mu^{y_{2}} \nu^{y_{2}-j}}{y_{2} !}\left[1+\frac{1}{\sigma(1-\nu)}\right] \Gamma\left(\frac{1}{\sigma} j\right), \mu=e^{\eta_{2}}$ and $\eta_{2}$ is the linear predictor of the outcome equation, $d=[1+\mu \sigma(1-\nu)]^{-\frac{1}{\sigma}}$ and $d^{\prime}=\left\{\frac{1}{\sigma^{2}} \log [1+\mu \sigma(1-\nu)]-\frac{1}{\sigma} \frac{1}{1+\mu \sigma(1-\nu)} \mu(1-\nu)\right\} d$ is the derivative of $d$ with respect to $\sigma . S^{\prime}$ indicates the derivative of $S$ and its subscript indicates the parameter with respect to which it was calculated. Obtaining analytical second derivatives for these expressions would be tedious.

Again, reparametrisation has to be made when calculating second derivatives with respect to $\theta^{*}, \sigma^{*}, \theta^{*}$. We will now discuss the trust region algorithm which will be used for optimisation using analytical Hessian.

\subsubsection{Trust algorithm}

The optim R function uses the BFGS algorithm to conduct optimisation. Unfortunately, optim cannot use an analytical Hessian. optimx function from the optimx package allows for BFGS with analytical Hessian, but the algorithm does not seem to work well both for numerical and analytical Hessian. The same can be said about the nlm function.

Line search methods, such as BFGS, find a search direction and then step length to move from the current iterate $x_{k}$ to the next one $x_{k+1}$. If the objective function is non-convex or flat, the algorithm will search $x_{k+1}$ which is far from $x_{k}$, but will typically yield a marginal improvement. However, it may happen that $x_{k+1}$ is too far from $x_{k}$. Hence, the objective function will not be evaluated and the algorithm will not converge (Braun, 2013).

On the other hand, the trust region algorithm (Sorensen, 1982) establishes a sphere around the 
current iterate $x_{k}$ within which the next iterate $x_{k+1}$ is to be found. If for the candidate for $x_{k+1}$ the model function is not close enough to the objective, then the region is shrunk and a new candidate found. Because the candidate points never lie beyond the region, the algorithm will not run too far from the current point. If the objective function is undefined or indeterminate at an iteration, then line search methods like BFGS will fail. On the other hand, the trust algorithm will reject the candidate $x_{k+1}$, reduce the radius of the region and try to find $x_{k+1}$ again (Geyer, 2011). Thus, the trust region algorithm turns out to be more stable than line search methods, especially for functions which are non-convex, ill-conditioned and have long plateaus (Braun, 2013).

As a starting point, let us recall the Taylor's approximation to the objective function $f(\boldsymbol{x})$ at iterate $k$ :

$$
\begin{aligned}
f(\boldsymbol{x}) & \approx m_{k}\left(\boldsymbol{p}_{\boldsymbol{k}}\right) \\
& =f_{k}+\boldsymbol{\nabla} \boldsymbol{f}_{\boldsymbol{k}}^{\top} \boldsymbol{p}_{\boldsymbol{k}}+\frac{1}{2} \boldsymbol{p}_{\boldsymbol{k}}^{\top} \boldsymbol{B}_{\boldsymbol{k}} \boldsymbol{p}_{\boldsymbol{k}}
\end{aligned}
$$

where $\boldsymbol{p}_{\boldsymbol{k}}=\boldsymbol{x}-\boldsymbol{x}_{\boldsymbol{k}}, \boldsymbol{\nabla} \boldsymbol{f}_{\boldsymbol{k}}$ is the gradient vector and $\boldsymbol{B}_{\boldsymbol{k}}$ is the Hessian matrix at $k$-th iteration. In the BFGS quasi-Newton approach we differentated $m_{k}\left(\boldsymbol{p}_{\boldsymbol{k}}\right)$ with respect to $\boldsymbol{p}_{\boldsymbol{k}}$ and set the derivative to zero. Then, the step length was chosen via a line search procedure.

In a trust region approach we choose the region within which $m_{k}$ can be trusted to be close to $f(\boldsymbol{x})$ and the step and search direction length to minimise $m_{k}$. If the step is not acceptable, the size of the trust region is reduced (Nocedal and Wright, 2006). The radius of the region is essential to the efficiency of the algorithm. If the radius is too small, the algorithm will miss a chance to move closer to the minimiser of $f(\boldsymbol{x})$ within the trust region. If the radius is too large, then the step may be rejected since the minimiser of $m_{k}$ will be too far from the minimiser of $f(\boldsymbol{x})$. As we will see, the size of the radius in the current iteration will depend on the radius in the previous iteration. Generally, if the minimiser of $m_{k}$ is close enough to $f(\boldsymbol{x})$ the radius is increased. Otherwise, the radius will be decreased or unchanged as compared to the previous iteration.

Let us now respecify $\boldsymbol{p}_{\boldsymbol{k}}=\boldsymbol{x}_{\boldsymbol{k}+\mathbf{1}}-\boldsymbol{x}_{\boldsymbol{k}}$. For obtaining $\boldsymbol{p}_{\boldsymbol{k}}$, at each iteration we seek the solution to the problem:

$$
\min _{p \in \mathbb{R}^{n}} m_{k}\left(\boldsymbol{p}_{\boldsymbol{k}}\right)=f_{k}+\nabla \boldsymbol{f}_{\boldsymbol{k}}^{\top} \boldsymbol{p}_{\boldsymbol{k}}+\frac{1}{2} \boldsymbol{p}_{\boldsymbol{k}}^{\top} \boldsymbol{B}_{\boldsymbol{k}} \boldsymbol{p}_{\boldsymbol{k}} \quad \text { s.t. }\left\|\boldsymbol{p}_{\boldsymbol{k}}\right\| \leq \triangle_{k}
$$


where $\|\cdot\|$ is the Euclidean norm and $\triangle_{k}$ is the radius of the trust-region at iteration $k$. If $\boldsymbol{B}_{\boldsymbol{k}}$ is the analytical Hessian, then the algorithm becomes a trust-region Newton method. In this case, $m_{k}$ will agree with the Taylor series to three terms. Therefore, the difference between the model function and the objective function will be

$$
\begin{aligned}
f\left(\boldsymbol{x}_{\boldsymbol{k}+\mathbf{1}}\right)-m_{k}\left(\boldsymbol{p}_{\boldsymbol{k}}\right) & =\left(f_{k}+\nabla \boldsymbol{f}_{\boldsymbol{k}}^{\top} \boldsymbol{p}_{\boldsymbol{k}}+\frac{1}{2} \boldsymbol{p}_{\boldsymbol{k}}^{\top} \boldsymbol{B}_{\boldsymbol{k}} \boldsymbol{p}_{\boldsymbol{k}}+O\left(\|\boldsymbol{p}\|^{3}\right)\right)-\left(f_{k}+\nabla \boldsymbol{f}_{\boldsymbol{k}}^{\top} \boldsymbol{p}_{\boldsymbol{k}}+\frac{1}{2} \boldsymbol{p}_{\boldsymbol{k}}^{\top} \boldsymbol{B}_{\boldsymbol{k}} \boldsymbol{p}_{\boldsymbol{k}}\right) \\
& =O\left(\|\boldsymbol{p}\|^{3}\right),
\end{aligned}
$$

where $O\left(\|\boldsymbol{p}\|^{3}\right)$ denotes the remainder (or the approximation error) of the Taylor series from the fourth term onwards. The approximation will be especially accurate when $\boldsymbol{p}$ is small (Nocedal and Wright, 2006).

Once the radius of the trust region is defined and the minimiser of $m$ found at iteration $k$ it is necessary to measure closeness between the objective function and the model. The closeness will be a benchmark for deciding about the trust region radius and parameter vector in the next iteration. The numerator of

$$
\rho_{k}=\frac{f\left(\boldsymbol{x}_{\boldsymbol{k}}\right)-f\left(\boldsymbol{x}_{\boldsymbol{k}}+\boldsymbol{p}_{\boldsymbol{k}}\right)}{m(\mathbf{0})-m\left(\boldsymbol{p}_{\boldsymbol{k}}\right)}
$$

will be the actual reduction of the objective function and the denominator will be the predicted reduction. The first terms of the numerator and denominator are evaluated at iteration $k$ i.e. $\boldsymbol{p}_{\boldsymbol{k}}=\boldsymbol{x}_{\boldsymbol{k}}-\boldsymbol{x}_{\boldsymbol{k}}=\mathbf{0}$ and the second terms are evaluated at iteration $k+1$ i.e. $\boldsymbol{p}_{\boldsymbol{k}}=\boldsymbol{x}_{\boldsymbol{k}+\mathbf{1}}-\boldsymbol{x}_{\boldsymbol{k}}$. Note that since $\boldsymbol{p}_{\boldsymbol{k}}$ is obtained by minimising over the region which includes the step $\boldsymbol{p}_{\boldsymbol{k}}=\mathbf{0}$, $m(\mathbf{0})-m\left(\boldsymbol{p}_{\boldsymbol{k}}\right) \geq 0$. Thus, if $\rho$ is negative or close to zero, then there will be no agreement between the model $m\left(\boldsymbol{p}_{\boldsymbol{k}}\right)$ and the objective function $f\left(\boldsymbol{x}_{\boldsymbol{k}}+\boldsymbol{p}_{\boldsymbol{k}}\right)$ - the step will be rejected and the trust region radius reduced at the next iteration. However, if $\rho$ is close to unity, then there will be a good agreement between the model and the objective function - the step will be accepted and the radius increased at the next iteration. Otherwise, the step will be accepted and the radius will remain unchanged.

Figure 5.7 compares the algorithm against line search methods based on a two parameter likelihood function (extracted from: Nocedal and Wright, 2006). The current point lies in upper end 
while the minimum point lies in the lower end of the valley of $f(\boldsymbol{x})$ contours. The quadratic model $m_{k}$, whose elliptical contours are shown by the dashed lines, is based on the information of $f(\boldsymbol{x})$ and its first and second derivatives. As shown by the longer arrow, a line search method based on this model searches along the step to the minimiser of $m_{k}$. This direction, however, allows only a small reduction in $f(\boldsymbol{x})$ even if an optimal step is taken. A trust-region method, as shown by the shorter arrow, shifts to the minimiser of $m_{k}$ within the dotted circle, which yields a more significant reduction in $f(\boldsymbol{x})$ and a better step.

Figure 5.7: Comparison of the algorithm against line search methods based on a two parameter likelihood function (extracted from: Nocedal and Wright, 2006). The current point lies in upper end while the minimum point lies in the lower end of the valley of $f(\boldsymbol{x})$ contours. The quadratic model $m_{k}$, whose elliptical contours are shown by the dashed lines, is based on the information of $f(\boldsymbol{x})$ and its first and second derivatives. As shown by the longer arrow, a line search method based on this model searches along the step to the minimiser of $m_{k}$. This direction, however, allows only a small reduction in $f(\boldsymbol{x})$ even if an optimal step is taken. A trust-region method, as shown by the shorter arrow, shifts to the minimiser of $m_{k}$ within the dotted circle, which yields a more significant reduction in $f(\boldsymbol{x})$ and a better step.

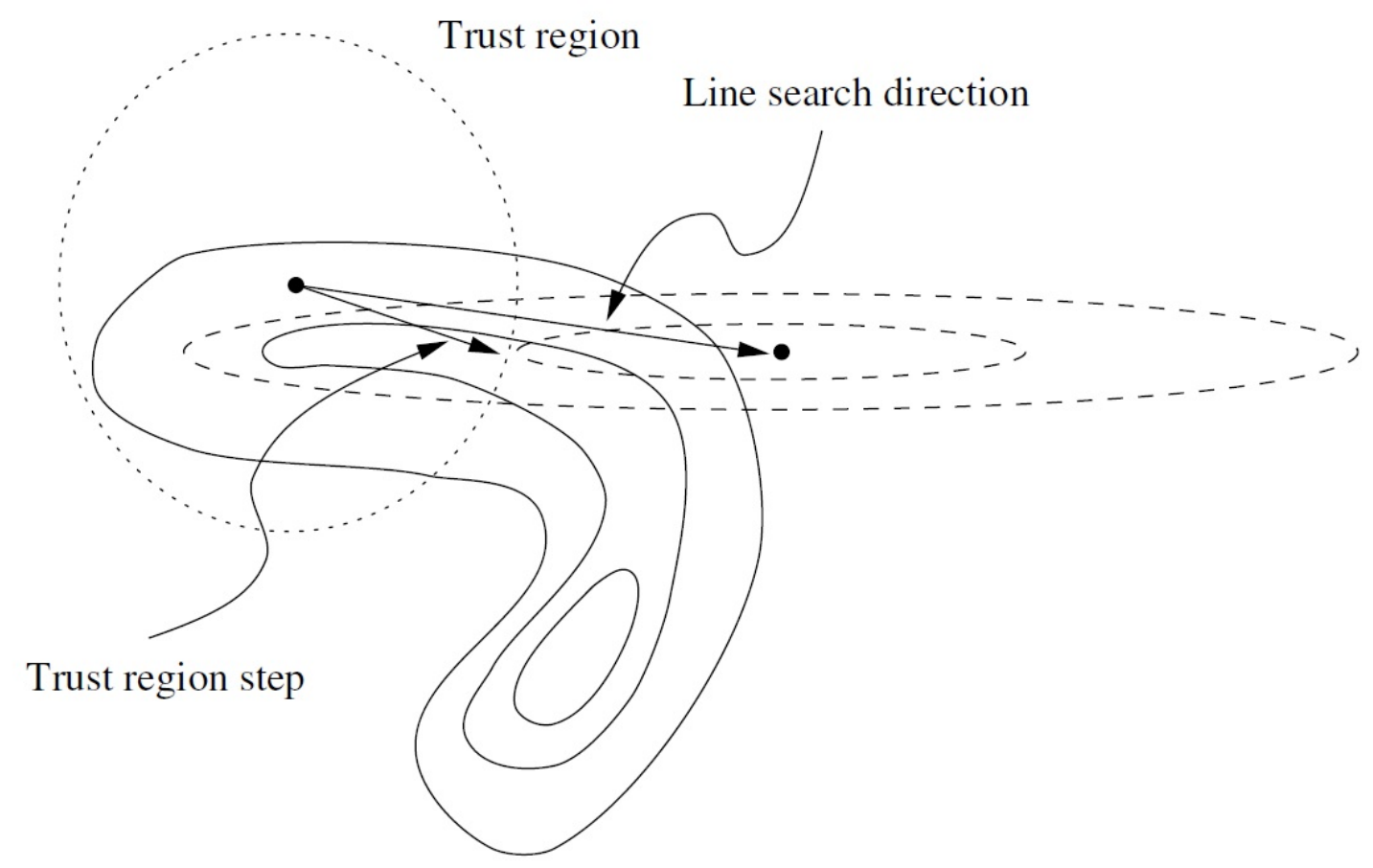

Summarising, given $\bar{\triangle}>0, \triangle_{0} \in(0, \bar{\triangle})$ and $\eta \in\left(0, \frac{1}{4}\right]$, loop the following for $k=0,1,2 \ldots$ :

1. Obtain $\boldsymbol{p}_{\boldsymbol{k}}$ by solving (5.9.1);

2. Evaluate $\rho_{k}$. 
3. If $\rho_{k}<\frac{1}{4}$

$$
\triangle_{k+1}=\frac{1}{4}\left\|\boldsymbol{p}_{\boldsymbol{k}}\right\|
$$

else

if $\rho_{k}>\frac{3}{4}$ and $\left\|\boldsymbol{p}_{\boldsymbol{k}}\right\|=\triangle_{k}$,

$\triangle_{k+1}=\min \left(2 \triangle_{k+1}, \bar{\triangle}>0\right)$,

else $\triangle_{k+1}=\triangle_{k}$.

4. If $\rho_{k}>\eta$

$x_{k+1}=x_{k}+p_{k}$,

else

$x_{k+1}=x_{k}$.

$\bar{\triangle}$ is the overall bound on the step lengths and $\eta$ is a constant which is set to $\frac{1}{4}$ in trust $\mathrm{R}$ package (Geyer, 2011). It is worth mentioning that the radius will be only increased when the step length reaches the size of the radius i.e. $\left\|\boldsymbol{p}_{\boldsymbol{k}}\right\|=\triangle_{k}$. Otherwise, since the accepted step length will act within the trust region, the radius will not interfere with the algorithm and thus will be left unchanged.

\subsubsection{Hessian checks}

We will now compare the analytical Hessian to the numerical one. First, we will use the hessian function from the numDeriv package to compare the analytical to the numerical Hessian component values. system.time will be used to compare the computational time for the cases with analytical and numerical Hessians.

Table 5.7 shows the percentage of times the analytical and numerical Hessian were close enough to each other. Similarly as in the case of gradient, "closeness" means 1 per cent difference relatively to the numerical Hessian. All Hessians are close enough for all copula and margin combinations apart from the bivariate normal and Frank copulas for negative binomial, Delaporte, Poisson inverse Gaussian and Sichel distributions. The reason is the same as for the gradients. In brief, some copula cdfs which are very small are evaluated as zeros. Hence, setting them to $10^{-8}$ will change the evaluation of Hessian components. It is worth mentioning that the bivariate normal copula had a high but permissable discrepancy for Poisson distribution. Despite that, in most cases the 
analytical Hessian seems to be more precise than the numerical one and hence can be used for the trust region algorithm. 


\begin{tabular}{|c|c|c|c|}
\hline Outcome distribution & Copula & $n=1000$ & $n=5000$ \\
\hline \multirow[t]{7}{*}{ Poisson } & FGM & 100 & 100 \\
\hline & Bivariate normal & 100 & 100 \\
\hline & $\mathrm{AMH}$ & 100 & 100 \\
\hline & Clayton & 100 & 100 \\
\hline & Frank & 100 & 100 \\
\hline & Joe & 100 & 100 \\
\hline & Gumbel & 100 & 100 \\
\hline \multirow[t]{7}{*}{ Negative binomial } & FGM & 100 & 100 \\
\hline & Bivariate normal & 30 & 0 \\
\hline & $\mathrm{AMH}$ & 100 & 100 \\
\hline & Clayton & 100 & 100 \\
\hline & Frank & 90 & 80 \\
\hline & Joe & 100 & 100 \\
\hline & Gumbel & 100 & 100 \\
\hline \multirow[t]{7}{*}{ Delaporte } & FGM & 100 & 100 \\
\hline & Bivariate normal & 60 & 20 \\
\hline & $\mathrm{AMH}$ & 100 & 100 \\
\hline & Clayton & 100 & 100 \\
\hline & Frank & 100 & 80 \\
\hline & Joe & 100 & 100 \\
\hline & Gumbel & 100 & 100 \\
\hline \multirow[t]{7}{*}{ Poisson inverse Gaussian } & FGM & 100 & 100 \\
\hline & Bivariate normal & 50 & 0 \\
\hline & $\mathrm{AMH}$ & 100 & 100 \\
\hline & Clayton & 100 & 100 \\
\hline & Frank & 80 & 60 \\
\hline & Joe & 100 & 100 \\
\hline & Gumbel & 100 & 100 \\
\hline \multirow[t]{7}{*}{ Sichel } & FGM & 100 & 100 \\
\hline & Bivariate normal & 30 & 0 \\
\hline & $\mathrm{AMH}$ & 100 & 100 \\
\hline & Clayton & 100 & 100 \\
\hline & Frank & 100 & 100 \\
\hline & Joe & 100 & 100 \\
\hline & Gumbel & 100 & 100 \\
\hline
\end{tabular}

Table 5.7: Numerical versus analytical Hessian check: percentage of times all Hessian elements were close enough.

Regarding computational times, optim was used in the case of analytical gradient only, whereas trust was used in the case of analytical gradient and Hessian. The reason for using optim is that it provides a benchmark for comparing with the previous results. Also, trust does not have an internal function approximating the Hessian. For Poisson, negative binomial and Delaporte distributions optim is twice as fast as the trust algorithm. For Poisson inverse Gaussian distribution and AMH copula, trust is generally faster than optim. Sometimes trust turned out to be faster for Poisson inverse Gaussian combinations when system.time was re-run. Despite the fact that the 
computational efficiency of trust depends on the choice of outcome distribution, we will still select the trust algorithm. Based on arguments from Section 5.9.2, trust turns out to be more reliable in numerical optimisation than optim. For instance, as we will see in the next section, for the vast majority of copula and outcome distribution combinations default algorithm settings can be used, which provide satisfactory results.

\subsubsection{Simulations}

In the simulation procedure the initial radius was set 1 for all copula and outcome combinations. The exceptions were the Frank copula and negative binomial case for the same covariates and instrumental variable, and Frank copula and Poisson inverse Gaussian with the same covariates, where the initial radius was set to $10^{-5}, 0.1,10^{-4}$ respectively. The reason for setting a small radius is that the simulation returned an error when a greater initial radius was determined. No parameter scaling was used.

All simulation boxplots can be found in Appendix J. All boxplots are symmetric and their medians are close to the reference lines. However, for the Sichel distribution and Frank margin the instrumental variable boxplots are more symmetric than those for the same set of covariates. Apparently, there are some exclusion restriction problems associated with the fact that no instrumental variable is used. This suggests that the identification will depend on the choice of covariates. The next section will elaborate on this matter.

\subsubsection{Exclusion restriction}

In the chapter on estimation we have seen that for the two-step approach it is easy to intuitively explain the problem of exclusion restriction. In the case of maximum likelihood, according to the author's best knowledge, no formal proof or intuitive explanation was demonstrated. In this section we will explore this issue empirically via a simulation study. The limitation of this approach is that it is restricted to the specifications used with only a few covariates and a more laborious simulation study with multiple settings needs to be conducted. 
In simulations, we specify

$$
\begin{aligned}
& \eta_{1}=\alpha_{0}+\alpha_{1} x_{1}+\alpha_{2} x_{2}+\alpha_{3} x_{3}, \\
& \eta_{2}=\alpha_{4}+\alpha_{5} x_{1}+\alpha_{6} x_{2},
\end{aligned}
$$

where $\eta_{1}$ and $\eta_{2}$ are the linear predictors of the selection and outcome equations respectively; $\boldsymbol{\alpha}=\left[\alpha_{0}, \alpha_{1}, \alpha_{2}, \alpha_{3}, \alpha_{4}, \alpha_{5}, \alpha_{6}\right]^{\top}$ is the parameter vector and $\boldsymbol{x}=\left[x_{1}, x_{2}, x_{3}\right]$ is the vector of covariates. When performing simulations using trust for arbitrary values of $\boldsymbol{\alpha}$, outcomes and copulas, the boxplots do not generally indicate any abnormal shapes both for IV and non-IV cases. However, there are two specifications where the instrumental variable helps in making boxplots more symmetric and closer to the reference values. To diagnose the problem of abnormal boxplots we will use the profile likelihood of each parameter.

In the first specification, the combination of Sichel distribution and Frank copula was used with $\boldsymbol{\alpha}=[1,2,-2,0.3,1.1,1.8,-1.9]^{\top}$, Kendall's $\tau$ and sample size equal to 0.5 and 1000 . When starting values are used to fix parameters, no substantial diferences can be found between corresponding shapes. On the other hand, when terminal optimisation values are used to fix parameters, the profile likelihood of $\theta^{*}$ differs for the case with and without instrumental variable. Other parameters remain unaffacted by the presence of an instrumental variable. Figures 5.8 and 5.9 show profile likelihoods for $\theta^{*}$ for the case with and without instrumental variable and other parameters fixed at terminal values. The shape of profile likelihoods does not differ much for the remaining parameters. The same can be said about the second specification where the negative binomial distribution and Frank copula were used with $\boldsymbol{\alpha}=[1,2.5,-3,0.5,3,-1,-2]^{\top}$, Kendall's $\tau$ and sample size equal to 0.5 and 1000. Figures 5.10 and 5.11 show $\theta^{*}$ profile likelihoods with other parameters fixed at terminal values for the case with and without instrumental variable. 
Figure 5.8: Profile likelihood of Sichel Frank model with IV. Kendall's $\tau=0.5$ and $\theta=\theta^{*}=5.7$. The shape of the profile likelihood for the remaining parameters is the same for the case with and without instrumental variable.

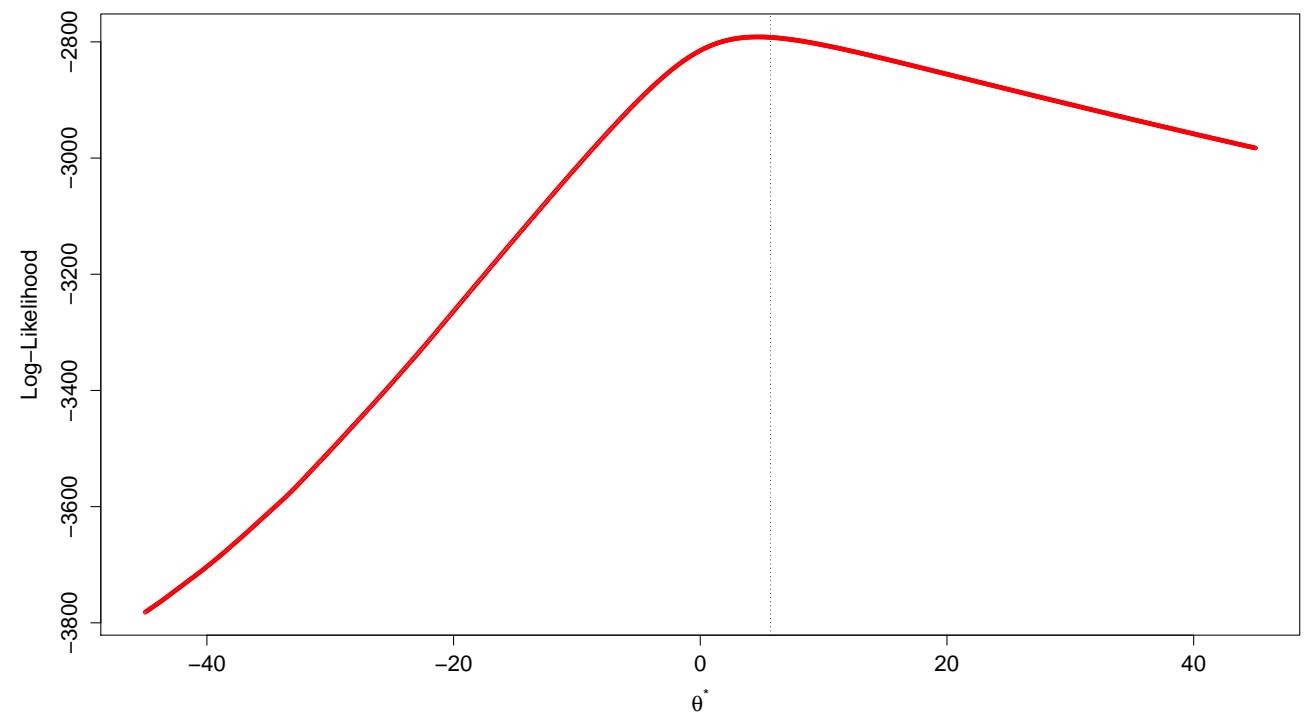

Figure 5.9: Profile likelihood of Sichel Frank model without IV. Kendall's $\tau=0.5$ and $\theta=\theta^{*}=5.7$. The shape of the profile likelihood for the remaining parameters is the same for the case with and without instrumental variable. The difference in shapes as compared to Figure 5.8 is likely to cause identification problems.

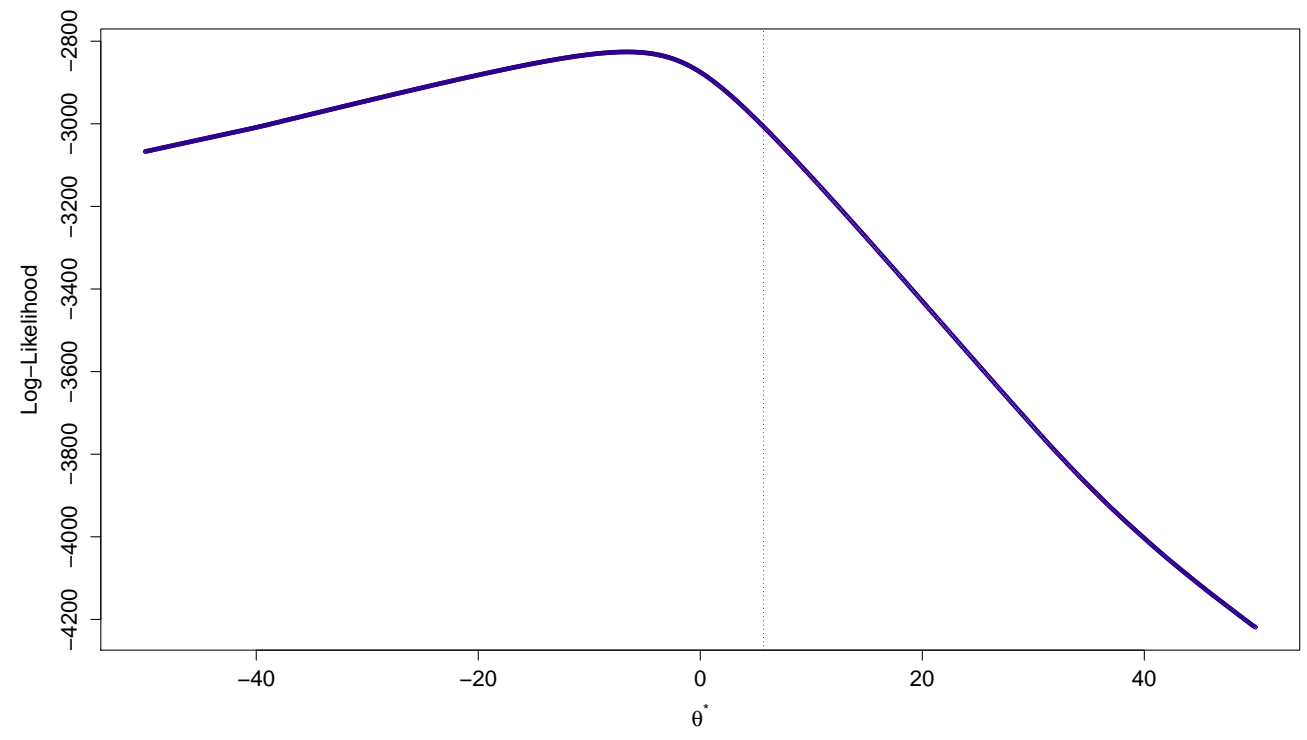


Figure 5.10: Profile likelihood of Negative binomial Frank model with IV. Kendall's $\tau=0.5$ and $\theta=\theta^{*}=5.7$. The shape of the profile likelihood for the remaining parameters is the same for the case with and without instrumental variable.

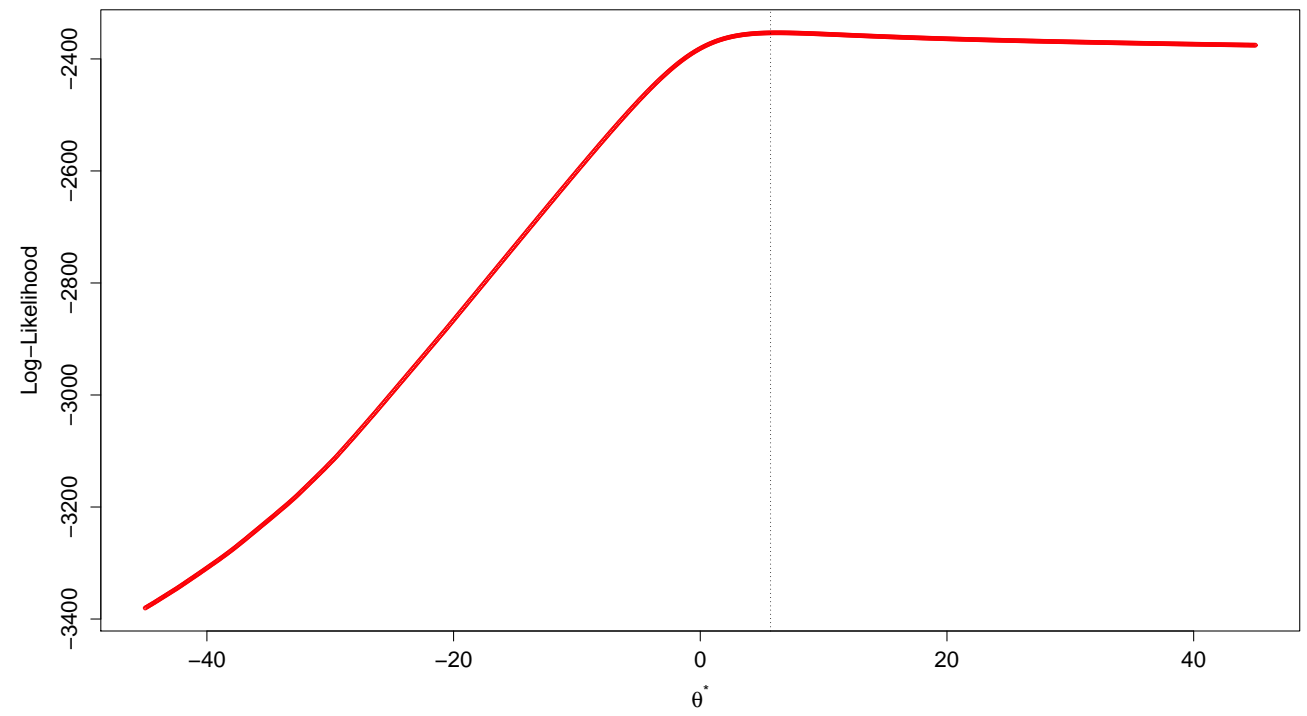

Figure 5.11: Profile likelihood of Negative binomial Frank model without IV. Kendall's $\tau=0.5$ and $\theta=\theta^{*}=5.7$. The shape of the profile likelihood for the remaining parameters is the same for the case with and without instrumental variable. The difference in shapes as compared to Figure 5.10 is likely to cause identification problems.

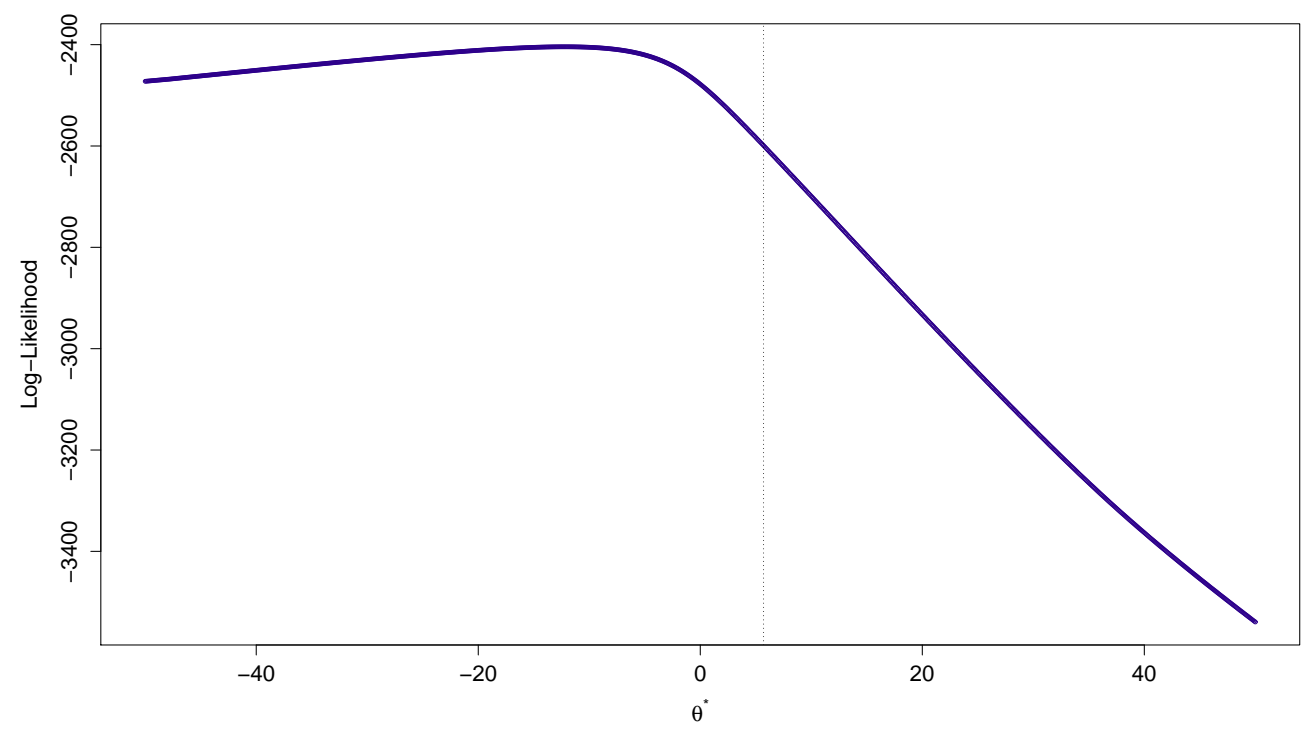


Intuitively, failure to arrive at the maximum is due to the shape of $\theta^{*}$ since for both specifications $\theta^{*}$ profile likelihood differed when comparing cases with and without IV. Presumably, the shape of the likelihood function was too "bumpy" at some stage of optimisation. This hypothesis cannot be verified since we cannot visualise the likelihood in more than two dimensions.

\subsection{Copula rotations}

As we have seen previously, some copulas do not have full coverage. For instance, Clayton copula can only capture Kendall's $\tau$ between 0 and 1 . If in a preliminary analysis Kendall's $\tau$ turns out to be negative, then copulas like Clayton would be of little use. This can be rectified by copula rotations. We will now present copula rotations of Clayton, Joe and Gumbel by 90, 180 and 270 degrees. They are defined as

$$
\begin{aligned}
C_{90} & =v-C(1-u, v) \\
C_{180} & =u+v-1+C(1-u, 1-v) \\
C_{270} & =u-C(u, 1-v)
\end{aligned}
$$

where $C$ denotes the unrotated copula and $u$ and $v$ are the margins (Brechmann and Schepsmeier, 2013). The manual derivations of first and second derivatives are provided in Appendix K.

Figure 5.12 provides contours of Clayton with normal margins unrotated and rotated by 90, 180 and 270 degrees with Kendall's $\tau$ equal to $0.5,-0.5,0.5$ and -0.5 respectively. The 90 and 270 degrees copulae show negative dependence. The 180 degrees (survival) copula still shows only positive dependence, but greater tail dependence now turns out to be in the upper right corner as compared to the unrotated copula.

Analytical gradients and Hessians were checked against the numerical ones. Overall, analytical gradients and Hessians turned out to be close enough to the numerical ones and hence the analytical quantities can be used in further analysis. Simulations for rotated copulas can be found in Appendix L. The same specifaction was used as for the simulations in the previous sections. Two scenarios were considered - one with and one without instrumental variable. Since the boxplots are symmetric and close to the reference lines, rotated copulas seem to work well. 
Figure 5.12: Contours of original Clayton and rotated copulae by 90, 180, 270 degrees with normal margins. Kendall's $\tau$ is equal to $0.5,-0.5,0.5$ and -0.5 respectively. The 90 and 270 degrees copulae show negative dependence. The 180 degrees (survival) copula still shows only positive dependence, but greater tail dependence now turns out to be in the upper right corner as compared to the unrotated copula.

Clayton

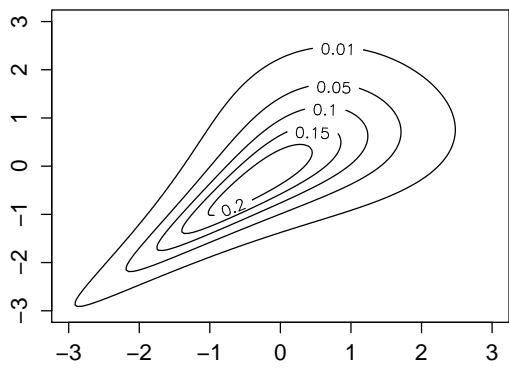

Clayton 180

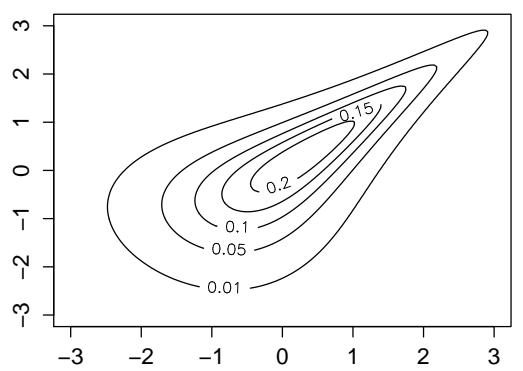

Clayton 90

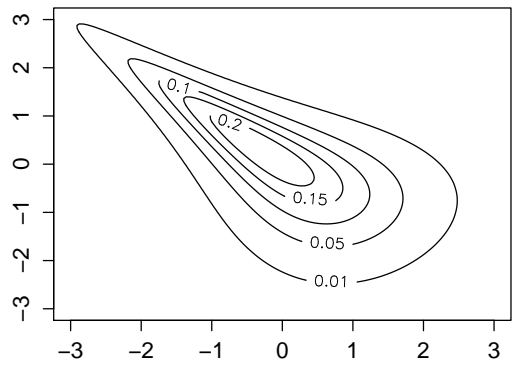

Clayton 270

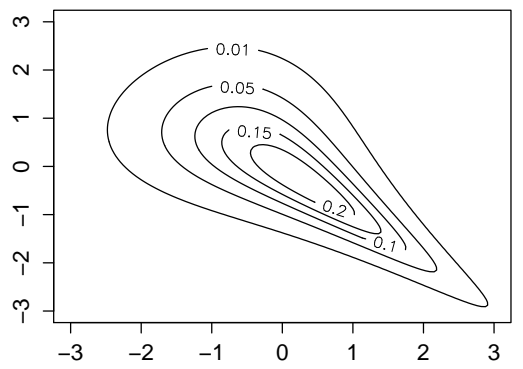




\subsection{Remarks on using Kendall's $\tau$ for a discrete and continuous margin}

The approach introduced in this chapter seems to be a useful tool in modelling discrete outcomes suffering from non-random sample selection. However, Genest and Neslehova (2007) argue that the dependence in terms of Kendall's $\tau$ between two variables is no longer a function of copula alone, but depends on the margins as well. This means that probabilistic and copula-based definitions of concordance measures no longer coincide - in the probabilistic case, Kendall's $\tau$ is no longer invariant to non-linear transformation of random variables. Hence, one should be careful with the interpretation of Kendall's $\tau$. The same authors argue that the dependence of Kendall's $\tau$ on the margins is a result of non-zero probability of ties. Hence, they propose using Kendall's $\tau_{b}$ as an alternative, which, as mentioned in Section 3.3, accounts for ties.

Genest and Neslehova (2007) also argue that the inferences for the parameter $\theta$ should not be based on conversion from Kendall's $\tau$ and Spearman's $\rho$. This may lead to serious bias and inconsistency in the estimation.

None of the arguments above apply to our approach - in our case, all estimates are estimated as $\theta$ or $\theta^{*}$ and hence converted to Kendall's $\tau$. In fact, the simulations do not show that there could be any bias related to Kendall's $\tau$.

The next chapter will introduce splines in the sample selection modelling framework. Hence, inferential methods will be discussed and simulations conducted. 


\section{Chapter 6}

\section{Introducing splines}

\subsection{Overview of splines}

In this chapter we will introduce splines in the sample selection modelling framework. These can be commonly found in the context of Generalized Addtive Models (GAMs; e.g. Hastie and Tibshirani, 1990). Thus, in this section we will give a brief overview of GAM theory. The notation and explanation will follow the one by Wood (2006). Note that everything presented in this section constitutes a revision of GAM theory and there are no new contributions to the topic per se. A generalized additive model can be represented as

$$
g(\mu)=\boldsymbol{x}^{\top} \boldsymbol{\beta}+f_{1}\left(z_{1}\right)+f_{2}\left(z_{2}\right)+f_{3}\left(z_{3}, z_{4}\right)
$$

where $\boldsymbol{x}$ is the vector of pre-specified continuous and binary covariates, $\boldsymbol{\beta}$ is the vector of parameters associated with the parametric component and $f_{1}, f_{2}$ and $f_{3}$ are the smooth (non-parametric) functions of the covariates $z_{1}, z_{2}, z_{3}$ and $z_{4}$ (Wood, 2006; Hastie and Tibshirani, 1986). $\mu=E(Y$ ) and response $Y$ typically follows a distribution from the exponential family. By introducing functions similar to $f$ we will be able to model continuous covariates more flexibly.

For simplicity, we will focus on the simplest possible GAM defined as

$$
y=f(z)+\epsilon
$$

where $y$ is the response variable, $z$ is the continuous covariate, $f$ is a smooth function and $\epsilon \sim$ 
$N\left(0, \sigma^{2}\right)$. Function $f$ in the linear predictor is typically represented by

$$
f(z)=\sum_{j=1}^{q} b_{j}(z) \alpha_{j},
$$

where $b_{j}(z)$ is the basis to be chosen and $\alpha_{j}$ is an unknown parameter to be estimated for the $j$-th basis function. One of the simplest bases that can be imagined is the third order polynomial basis where $b_{1}(z)=1, b_{2}(z)=z, b_{3}(z)=z^{2}$ and $b_{4}(z)=z^{3}$. Thus, (6.1.1) becomes

$$
y=\alpha_{1}+\alpha_{2} z+\alpha_{3} z^{2}+\alpha_{4} z^{3}+\epsilon .
$$

Another commonly used family of bases are splines. A spline is a curve made up of sections of polynomial joined together, which are continuous in value and their first and second derivatives (Wood, 2006). The sections of the splines are joined by knots, which are typically chosen by the modeller. For instance, the knots can be evenly spaced across the range of the explanatory variable or placed at its quantiles. Thus, their practical advantage towards polynomal bases is that they act on the function locally. A commonly known type of splines are the cubic splines, which are composed of cubic polynomials.

Let us explain the theoretical rationale behind splines. Consider a set of points where $\left\{x_{i}, y_{i}\right.$ : $i=1, \ldots, n\}$ with $x_{i}<x_{i+1}$. Natural cubic splines, $g(x)$, interpolating these points are made up of cubic polynomial sections for each $\left[x_{i}, x_{i+1}\right]$. These are joined together in a way that the whole spline is continuous to second derivative. Furthermore, $g\left(x_{i}\right)=y_{i}$ and the second derivatives of the endpoints are set to zero i.e. $g^{\prime \prime}\left(x_{1}\right)=g^{\prime \prime}\left(x_{n}\right)=0$. It can be shown that out of all functions that are continuous on $\left[x_{1}, x_{n}\right]$ and interpolate points, $g(x)$ is the one that is the smoothest in a sense of minimising (Wood, 2006)

$$
J(f)=\int_{x_{1}}^{x_{n}} f^{\prime \prime}(x)^{2} d x .
$$

The following proof is provided by Green and Silverman (1994) and reiterated by (Wood, 2006). Let $f(x)$ be an interpolant of $\left\{x_{i}, y_{i}\right\}$, where $f(x) \neq g(x)$ and let $h(x)=f(x)-g(x)$. The aim is to 
express $J(f)$ in terms of $J(g)$. Hence,

$$
\begin{aligned}
\int_{x_{1}}^{x_{n}} f^{\prime \prime}(x)^{2} d x & =\int_{x_{1}}^{x_{n}}\left(g^{\prime \prime}(x)+h^{\prime \prime}(x)\right)^{2} d x \\
& =\int_{x_{1}}^{x_{n}} g^{\prime \prime}(x)^{2} d x+2 \int_{x_{1}}^{x_{n}} g^{\prime \prime}(x) h^{\prime \prime}(x) d x+\int_{x_{1}}^{x_{n}} h^{\prime \prime}(x)^{2} d x .
\end{aligned}
$$

Integrating out the second term by parts yields

$$
\begin{aligned}
\int_{x_{1}}^{x_{n}} g^{\prime \prime}(x) h^{\prime \prime}(x) d x & =g^{\prime \prime}\left(x_{n}\right) h^{\prime}\left(x_{n}\right)-g^{\prime \prime}\left(x_{1}\right) h^{\prime}\left(x_{1}\right)-\int_{x_{1}}^{x_{n}} g^{\prime \prime \prime}(x) h^{\prime}(x) d x \\
& =-\int_{x_{1}}^{x_{n}} g^{\prime \prime \prime}(x) h^{\prime}(x) d x \\
& =-\sum_{i=1}^{n-1} g^{\prime \prime \prime}\left(x_{i}^{+}\right) \int_{x_{i}}^{x_{i+1}} h^{\prime}(x) d x \\
& =-\sum_{i=1}^{n-1} g^{\prime \prime \prime}\left(x_{i}^{+}\right)\left(h\left(x_{i+1}\right)-h\left(x_{i}\right)\right) d x \\
& =0
\end{aligned}
$$

The first two terms in the first line are equal to zero since $g^{\prime \prime}\left(x_{1}\right)=g^{\prime \prime}\left(x_{n}\right)=0$. The summation in line three results from the fact that $g(x)$ is made up of cubic polynomials. Hence, $g^{\prime \prime \prime}(x)$ is constant over any interval $\left(x_{i}, x_{i+1}\right)$ and $x_{i}^{+}$denotes an element of such an interval. The transition from line 4 to 5 follows from the fact that $f(x)$ and $g(x)$ are interpolants and thus, equal at each point i.e. $h\left(x_{i}\right)=h\left(x_{i+1}\right)=0$.

Therefore, it follows that (Wood, 2006)

$$
\begin{aligned}
\int_{x_{1}}^{x_{n}} f^{\prime \prime}(x)^{2} d x & =\int_{x_{1}}^{x_{n}} g^{\prime \prime}(x)^{2} d x+\int_{x_{1}}^{x_{n}} h^{\prime \prime}(x)^{2} d x \\
& \geq \int_{x_{1}}^{x_{n}} g^{\prime \prime}(x)^{2} d x
\end{aligned}
$$

The equality only holds if $h^{\prime \prime}(x)=0$ for $x_{1}<x<x_{n}$. However, note that $h\left(x_{1}\right)=h\left(x_{n}\right)=0$. So we have equality if and only if $h(x)=0$ on $\left[x_{1}, x_{n}\right]$. This implies that any interpolant not identical to $g(x)$ will have a higher integrated squared second derivative. Therefore, the cubic spline will be the smoothest possible interpolant for any set of data (Wood, 2006).

Since $y_{i}$ is measured with noise, it is more natural to smooth $x_{i}, y_{i}$ data, rather than interpolate 
it. Hence, instead of setting $g\left(x_{i}\right)$ to $y_{i}$, we will treat $g\left(x_{i}\right)$ as $n$ free parameters of the cubic spline. To estimate them, one has to minimise

$$
\sum_{n=1}^{n}\left\{y_{i}-g\left(x_{i}\right)\right\}^{2}+\lambda \int g^{\prime \prime}(x) d x
$$

where $\lambda$ is the tuning parameter controlling for the trade-off between smoothing and data fit. The resulting $g(x)$ is a smoothing spline. In fact, of all functions $f$, that are continuous on $\left[x_{1}, x_{n}\right]$ and have continuous first derivatives, $g(x)$ is the function minimising

$$
\sum_{n=1}^{n}\left\{y_{i}-f\left(x_{i}\right)\right\}^{2}+\lambda \int f^{\prime \prime}(x) d x
$$

The proof of this is as follows. Suppose that there is a different function than $g(x)$ minimising (6.1.3), say $t(x)$. In this case, $\left\{x_{i}, t\left(x_{i}\right)\right\}$ could be interpolated using a cubic spline $g(x)$. Thus, $t(x)$ and $g(x)$ have the same sum of squares in (6.1.3), but by the properties of natural cubic splines discussed in the previous paragraphs, $g(x)$ will have the lower integrated squared second derivative. Therefore, $g(x)$ will have a lower (6.1.3) than $t(x)$, which yields a contradiction unless $t=g$.

As we can see, the cubic spline emerges naturally from the definition of (6.1.3). In that sense, they seem to be ideal smoothers. However, since we are dealing with $n$ parameters, the computational burden can be large - particularly, in a multivariate case. A potential solution to this would be the penalized regression spline approach. Generally, this consists of creating spline bases and penalties for a much smaller data set than the one to be analysed. These bases and penalties are used to model the original values. The covariate values should be arranged to nicely cover the distribution of covariate values in the original data set.

To exemplify penalized regression splines consider B-splines bases. The B-splines act strictly locally i.e. each basis function is non-zero only between $m+3$ adjacent knots, where $m+1$ is the order of the basis. For a $k$ parameter B-spline, one needs to define $k+m+1$ knots. We will denote them as $x$ and $x_{1}<x_{2}<\ldots<x_{k+m+1}$. The interval over which spline is to be evaluated lies within $\left[x_{m+2}, x_{k}\right]$. In essence, this means that the locations of the first and the last $m+1$ knots are 
arbitrary. An $(m+1)^{t h}$ order spline can be written as

$$
f(x)=\sum_{i=1}^{k} B_{i}^{m}(x) \beta_{i}
$$

where the B-spline basis functions $B^{m}$ are recursively defined as

$$
B_{i}^{m}(x)=\frac{x-x_{i}}{x_{i+m+1}-x_{i}} B_{i}^{m-1}(x)+\frac{x_{i+m+2}-x}{x_{i+m+2}-x_{i+1}} B_{i}^{m-1}(x) \quad i=1, \ldots, k
$$

and

$$
B_{i}^{-1}(x)= \begin{cases}1, & \text { if } x_{i} \leq x \leq x_{1+i}, \\ 0, & \text { otherwise. }\end{cases}
$$

For detailed description of B-plines see De Boor (1978). An example of B-splines is illustrated in Figure 6.1 (Racine, 2014). 7 equally spaced basis functions are depicted on the $[0,1]$ interval, where the knots are given by $[0,0.4,0.6,0.8,1]$ and $m=2$ (cubic B-spline). As one one can see, each B-spline basis function is non-zero between 5 adjacent knots (or on 4 intervals). The boundary knots are given by $[-0.6,1.6]$.

Figure 6.1: Illustration of a B-spline. 7 equally spaced basis functions are depicted on the $[0,1]$ interval, where the knots are given by $[0,0.4,0.6,0.8,1]$ and $m=2$ (cubic B-spline). As one one can see, each B-spline basis function is non-zero between 5 adjacent knots (or on 4 intervals). The boundary knots are given by $[-0.6,1.6]$.

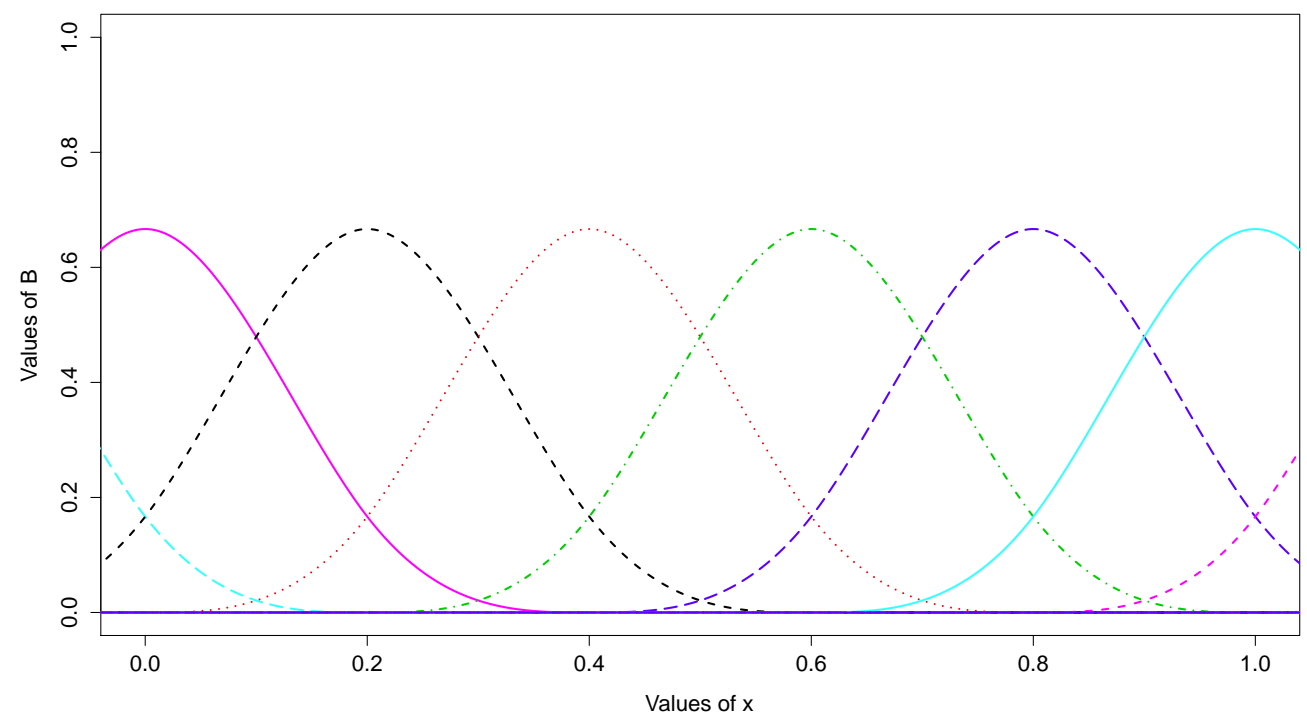


Originally, B-plines were established as a very stable basis for large scale spline interpolation (see: De Boor, 1978). As an alternative, Eilers and Marx (1996) developed P-splines, which also ensure computational efficiency and stability. P-splines are low-rank smoothers using B-spline bases. These are defined on evenly spaced knots and a difference penalty is applied directly to the parameters to account for function wiggliness. Let us consider the following example. Suppose we want to penalize the squared difference between adjacent parameter values, $\beta_{i}$ and $\beta_{i+1}$. The penalty would be

$$
\begin{aligned}
P & =\sum_{i=1}^{k-1}\left(\beta_{i+1}-\beta_{i}\right)^{2} \\
& =\beta_{1}^{2}-2 \beta_{1} \beta_{2}+2 \beta_{2}^{2}-2 \beta_{2} \beta_{3}+\ldots+\beta_{k}
\end{aligned}
$$

where $k$ denotes the basis dimension. P-splines are easy to set up and use and allow for a lot of flexibility, in a sense that any order of penalty can be combined with any order of B-spline basis. Their disadvantage is that they are less simple in their form when uneven knot spacing is required and their penalties are more difficult to interpret than the usual spline penalties (Wood, 2006).

Other examples of cubic splines include cubic smoothing splines, cubic regression splines and cyclic cubic regression splines. The literature on cubic splines is vast and we will not further elaborate on them (see e.g. Hastie and Tibshirani, 1990).

An alternative to cubic splines are thin plate splines. Let us consider

$$
y=f(\boldsymbol{z})+\epsilon
$$

The problem is the same as in equation (6.1.1), but single covariate $z$ was replaced with $d$ dimensional vector $\boldsymbol{z}$. The thin plate spline estimates $f$ by finding $\hat{g}$, which minimises

$$
\|\boldsymbol{y}-\boldsymbol{g}\|^{2}+\lambda J_{m d}(g)
$$

where $\boldsymbol{y}$ is a vector of $y_{i}$ response and $\boldsymbol{g}=\left[g\left(\boldsymbol{z}_{1}\right), g\left(\boldsymbol{z}_{2}\right), \ldots, g\left(\boldsymbol{z}_{n}\right)\right]^{\boldsymbol{\top}} ; n$ is the number of observations and $i$ indexes each observation. $J_{m d}(g)$ is the penalty functional which measures the "wiggliness" of $g$ and $\lambda$ is the smoothing parameter, which controls for the compromise between data fitting and 
smoothness of $g . J_{m d}(g)$ is defined as

$$
\int \ldots \int_{\mathbb{R}^{d}} \sum_{\nu_{1}+\ldots+\nu_{d}=m} \frac{m !}{\nu_{1} ! \ldots \nu_{d} !}\left(\frac{\partial^{m} g}{\partial z_{1}^{\nu_{1}} \ldots \partial z_{d}^{\nu_{d}}}\right)^{2} d z_{1} \ldots d z_{d}
$$

where $m$ indicates the derivative order with respect to which smoothness is measured. For example, a penalty of two smooth predictors whose wiggliness is measured in terms of second derivatives would assume the form of

$$
J_{22}(g)=\iint\left(\frac{\partial^{2} g}{\partial z_{1}^{2}}\right)^{2}+2\left(\frac{\partial^{2} g}{\partial z_{1} \partial z_{2}}\right)^{2}+\left(\frac{\partial^{2} g}{\partial z_{2}^{2}}\right)^{2} d z_{1} d z_{2}
$$

By setting the restriction of $2 m>d$, it can be shown that $\hat{g}$ minimising (6.1.5) can be written as

$$
\hat{g}(\boldsymbol{z})=\sum_{i=1}^{n} \delta_{i} \eta_{m d}\left(\left\|\boldsymbol{z}-\boldsymbol{z}_{i}\right\|\right)+\sum_{j=1}^{M} \alpha_{j} \phi_{j}(\boldsymbol{z})
$$

where $\boldsymbol{\delta}$ and $\boldsymbol{\alpha}$ are vectors of coefficients to be estimated, $\boldsymbol{\delta}$ being subject to the linear constraints that $\mathbf{T}^{\top} \boldsymbol{\delta}=\mathbf{0}$ and $T_{i j}=\phi_{j}\left(\boldsymbol{z}_{i}\right)$. The $M=\left(\begin{array}{c}m+d-1 \\ d\end{array}\right)$ functions, $\phi_{i}$, are linearly independent polynomials spanning the space of polynomials in $\mathbb{R}^{d}$ of degree less than $m$. The $\phi_{i}$ span the space of functions for which $J_{m d}$ is zero (the null space of $J_{m d}$ ) - functions which are considered "completely smooth". For instance, for $m=d=2$ these functions are $\phi_{1}(\boldsymbol{z})=1, \phi_{2}(\boldsymbol{z})=z_{1}$ and $\phi_{3}(\boldsymbol{z})=z_{2}$. Also,

$$
\eta_{m d}(r)= \begin{cases}\frac{(-1)^{m+1+d / 2}}{2^{2 m-1} \pi^{d / 2}(m-1) !(m-d / 2) !} r^{2 m-d} \log (r), & \text { if } d \text { is even } \\ \frac{\Gamma(d / 2-m)}{2^{2 m} \pi^{d / 2}(m-1) !} r^{2 m-d}, & \text { if } d \text { is odd. }\end{cases}
$$

Defining $n \times n$ matrix $\mathbf{E}$, where $E_{i j} \equiv \eta_{m d}\left(\left\|\boldsymbol{z}_{i}-\boldsymbol{z}_{j}\right\|\right)$ we have that

$$
g=\mathbf{E} \boldsymbol{\delta}+\mathbf{T} \boldsymbol{\alpha}
$$

and the fitting problem in (6.1.5) becomes

$$
\min \|\boldsymbol{y}-\mathbf{E} \boldsymbol{\delta}-\mathbf{T} \boldsymbol{\alpha}\|^{2}+\lambda \boldsymbol{\delta}^{\top} \mathbf{E} \boldsymbol{\delta} \text { subject to } \mathbf{T}^{\top} \boldsymbol{\delta}=\mathbf{0}
$$

with respect to $\boldsymbol{\delta}$ and $\boldsymbol{\alpha}$ (for more details see: Green and Silverman, 1994). Note that the choice of 
knot locations and bases emerges from the definition of the smoothing problem and hence is done in an automatic way. Furthermore, the bases are not restricted to only one predictor and many smooths can be represented (Wood, 2006). Thus, we will be using thin plate regression splines to enhance the existing copula count sample selection model.

Unfortunataly, estimating thin plate splines has a high computational cost since there are as many unknown parameters as there are data. Apart from the single covariate case, the cost is proportional to the cube of parameters. At the same time, it seems intuitive that many parameters are redundant. Hence, to reduce the number of components related to $\boldsymbol{\delta}$ while leaving zero wiggliness i.e. $\boldsymbol{\alpha}$ components unchanged we will use thin plate regression splines.

Let $\mathbf{E}=\mathbf{U D}^{\top} \mathbf{U}$ be the eigen-decomposition of $\mathbf{E}$, where $\mathbf{D}$ is a diagonal matrix of eigenvalues of E arranged in descending order and the columns of $\mathbf{U}$ are the corresponding eigenvectors. Let $\mathbf{U}_{k}$ denote the matrix consisting of the first $k$ columns of $\mathbf{U}$ and let $\mathbf{D}_{k}$ be the top left $k \times k$ submatrix of $\mathbf{D}$. Restricting $\boldsymbol{\delta}$ to the column space of $\mathbf{U}_{k}$, by writing $\boldsymbol{\delta}=\mathbf{U}_{k} \boldsymbol{\delta}_{k}$, means that (6.1.10) becomes

$$
\min \left\|\boldsymbol{y}-\mathbf{U}_{k} \mathbf{D}_{k} \boldsymbol{\delta}_{k}-\mathbf{T} \boldsymbol{\alpha}\right\|^{2}+\lambda \boldsymbol{\delta}_{k}^{\top} \mathbf{D}_{k} \boldsymbol{\delta}_{k} \text { subject to } \mathbf{T}^{\top} \mathbf{U}_{k} \boldsymbol{\delta}_{k}=\mathbf{0},
$$

with respect to $\boldsymbol{\delta}_{k}$ and $\boldsymbol{\alpha}$. To absorb the constraints we first find any orthogonal column basis $\mathbf{Z}_{k}$ such that $\mathbf{T}^{\top} \mathbf{U}_{k} \mathbf{Z}_{k}=\mathbf{0}$. This can be done by conducting QR decomposition of $\mathbf{U}_{k}^{\top} \mathbf{T}$ and the final $M$ columns of the orthogonal factor yield $\mathbf{Z}_{k}$. Restricting $\boldsymbol{\delta}_{k}$ to this space, let us rewrite $\boldsymbol{\delta}_{k}=\mathbf{Z}_{k} \overline{\boldsymbol{\delta}}$. It follows that

$$
\min \left\|\boldsymbol{y}-\mathbf{U}_{k} \mathbf{D}_{k} \mathbf{Z}_{k} \overline{\boldsymbol{\delta}}-\mathbf{T} \boldsymbol{\alpha}\right\|^{2}+\lambda \overline{\boldsymbol{\delta}}^{\top} \mathbf{Z}_{k}^{\top} \mathbf{D}_{k} \mathbf{Z}_{k} \overline{\boldsymbol{\delta}}
$$

yields an unconstrained problem of rank $k$ approximation to the smoothing spline with respect to $\overline{\boldsymbol{\delta}}$ and $\boldsymbol{\alpha}$. The computational cost of model estimation will be $O\left(k^{3}\right)$. Once the model is fitted, to evaluate (6.1.8) one needs to calculate $\boldsymbol{\delta}=\mathbf{U}_{k} \mathbf{Z}_{k} \overline{\boldsymbol{\delta}}$. The matrices $\mathbf{U}_{k}$ and $\mathbf{D}_{k}$ can be obtained cheaply at the computational cost of $O\left(n^{2} k\right)$ using Lanczos iterations. In brief, Lanczos algorithm aims at conducting a rank $k$ eigen-decomposition of a symmetric matrix. This is done by iteratively constructing a tri-diagonal matrix whose eigenvalues converge to those required as iteration proceeds (Wood, 2006). 
In the next section we will introduce thin plate regression splines to the count copula sample selection models.

\subsection{Introducing non-parametric terms in sample selection models}

\subsubsection{Model specification}

In the previous chapters the model predictors had prespecified effects. In this section we will introduce the smooth terms described previously. The selection equation from Chapter 3 is modified as follows:

$$
Y_{1}^{*}=\eta_{1}+\epsilon_{1}=\boldsymbol{\gamma}^{\top} \boldsymbol{z}+\sum_{k_{1}=1}^{K_{1}} f_{1 k_{1}}\left(u_{1 k_{1}}\right)+\epsilon_{1}
$$

where $Y_{1}^{*} \sim N\left(\eta_{1}, 1\right), \eta_{1}$ is the linear predictor, $\gamma$ is the parameter vector of all parametric components, $\boldsymbol{z}$ is a vector of covariates and $f_{1 k_{1}}\left(u_{1 k_{1}}\right)$ are the unknown smooth functions of $K_{1}$ continuous covariates.

On the other hand, the outcome equation takes the following form:

$$
Y_{2} \sim \mathcal{F}(\mu, \sigma, \nu)
$$

where $\mathcal{F}$ is a discrete distribution, $E\left(Y_{2}\right)=\mu=\exp \left(\eta_{2}\right)=\exp \left(\boldsymbol{\beta}^{\top} \boldsymbol{x}+\sum_{k_{2}=1}^{K_{2}} f_{2 k_{2}}\left(u_{2 k_{2}}\right)\right)$, $\eta_{2}$ is the linear predictor, $\sigma$ and $\nu$ are the scale and shape parameters. Similarly, $\boldsymbol{\beta}$ is the parameter vector of all parametric components, $\boldsymbol{x}$ is a vector of covariates and $f_{2 k_{2}}\left(u_{2 k_{2}}\right)$ are the unknown smooth functions of $K_{2}$ continuous covariates.

The non-parametric components $f_{v k_{v}}\left(u_{v k_{v}}\right)$ are represented as regression splines. Specifically,

$$
\begin{aligned}
f_{v k_{v}}\left(u_{v k_{v}}\right) & =\sum_{j=1}^{J_{v k_{v}}} \alpha_{v k_{v} j} b_{v k_{v} j}\left(u_{v k_{v}}\right) \\
& =\mathbf{B}\left(u_{v k_{v}}\right)^{\top} \boldsymbol{\alpha}_{v k_{v}},
\end{aligned}
$$

where $b_{v k_{v} j}\left(u_{v k_{v}}\right)$ is the known spline basis function, $\alpha_{v k_{v} j}$ are the associated regression parameters, $J_{v k_{v}}$ is the number of bases, $\mathbf{B}\left(u_{v k_{v}}\right)=\left[b_{v k_{v} 1}, b_{v k_{v} 2}, \ldots, b_{v k_{v} J_{v k_{v}}}\right]^{\top}$ is the vector of dimension $J_{v k_{v}}$ 
containing basis functions and $\boldsymbol{\alpha}_{v k_{v}}$ is the corresponding parameter vector. Evaluating basis functions yields $J_{v k_{v}}$ curves. These are multiplied by $\boldsymbol{\alpha}_{v k_{v}}$ and summed to give an estimate of $f_{v k_{v}}\left(u_{v k_{v}}\right)$ (Ruppert et al., 2003).

The model above may not be identifiable and hence the smooth functions are subject to identi-

fiability constraint $\sum_{k_{v}=1}^{K_{v}} f_{v k_{v}}\left(u_{v k_{v}}\right)=0, v=1,2$ and $k_{v}=1, \ldots, K_{v}$. We achieve this by employing the parsimonious approach by Wood (2006).

For the basis functions we will employ thin plate regression splines since they offer computational and practical advantages. First, thin plate regression splines truncate the space of wiggly components of the ordinary thin plate spline making estimation computationally efficient. Second, choosing knot locations or basis functions in the thin plate spline framework is automatic - all emerges from defining the mathematical statement of the smoothing problem (Wood, 2006). Third, thin plate regression spline smooths can incorporate many predictors. Hence, the linear predictors can be defined as

$$
\begin{aligned}
& \eta_{1}=\boldsymbol{\gamma}^{\top} \boldsymbol{z}+\mathbf{B}_{1}^{\top} \boldsymbol{\alpha}_{1} \\
& \eta_{2}=\boldsymbol{\beta}^{\top} \boldsymbol{x}+\mathbf{B}_{2}^{\top} \boldsymbol{\alpha}_{2},
\end{aligned}
$$

where $\mathbf{B}_{v}^{\top}=\left[\mathbf{B}\left(u_{v 1}\right)^{\top}, \ldots, \mathbf{B}\left(u_{v K_{v}}\right)^{\top}\right]$ and $\boldsymbol{\alpha}_{v}^{\top}=\left(\boldsymbol{\alpha}_{v 1}^{\top}, \ldots, \boldsymbol{\alpha}_{v K_{v}}^{\top}\right)$. Let us define $\mathbf{X}_{1}=\left(\boldsymbol{z}^{\top}, \mathbf{B}_{1}^{\top}\right)^{\top}$ and $\mathbf{X}_{2}=\left(\boldsymbol{x}^{\top}, \mathbf{B}_{2}^{\top}\right)^{\top}$. Thus, we have that

$$
\begin{aligned}
& \eta_{1}=\mathbf{X}_{1}^{\top} \boldsymbol{\delta}_{1} \\
& \eta_{2}=\mathbf{X}_{2}^{\top} \boldsymbol{\delta}_{2},
\end{aligned}
$$

where $\boldsymbol{\delta}_{1}^{\top}=\left(\boldsymbol{\gamma}^{\top}, \boldsymbol{\alpha}_{1}^{\top}\right)$ and $\boldsymbol{\delta}_{2}^{\top}=\left(\boldsymbol{\gamma}^{\top}, \boldsymbol{\alpha}_{2}^{\top}\right)$.

\subsubsection{Deriving penalized linear working model}

It is more computationally efficient to transform the penalized likelihood function into a penalized iterated reweighted least squares (P-IRLS) scheme. As we will see in Section 6.2.3, the P-IRLS scheme will facilitate the derivation of the smoothing parameter selection criterion. We will first focus on the non-penalized model and the iterated reweighted least squares (IRLS). The penalized 
case will follow immediately and will differ from IRLS by inclusion of the penalty term in the model framework. The derivation of equations in this section is based on Green (1984) and the conclusions can be found in Wood (2006), for instance.

Let $\ell$ be the log-likelihood of the model described previously and $\boldsymbol{\delta}=\left(\boldsymbol{\delta}_{\mathbf{1}}, \boldsymbol{\delta}_{\mathbf{2}}, \theta^{*}, \sigma^{*}, \nu^{*}\right)^{\top}$, where $\boldsymbol{\delta}_{1}, \boldsymbol{\delta}_{\mathbf{2}}$ are the parameters of the selection and outcome linear predictor respectively, $\theta^{*}$ is the association parameter and $\sigma^{*}$ and $\nu^{*}$ are the remaining parameters of the outcome. The star superscript indicates the reparametrisation mentioned in Chapter 5. We will use the most general derivation possible, but the same will apply for specifications without $\sigma^{*}$ or $\nu^{*}$. The first derivative of $\ell$ with respect to $\delta$ can be decomposed as follows (Green, 1984)

$$
\frac{\partial \ell}{\partial \boldsymbol{\delta}}=\widetilde{\mathbf{X}}^{\top} \mathbf{u}
$$

where $\mathbf{u}$ is a vector consisting of $n$ subvectors $\frac{\partial \ell}{\partial \boldsymbol{\eta}_{\boldsymbol{i}}}=\left(\frac{\partial \ell}{\partial \eta_{1 i}}, \frac{\partial \ell}{\partial \eta_{2 i}}, \frac{\partial \ell}{\partial \theta^{*}}, \frac{\partial \ell}{\partial \sigma^{*}}, \frac{\partial \ell}{\partial \nu^{*}}\right)^{\top} ; \widetilde{\mathbf{X}}$ is the $5 n \times p$ matrix consisting of $5 \times p$ submatrices $\widetilde{\mathbf{X}}_{i}=\operatorname{diag}\left(\widetilde{\mathbf{X}}_{1 i}^{\top}, \widetilde{\mathbf{X}}_{\mathbf{2} i}^{\top}, 1,1,1\right)$ i.e.

$$
\widetilde{\mathbf{X}}=\left[\begin{array}{c}
\widetilde{\mathbf{X}}_{1} \\
\vdots \\
\widetilde{\mathbf{X}}_{n}
\end{array}\right]
$$

Let us denote $\frac{\partial \boldsymbol{\ell}}{\partial \boldsymbol{\delta}^{*}}$ as the updated score vector at the next iteration. The first order Taylor expansion for $\frac{\partial \ell}{\partial \boldsymbol{\delta}^{*}}$ about $\boldsymbol{\delta}$ yields

$$
\frac{\partial \ell}{\partial \boldsymbol{\delta}^{*}}=\frac{\partial \ell}{\partial \boldsymbol{\delta}}+\left(\boldsymbol{\delta}^{*}-\boldsymbol{\delta}\right) \frac{\partial^{2} \ell}{\partial \boldsymbol{\delta} \partial \boldsymbol{\delta}^{\top}}
$$

where $\frac{\partial^{2} \ell}{\partial \boldsymbol{\delta} \partial \boldsymbol{\delta}}$ is the Hessian. Setting (6.2.2) to zero and rearranging yields

$$
-\frac{\partial^{2} \ell}{\partial \boldsymbol{\delta} \partial \boldsymbol{\delta}^{\boldsymbol{\top}}}\left(\boldsymbol{\delta}^{*}-\boldsymbol{\delta}\right)=\frac{\partial \ell}{\partial \boldsymbol{\delta}}
$$


Note that

$$
\begin{aligned}
-\frac{\partial^{2} \ell}{\partial \boldsymbol{\delta} \partial \boldsymbol{\delta}^{\top}} & =-\frac{\partial}{\partial \boldsymbol{\delta}}\left(\left(\frac{\partial \boldsymbol{\eta}}{\partial \boldsymbol{\delta}}\right)^{\top} \frac{\partial \ell}{\partial \boldsymbol{\eta}}\right) \\
& =-\left(\frac{\partial \boldsymbol{\eta}}{\partial \boldsymbol{\delta}}\right)^{\top} \frac{\partial^{2} \ell}{\partial \boldsymbol{\eta} \partial \boldsymbol{\eta}}\left(\frac{\partial \boldsymbol{\eta}}{\partial \boldsymbol{\delta}}\right)
\end{aligned}
$$

where $\frac{\partial^{2} \ell}{\partial \boldsymbol{\eta} \partial \boldsymbol{\eta}}$ is a $5 n \times 5 n$ block diagonal matrix having $n$

$$
\frac{\partial^{2} \ell}{\partial \boldsymbol{\eta}_{i} \partial \boldsymbol{\eta}_{i}}=\left[\begin{array}{ccccc}
\frac{\partial^{2} \ell}{\partial \boldsymbol{\eta}_{1 i} \partial \boldsymbol{\eta}_{1 i}} & \cdot & \cdot & \cdot & \cdot \\
\frac{\partial^{2} \ell}{\partial \boldsymbol{\eta}_{1 i} \partial \boldsymbol{\eta}_{2 i}} & \frac{\partial^{2} \ell}{\partial \boldsymbol{\eta}_{2 i} \partial \boldsymbol{\eta}_{2 i}} & \cdot & \cdot & \cdot \\
\frac{\partial^{2} \ell}{\partial \boldsymbol{\eta}_{1 i} \partial \theta^{*}} & \frac{\partial^{2} \ell}{\partial \boldsymbol{\eta}_{2 i} \partial \theta^{*}} & \frac{\partial^{2} \ell}{\partial \theta^{* 2}} & \cdot & \cdot \\
\frac{\partial^{2} \ell}{\partial \boldsymbol{\eta}_{1 i} \partial \sigma^{*}} & \frac{\partial^{2} \ell}{\partial \boldsymbol{\eta}_{2 i} \partial \sigma^{*}} & \frac{\partial^{2} \ell}{\partial \theta^{*} \partial \sigma^{*}} & \frac{\partial^{2} \ell}{\partial \sigma^{* 2}} & . \\
\frac{\partial^{2} \ell}{\partial \boldsymbol{\eta}_{1 i} \partial \nu^{*}} & \frac{\partial^{2} \ell}{\partial \boldsymbol{\eta}_{2 i} \partial \nu^{*}} & \frac{\partial^{2} \ell}{\partial \theta^{*} \partial \nu^{*}} & \frac{\partial^{2} \ell}{\partial \sigma^{*} \partial \nu^{*}} & \frac{\partial^{2} \ell}{\partial \nu^{* 2}}
\end{array}\right]
$$

symmetric submatrices on the main diagonal. Let (e.g. Green, 1984)

$$
E\left(-\frac{\partial^{2} \ell}{\partial \boldsymbol{\eta} \partial \boldsymbol{\eta}}\right)=\mathbf{W}
$$

As we will see below, the square root of $\mathbf{W}$ will need to be found. Substituting $-\frac{\partial^{2} \ell}{\partial \boldsymbol{\eta} \partial \boldsymbol{\eta}}$ with its expectation will ensure that $\mathbf{W}$ is postive definite. Thus,

$$
\begin{aligned}
-\frac{\partial^{2} \ell}{\partial \boldsymbol{\delta} \partial \boldsymbol{\delta}^{\top}}\left(\boldsymbol{\delta}^{*}-\boldsymbol{\delta}\right) & =\frac{\partial \ell}{\partial \boldsymbol{\delta}} \\
-\left(\frac{\partial \boldsymbol{\eta}}{\partial \boldsymbol{\delta}}\right)^{\top} \frac{\partial^{2} \ell}{\partial \boldsymbol{\eta} \partial \boldsymbol{\eta}}\left(\frac{\partial \boldsymbol{\eta}}{\partial \boldsymbol{\delta}}\right)\left(\boldsymbol{\delta}^{*}-\boldsymbol{\delta}\right) & =\frac{\partial \ell}{\partial \boldsymbol{\delta}} .
\end{aligned}
$$

Substituting yields

$$
\begin{aligned}
\widetilde{\mathbf{X}}^{\top} \mathbf{W} \widetilde{\mathbf{X}}\left(\boldsymbol{\delta}^{*}-\boldsymbol{\delta}\right) & =\widetilde{\mathbf{X}}^{\top} \mathbf{u} \\
\widetilde{\mathbf{X}}^{\top} \mathbf{W} \widetilde{\mathbf{X}} \boldsymbol{\delta}^{*} & =\widetilde{\mathbf{X}}^{\top} \mathbf{W} \mathbf{W}^{-1} \mathbf{u}+\widetilde{\mathbf{X}}^{\top} \mathbf{W} \widetilde{\mathbf{X}} \boldsymbol{\delta} \\
\boldsymbol{\delta}^{*} & =\left(\widetilde{\mathbf{X}}^{\top} \mathbf{W} \widetilde{\mathbf{X}}\right)^{-1} \widetilde{\mathbf{X}}^{\top} \mathbf{W}\left(\mathbf{W}^{-1} \mathbf{u}+\widetilde{\mathbf{X}} \boldsymbol{\delta}\right) .
\end{aligned}
$$


$\boldsymbol{\delta}^{*}$ turns out to be the solution to the iterated re-weighted least squares (IRLS) problem:

$$
\min _{\delta^{*} \in \mathbb{R}^{n}}\left\|\sqrt{\mathbf{W}}\left(\mathbf{z}-\tilde{\mathbf{X}} \boldsymbol{\delta}^{*}\right)\right\|^{2}
$$

where $\mathbf{z}=\mathbf{W}^{-1} \mathbf{u}+\widetilde{\mathbf{X}} \boldsymbol{\delta}$ is the pseudo-data vector. In other words, we regress $\mathbf{W}^{-1} \mathbf{u}+\widetilde{\mathbf{X}} \boldsymbol{\delta}$ onto $\widetilde{\mathbf{X}}$ using weight matrix W (Green, 1984).

The P-IRLS generalises IRLS. Note that (Wood, 2006)

$$
\ell_{p}=\ell-\frac{1}{2} \boldsymbol{\delta}^{\top} \mathbf{S}_{\lambda} \boldsymbol{\delta}
$$

where $\mathbf{S}_{\lambda}$ is the overall penalty matrix defined as $\operatorname{diag}\left(\mathbf{0}_{P_{1}}^{\top}, \lambda_{1 k_{1}} \mathbf{S}_{1 k_{1}}, \ldots, \lambda_{1 K_{1}} \mathbf{S}_{1 K_{1}}, \mathbf{0}_{P_{2}}^{\top}, \lambda_{2 k_{2}} \mathbf{S}_{2 k_{2}}, \ldots, \lambda_{2 K_{2}} \mathbf{S}_{2 K_{2}}\right)$, where $P_{v}$ indexes the strictly parametric components of the $v$-th equation. Also, (Green, 1984)

$$
\begin{aligned}
\frac{\partial \ell_{p}}{\partial \boldsymbol{\delta}} & =\frac{\partial \ell}{\partial \boldsymbol{\delta}}-\mathbf{S}_{\lambda} \boldsymbol{\delta} \\
& =\widetilde{\mathbf{X}}^{\top} \mathbf{u}-\mathbf{S}_{\lambda} \boldsymbol{\delta}
\end{aligned}
$$

and

$$
\begin{aligned}
\frac{\partial^{2} \ell_{p}}{\partial \boldsymbol{\delta} \partial \boldsymbol{\delta}^{\top}} & =\frac{\partial^{2} \ell}{\partial \boldsymbol{\delta} \partial \boldsymbol{\delta}^{\top}}-\mathbf{S}_{\lambda} \\
& =\left(\frac{\partial \boldsymbol{\eta}}{\partial \boldsymbol{\delta}}\right)^{\top} \frac{\partial^{2} \ell}{\partial \boldsymbol{\eta} \partial \boldsymbol{\eta}}\left(\frac{\partial \boldsymbol{\eta}}{\partial \boldsymbol{\delta}}\right)-\mathbf{S}_{\lambda} \\
& =-\widetilde{\mathbf{X}}^{\top} \mathbf{W} \widetilde{\mathbf{X}}-\mathbf{S}_{\lambda} .
\end{aligned}
$$

By replacing $\ell$ with $\ell_{p}$ in (6.2.3) we obtain

$$
\begin{aligned}
-\frac{\partial^{2} \ell_{p}}{\partial \boldsymbol{\delta} \partial \boldsymbol{\delta}^{\top}}\left(\boldsymbol{\delta}^{*}-\boldsymbol{\delta}\right) & =\frac{\partial \ell_{p}}{\partial \boldsymbol{\delta}} \\
\left(\widetilde{\mathbf{X}}^{\top} \mathbf{W} \widetilde{\mathbf{X}}+\mathbf{S}_{\lambda}\right)\left(\boldsymbol{\delta}^{*}-\boldsymbol{\delta}\right) & =\widetilde{\mathbf{X}}^{\top} \mathbf{u}-\mathbf{S}_{\lambda} \boldsymbol{\delta} \\
\left(\widetilde{\mathbf{X}}^{\top} \mathbf{W} \widetilde{\mathbf{X}}+\mathbf{S}_{\lambda}\right) \boldsymbol{\delta}^{*}-\left(\widetilde{\mathbf{X}}^{\top} \mathbf{W} \widetilde{\mathbf{X}}+\mathbf{S}_{\lambda}\right) \boldsymbol{\delta} & =\widetilde{\mathbf{X}}^{\top} \mathbf{u}-\mathbf{S}_{\lambda} \boldsymbol{\delta} \\
\left(\widetilde{\mathbf{X}}^{\top} \mathbf{W} \widetilde{\mathbf{X}}+\mathbf{S}_{\lambda}\right) \boldsymbol{\delta}^{*}-\widetilde{\mathbf{X}}^{\top} \mathbf{W} \widetilde{\mathbf{X}} \boldsymbol{\delta} & =\widetilde{\mathbf{X}}^{\top} \mathbf{u} . \\
\left(\widetilde{\mathbf{X}}^{\top} \mathbf{W} \widetilde{\mathbf{X}}+\mathbf{S}_{\lambda}\right) \boldsymbol{\delta}^{*}-\widetilde{\mathbf{X}}^{\top} \mathbf{W} \widetilde{\mathbf{X}} \boldsymbol{\delta} & =\widetilde{\mathbf{X}}^{\top} \mathbf{W} \mathbf{W}^{-1} \mathbf{u} .
\end{aligned}
$$


Rearranging the last line of (6.2.6) yields

$$
\boldsymbol{\delta}^{*}=\left(\widetilde{\mathbf{X}}^{\top} \mathbf{W} \widetilde{\mathbf{X}}+\mathbf{S}_{\lambda}\right)^{-1} \widetilde{\mathbf{X}}^{\top} \mathbf{W}\left(\mathbf{W}^{-1} \mathbf{u}+\widetilde{\mathbf{X}} \boldsymbol{\delta}\right)
$$

Similarly, $\boldsymbol{\delta}^{*}$ turns out to be the solution to the P-IRLS problem

$$
\min _{\delta^{*} \in \mathbb{R}^{n}}\left\|\sqrt{\mathbf{W}}\left(\mathbf{z}-\tilde{\mathbf{X}} \boldsymbol{\delta}^{*}\right)\right\|^{2}+\boldsymbol{\delta}^{* \mathbf{T}} \mathbf{S}_{\lambda} \boldsymbol{\delta}^{*}
$$

where $\mathbf{z}=\mathbf{W}^{-1} \mathbf{u}+\widetilde{\mathbf{X}} \boldsymbol{\delta}$ is the pseudo-data vector (Wood, 2006).

\subsubsection{Smoothing parameter selection}

In the spline context it is essential to have an automatic way of selecting smoothing parameter $\boldsymbol{\lambda}$ in a computationally efficient way. At the same time, optimisation of all parameters including $\boldsymbol{\lambda}$ will

lead to overfitting since the highest value of $\hat{\ell}_{p}$ will be when $\boldsymbol{\lambda}=\mathbf{0}$. Thus, it is intuitive to select $\boldsymbol{\lambda}$ in a way that the smooths are as close as possible to the true functions.

We will use the approach by Radice et al. (2015) which employs the unbiased risk estimator (UBRE; Craven and Wahba, 1978). The final result of this section originates from Radice et al. (2015), whereas most of expressions are extended versions of the ones in Wood (2006). UBRE can be viewed as an estimate of the expected mean squared error $\kappa$ :

$$
E(\kappa)=E\left(\frac{1}{n^{*}}\|\boldsymbol{\mu}-\hat{\boldsymbol{\mu}}\|^{2}\right)
$$

where $n^{*}=5 n$. Intuitively, by taking the difference between the true model predictions, $\boldsymbol{\mu}$ and prediction estimates, $\hat{\boldsymbol{\mu}}$, we want to minimise the distance between the true model and the estimated model. Unfortunately, $\boldsymbol{\mu}$ is unknown in practice, but it can be estimated. Hence, it is natural to take the expectation of the difference between $\hat{\boldsymbol{\mu}}$ and $\boldsymbol{\mu}$. Let $\boldsymbol{\mu}=\sqrt{\mathbf{W}} \widetilde{\mathbf{X}} \boldsymbol{\delta}^{*}$ and $\hat{\boldsymbol{\mu}}=\sqrt{\mathbf{W}} \widetilde{\mathbf{X}} \hat{\boldsymbol{\delta}}^{*}$. 
Expanding $\kappa$ in (6.2.9) yields (Wood, 2006)

$$
\begin{aligned}
& \kappa=\frac{1}{n^{*}}\|\boldsymbol{\mu}-\hat{\boldsymbol{\mu}}\|^{2} \\
& \left.=\frac{1}{n^{*}} \| \sqrt{\mathbf{W}} \widetilde{\mathbf{X}} \boldsymbol{\delta}^{*}-\sqrt{\mathbf{W}} \widetilde{\mathbf{X}} \hat{\boldsymbol{\delta}}^{*}\right) \|^{2} \\
& =\frac{1}{n^{*}}\left\|\sqrt{\mathbf{W}} \widetilde{\mathbf{X}} \boldsymbol{\delta}^{*}-\sqrt{\mathbf{W}} \mathbf{A} \mathbf{z}\right\|^{2} \\
& =\frac{1}{n^{*}}\|\sqrt{\mathbf{W}} \mathbf{z}-\sqrt{\mathbf{W}} \mathbf{A} \mathbf{z}-\boldsymbol{\epsilon}\|^{2} \\
& =\frac{1}{n^{*}}\left(\|\sqrt{\mathbf{W}} \mathbf{z}-\sqrt{\mathbf{W}} \mathbf{A} \boldsymbol{z}\|^{2}-2 \boldsymbol{\epsilon}^{\top}(\sqrt{\mathbf{W}} \mathbf{z}-\sqrt{\mathbf{W}} \mathbf{A} \boldsymbol{z})+\boldsymbol{\epsilon}^{\top} \boldsymbol{\epsilon}\right)
\end{aligned}
$$

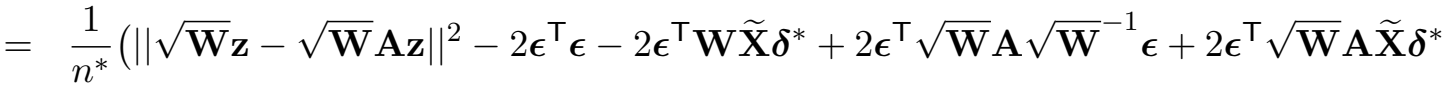

$$
\begin{aligned}
& \left.+\boldsymbol{\epsilon}^{\top} \boldsymbol{\epsilon}\right) \\
& =\frac{1}{n^{*}}\left(\|\sqrt{\mathbf{W}} \mathbf{z}-\sqrt{\mathbf{W}} \mathbf{A} \mathbf{z}\|^{2}-\boldsymbol{\epsilon}^{\top} \boldsymbol{\epsilon}-2 \boldsymbol{\epsilon}^{\top} \mathbf{W} \widetilde{\mathbf{X}} \boldsymbol{\delta}^{*}+2 \boldsymbol{\epsilon}^{\top} \sqrt{\mathbf{W}} \mathbf{A} \sqrt{\mathbf{W}^{-1}} \boldsymbol{\epsilon}+2 \boldsymbol{\epsilon}^{\top} \sqrt{\mathbf{W}} \mathbf{A} \tilde{\mathbf{X}} \boldsymbol{\delta}^{*}\right),
\end{aligned}
$$

where $\boldsymbol{\epsilon}=\sqrt{\mathbf{W}} \mathbf{z}-\sqrt{\mathbf{W}} \widetilde{\mathbf{X}} \boldsymbol{\delta}^{*}$ and $\sqrt{\mathbf{W}} \mathbf{A}=\sqrt{\mathbf{W}} \widetilde{\mathbf{X}}\left(\widetilde{\mathbf{X}}{ }^{\top} \mathbf{W} \widetilde{\mathbf{X}}+\mathbf{S}_{\lambda}\right)^{-1} \widetilde{\mathbf{X}}^{\top} \mathbf{W}$ is the hat matrix. Note that

$$
\sqrt{\mathbf{W}} \mathbf{z}=\sqrt{\mathbf{W}}\left(\mathbf{W}^{-1} \mathbf{u}+\widetilde{\mathbf{X}} \delta\right)
$$

We know that

$$
\begin{aligned}
E(\mathbf{u}) & =E\left(\frac{\partial \ell}{\partial \boldsymbol{\eta}}\right) \\
& =\int_{-\infty}^{\infty} f(\mathrm{z}) \frac{\partial \ell}{\partial \boldsymbol{\eta}} d \mathrm{z} \\
& =\int_{-\infty}^{\infty} f(\mathrm{z}) \frac{\partial}{\partial \boldsymbol{\eta}} \log L d \mathrm{z} \\
& =\int_{-\infty}^{\infty} f(\mathrm{z}) \frac{1}{L} \frac{\partial L}{\partial \boldsymbol{\eta}} d \mathrm{z} \\
& =\int_{-\infty}^{\infty} f(\mathrm{z}) \frac{1}{f(\mathrm{z})} \frac{\partial L}{\partial \boldsymbol{\eta}} d \mathrm{z} \\
& =\int_{-\infty}^{\infty} \frac{\partial L}{\partial \boldsymbol{\eta}} d \mathrm{z} \\
& =\frac{\partial}{\partial \boldsymbol{\eta}} \int_{-\infty}^{\infty} f(\mathrm{z}) d \mathrm{z} \\
& =\frac{\partial}{\partial \boldsymbol{\eta}} 1 \\
& =\mathbf{0}
\end{aligned}
$$


Hence, (Wood, 2006)

$$
\begin{aligned}
E(\sqrt{\mathbf{W}} \mathbf{z}) & =E\left(\sqrt{\mathbf{W}}\left(\mathbf{W}^{-1} \mathbf{u}+\widetilde{\mathbf{X}} \boldsymbol{\delta}\right)\right) \\
& =\sqrt{\mathbf{W}} \widetilde{\mathbf{X}} \boldsymbol{\delta} .
\end{aligned}
$$

Also, we have $\operatorname{Var}(\mathbf{u})=E\left(\mathbf{u u}^{\boldsymbol{\top}}\right)-(E(\mathbf{u}))^{2}=E\left(\mathbf{u u}^{\boldsymbol{\top}}\right)=\mathbf{W}$. Hence, $($ Wood, 2006)

$$
\begin{aligned}
\operatorname{Var}(\sqrt{\mathbf{W}} \mathbf{z}) & =\operatorname{Var}\left(\sqrt{\mathbf{W}}\left(\mathbf{W}^{-1} \mathbf{u}+\widetilde{\mathbf{X}} \boldsymbol{\delta}\right)\right) \\
& =\operatorname{Var}\left(\sqrt{\mathbf{W}}^{-1} \mathbf{u}+\sqrt{\mathbf{W}} \widetilde{\mathbf{X}} \boldsymbol{\delta}\right) \\
& =\operatorname{Var}\left(\sqrt{\mathbf{W}}^{-1} \mathbf{u}\right) \\
& =\sqrt{\mathbf{W}}^{-1} \mathbf{W} \sqrt{\mathbf{W}}^{-1} \\
& =\mathbf{I} .
\end{aligned}
$$

From likelihood theory we also know that since the likelihood is composed of i.i.d. random variables, the distribution of the score vector by the central limit theorem, as $n \rightarrow \infty$, will be normal (e.g. Wood, 2006). That is

$$
\mathbf{u} \sim N(\mathbf{0}, \mathbf{W}) .
$$

Hence,

$$
\begin{aligned}
\sqrt{\mathbf{W}}^{-1} \mathbf{u} & \sim N(\mathbf{0}, \mathbf{I}), \\
\mathbf{W}^{-1} \mathbf{u} & \sim N\left(\mathbf{0}, \mathbf{W}^{-1}\right), \\
\mathbf{W}^{-1} \mathbf{u}+\widetilde{\mathbf{X}} \boldsymbol{\delta} & \sim N\left(\widetilde{\mathbf{X}} \boldsymbol{\delta}, \mathbf{W}^{-1}\right), \\
\mathbf{z} & \sim N\left(\widetilde{\mathbf{X}} \boldsymbol{\delta}, \mathbf{W}^{-1}\right), \\
\sqrt{\mathbf{W}} \mathbf{z} & \sim N(\sqrt{\mathbf{W}} \widetilde{\mathbf{X}} \boldsymbol{\delta}, \mathbf{I}),
\end{aligned}
$$

or

$$
\sqrt{\mathbf{W}} \mathbf{z}-\sqrt{\mathbf{W}} \widetilde{\mathbf{X}} \boldsymbol{\delta} \sim N(\mathbf{0}, \mathbf{I})
$$


Taking the expectation of $\kappa$ yields (Wood, 2006)

$$
\begin{aligned}
E(\kappa) & =\frac{1}{n^{*}} E\left(\|\sqrt{\mathbf{W}} \mathbf{z}-\sqrt{\mathbf{W}} \mathbf{A} \mathbf{z}\|^{2}\right)-\frac{1}{n^{*}} E\left(\boldsymbol{\epsilon}^{\top} \boldsymbol{\epsilon}\right)-\frac{1}{n^{*}} E\left(2 \boldsymbol{\epsilon}^{\top} \mathbf{W} \widetilde{\mathbf{X}} \boldsymbol{\delta}^{*}\right) \\
& +\frac{1}{n^{*}} E\left(2 \boldsymbol{\epsilon}^{\top} \sqrt{\mathbf{W}} \mathbf{A} \sqrt{\mathbf{W}^{-1}} \boldsymbol{\epsilon}\right)+\frac{1}{n^{*}} E\left(2 \boldsymbol{\epsilon}^{\top} \sqrt{\mathbf{W}} \mathbf{A} \widetilde{\mathbf{X}} \boldsymbol{\delta}^{*}\right)
\end{aligned}
$$

The third and the fifth term are equal to zero since $E(\boldsymbol{\epsilon})=\mathbf{0}$. Also,

$$
E\left(\epsilon^{\top} \epsilon\right)=E\left(\sum_{i=1}^{n^{*}} \epsilon_{i}^{2}\right)=n^{*}
$$

and

$$
\begin{aligned}
E\left(\boldsymbol{\epsilon}^{\top} \sqrt{\mathbf{W}} \mathbf{A} \sqrt{\mathbf{W}}^{-1} \boldsymbol{\epsilon}\right) & =E\left(\operatorname{tr}\left(\boldsymbol{\epsilon}^{\top} \sqrt{\mathbf{W}} \mathbf{A} \sqrt{\mathbf{W}}^{-1} \boldsymbol{\epsilon}\right)\right) \\
& =E\left(\operatorname{tr}\left(\sqrt{\mathbf{W}} \sqrt{\mathbf{W}}^{-1} \mathbf{A} \boldsymbol{\epsilon} \boldsymbol{\epsilon}^{\top}\right)\right) \\
& \left.=\operatorname{tr}\left(\mathbf{A} E\left(\boldsymbol{\epsilon} \boldsymbol{\epsilon}^{\top}\right)\right)\right) \\
& =\operatorname{tr}(\mathbf{A I}) \\
& =\operatorname{tr}(\mathbf{A}) .
\end{aligned}
$$

The first line of (6.2.10) is justified by the fact that a scalar is its own trace. The remaining lines follow from the properties of the trace of a matrix. Thus,

$$
E(\kappa)=\frac{1}{n^{*}} E\left(\|\sqrt{\mathbf{W}} \mathbf{z}-\sqrt{\mathbf{W}} \mathbf{A} \mathbf{z}\|^{2}\right)-1+\frac{2}{n^{*}} \operatorname{tr}(\mathbf{A})
$$

Therefore, UBRE will be the estimate of $E(\kappa)$ equal to (Radice et al., 2015)

$$
\mathcal{V}_{u}(\boldsymbol{\lambda})=\frac{1}{n^{*}}\left\|\sqrt{\mathbf{W}}\left(\mathbf{z}-\widetilde{\mathbf{X}} \hat{\boldsymbol{\delta}}^{*}\right)\right\|^{2}-1+\frac{2}{n^{*}} \operatorname{tr}(\mathbf{A})
$$

where $n^{*}=5 n, \sqrt{\mathbf{W}} \mathbf{A}=\sqrt{\mathbf{W}} \widetilde{\mathbf{X}}\left(\widetilde{\mathbf{X}}^{\top} \mathbf{W} \widetilde{\mathbf{X}}+\mathbf{S}_{\lambda}\right)^{-1} \widetilde{\mathbf{X}}^{\top} \mathbf{W}$ is the is the hat matrix.

In summary, the following two steps are iterated until convergence:

1. Using starting values $\boldsymbol{\delta}^{[a]}$ and fixing the smoothing parameter vector at $\boldsymbol{\lambda}^{[a]}$, find the estimate 
of $\delta$ :

$$
\boldsymbol{\delta}^{[a+1]}=\arg _{\boldsymbol{\delta}} \max \ell_{p}(\boldsymbol{\delta})
$$

2. Using $\boldsymbol{\delta}^{[a+1]}$ construct the working linear model components from (6.2.11) and find the next iteration estimate of $\boldsymbol{\lambda}$ :

$$
\boldsymbol{\lambda}^{[a+1]}=\arg _{\boldsymbol{\lambda}} \min \mathcal{V}_{u}(\boldsymbol{\lambda})
$$

The algorithm can be seen as an adaptation of the outer iteration scheme by O'Sullivan et al. (1986). As an alternative, one could use performance iteration by $\mathrm{Gu}$ (1992). The difference between both methods is that performance iteration requires less iterations until convergence. In fact, it typically requires no more iterations from step 1 than are required to fit a model with known smoothing parameters. Hence, performance iteration methods are computationally efficient.

Nevertheless, performance iteration scheme may fail on some occasions. For instance, suppose that for a given set of iterations we obtain $\tilde{\boldsymbol{\delta}}$ and $\tilde{\boldsymbol{\lambda}}$. However, for the consecutive set of iterations $\hat{\boldsymbol{\delta}}$ and $\hat{\boldsymbol{\lambda}}$ are obtained. In the following iteration, a new set of estimates is obtained, but these are the same as $\tilde{\boldsymbol{\delta}}$ and $\tilde{\boldsymbol{\lambda}}$. This cycling does not end and hence convergence does not occur. This problem is particularily common in the presence of concurvity. For instance, suppose that a model includes terms such as $f_{1}\left(x_{1}\right)+f_{2}\left(x_{2}\right)$, but $x_{2}$ is a smooth function of $x_{1}$. Hence the smooth functions, $f_{1}$ and $f_{2}$, are confounded, and there may be very little information in the UBRE score from which to estimate their smoothing parameters separately. Concurvity problems like this are very common in models which include a smooth of spatial location and a number of covariates which themselves vary smoothly over space (Wood, 2006).

Another problem that the performance iteration algorithm may suffer from is the divergence of step 1. In the context of GAMs, divergence is not easily detected because the model likelihood, UBRE and penalized likelihood may all legitimately increase or decrease from one iteration to the other.

None of the problems above apply to outer iteration scheme. Despite the fact that it is computationally expensive, we will still proceed with outer iterations. 


\subsection{Simulations}

In this section, we will use simulations to check whether our spline implementation can recover the true functional forms of continuous covariates. The implementation of estimating functions can be found in SemiParSampleSel package (Marra et al., 2015).

The simulation setup will be similar to the one in Chapter 5. The first batch of simulations included 7 copula models for each of the 5 distributions. The copulas were FGM, bivariate normal, AMH, Clayton, Frank, Joe and Gumbel. The second batch of simulations included copula rotations of Clayton, Joe and Gumbel by 90, 180 and 270 degrees. Again, two model specifications were considered. The first specification had an intercept and two explanatory variables, continuous $x_{1}$ and binary $x_{2}$, in both equations. The second specification included an additional binary explanatory variable $x_{3}$ in the selection equation. The parameter settings for the binary variables, intercepts, $\sigma$ and $\nu$ were the same as in Chapter 5. The specification of the test function of $x_{1}$ in the selection and outcome equation was $f_{1}\left(x_{1}\right)=0.4\left[-4-\left(5.5 x_{1}-2.9\right)+3\left(4.5 x_{1}-2.3\right)^{2}-\left(4.5 x_{1}-2.3\right)^{3}\right]$ and $f_{2}\left(x_{1}\right)=x_{1} \sin \left(8 x_{1}\right)$ respectively. These were estimated using thin plate regression splines. The reason for choosing this specification is the wiggly shape of the functions and they are not monotonically increasing or decreasing. Both test functions are illustrated in Figure 6.2 on the domain $[0,1]$. 
Figure 6.2: Test functions, $f_{1}\left(x_{1}\right)=0.4\left[-4-\left(5.5 x_{1}-2.9\right)+3\left(4.5 x_{1}-2.3\right)^{2}-\left(4.5 x_{1}-2.3\right)^{3}\right]$ and $f_{2}\left(x_{1}\right)=x_{1} \sin \left(8 x_{1}\right)$, for the selection and outcome equation respectively. Both functions are neither monotonically increasing nor decreasing.
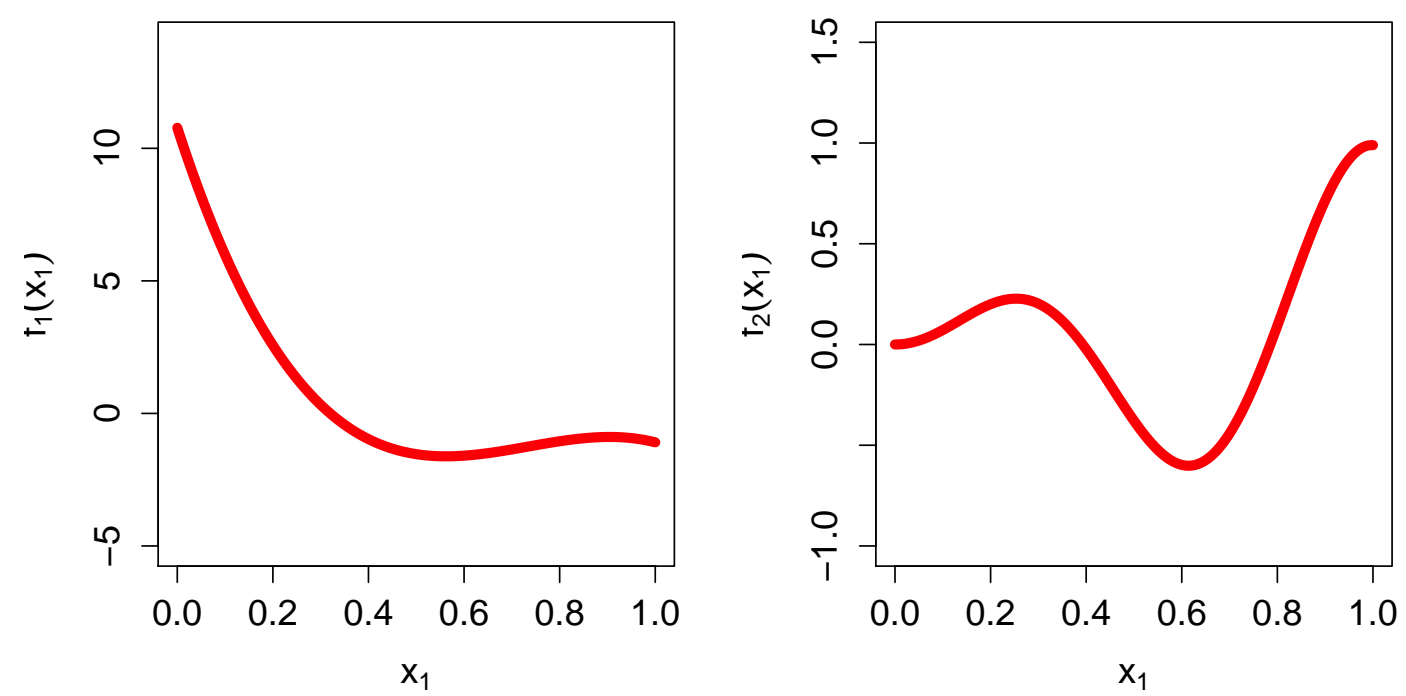

For each model combination 100 simulations were run with 3000 observations each. First, the two (three) covariates were generated from a trivariate normal distribution with the zero mean vector, covariance matrix with ones on the main diagonal and Pearson's correlation coefficient equal to 0.5. Then, the covariates were transformed using a standard normal cdf. Hence, the unconditional expected mean values were calculated and the parameters $\mu, \sigma$ and $\nu$ were inserted into the mvdc() function which created random outcome responses from a previously defined copula. The $\mathrm{R}$ code for this can be found in Appendix M. For copulas, Kendall's $\tau$ between the outcome and the selection equation was fixed at 0.2 for FGM, 0.3 for AMH, - 0.5 for 90 and 270 degrees rotated models and 0.5 for the remainder.

Figures in Appendix N-Q show the plots of smooths for every combination of copula and margin. The thin black lines indicate smooth plots for each replicate whereas the thick red line represents the true functional form. Since the thin lines are close to the true function, the models can, on average, recapture the true functional form of the covariate. Hence, the spline implementation can be used in practice when the functional form of the continuous covariate is unknown. 


\subsection{Inference}

\subsubsection{Frequentist confidence intervals}

From Section 6.2.3 we know that

$$
\sqrt{\mathbf{W}} \mathbf{z} \sim N(\sqrt{\mathbf{W}} \widetilde{\mathbf{X}} \boldsymbol{\delta}, \mathbf{I})
$$

To obtain the distribution of $\boldsymbol{\delta}^{*}$ we need to multiply $\sqrt{\mathbf{W}} \mathbf{z}$ by $\left(\widetilde{\mathbf{X}}^{\top} \mathbf{W} \widetilde{\mathbf{X}}+\mathbf{S}_{\lambda}\right)^{-1} \widetilde{\mathbf{X}}^{\top} \sqrt{\mathbf{W}}$. Hence

$$
\begin{aligned}
& \left(\widetilde{\mathbf{X}}^{\top} \mathbf{W} \widetilde{\mathbf{X}}+\mathbf{S}_{\lambda}\right)^{-1} \widetilde{\mathbf{X}}^{\top} \mathbf{W} \mathbf{z} \\
\sim & N\left(\left(\widetilde{\mathbf{X}}^{\top} \mathbf{W} \widetilde{\mathbf{X}}+\mathbf{S}_{\lambda}\right)^{-1} \widetilde{\mathbf{X}}^{\top} \mathbf{W} \widetilde{\mathbf{X}} \boldsymbol{\delta},\left(\widetilde{\mathbf{X}}^{\top} \mathbf{W} \widetilde{\mathbf{X}}+\mathbf{S}_{\lambda}\right)^{-1} \widetilde{\mathbf{X}}^{\top} \mathbf{W} \widetilde{\mathbf{X}}\left(\widetilde{\mathbf{X}}^{\top} \mathbf{W} \widetilde{\mathbf{X}}+\mathbf{S}_{\lambda}\right)^{-1}\right)
\end{aligned}
$$

or

$$
\hat{\boldsymbol{\delta}}^{*} \sim N\left(\left(\widetilde{\mathbf{X}}^{\top} \mathbf{W} \widetilde{\mathbf{X}}+\mathbf{S}_{\lambda}\right)^{-1} \widetilde{\mathbf{X}}^{\top} \mathbf{W} \widetilde{\mathbf{X}} \boldsymbol{\delta}, \mathbf{H}_{p}^{-1} \mathbf{H H}_{p}^{-1}\right)
$$

where $\mathbf{H}=\widetilde{\mathbf{X}}^{\top} \mathbf{W} \widetilde{\mathbf{X}}$ and $\mathbf{H}_{p}=\widetilde{\mathbf{X}}^{\top} \mathbf{W} \widetilde{\mathbf{X}}+\mathbf{S}_{\lambda}$. Note that the confidence intervals above are derived for transformations $\theta^{*}, \sigma^{*}$ and $\nu^{*}$. To obtain confidence intervals for the non-transformed parameters (e.g. $\left.\sigma=\exp \left(\sigma^{*}\right)\right)$, one needs to use the delta method. In brief, the delta method states that if

$$
\sqrt{n}(\boldsymbol{G}-\boldsymbol{\gamma}) \stackrel{D}{\rightarrow} N(\mathbf{0}, \boldsymbol{\Sigma}),
$$

where $\boldsymbol{G}$ is the random variable, $\gamma$ is the mean and $\boldsymbol{\Sigma}$ is the covariance matrix, then for any function $h$ there exists

$$
\sqrt{n}(h(\boldsymbol{G})-h(\boldsymbol{\gamma})) \stackrel{D}{\rightarrow} N\left(\mathbf{0}, \nabla h(\boldsymbol{\gamma})^{\top} \boldsymbol{\Sigma} \nabla h(\boldsymbol{\gamma})\right),
$$

where $\nabla h(\gamma)$ denotes the gradient of $h(\gamma)$. 


\subsubsection{Bayesian confidence intervals (credible intervals)}

It seems that the derivations above provide a sufficient result to construct confidence intervals. However, Marra and Wood (2012) show in a simulation study that the frequentist confidence intervals can have a poorer component-wise coverage than their Bayesian counterparts. They argue that, unlike Bayesian confidence intervals, the frequentist ones do not include a bias component.

Based on previous results, the distribution of $\boldsymbol{\delta}^{*}$ conditioned on the pseudodata will be derived. This will create the baseline for constructing Bayesian confidence intervals, which in simulation by Marra and Wood (2012) had close to nominal coverage probabilities. They argue that, unlike the frequentist ones, the Bayesian confidence intervals contain both the bias and variance component in the frequentist sense. The derivations and results in this section are all available in the literature as referenced. These are used in the implementation of SemiParSampleSel R package.

The idea behind Bayesian confidence intervals is that by imposing a particular penalty, we effectively impose some prior beliefs about the characteristics of the true model. The modelling approach enables more flexibility than it is initially believed and we choose a certain penalty for models that are too wiggly (Wood, 2006). It is natural to set a prior distribution on $\boldsymbol{\delta}^{*}$. Let the prior for $\boldsymbol{\delta}^{*}$ be

$$
f_{\boldsymbol{\delta}^{*}}\left(\boldsymbol{\delta}^{*}\right) \propto e^{-\frac{1}{2} \boldsymbol{\delta}^{* \top} \mathbf{S}_{\lambda} \boldsymbol{\delta}^{*} .}
$$

As we will see, the choice of prior in (6.4.3) will ensure conjugacy. Also, (6.4.3) is appropriate since it expresses the belief that smooth models are more likely than wiggly ones, but it gives equal probability density to all models of equal smoothness (Wood, 2006). Recall (6.4.1):

$$
\begin{aligned}
\sqrt{\mathbf{W}} \mathbf{z} & \sim N(\sqrt{\mathbf{W}} \widetilde{\mathbf{X}} \boldsymbol{\delta}, \mathbf{I}) \\
\mathbf{z} & \sim N\left(\widetilde{\mathbf{X}} \boldsymbol{\delta}, \mathbf{W}^{-1}\right) .
\end{aligned}
$$

Therefore,

$$
f\left(\mathbf{z} \mid \boldsymbol{\delta}^{*}\right) \propto e^{-\frac{1}{2}\left(\mathbf{z}-\widetilde{\mathbf{X}} \boldsymbol{\delta}^{*}\right)^{\top} \mathbf{W}\left(\mathbf{z}-\widetilde{\mathbf{X}} \boldsymbol{\delta}^{*}\right)} .
$$


Hence, the posterior will be

$$
\begin{aligned}
f\left(\boldsymbol{\delta}^{*} \mid \mathbf{z}\right) & \propto e^{-\frac{1}{2}\left[\left(\mathbf{z}-\widetilde{\mathbf{X}} \boldsymbol{\delta}^{*}\right)^{\top} \mathbf{W}\left(\mathbf{z}-\widetilde{\mathbf{X}} \boldsymbol{\delta}^{*}\right)+\boldsymbol{\delta}^{* \top} \mathbf{S}_{\lambda} \boldsymbol{\delta}^{*}\right]} \\
& =e^{-\frac{1}{2}\left(\mathbf{z}^{\top} \mathbf{z}-2 \boldsymbol{\delta}^{*} \widetilde{\mathbf{X}}^{\top} \mathbf{W} \mathbf{z}+\boldsymbol{\delta}^{* \top} \widetilde{\mathbf{X}}^{\top} \mathbf{W} \widetilde{\mathbf{X}} \boldsymbol{\delta}^{*}+\boldsymbol{\delta}^{* \top} \mathbf{S}_{\lambda} \boldsymbol{\delta}^{*}\right)} \\
& =e^{-\frac{1}{2}\left[\mathbf{z}^{\top} \mathbf{z}-2 \boldsymbol{\delta}^{*} \widetilde{\mathbf{X}}^{\top} \mathbf{W} \mathbf{z}+\boldsymbol{\delta}^{* \top}\left(\widetilde{\mathbf{X}}{ }^{\top} \mathbf{W} \widetilde{\mathbf{X}}+\mathbf{S}_{\lambda}\right) \boldsymbol{\delta}^{*}\right]} \\
& =e^{-\frac{1}{2}\left[-2 \boldsymbol{\delta}^{*} \widetilde{\mathbf{X}}^{\top} \mathbf{W} \mathbf{z}+\boldsymbol{\delta}^{* \top}\left(\widetilde{\mathbf{X}}{ }^{\top} \mathbf{W} \widetilde{\mathbf{X}}+\mathbf{S}_{\lambda}\right) \boldsymbol{\delta}^{*}\right]}
\end{aligned}
$$

To see that $\boldsymbol{\delta}^{*} \mid \mathbf{z}$ is normally distributed, consider

$$
\boldsymbol{\alpha} \sim N\left(\left[\widetilde{\mathbf{X}}^{\top} \mathbf{W} \widetilde{\mathbf{X}}+\mathbf{S}_{\lambda}\right]^{-1} \widetilde{\mathbf{X}}^{\top} \mathbf{W} \mathbf{z},\left[\widetilde{\mathbf{X}}^{\top} \mathbf{W} \widetilde{\mathbf{X}}+\mathbf{S}_{\lambda}\right]^{-1}\right)
$$

Thus,

$$
\begin{aligned}
& f(\boldsymbol{\alpha}) \propto e^{-\frac{1}{2}\left(\boldsymbol{\alpha}-\left[\tilde{\mathbf{X}}^{\top} \mathbf{W} \widetilde{\mathbf{X}}+\mathbf{S}_{\lambda}\right]^{-1} \widetilde{\mathbf{X}}^{\top} \mathbf{W} \mathbf{z}\right)^{\top}\left(\tilde{\mathbf{X}}^{\top} \mathbf{W} \tilde{\mathbf{X}}+\mathbf{S}_{\lambda}\right)\left(\boldsymbol{\alpha}-\left[\widetilde{\mathbf{X}}^{\top} \mathbf{W} \widetilde{\mathbf{X}}+\mathbf{S}_{\lambda}\right]^{-1} \widetilde{\mathbf{X}}^{\top} \mathbf{W} \mathbf{z}\right)} \\
& f(\boldsymbol{\alpha})=e^{-\frac{1}{2}\left(\boldsymbol{\alpha}^{\top}\left(\widetilde{\mathbf{X}}^{\top} \mathbf{W} \widetilde{\mathbf{X}}+\mathbf{S}_{\lambda}\right) \boldsymbol{\alpha}-\boldsymbol{\alpha} \widetilde{\mathbf{X}}^{\top} \mathbf{W} \mathbf{z}-\boldsymbol{\alpha} \widetilde{\mathbf{X}}^{\top} \mathbf{W} \mathbf{z}+\left(\widetilde{\mathbf{X}}^{\top} \mathbf{W} \widetilde{\mathbf{X}}+\mathbf{S}_{\lambda}\right)^{-1}\left(\widetilde{\mathbf{X}}^{\top} \mathbf{W} \mathbf{z}\right)\left(\tilde{\mathbf{X}}^{\top} \mathbf{W} \mathbf{z}\right)^{\top}\right)} \\
& f(\boldsymbol{\alpha}) \propto e^{-\frac{1}{2}\left(\boldsymbol{\alpha}^{\top}\left(\widetilde{\mathbf{X}}^{\top} \mathbf{W} \widetilde{\mathbf{X}}+\mathbf{S}_{\lambda}\right) \boldsymbol{\alpha}-2 \boldsymbol{\alpha} \widetilde{\mathbf{X}}^{\top} \mathbf{W} \mathbf{z}\right)}
\end{aligned}
$$

By comparing $f(\boldsymbol{\alpha})$ and $f\left(\boldsymbol{\delta}^{*} \mid \mathbf{z}\right)$, we see that the last rows will be identical if $\boldsymbol{\alpha}=\boldsymbol{\delta}^{*}$. Thus, $\boldsymbol{\alpha}$ will have the same distribution as $\boldsymbol{\delta}^{*}$. Therefore,

$$
\boldsymbol{\delta}^{*} \mid \mathbf{z} \sim N\left(\left[\widetilde{\mathbf{X}}^{\top} \mathbf{W} \widetilde{\mathbf{X}}+\mathbf{S}_{\lambda}\right]^{-1} \widetilde{\mathbf{X}}^{\top} \mathbf{W} \mathbf{z},\left[\widetilde{\mathbf{X}}^{\top} \mathbf{W} \widetilde{\mathbf{X}}+\mathbf{S}_{\lambda}\right]^{-1}\right)
$$

Recall that $\mathbf{z}=\mathbf{W}^{-1} \mathbf{u}+\widetilde{\mathbf{X}} \boldsymbol{\delta}$. As the number of observations grows large, by law of large numbers the gradient $\mathbf{u}$ will converge to its expected value i.e. $E(\mathbf{u})=\mathbf{0}$. Thus, $\mathbf{z}=\widetilde{\mathbf{X}} \boldsymbol{\delta}$. Hence,

$$
\boldsymbol{\delta}^{*} \mid \mathbf{z} \sim N\left(\left[\widetilde{\mathbf{X}}^{\top} \mathbf{W} \widetilde{\mathbf{X}}+\mathbf{S}_{\lambda}\right]^{-1} \widetilde{\mathbf{X}}^{\top} \mathbf{W} \widetilde{\mathbf{X}} \boldsymbol{\delta}, \mathbf{H}_{p}^{-1}\right)
$$

where $\mathbf{H}_{p}=\widetilde{\mathbf{X}}^{\top} \mathbf{W} \widetilde{\mathbf{X}}+\mathbf{S}_{\lambda}$. By comparing (6.4.4) with its frequentist counterpart in (6.4.2), note that the difference lies in the covariances of $\hat{\boldsymbol{\delta}}^{*}$ and $\boldsymbol{\delta}^{*}$. For instance, the confidence interval for the 
smooth components will be

$$
\mathbf{B}\left(u_{k}\right)^{\mathbf{T}} \hat{\boldsymbol{\alpha}}_{k} \sim N\left(\mathbf{B}\left(u_{k}\right)^{\mathbf{T}} \boldsymbol{\alpha}_{k}, \mathbf{B}\left(u_{k}\right)^{\mathbf{T}} \mathbf{V}_{\delta \mathbf{k}}^{*} \mathbf{B}\left(u_{k}\right)\right)
$$

where $\mathbf{V}_{\delta \mathbf{k}}^{*}$ is the covariance matrix in (6.4.4) constrained to the $k$-th smooth. Intervals for nontransformed parameters (i.e. $\sigma, \nu, \theta$ ) can be obtained by simulating from the posterior distribution of $\boldsymbol{\delta}^{*}$ as follows:

1. Draw $n_{\text {sim }}$ random vectors from (6.4.4).

2. Calculate $n_{\text {sim }}$ simulated realisations of the function of interest. For example, since $\sigma=$ $\exp \left(\sigma^{*}\right), \boldsymbol{\sigma}^{\text {sim }}=\left(\sigma_{1}^{\text {sim }}, \sigma_{2}^{\text {sim }}, \ldots, \sigma_{n_{s i m}}^{\text {sim }}\right)$ where $\sigma_{o}^{\text {sim }}=\exp \left(\sigma_{o}^{* s i m}\right), o=1,2, \ldots, n_{\text {sim }}$.

3. Using $\boldsymbol{\sigma}^{\text {sim }}$, calculate the lower, $\xi / 2$, and upper, $1-\xi / 2$, quantiles. For 95 per cent Bayesian confidence intervals, $\xi$ is set to 0.05 .

Ruppert et al. (2003) argue that the result in (6.4.5) is inappropriate for testing the null hypothesis of a smooth term being equal to zero, since it only considers marginal distributions of the smooth function vector. Thus, the joint distribution of the smooth function vector needs to be considered. For this reason, we will use the test derived by Wood (2012) and discussed by Marra (2013) in the context of equation system models. Let $\hat{\mathbf{s}}_{k}=\mathbf{B}\left(\mathbf{u}_{k}\right)^{\mathbf{T}} \hat{\boldsymbol{\alpha}}_{k}, \mathbf{V}_{\mathbf{s}_{k}}^{*}=\mathbf{B}\left(\mathbf{u}_{k}\right)^{\mathrm{T}} \mathbf{V}_{\delta \mathbf{k}}^{*} \mathbf{B}\left(\mathbf{u}_{k}\right)$, where $\mathbf{B}\left(\mathbf{u}_{k}\right)$ is a full rank matrix with $\mathbf{u}_{k}=\left[u_{k 1}, u_{k 1}, \ldots, u_{k n}\right]^{\top}$. Hence,

$$
T_{r_{k}}=\hat{\mathbf{s}}_{k}^{\top} \mathbf{V}_{\mathbf{s}_{k}}^{r_{k}{ }^{*-}} \hat{\mathbf{s}}_{k} \sim \chi_{r k}^{2}
$$

is the statistic for obtaining test p-value, where $\mathbf{V}_{\mathbf{s}_{k}}^{r_{k^{*-}}}$ is the Moore-Penrose pseudoinverse of $\mathbf{V}_{\mathbf{s}_{k}}^{*}$. The $r_{k}$ parameter is chosen using the effective degrees of freedom $(e d f)$ equal to $\operatorname{tr}\left(\hat{\mathbf{A}}_{\hat{\lambda}}\right)$. Since $e d f$ is not an integer the following decision rules apply (Wood, 2012)

$$
r_{k}= \begin{cases}e d f_{k}, & \text { if } e d f_{k}<\operatorname{floor}\left(e d f_{k}\right)+0.05 \\ e d f_{k}+1 & \text { otherwise. }\end{cases}
$$

This also turned to be effective for semiparametric bivariate probit models (Marra, 2013). Since our modelling approach is similar to the one by Marra (2013), we will use the readily available result 
above.

\subsection{Predictions}

In this section we will obtain some results that will be useful for predictions and their corresponding confidence intervals. The Bayesian distributional result from Section 6.4.2 implies that

$$
\boldsymbol{\delta}^{*} \sim N\left(\left[\widetilde{\mathbf{X}}^{\top} \mathbf{W} \widetilde{\mathbf{X}}+\mathbf{S}_{\lambda}\right]^{-1} \widetilde{\mathbf{X}}^{\top} \mathbf{W} \widetilde{\mathbf{X}} \boldsymbol{\delta}, \mathbf{H}_{p}^{-1}\right)
$$

where $\mathbf{H}_{p}=\widetilde{\mathbf{X}}^{\top} \mathbf{W} \widetilde{\mathbf{X}}+\mathbf{S}_{\lambda}$. Suppose that we truncate the $\boldsymbol{\delta}^{*}$ parameter space to the parameters associated with the outcome. By doing so, we will only deal with parameters that enter the expectation of the outcome. The same will be done for $\widetilde{\mathbf{X}}, \mathbf{W}$ and $\mathbf{S}_{\lambda}$. To avoid clutter we will avoid any subscripts. Also,

$$
\widetilde{\mathbf{X}} \boldsymbol{\delta}^{*} \sim N\left(\widetilde{\mathbf{X}}\left[\widetilde{\mathbf{X}}^{\top} \mathbf{W} \widetilde{\mathbf{X}}+\mathbf{S}_{\lambda}\right]^{-1} \widetilde{\mathbf{X}}^{\top} \mathbf{W} \widetilde{\mathbf{X}} \boldsymbol{\delta}, \widetilde{\mathbf{X}} \mathbf{H}_{p}^{-1} \widetilde{\mathbf{X}}^{\top}\right)
$$

Since $E\left(Y_{2}\right)=e^{\eta_{2}}$ for all outcome margins, we need to exponentiate $\widetilde{\mathbf{X}} \boldsymbol{\delta}^{*}$. Using the delta method, we can obtain the (approximated) prediction distribution for a vector of observations:

$$
\begin{aligned}
& e^{\widetilde{\mathbf{X}} \boldsymbol{\delta}^{*}} \sim N\left(e^{\widetilde{\mathbf{X}}\left[\widetilde{\mathbf{X}}^{\top} \mathbf{W} \widetilde{\mathbf{X}}+\mathbf{S}_{\lambda}\right]^{-1} \widetilde{\mathbf{X}}^{\top} \mathbf{W} \widetilde{\mathbf{X}} \boldsymbol{\delta}}, \mathbf{L} \widetilde{\mathbf{X}} \mathbf{H}_{p}^{-1} \widetilde{\mathbf{X}}^{\top} \mathbf{L}\right), \\
& e^{\tilde{\mathbf{X}} \boldsymbol{\delta}^{*}} \sim N\left(e^{\widetilde{\mathbf{X}}\left[\tilde{\mathbf{X}}^{\top} \mathbf{W} \tilde{\mathbf{X}}+\mathbf{S}_{\lambda}\right]^{-1} \widetilde{\mathbf{X}}^{\top} \mathbf{W} \tilde{\mathbf{X}} \boldsymbol{\delta}}, \mathbf{V}\right),
\end{aligned}
$$

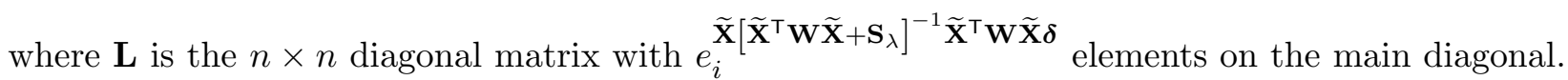
Finally, we can obtain the distribution for the average predicted value:

$$
\frac{\sum e_{i}^{\widetilde{\mathbf{X}} \delta^{*}}}{n} \sim N\left(\frac{\sum e_{i}^{\widetilde{\mathbf{X}}\left[\widetilde{\mathbf{X}}^{\top} \mathbf{W} \widetilde{\mathbf{X}}+\mathbf{S}_{\lambda}\right]^{-1} \widetilde{\mathbf{X}}^{\top} \mathbf{W} \widetilde{\mathbf{X}} \boldsymbol{\delta}}}{n}, \frac{\sum V_{i i}}{n^{2}}\right)
$$

where $V_{i i}$ are the leading diagonal elements of $\mathbf{V}$. 


\subsection{Model selection}

In literature, one can find several ways of comparing competing nested or non-nested models. Methods presented in this section can be also applied to our modelling approach. From now on we will use $\boldsymbol{\delta}$ and $\hat{\boldsymbol{\delta}}$ to denote the parameters and their estimators respectively. The first two are commonly known as the Akaike and Bayesian Information Criteria (Akaike, 1974; Schwarz, 1978). In our case, these are defined as

$$
\begin{aligned}
\mathrm{AIC} & =-2 \ell(\hat{\boldsymbol{\delta}})+2 e d f \\
\mathrm{BIC} & =-2 \ell(\hat{\boldsymbol{\delta}})+\log (n) e d f
\end{aligned}
$$

where $\ell(\hat{\boldsymbol{\delta}})$ is the likelihood of a model evaluated at estimates and the effective degrees of freedom $(e d f)$ are defined by $\operatorname{tr}\left(\hat{\mathbf{A}}_{\hat{\boldsymbol{\lambda}}}\right)$. Note that if only strictly parametric terms are used, then the effective degrees of freedom will be reduced to the total number of parameters.

The two alternative approaches are based on likelihood ratio tests. The first one was proposed by Vuong (1989). The null hypothesis of Vuong test is

$$
H_{0}: \mathrm{E}\left(q_{i}\right)=0 \quad \forall i=1, . ., n,
$$

where $n$ denotes the sample size, $i$ indicates each observation and $q_{i}$ is the log-likelihood ratio of the two models. The null hypothesis simply states that two models are equally close to the actual model. The alternative hypothesis implies that one model is closer than the other. Let $\ell_{1}\left(\hat{\boldsymbol{\delta}}_{\mathbf{1}}\right)$ and $\ell_{2}\left(\hat{\boldsymbol{\delta}}_{\mathbf{2}}\right)$ describe the likelihood of two competing models, say 1 and 2, evaluated at their estimates. The test statistic is

$$
V=\frac{\ell_{1}\left(\hat{\boldsymbol{\delta}}_{\mathbf{1}}\right)-\ell_{2}\left(\hat{\boldsymbol{\delta}}_{\mathbf{2}}\right)-\left\{\frac{e d f_{1}-e d f_{2}}{2} \log (n)\right\}}{\sqrt{\sum_{i=1}^{n}\left(q_{i}-\bar{q}\right)^{2}}} \underset{H_{0}}{\stackrel{d}{\longrightarrow}} \mathcal{N}(0,1) .
$$

For significance level $\eta$, if $V>\Phi^{-1}\left(1-\frac{\eta}{2}\right)$ then model 1 is preferred over model 2 and vice versa when $V<\Phi^{-1}\left(\frac{\eta}{2}\right)$. Otherwise, we cannot prefer one model over another and hence $H_{0}$ cannot be rejected. 
The second inferential approach is the Clarke test (Clarke, 2007) with

$$
H_{0}: \mathbb{P}\left(q_{i}>0\right)=0.5 \quad \forall i=1, . ., n
$$

meaning that if the two models are equivalent the likelihood ratios of all observations should be evenly distributed and 50 per cent of ratios should be greater than zero. The test statistic is

$$
B=\sum_{i=1}^{n} \mathbf{1}\left(q_{i}-\left\{\frac{e d f_{1}-e d f_{2}}{2 n}\right\} \log (n)\right) \underset{H_{0}}{\stackrel{d}{\longrightarrow}} \operatorname{Bin}(n, 0.5) .
$$

Model 1 is preferred over model 2 if $B$ is significantly larger than the expected value under the null hypothesis and vice versa. For further details see Clarke (2007).

\subsection{Asymptotic considerations}

In this section we will prove the ML estimator consistency of the count copula sample selection model. The approach will be based on the one by Radice et al. (2015). Let $\hat{\boldsymbol{\delta}}$ be the penalized ML estimator defined as

$$
\hat{\boldsymbol{\delta}}=\underset{\boldsymbol{\delta}}{\arg \max } \ell_{p}(\boldsymbol{\delta})
$$

where $\ell_{p}(\boldsymbol{\delta})$ is the penalized likelihood. Note that we are considering a situation in which the number of bases is fixed. In this case, the unknown smooth functions may not have an exact representation as linear combinations of given basis functions. Consequently, the unknown functions and parameters may not be asymptotically identified by their estimators as the sample size grows (Radice et al., 2015). However, in practice the number of bases functions is fixed. Assuming that the number of bases is high, it is possible to assume heuristically that the approximation bias is negligible compared to estimation variability (Kauermann, 2005).

Let $L^{t}$ be the likelihood function of the true model which contains all parameters from the outcome and selection equation as well as parameters $\sigma^{*}, \nu^{*}$ and $\theta^{*}$. The smooth terms $\sum_{k_{1}=1}^{K_{1}} f_{1 k_{1}}\left(u_{1 k_{1}}\right)$ and $\sum_{k_{2}=1}^{K_{2}} f_{2 k_{2}}\left(u_{2 k_{2}}\right)$ are replaced with their approximations $\mathbf{B}_{1}^{\mathbf{T}} \boldsymbol{\alpha}_{1}$ and $\mathbf{B}_{2}^{\mathbf{T}} \boldsymbol{\alpha}_{2}$. 
Thus, the Kullback-Leibler distance between $L^{t}$ and $L(\boldsymbol{\delta})$ is

$$
\mathrm{KL}\left(L^{t} \| L(\boldsymbol{\delta})\right)=E\left(\ell^{\top}-\ell(\boldsymbol{\delta})\right)
$$

where the expectation is taken with respect to the true model distribution where

$$
\boldsymbol{\delta}=\left(\boldsymbol{\delta}_{\mathbf{1}}^{\top}, \boldsymbol{\delta}_{\mathbf{2}}^{\top}, \theta^{*}, \sigma^{*}, \nu^{*}\right)^{\top}
$$

Let us define the minimiser of $\boldsymbol{\delta}^{0}=\left(\boldsymbol{\delta}_{\mathbf{1}}{ }^{0}, \boldsymbol{\delta}_{\mathbf{2}}{ }^{0}, \theta^{0 *}, \sigma^{0 *}, \nu^{0 *}\right)$. Thus,

$$
\boldsymbol{\delta}^{0}=\underset{\boldsymbol{\delta}}{\arg \min } \mathrm{KL}\left(L^{t} \| L(\boldsymbol{\delta})\right)
$$

It follows that $\boldsymbol{\delta}^{0}$ is the minimiser of the expected unpenalized $\log$-likelihood $\ell(\cdot)$ and hence $E\left(\mathbf{g}\left(\boldsymbol{\delta}^{0}\right)\right)=$ 0. Recall that $\mathbf{g}(\boldsymbol{\delta})$ and $\mathbf{H}(\boldsymbol{\delta})$ are the gradient and Hessian functions of $\ell(\cdot)$ respectively and $\mathbf{g}_{p}(\boldsymbol{\delta})=\mathbf{g}(\boldsymbol{\delta})-\mathbf{S}_{\lambda} \boldsymbol{\delta}$ and $\mathbf{H}_{p}(\boldsymbol{\delta})=\mathbf{H}(\boldsymbol{\delta})-\mathbf{S}_{\lambda}$ are the penalized versions of these. Following Kauermann (2005), the following assumptions are made

1. $\mathbf{g}\left(\boldsymbol{\delta}^{0}\right)=O_{P}\left(n^{1 / 2}\right)$

2. $E\left(\mathbf{H}\left(\boldsymbol{\delta}^{0}\right)\right)=O(n)$

3. $\mathbf{H}\left(\boldsymbol{\delta}^{0}\right)-E\left(\mathbf{H}\left(\boldsymbol{\delta}^{0}\right)\right)=O_{P}\left(n^{1 / 2}\right)$

4. $\mathbf{S}_{\lambda}=o\left(n^{1 / 2}\right)$.

Asymptotic notation of $O_{P}, O, o_{P}$, and $o$ was adopted from Barndorff-Nielsen and Cox (1989). For instance, suppose that $\left\{a_{t}\right\}$ and $\left\{b_{t}\right\}$ are two sequences of real numbers, $O$ and $o$ imply that

$$
\begin{aligned}
a_{t}=o\left(b_{t}\right) & \Longleftrightarrow \lim _{t \rightarrow \infty} \frac{a_{t}}{b_{t}}=0, \\
a_{t}=O\left(b_{t}\right) & \Longleftrightarrow \lim _{t \rightarrow \infty} \frac{a_{t}}{b_{t}}=A<\infty .
\end{aligned}
$$

Suppose now we have a sequence of random variables $\left\{X_{t}\right\}$. We say

$$
X_{t}=o_{P}\left(a_{t}\right) \Longleftrightarrow \frac{X_{t}}{a_{t}} \stackrel{p}{\rightarrow} 0
$$


In other words, $X_{t}=o_{P}\left(a_{t}\right)$ means that $\frac{X_{t}}{a_{t}}$ converges in probability to zero. That is, for any $\epsilon$, there exist $\delta$ and $t_{0}$ such that $\operatorname{Pr}\left(\left|\frac{X_{t}}{a_{t}}\right|>\epsilon\right)<\delta$ for all $t>t_{0}$. On the other hand, $X_{t}=O_{P}\left(a_{t}\right)$ implies that $\frac{X_{t}}{a_{t}}$ is bounded in probability. More precisely, there exists an $M<\infty$, and for any $\epsilon$, a $t_{0}$, such that

$$
\operatorname{Pr}\left(\frac{X_{t}}{a_{t}}>M\right)<\epsilon, \text { for all } t>t_{0}
$$

Conditions 1-3 are the usual assumptions of asymptotics. Conditions 1 and 3 imply that since $\mathbf{g}\left(\boldsymbol{\delta}^{0}\right)$ and $\mathbf{H}\left(\boldsymbol{\delta}^{0}\right)$ are made up of sums of iid random variables, $\frac{1}{n} \mathbf{g}\left(\boldsymbol{\delta}^{0}\right)$ and $\frac{1}{n} \mathbf{H}\left(\boldsymbol{\delta}^{0}\right)$ will converge in probability to their expected values at the rate of $n^{-1 / 2}$.

In order to prove consistency, we need to show that the difference between the penalized ML estimator $\hat{\boldsymbol{\delta}}$ and the true value $\boldsymbol{\delta}^{0}$ converges in probability at the rate $n^{-1 / 2}$ as $n$ tends to infinity. That is,

$$
\hat{\boldsymbol{\delta}}-\boldsymbol{\delta}^{0}=O_{P}\left(n^{-1 / 2}\right) \quad \text { as } n \rightarrow \infty
$$

First, one needs to show that

$$
\hat{\boldsymbol{\delta}}-\boldsymbol{\delta}^{0} \approx\left(-E\left(\mathbf{H}\left(\boldsymbol{\delta}^{0}\right)\right)+\mathbf{S}_{\lambda}\right)^{-1}\left(\mathbf{g}\left(\boldsymbol{\delta}^{0}\right)-\mathbf{S}_{\lambda} \boldsymbol{\delta}^{0}\right)
$$

As shown in Kauermann (2005), this will imply convergence in probability at the rate of $n^{-1 / 2}$ and hence consistency. Let us formulate the Taylor expansion of $\mathbf{g}_{p}(\hat{\boldsymbol{\delta}})$ at point $\boldsymbol{\delta}^{0}$ :

$$
\mathbf{g}_{p}(\hat{\boldsymbol{\delta}})=\mathbf{g}_{p}\left(\boldsymbol{\delta}^{0}\right)+\mathbf{H}_{p}\left(\boldsymbol{\delta}^{0}\right)\left(\hat{\boldsymbol{\delta}}-\boldsymbol{\delta}^{0}\right)+\text { higher order terms }
$$

Note that $\mathbf{g}_{p}(\hat{\boldsymbol{\delta}})=0$. Rearranging and inverting the series yields

$$
\begin{aligned}
\hat{\boldsymbol{\delta}}-\boldsymbol{\delta}^{0} & =-\mathbf{H}_{p}\left(\boldsymbol{\delta}^{0}\right)^{-1} \mathbf{g}_{p}\left(\boldsymbol{\delta}^{0}\right) \\
& =-\mathbf{H}_{p}\left(\boldsymbol{\delta}^{0}\right)^{-1}\left(\mathbf{g}\left(\boldsymbol{\delta}^{0}\right)-\mathbf{S}_{\lambda} \boldsymbol{\delta}^{0}\right)
\end{aligned}
$$


By expanding $\mathbf{H}\left(\boldsymbol{\delta}^{0}\right)$ we obtain

$$
\begin{aligned}
\mathbf{H}_{p}\left(\boldsymbol{\delta}^{0}\right) & =\mathbf{H}\left(\boldsymbol{\delta}^{0}\right)-\mathbf{S}_{\lambda} \\
& =\left(\mathbf{H}\left(\boldsymbol{\delta}^{0}\right)-E\left(\mathbf{H}\left(\boldsymbol{\delta}^{0}\right)\right)\right)+\left(E\left(\mathbf{H}\left(\boldsymbol{\delta}^{0}\right)\right)-\mathbf{S}_{\lambda}\right) \\
& =\mathbf{R}-\mathbf{F}(\lambda)
\end{aligned}
$$

where $\mathbf{R}=\mathbf{H}\left(\boldsymbol{\delta}^{0}\right)-E\left(\mathbf{H}\left(\boldsymbol{\delta}^{0}\right)\right)$ and $\mathbf{F}(\lambda)=-E\left(\mathbf{H}\left(\boldsymbol{\delta}^{0}\right)\right)+\mathbf{S}_{\lambda}$. Let $f(\cdot)=(\cdot-\mathbf{F}(\lambda))^{-1}$ be an auxiliary function of a matrix argument. A Taylor expansion of $f(\mathbf{R})=\mathbf{H}_{p}^{-1}\left(\boldsymbol{\delta}^{0}\right)$ at $\mathbf{0}$ yields

$$
\begin{aligned}
\mathbf{H}_{p}\left(\boldsymbol{\delta}^{0}\right)^{-1} & =f(\mathbf{R}) \\
& =f(\mathbf{0})+f^{\prime}(\mathbf{0})(\mathbf{R}-\mathbf{0})+\ldots \\
& =-\mathbf{F}(\lambda)^{-1}-\mathbf{F}(\lambda)^{-1} \mathbf{R F}(\lambda)^{-1}+\ldots \\
& =-\mathbf{F}(\lambda)^{-1}\left(\mathbf{I}+\mathbf{R F}(\lambda)^{-1}+\ldots\right),
\end{aligned}
$$

where $\mathbf{I}$ is the identity matrix. From assumptions $1-4$ it follows that $\mathbf{R}=O_{P}\left(n^{1 / 2}\right), \mathbf{F}(\lambda)=$ $O(n)+o\left(n^{1 / 2}\right)=O(n)$. Hence, $\mathbf{R F}(\lambda)^{-1}=O_{P}\left(n^{1 / 2}\right) O\left(n^{-1}\right)=O_{P}\left(n^{-1 / 2}\right)$. The remaining terms of the expansion are negligible. Thus,

$$
\begin{aligned}
\mathbf{H}_{p}\left(\boldsymbol{\delta}^{0}\right)^{-1} & =-\mathbf{F}(\lambda)^{-1}\left(\mathbf{I}+\mathbf{R F}(\lambda)^{-1}+\ldots\right) \\
& =-\mathbf{F}(\lambda)^{-1}\left(\mathbf{I}+O_{P}\left(n^{-1 / 2}\right)\right)
\end{aligned}
$$

Therefore,

$$
\hat{\boldsymbol{\delta}}-\boldsymbol{\delta}^{0}=\mathbf{F}(\lambda)^{-1}\left(\mathbf{g}\left(\boldsymbol{\delta}^{0}\right)-\mathbf{S}_{\lambda} \boldsymbol{\delta}^{0}\right)\left(\mathbf{I}+o_{P}(1)\right)
$$

which proves (6.7.2) and hence

$$
\hat{\boldsymbol{\delta}}-\boldsymbol{\delta}^{0}=O_{P}\left(n^{-1 / 2}\right) \quad \text { as } n \rightarrow \infty .
$$

Several remarks need to be made. 
Remark 1: Note that the asymptotic bias and covariance can be written as

$$
\begin{aligned}
\operatorname{bias}(\hat{\boldsymbol{\delta}}) & =E\left(\hat{\boldsymbol{\delta}}-\boldsymbol{\delta}^{0}\right) \\
& \approx E\left(\mathbf{F}(\lambda)^{-1}\left(\mathbf{g}\left(\boldsymbol{\delta}^{0}\right)-\mathbf{S}_{\lambda} \boldsymbol{\delta}^{0}\right)\right) \\
& =\mathbf{F}(\lambda)^{-1}\left(\mathbf{0}-\mathbf{S}_{\lambda} \boldsymbol{\delta}^{0}\right) \\
& =-\mathbf{F}(\lambda)^{-1} \mathbf{S}_{\lambda} \boldsymbol{\delta}^{0}
\end{aligned}
$$

and

$$
\begin{aligned}
\operatorname{Cov}(\hat{\boldsymbol{\delta}}) & \approx \operatorname{Cov}\left(\mathbf{F}(\lambda)^{-1}\left(\mathbf{g}\left(\delta^{0}\right)-\mathbf{S}_{\lambda} \delta^{0}\right)+\delta^{0}\right) \\
& =\operatorname{Cov}\left(\mathbf{F}(\lambda)^{-1}\left(\mathbf{g}\left(\delta^{0}\right)-\mathbf{S}_{\lambda} \delta^{0}\right)\right) \\
& =\operatorname{Cov}\left(\mathbf{F}(\lambda)^{-1} \mathbf{g}\left(\delta^{0}\right)\right) \\
& =\mathbf{F}(\lambda)^{-1} \operatorname{Cov}\left(\mathbf{g}\left(\delta^{0}\right)\right) \mathbf{F}(\lambda)^{-1}
\end{aligned}
$$

Since

$$
\begin{aligned}
\operatorname{Cov}\left(\mathbf{g}\left(\boldsymbol{\delta}^{0}\right)\right) & =E\left(\mathbf{g}\left(\boldsymbol{\delta}^{0}\right) \mathbf{g}\left(\boldsymbol{\delta}^{0}\right)^{\top}\right)-E\left(\mathbf{g}\left(\boldsymbol{\delta}^{0}\right)\right) E\left(\mathbf{g}\left(\boldsymbol{\delta}^{0}\right)\right)^{\top} \\
& =E\left(\mathbf{g}\left(\boldsymbol{\delta}^{0}\right) \mathbf{g}\left(\boldsymbol{\delta}^{0}\right)^{\top}\right) \\
& =-E\left(\mathbf{H}\left(\boldsymbol{\delta}^{0}\right)\right)
\end{aligned}
$$

we have that

$$
\operatorname{Cov}(\hat{\boldsymbol{\delta}})=-\mathbf{F}(\lambda)^{-1} E\left(\mathbf{H}\left(\boldsymbol{\delta}^{0}\right)\right) \mathbf{F}(\lambda)^{-1}
$$

In addition, conditions 2 and 4 imply that

$$
\begin{aligned}
\operatorname{bias}(\hat{\boldsymbol{\delta}}) & =-\mathbf{F}(\lambda)^{-1} \mathbf{S}_{\lambda} \boldsymbol{\delta}^{0} \\
& =-O\left(n^{-1}\right) o\left(n^{1 / 2}\right) \boldsymbol{\delta}^{0} \\
& =o\left(n^{-1 / 2}\right)
\end{aligned}
$$


and

$$
\begin{aligned}
\operatorname{Cov}(\hat{\boldsymbol{\delta}}) & =-\mathbf{F}(\lambda)^{-1} E\left(\mathbf{H}\left(\boldsymbol{\delta}^{0}\right)\right) \mathbf{F}(\lambda)^{-1} \\
& =O\left(n^{-1}\right) O(n) O\left(n^{-1}\right) \\
& =O\left(n^{-1}\right)
\end{aligned}
$$

Remark 2: Assumption 4 implies that

$$
\begin{aligned}
\sqrt{n} \operatorname{Cov}(\hat{\boldsymbol{\delta}}) & =-\sqrt{n} \mathbf{F}(\lambda)^{-1} E\left(\mathbf{H}\left(\boldsymbol{\delta}^{0}\right)\right) \mathbf{F}(\lambda)^{-1} \\
& \approx\left(\frac{1}{\sqrt{n}} E\left(-\mathbf{H}\left(\boldsymbol{\delta}^{0}\right)\right)\right)^{-1} .
\end{aligned}
$$

Also, by the same assumption

$$
\sqrt{n} \mathbf{V}_{\boldsymbol{\delta}} \approx\left(-\frac{1}{\sqrt{n}} \mathbf{H}\left(\boldsymbol{\delta}^{0}\right)\right)^{-1}
$$

where $\mathbf{V}_{\boldsymbol{\delta}}=-\mathbf{H}_{p}^{-1}$ is the Bayesian approximation of the covariance matrix. Thus, the frequentist and the Bayesian approximation become equivalent as the sample size grows to infinity.

Remark 3: Recall that $\mathbf{g}\left(\boldsymbol{\delta}^{0}\right)$ is a sum of iid components. From standard maximum likelihood asymptotics, it follows that $\left(-E\left(\mathbf{H}\left(\boldsymbol{\delta}^{0}\right)\right)\right)^{-1 / 2} \mathbf{g}\left(\boldsymbol{\delta}^{0}\right) \rightarrow \mathcal{N}(\mathbf{0}, \mathbf{I})$ (see Wood, 2006). Thus, the approximation in (6.7.2) also implies asymptotic normality of the normalised estimator $\hat{\boldsymbol{\delta}}$. 


\section{Chapter 7}

\section{Data illustration using the SemiParSampleSel package}

In Chapter 4 we conducted some preliminary analysis on data originating from the United States Veterans Administration and German Socio-Economic Panel. It turned out that non-random sample selection is likely to be present for both data sets. In addition, the Poisson Inverse Gaussian and Sichel distributions provided the best fit for non-missing response in VA data and Delaporte was the most suitable distribution for SOEP data.

In this chapter, we will illustrate the semi-parametric sample selection model for discrete responses on the previously explored data sets. All functions are implemented in SemiParSampleSel. At each step of the analysis, we will provide an example code used to generate the results. For each response, we will estimate three models with the best-fitting margin found in Chapter 4 for three different copulas. Alternating copulas for each model will serve as a sensitivity analysis to the initial joint distribution assumption. For each model, the average predicted values and association parameters will be reported with their respective confidence intervals. Furthermore, Akaike and Bayesian information criteria will be provided. Finally, smooth terms of each model will be depicted. 


\subsection{Veterans Administration data}

The data set on outpatient and inpatient visits originates from the 2001 National Survey of Veterans. Recall that the outpatients are individuals who do not stay overnight in the hospital whereas inpatients stay overnight in the hospital. In this setup, a veteran decides whether or not to use medical services, and conditional on a positive decision, he decides whether to go to a VA or non-VA health care facility and the amounts from each source. That is, the decision on whether or not to go to a VA or non-VA facility and the extent of services used is conditional on the decision to seek treatment first (Lahiri and Xing, 2004). Here, non-random sample selection arises due to the fact that individuals decide whether they should be treated or not. In this case the unmeasured confounders based on which non-random sample selection might occur are, for instance, attitudes towards health risks (e.g. smoking or drinking).

The dataset contains 20,041 observations and 39 relevant variables including responses. Recall the four responses summarised in Table 7.1. Again, we will only focus on the response NUMVAOUT. Since the best-fitting outcome for NUMVAOUT was Poisson inverse Gaussian in preliminary analysis, we will use it for model illustration. Frank model was fitted to see whether positive or negative correlation between the two equations was present. Since the correlation turned out to be negative, Clayton 90 and Gumbel 270 copula models were fitted to check how sensitive is the initial model to the Frank copula assumption. The selection and outcome equations are

$$
\begin{aligned}
Y_{1}^{*}= & \gamma_{0}+f_{11}(\text { VETSAGE })+\gamma_{1} \text { EXTSEX }+\gamma_{2} \text { DISACOND }+ \\
& \gamma_{3} \text { HIGHBP }+\gamma_{4} \text { LUNG }+\gamma_{5} \text { HEAR }+ \\
& \gamma_{6} \text { ENT }+\gamma_{7} \text { EYE }+\gamma_{8} \text { CANCER }+\gamma_{9} \text { HEART }+ \\
& \gamma_{10} \text { STROKE }+\gamma_{11} \text { KIDNEY }+\gamma_{12} \text { RHEUM }+\gamma_{13} \text { LIVER }+ \\
& \gamma_{14} \text { HIV }+\gamma_{15} \text { DIABETES }+\gamma_{16} \text { STOMACH }+\gamma_{17} \text { CHRONIC }+ \\
& \gamma_{18} \text { DRUGS }+\gamma_{19} \text { PTSD }+\gamma_{20} \text { MENTAL }+\gamma_{21} \text { INJURY }+ \\
& \gamma_{22} \text { TXOTH },
\end{aligned}
$$




$$
\begin{aligned}
\mu= & e^{\eta_{2}} \\
= & \exp \left(\beta_{0}+f_{21}(\text { VETSAGE })+\beta_{1} \text { WHITE }+\beta_{2} \text { DISACOND }+\right. \\
& \beta_{3} \text { MEDICAID }+\beta_{4} \text { MEDICARE }+\beta_{5} \text { HIGHBP }+\beta_{6} \text { LUNG }+ \\
& \beta_{7} \text { HEAR }+\beta_{8} \text { ENT }+\beta_{9} \text { EYE }+\beta_{10} \text { CANCER }+ \\
& \beta_{11} \text { HEART }+\beta_{12} \text { STROKE }+\beta_{13} \text { KIDNEY }+\beta_{14} \text { RHEUM }+ \\
& \beta_{15} \text { LIVER }+\beta_{16} \text { HIV }+\beta_{17} \text { DIABETES }+\beta_{18} \text { STOMACH }+ \\
& \beta_{19} \text { CHRONIC }+\beta_{20} \text { DRUGS }+\beta_{21} \text { PTSD }+\beta_{22} \text { MENTAL }+ \\
& \left.\beta_{23} \text { INJURY }+\beta_{24} \text { TXOTH }\right),
\end{aligned}
$$

where $Y_{1}^{*}$ is the latent selection variable and VETSAGE will be modelled using a thin plate regression spline; the remaining variables are binary.

\begin{tabular}{|c|c|}
\hline Response & Meaning \\
\hline NUMVAOUT & Number of VA outpatients \\
\hline NUMOTH & Number of non-VA outpatients \\
\hline VANITES & Nights hospitalised in VA facilities \\
\hline OTHNITES & Nights hospitalised in non-VA facilities \\
\hline
\end{tabular}

Table 7.1: Responses in the VA data.

The code needed to estimate the sample selection model for Gumbel 270 and Poisson inverse Gaussian margin was:

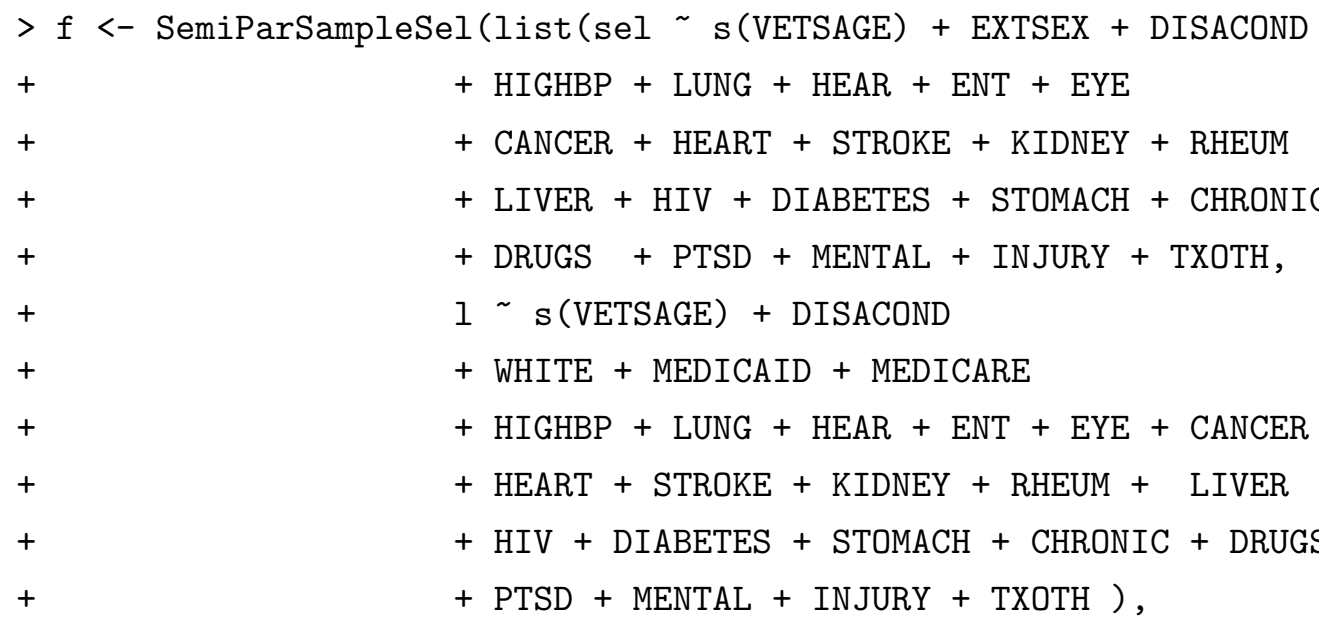$$
+
$$$$
+
$$$$
+
$$$$
+
$$$$
+
$$$$
+
$$$$
+
$$$$
+
$$$$
+
$$$$
+
$$ 


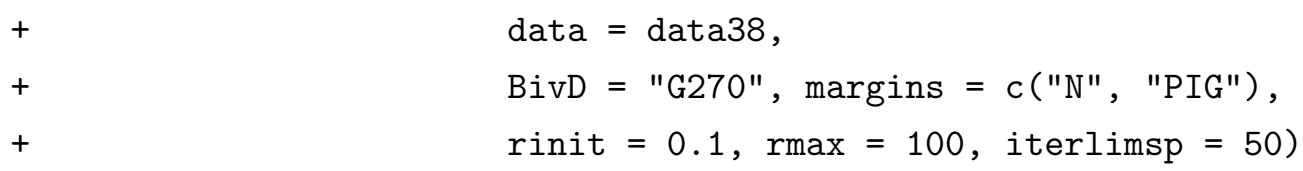

All covariates were plugged into the formulas encapsulated in a list. BivD indicates the copula used, in this case it was Gumbel 270. The margins argument contains a vector indicating selection and outcome margins; "N" and "PIG" denote normal and Poisson inverse Gaussian margins respectively. rinit denotes the initial radius of the trust algorithm reduced from its default equal to 1 to 0.1 to ensure convergence. rmax stands for the maximum radius (set to default); iterlimsp specifies the maximum number of loops before the smoothing parameter step is terminated (set to default).

Before looking at the outputs, one needs to check convergence using conv. check():

$>\operatorname{conv} \cdot \operatorname{check}(\mathrm{f})$

Largest absolute gradient value: 0.01330095

Observed information matrix is positive definite

Eigenvalue range: [0.2323656,528039.6]

Trust region iterations before smoothing parameter estimation: 39

Loops for smoothing parameter estimation: 4

Trust region iterations within smoothing loops: 43

As we can see, the algorithm converged successfully since the Hessian is positive definite. Additional information is provided in the last three lines.

The summary () function can be used to return the main model output including coefficients and their corresponding p-values. The average prediction for the univariate and sample selection model can be obtained by

$>\operatorname{aver}(f$, univariate $=$ TRUE $)$

Estimated average with $95 \%$ confidence interval:

$3.65(3.31,3.99)$

$>\operatorname{aver}(f$, univariate $=$ FALSE $)$

Estimated average with $95 \%$ confidence interval: 


\section{$3.97(3.57,4.38)$}

Table 7.2 shows the average predictions and association parameters for each model fitted to each of the responses. The values in brackets indicate $95 \%$ confidence interval bounds. Since all

confidence intervals of $\hat{\theta}$ do not include the borderline values, it is likely that mild non-random sample selection is present in the data. For outpatient responses, the predicted values do not vary substantially. However, the confidence intervals for Frank and Gumbel 270 in NUMOTH do not overlap. For inpatient responses, there are considerable differences in average predicted values between Frank and the remaining models. Note that the average predictions of the univariate model for NUMOTH and OTHNITES are smaller than the ones of the sample selection models. As we can see, the choice of copula can have an impact on the predicted values and should be taken into account by the practitioner. 


\begin{tabular}{|c|c|c|c|}
\hline \multicolumn{4}{|c|}{ "Number of outpatient visits } \\
\hline Response & Model & $\bar{y}$ & $\hat{\theta}$ \\
\hline \multirow{8}{*}{$\begin{array}{l}\text { NUMVAOUT } \\
\text { (Poisson i. Gaussian) }\end{array}$} & Univariate & 3.65 & - \\
\hline & & $(3.31,3.99)$ & - \\
\hline & Frank & 3.77 & -1.87 \\
\hline & & $(3.39,4.16)$ & $(-2.50,-1.26)$ \\
\hline & Clayton 90 & 3.70 & -1.14 \\
\hline & & $(3.36,4.05)$ & $(-1.51,-0.86)$ \\
\hline & Gumbel 270 & 3.97 & -1.36 \\
\hline & & $(3.57,4.38)$ & $(-1.52,-1.24)$ \\
\hline \multirow{9}{*}{$\begin{array}{l}\text { NUMOTH } \\
\text { (Sichel) }\end{array}$} & Univariate & 5.16 & - \\
\hline & & $(5.00,5.32)$ & - \\
\hline & Frank & 9.34 & -11.62 \\
\hline & & $(8.75,9.92)$ & $(-12.64,-10.63)$ \\
\hline & Clayton 90 & 8.89 & -7.51 \\
\hline & & $(8.40,9.38)$ & $(-8.27,-6.81)$ \\
\hline & Gumbel 270 & 11.6 & -3.96 \\
\hline & & $(10.3,12.8)$ & $(-4.30,-3.67)$ \\
\hline & \multicolumn{3}{|c|}{ Number of days hospitalised } \\
\hline Response & Model & $\bar{y}$ & $\hat{\theta}$ \\
\hline \multirow{8}{*}{$\begin{array}{l}\text { VANITES } \\
\text { (Sichel) }\end{array}$} & Univariate & 11.86 & - \\
\hline & & $(9.17,14.54)$ & - \\
\hline & Frank & 23.1 & -1.81 \\
\hline & & $(10.6,35.6)$ & $(-3.00,-0.63)$ \\
\hline & Clayton 90 & 104 & -0.85 \\
\hline & & $(49,158)$ & $(-1.07,-0.65)$ \\
\hline & Gumbel 270 & 115.9 & -1.56 \\
\hline & & $(59.8,171.9)$ & $(-1.71,-1.42)$ \\
\hline \multirow{8}{*}{$\begin{array}{l}\text { OTHNITES } \\
\text { (Poisson i. Gaussian) }\end{array}$} & Univariate & 5.65 & - \\
\hline & & $(5.32,5.98)$ & - \\
\hline & Frank & 19.5 & -4.45 \\
\hline & & $(12.7,26.3)$ & $(-5.31,-3.59)$ \\
\hline & Clayton 90 & 24.7 & -1.24 \\
\hline & & $(20.2,29.3)$ & $(-1.36,-1.12)$ \\
\hline & Gumbel 270 & 33.1 & -1.88 \\
\hline & & $(23.3,42.9)$ & $(-2.01,-1.77)$ \\
\hline
\end{tabular}

Table 7.2: Average predictions and association parameters for Frank, Clayton 90 and Gumbel 270 models. The values in brackets indicate $95 \%$ confidence intervals. For outpatient responses, the predicted values do not vary substantially. However, the confidence intervals for Frank and Gumbel 270 in NUMOTH do not overlap. For inpatient responses, there are considerable differences in average predicted values between Frank and the remaining models. Note that the average predictions of the univariate model for NUMOTH and OTHNITES are smaller than the ones of the sample selection models. As we can see, the choice of copula can have an impact on the predicted values. 
AIC() and BIC() commands return the Akaike and Bayesian Information Criteria:

$>\operatorname{AIC}(f)$

[1] 50879.25

$>\mathrm{BIC}(\mathrm{f})$

[1] 51345.13

Table 7.3 shows the Akaike and Bayesian Information Criteria for all models. For example, the lowest AIC and BIC values for NUMVAOUT can be found for the Poisson inverse Gaussian Clayton 90 model. Hence, Clayton 90 would be preferred over the Frank and Gumbel 270 models. For none of the responses Frank is the favoured model.

\begin{tabular}{|c|c|c|c|}
\hline Response & Model & $\overline{\mathrm{AIC}}$ & BIC \\
\hline \multirow{3}{*}{$\begin{array}{l}\text { NUMVAOUT } \\
\text { (Poisson i. Gaussian) }\end{array}$} & Frank & 50884.5 & 51350.2 \\
\hline & Clayton 90 & 50846.4 & 51313.1 \\
\hline & Gumbel 270 & 50879.3 & 51345.1 \\
\hline \multirow{3}{*}{$\begin{array}{l}\text { NUMOTH } \\
\text { (Sichel) }\end{array}$} & Frank & 77226.3 & 77688.7 \\
\hline & Clayton 90 & 77210.5 & 77672.2 \\
\hline & Gumbel 270 & 77251.4 & 77715.6 \\
\hline \multirow{3}{*}{$\begin{array}{l}\text { VANITES } \\
\text { (Sichel) }\end{array}$} & Frank & 8537.2 & 8986.7 \\
\hline & Clayton 90 & 8523.7 & 8933.7 \\
\hline & Gumbel 270 & 8525.9 & 8935.3 \\
\hline \multirow{3}{*}{$\begin{array}{l}\text { OTHNITES } \\
\text { (Poisson i. Gaussian) }\end{array}$} & Frank & 21205.4 & 21626.0 \\
\hline & Clayton 90 & 21155.2 & 21575.9 \\
\hline & Gumbel 270 & 21164.2 & 21584.8 \\
\hline
\end{tabular}

Table 7.3: Akaike and Bayesian information criteria for Frank, Clayton 90 and Gumbel 270 models. For example, the lowest AIC and BIC values for NUMVAOUT can be found for the Poisson inverse Gaussian Clayton 90 model. Hence, Clayton 90 would be preferred over the Frank and Gumbel 270 models. For none of the responses Frank is the favoured model. 
To obtain smooth plots for each equation one needs to submit the following code:

$>\operatorname{plot}(f$, eq $=1)$

$>\operatorname{plot}(f$, eq $=2)$

Figures 7.1-7.3 show the smooth term plots of Frank, Joe 90 and Gumbel 270 models for the selection and outcome equations respctively. The remainder of the plots can be found in Appendix R. Since the shape of the smooths remains roughly the same as compared to one another, the smooths do not seem to be sensitive to the choice of bivariate distribution. For the selection smooth, the probability of having an outpatient VA visit increases up to the age of 65 ; then it decreases gradually. The shape of the outcome equation smooth is first decreasing, then increasing and then decreasing again. This suggests that the patients first seek standard low-cost health care methods by visiting the VA administration and later require more specialised health care and hence address non-VA inpatient facilities. Alternatively, one can speculate that the 45-year old patients overutilise the VA health care system. 
Figure 7.1: Selection (left) and outcome (right) smooth plots for Poisson inverse Gaussian Frank model for NUMVAOUT. The horizontal axis represents veterans' age and the vertical axis shows the smooth value on the linear predictor scale. The numbers in brackets on the vertical axis are the effective degrees of freedom of the smooth. For the selection smooth, the probability of having an outpatient VA visit increases up to the age of 65 ; then it decreases gradually. The shape of the outcome equation smooth is first decreasing, then increasing and then decreasing again. This suggests that the patients first seek standard low-cost health care methods by visiting the VA administration and later require more specialised health care and hence address non-VA inpatient facilities. Alternatively, one can speculate that the 45-year old patients overutilise the VA health care system.
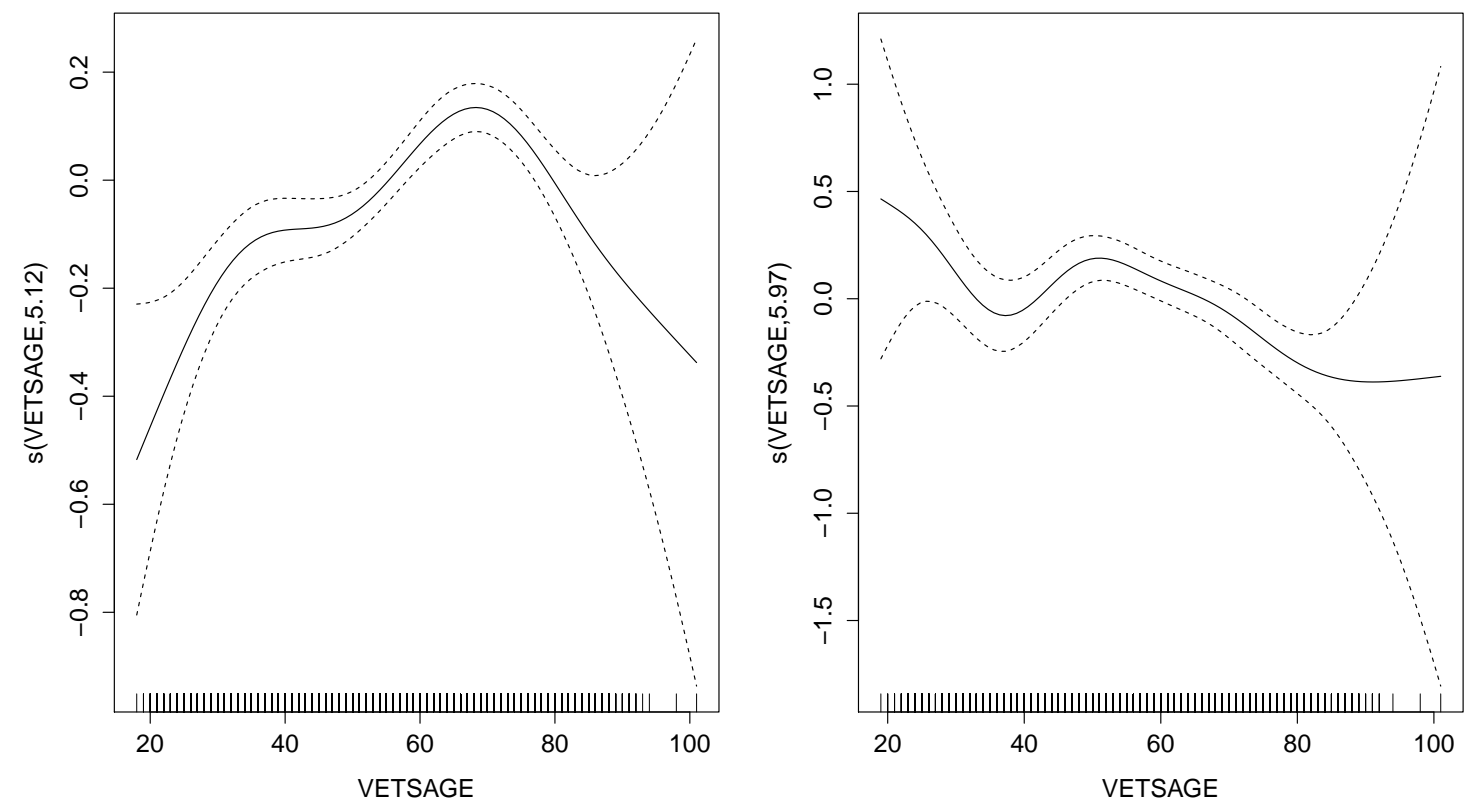
Figure 7.2: Selection (left) and outcome (right) smooth plots for Poisson inverse Gaussian Clayton 90 model for NUMVAOUT. The horizontal axis represents veterans' age and the vertical axis shows the smooth value on the linear predictor scale. The numbers in brackets on the vertical axis are the effective degrees of freedom of the smooth. For the selection smooth, the probability of having an outpatient VA visit increases up to the age of 65 ; then it decreases gradually. The shape of the outcome equation smooth is first decreasing, then increasing and then decreasing again. This suggests that the patients first seek standard low-cost health care methods by visiting the VA administration and later require more specialised health care and hence address non-VA inpatient facilities. Alternatively, one can speculate that the 45-year old patients overutilise the VA health care system.
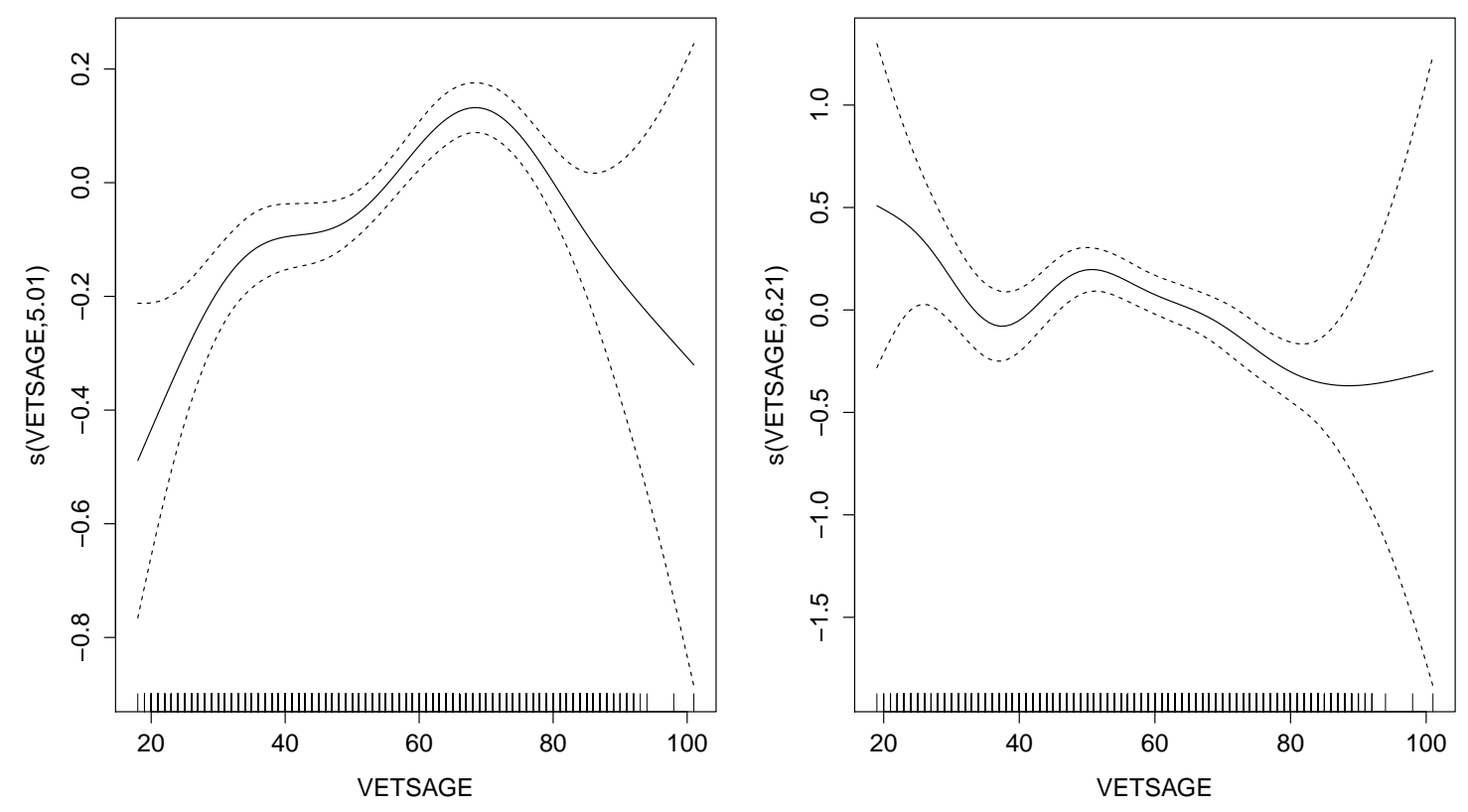
Figure 7.3: Selection (left) and outcome (right) smooth plots for Poisson inverse Gaussian Gumbel 270 model for NUMVAOUT. The horizontal axis represents veterans' age and the vertical axis shows the smooth value on the linear predictor scale. The numbers in brackets on the vertical axis are the effective degrees of freedom of the smooth. For the selection smooth, the probability of having an outpatient VA visit increases up to the age of 65 ; then it decreases gradually. The shape of the outcome equation smooth is first decreasing, then increasing and then decreasing again. This suggests that the patients first seek standard low-cost health care methods by visiting the VA administration and later require more specialised health care and hence address non-VA inpatient facilities. Alternatively, one can speculate that the 45-year old patients overutilize the VA health care system.
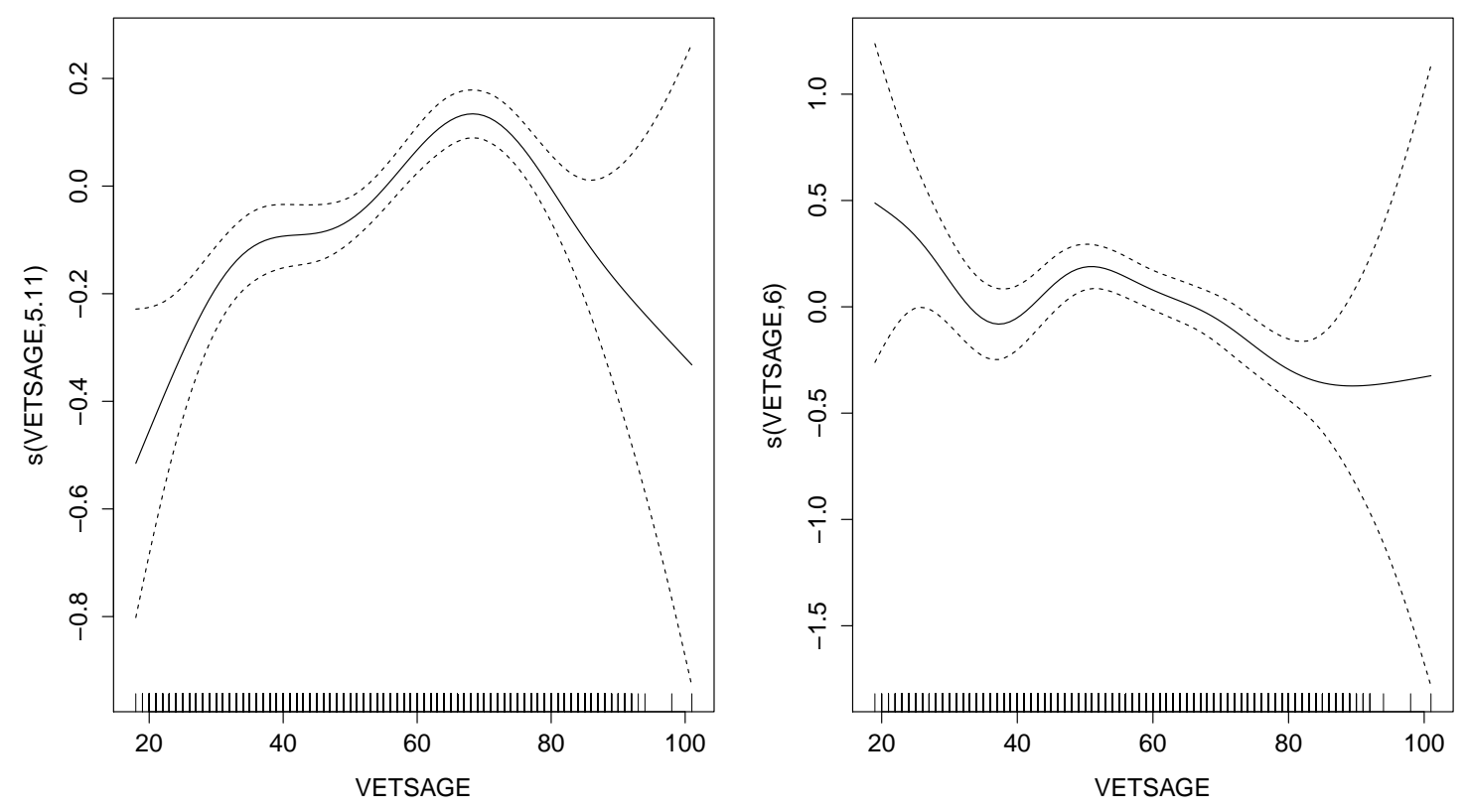


\subsection{German Socio-Economic Panel data}

The data set is from the German Socio-Economic Panel survey of 1984 (SOEP v28). Following Winkelmann (1998) the response of interest will be 'direct job changes' (DJC) of German male workers aged between 35 and 60 over ten years which is defined as the difference between job changes and the number of unemployment periods. This is supposed to stand for 'voluntary' mobility (Winkelmann, 1998). The individuals decide whether or not they should be part of the labour market as dependent workers. If they decide to be active, then they may voluntarily change jobs a number of times. Individuals who decide to be active can differ from those who are inactive on unobserved characteristics such as market wage. In this case, the parameter estimates will be biased and inconsistent. To fix ideas, we will employ the proposed sample selection modelling approach.

The sample consists of 2651 observations. The covariates are recalled in Table 7.4. Since in Chapter 4 the Delaporte distribution turned out to have the best fit, we will use it for the outcome margin. Three copulas will be considered: Frank, Joe 90 and Clayton 270. Replicating the original modelling approach by Winkelmann (1998) we selected two explanatory variables for the selection equation and three regressors for the outcome equation. According to Winkelmann (1998), unionised labour market participants are less likely to be mobile. The effect of education and employment status remains open. Despite the specification by Winkelmann (1998), it is not clear whether length of education should be modelled as a low degree polynomial or not. The same applies to full-time experience which is a regressor of the probability of labour market participation. For this reason, we will apply thin plate regression splines to EXPFT and LEduc in the selection and outcome equations. Formally, the selection equation may be expressed as follows:

$$
Y_{1}^{*}=\gamma_{0}+f_{11}(\text { EXPFT })+\gamma_{1} \text { Single }
$$

where $f$ is the spline component and $Y_{1}^{*}$ is the latent variable. The mean of the outcome is defined as

$$
\begin{aligned}
\mu & =e^{\eta_{2}} \\
& =\exp \left(\beta_{0}+f_{21}(\text { LEduc })+\beta_{1} \text { WhiteCollar }+\beta_{2} \text { SPDSup }\right)
\end{aligned}
$$




\begin{tabular}{|c|c|}
\hline Variable & Description \\
\hline EXPFT & Full-time employment working experience \\
\hline Single & 1 if the individual is single; 0 otherwise \\
\hline WhiteCollar & Length of education in years \\
\hline LEduc & indivdual is a white collar worker; 0 otherwise \\
\hline SPDSup & 1 if the indivdual is a strong or a very strong supporter of SPD; 0 otherwise \\
\hline
\end{tabular}

Table 7.4: Variables used for sample selection model for SOEP data

The code needed to estimate the sample selection model for Clayton 270 and Delaporte margin is

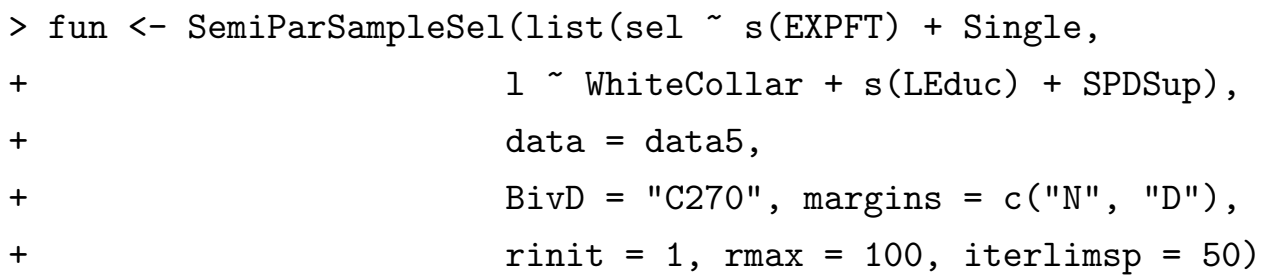

The BivD argument indicates Clayton 270 and margins implies normal and Delaporte distributions. rinit, rmax and iterlimsp were set to their defaults.

Again, using conv.check we can conclude that the algorithm converged successfully:

$>\operatorname{conv} \cdot \operatorname{check}(\mathrm{fun})$

Largest absolute gradient value: 9.346371e-05

Observed information matrix is positive definite

Eigenvalue range: [0.8425917,6140.916]

Trust region iterations before smoothing parameter estimation: 9

Loops for smoothing parameter estimation: 51

Trust region iterations within smoothing loops: 108

Smoothing algorithm reached the max. number of iterations allowed.

The summary () function can be used to return the model output. AIC and BIC can be obtained using the command

$>\operatorname{AIC}$ (fun) 
[1] 6145.886

$>$ BIC (fun)

[1] 6238.909

Table 7.5 shows AIC and BIC values for the three models. The lowest AIC and BIC scores can be obtained with Delaporte Frank. Hence, if the modeller is interested in making predictions of the number of job changes, he may consider Delaporte Frank.

\begin{tabular}{lcc}
\hline \hline $\begin{array}{l}\text { Model } \\
\text { (Delaporte) }\end{array}$ & AIC & BIC \\
\hline Frank & 6137.9 & 6231.9 \\
& 6145.6 & 6238.2 \\
Joe 90 & 6145.9 & 6238.9 \\
Clayton 270 & & \\
\hline \hline
\end{tabular}

Table 7.5: Akaike and Bayesian information criteria for the Delaporte models for SOEP data. The lowest AIC and BIC scores can be obtained with Delaporte Frank. Hence, if the modeller is interested in making predictions of the number of job changes, he may consider Delaporte Frank.

The average prediction for the univariate and sample selection model is obtained using aver():

$>$ aver (fun, univariate $=$ TRUE)

Estimated average with $95 \%$ confidence interval:

$0.526(0.484,0.568)$

$>\operatorname{aver}($ fun, univariate $=$ FALSE)

Estimated average with $95 \%$ confidence interval:

$1.03(0.70,1.35)$

Table 7.6 shows the average predictions of job changes and estimates of association parameters for three models. The values in brackets indicate $95 \%$ confidence interval bounds. For all three models, the confidence intervals of $\hat{\theta}$ do not reach their bound, which implies that non-random sample selection is likely to be present. The average predictions do not differ substantially from another. In fact, their confidence intervals of all three models overlap. Thus, the copula assumption does not seem to have a major impact on the predictions. Note that the confidence interval of the 
univariate model ignoring non-random sample selection does not overlap in predictions with the sample selection models. This suggests that non-random sample selection needs to be accounted for.

\begin{tabular}{lcc}
\hline \hline & $\bar{y}$ & $\hat{\theta}$ \\
\hline Univariate & 0.53 & - \\
& $(0.48,0.57)$ & - \\
Frank & 0.75 & -15.25 \\
& $(0.60,0.89)$ & $(-25.93,-3.02)$ \\
Joe 90 & 1.02 & -2.52 \\
& $(0.69,1.35)$ & $(-3.94,-1.82)$ \\
Clayton 270 & 1.03 & -1.76 \\
& $(0.70,1.35)$ & $(-3.19,-1.01)$ \\
\hline \hline
\end{tabular}

Table 7.6: Average predictions of job changes and estimates of association parameters for Delaporte model and Frank, Joe 90 and Clayton 270. The values in brackets indicate $95 \%$ confidence interval bounds. For all three models, the confidence intervals of $\theta$ do not reach their bound, which implies that non-random sample selection is likely to be present. The average predictions do not differ substantially from another. In fact, their confidence intervals of all three models overlap. Thus, the copula assumption does not seem to have a major impact on the predictions. Note that the confidence interval of the univariate model ignoring non-random sample selection does not overlap in predictions with the sample selection models. This suggests that non-random sample selection needs to be accounted for.

Figures 7.4 - 7.6 show the smoothing component plots for the selection and outcome equation of each of the three combinations. It is worth noticing that the plots do not differ substantially in shape. In fact, the shape seems to be similar to the one in Figure 4.9 in Chapter 4 . However, the difference lies in the fact that the graphs above are more extended along the vertical axis. The interpretation of the smooths remains the same as for Figure 4.9. The selection equation smooth suggests that the likelihood of being active on the labor market as a dependent worker increases when gaining full time work experience. Then, the likelihood of being active gradually decreases as individuals start to receive pensions or enter into self-employment. The outcome smooth suggests that the longer the education of an individual lasts the less likely he is to change his job. This is due to increasing specialisation in his professional area as he becomes more qualified. Therefore, it is harder for him to find an alternative occupation or there are no financial incentives for changing jobs. 
Figure 7.4: Selection (left) and outcome (right) equation smooth for Delaporte outcome and Frank copula. The horizontal axis represents full time experience and length of education. The vertical axis shows the smooth value on the linear predictor scale. The numbers in brackets on the vertical axis are the effective degrees of freedom of the smooth. The plots do not differ substantially in shape as compared to Figure 4.9. The selection equation smooth suggests that the likelihood of being active on the labor market as a dependent worker increases when gaining full time work experience. Then, the likelihood of being active gradually decreases as individuals start to receive pensions or enter into self-employment. The outcome smooth suggests that the longer the education of an individual lasts the less likely he is to change his job. This is due to increasing specialisation in his professional area as he becomes more qualified. Therefore, it is harder for him to find an alternative occupation or there are no financial incentives for changing jobs.
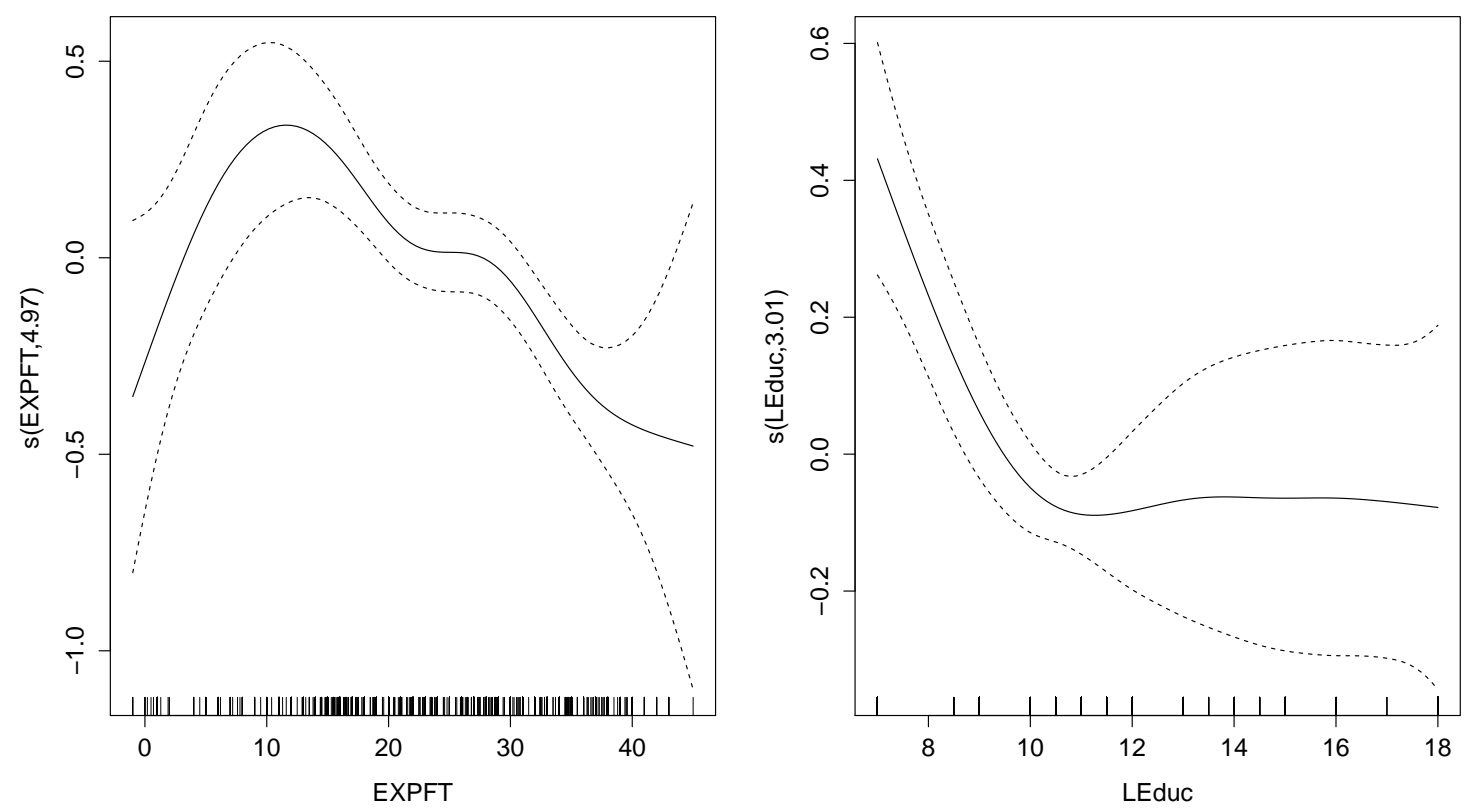
Figure 7.5: Selection (left) and outcome (right) equation smooth for Delaporte outcome and Joe 90 copula. The horizontal axis represents full time experience and length of education. The vertical axis shows the smooth value on the linear predictor scale. The numbers in brackets on the vertical axis are the effective degrees of freedom of the smooth. The plots do not differ substantially in shape as compared to Figure 4.9. The selection equation smooth suggests that the likelihood of being active on the labor market as a dependent worker increases when gaining full time work experience. Then, the likelihood of being active gradually decreases as individuals start to receive pensions or enter into self-employment. The outcome smooth suggests that the longer the education of an individual lasts the less likely he is to change his job. This is due to increasing specialisation in his professional area as he becomes more qualified. Therefore, it is harder for him to find an alternative occupation or there are no financial incentives for changing jobs.
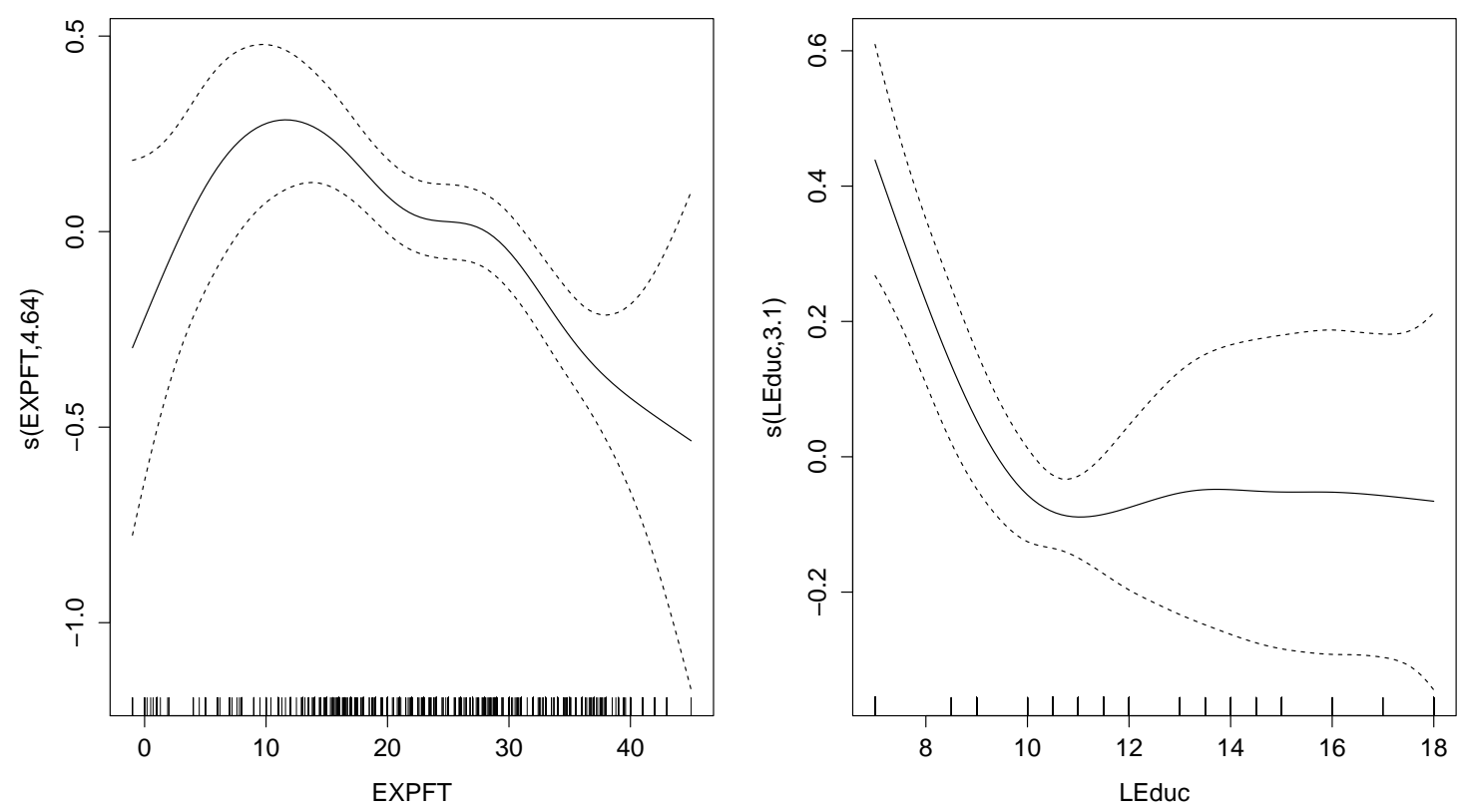
Figure 7.6: Selection (left) and outcome (right) equation smooth for Delaporte outcome and Clayton 90 copula. The horizontal axis represents full time experience and length of education. The vertical axis shows the smooth value on the linear predictor scale. The numbers in brackets on the vertical axis are the effective degrees of freedom of the smooth. The plots do not differ substantially in shape as compared to Figure 4.9. The selection equation smooth suggests that the likelihood of being active on the labor market as a dependent worker increases when gaining full time work experience. Then, the likelihood of being active gradually decreases as individuals start to receive pensions or enter into self-employment. The outcome smooth suggests that the longer the education of an individual lasts the less likely he is to change his job. This is due to increasing specialisation in his professional area as he becomes more qualified. Therefore, it is harder for him to find an alternative occupation or there are no financial incentives for changing jobs.
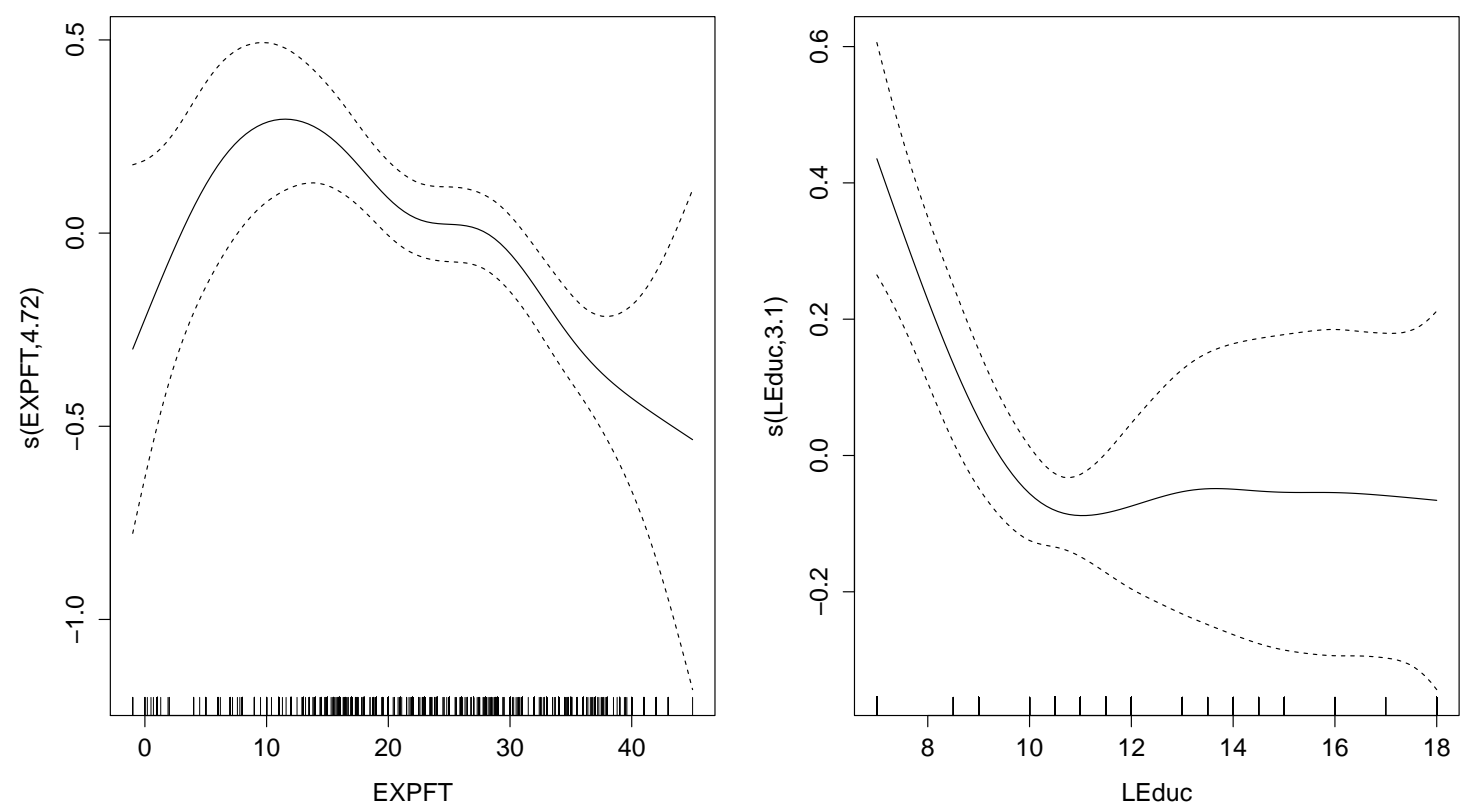


\section{Chapter 8}

\section{Discussion}

In this thesis we introduced a modelling framework that allows for correcting for non-random sample selection when the response is discrete. In Chapter 2 we defined the problem of non-random sample selection and reviewed the relevant imputation methods. We also gave a brief of overview of sample selection models applications.

In Chapter 3 we presented some sample selection models relevant to our framework. We discussed the Heckman and Greene's model together with the copula approach. Hence, we justfied the choice of the copula approach and the frequentist framework.

In Chapter 4 we explored the VA and SOEP data. The analysis showed that both data sets suffered from non-random sample selection. Simultaneously, the best distribution for non-missing data turned out to be Poisson inverse Gaussian and Sichel for VA and Delaporte for SOEP data.

In Chapter 5 we introduced the sample selection model for count responses with fixed covariate effects. We elaborated upon the theory behind the model. The numerical aspects such as starting values and the algorithm were presented. The analytical gradient and Hessian were checked against the numerical one. Finally, simulations were performed for two different specifications.

Chapter 6 reviewed the spline methods. The working linear model was derived for the parametric and extended for the non-parametric case. The un-biased risk estimator was derived and the algorithm presented. Inferential methods were introduced together with model selection methods. Finally, we proved consistency of penalized ML estimators.

In Chapter 7 we revisited the VA and SOEP data, where the sample selection models were applied. The models were based on the best-fitting distribution from Chapter 4 . The estimation 
results were presented and the presence of non-random sample selection was confirmed.

For further research, it is recommended to derive models for outcome margins with finite discrete outcomes such as binomial distribution. The likelihood function does not need to be modified since it is already adjusted for discrete responses. In fact, the most recent version of SemiParSampleSel incorporates 18 new discrete margins, whose gradients and Hessian were tested in the same manner as in Chapter 5. These are listed in Table 8.1.

Up until now, for the outcome distribution, the linear predictor is only defined in terms of $\mu$. One could introduce linear predictors for $\sigma, \nu$ and $\theta$ (see: Kneib, 2013). For example, if the outcome is Sichel and the copula is AMH, then

$$
\begin{aligned}
\mu & =e^{\eta_{2}} \\
& =\exp \left(\beta_{0}+f\left(x_{1}\right)+\beta_{1} x_{2}+\beta_{2} x_{3}\right) \\
\sigma & =e^{\eta_{\sigma}} \\
& =\exp \left(\gamma_{0}+f\left(x_{4}\right)+\gamma_{1} x_{2}\right) \\
\nu & =\eta_{\nu} \\
& =\zeta_{0}+\zeta_{1} x_{5} \\
\theta & =\tanh \left(\eta_{\theta}\right) \\
& =\tanh \left(\omega_{0}+\omega_{1} x_{3}\right)
\end{aligned}
$$

In order to modify the currently existing approach one needs to respecify the design matrix $\widetilde{\mathbf{X}}$ consisting of $5 \times p$ submatrices $\widetilde{\mathbf{X}}_{i}=\operatorname{diag}\left(\widetilde{\mathbf{X}}_{\mathbf{1}}^{\top}, \widetilde{\mathbf{X}}_{\mathbf{2} i}^{\top}, 1,1,1\right)$. Instead, the submatrices will be defined as $\widetilde{\mathbf{X}}_{i}=\operatorname{diag}\left(\widetilde{\mathbf{X}}_{\mathbf{1}}^{\top}, \widetilde{\mathbf{X}}_{\mathbf{2} i}^{\top}, \widetilde{\mathbf{X}}_{\mathbf{3} i}^{\top}, \widetilde{\mathbf{X}}_{\mathbf{4} i}^{\top}, \widetilde{\mathbf{X}}_{\mathbf{5} i}^{\top}\right)$. Analogicallly, the subvectors of $\mathbf{u}$ would have be respecified as $\frac{\partial \ell}{\partial \boldsymbol{\eta}_{i}}=\left(\frac{\partial \ell}{\partial \eta_{1 i}}, \frac{\partial \ell}{\partial \eta_{2 i}}, \frac{\partial \ell}{\partial \eta_{3 i}}, \frac{\partial \ell}{\partial \eta_{4 i}}, \frac{\partial \ell}{\partial \eta_{5 i}}\right)^{\top}$. The derivation of the penalized linear working model and inferential methods will be the same as in Chapter 6. Asymptotic proofs will follow the same reasoning as in Section 6.7. In fact, the latest implementation of SemiParSampleSel package supports the specifications like the one presented above. However, since the interpretation of the parameters becomes more problematic, using such specifications has to be well-motivated by the application. 


\begin{tabular}{|c|c|c|c|c|}
\hline Distribution & $\begin{array}{c}\mu \text { link } \\
(\mu \text { range })\end{array}$ & $\begin{array}{c}\sigma \text { link } \\
(\sigma \text { range })\end{array}$ & $\begin{array}{c}\nu \text { link } \\
(\nu \text { range })\end{array}$ & $\begin{array}{c}\text { Binomial } \\
\text { denominator }\end{array}$ \\
\hline Beta binomial & $\begin{array}{l}\text { logit } \\
{[0,1]}\end{array}$ & $\begin{array}{c}\log \\
{[0, \infty)}\end{array}$ & - & $q$ \\
\hline Binomial & $\begin{array}{l}\text { logit } \\
{[0,1]}\end{array}$ & - & - & $q$ \\
\hline Geometric & $\begin{array}{c}\log \\
{[0, \infty)}\end{array}$ & - & - & - \\
\hline Logarithmic & $\begin{array}{l}\text { logit } \\
{[0,1]}\end{array}$ & - & - & - \\
\hline $\begin{array}{c}\text { Negative } \\
\text { binomial } \\
\text { type II }\end{array}$ & $\begin{array}{c}\log \\
{[0, \infty)}\end{array}$ & $\begin{array}{c}\log \\
{[0, \infty)}\end{array}$ & - & - \\
\hline Waring & $\begin{array}{c}\log \\
{[0, \infty)}\end{array}$ & $\begin{array}{c}\log \\
{[0, \infty)}\end{array}$ & - & - \\
\hline Yule & $\begin{array}{c}\log \\
{[0, \infty)}\end{array}$ & - & - & - \\
\hline $\begin{array}{l}\text { Zero inflated } \\
\text { beta binomial }\end{array}$ & $\begin{array}{l}\operatorname{logit} \\
{[0,1]}\end{array}$ & $\begin{array}{c}\log \\
{[0, \infty)}\end{array}$ & $\begin{array}{l}\text { logit } \\
{[0,1]}\end{array}$ & $q$ \\
\hline $\begin{array}{l}\text { Zero altered } \\
\text { beta binomial }\end{array}$ & $\begin{array}{l}\text { logit } \\
{[0,1]}\end{array}$ & $\begin{array}{c}\log \\
{[0, \infty)}\end{array}$ & $\begin{array}{l}\text { logit } \\
{[0,1]}\end{array}$ & $q$ \\
\hline $\begin{array}{l}\text { Zero inflated } \\
\text { binomial }\end{array}$ & $\begin{array}{l}\text { logit } \\
{[0,1]}\end{array}$ & $\begin{array}{l}\text { logit } \\
{[0,1]}\end{array}$ & - & $q$ \\
\hline $\begin{array}{l}\text { Zero altered } \\
\text { binomial }\end{array}$ & $\begin{array}{l}\text { logit } \\
{[0,1]}\end{array}$ & $\begin{array}{l}\text { logit } \\
{[0,1]}\end{array}$ & - & $q$ \\
\hline $\begin{array}{l}\text { Zero altered } \\
\text { logarithmic }\end{array}$ & $\begin{array}{l}\text { logit } \\
{[0,1]}\end{array}$ & $\begin{array}{l}\text { logit } \\
{[0,1]}\end{array}$ & - & - \\
\hline $\begin{array}{c}\text { Zero inflated } \\
\text { negative binomial }\end{array}$ & $\begin{array}{c}\log \\
{[0, \infty)}\end{array}$ & $\begin{array}{c}\log \\
{[0, \infty)}\end{array}$ & $\begin{array}{l}\operatorname{logit} \\
{[0,1]}\end{array}$ & - \\
\hline $\begin{array}{c}\text { Zero altered } \\
\text { negative binomial }\end{array}$ & $\begin{array}{c}\log \\
{[0, \infty)}\end{array}$ & $\begin{array}{c}\log \\
{[0, \infty)}\end{array}$ & $\begin{array}{l}\text { logit } \\
{[0,1]}\end{array}$ & - \\
\hline $\begin{array}{l}\text { Zero altered } \\
\text { Poisson }\end{array}$ & $\begin{array}{c}\log \\
{[0, \infty)}\end{array}$ & $\begin{array}{l}\operatorname{logit} \\
{[0,1]}\end{array}$ & - & - \\
\hline $\begin{array}{l}\text { Zero inflated } \\
\text { Poisson }\end{array}$ & $\begin{array}{c}\log \\
{[0, \infty)}\end{array}$ & $\begin{array}{l}\text { logit } \\
{[0,1]}\end{array}$ & - & - \\
\hline $\begin{array}{c}\text { Zero inflated } \\
\text { Poisson type II }\end{array}$ & $\begin{array}{c}\log \\
{[0, \infty)}\end{array}$ & $\begin{array}{l}\text { logit } \\
{[0,1]}\end{array}$ & - & - \\
\hline $\begin{array}{c}\text { Zero inflated } \\
\text { Poisson inverse } \\
\text { Gaussian }\end{array}$ & $\begin{array}{c}\log \\
{[0, \infty)}\end{array}$ & $\begin{array}{c}\log \\
{[0, \infty)}\end{array}$ & $\begin{array}{l}\operatorname{logit} \\
{[0,1]}\end{array}$ & - \\
\hline
\end{tabular}

Table 8.1: Additional discrete distributions implemented in SemiParSampleSel R package. The brackets underneath the link function indicate the parameter range. For binomial-type distributions, the binomial denominator is determined by the modeller. Note that the support of the logarithmic distribution does not include zero. 
Finally, sample selection models for count responses can be proposed for a wider variety of fields. For instance, in medicine, patients who are newly referred to a specialist by a general practicioner often have to be assessed using ultrasound or magnetic resonance imaging devices (see Ciurtin et al., 2015). Unfortuntely, the public health systems cannot afford an assessment for every patient. At the same time, non-random sample selection may be present in assessment scores, since patients can be referred based on characteristics that cannot be included in the model e.g. fatigue of the consultant, patients personality. There is a potential to obtain a data set which would include patients with ultrasound scans and those who did not have one. Based on this, sample selection models can be estimated to check presence of non-random sample selection. Eventually, these can be used for predicting ultrasound scores of newly referred patients. 


\section{Appendix A}

\section{Derivation of the conditional expected value for the sample selection model with continuous response}

We will derive the conditional expected value stated in (3.1.3). Let us first specify the conditional truncated cumulative density function:

$$
\begin{aligned}
F_{2 \mid 1}\left(y_{2} \mid y_{1}^{*}>0\right) & =\operatorname{Pr}\left(Y_{2} \leq y_{2} \mid Y_{1}^{*}>0\right) \\
& =\frac{\operatorname{Pr}\left(Y_{2} \leq y_{2} \cap Y_{1}^{*}>0\right)}{\operatorname{Pr}\left(Y_{1}^{*}>0\right)} \\
& =\frac{F\left(1, y_{2}\right)}{F_{1}(1)} \\
& =\frac{F\left(1, y_{2}\right)}{1-F_{1}(0)} .
\end{aligned}
$$

By differentiating (A.0.1) with respect to $y_{2}$ we obtain the conditional incidentally truncated density function:

$$
\begin{aligned}
f_{2 \mid 1}\left(y_{2} \mid y_{1}^{*}>0\right) & =\frac{\partial}{\partial y_{2}} \frac{F\left(1, y_{2}\right)}{1-F_{1}(0)} \\
& =\frac{1}{1-F_{1}(0)} \frac{\partial}{\partial y_{2}} F\left(1, y_{2}\right) .
\end{aligned}
$$


The element $\frac{\partial}{\partial y_{2}} F\left(1, y_{2}\right)$ can be re-expressed as

$$
\begin{aligned}
\frac{\partial}{\partial y_{2}} F\left(1, y_{2}\right) & =\frac{\partial}{\partial y_{2}}\left(\int_{-\infty}^{y_{2}} \int_{-\boldsymbol{\gamma}^{\top} \boldsymbol{z}}^{\infty} f\left(y_{2}, \epsilon_{1}\right) d \epsilon_{1} d y_{2}\right) \\
& =\frac{\partial}{\partial y_{2}}\left(\int_{-\infty}^{y_{2}} \int_{-\boldsymbol{\gamma}^{\top} \boldsymbol{z}}^{\infty} f\left(y_{2} \mid \epsilon_{1}\right) f\left(\epsilon_{1}\right) d \epsilon_{1} d y_{2}\right) \\
& =\int_{-\boldsymbol{\gamma}^{\top} \boldsymbol{z}}^{\infty} f\left(y_{2} \mid \epsilon_{1}\right) f\left(\epsilon_{1}\right) d \epsilon_{1} .
\end{aligned}
$$

Therefore, the general form of the conditional truncated density function is

$$
f_{2 \mid 1}\left(y_{2} \mid y_{1}^{*}>0\right)=\frac{1}{1-F_{1}(0)} \int_{-\gamma^{\top} \boldsymbol{z}}^{\infty} f\left(y_{2} \mid \epsilon_{1}\right) f\left(\epsilon_{1}\right) d \epsilon_{1}
$$

The conditional expected value is

$$
\begin{aligned}
E\left(Y_{2} \mid-\boldsymbol{\gamma}^{\top} \boldsymbol{z}<\epsilon_{1}\right) & =\int_{-\infty}^{\infty} y_{2} f_{2 \mid 1}\left(y_{2} \mid-\boldsymbol{\gamma}^{\top} \boldsymbol{z}<\epsilon_{1}\right) d y_{2} \\
& =\frac{1}{1-F_{1}(0)} \int_{-\infty}^{\infty} y_{2} \int_{-\boldsymbol{\gamma}^{\top} \boldsymbol{z}}^{\infty} f\left(y_{2} \mid \epsilon_{1}\right) f\left(\epsilon_{1}\right) d \epsilon_{1} d y_{2} \\
& =\frac{1}{1-F_{1}(0)} \int_{-\boldsymbol{\gamma}^{\top} \boldsymbol{z}}^{\infty} \int_{-\infty}^{\infty} y_{2} f\left(y_{2} \mid \epsilon_{1}\right) d y_{2} f\left(\epsilon_{1}\right) d \epsilon_{1}
\end{aligned}
$$

One can see that the internal integral of (A.0.4) is the mean of the conditional normal distribution i.e. $E\left(Y_{2} \mid \epsilon_{1}\right)=\boldsymbol{\beta}^{\top} \boldsymbol{x}+\rho \sigma_{2} \epsilon_{1}$. Also, $1-F_{0}=1-\operatorname{Pr}\left(Y_{1}^{*} \leq 0\right)=1-\operatorname{Pr}\left(\boldsymbol{\gamma}^{\top} \boldsymbol{z}+\epsilon_{1} \leq 0\right)=$ $1-\operatorname{Pr}\left(-\boldsymbol{\gamma}^{\top} \boldsymbol{z} \geq \epsilon_{1}\right)=1-\Phi\left(-\boldsymbol{\gamma}^{\top} \boldsymbol{z}\right)$, where $\Phi(\cdot)$ is the cumulative distribution function of a standard normal distribution. Thus,

$$
\begin{aligned}
E\left(Y_{2} \mid-\boldsymbol{\gamma}^{\top} \boldsymbol{z}<\epsilon_{1}\right) & =\frac{1}{1-\Phi\left(-\boldsymbol{\gamma}^{\top} \boldsymbol{z}\right)} \int_{-\gamma^{\top} z}^{\infty}\left(\boldsymbol{\beta}^{\top} \boldsymbol{x}+\rho \sigma_{2} \epsilon_{1}\right) f\left(\epsilon_{1}\right) d \epsilon_{1} \\
& =\frac{\boldsymbol{\beta}^{\top} \boldsymbol{x}}{1-\Phi\left(-\boldsymbol{\gamma}^{\top} \boldsymbol{z}\right)} \int_{-\boldsymbol{\gamma}^{\top} \boldsymbol{z}}^{\infty} f\left(\epsilon_{1}\right) d \epsilon_{1}+\frac{\rho \sigma_{2}}{1-\Phi\left(-\boldsymbol{\gamma}^{\top} \boldsymbol{z}\right)} \int_{-\boldsymbol{\gamma}^{\top} \boldsymbol{z}}^{\infty} \epsilon_{1} f\left(\epsilon_{1}\right) d \epsilon_{1} \\
& =\boldsymbol{\beta}^{\top} \boldsymbol{x}+\frac{\rho \sigma_{2}}{1-\Phi\left(-\boldsymbol{\gamma}^{\top} \boldsymbol{z}\right)} \int_{-\boldsymbol{\gamma}^{\top} \boldsymbol{z}}^{\infty} \epsilon_{1} f\left(\epsilon_{1}\right) d \epsilon_{1} \\
& =\boldsymbol{\beta}^{\top} \boldsymbol{x}+\frac{\rho \sigma_{2}}{1-\Phi\left(-\boldsymbol{\gamma}^{\top} \boldsymbol{z}\right)} \int_{-\boldsymbol{\gamma}^{\top} \boldsymbol{z}}^{\infty} \frac{1}{2 \pi} \epsilon_{1} e^{-\epsilon_{1}^{2} / 2} d \epsilon_{1}
\end{aligned}
$$

Note that

$$
\frac{d}{d \epsilon_{1}}\left(e^{-\epsilon_{1}^{2} / 2}\right)=-\epsilon_{1} e^{-\epsilon_{1}^{2} / 2}
$$


So,

$$
\begin{aligned}
E\left(Y_{2} \mid-\boldsymbol{\gamma}^{\top} \boldsymbol{z}<\epsilon_{1}\right) & =\boldsymbol{\beta}^{\top} \boldsymbol{x}+\frac{\rho \sigma_{2}}{1-\Phi\left(-\boldsymbol{\gamma}^{\top} \boldsymbol{z}\right)}\left(-\left.\frac{1}{2 \pi} e^{-\epsilon_{1}^{2} / 2}\right|_{-\boldsymbol{\gamma}^{\top} \boldsymbol{z}} ^{\infty}\right) \\
& =\boldsymbol{\beta}^{\top} \boldsymbol{x}+\frac{\rho \sigma_{2}}{1-\Phi\left(-\boldsymbol{\gamma}^{\top} \boldsymbol{z}\right)} \phi\left(-\boldsymbol{\gamma}^{\top} \boldsymbol{z}\right) .
\end{aligned}
$$

Therefore,

$$
\begin{aligned}
E\left(Y_{2} \mid Y_{1}^{*}>0\right) & =\boldsymbol{\beta}^{\top} \boldsymbol{x}+\rho \sigma_{2} \frac{\phi\left(-\boldsymbol{\gamma}^{\top} \boldsymbol{z}\right)}{1-\Phi\left(-\boldsymbol{\gamma}^{\top} \boldsymbol{z}\right)} \\
& =\boldsymbol{\beta}^{\top} \boldsymbol{x}+\rho \sigma_{2} \lambda\left(-\boldsymbol{\gamma}^{\top} \boldsymbol{z}\right)
\end{aligned}
$$

where $\lambda\left(-\boldsymbol{\gamma}^{\top} \boldsymbol{z}\right)=\frac{\phi\left(-\boldsymbol{\gamma}^{\top} \boldsymbol{z}\right)}{1-\Phi\left(-\boldsymbol{\gamma}^{\top} \boldsymbol{z}\right)}$. 


\section{Appendix B}

\section{Derivation of the likelihood function for the sample selection model with continuous response and bivariate normal distribution}

Let us denote the likelihood for all observations with observed outcomes $f\left(y_{2}, y_{1}^{*}>0\right)$ and all observations with missing outcomes $\operatorname{Pr}\left(Y_{1}^{*} \leq 0\right)$. We will derive the two expressions for the case when the margins are normally distributed and the bivariate distribution is normal. Let us start 
with the former:

$$
\begin{aligned}
f\left(y_{2}, y_{1}^{*}>0\right) & =\int_{0}^{\infty} f\left(y_{2}, y_{1}^{*}\right) d y_{1}^{*} \\
& =\int_{-\boldsymbol{\gamma}^{\top} \boldsymbol{z}}^{\infty} f\left(\epsilon_{2}, \epsilon_{1}\right) d \epsilon_{1} \\
& =\int_{-\boldsymbol{\gamma}^{\top} \boldsymbol{z}}^{\infty} f\left(\epsilon_{1} \mid \epsilon_{2}\right) f\left(\epsilon_{2}\right) d \epsilon_{1} \\
& =f\left(\epsilon_{2}\right) \int_{-\boldsymbol{\gamma}^{\top} \boldsymbol{z}}^{\infty} f\left(\epsilon_{1} \mid \epsilon_{2}\right) d \epsilon_{1} \\
& =f\left(y_{2}\right) \int_{-\boldsymbol{\gamma}^{\top} \boldsymbol{z}}^{\infty} f\left(\epsilon_{1} \mid \epsilon_{2}\right) d \epsilon_{1} \\
& \left.=\frac{1}{\sigma} \phi\left(\frac{y_{2}-\boldsymbol{\beta}^{\top} \boldsymbol{x}}{\sigma}\right) \int_{-\boldsymbol{\gamma}^{\top} \boldsymbol{z}}^{\infty} \frac{f\left(\epsilon_{1} \mid \epsilon_{2}\right) d \epsilon_{1}}{\sigma}\right) \int_{-\boldsymbol{\gamma}^{\top} \boldsymbol{z}}^{\infty} \frac{1}{\sqrt{1-\rho^{2}}} \phi\left(\frac{\epsilon_{1}-\frac{\rho}{\sigma}\left(y_{2}-\boldsymbol{\beta}^{\top} \boldsymbol{x}\right)}{\sqrt{1-\rho^{2}}}\right) d \epsilon_{1} \\
& =\frac{1}{\sigma} \phi\left(\frac{y_{2}-\boldsymbol{\beta}^{\top} \boldsymbol{x}}{\sigma}\right) \\
& =\frac{1}{\sigma} \phi\left(\frac{y_{2}-\boldsymbol{\beta}^{\top} \boldsymbol{x}}{\sigma}\right) \Phi\left(\frac{\boldsymbol{\gamma}^{\top} \boldsymbol{z}+\frac{\rho}{\sigma}\left(y_{2}-\boldsymbol{\beta}^{\top} \boldsymbol{x}\right)}{\sqrt{1-\rho^{2}}}\right) .
\end{aligned}
$$

The latter expression can be derived as follows:

$$
\begin{aligned}
\operatorname{Pr}\left(Y_{1}^{*} \leq 0\right) & =P\left(\epsilon_{1} \leq-\boldsymbol{\gamma}^{\top} \boldsymbol{z}\right) \\
& =\Phi\left(-\boldsymbol{\gamma}^{\top} \boldsymbol{z}\right) \\
& \left(=1-\Phi\left(\boldsymbol{\gamma}^{\top} \boldsymbol{z}\right)\right) .
\end{aligned}
$$

Therefore, the likelihood function will be

$$
L=\prod_{0} \Phi\left(-\boldsymbol{\gamma}^{\top} \boldsymbol{z}\right) \prod_{1} \frac{1}{\sigma} \phi\left(\frac{y_{2}-\boldsymbol{\beta}^{\boldsymbol{\top}} \boldsymbol{x}}{\sigma}\right) \Phi\left(\frac{\boldsymbol{\gamma}^{\top} \boldsymbol{z}+\frac{\rho}{\sigma}\left(y_{2}-\boldsymbol{\beta}^{\boldsymbol{\top}} \boldsymbol{x}\right)}{\sqrt{1-\rho^{2}}}\right) .
$$

0 and 1 under $\prod$ index whether the observation's outcome is not observed or observed respectively. 


\section{Appendix $\mathrm{C}$}

\section{Derivation of the conditional expected value for the Greene count model}

We know from the Greene count data model described in Subsection 3.2 that

$$
e^{\epsilon_{2}} \sim L N\left(0, \sigma^{2}\right)
$$

or equivalently

$$
\epsilon_{2} \sim N\left(0, \sigma^{2}\right) .
$$

Also we know that,

$$
E\left(Y_{2} \mid \epsilon_{2}\right)=\omega\left(\epsilon_{2}\right)=\exp \left(\boldsymbol{\beta}^{\boldsymbol{\top}} \boldsymbol{x}+\epsilon_{2}\right) .
$$

Based on (C.0.1) we can write

$$
Y_{2}=\exp \left(\boldsymbol{\beta}^{\top} \boldsymbol{x}+\epsilon_{2}\right)+\eta
$$


where $E\left(\eta \mid \epsilon_{2}\right)=0$. Therefore,

$$
\begin{aligned}
E\left(Y_{2} \mid Y_{1}=1\right) & =E\left(e^{\boldsymbol{\beta}^{\boldsymbol{\top}} \boldsymbol{x}+\epsilon_{2}}+\eta \mid Y_{1}^{*}>0\right) \\
& =e^{\boldsymbol{\beta}^{\boldsymbol{\top}} \boldsymbol{x}} E\left(e^{\epsilon_{2}} \mid Y_{1}^{*}>0\right) \\
& =e^{\boldsymbol{\beta}^{\boldsymbol{\top}} \boldsymbol{x}} E\left(e^{\epsilon_{2}} \mid-\boldsymbol{\gamma}^{\boldsymbol{\top}} \boldsymbol{z}<0\right) .
\end{aligned}
$$

For the expected value in (C.0.2) we can use the general expression derived in appendix A (see: equation A.0.4). That is

$$
e^{\boldsymbol{\beta}^{\top} \boldsymbol{x}} E\left(e^{\epsilon_{2}} \mid-\boldsymbol{\gamma}^{\top} \boldsymbol{z}<0\right)=e^{\boldsymbol{\beta}^{\top} \boldsymbol{x}} \frac{1}{F_{1}(1)} \int_{-\boldsymbol{\gamma}^{\top} \boldsymbol{z}}^{\infty} \int_{-\infty}^{\infty} e^{\epsilon_{2}} f\left(e^{\epsilon_{2}} \mid \epsilon_{1}\right) d\left(e^{\epsilon_{2}}\right) f\left(\epsilon_{1}\right) d \epsilon_{1}
$$

where $F_{1}(1)=\Phi\left(\boldsymbol{\gamma}^{\top} \boldsymbol{z}\right)$. We need to calculate $E\left(e^{\epsilon_{2}} \mid \epsilon_{1}\right)$ which is the inner integral of (C.0.3). The derivation of $E\left(e^{\epsilon_{2}} \mid \epsilon_{1}\right)$ can be found in Terza (1998, p. 143-144). He shows that

$$
E\left(e^{\epsilon_{2}} \mid \epsilon_{1}\right)=e^{\rho \sigma \epsilon_{1}+\frac{1}{2} \sigma^{2}\left(1-\rho^{2}\right)} .
$$

So,

$$
\begin{aligned}
E\left(Y_{2} \mid Y_{1}=1\right) & =e^{\boldsymbol{\beta}^{\top} \boldsymbol{x}} \frac{1}{\Phi\left(\boldsymbol{\gamma}^{\top} \boldsymbol{z}\right)} \int_{-\boldsymbol{\gamma}^{\top} \boldsymbol{z}}^{\infty} e^{\rho \sigma \epsilon_{1}+\frac{1}{2} \sigma^{2}\left(1-\rho^{2}\right)} f\left(\epsilon_{1}\right) d \epsilon_{1} \\
& =e^{\boldsymbol{\beta}^{\boldsymbol{\top}} \boldsymbol{x}+\frac{1}{2} \sigma^{2}} \frac{1}{\Phi\left(\boldsymbol{\gamma}^{\top} \boldsymbol{z}\right)} \int_{-\boldsymbol{\gamma}^{\top} \boldsymbol{z}}^{\infty} e^{\rho \sigma \epsilon_{1}+\frac{1}{2} \sigma^{2}\left(1-\rho^{2}\right)} \frac{1}{\sqrt{2 \pi}} e^{-\frac{\epsilon^{2}}{2}} d \epsilon_{1} \\
& =e^{\boldsymbol{\beta}^{\top} \boldsymbol{x}+\frac{1}{2} \sigma^{2}} \frac{1}{\Phi\left(\boldsymbol{\gamma}^{\top} \boldsymbol{z}\right)} \int_{-\boldsymbol{\gamma}^{\top} \boldsymbol{z}}^{\infty} \frac{1}{\sqrt{2 \pi}} e^{-\frac{\epsilon_{1}^{2}-2 \rho \sigma \epsilon_{1}+\sigma^{2} \rho^{2}}{2}} d \epsilon_{1} \\
& =e^{\boldsymbol{\beta}^{\boldsymbol{\top}} \boldsymbol{x}+\frac{1}{2} \sigma^{2}} \frac{1}{\Phi\left(\boldsymbol{\gamma}^{\top} \boldsymbol{z}\right)} \int_{-\boldsymbol{\gamma}^{\top} \boldsymbol{z}}^{\infty} \frac{1}{\sqrt{2 \pi}} e^{-\frac{\left(\epsilon_{1}-\kappa\right)^{2}}{2}} d \epsilon_{1} \\
& =e^{\boldsymbol{\beta}^{\boldsymbol{\top}} \boldsymbol{x}+\frac{1}{2} \sigma^{2}} \frac{\Phi\left(\boldsymbol{\gamma}^{\top} \boldsymbol{z}+\kappa\right)}{\Phi\left(-\boldsymbol{\gamma}^{\top} \boldsymbol{z}\right)}
\end{aligned}
$$

where $\kappa=\sigma \rho$. 
Appendix D

gamlss diagnostic plots for VA and SOEP data 
Figure D.1: Poisson model diagnostics for the NUMVAOUT response.
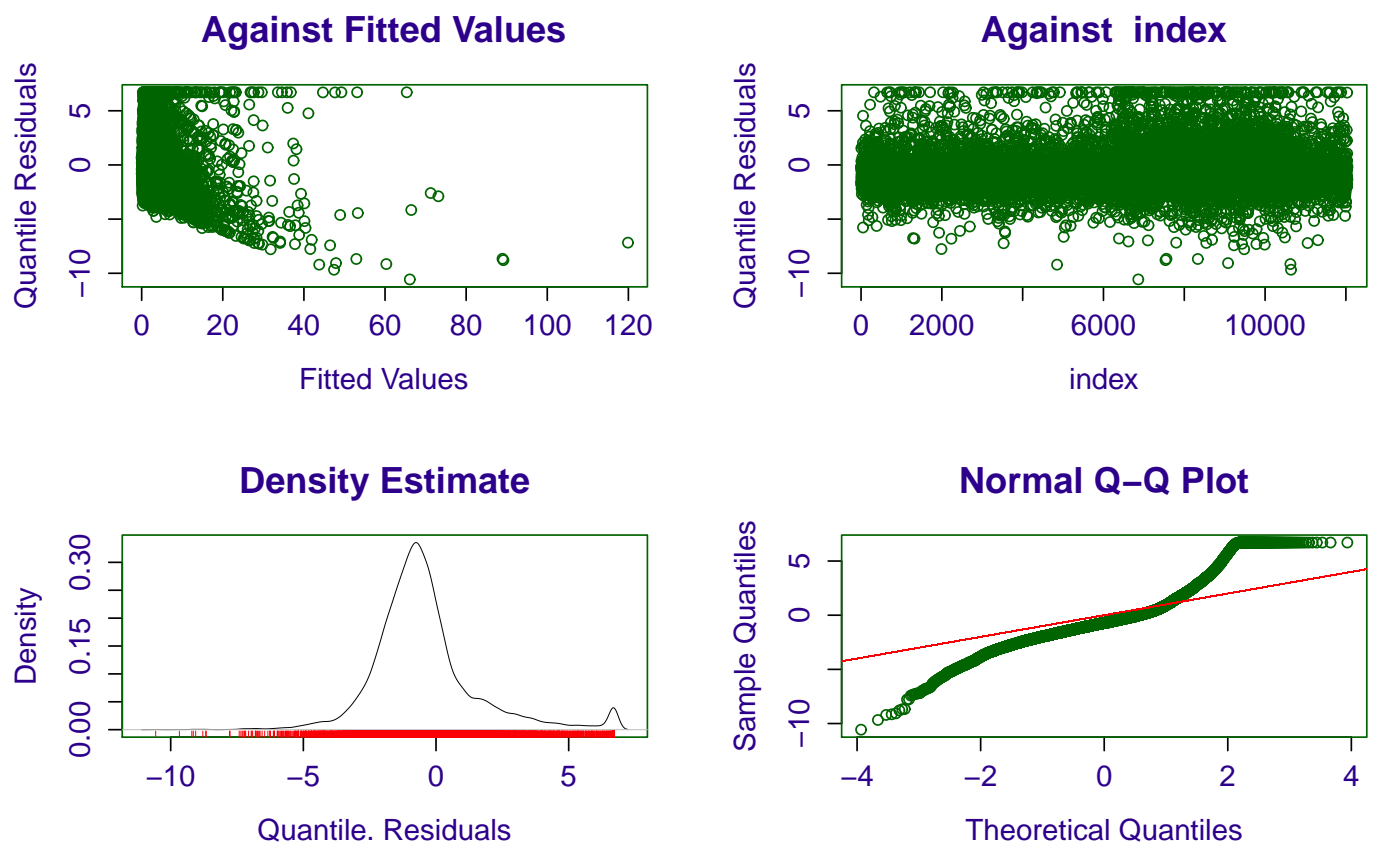

Figure D.2: Negative binomial model diagnostics for the NUMVAOUT response.
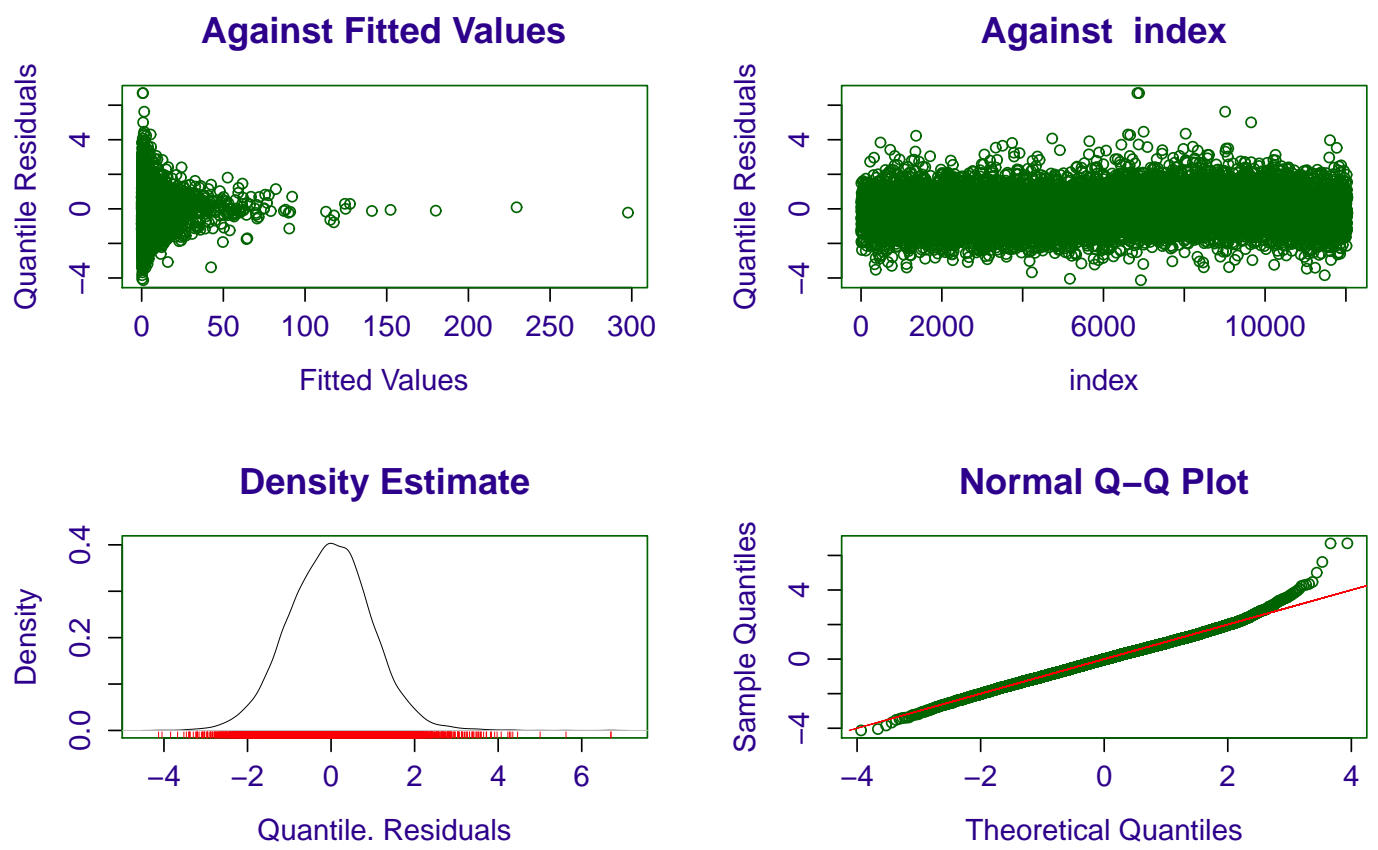
Figure D.3: Delaporte model diagnostics for the NUMVAOUT response.
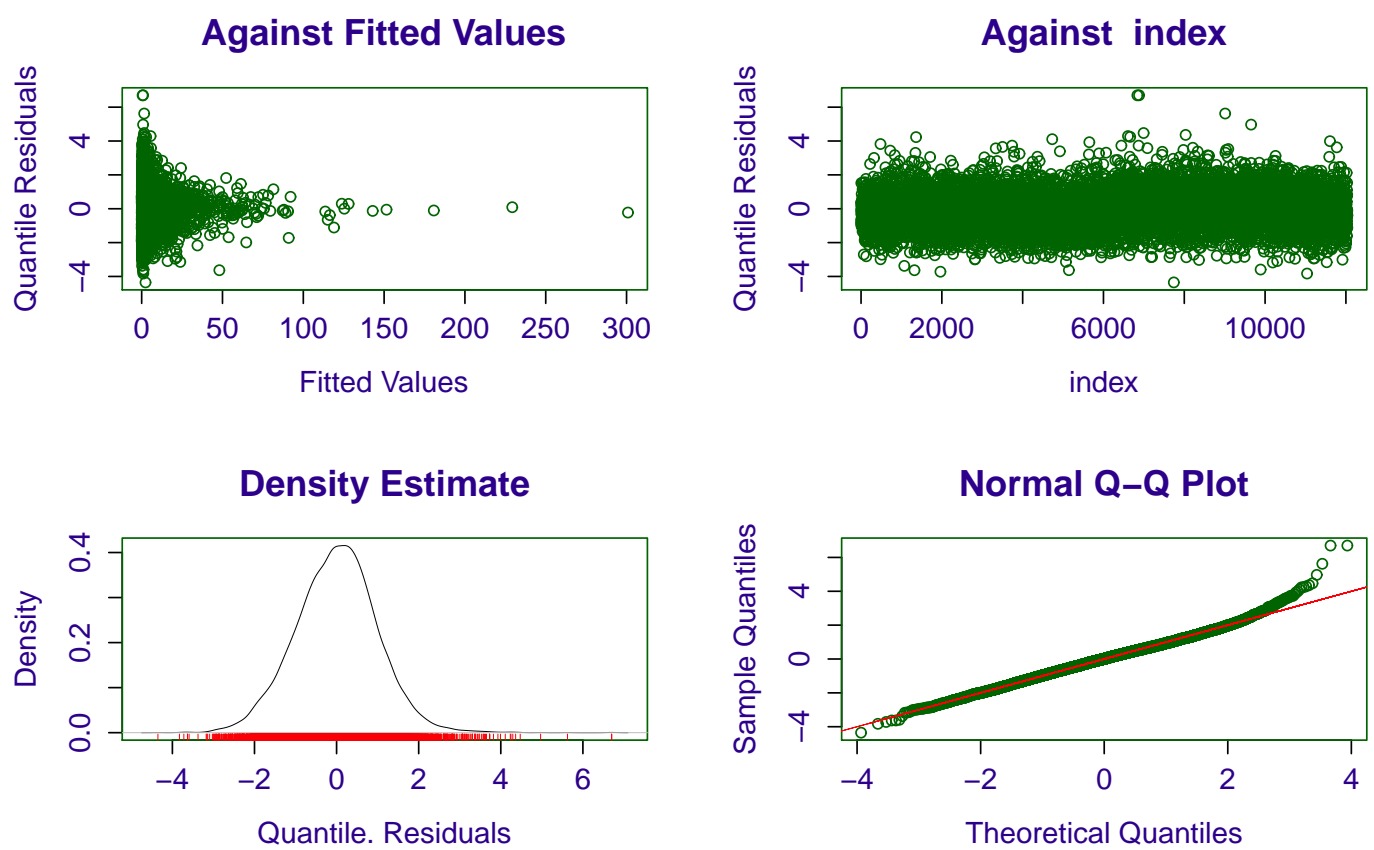

Figure D.4: Sichel model diagnostics for the NUMVAOUT response.
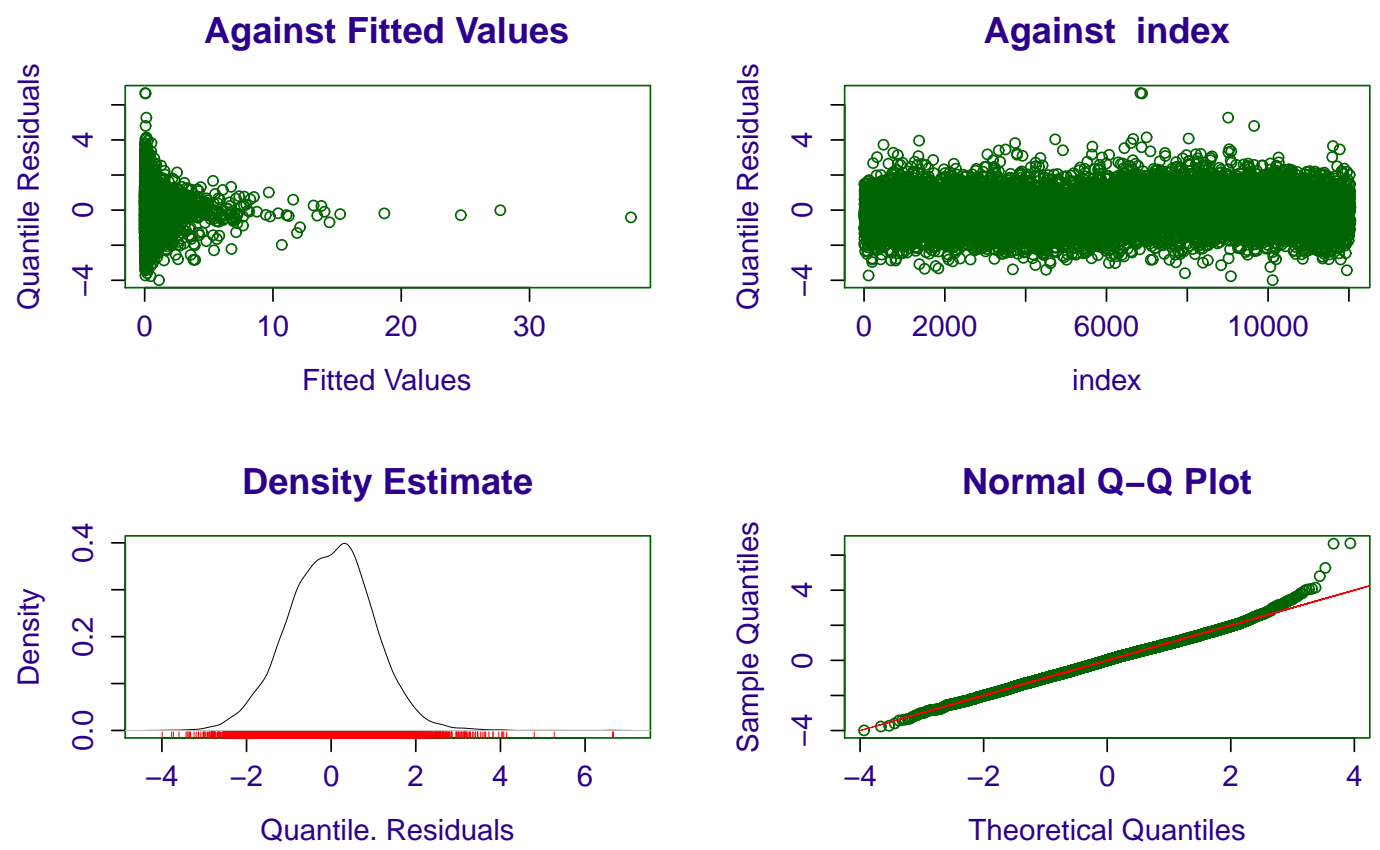
Figure D.5: Poisson model diagnostics for the NUMOTH response.

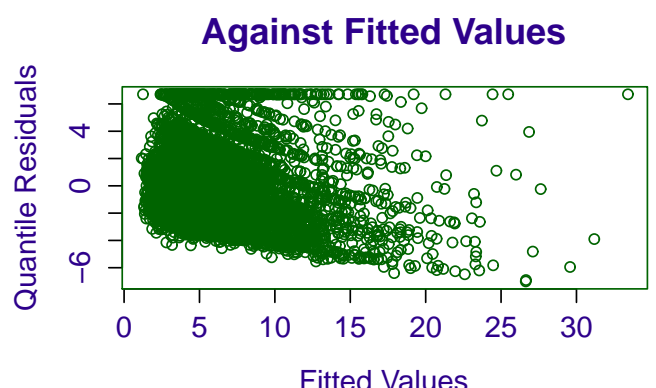

Density Estimate

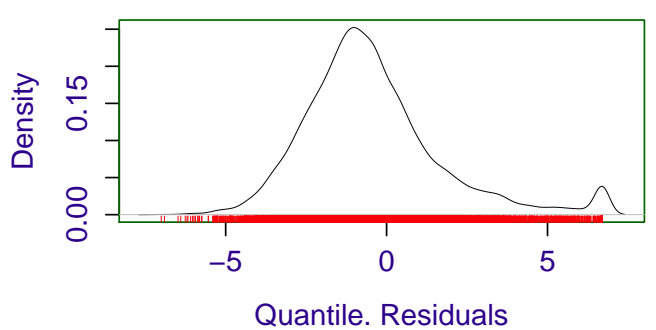

Against index

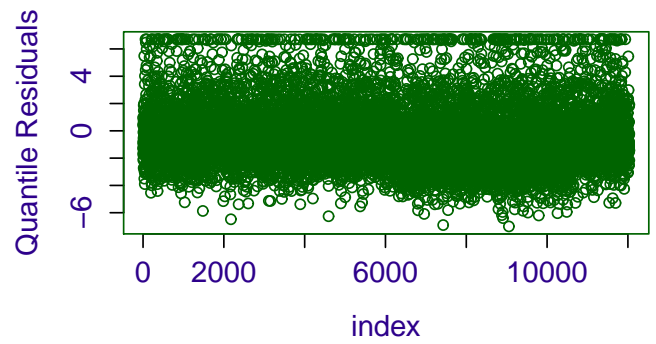

Normal Q-Q Plot

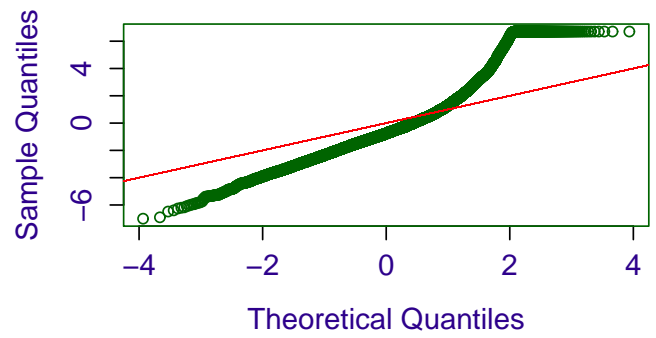

Figure D.6: Negative binomial model diagnostics for the NUMOTH response.

Against Fitted Values

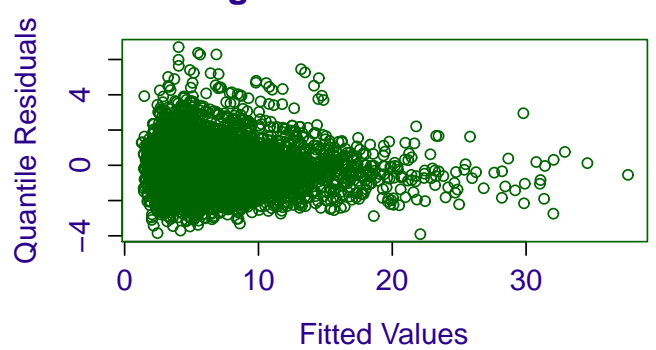

Density Estimate

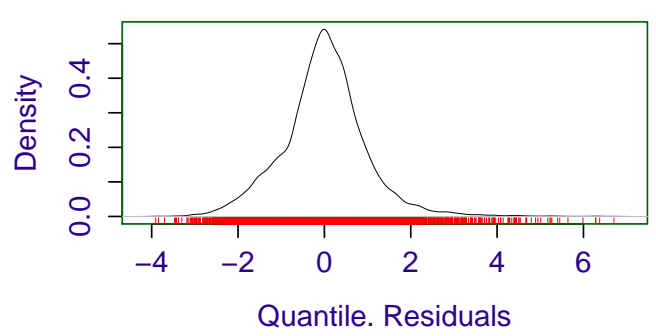

Against index

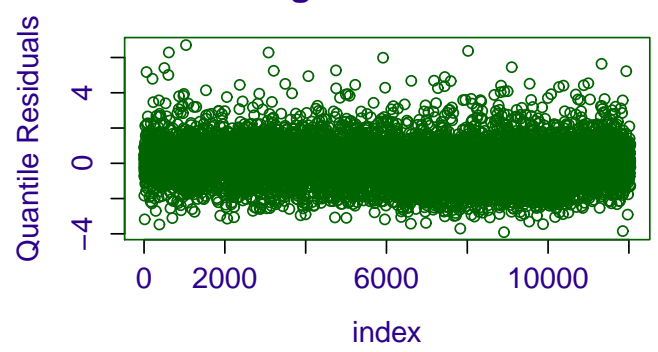

Normal Q-Q Plot

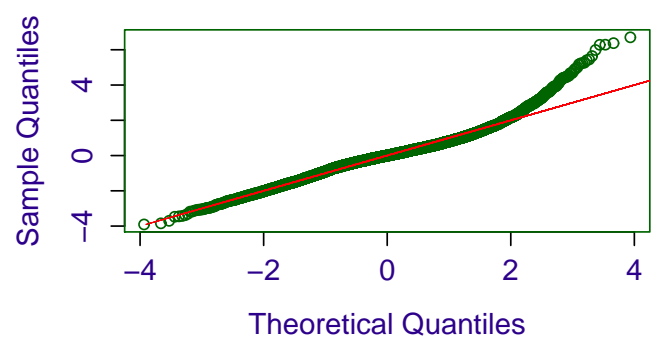


Figure D.7: Delaporte model diagnostics for the NUMOTH response.

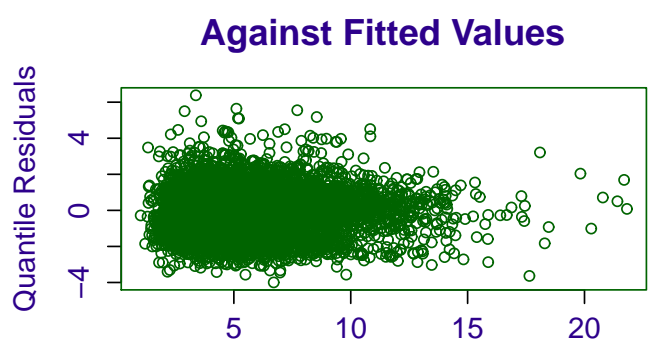

Fitted Values

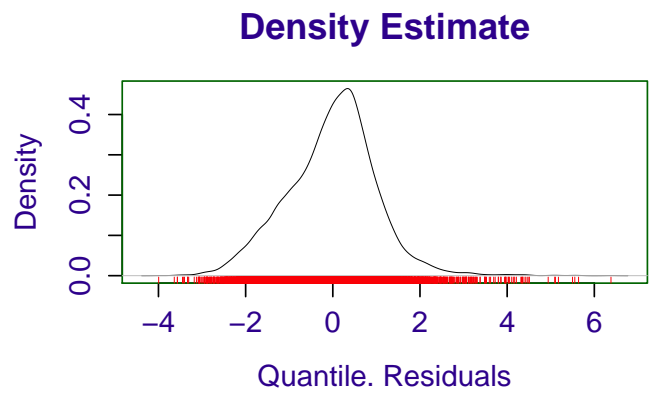

Against index

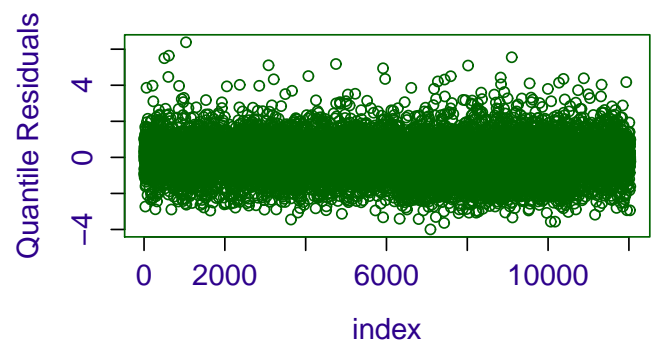

Normal Q-Q Plot

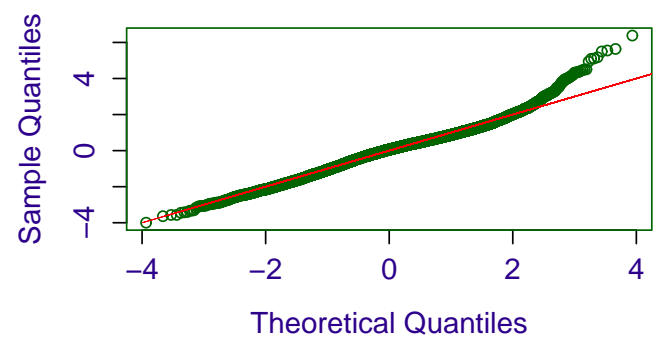

Figure D.8: Poisson Inverse Gaussian model diagnostics for the NUMOTH response.

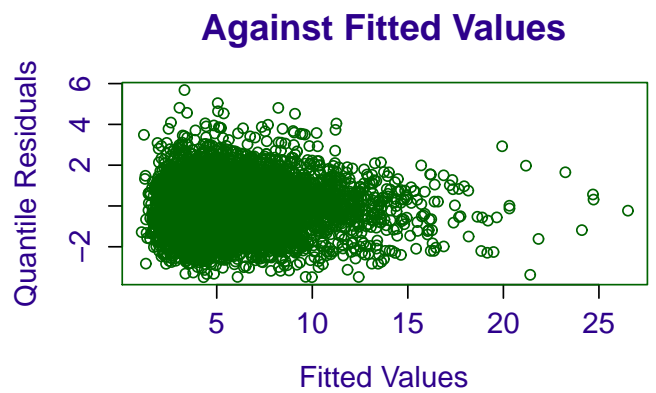

Density Estimate

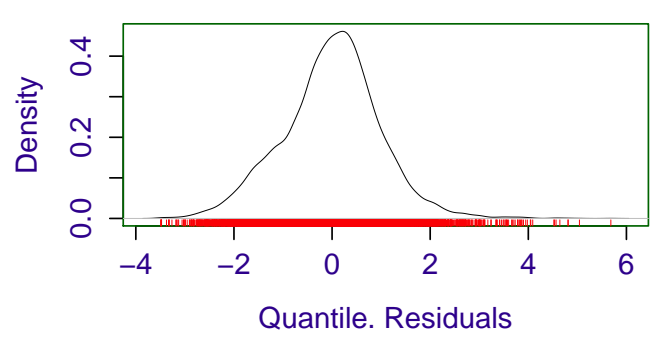

Against index

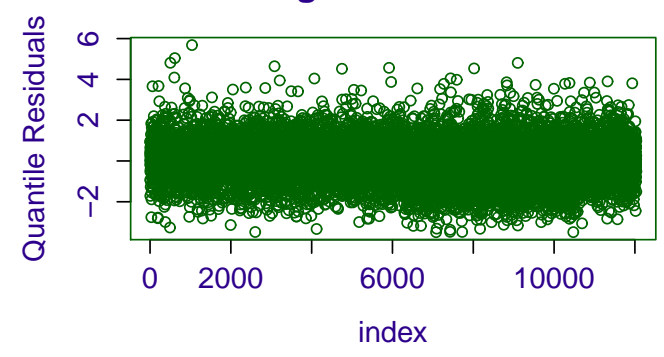

Normal Q-Q Plot

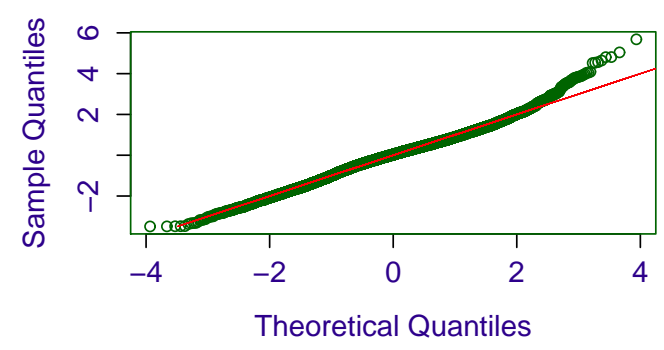


Figure D.9: Poisson model diagnostics for the VANITES response.
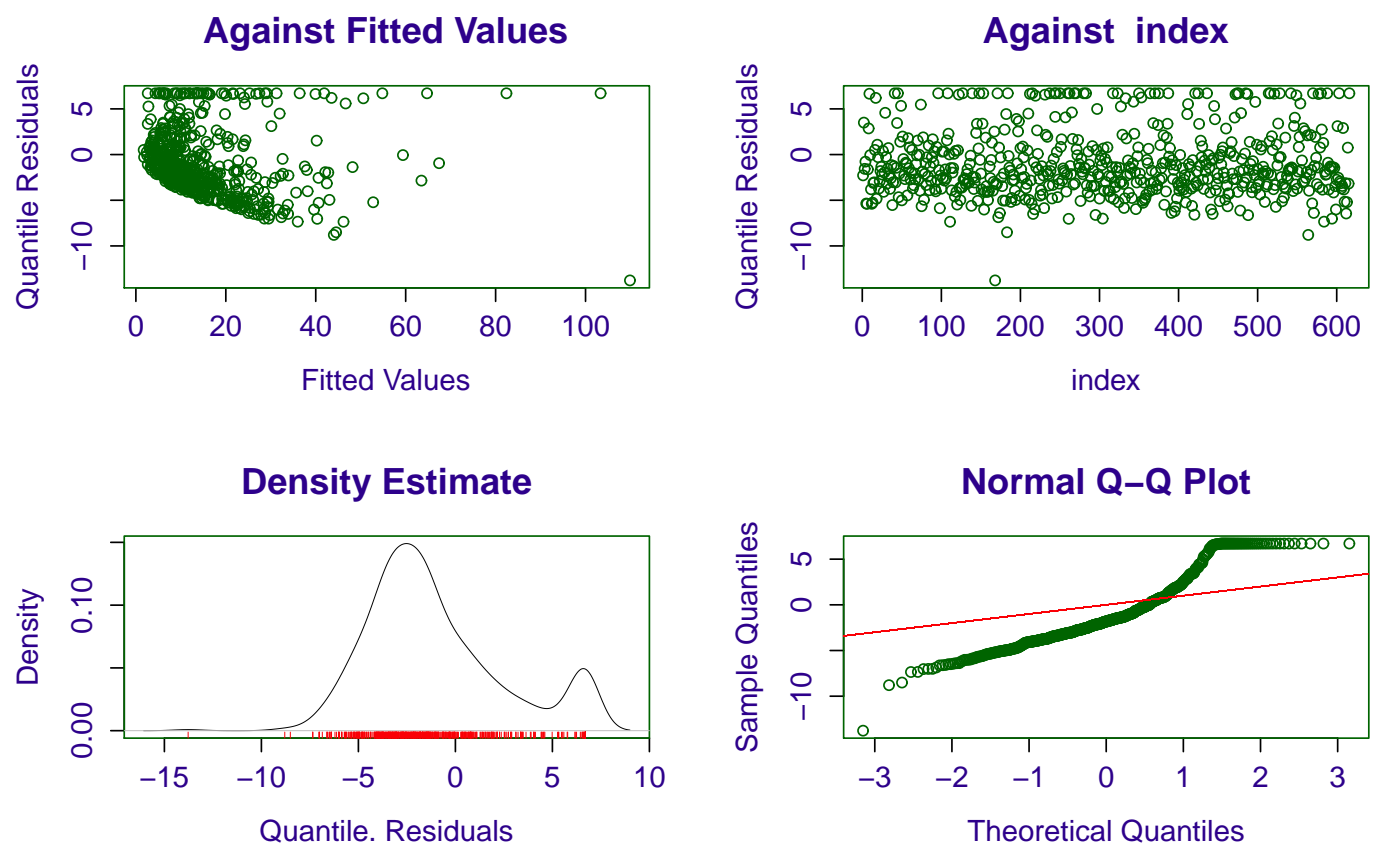

Figure D.10: Negative binomial model diagnostics for the VANITES response.
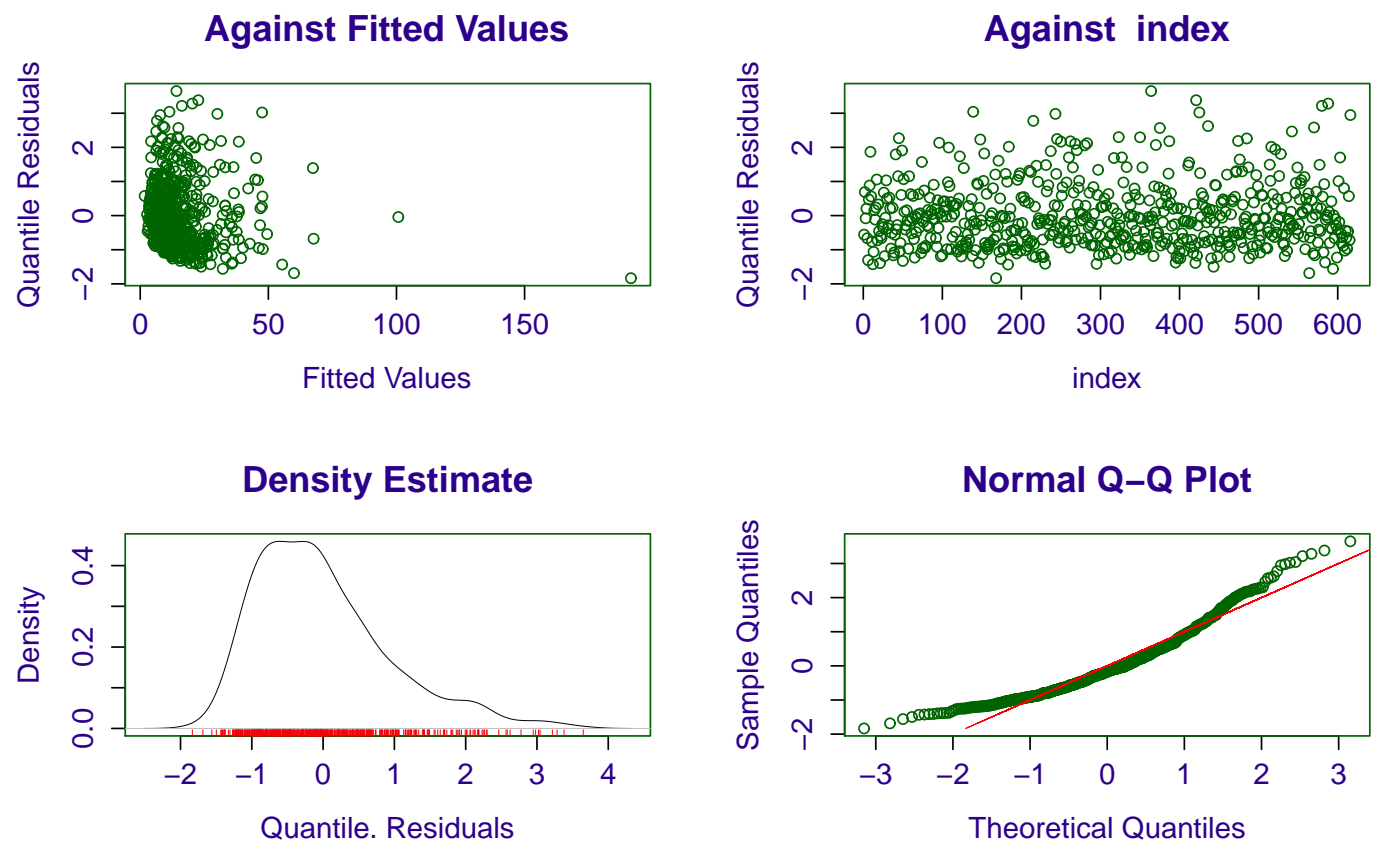
Figure D.11: Delaporte model diagnostics for the VANITES response.
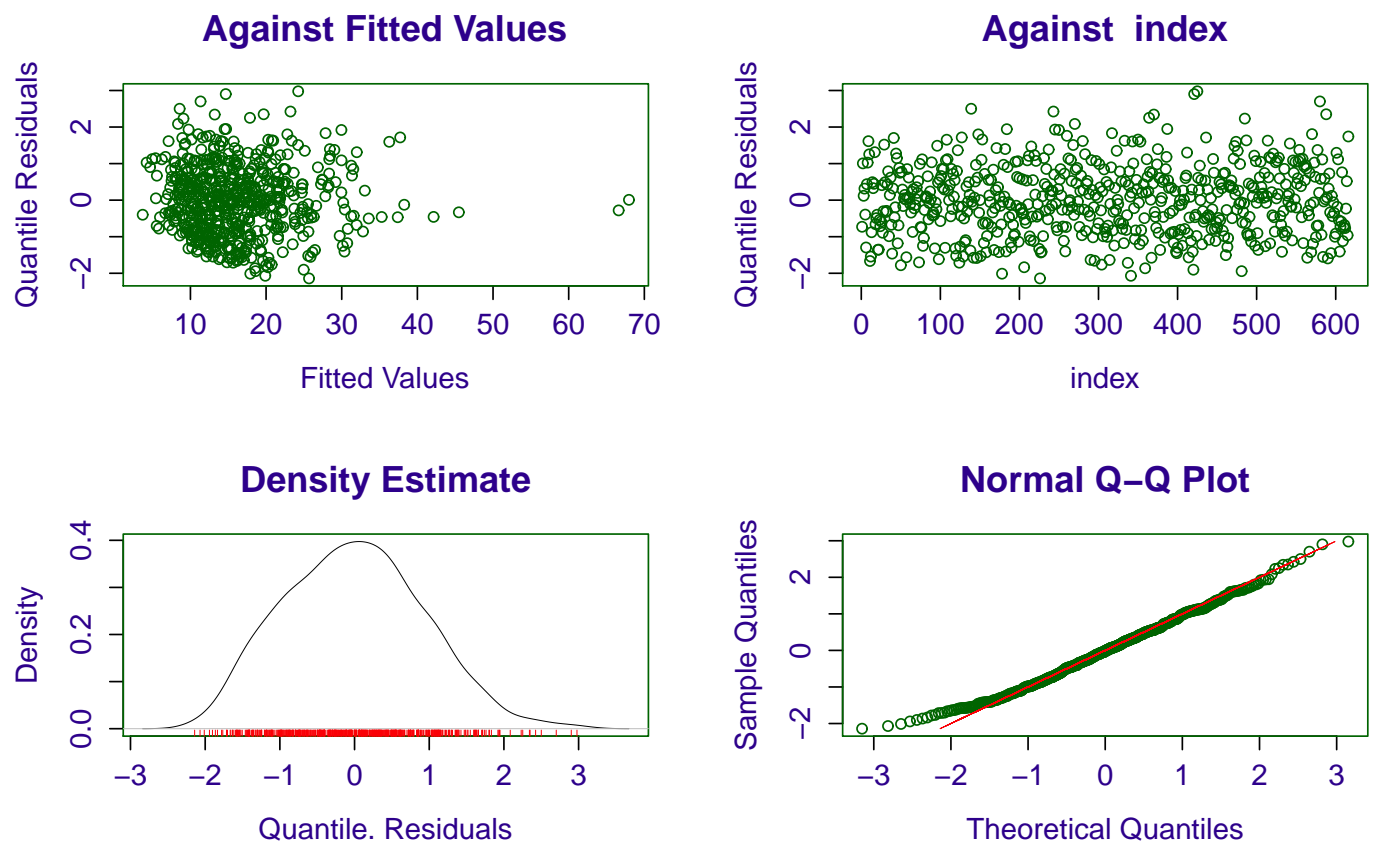

Figure D.12: Poisson Inverse Gaussian model diagnostics for the VANITES response.
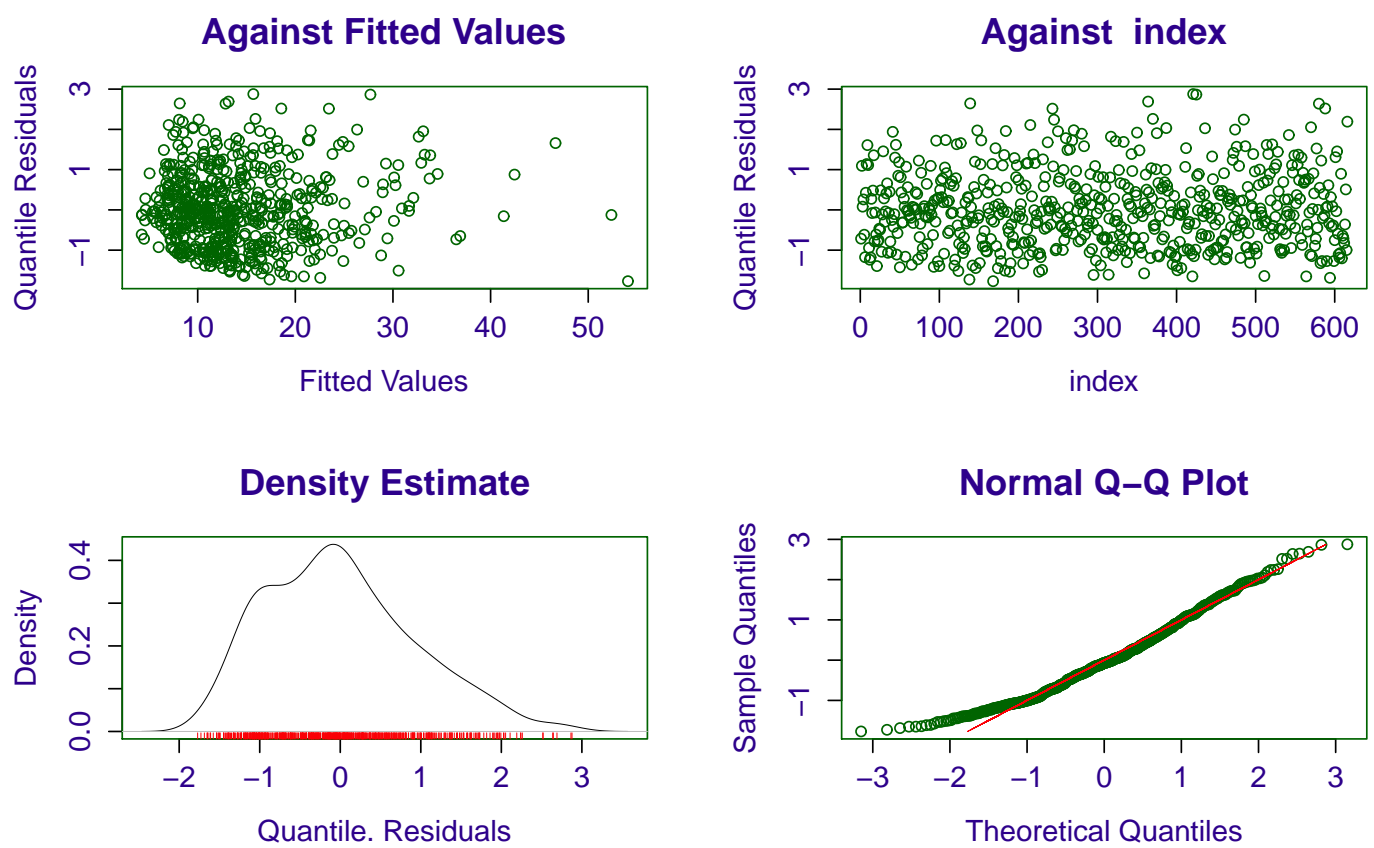
Figure D.13: Poisson model diagnostics for the OTHNITES response.
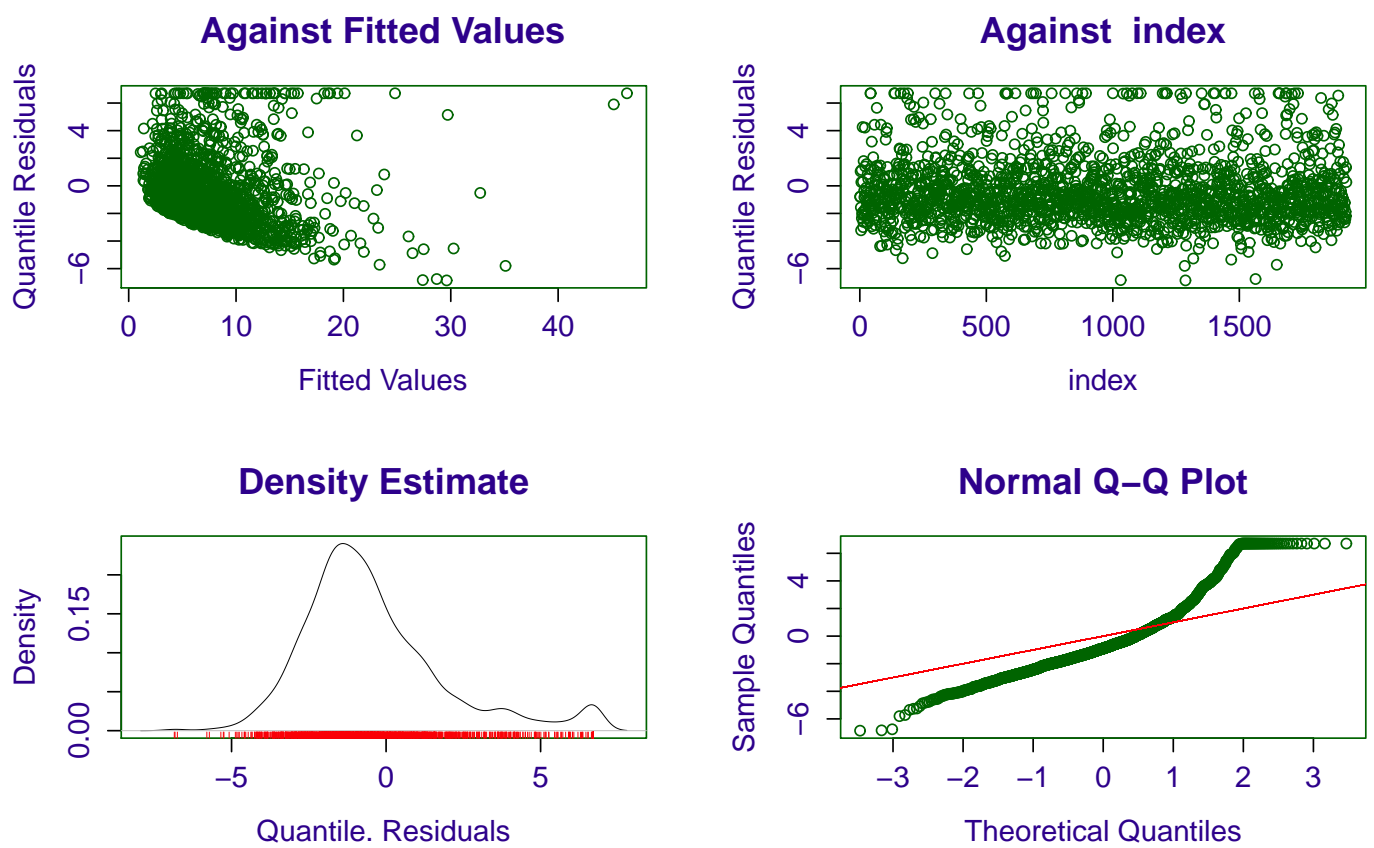

Figure D.14: Negative binomial model diagnostics for the OTHNITES response.
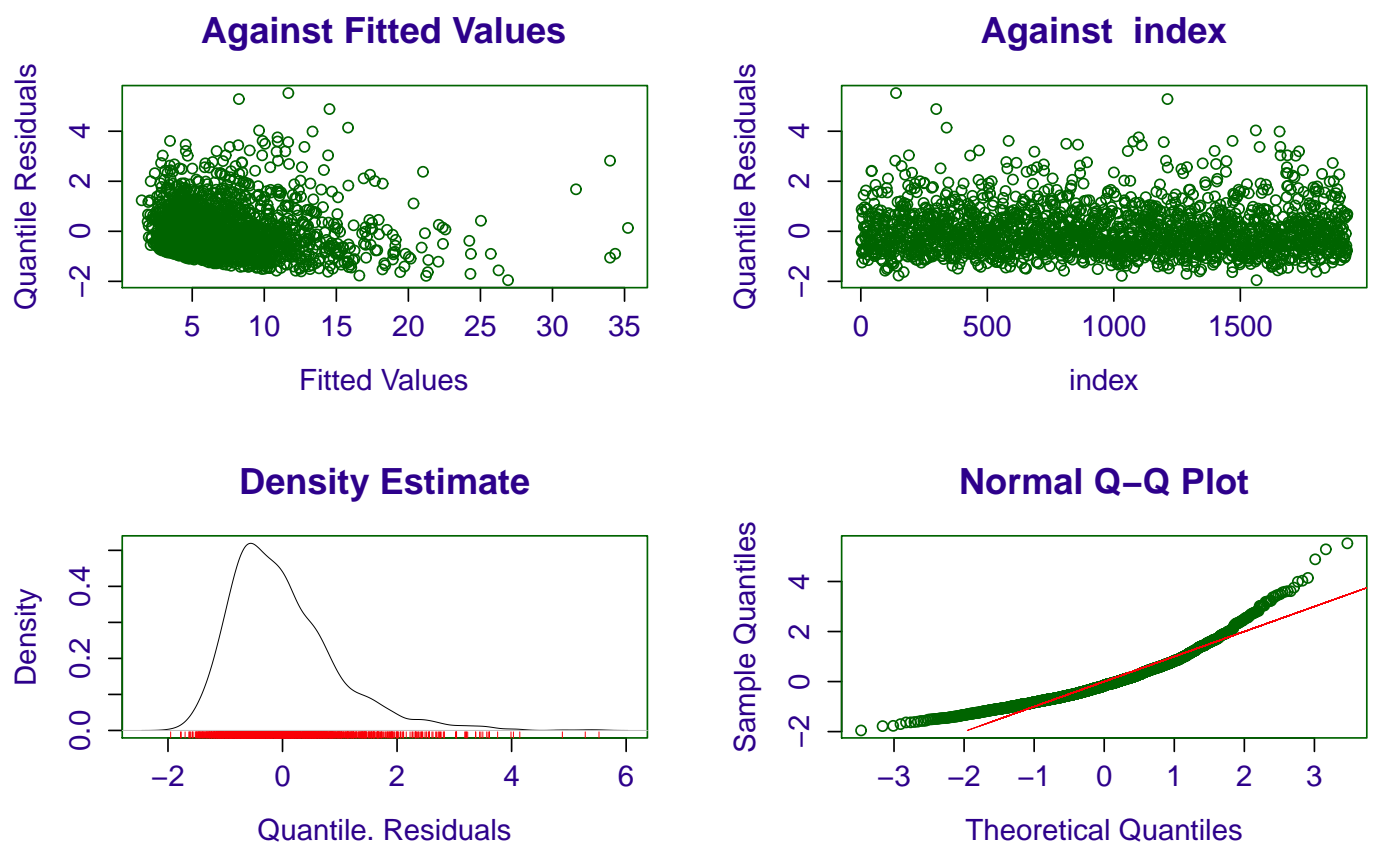
Figure D.15: Delaporte model diagnostics for the OTHNITES response.

Against Fitted Values

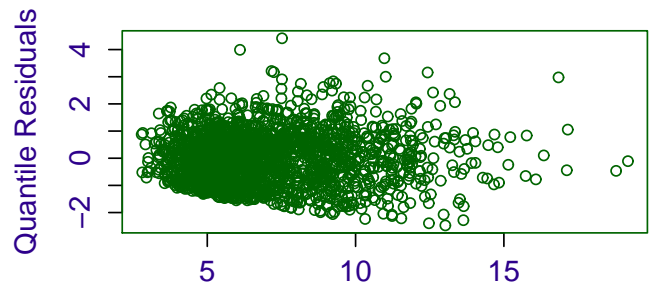

Fitted Values

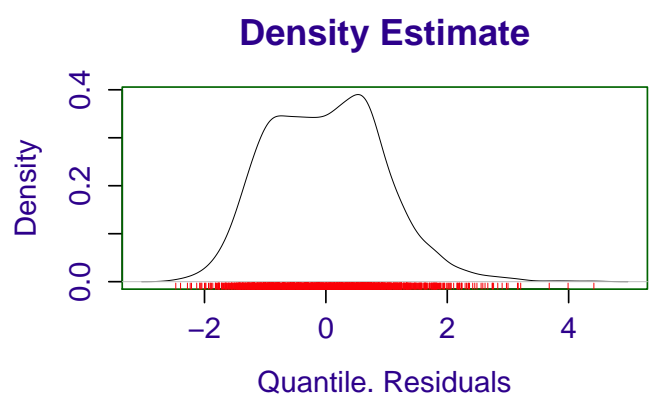

Against index

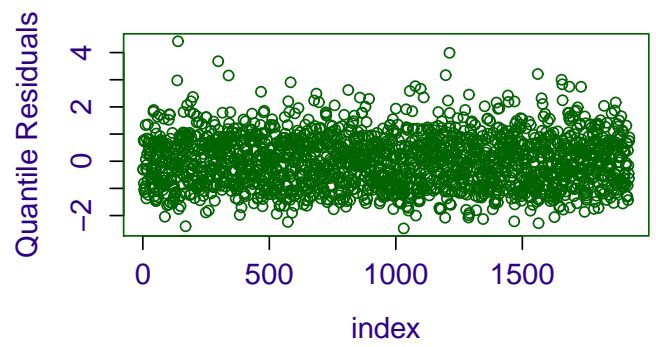

Normal Q-Q Plot

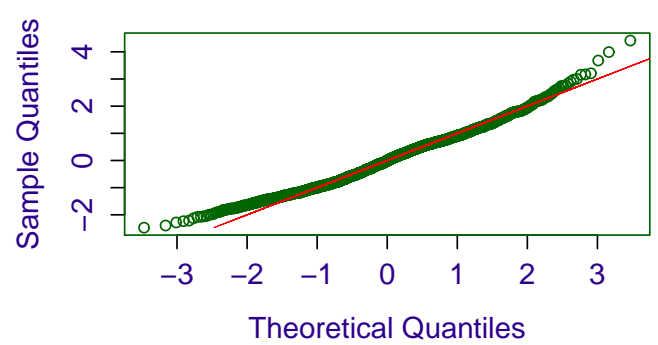

Figure D.16: Sichel model diagnostics for the OTHNITES response.
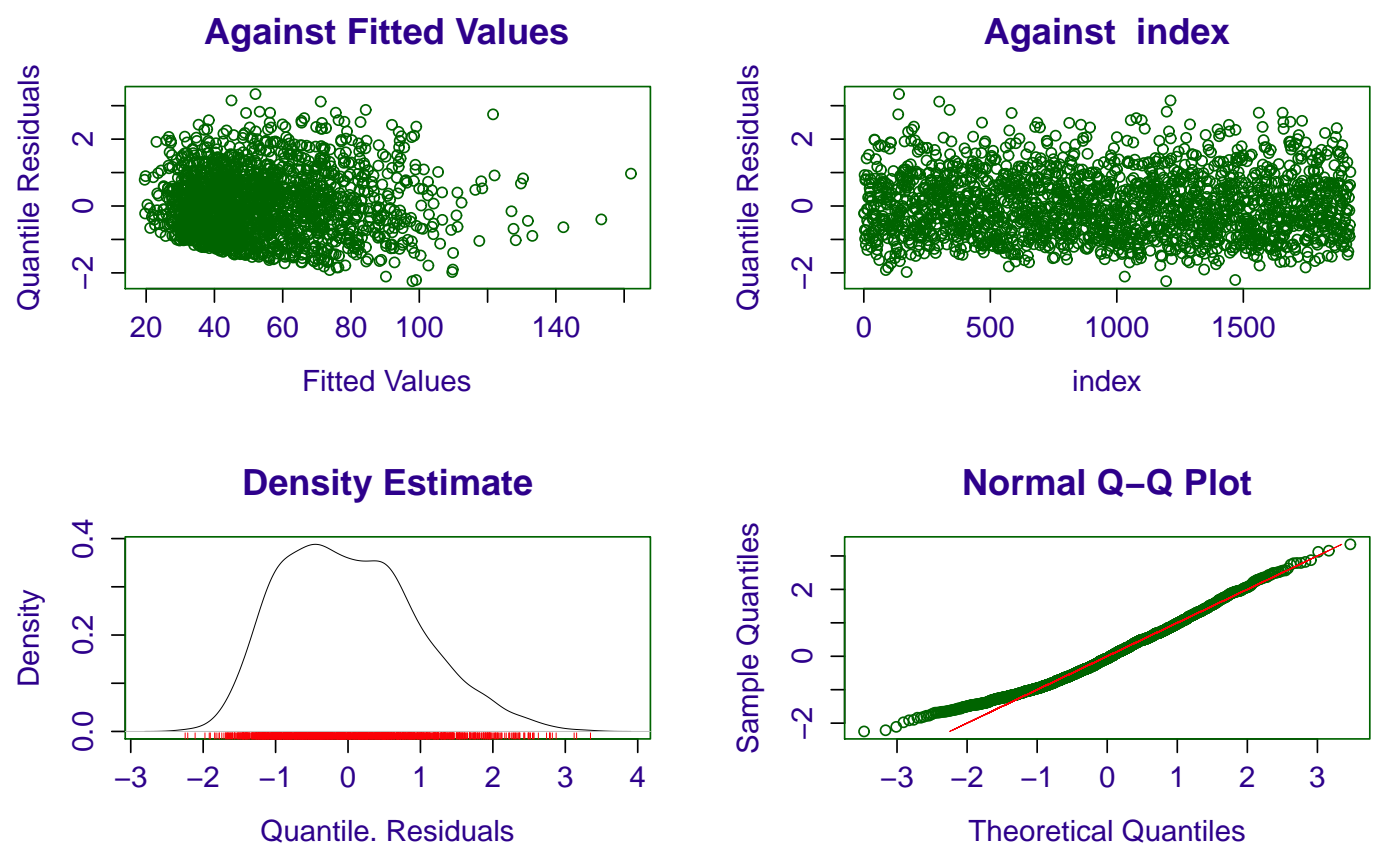
Figure D.17: Poisson model diagnostics for the SOEP response.
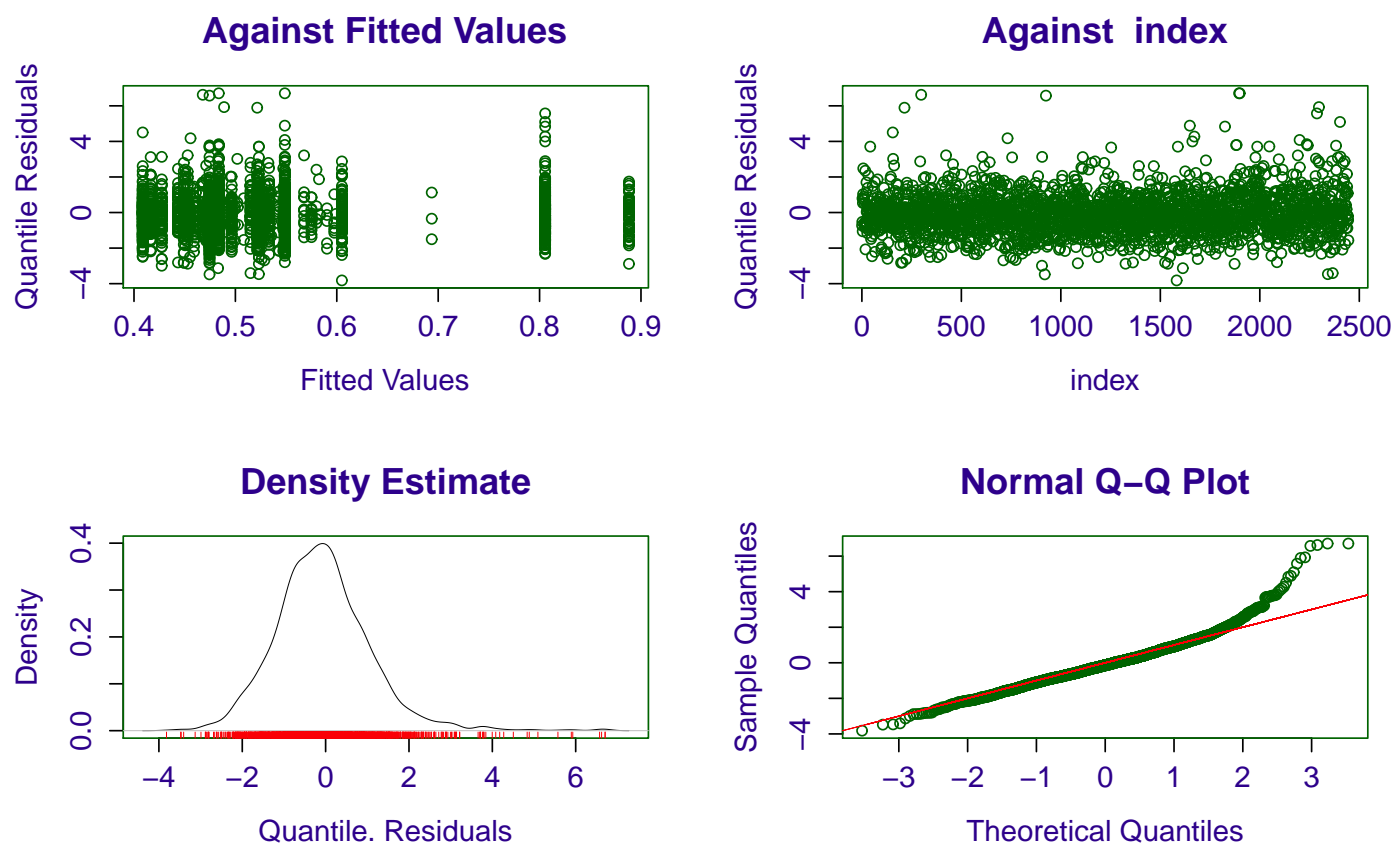

Figure D.18: Negative binomial model diagnostics for the SOEP response.
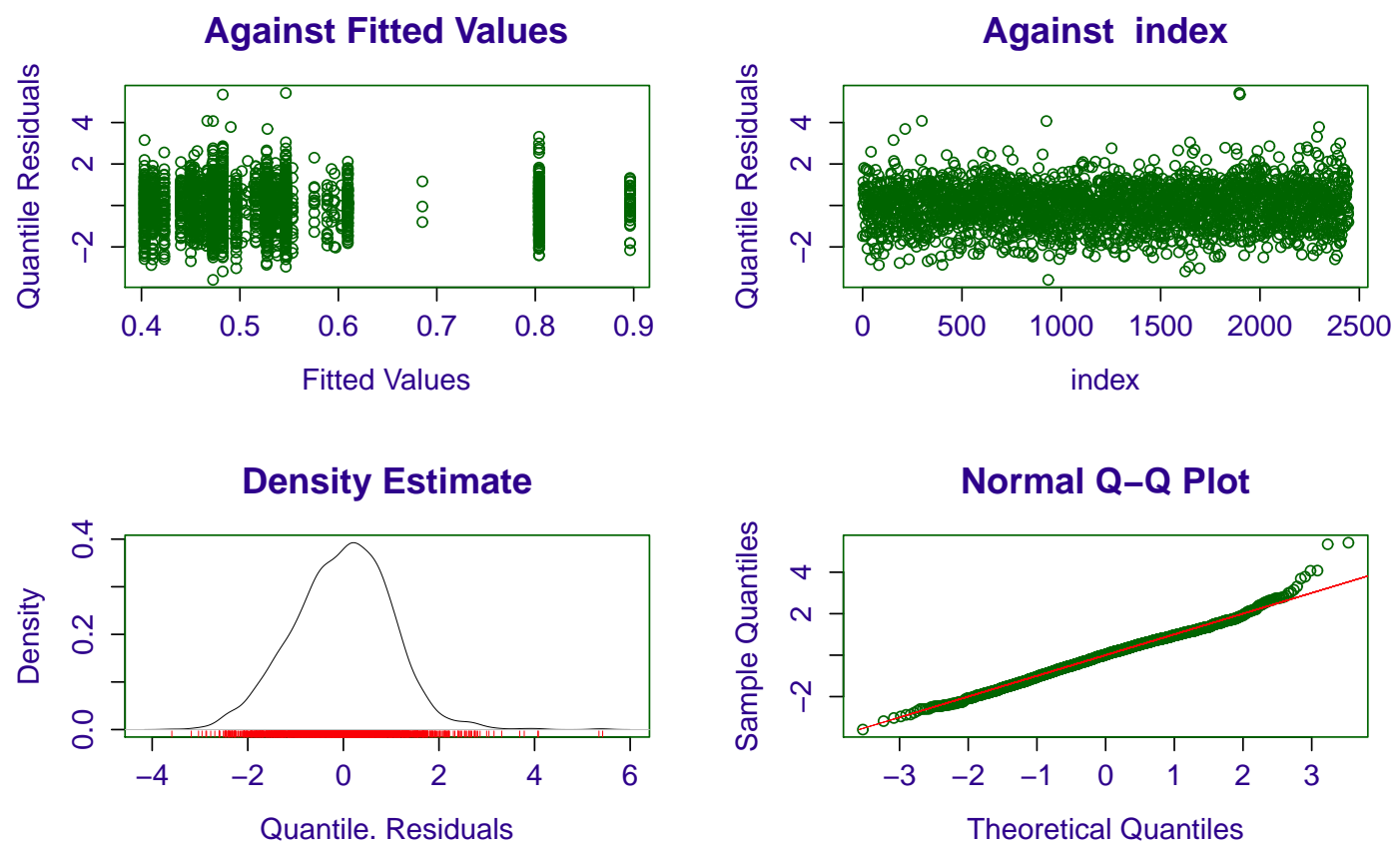
Figure D.19: Poisson Inverse Gaussian model diagnostics for the SOEP response.

Against Fitted Values

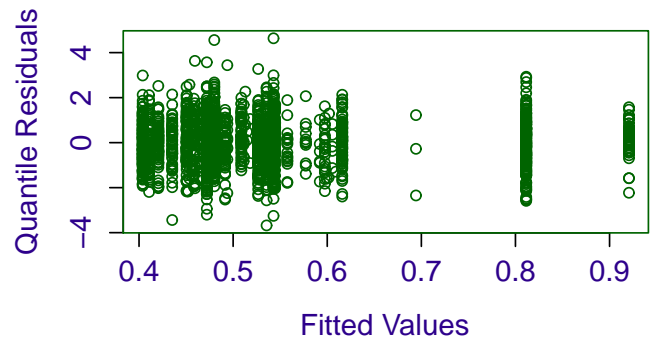

Density Estimate

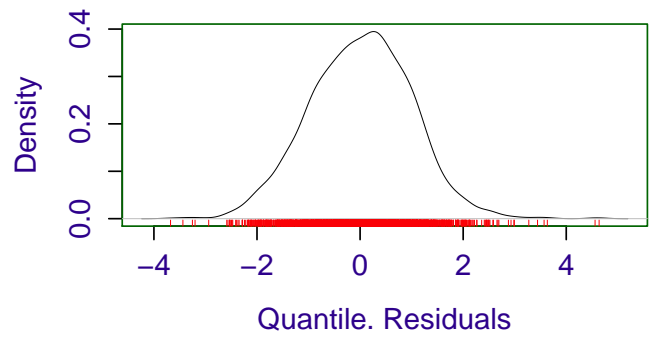

Against index

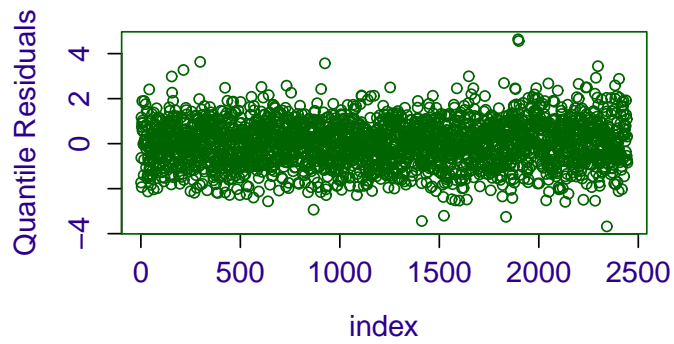

Normal Q-Q Plot

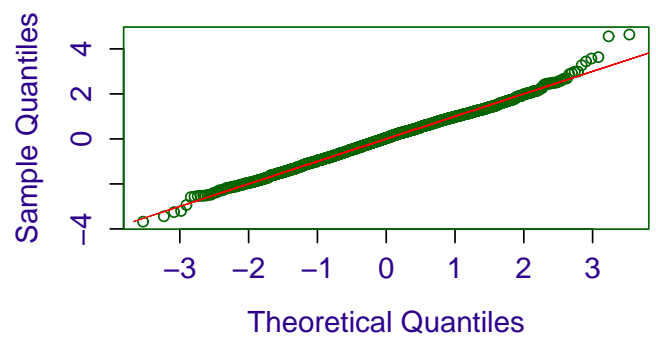

Figure D.20: Sichel model diagnostics for the SOEP response.
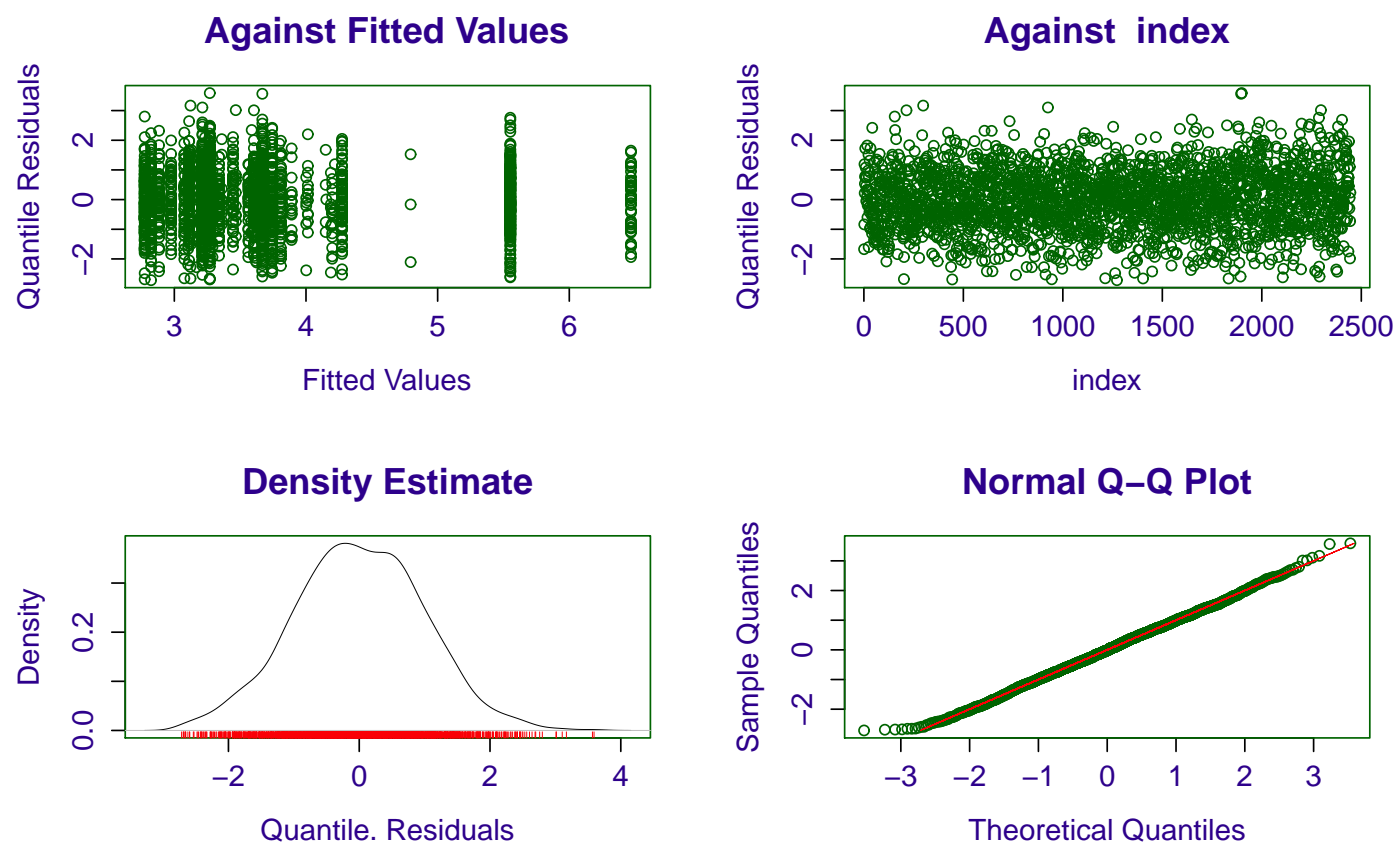


\section{Appendix E}

\section{Starting value for $\theta^{*}$ and data simulation $R$ code}

$\underline{\theta^{*} \text { starting value } \mathrm{R} \text { code }}$

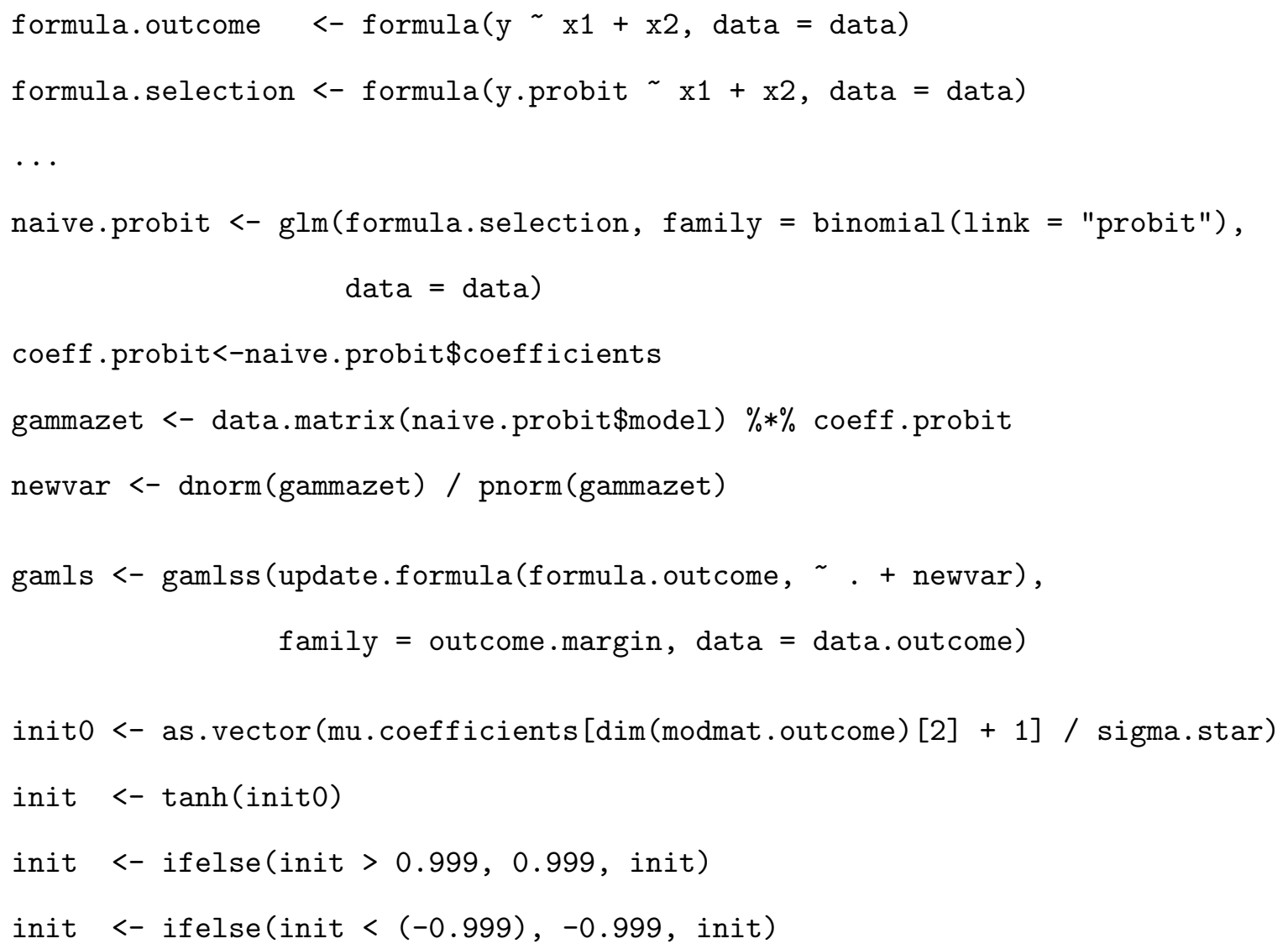

$\underline{\text { Data simulation } \mathrm{R} \text { code }}$ 


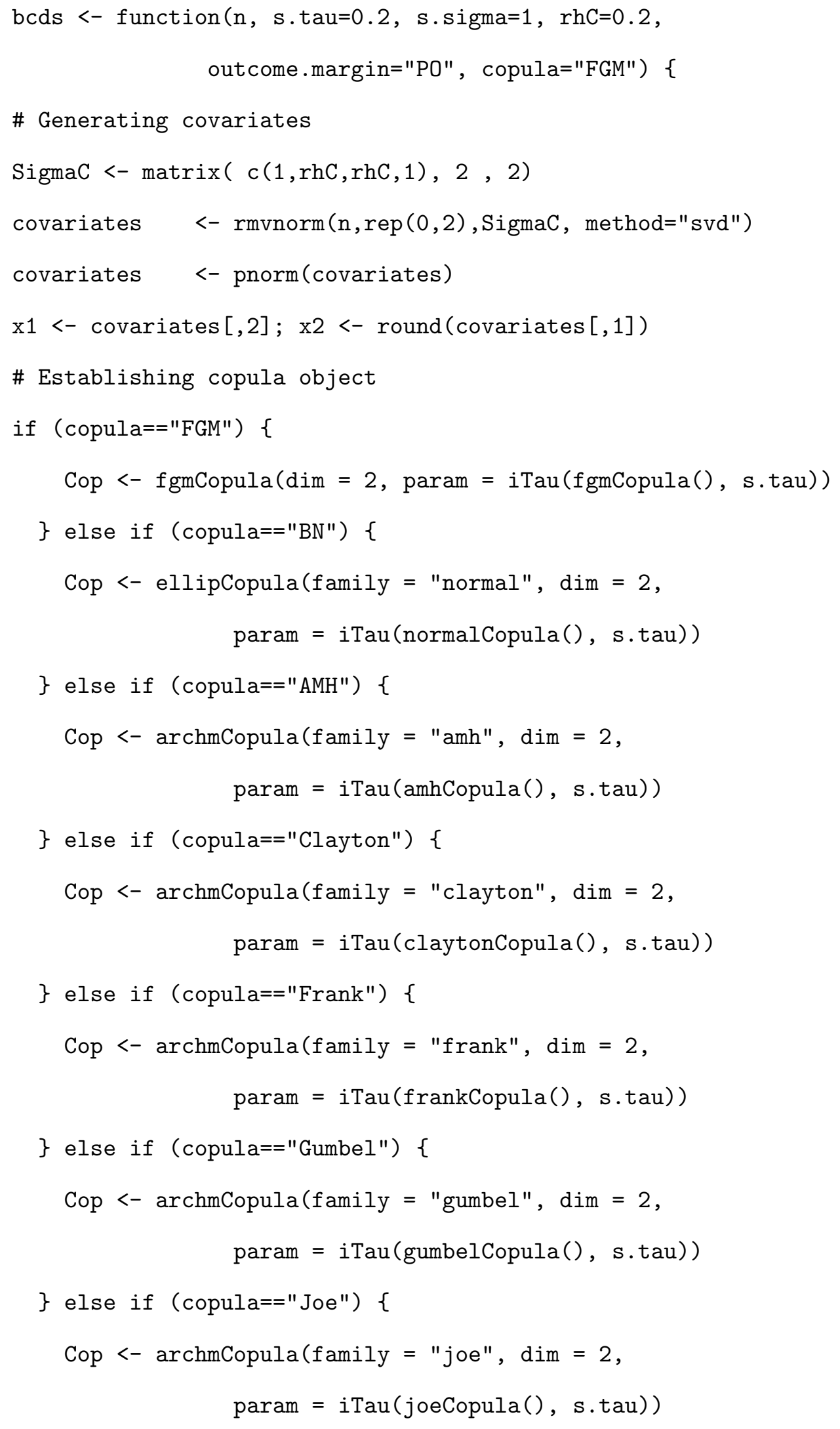




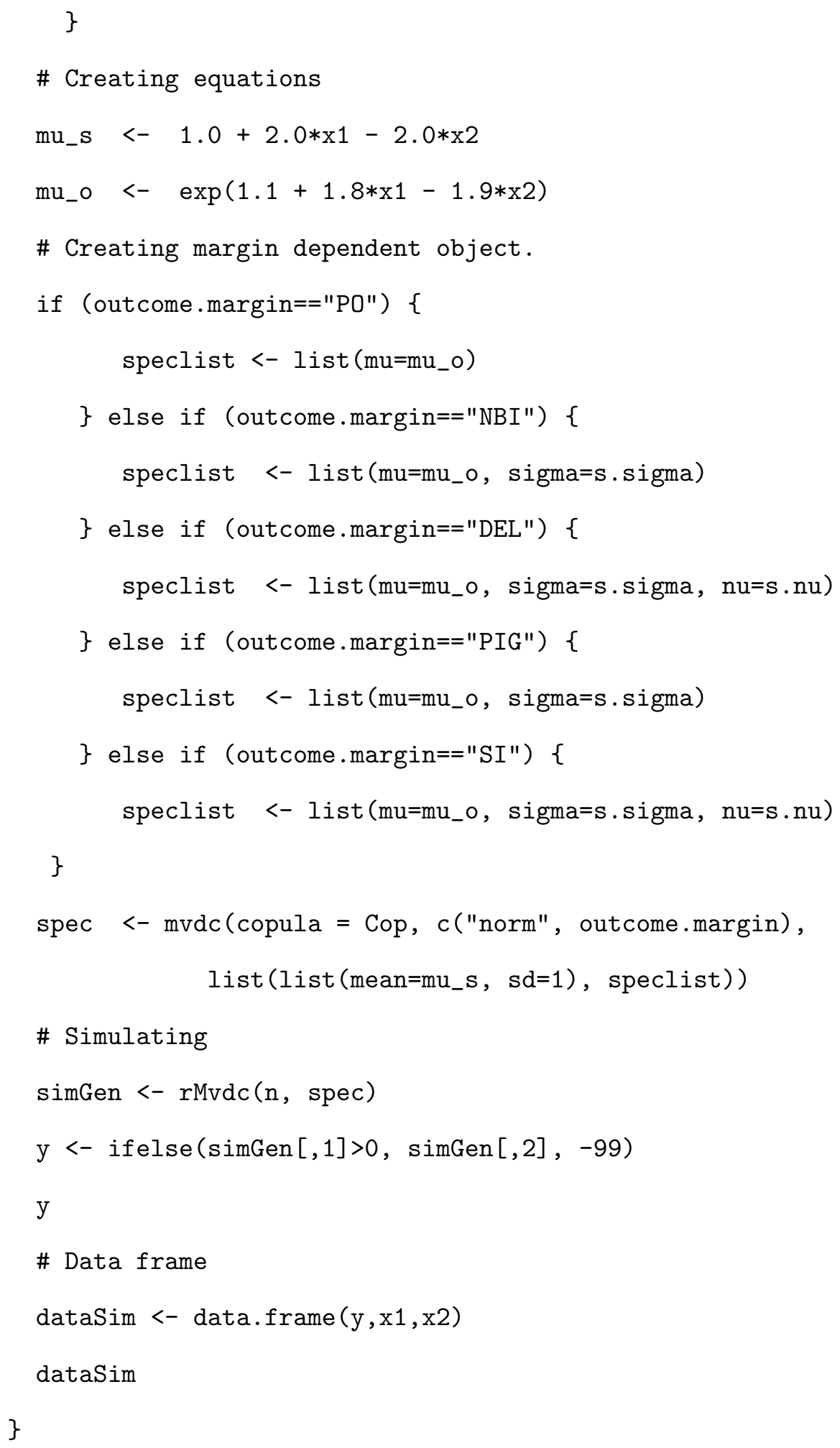




\section{Appendix F}

\section{Derivation of the score vector}

\section{components}

Recall section 5.8.1. We will now calculate first derivatives of copulas with respect to their margins and $\theta$. We will denote margins as $u$ and $v$. Afterwards, we will proceed to the derivatives of pmfs and cdfs with respect to $\boldsymbol{\delta}_{\mathbf{1}}, \boldsymbol{\delta}_{\mathbf{2}}, \sigma$ and $\nu$.

$\underline{\text { First derivatives with respect to copulas }}$

Clayton: $C_{\theta}(u, v)=\left(u^{-\theta}+v^{-\theta}-1\right)^{-\frac{1}{\theta}}$

$$
\begin{aligned}
\frac{\partial C_{\theta}}{\partial u}= & \frac{1}{\theta}\left(u^{-\theta}+v^{-\theta}-1\right)^{-\frac{1}{\theta}-1} \theta u^{-\theta-1} \\
= & u^{-\theta-1}\left(u^{-\theta}+v^{-\theta}-1\right)^{-\frac{1}{\theta}-1} \\
\frac{\partial C_{\theta}}{\partial v}= & v^{-\theta-1}\left(u^{-\theta}+v^{-\theta}-1\right)^{-\frac{1}{\theta}-1} \\
\frac{\partial C_{\theta}}{\partial \theta}= & \frac{1}{\theta^{2}} \log \left(u^{-\theta}+v^{-\theta}-1\right)\left(u^{-\theta}+v^{-\theta}-1\right)^{-\frac{1}{\theta}} \\
& +\frac{1}{\theta}\left(u^{-\theta} \log (u)+v^{-\theta} \log (v)\right)\left(u^{-\theta}+v^{-\theta}-1\right)^{-\frac{1}{\theta}-1}
\end{aligned}
$$


AMH: $C_{\theta}(u, v)=u v /[1-\theta(1-u)(1-v)]$

$$
\begin{aligned}
\frac{\partial C_{\theta}}{\partial u} & =\frac{v[1-\theta(1-u)(1-v)]-u v \theta(1-v)}{[1-\theta(1-u)(1-v)]^{2}} \\
\frac{\partial C_{\theta}}{\partial v} & =\frac{u[1-\theta(1-u)(1-v)]-u v \theta(1-u)}{[1-\theta(1-u)(1-v)]^{2}} \\
\frac{\partial C_{\theta}}{\partial \theta} & =\frac{u v}{[1-\theta(1-u)(1-v)]^{2}}(1-u)(1-v)
\end{aligned}
$$

Gumbel: $C_{\theta}(u, v)=\exp \left\{-\left[(-\log u)^{\theta}+(-\log v)^{\theta}\right]^{\frac{1}{\theta}}\right\}$

$$
\begin{aligned}
\frac{\partial C_{\theta}}{\partial u}= & \exp \left\{-\left[(-\log u)^{\theta}+(-\log v)^{\theta}\right]^{\frac{1}{\theta}}\right\}\left\{-\frac{1}{\theta}\left[(-\log u)^{\theta}+(-\log v)^{\theta}\right]^{\frac{1}{\theta}-1}\right\}\left[\theta(-\log u)^{\theta-1}\right]\left(-\frac{1}{u}\right) \\
\frac{\partial C_{\theta}}{\partial v}= & \exp \left\{-\left[(-\log u)^{\theta}+(-\log v)^{\theta}\right]^{\frac{1}{\theta}}\right\}\left\{-\frac{1}{\theta}\left[(-\log u)^{\theta}+(-\log v)^{\theta}\right]^{\frac{1}{\theta}-1}\right\}\left[\theta(-\log v)^{\theta-1}\right]\left(-\frac{1}{v}\right) \\
\frac{\partial C_{\theta}}{\partial \theta}= & -\exp \left\{-\left[(-\log u)^{\theta}+(-\log v)^{\theta}\right]^{\frac{1}{\theta}}\right\}\left[(-\log u)^{\theta}+(-\log v)^{\theta}\right]^{\frac{1}{\theta}}\left\{-\frac{1}{\theta^{2}} \log \left[(-\log u)^{\theta}\right.\right. \\
& \left.\left.+(-\log v)^{\theta}\right]\right\}+\frac{1}{\theta} \frac{1}{(-\log u)^{\theta}+(-\log v)^{\theta}}\left[(-\log u)^{\theta} \log (-\log u)+(-\log v)^{\theta} \log (-\log v)\right] \\
& {\left[(-\log u)^{\theta}+(-\log v)^{\theta}\right]^{\frac{1}{\theta}}\left\{-\exp \left\{-\left[(-\log u)^{\theta}+(-\log v)^{\theta}\right]^{\frac{1}{\theta}}\right\}\right\} }
\end{aligned}
$$

Joe: $C_{\theta}(u, v)=1-\left[(1-u)^{\theta}+(1-v)^{\theta}-(1-u)^{\theta}(1-v)^{\theta}\right]^{\frac{1}{\theta}}$

$$
\begin{aligned}
\frac{\partial C_{\theta}}{\partial u}= & -\frac{1}{\theta}\left[(1-u)^{\theta}+(1-v)^{\theta}-(1-u)^{\theta}(1-v)^{\theta}\right]^{\frac{1}{\theta}-1}\left[-\theta(1-u)^{\theta-1}+\theta(1-v)^{\theta}(1-u)^{\theta-1}\right] \\
\frac{\partial C_{\theta}}{\partial v}= & -\frac{1}{\theta}\left[(1-u)^{\theta}+(1-v)^{\theta}-(1-u)^{\theta}(1-v)^{\theta}\right]^{\frac{1}{\theta}-1}\left[-\theta(1-v)^{\theta-1}+\theta(1-v)^{\theta-1}(1-u)^{\theta}\right] \\
\frac{\partial C_{\theta}}{\partial \theta}= & \frac{1}{\theta^{2}}\left[(1-u)^{\theta}+(1-v)^{\theta}-(1-u)^{\theta}(1-v)^{\theta}\right]^{\frac{1}{\theta}} \log \left((1-u)^{\theta}+(1-v)^{\theta}-(1-u)^{\theta}(1-v)^{\theta}\right) \\
& -\frac{1}{\theta}\left[(1-u)^{\theta}+(1-v)^{\theta}-(1-u)^{\theta}(1-v)^{\theta}\right]^{\frac{1}{\theta}-1}\left[(1-u)^{\theta} \log (1-u)\right. \\
& \left.+(1-v)^{\theta} \log (1-v)-(1-u)^{\theta} \log (1-u)(1-v)^{\theta}-(1-v)^{\theta} \log (1-v)(1-u)^{\theta}\right]
\end{aligned}
$$


Frank: $C_{\theta}(u, v)=-\theta^{-1} \log \left[1+\left(e^{-\theta u}-1\right)\left(e^{-\theta v}-1\right) /\left(e^{-\theta}-1\right)\right]$

$$
\begin{aligned}
\frac{\partial C_{\theta}}{\partial u}= & -\theta^{-1} \frac{1}{1+\left(e^{-\theta u}-1\right)\left(e^{-\theta v}-1\right) /\left(e^{-\theta}-1\right)}\left(-\theta e^{-\theta u} \frac{e^{-\theta v}-1}{e^{-\theta}-1}\right) \\
\frac{\partial C_{\theta}}{\partial v}= & -\theta^{-1} \frac{1}{1+\left(e^{-\theta u}-1\right)\left(e^{-\theta v}-1\right) /\left(e^{-\theta}-1\right)}\left(-\theta e^{-\theta v} \frac{e^{-\theta u}-1}{e^{-\theta}-1}\right) \\
\frac{\partial C_{\theta}}{\partial \theta}= & \frac{1}{\theta^{2}} \log \left[1+\left(e^{-\theta u}-1\right)\left(e^{-\theta v}-1\right) /\left(e^{-\theta}-1\right)\right] \\
& -\frac{1}{\theta} \frac{1}{1+\left(e^{-\theta u}-1\right)\left(e^{-\theta v}-1\right) /\left(e^{-\theta}-1\right)} \\
& \frac{\left[-e^{-\theta u} u\left(e^{-\theta v}-1\right)-e^{-\theta v} v\left(e^{-\theta u}-1\right)\right]\left(e^{-\theta}-1\right)+\left(e^{-\theta u}-1\right)\left(e^{-\theta v}-1\right) e^{-\theta}}{\left(e^{-\theta}-1\right)^{2}}
\end{aligned}
$$

FGM: $C_{\theta}(u, v)=u v[1+\theta(1-u)(1-v)]$

$$
\begin{aligned}
& \frac{\partial C_{\theta}}{\partial u}=v\{[1+\theta(1-u)(1-v)]-u \theta(1-v)\} \\
& \frac{\partial C_{\theta}}{\partial v}=u\{[1+\theta(1-u)(1-v)]-v \theta(1-u)\} \\
& \frac{\partial C_{\theta}}{\partial \theta}=(1-u)(1-v) u v
\end{aligned}
$$

Bivariate normal: $C_{\theta}(u, v)=\Phi_{2}\left(\Phi^{-1}(u), \Phi^{-1}(v) ; \theta\right)$

$$
\begin{aligned}
\frac{\partial C_{\theta}}{\partial u} & =\frac{\partial C_{\theta}(u, v)}{\partial \Phi^{-1}(u)} \frac{\Phi^{-1}(u)}{\partial u} \\
& =\frac{\partial C_{\theta}(u, v)}{\partial \Phi^{-1}(u)} \frac{1}{\phi\left(\Phi^{-1}(u)\right)} \\
& =\Phi\left(\left(\Phi^{-1}(v)-\theta \Phi^{-1}(u)\right) / \sqrt{1-\theta^{2}}\right) \\
\frac{\partial C_{\theta}}{\partial v} & =\frac{\partial C_{\theta}(u, v)}{\partial \Phi^{-1}(v)} \frac{\Phi^{-1}(v)}{\partial v} \\
& =\frac{\partial C_{\theta}(u, v)}{\partial \Phi^{-1}(v)} \frac{1}{\phi\left(\Phi^{-1}(v)\right)} \\
& =\Phi\left(\left(\Phi^{-1}(u)-\theta \Phi^{-1}(v)\right) / \sqrt{1-\theta^{2}}\right) \\
\frac{\partial C_{\theta}}{\partial \theta} & =\phi_{2}\left(\Phi^{-1}(u), \Phi^{-1}(v) ; \theta\right)
\end{aligned}
$$

(compare: Lee, 1983). Let us now consider derivatives of univariate pmfs and cdfs. Beforehand, it is important state that $\frac{\partial F_{1}}{\partial \boldsymbol{\delta}_{\mathbf{1}}}=\frac{\partial}{\partial \boldsymbol{\delta}_{\mathbf{1}}} \Phi\left(-\eta_{1}\right)=\frac{\partial F_{1}}{\partial \eta_{1}} \frac{\partial \eta_{1}}{\partial \boldsymbol{\delta}_{\mathbf{1}}}=-\phi\left(-\eta_{1}\right) \mathbf{X}_{\mathbf{1}}$. The relationship between the 
discrete univariate cdfs and pmfs can be mathematically described as

$$
\begin{aligned}
F(x) & =\sum_{0}^{x} P\left(X=x_{i}\right) \\
& =\sum_{0}^{x} f\left(x_{i}\right)
\end{aligned}
$$

Hence, it follows that

$$
\frac{\partial F(x)}{\partial \theta}=\sum_{0}^{x} \frac{\partial f\left(x_{i}\right)}{\partial \theta}
$$

where $\theta$ is a parameter which defines the distribution. Thus, we will be now considering the derivatives of pmfs with respect to their parameters. The derivations for the Poisson, negative binomial, Delaporte, Poisson inverse Gaussian and Sichel are listed below.

Poisson: $f\left(y_{2}\right)=\frac{e^{-\mu} \mu_{2}}{\Gamma\left(y_{2}+1\right)}$ where $\mu=e^{\eta_{2}}$ and $\eta_{2}$ is the linear predictor of the outcome equation.

$$
\frac{\partial f_{2}}{\partial \delta_{2}}=f_{2}\left(-\mu+y_{2}\right) \mathbf{X}_{2}
$$

Negative binomial: $f\left(y_{2}\right)=\frac{\Gamma\left(y_{2}+\frac{1}{\sigma}\right)}{\Gamma\left(y_{2}+1\right) \Gamma\left(\frac{1}{\sigma}\right)} \frac{(\mu \sigma)^{y_{2}}}{(\mu \sigma+1)^{y_{2}+\frac{1}{\sigma}}}$ where $\mu=e^{\eta_{2}}$ and $\eta_{2}$ is the linear predictor of the outcome equation. Note that $\Gamma^{\prime}(x)=\psi(x) \Gamma(x)$ where $\psi(x)=\frac{d}{d x} \log \Gamma(x)$.

$$
\begin{aligned}
\frac{\partial f_{2}}{\partial \delta_{2}}= & f_{2}\left[y_{2}(\mu \sigma)^{-1} \mu \sigma-\left(y_{2}+\frac{1}{\sigma}\right)(\mu \sigma+1)^{-1} \mu \sigma\right] \mathbf{X}_{\mathbf{2}} \\
\frac{\partial f_{2}}{\partial \sigma}= & f_{2}\left[\psi\left(y_{2}+\frac{1}{\sigma}\right)\left(-\frac{1}{\sigma^{2}}\right)+y_{2}(\mu \sigma)^{-1} \mu-\left(\psi\left(\frac{1}{\sigma}\right)\left(-\frac{1}{\sigma^{2}}\right)+\left(-\frac{1}{\sigma^{2}} \log (\mu \sigma+1)\right.\right.\right. \\
& \left.\left.\left.+\left(y_{2}+\frac{1}{\sigma}\right) \frac{1}{\mu \sigma+1} \mu\right)\right)\right]
\end{aligned}
$$

Delaporte: $f\left(y_{2}\right)=\frac{e^{-\mu \nu}}{y_{2} !}\left[1+\mu \sigma(1-\nu)^{-\frac{1}{\sigma}}\right] S$ where $S=\sum_{j=0}^{y_{2}}\left(\begin{array}{c}y_{2} \\ j\end{array}\right) \frac{\mu^{y_{2}} \nu^{y_{2}-j}}{y_{2} !}\left[1+\frac{1}{\sigma(1-\nu)}\right] \Gamma\left(\frac{1}{\sigma} j\right), \mu=e^{\eta_{2}}$ 
and $\eta_{2}$ is the linear predictor of the outcome equation.

$$
\begin{aligned}
\frac{\partial f_{2}}{\partial \delta_{2}} & =f_{2}\left[(-\mu \nu)-\frac{1}{\sigma} \mu \sigma(1-\nu)(1+\mu \sigma(1-\nu))^{-1}+S_{\boldsymbol{\delta}_{\mathbf{2}}}^{\prime} / S\right] \mathbf{X}_{\mathbf{2}} \\
\frac{\partial f_{2}}{\partial \sigma} & =f_{2}\left[d^{\prime} / d+\psi\left(\frac{1}{\sigma}\right)\left(\frac{1}{\sigma^{2}}\right)+S_{\sigma}^{\prime} / S\right] \\
\frac{\partial f_{2}}{\partial \nu} & =f_{2}\left[-\mu+\mu(1+\mu \sigma(1-\nu))^{-1}+S_{\nu}^{\prime} / S\right]
\end{aligned}
$$

where $d=[1+\mu \sigma(1-\nu)]^{-\frac{1}{\sigma}}$ and $d^{\prime}=\left\{\frac{1}{\sigma^{2}} \log [1+\mu \sigma(1-\nu)]-\frac{1}{\sigma} \frac{1}{1+\mu \sigma(1-\nu)} \mu(1-\nu)\right\} d$ is the derivative of $d$ with respect to $\sigma$. $S^{\prime}$ indicates the derivative of $S$ and its subscript indicates the parameter with respect to which it was calculated.

The derivatives of Poisson inverse Gaussian $\left(f\left(y_{2}\right)=\left(\frac{2 \alpha}{\pi}\right)^{\frac{1}{2}} \frac{\mu^{y_{2}} e^{\frac{1}{\sigma}} K_{y_{2}-\frac{1}{2}}(\alpha)}{(\alpha \sigma)^{y_{2}} y_{2} !}\right.$, where $\mu=e^{\eta_{2}}, \alpha^{2}=$ $\frac{1}{\sigma^{2}}+\frac{2 \mu}{\sigma}$ and $K$ is the Bessel function of third kind) and Sichel $\left(f\left(y_{2}\right)=\frac{\mu^{y_{2}} K_{y_{2}+\nu}(\alpha)}{(\alpha \sigma)^{y_{2}+\nu_{2} ! K_{\nu}\left(\frac{1}{\sigma}\right)}}\right.$, where $\mu=e^{\eta_{2}}, \alpha^{2}=\frac{1}{\sigma^{2}}+\frac{2 \mu}{\sigma}$ and $K$ is the Bessel function of third kind) distributions with respect to their parameters were approximated numerically due to numerical difficulties of approximating the Bessel function of third kind. For the Poisson inverse Gaussian distribution, the derivatives of pmfs with respect to their parameters are as follows

$$
\begin{aligned}
\frac{\partial f_{2}}{\partial \delta_{2}} & =\frac{f\left(y_{2} \mid \eta_{2}+\epsilon, \sigma\right)-f\left(y_{2} \mid \eta_{2}, \sigma\right)}{\epsilon} \mathbf{X}_{\mathbf{2}} \\
\frac{\partial f_{2}}{\partial \sigma} & =\frac{f\left(y_{2} \mid \eta_{2}, \sigma+\epsilon\right)-f\left(y_{2} \mid \eta_{2}, \sigma\right)}{\epsilon},
\end{aligned}
$$

and for Sichel

$$
\begin{aligned}
& \frac{\partial f_{2}}{\partial \delta_{2}}=\frac{f\left(y_{2} \mid \eta_{2}+\epsilon, \sigma, \nu\right)-f\left(y_{2} \mid \eta_{2}, \sigma, \nu\right)}{\epsilon} \mathbf{X}_{\mathbf{2}} \\
& \frac{\partial f_{2}}{\partial \sigma}=\frac{f\left(y_{2} \mid \eta_{2}, \sigma+\epsilon, \nu\right)-f\left(y_{2} \mid \eta_{2}, \sigma, \nu\right)}{\epsilon} \\
& \frac{\partial f_{2}}{\partial \nu}=\frac{f\left(y_{2} \mid \eta_{2}, \sigma, \nu+\epsilon\right)-f\left(y_{2} \mid \eta_{2}, \sigma, \nu\right)}{\epsilon},
\end{aligned}
$$

where $\epsilon$ is set to $10^{-7}$. 
Appendix G

Simulations for likelihood function using optim for numerical and anaytical gradient and specification with and without instrumental variable 


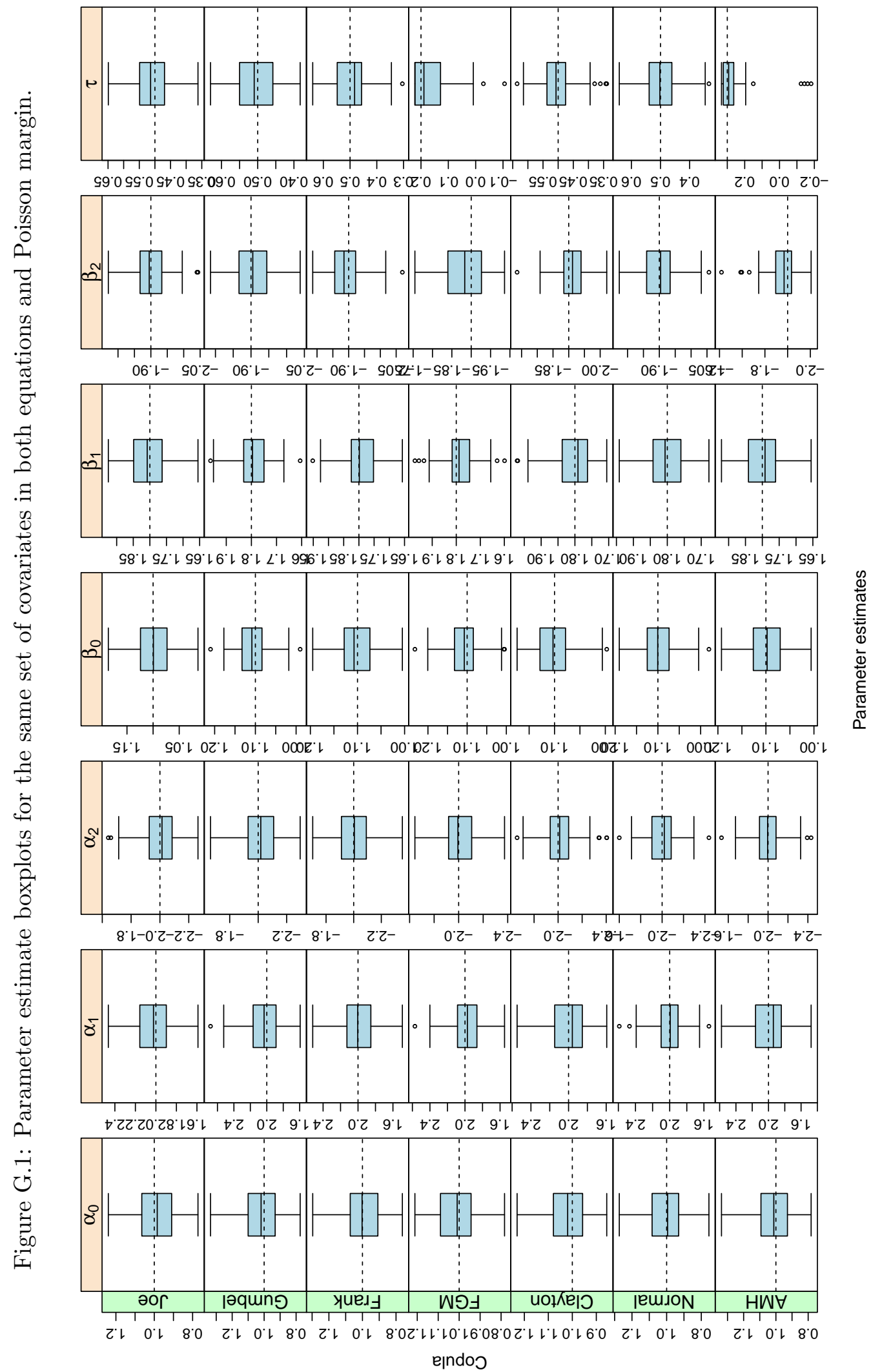




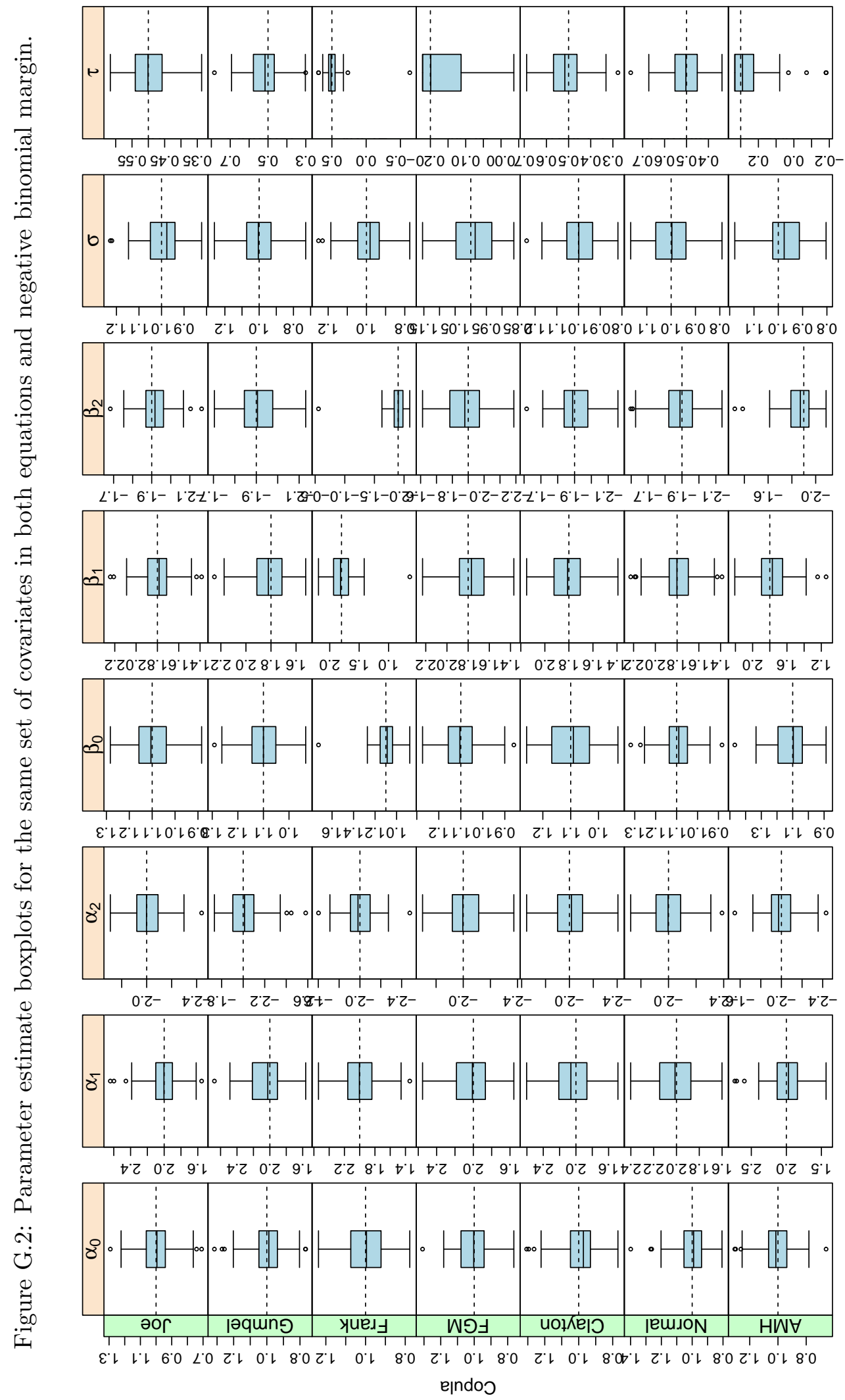




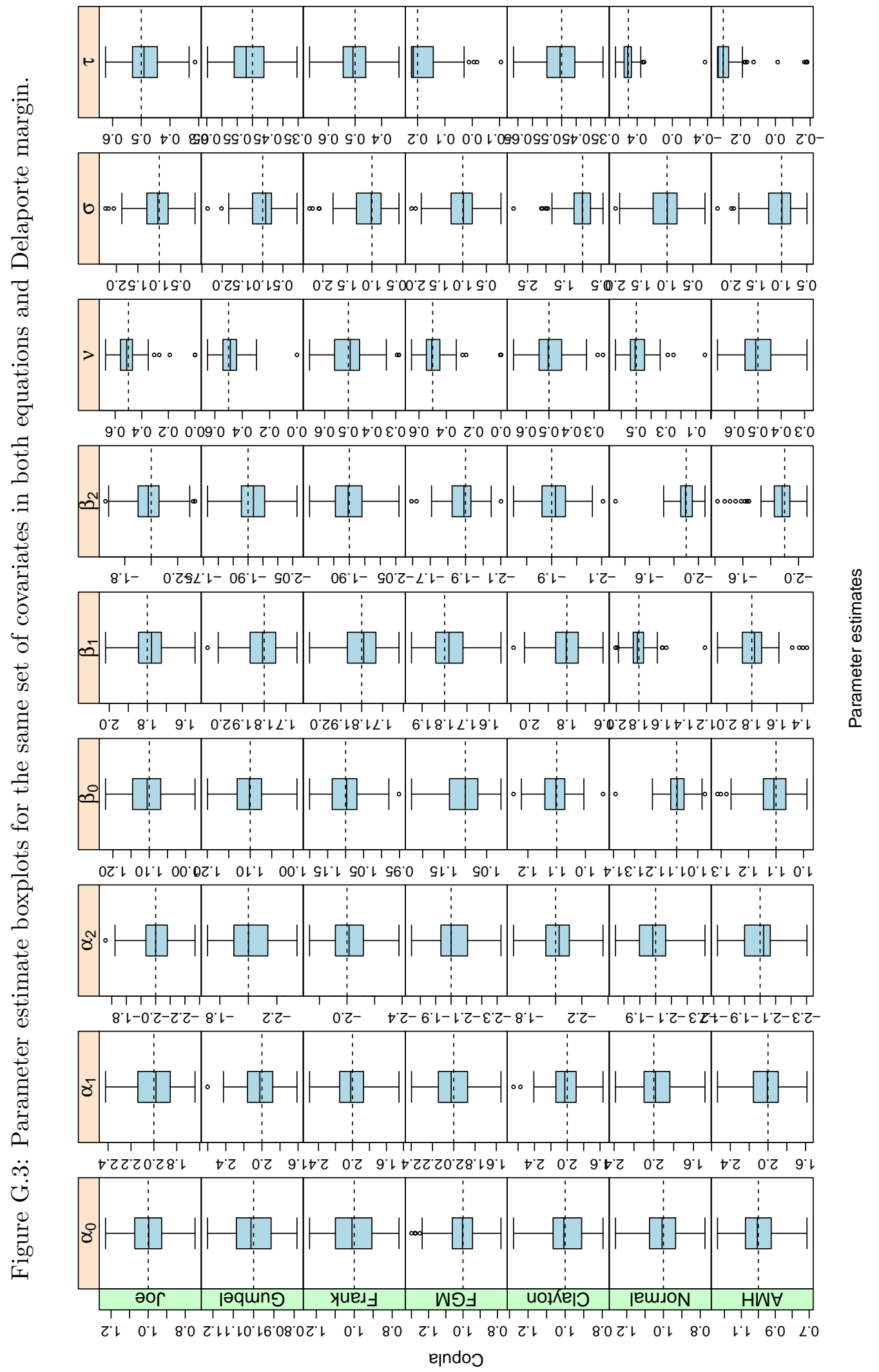



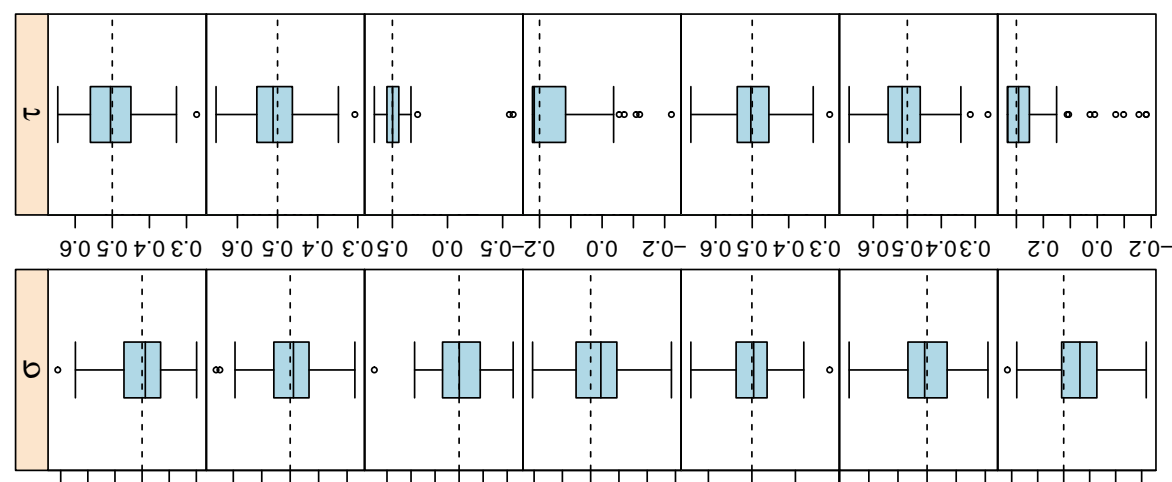

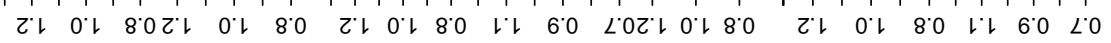

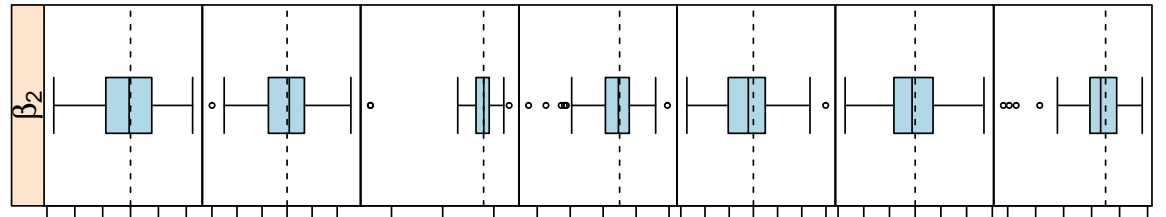

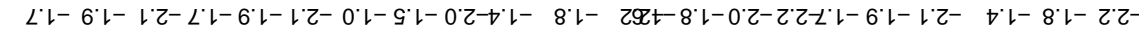

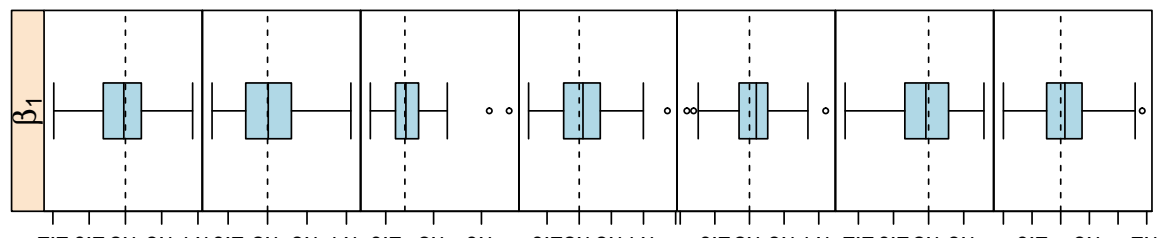

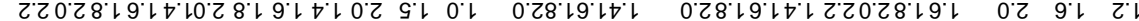

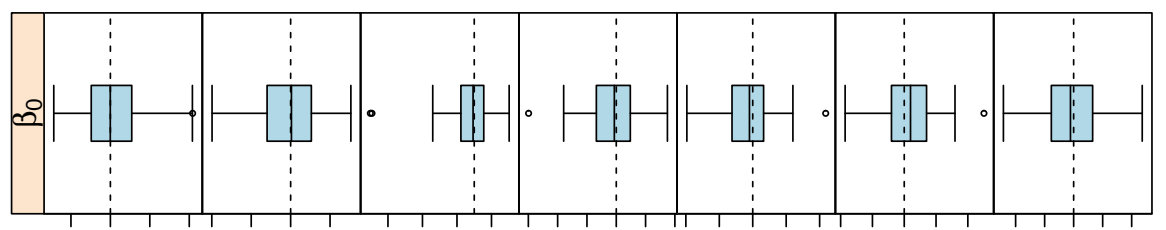

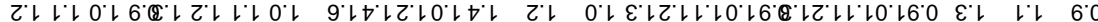
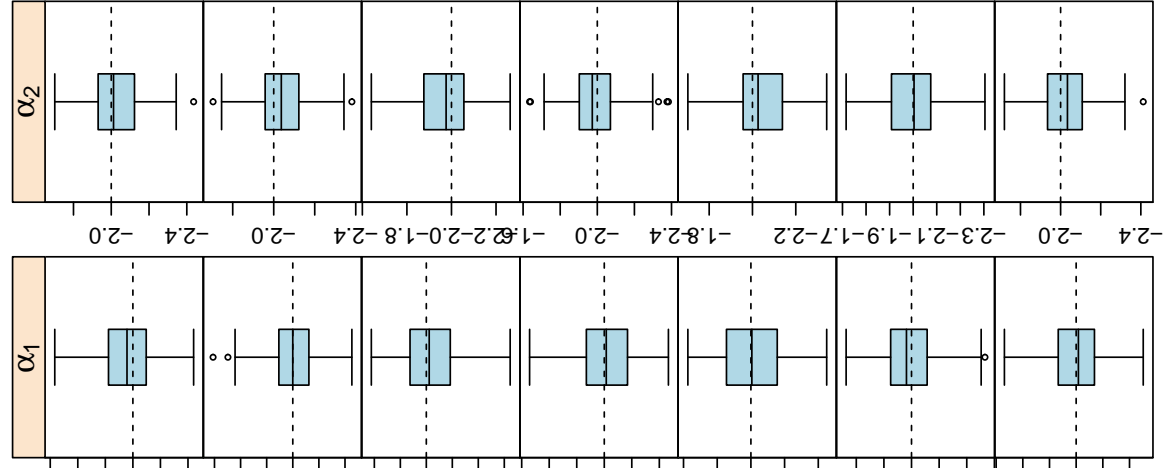

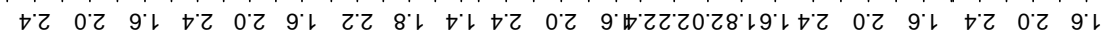

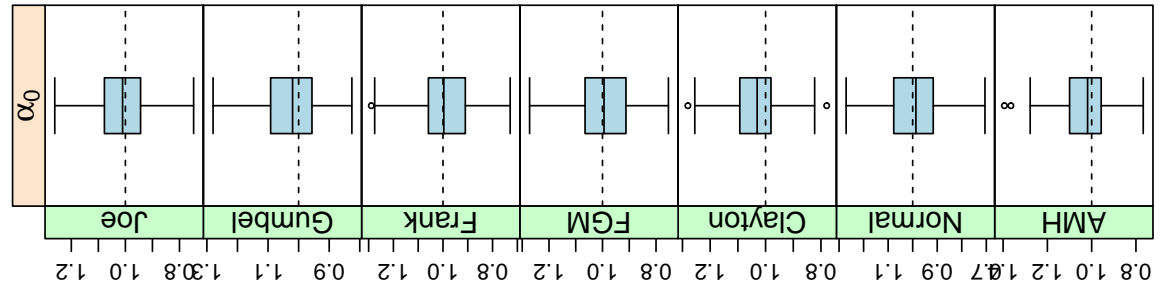

ejndoo 


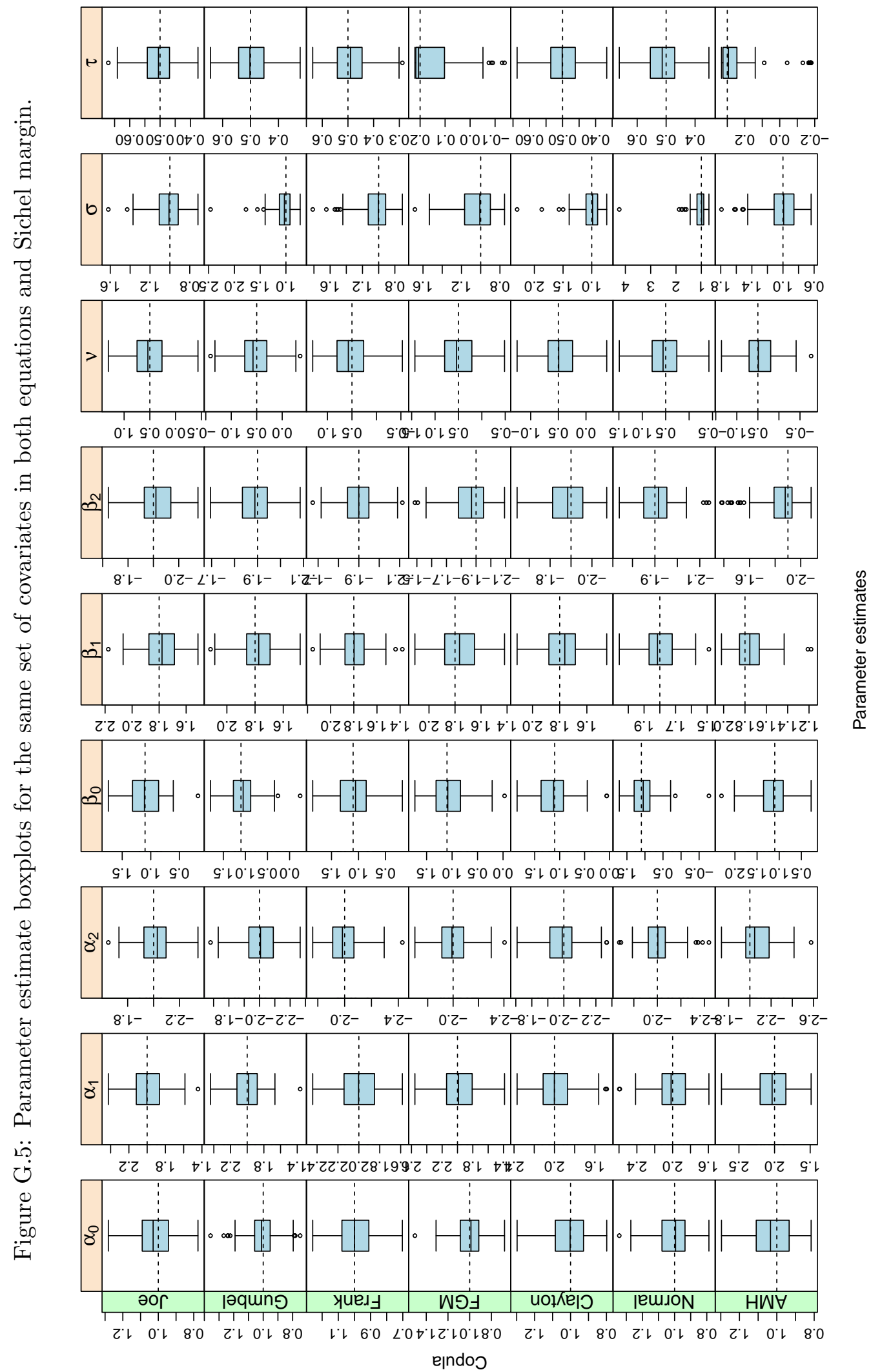




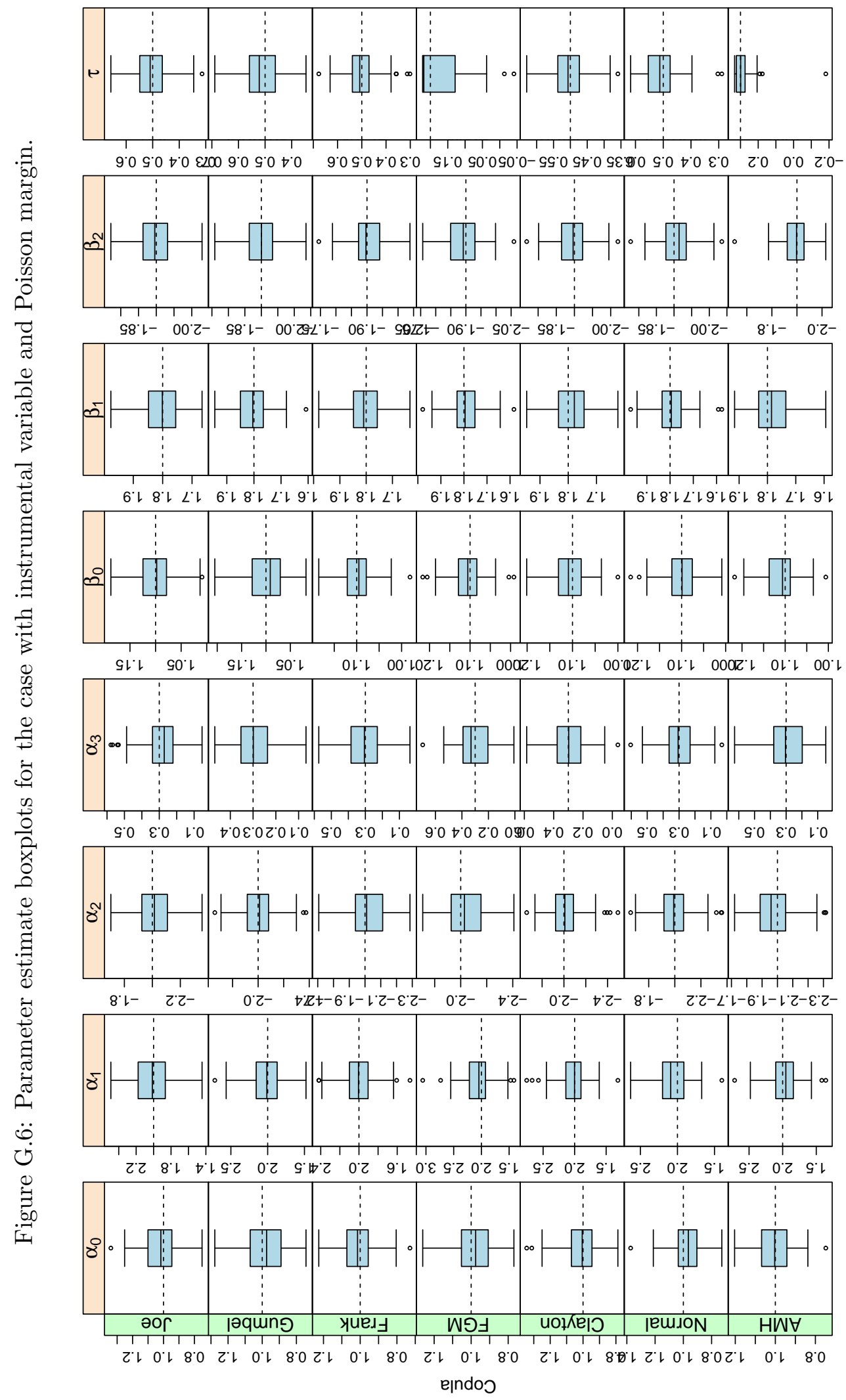

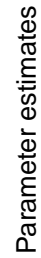




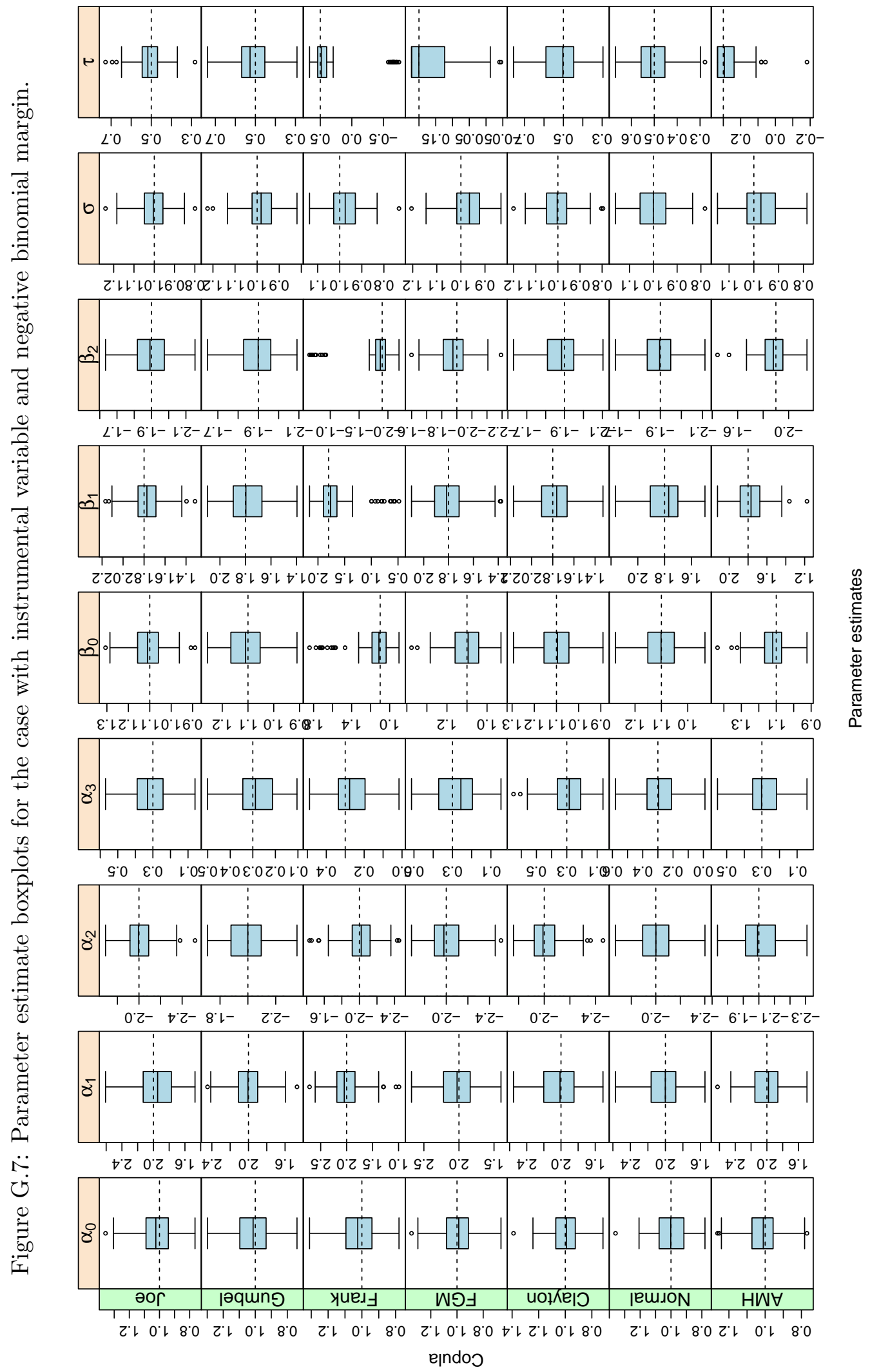




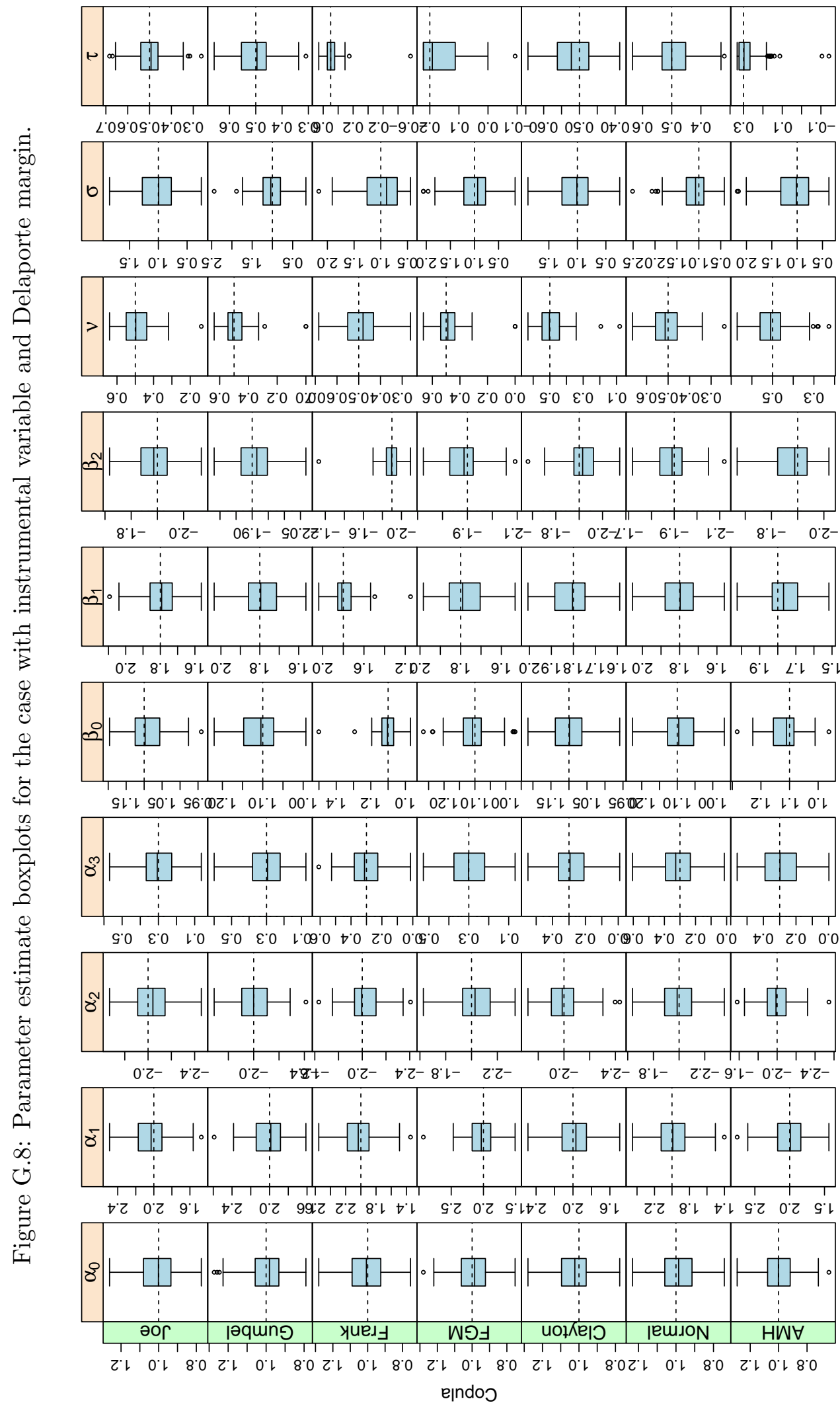

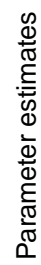




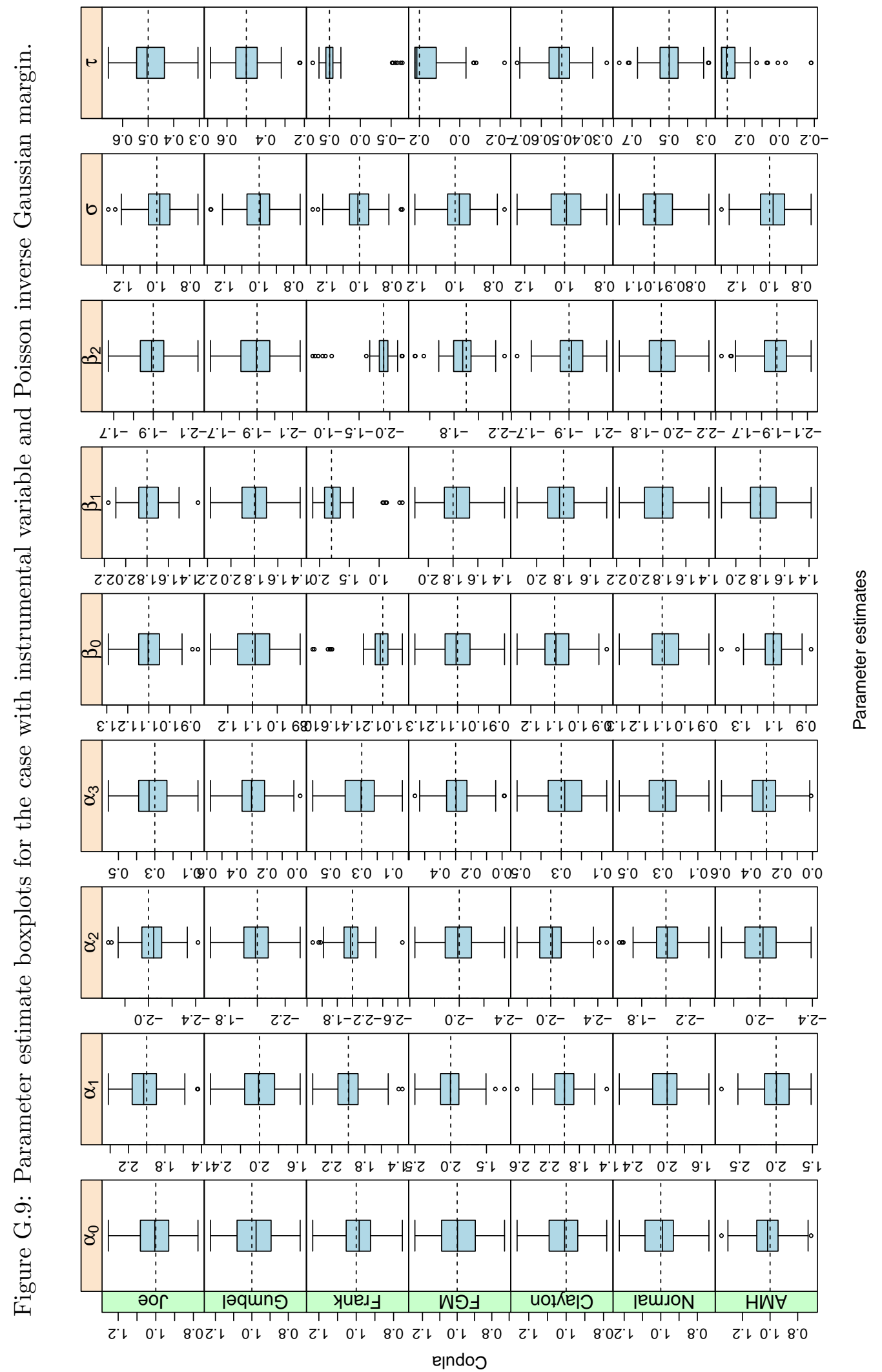




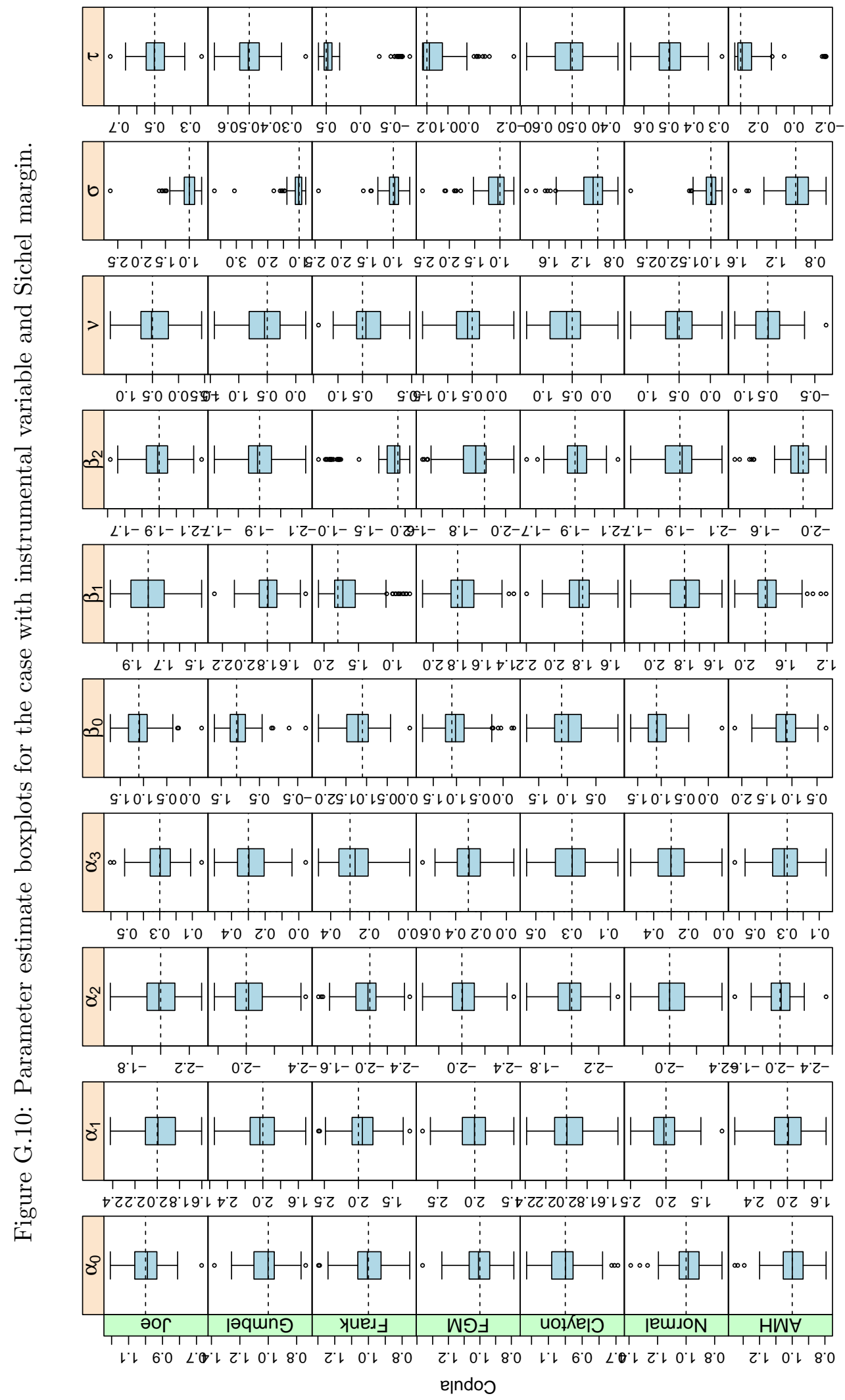



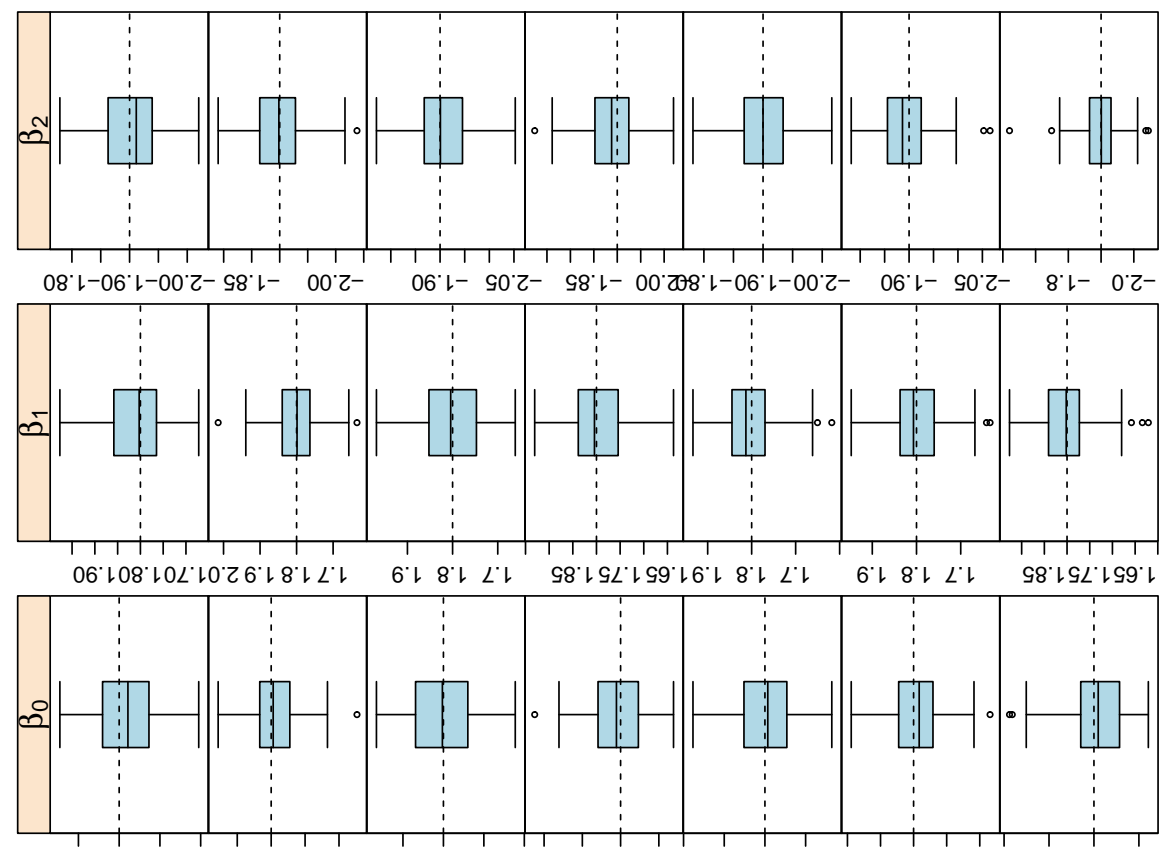

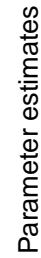
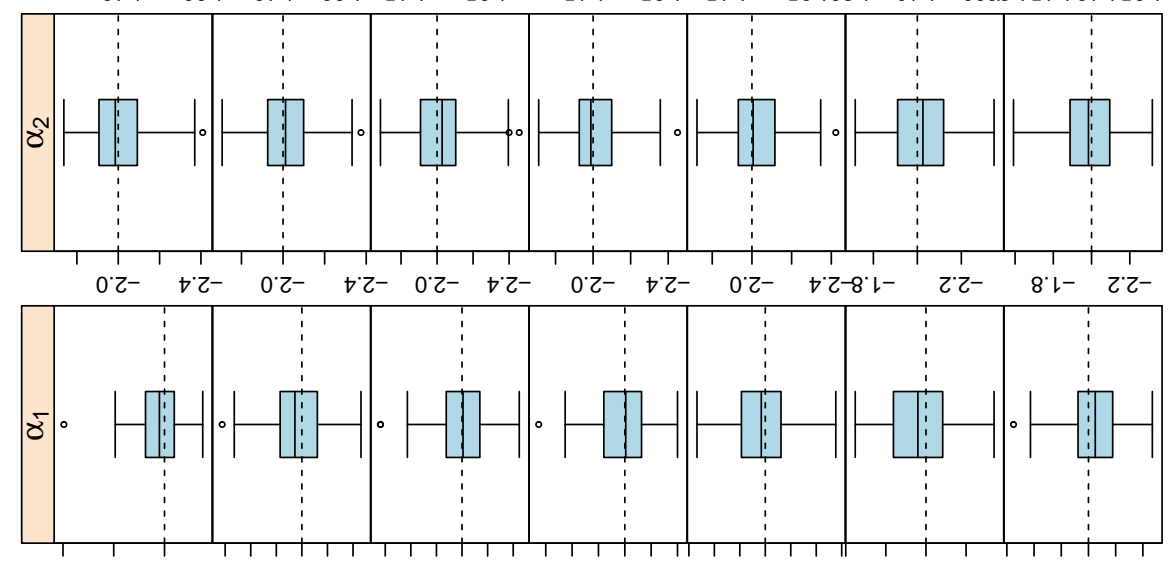

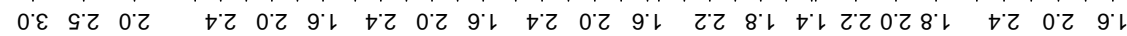

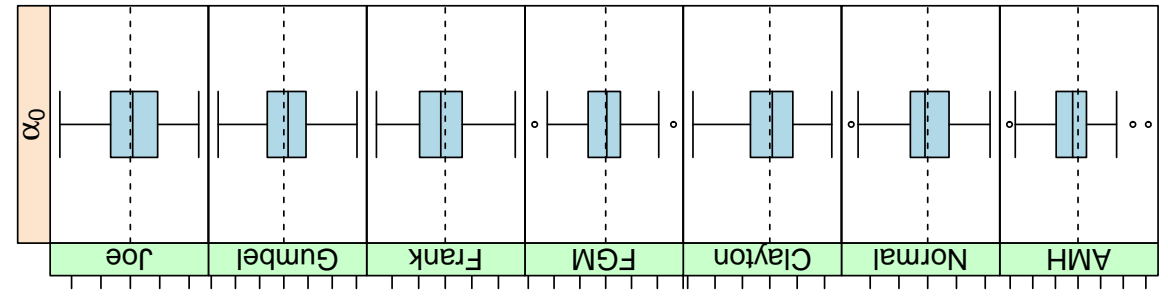

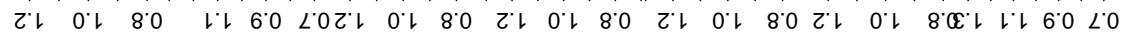

ejndoo 

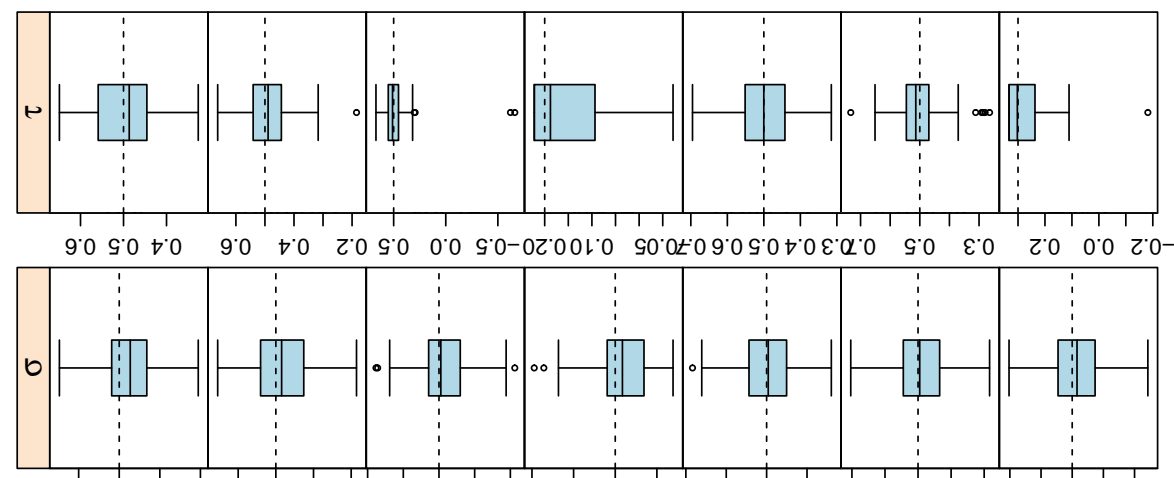

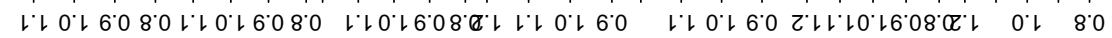
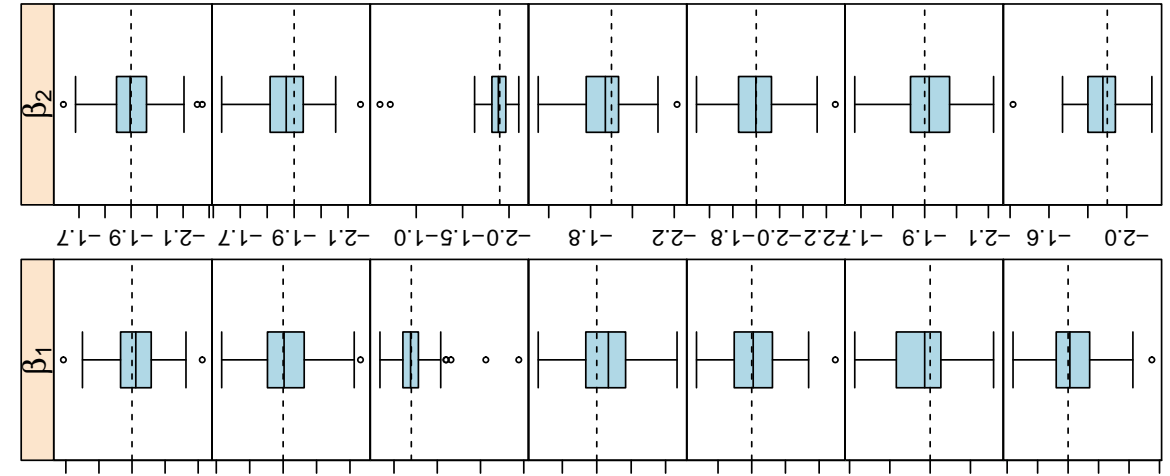

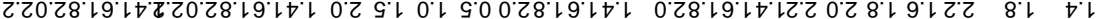

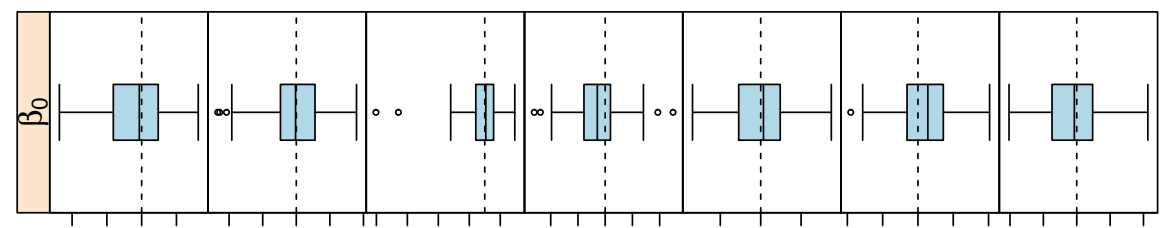

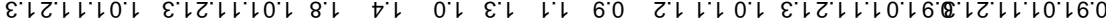
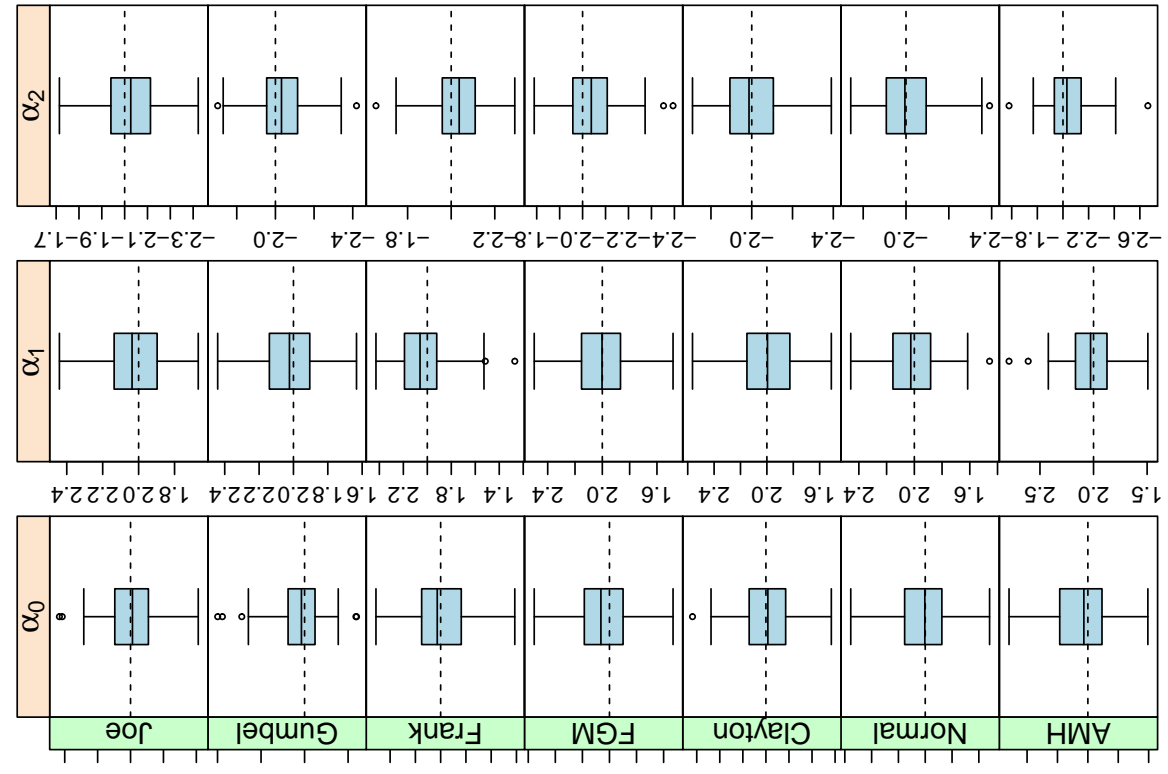

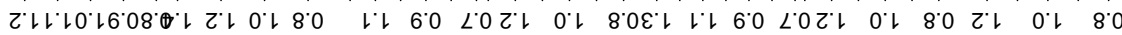
ejndoo 


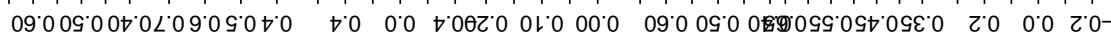

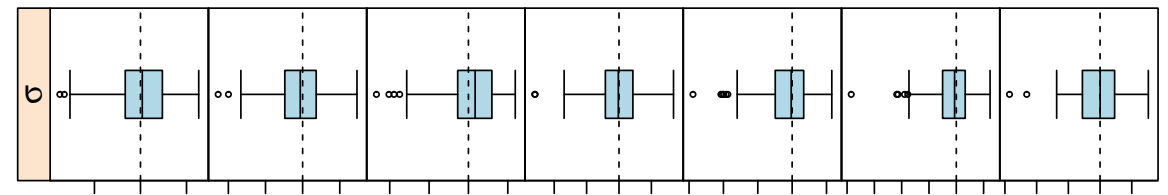

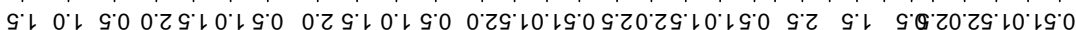

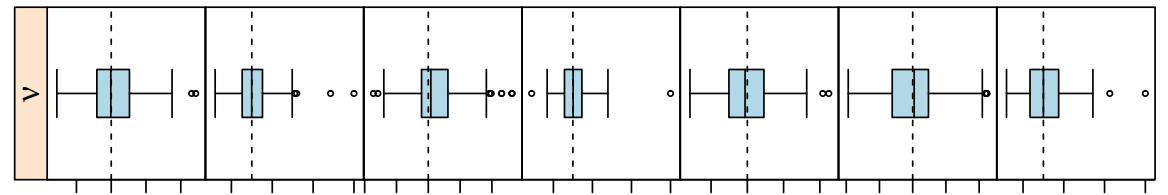

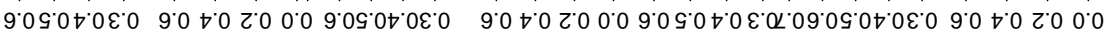
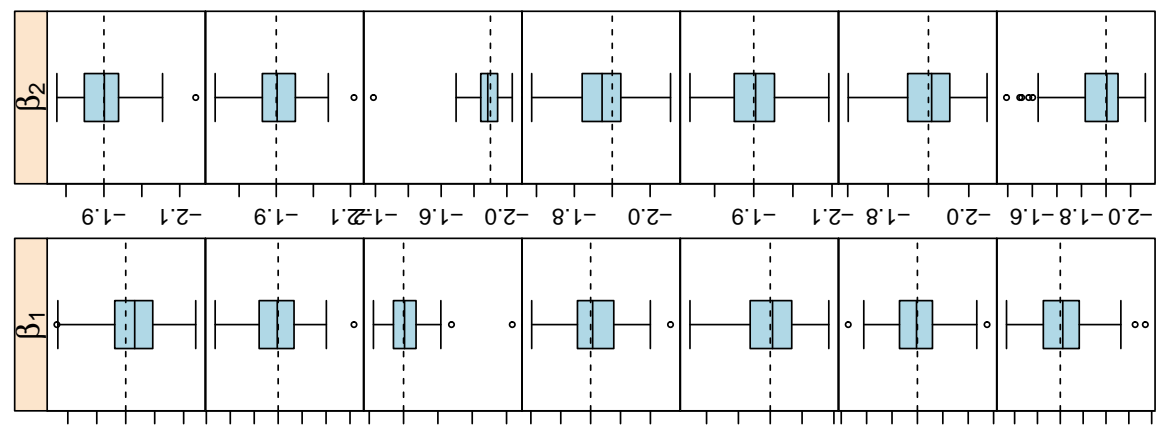

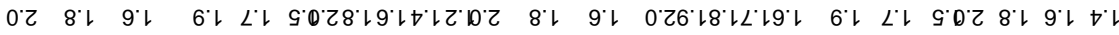

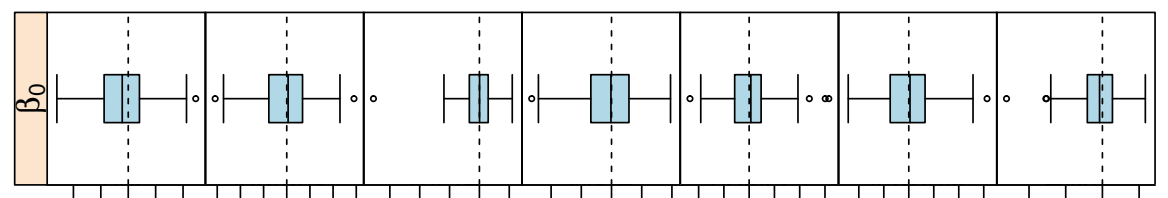

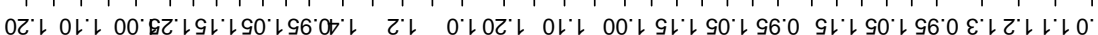
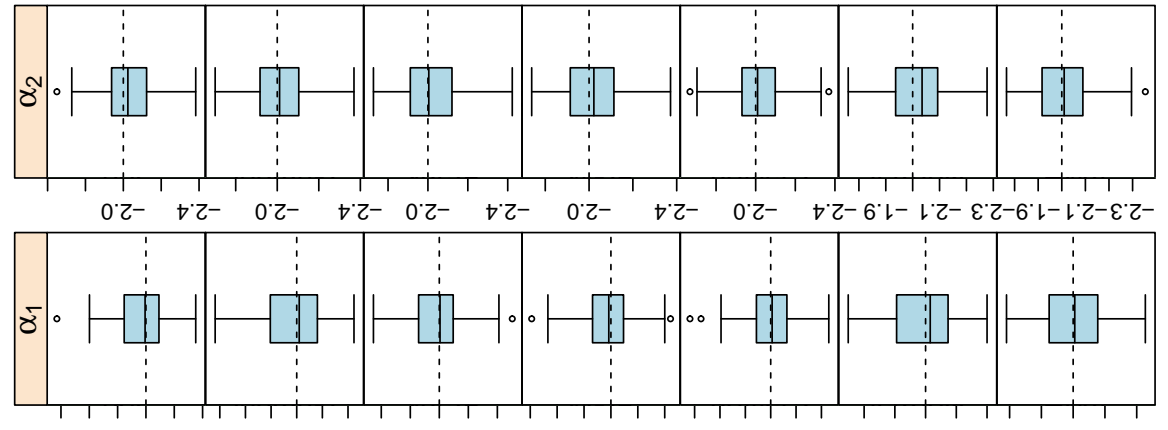

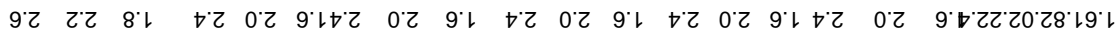

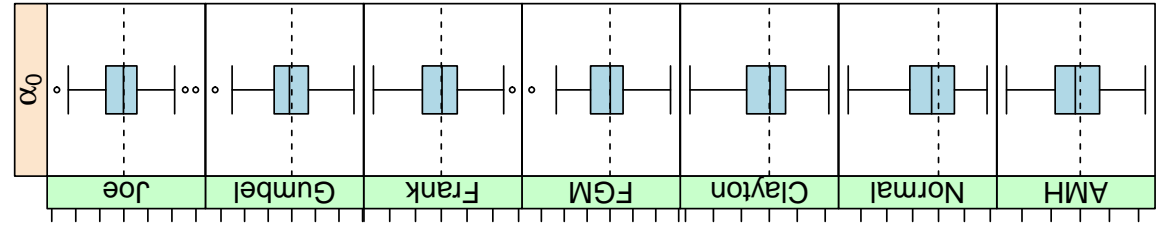

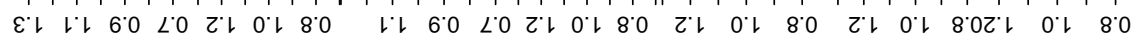
ejndoo 


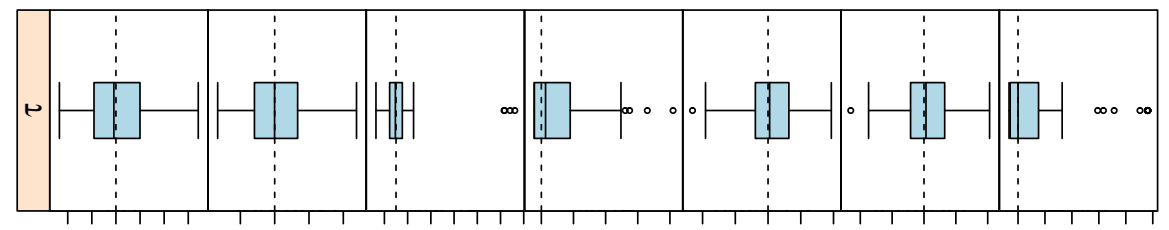

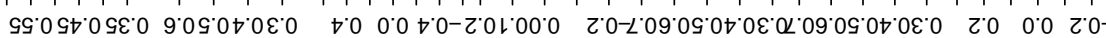

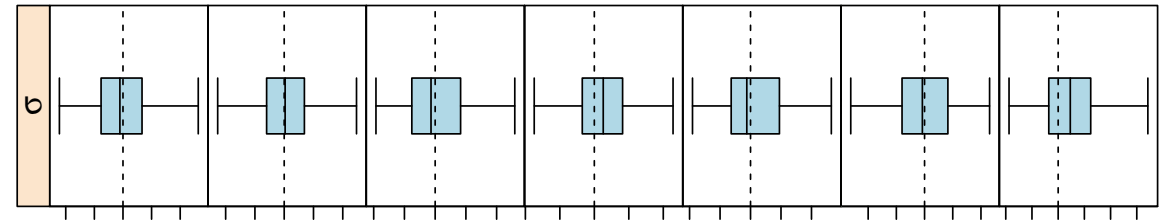

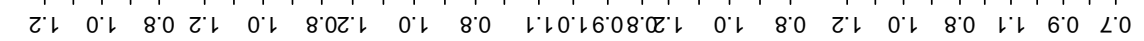
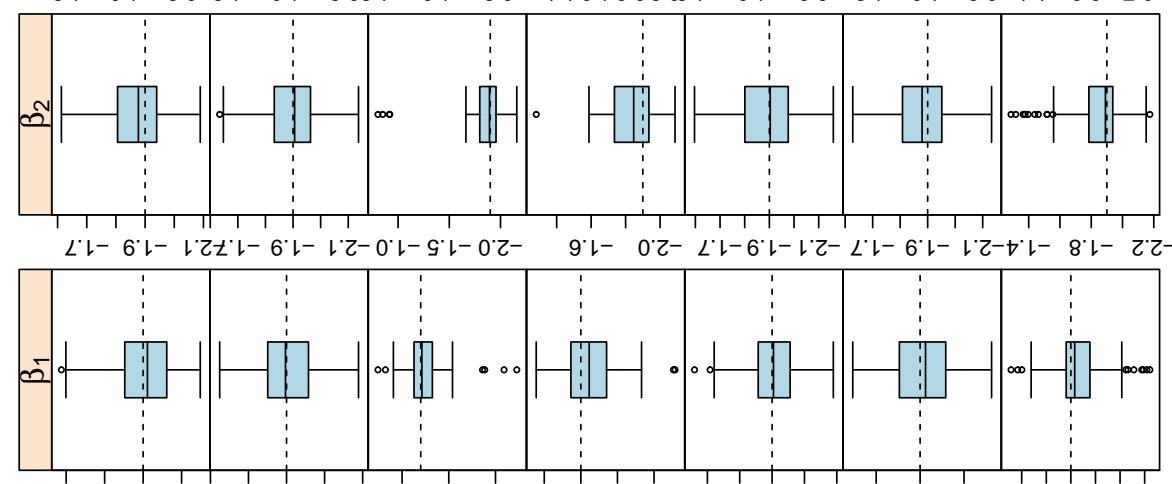

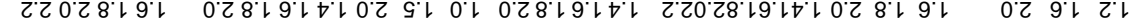
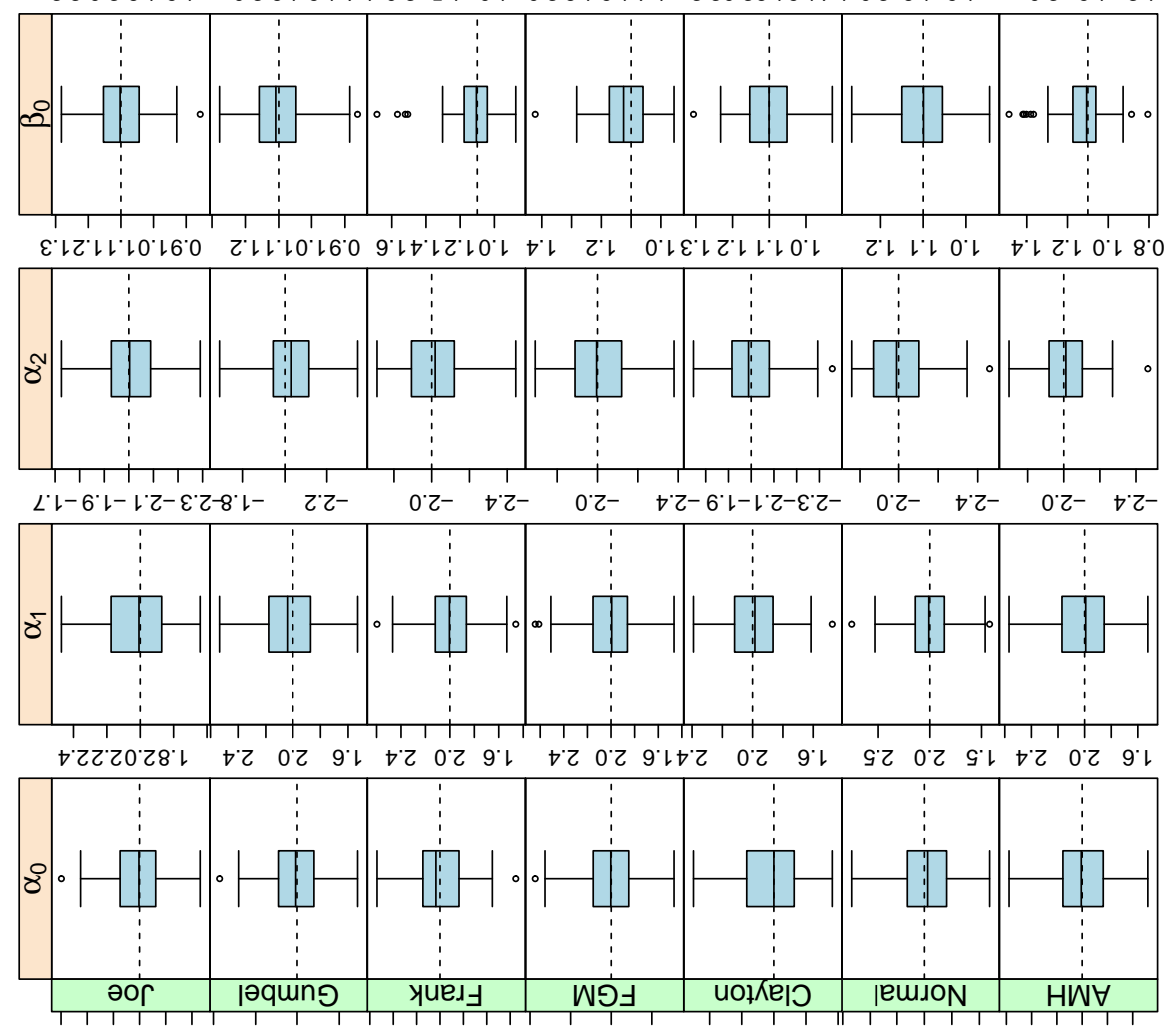

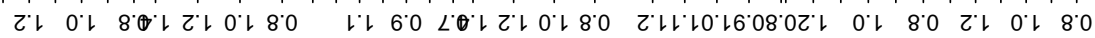
ejndoo 


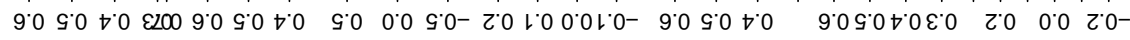

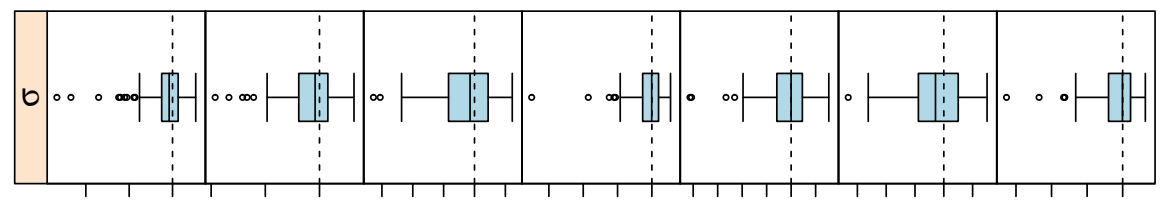

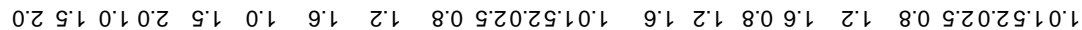

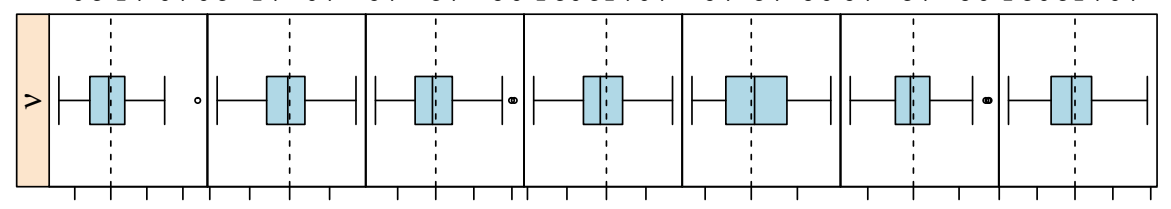

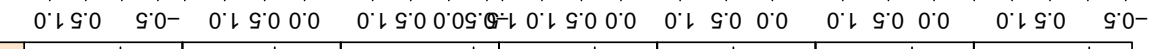
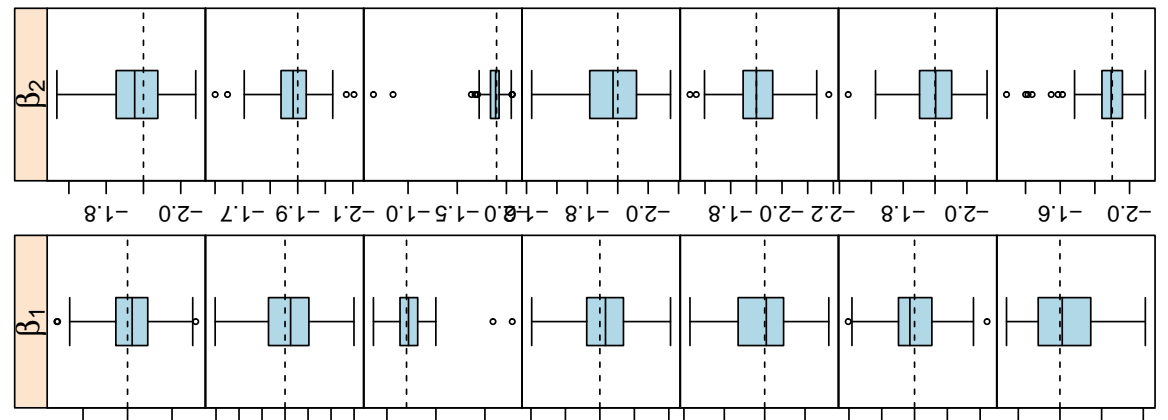

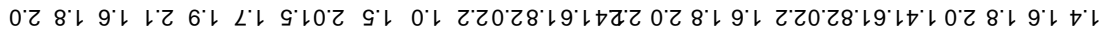

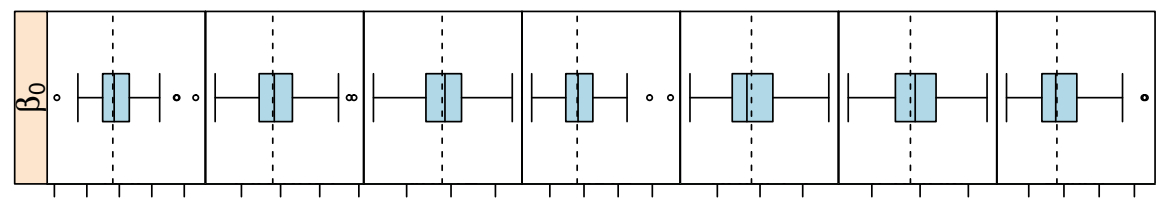

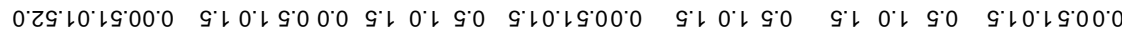
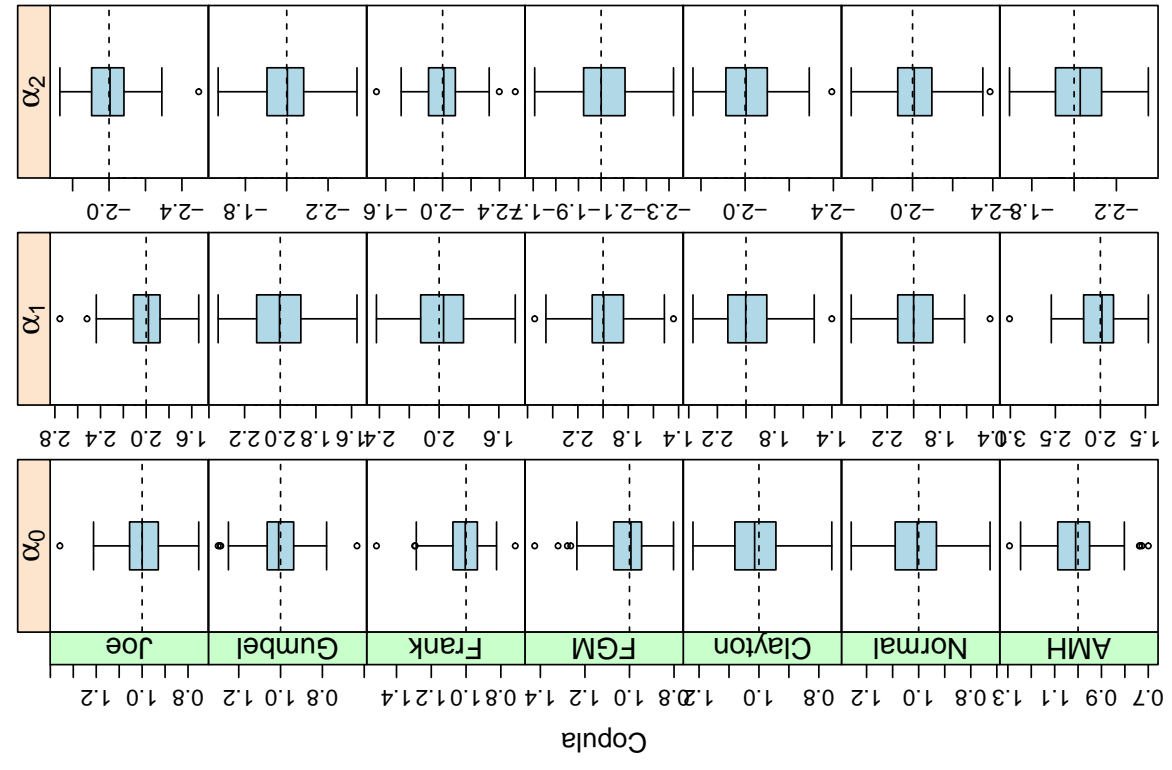


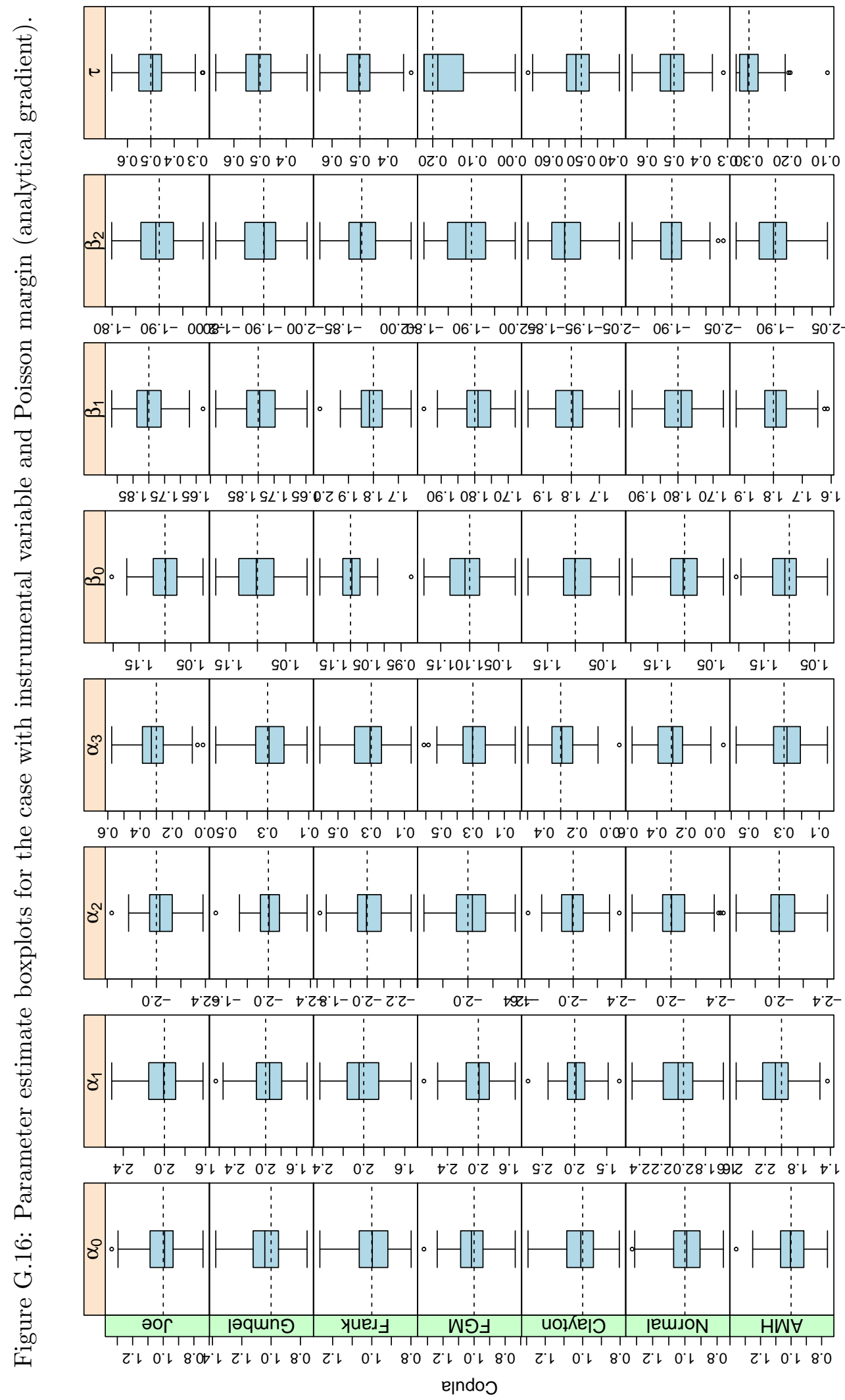




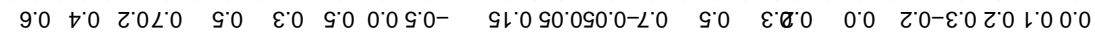

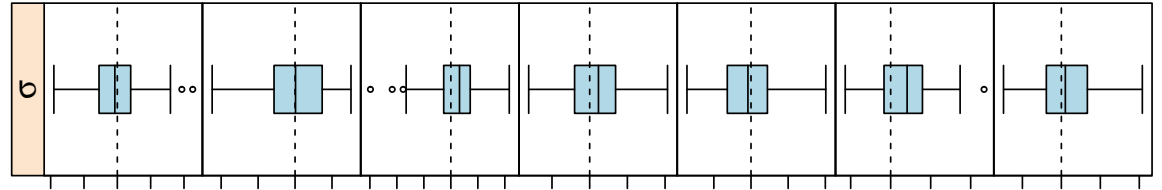

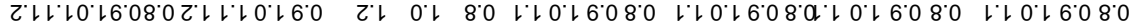
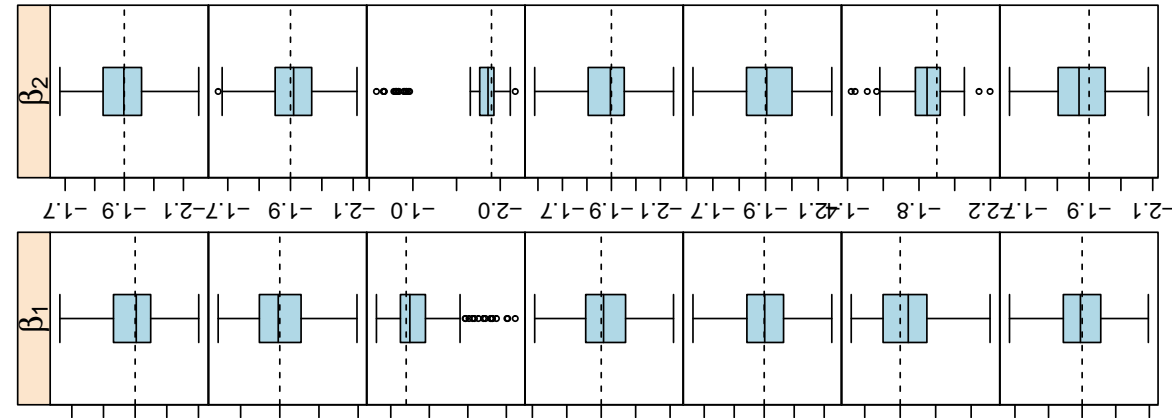

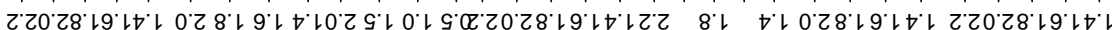

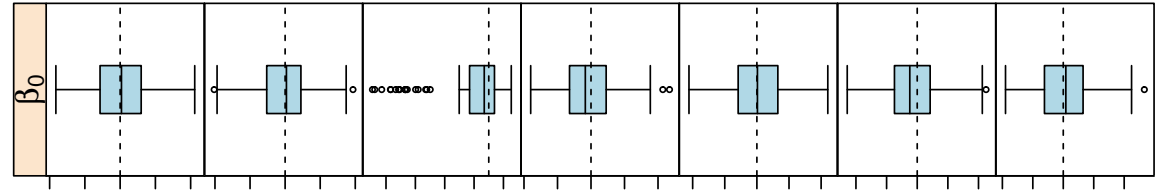

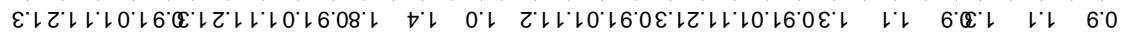
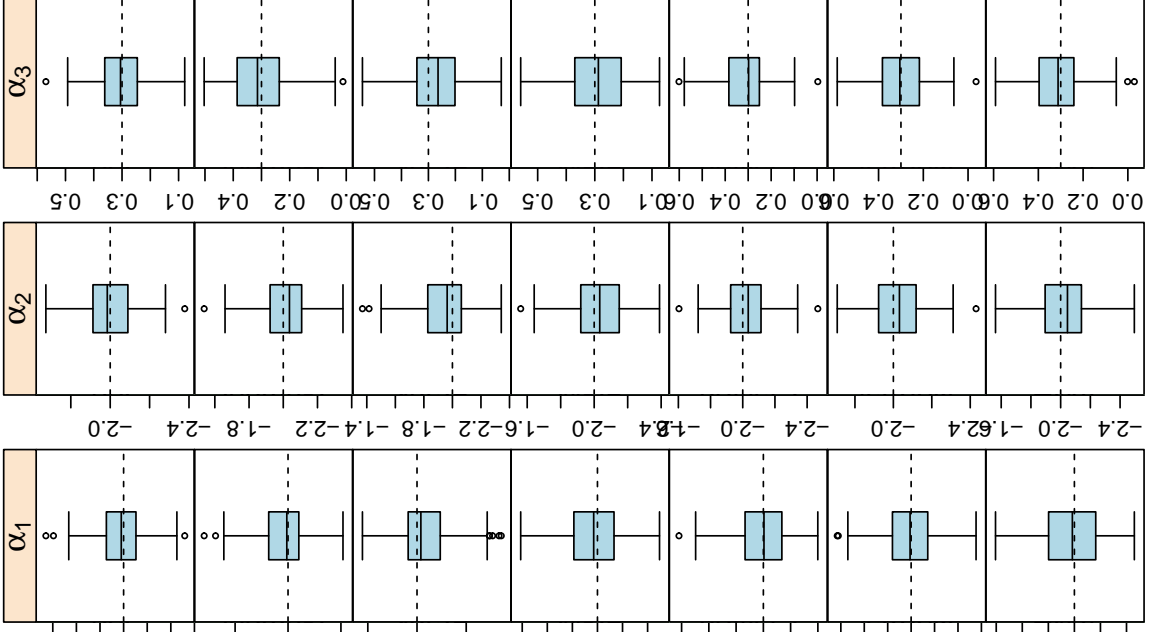

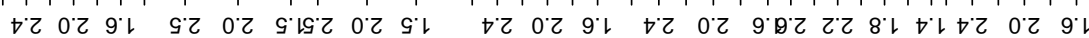

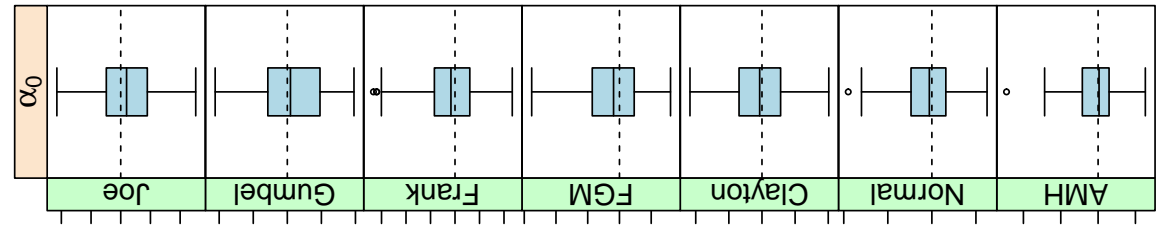

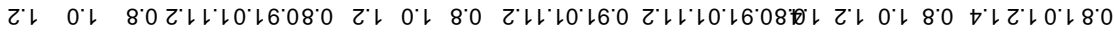
ejndoo 


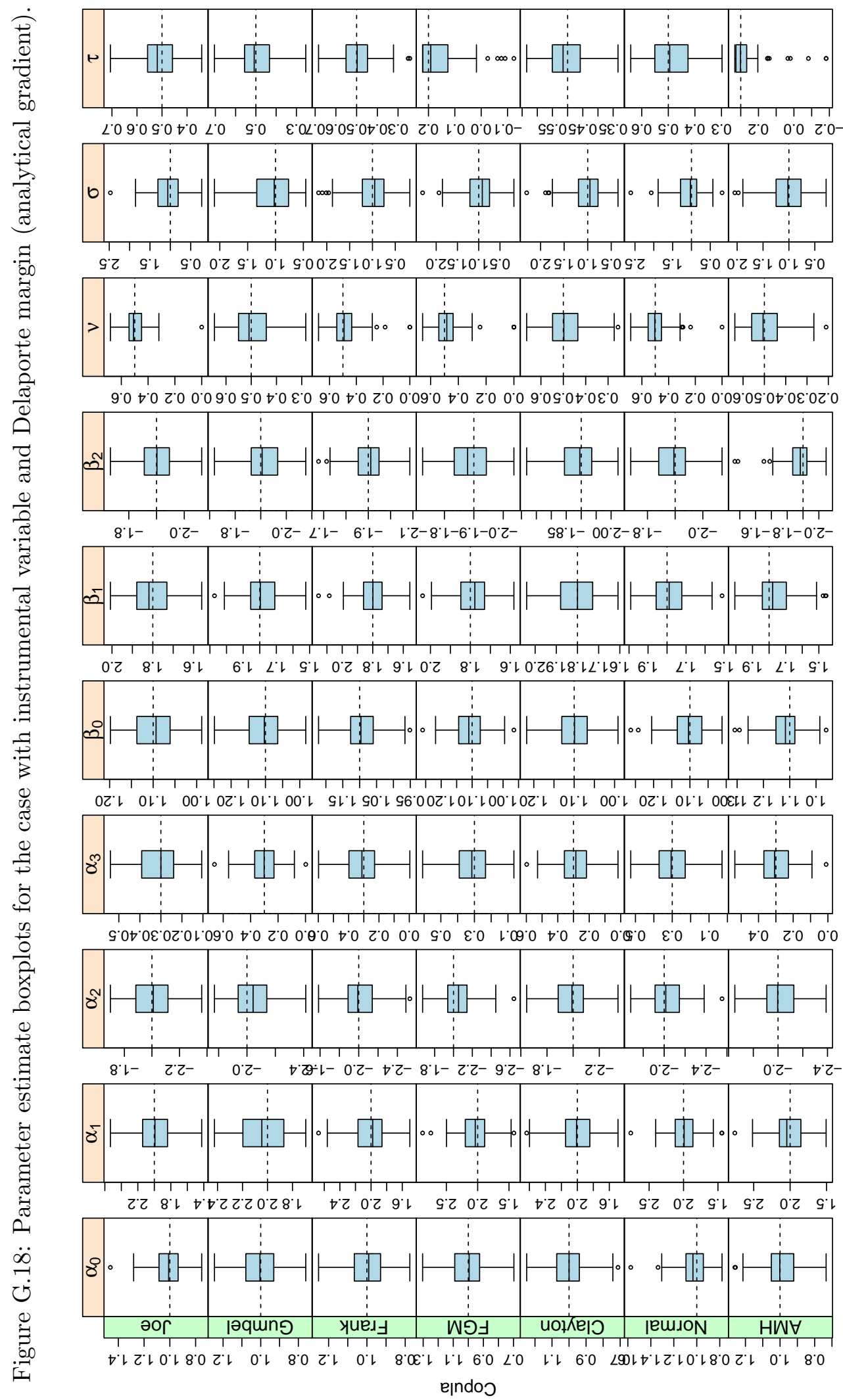




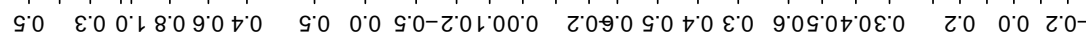
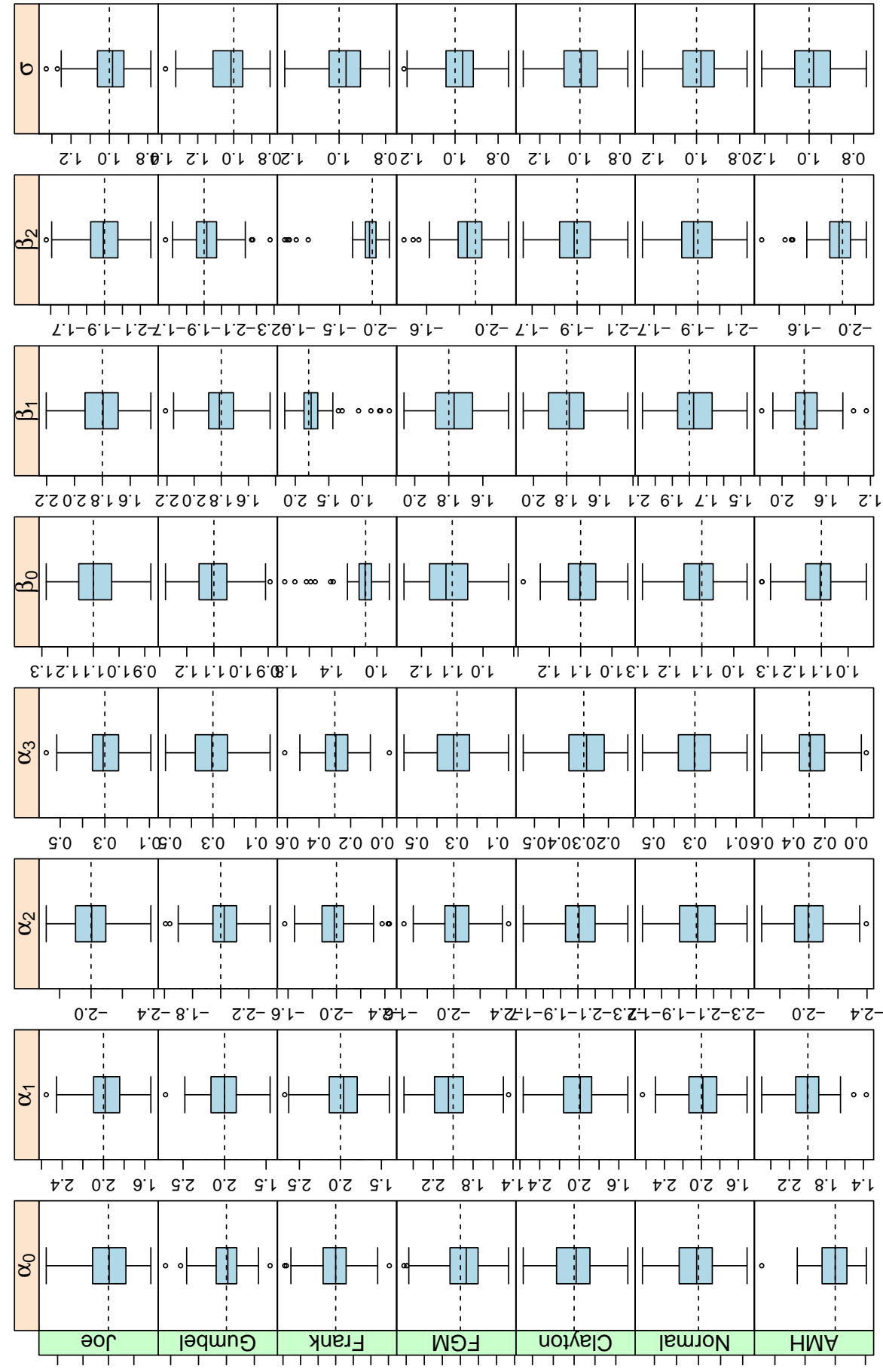

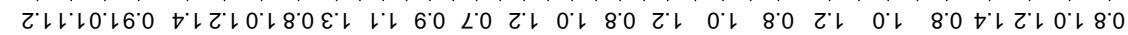
ejndo 


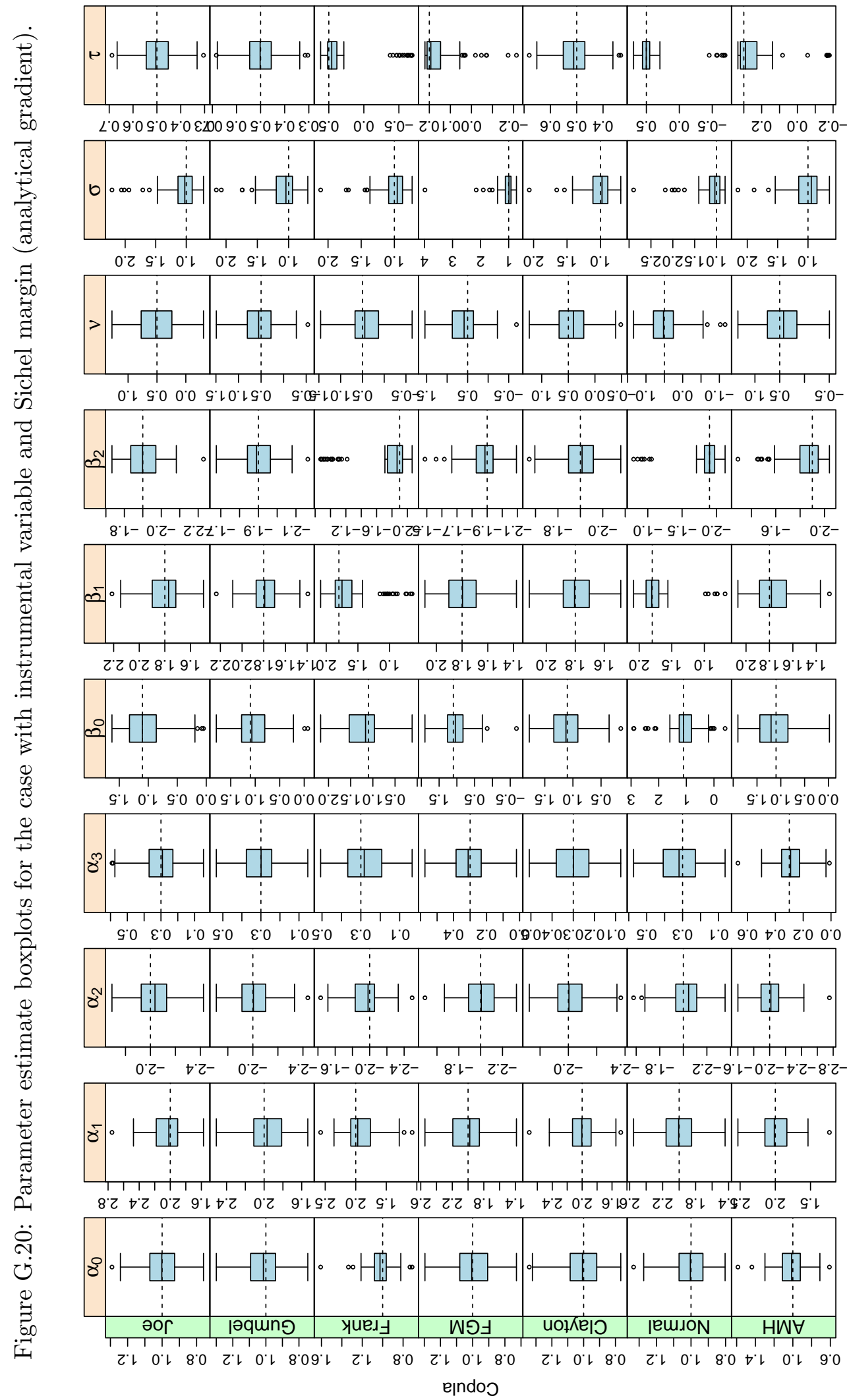




\section{Appendix $\mathbf{H}$}

\section{General form of analytical Hessian}

$$
\begin{aligned}
\frac{\partial^{2} \ell}{\partial \boldsymbol{\delta}_{\mathbf{1}} \partial \boldsymbol{\delta}_{\mathbf{1}}} & =\sum_{0}-\frac{1}{F_{1}^{2}}\left(\frac{\partial F_{1}}{\partial \boldsymbol{\delta}_{\mathbf{1}}}\right)+\frac{1}{F_{1}} \frac{\partial^{2} F_{1}}{\partial \boldsymbol{\delta}_{\mathbf{1}} \partial \boldsymbol{\delta}_{\mathbf{1}}} \\
& +\sum_{1}-\frac{1}{\left(f_{2}-F\left(0, y_{2}\right)+F\left(0, y_{2}-1\right)\right)^{2}}\left(\frac{\partial}{\partial \boldsymbol{\delta}_{\mathbf{1}}}\left(-F\left(0, y_{2}\right)+F\left(0, y_{2}-1\right)\right)\right)^{2} \\
& +\quad \frac{1}{f_{2}-F\left(0, y_{2}\right)+F\left(0, y_{2}-1\right)} \frac{\partial^{2}}{\partial \boldsymbol{\delta}_{\mathbf{1}} \partial \boldsymbol{\delta}_{\mathbf{1}}}\left(-F\left(0, y_{2}\right)+F\left(0, y_{2}-1\right)\right) . \\
\frac{\partial^{2} \ell}{\partial \boldsymbol{\delta}_{\mathbf{2}} \partial \boldsymbol{\delta}_{\mathbf{2}}}= & \sum_{1}-\frac{1}{\left(f_{2}-F\left(0, y_{2}\right)+F\left(0, y_{2}-1\right)\right)^{2}} \\
& \left(\frac{\partial f_{2}}{\partial \boldsymbol{\delta}_{\mathbf{2}}}-\frac{\partial F\left(0, y_{2}\right)}{\partial F_{2}} \frac{\partial F_{2}}{\partial \boldsymbol{\delta}_{\mathbf{2}}}+\frac{\partial F\left(0, y_{2}-1\right)}{\partial\left(F_{2}-f_{2}\right)} \frac{\partial\left(F_{2}-f_{2}\right)}{\partial \boldsymbol{\delta}_{\mathbf{2}}}\right)^{2} \\
+ & \frac{1}{f_{2}-F\left(0, y_{2}\right)+F\left(0, y_{2}-1\right)}\left(\frac{\partial^{2} f_{2}}{\partial \boldsymbol{\delta}_{\mathbf{2}} \boldsymbol{\delta}_{\mathbf{2}}}-\left(\frac{\partial^{2} F\left(0, y_{2}\right)}{\partial F_{2}^{2}}\left(\frac{\partial F_{2}}{\partial \boldsymbol{\delta}_{\mathbf{2}}}\right)^{2}+\frac{\partial F\left(0, y_{2}\right)}{\partial F_{2}} \frac{\partial^{2} F_{2}}{\partial \boldsymbol{\delta}_{\mathbf{2}} \boldsymbol{\delta}_{\mathbf{2}}}\right)\right. \\
+ & \left.\frac{\partial^{2} F\left(0, y_{2}-1\right)}{\partial\left(F_{2}-f_{2}\right)^{2}}\left(\frac{\partial\left(F_{2}-f_{2}\right)}{\partial \boldsymbol{\delta}_{\mathbf{2}}}\right)^{2}+\frac{\partial F\left(0, y_{2}-1\right)}{\partial\left(F_{2}-f_{2}\right)} \frac{\partial^{2}\left(F_{2}-f_{2}\right)}{\partial \boldsymbol{\delta}_{\mathbf{2}} \boldsymbol{\delta}_{\mathbf{2}}}\right)^{2}
\end{aligned}
$$




$$
\begin{aligned}
\frac{\partial^{2} \ell}{\partial \boldsymbol{\delta}_{\mathbf{1}} \partial \boldsymbol{\delta}_{\mathbf{2}}=} & \sum_{1}-\frac{1}{\left(f_{2}-F\left(0, y_{2}\right)+F\left(0, y_{2}-1\right)\right)^{2}} \\
& \left(-\frac{\partial F\left(0, y_{2}\right)}{\partial F_{1}} \frac{\partial F_{1}}{\partial \boldsymbol{\delta}_{\mathbf{1}}}+\frac{\partial F\left(0, y_{2}-1\right)}{\partial F_{1}} \frac{\partial F_{1}}{\partial \boldsymbol{\delta}_{\mathbf{1}}}\right) \\
& \left(\frac{\partial f_{2}}{\partial \boldsymbol{\delta}_{\mathbf{2}}}-\frac{\partial F\left(0, y_{2}\right)}{\partial F_{2}} \frac{\partial F_{2}}{\partial \boldsymbol{\delta}_{\mathbf{2}}}+\frac{\partial F\left(0, y_{2}-1\right)}{\partial\left(F_{2}-f_{2}\right)} \frac{\partial\left(F_{2}-f_{2}\right)}{\partial \boldsymbol{\delta}_{\mathbf{2}}}\right) \\
+ & \frac{1}{f_{2}-F\left(0, y_{2}\right)+F\left(0, y_{2}-1\right)} \\
& \left(-\frac{\partial^{2} F\left(0, y_{2}\right)}{\partial F_{1} \partial F_{2}} \frac{\partial F_{2}}{\partial \boldsymbol{\delta}_{\mathbf{2}}} \frac{\partial F_{1}}{\partial \boldsymbol{\delta}_{\mathbf{1}}}+\frac{\partial^{2} F\left(0, y_{2}-1\right)}{\partial F_{1} \partial\left(F_{2}-f_{2}\right)} \frac{\partial\left(F_{2}-f_{2}\right)}{\partial \boldsymbol{\delta}_{\mathbf{2}}} \frac{\partial F_{1}}{\partial \boldsymbol{\delta}_{\mathbf{1}}}\right) .
\end{aligned}
$$

$$
\begin{aligned}
\frac{\partial^{2} \ell}{\partial \theta^{2}} & =\sum_{1}-\frac{1}{\left(f_{2}-F\left(0, y_{2}\right)+F\left(0, y_{2}-1\right)\right)^{2}}\left(-\frac{\partial F\left(0, y_{2}\right)}{\partial \theta}+\frac{\partial F\left(0, y_{2}-1\right)}{\partial \theta}\right)^{2} \\
& +\frac{1}{f_{2}-F\left(0, y_{2}\right)+F\left(0, y_{2}-1\right)}\left(-\frac{\partial^{2} F\left(0, y_{2}\right)}{\partial \theta^{2}}+\frac{\partial^{2} F\left(0, y_{2}-1\right)}{\partial \theta^{2}}\right)
\end{aligned}
$$

$$
\begin{aligned}
\frac{\partial^{2} \ell}{\partial \boldsymbol{\delta}_{\mathbf{1}} \partial \theta}= & \sum_{1}-\frac{1}{\left(f_{2}-F\left(0, y_{2}\right)+F\left(0, y_{2}-1\right)\right)^{2}} \\
& \left(-\frac{\partial F\left(0, y_{2}\right)}{\partial \theta}+\frac{\partial F\left(0, y_{2}-1\right)}{\partial \theta}\right)\left(-\frac{\partial F\left(0, y_{2}\right)}{\partial F_{1}} \frac{\partial F_{1}}{\partial \boldsymbol{\delta}_{\mathbf{1}}}+\frac{\partial F\left(0, y_{2}-1\right)}{\partial F_{1}} \frac{\partial F_{1}}{\partial \boldsymbol{\delta}_{\mathbf{1}}}\right) \\
+ & \frac{1}{f_{2}-F\left(0, y_{2}\right)+F\left(0, y_{2}-1\right)}\left(-\frac{\partial F\left(0, y_{2}\right)}{\partial \theta \partial F_{1}} \frac{\partial F_{1}}{\partial \boldsymbol{\delta}_{\mathbf{1}}}+\frac{\partial F\left(0, y_{2}-1\right)}{\partial \theta \partial F_{1}} \frac{\partial F_{1}}{\partial \boldsymbol{\delta}_{\mathbf{1}}}\right) .
\end{aligned}
$$

$$
\begin{aligned}
\frac{\partial^{2} \ell}{\partial \boldsymbol{\delta}_{\mathbf{2}} \partial \theta}= & \sum_{1}-\frac{1}{\left(f_{2}-F\left(0, y_{2}\right)+F\left(0, y_{2}-1\right)\right)^{2}} \\
& \left(-\frac{\partial F\left(0, y_{2}\right)}{\partial \theta}+\frac{\partial F\left(0, y_{2}-1\right)}{\partial \theta}\right)\left(\frac{\partial f_{2}}{\boldsymbol{\delta}_{\mathbf{2}}}-\frac{\partial F\left(0, y_{2}\right)}{\partial F_{2}} \frac{\partial F_{2}}{\partial \boldsymbol{\delta}_{\mathbf{2}}}+\frac{\partial F\left(0, y_{2}-1\right)}{\partial\left(F_{2}-f_{2}\right)} \frac{\partial\left(F_{2}-f_{2}\right)}{\partial \boldsymbol{\delta}_{\mathbf{2}}}\right) \\
+ & \frac{1}{f_{2}-F\left(0, y_{2}\right)+F\left(0, y_{2}-1\right)}\left(-\frac{\partial F\left(0, y_{2}\right)}{\partial \theta \partial F_{2}} \frac{\partial F_{2}}{\partial \boldsymbol{\delta}_{\mathbf{2}}}+\frac{\partial F\left(0, y_{2}-1\right)}{\partial \theta \partial\left(F_{2}-f_{2}\right)} \frac{\partial\left(F_{2}-f_{2}\right)}{\partial \boldsymbol{\delta}_{\mathbf{2}}}\right) .
\end{aligned}
$$




$$
\begin{aligned}
\frac{\partial^{2} \ell}{\partial \sigma^{2}}= & \sum_{1}-\frac{1}{\left(f_{2}-F\left(0, y_{2}\right)+F\left(0, y_{2}-1\right)\right)^{2}} \\
& \left(\frac{\partial f_{2}}{\partial \sigma}-\frac{\partial F\left(0, y_{2}\right)}{\partial F_{2}} \frac{\partial F_{2}}{\partial \sigma}+\frac{\partial F\left(0, y_{2}-1\right)}{\partial\left(F_{2}-f_{2}\right)} \frac{\partial\left(F_{2}-f_{2}\right)}{\partial \sigma}\right)^{2} \\
+ & \frac{1}{f_{2}-F\left(0, y_{2}\right)+F\left(0, y_{2}-1\right)}\left(\frac{\partial^{2} f_{2}}{\partial \sigma^{2}}-\left(\frac{\partial^{2} F\left(0, y_{2}\right)}{\partial F_{2}^{2}}\left(\frac{\partial F_{2}}{\partial \sigma}\right)^{2}+\frac{\partial F\left(0, y_{2}\right)}{\partial F_{2}} \frac{\partial^{2} F_{2}}{\partial \sigma^{2}}\right)\right. \\
+ & \left.\frac{\partial^{2} F\left(0, y_{2}-1\right)}{\partial\left(F_{2}-f_{2}\right)^{2}}\left(\frac{\partial\left(F_{2}-f_{2}\right)}{\partial \sigma}\right)^{2}+\frac{\partial F\left(0, y_{2}-1\right)}{\partial\left(F_{2}-f_{2}\right)} \frac{\partial^{2}\left(F_{2}-f_{2}\right)}{\partial \sigma^{2}}\right) .
\end{aligned}
$$

An analogical result follows for $\frac{\partial^{2} \ell}{\partial \nu^{2}}$.

$$
\begin{aligned}
\frac{\partial^{2} \ell}{\partial \boldsymbol{\delta}_{1} \partial \sigma}= & \sum_{1}-\frac{1}{\left(f_{2}-F\left(0, y_{2}\right)+F\left(0, y_{2}-1\right)\right)^{2}} \\
& \left(-\frac{\partial F\left(0, y_{2}\right)}{\partial F_{1}} \frac{\partial F_{1}}{\partial \boldsymbol{\delta}_{\mathbf{1}}}+\frac{\partial F\left(0, y_{2}-1\right)}{\partial F_{1}} \frac{\partial F_{1}}{\partial \boldsymbol{\delta}_{\mathbf{1}}}\right) \\
& \left(\frac{\partial f_{2}}{\partial \sigma}-\frac{\partial F\left(0, y_{2}\right)}{\partial F_{2}} \frac{\partial F_{2}}{\partial \sigma}+\frac{\partial F\left(0, y_{2}-1\right)}{\partial\left(F_{2}-f_{2}\right)} \frac{\partial\left(F_{2}-f_{2}\right)}{\partial \sigma}\right) \\
+ & \frac{1}{f_{2}-F\left(0, y_{2}\right)+F\left(0, y_{2}-1\right)} \\
& \left(-\frac{\partial^{2} F\left(0, y_{2}\right)}{\partial F_{1} \partial F_{2}} \frac{\partial F_{2}}{\partial \sigma} \frac{\partial F_{1}}{\partial \boldsymbol{\delta}_{\mathbf{1}}}+\frac{\partial^{2} F\left(0, y_{2}-1\right)}{\partial F_{1} \partial\left(F_{2}-f_{2}\right)} \frac{\partial\left(F_{2}-f_{2}\right)}{\partial \sigma} \frac{\partial F_{1}}{\partial \boldsymbol{\delta}_{\mathbf{1}}}\right) .
\end{aligned}
$$

An analogical result follows for $\frac{\partial^{2} \ell}{\partial \delta_{1} \partial \nu}$.

$$
\begin{aligned}
\frac{\partial^{2} \ell}{\partial \boldsymbol{\delta}_{\mathbf{2}} \partial \sigma}= & \sum_{1}-\frac{1}{\left(f_{2}-F\left(0, y_{2}\right)+F\left(0, y_{2}-1\right)\right)^{2}} \\
& \left(\frac{\partial f_{2}}{\partial \sigma}-\frac{\partial F\left(0, y_{2}\right)}{\partial F_{2}} \frac{\partial F_{2}}{\partial \sigma}+\frac{\partial F\left(0, y_{2}-1\right)}{\partial\left(F_{2}-f_{2}\right)} \frac{\partial\left(F_{2}-f_{2}\right)}{\partial \sigma}\right) \\
& \left(\frac{\partial f_{2}}{\partial \boldsymbol{\delta}_{\mathbf{2}}}-\frac{\partial F\left(0, y_{2}\right)}{\partial F_{2}} \frac{\partial F_{2}}{\partial \boldsymbol{\delta}_{\mathbf{2}}}+\frac{\partial F\left(0, y_{2}-1\right)}{\partial\left(F_{2}-f_{2}\right)} \frac{\partial\left(F_{2}-f_{2}\right)}{\partial \boldsymbol{\delta}_{\mathbf{2}}}\right) \\
+ & \frac{1}{f_{2}-F\left(0, y_{2}\right)+F\left(0, y_{2}-1\right)}\left(\frac{\partial^{2} f_{2}}{\partial \boldsymbol{\delta}_{\mathbf{2}} \sigma}-\left(\frac{\partial^{2} F\left(0, y_{2}\right)}{\partial F_{2}^{2}} \frac{\partial F_{2}}{\partial \sigma} \frac{\partial F_{2}}{\partial \boldsymbol{\delta}_{\mathbf{2}}}+\frac{\partial F\left(0, y_{2}\right)}{\partial F_{2}} \frac{\partial^{2} F_{2}}{\partial \boldsymbol{\delta}_{\mathbf{2}} \sigma}\right)\right. \\
+ & \left.\frac{\partial^{2} F\left(0, y_{2}-1\right)}{\partial\left(F_{2}-f_{2}\right)^{2}} \frac{\partial\left(F_{2}-f_{2}\right)}{\partial \boldsymbol{\delta}_{\mathbf{2}}} \frac{\partial\left(F_{2}-f_{2}\right)}{\partial \sigma}+\frac{\partial F\left(0, y_{2}-1\right)}{\partial\left(F_{2}-f_{2}\right)} \frac{\partial^{2}\left(F_{2}-f_{2}\right)}{\partial \boldsymbol{\delta}_{\mathbf{2}} \sigma}\right) .
\end{aligned}
$$


An analogical result follows for $\frac{\partial^{2} \ell}{\partial \boldsymbol{\delta}_{2} \partial \nu}$.

$$
\begin{aligned}
\frac{\partial^{2} \ell}{\partial \sigma \partial \theta}= & \sum_{1}-\frac{1}{\left(f_{2}-F\left(0, y_{2}\right)+F\left(0, y_{2}-1\right)\right)^{2}} \\
& \left(-\frac{\partial F\left(0, y_{2}\right)}{\partial \theta}+\frac{\partial F\left(0, y_{2}-1\right)}{\partial \theta}\right)\left(\frac{\partial f_{2}}{\sigma}-\frac{\partial F\left(0, y_{2}\right)}{\partial F_{2}} \frac{\partial F_{2}}{\partial \sigma}+\frac{\partial F\left(0, y_{2}-1\right)}{\partial\left(F_{2}-f_{2}\right)} \frac{\partial\left(F_{2}-f_{2}\right)}{\partial \sigma}\right) \\
+ & \frac{1}{f_{2}-F\left(0, y_{2}\right)+F\left(0, y_{2}-1\right)}\left(-\frac{\partial F\left(0, y_{2}\right)}{\partial \theta \partial F_{2}} \frac{\partial F_{2}}{\partial \sigma}+\frac{\partial F\left(0, y_{2}-1\right)}{\partial \theta \partial\left(F_{2}-f_{2}\right)} \frac{\partial\left(F_{2}-f_{2}\right)}{\partial \sigma}\right) .
\end{aligned}
$$

An analogical result follows for $\frac{\partial^{2} \ell}{\partial \nu \partial \theta}$.

$$
\begin{aligned}
\frac{\partial^{2} \ell}{\partial \nu \partial \sigma}= & \sum_{1}-\frac{1}{\left(f_{2}-F\left(0, y_{2}\right)+F\left(0, y_{2}-1\right)\right)^{2}} \\
& \left(\frac{\partial f_{2}}{\partial \sigma}-\frac{\partial F\left(0, y_{2}\right)}{\partial F_{2}} \frac{\partial F_{2}}{\partial \sigma}+\frac{\partial F\left(0, y_{2}-1\right)}{\partial\left(F_{2}-f_{2}\right)} \frac{\partial\left(F_{2}-f_{2}\right)}{\partial \sigma}\right) \\
& \left(\frac{\partial f_{2}}{\partial \nu}-\frac{\partial F\left(0, y_{2}\right)}{\partial F_{2}} \frac{\partial F_{2}}{\partial \nu}+\frac{\partial F\left(0, y_{2}-1\right)}{\partial\left(F_{2}-f_{2}\right)} \frac{\partial\left(F_{2}-f_{2}\right)}{\partial \nu}\right) \\
+ & \frac{1}{f_{2}-F\left(0, y_{2}\right)+F\left(0, y_{2}-1\right)}\left(\frac{\partial^{2} f_{2}}{\partial \nu \partial \sigma}-\left(\frac{\partial^{2} F\left(0, y_{2}\right)}{\partial F_{2}^{2}} \frac{\partial F_{2}}{\partial \sigma} \frac{\partial F_{2}}{\partial \nu}+\frac{\partial F\left(0, y_{2}\right)}{\partial F_{2}} \frac{\partial^{2} F_{2}}{\partial \nu \partial \sigma}\right)\right. \\
+ & \left.\frac{\partial^{2} F\left(0, y_{2}-1\right)}{\partial\left(F_{2}-f_{2}\right)^{2}} \frac{\partial\left(F_{2}-f_{2}\right)}{\partial \nu} \frac{\partial\left(F_{2}-f_{2}\right)}{\partial \sigma}+\frac{\partial F\left(0, y_{2}-1\right)}{\partial\left(F_{2}-f_{2}\right)} \frac{\partial^{2}\left(F_{2}-f_{2}\right)}{\partial \nu \partial \sigma}\right) .
\end{aligned}
$$

Note that $\frac{\partial^{2} F_{1}}{\partial \boldsymbol{\delta}_{1} \partial \boldsymbol{\delta}_{1}}=\frac{\partial}{\boldsymbol{\delta}_{\mathbf{1}}}\left(-\phi\left(-\eta_{1}\right) \mathbf{X}_{\mathbf{1}}\right)=-\phi\left(-\eta_{1}\right) \eta_{1} \mathbf{X}_{\mathbf{1}} \mathbf{X}_{\mathbf{1}}^{\top}$. Since for optimisation we are using $\theta^{*}$,

$$
\begin{aligned}
\frac{\partial^{2} \ell}{\partial^{2} \theta^{*}} & =\frac{\partial}{\partial \theta^{*}}\left(\frac{\partial \ell}{\partial \theta} \frac{\partial \theta}{\partial \theta^{*}}\right) \\
& =\frac{\partial^{2} \ell}{\partial \theta^{2}}\left(\frac{\partial \theta}{\partial \theta^{*}}\right)^{2}+\frac{\partial \ell}{\partial \theta} \frac{\partial^{2} \theta}{\partial \theta^{* 2}}
\end{aligned}
$$

For $\sigma^{*}$

$$
\begin{aligned}
\frac{\partial^{2} \ell}{\partial^{2} \sigma^{*}} & =\frac{\partial}{\partial \sigma^{*}}\left(\frac{\partial \ell}{\partial \sigma} \frac{\partial \sigma}{\partial \sigma^{*}}\right) \\
& =\frac{\partial^{2} \ell}{\partial \sigma^{2}} \sigma^{2}+\frac{\partial \ell}{\partial \sigma} \sigma
\end{aligned}
$$


and $\nu^{*}$ (Delaporte)

$$
\begin{aligned}
& \frac{\partial^{2} \ell}{\partial^{2} \nu^{*}}=\frac{\partial}{\partial \nu^{*}}\left(\frac{\partial \ell}{\partial \nu} \frac{\partial \nu}{\partial \nu^{*}}\right) \\
& =\frac{\partial}{\partial \nu^{*}}\left(\frac{\partial \ell}{\partial \nu} \frac{\frac{\nu}{1-\nu}\left(1+\frac{\nu}{1-\nu}\right)-\left(\frac{\nu}{1-\nu}\right)^{2}}{\left(1+\frac{\nu}{1-\nu}\right)^{2}}\right)
\end{aligned}
$$

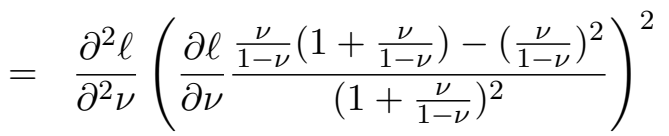

$$
\begin{aligned}
& +\frac{\partial \ell}{\partial \nu} \frac{\exp \left(\nu^{*}\right)\left(1-\exp \left(\nu^{*}\right)\right)^{2}-2\left(1-\exp \left(\nu^{*}\right)\right) \exp ^{2}\left(\nu^{*}\right)}{\left(1-\exp \left(\nu^{*}\right)\right)^{4}} \text {. }
\end{aligned}
$$




\section{Appendix I}

\section{Copula, pmf and cdf second derivatives}

All derivations below were conducted manually. According to the author's best knowledge, these cannot be found in the literature.

$\underline{\text { First derivatives with respect to copulas }}$

Clayton:

$$
\begin{aligned}
& \frac{\partial^{2} C}{\partial u^{2}}=(-\theta-1) u^{-\theta-2}\left(u^{-\theta}+v^{-\theta}-1\right)^{-\frac{1}{\theta}-1}+u^{2(-\theta-1)}\left(u^{-\theta}+v^{-\theta}-1\right)^{-\frac{1}{\theta}-2}(-\theta)\left(-\frac{1}{\theta}-1\right) \\
& \frac{\partial^{2} C}{\partial v^{2}}=(-\theta-1) v^{-\theta-2}\left(u^{-\theta}+v^{-\theta}-1\right)^{-\frac{1}{\theta}-1}+v^{2(-\theta-1)}\left(u^{-\theta}+v^{-\theta}-1\right)^{-\frac{1}{\theta}-2}(-\theta)\left(-\frac{1}{\theta}-1\right) \\
& \frac{\partial^{2} C}{\partial u \partial v}=u^{-\theta-1}\left(u^{-\theta}+v^{-\theta}-1\right)^{-\frac{1}{\theta}-2}\left(-\frac{1}{\theta}-1\right)(-\theta) v^{-\theta-1} \\
& \frac{\partial^{2} C}{\partial u \partial \theta}=u^{-\theta-1} \log (u)(-1)\left(u^{-\theta}+v^{-\theta}-1\right)^{-\frac{1}{\theta}-1} \\
&+u^{-\theta-1}\left(\frac{\partial C}{\partial \theta}\left(u^{-\theta}+v^{-\theta}-1\right)^{-1}+\left(u^{-\theta}+v^{-\theta}-1\right)^{-\frac{1}{\theta}-2}\left(u^{-\theta} \log (u)+v^{-\theta} \log (v)\right)\right)
\end{aligned}
$$




$$
\begin{aligned}
\frac{\partial^{2} C}{\partial v \partial \theta} & =v^{-\theta-1} \log (v)(-1)\left(u^{-\theta}+v^{-\theta}-1\right)^{-\frac{1}{\theta}-1} \\
& +v^{-\theta-1}\left(\frac{\partial C}{\partial \theta}\left(u^{-\theta}+v^{-\theta}-1\right)^{-1}+\left(u^{-\theta}+v^{-\theta}-1\right)^{-\frac{1}{\theta}-2}\left(u^{-\theta} \log (u)+v^{-\theta} \log (v)\right)\right) \\
\frac{\partial^{2} C}{\partial \theta^{2}}= & -\frac{2}{\theta^{3}} \log \left(u^{-\theta}+v^{-\theta}-1\right)\left(u^{-\theta}+v^{-\theta}-1\right)^{-\frac{1}{\theta}} \\
+ & \frac{1}{\theta^{2}}\left(\left(u^{-\theta}+v^{-\theta}-1\right)^{-\frac{1}{\theta}-1}\left(-u^{-\theta} \log (u)-v^{-\theta} \log (v)\right)+\log \left(u^{-\theta}+v^{-\theta}-1\right) \frac{\partial C}{\partial \theta}\right) \\
+ & \left(-\frac{1}{\theta^{2}}\left(u^{-\theta} \log (u)+v^{-\theta} \log (v)\right)+\frac{1}{\theta}\left(-u^{-\theta} \log ^{2}(u)-v^{-\theta} \log ^{2}(v)\right)\right)\left(u^{-\theta}+v^{-\theta}-1\right)^{-\frac{1}{\theta}-1} \\
+ & \frac{1}{\theta}\left(u^{-\theta} \log (u)+v^{-\theta} \log (v)\right) \\
& \left(\frac{\partial C}{\partial \theta}\left(u^{-\theta}+v^{-\theta}-1\right)^{-1}+\left(u^{-\theta}+v^{-\theta}-1\right)^{-\frac{1}{\theta}-2}\left(u^{-\theta} \log (u)+v^{-\theta} \log (v)\right)\right)
\end{aligned}
$$

AMH:

$$
\begin{aligned}
& \frac{\partial^{2} C}{\partial u^{2}}=\frac{(1-\theta(1-u)(1-v))^{2}}{(1-\theta(1-u)(1-v))^{4}} \\
& -\frac{2(v(1-\theta(1-u)(1-v))-u v \theta(1-v))(1-\theta(1-u)(1-v)) \theta(1-v)}{(1-\theta(1-u)(1-v))^{4}} \\
& \frac{\partial^{2} C}{\partial v^{2}}=\frac{(1-\theta(1-u)(1-v))^{2}}{(1-\theta(1-u)(1-v))^{4}} \\
& -\frac{2(u(1-\theta(1-u)(1-v))-u v \theta(1-u))(1-\theta(1-u)(1-v)) \theta(1-u)}{(1-\theta(1-u)(1-v))^{4}} \\
& \frac{\partial^{2} C}{\partial u \partial v}=\frac{((1-\theta(1-u)(1-v))+v \theta(1-u)-\theta(u-2 u v))(1-\theta(1-u)(1-v))^{2}}{(1-\theta(1-u)(1-v))^{4}} \\
& -\frac{2(v(1-\theta(1-u)(1-v))-u v \theta(1-v))(1-\theta(1-u)(1-v)) \theta(1-u)}{(1-\theta(1-u)(1-v))^{4}} \\
& \frac{\partial^{2} C}{\partial u \partial \theta}=\frac{(-v(1-u)(1-v)-u v(1-v))(1-\theta(1-u)(1-v))^{2}}{(1-\theta(1-u)(1-v))^{4}} \\
& -\frac{2(v(1-\theta(1-u)(1-v))-u v \theta(1-v))(1-\theta(1-u)(1-v))(-(1-u)(1-v))}{(1-\theta(1-u)(1-v))^{4}}
\end{aligned}
$$




$$
\begin{gathered}
\frac{\partial^{2} C}{\partial v \partial \theta}=\frac{(-u(1-u)(1-v)-u v(1-u))(1-\theta(1-u)(1-v))^{2}}{(1-\theta(1-u)(1-v))^{4}} \\
-\frac{2(u(1-\theta(1-u)(1-v))-u v \theta(1-u))(1-\theta(1-u)(1-v))(-(1-u)(1-v))}{(1-\theta(1-u)(1-v))^{4}} \\
\frac{\partial^{2} C}{\partial \theta^{2}}=2 u v(1-u)^{2}(1-v)^{2}(1-\theta(1-u)(1-v))^{-3}
\end{gathered}
$$

Gumbel:

$$
\begin{aligned}
& \frac{\partial^{2} C}{\partial u^{2}}=\frac{\partial C}{\partial u}\left(-\frac{1}{\theta}\left((-\log u)^{\theta}+(-\log v)^{\theta}\right)^{\frac{1}{\theta}-1}\right)\left(\theta(-\log u)^{\theta-1}\right)\left(-\frac{1}{u}\right) \\
& +C_{\theta}(u, v)\left(\left(-\frac{1}{\theta}\right)\left(\frac{1}{\theta}-1\right)\left((-\log u)^{\theta}+(-\log v)^{\theta}\right)^{\frac{1}{\theta}-2}\left(\theta(-\log u)^{\theta-1}\left(-\frac{1}{u}\right)\right)^{2}\right. \\
& \left.+\left(-\frac{1}{\theta}\left((-\log u)^{\theta}+(-\log v)^{\theta}\right)^{\frac{1}{\theta}-1}\right)\left(\theta(\theta-1)(-\log u)^{\theta-2}\left(\frac{1}{u^{2}}\right)+\theta(-\log u)^{\theta-1}\left(\frac{1}{u^{2}}\right)\right)\right) \\
& \frac{\partial^{2} C}{\partial v^{2}}=\frac{\partial C}{\partial v}\left(-\frac{1}{\theta}\left((-\log u)^{\theta}+(-\log v)^{\theta}\right)^{\frac{1}{\theta}-1}\right)\left(\theta(-\log v)^{\theta-1}\right)\left(-\frac{1}{v}\right) \\
& +C_{\theta}(u, v)\left(\left(-\frac{1}{\theta}\right)\left(\frac{1}{\theta}-1\right)\left((-\log u)^{\theta}+(-\log v)^{\theta}\right)^{\frac{1}{\theta}-2}\left(\theta(-\log v)^{\theta-1}\left(-\frac{1}{v}\right)\right)^{2}\right. \\
& \left.+\left(-\frac{1}{\theta}\left((-\log u)^{\theta}+(-\log v)^{\theta}\right)^{\frac{1}{\theta}-1}\right)\left(\theta(\theta-1)(-\log v)^{\theta-2}\left(\frac{1}{v^{2}}\right)+\theta(-\log v)^{\theta-1}\left(\frac{1}{v^{2}}\right)\right)\right) \\
& \frac{\partial^{2} C}{\partial u \partial v}=\frac{\partial C}{\partial v}\left(-\frac{1}{\theta}\left((-\log u)^{\theta}+(-\log v)^{\theta}\right)^{\frac{1}{\theta}-1}\right)\left(\theta(-\log u)^{\theta-1}\right)\left(-\frac{1}{u}\right) \\
& +C_{\theta}(u, v)\left(-\frac{1}{\theta}\right)\left(\frac{1}{\theta}-1\right)\left((-\log u)^{\theta}+(-\log v)^{\theta}\right)^{\frac{1}{\theta}-2} \times \\
& \times\left(\theta(-\log v)^{\theta-1}\left(-\frac{1}{v}\right)\right)\left(\theta(-\log u)^{\theta-1}\left(-\frac{1}{u}\right)\right) \\
& \frac{\partial^{2} C}{\partial u \partial \theta}=\frac{\partial C}{\partial \theta}\left(-\frac{1}{\theta}\left((-\log u)^{\theta}+(-\log v)^{\theta}\right)^{\frac{1}{\theta}-1}\right)\left(\theta(-\log u)^{\theta-1}\right)\left(-\frac{1}{u}\right) \\
& +\left(-\frac{1}{u}\right) C(u, v)\left(\left[\frac{1}{\theta^{2}}\left((-\log u)^{\theta}+(-\log v)^{\theta}\right)^{\frac{1}{\theta}-1}\right.\right. \\
& -\frac{1}{\theta}\left(\frac{\partial-\log C(u, v)}{\partial \theta}\left((-\log u)^{\theta}+(-\log v)^{\theta}\right)^{-1}-\left((-\log u)^{\theta}+(-\log v)^{\theta}\right)^{-\frac{1}{\theta}-2} \times\right. \\
& \left.\left.\left((-\log u)^{\theta} \log (-\log u)+(-\log v)^{\theta} \log (-\log v)\right)\right)\right]\left(\theta(-\log u)^{\theta-1}\right) \\
& \left.+\left(-\frac{1}{\theta}\right)\left((-\log u)^{\theta}+(-\log v)^{\theta}\right)^{\frac{1}{\theta}-1}\left((-\log u)^{\theta-1}+\theta(-\log u)^{\theta-1} \log (-\log u)\right)\right)
\end{aligned}
$$




$$
\begin{aligned}
& \frac{\partial^{2} C}{\partial v \partial \theta}=\frac{\partial C}{\partial \theta}\left(-\frac{1}{\theta}\left((-\log u)^{\theta}+(-\log v)^{\theta}\right)^{\frac{1}{\theta}-1}\right)\left(\theta(-\log v)^{\theta-1}\right)\left(-\frac{1}{v}\right) \\
& +\left(-\frac{1}{v}\right) C(u, v)\left(\left[\frac{1}{\theta^{2}}\left((-\log u)^{\theta}+(-\log v)^{\theta}\right)^{\frac{1}{\theta}-1}\right.\right. \\
& -\frac{1}{\theta}\left(\frac{\partial-\log C(u, v)}{\partial \theta}\left((-\log u)^{\theta}+(-\log v)^{\theta}\right)^{-1}-\left((-\log u)^{\theta}+(-\log v)^{\theta}\right)^{-\frac{1}{\theta}-2} \times\right. \\
& \left.\left.\left((-\log u)^{\theta} \log (-\log u)+(-\log v)^{\theta} \log (-\log v)\right)\right)\right]\left(\theta(-\log v)^{\theta-1}\right) \\
& \left.+\left(-\frac{1}{\theta}\right)\left((-\log u)^{\theta}+(-\log v)^{\theta}\right)^{\frac{1}{\theta}-1}\left((-\log v)^{\theta-1}+\theta(-\log v)^{\theta-1} \log (-\log v)\right)\right) \\
& \frac{\partial^{2} C}{\partial \theta^{2}}=-\frac{\partial C}{\partial \theta}\left((-\log u)^{\theta}+(-\log v)^{\theta}\right)^{\frac{1}{\theta}}\left(-\frac{1}{\theta^{2}} \log \left((-\log u)^{\theta}+(-\log v)^{\theta}\right)\right) \\
& -C(u, v)\left(\frac{\partial-\log C(u, v)}{\partial \theta}\left(-\frac{1}{\theta^{2}} \log \left((-\log u)^{\theta}+(-\log v)^{\theta}\right)\right)+\left((-\log u)^{\theta}+(-\log v)^{\theta}\right)^{\frac{1}{\theta}} \times\right. \\
& \times\left(\frac{2}{\theta^{3}} \log \left((-\log u)^{\theta}+(-\log v)^{\theta}\right)\right. \\
& \left.\left.-\frac{1}{\theta^{2}}\left((-\log u)^{\theta}+(-\log v)^{\theta}\right)^{-1}\left((-\log u)^{\theta} \log (-\log u)+(-\log v)^{\theta} \log (-\log v)\right)\right)\right) \\
& +\left(-\frac{1}{\theta^{2}}\left((-\log u)^{\theta}+(-\log v)^{\theta}\right)^{-1}-\frac{1}{\theta}\left((-\log u)^{\theta}+(-\log v)^{\theta}\right)^{-2} \times\right. \\
& \left.\times\left((-\log u)^{\theta} \log (-\log u)+(-\log v)^{\theta} \log (-\log v)\right)\right) \times \\
& \times\left((-\log u)^{\theta} \log (-\log u)+(-\log v)^{\theta} \log (-\log v)\right)\left((-\log u)^{\theta}+(-\log v)^{\theta}\right)^{\frac{1}{\theta}}(-C(u, v)) \\
& +\quad\left(( - C ( u , v ) ) \left[\left((-\log u)^{\theta} \log ^{2}(-\log u)+(-\log v)^{\theta} \log ^{2}(-\log v)\right)\left((-\log u)^{\theta}+(-\log v)^{\theta}\right)^{\frac{1}{\theta}}\right.\right. \\
& \left.+\frac{\partial-\log C(u, v)}{\partial \theta}\left((-\log u)^{\theta} \log (-\log u)+(-\log v)^{\theta} \log (-\log v)\right)\right] \\
& \left.+\left((-\log u)^{\theta}+(-\log v)^{\theta}\right)^{\frac{1}{\theta}}\left((-\log u)^{\theta} \log (-\log u)+(-\log v)^{\theta} \log (-\log v)\right)\left(-\frac{\partial C}{\partial \theta}\right)\right) \times \\
& \times \frac{1}{\theta}\left((-\log u)^{\theta}+(-\log v)^{\theta}\right)^{-1}
\end{aligned}
$$

Joe:

$$
\begin{aligned}
\frac{\partial^{2} C}{\partial u^{2}}= & -\frac{1}{\theta}\left(\frac{1}{\theta}-1\right)\left((1-u)^{\theta}+(1-v)^{\theta}-(1-u)^{\theta}(1-v)^{\theta}\right)^{\frac{1}{\theta}-2} \\
\times & \left(-\theta(1-u)^{\theta-1}+(1-v)^{\theta} \theta(1-u)^{\theta-1}\right)^{2} \\
& -\frac{1}{\theta}\left((1-u)^{\theta}+(1-v)^{\theta}-(1-u)^{\theta}(1-v)^{\theta}\right)^{\frac{1}{\theta}-1} \\
\times & \left(\theta(\theta-1)(1-u)^{\theta-2}-\theta(\theta-1)(1-u)^{\theta-2}(1-v)^{\theta}\right)
\end{aligned}
$$




$$
\begin{aligned}
\frac{\partial^{2} C}{\partial v^{2}}= & -\frac{1}{\theta}\left(\frac{1}{\theta}-1\right)\left((1-u)^{\theta}+(1-v)^{\theta}-(1-u)^{\theta}(1-v)^{\theta}\right)^{\frac{1}{\theta}-2} \\
\times & \left(-\theta(1-v)^{\theta-1}+(1-u)^{\theta} \theta(1-v)^{\theta-1}\right)^{2} \\
& -\frac{1}{\theta}\left((1-u)^{\theta}+(1-v)^{\theta}-(1-u)^{\theta}(1-v)^{\theta}\right)^{\frac{1}{\theta}-1} \\
\times & \left(\theta(\theta-1)(1-v)^{\theta-2}-\theta(\theta-1)(1-v)^{\theta-2}(1-u)^{\theta}\right)
\end{aligned}
$$

$$
\begin{aligned}
\frac{\partial^{2} C}{\partial u \partial v}= & -\frac{1}{\theta}\left(\frac{1}{\theta}-1\right)\left((1-u)^{\theta}+(1-v)^{\theta}-(1-u)^{\theta}(1-v)^{\theta}\right)^{\frac{1}{\theta}-2} \\
\times & \left(-\theta(1-u)^{\theta-1}+(1-v)^{\theta} \theta(1-u)^{\theta-1}\right)\left(-\theta(1-v)^{\theta-1}+(1-u)^{\theta} \theta(1-v)^{\theta-1}\right) \\
& -\frac{1}{\theta}\left((1-u)^{\theta}+(1-v)^{\theta}-(1-u)^{\theta}(1-v)^{\theta}\right)^{\frac{1}{\theta}-1} \\
\times & \left(-\theta^{2}(1-v)^{\theta-1}(1-u)^{\theta-1}\right)
\end{aligned}
$$

$$
\begin{aligned}
\frac{\partial^{2} C}{\partial u \partial \theta}= & \frac{1}{\theta^{2}}\left((1-u)^{\theta}+(1-v)^{\theta}-(1-u)^{\theta}(1-v)^{\theta}\right)^{\frac{1}{\theta}-1}\left(-\theta(1-u)^{\theta-1}+(1-v)^{\theta} \theta(1-u)^{\theta-1}\right) \\
- & \frac{1}{\theta}\left(\left(-\theta(1-u)^{\theta-1}+(1-v)^{\theta} \theta(1-u)^{\theta-1}\right)\right. \\
& \left(\frac{\partial(1-C)}{\partial \theta}\left((1-u)^{\theta}+(1-v)^{\theta}-(1-u)^{\theta}(1-v)^{\theta}\right)^{-1}\right. \\
& -\left((1-u)^{\theta}+(1-v)^{\theta}-(1-u)^{\theta}(1-v)^{\theta}\right)^{\frac{1}{\theta}-2} \times \\
& \times\left((1-u)^{\theta} \log (1-u)+(1-v)^{\theta} \log (1-v)\right. \\
& \left.\left.-(1-u)^{\theta} \log (1-u)(1-v)^{\theta}-(1-v)^{\theta} \log (1-v)(1-u)^{\theta}\right)\right) \\
& +\left((1-u)^{\theta}+(1-v)^{\theta}-(1-u)^{\theta}(1-v)^{\theta}\right)^{\frac{1}{\theta}-1} \times \\
& \times\left(-(1-u)^{\theta-1}-\theta(1-u)^{\theta-1} \log (1-u)+(1-v)^{\theta} \log (1-v) \theta(1-u)^{\theta-1}\right. \\
& \left.\left.+\left((1-u)^{\theta-1}+\theta(1-u)^{\theta-1} \log (1-u)(1-v)^{\theta}\right)\right)\right)
\end{aligned}
$$




$$
\begin{aligned}
\frac{\partial^{2} C}{\partial v \partial \theta}= & \frac{1}{\theta^{2}}\left((1-u)^{\theta}+(1-v)^{\theta}-(1-u)^{\theta}(1-v)^{\theta}\right)^{\frac{1}{\theta}-1}\left(-\theta(1-v)^{\theta-1}+(1-u)^{\theta} \theta(1-v)^{\theta-1}\right) \\
- & \frac{1}{\theta}\left(\left(-\theta(1-v)^{\theta-1}+(1-u)^{\theta} \theta(1-v)^{\theta-1}\right)\right. \\
& \left(\frac{\partial(1-C)}{\partial \theta}\left((1-u)^{\theta}+(1-v)^{\theta}-(1-u)^{\theta}(1-v)^{\theta}\right)^{-1}\right. \\
& -\left((1-u)^{\theta}+(1-v)^{\theta}-(1-u)^{\theta}(1-v)^{\theta}\right)^{\frac{1}{\theta}-2} \times \\
& \times\left((1-u)^{\theta} \log (1-u)+(1-v)^{\theta} \log (1-v)\right. \\
& \left.\left.-(1-u)^{\theta} \log (1-u)(1-v)^{\theta}-(1-v)^{\theta} \log (1-v)(1-u)^{\theta}\right)\right) \\
& +\left((1-u)^{\theta}+(1-v)^{\theta}-(1-u)^{\theta}(1-v)^{\theta}\right)^{\frac{1}{\theta}-1} \times \\
& \times\left(-(1-v)^{\theta-1}-\theta(1-v)^{\theta-1} \log (1-v)+(1-u)^{\theta} \log (1-u) \theta(1-v)^{\theta-1}\right. \\
& \left.\left.+\left((1-v)^{\theta-1}+\theta(1-v)^{\theta-1} \log (1-v)(1-u)^{\theta}\right)\right)\right)
\end{aligned}
$$




$$
\begin{aligned}
& \frac{\partial^{2} C}{\partial \theta^{2}}=-\frac{2}{\theta^{3}} \log \left((1-u)^{\theta}+(1-v)^{\theta}-(1-u)^{\theta}(1-v)^{\theta}\right)\left((1-u)^{\theta}+(1-v)^{\theta}-(1-u)^{\theta}(1-v)^{\theta}\right)^{\frac{1}{\theta}} \\
& +\frac{1}{\theta^{2}}\left(\left((1-u)^{\theta}+(1-v)^{\theta}-(1-u)^{\theta}(1-v)^{\theta}\right)^{\frac{1}{\theta}-1}\right. \\
& \times\left((1-u)^{\theta} \log (1-u)+(1-v)^{\theta} \log (1-v)\right. \\
& \left.-(1-u)^{\theta} \log (1-u)(1-v)^{\theta}-(1-v)^{\theta} \log (1-v)(1-u)^{\theta}\right) \\
& \left.+\log \left((1-u)^{\theta}+(1-v)^{\theta}-(1-u)^{\theta}(1-v)^{\theta}\right) \frac{\partial(1-C)}{\partial \theta}\right) \\
& +\frac{1}{\theta^{2}}\left((1-u)^{\theta}+(1-v)^{\theta}-(1-u)^{\theta}(1-v)^{\theta}\right)^{\frac{1}{\theta}-1} \\
& \times\left((1-u)^{\theta} \log (1-u)+(1-v)^{\theta} \log (1-v)\right. \\
& \left.\left.-(1-u)^{\theta} \log (1-u)(1-v)^{\theta}-(1-v)^{\theta} \log (1-v)(1-u)^{\theta}\right)\right) \\
& -\frac{1}{\theta}\left(\left((1-u)^{\theta}+(1-v)^{\theta}-(1-u)^{\theta}(1-v)^{\theta}\right)^{\frac{1}{\theta}-1} \times\right. \\
& \times\left((1-u)^{\theta} \log ^{2}(1-u)+(1-v)^{\theta} \log ^{2}(1-v)\right. \\
& -(1-u)^{\theta} \log ^{2}(1-u)(1-v)^{\theta}-(1-v)^{\theta} \log ^{2}(1-v)(1-u)^{\theta} \\
& \left.-2(1-u)^{\theta} \log (1-u)(1-v)^{\theta} \log (1-v)\right) \\
& +\left((1-u)^{\theta} \log (1-u)+(1-v)^{\theta} \log (1-v)\right. \\
& \left.-(1-u)^{\theta} \log (1-u)(1-v)^{\theta}-(1-v)^{\theta} \log (1-v)(1-u)^{\theta}\right) \\
& \left(\frac{\partial(1-C)}{\partial \theta}\left((1-u)^{\theta}+(1-v)^{\theta}-(1-u)^{\theta}(1-v)^{\theta}\right)^{-1}\right. \\
& -\left((1-u)^{\theta}+(1-v)^{\theta}-(1-u)^{\theta}(1-v)^{\theta}\right)^{\frac{1}{\theta}-2} \times \\
& \times\left((1-u)^{\theta} \log (1-u)+(1-v)^{\theta} \log (1-v)\right. \\
& \left.\left.\left.-(1-u)^{\theta} \log (1-u)(1-v)^{\theta}-(1-v)^{\theta} \log (1-v)(1-u)^{\theta}\right)\right)\right)
\end{aligned}
$$

Frank:

$$
\begin{aligned}
\frac{\partial^{2} C}{\partial u^{2}} & =\frac{\frac{e^{-\theta v}-1}{e^{-\theta}-1} e^{-\theta u}(-\theta)\left(1+\left(e^{-\theta u}-1\right)\left(e^{-\theta v}-1\right) /\left(e^{-\theta}-1\right)\right)-\left(\frac{e^{-\theta v}-1}{e^{-\theta}-1} e^{-\theta u}\right)^{2}(-\theta)}{\left(1+\left(e^{-\theta u}-1\right)\left(e^{-\theta v}-1\right) /\left(e^{-\theta}-1\right)\right)^{2}} \\
\frac{\partial^{2} C}{\partial v^{2}} & =\frac{\frac{e^{-\theta u}-1}{e^{-\theta}-1} e^{-\theta v}(-\theta)\left(1+\left(e^{-\theta u}-1\right)\left(e^{-\theta v}-1\right) /\left(e^{-\theta}-1\right)\right)-\left(\frac{e^{-\theta u}-1}{e^{-\theta}-1} e^{-\theta v}\right)^{2}(-\theta)}{\left(1+\left(e^{-\theta u}-1\right)\left(e^{-\theta v}-1\right) /\left(e^{-\theta}-1\right)\right)^{2}}
\end{aligned}
$$




$$
\begin{aligned}
& \frac{\partial^{2} C}{\partial u \partial v}=\frac{\frac{e^{-\theta u}}{e^{-\theta}-1} e^{-\theta v}(-\theta)\left(1+\left(e^{-\theta u}-1\right)\left(e^{-\theta v}-1\right) /\left(e^{-\theta}-1\right)\right)-\frac{e^{-\theta v}-1}{e^{-\theta}-1} e^{-\theta u} \frac{e^{-\theta u}-1}{e^{-\theta}-1} e^{-\theta v}(-\theta)}{\left(1+\left(e^{-\theta u}-1\right)\left(e^{-\theta v}-1\right) /\left(e^{-\theta}-1\right)\right)^{2}} \\
& \frac{\partial^{2} C}{\partial u \partial \theta}=\frac{\frac{\left(e^{-\theta}-1\right)\left(\left(e^{-\theta v}-1\right) e^{-\theta u}(-u)+e^{-\theta u-\theta v}(-v)\right)+\left(e^{-\theta v}-1\right) e^{-\theta u-\theta}}{\left(e^{-\theta}-1\right)^{2}} \times}{\left(1+\left(e^{-\theta u}-1\right)\left(e^{-\theta v}-1\right) /\left(e^{-\theta}-1\right)\right)^{2}} \\
& \frac{\times\left(1+\left(e^{-\theta u}-1\right)\left(e^{-\theta v}-1\right) /\left(e^{-\theta}-1\right)\right)}{\left(1+\left(e^{-\theta u}-1\right)\left(e^{-\theta v}-1\right) /\left(e^{-\theta}-1\right)\right)^{2}} \\
& -\frac{\frac{e^{-\theta v}-1}{e^{-\theta}-1} e^{-\theta u} \frac{\left(e^{-\theta}-1\right)\left(\left(e^{-\theta v}-1\right) e^{-\theta u}(-u)+\left(e^{-\theta u}-1\right) e^{-\theta v}(-v)\right)+\left(e^{-\theta v}-1\right)\left(e^{-\theta u}-1\right) e^{-\theta}}{\left(e^{-\theta}-1\right)^{2}}}{\left(1+\left(e^{-\theta u}-1\right)\left(e^{-\theta v}-1\right) /\left(e^{-\theta}-1\right)\right)^{2}} \\
& \frac{\partial^{2} C}{\partial v \partial \theta}=\frac{\frac{\left(e^{-\theta}-1\right)\left(\left(e^{-\theta u}-1\right) e^{-\theta v}(-v)+e^{-\theta v-\theta u}(-u)\right)+\left(e^{-\theta u}-1\right) e^{-\theta v-\theta}}{\left(e^{-\theta}-1\right)^{2}} \times}{\left(1+\left(e^{-\theta u}-1\right)\left(e^{-\theta v}-1\right) /\left(e^{-\theta}-1\right)\right)^{2}} \\
& \frac{\times\left(1+\left(e^{-\theta u}-1\right)\left(e^{-\theta v}-1\right) /\left(e^{-\theta}-1\right)\right)}{\left(1+\left(e^{-\theta u}-1\right)\left(e^{-\theta v}-1\right) /\left(e^{-\theta}-1\right)\right)^{2}} \\
& -\frac{\frac{e^{-\theta u}-1}{e^{-\theta}-1} e^{-\theta v} \frac{\left(e^{-\theta}-1\right)\left(\left(e^{-\theta v}-1\right) e^{-\theta u}(-u)+\left(e^{-\theta u}-1\right) e^{-\theta v}(-v)\right)+\left(e^{-\theta v}-1\right)\left(e^{-\theta u}-1\right) e^{-\theta}}{\left(e^{-\theta}-1\right)^{2}}}{\left(1+\left(e^{-\theta u}-1\right)\left(e^{-\theta v}-1\right) /\left(e^{-\theta}-1\right)\right)^{2}} \\
& \frac{\partial^{2} C}{\partial \theta^{2}}=-\frac{2}{\theta^{3}} \log \left[1+\left(e^{-\theta u}-1\right)\left(e^{-\theta v}-1\right) /\left(e^{-\theta}-1\right)\right]+\frac{2}{\theta^{2}} \frac{1}{1+\left(e^{-\theta u}-1\right)\left(e^{-\theta v}-1\right) /\left(e^{-\theta}-1\right)} \times \\
& \times \frac{\left(e^{-\theta}-1\right)\left(-e^{-\theta u} u\left(e^{-\theta v}-1\right)-e^{-\theta v} v\left(e^{-\theta u}-1\right)\right)+\left(e^{-\theta u}-1\right)\left(e^{-\theta v}-1\right) e^{-\theta}}{\left(e^{-\theta}-1\right)^{2}} \\
& -\frac{1}{\theta}\left(-\frac{\left(\left(e^{-\theta}-1\right)\left(-e^{-\theta u} u\left(e^{-\theta v}-1\right)-e^{-\theta v} v\left(e^{-\theta u}-1\right)\right)+\left(e^{-\theta u}-1\right)\left(e^{-\theta v}-1\right) e^{-\theta}\right)^{2}}{\left(1+\left(e^{-\theta u}-1\right)\left(e^{-\theta v}-1\right) /\left(e^{-\theta}-1\right)\right)^{2}\left(e^{-\theta}-1\right)^{4}}\right. \\
& +\frac{\left(e^{-\theta}-1\right)^{3}\left(\left(e^{-\theta v}-1\right) e^{-\theta u} u^{2}+2 e^{-\theta u-\theta v} u v+\left(e^{-\theta u}-1\right) e^{-\theta v} v^{2}\right)}{\left(1+\left(e^{-\theta u}-1\right)\left(e^{-\theta v}-1\right) /\left(e^{-\theta}-1\right)\right)\left(e^{-\theta}-1\right)^{4}} \\
& +\frac{-\left(-e^{-\theta u} u\left(e^{-\theta v}-1\right)-e^{-\theta v} v\left(e^{-\theta u}-1\right)\right) e^{-\theta}}{\left(1+\left(e^{-\theta u}-1\right)\left(e^{-\theta v}-1\right) /\left(e^{-\theta}-1\right)\right)\left(e^{-\theta}-1\right)^{4}} \\
& +\frac{\left(e^{-\theta u-\theta v-\theta}(-u-v-1)+e^{-\theta u-\theta}(-u-1)+e^{-\theta v-\theta}(-v-1)-e^{-\theta}\right)}{\left(1+\left(e^{-\theta u}-1\right)\left(e^{-\theta v}-1\right) /\left(e^{-\theta}-1\right)\right)\left(e^{-\theta}-1\right)^{4}} \\
& -\frac{2\left(e^{-\theta}-1\right) e^{-\theta}}{\left(1+\left(e^{-\theta u}-1\right)\left(e^{-\theta v}-1\right) /\left(e^{-\theta}-1\right)\right)\left(e^{-\theta}-1\right)^{4}} \\
& \left.\times\left(\left(e^{-\theta}-1\right)\left(-e^{-\theta u} u\left(e^{-\theta v}-1\right)-e^{-\theta v} v\left(e^{-\theta u}-1\right)\right)+\left(e^{-\theta u}-1\right)\left(e^{-\theta v}-1\right) e^{-\theta}\right)\right)
\end{aligned}
$$


FGM:

$$
\begin{aligned}
& \frac{\partial^{2} C}{\partial u^{2}}=v(-2 \theta(1-v)) \\
& \frac{\partial^{2} C}{\partial v^{2}}=u(-2 \theta(1-u)) \\
& \frac{\partial^{2} C}{\partial u \partial v}=((1+\theta(1-u)(1-v))-u \theta(1-v))+v(-\theta(1-u)+u \theta) \\
& \frac{\partial^{2} C}{\partial u \partial \theta}=v((1-u)(1-v)-u(1-v)) \\
& \frac{\partial^{2} C}{\partial v \partial \theta}=u((1-u)(1-v)-v(1-u)) \\
& \frac{\partial^{2} C}{\partial \theta^{2}}=0
\end{aligned}
$$

Bivariate normal:

$$
\begin{gathered}
\frac{\partial^{2} C}{\partial u^{2}}=\phi\left(\frac{\Phi^{-1}(v)-\theta \Phi^{-1}(u)}{\sqrt{1-\theta^{2}}}\right)\left(-\theta\left(\phi\left(\Phi^{-1}(u)\right)\right)^{-1} \frac{1}{\sqrt{1-\theta^{2}}}\right) \\
\frac{\partial^{2} C}{\partial v^{2}}=\phi\left(\frac{\Phi^{-1}(u)-\theta \Phi^{-1}(v)}{\sqrt{1-\theta^{2}}}\right)\left(-\theta\left(\phi\left(\Phi^{-1}(v)\right)\right)^{-1} \frac{1}{\sqrt{1-\theta^{2}}}\right) \\
\frac{\partial^{2} C}{\partial u \partial v}=\phi\left(\frac{\Phi^{-1}(v)-\theta \Phi^{-1}(u)}{\sqrt{1-\theta^{2}}}\right)\left(\phi\left(\Phi^{-1}(v)\right)\right)^{-1} \frac{1}{\sqrt{1-\theta^{2}}} \\
\frac{\partial^{2} C}{\partial u \partial \theta}=\phi\left(\frac{\Phi^{-1}(v)-\theta \Phi^{-1}(u)}{\sqrt{1-\theta^{2}}}\right) \frac{-\Phi^{-1}(u) \sqrt{1-\theta^{2}}+\theta\left(\Phi^{-1}(v)-\theta \Phi^{-1}(u)\right)\left(1-\theta^{2}\right)^{-\frac{1}{2}}}{1-\theta^{2}}
\end{gathered}
$$




$$
\begin{gathered}
\frac{\partial^{2} C}{\partial v \partial \theta}=\phi\left(\frac{\Phi^{-1}(u)-\theta \Phi^{-1}(v)}{\sqrt{1-\theta^{2}}}\right) \frac{-\Phi^{-1}(v) \sqrt{1-\theta^{2}}+\theta\left(\Phi^{-1}(u)-\theta \Phi^{-1}(v)\right)\left(1-\theta^{2}\right)^{-\frac{1}{2}}}{1-\theta^{2}} \\
\frac{\partial^{2} C}{\partial \theta^{2}}=\left(1-\theta^{2}\right)^{-1} \theta \frac{\partial C}{\partial \theta}+\frac{\partial C}{\partial \theta}\left(-\frac{-2 \Phi^{-1}(u) \Phi^{-1}(v)\left(1-\theta^{2}\right)+2 \theta z}{2\left(1-\theta^{2}\right)^{2}}\right)
\end{gathered}
$$

where $z=\left(\Phi^{-1}(u)\right)^{2}-2 \theta \Phi^{-1}(u) \Phi^{-1}(v)+\left(\Phi^{-1}(v)\right)^{2}$. 
First derivatives with respect to pmfs and cdfs

The second derivatives of outcome margins with respect to their parameters i.e. $\frac{\partial^{2} F}{\partial \delta_{2}^{2}}, \frac{\partial^{2} F}{\partial \sigma^{2}}, \frac{\partial^{2} F}{\partial \nu^{2}}$, $\frac{\partial^{2} F}{\partial \boldsymbol{\delta}_{2} \partial \sigma}, \frac{\partial^{2} F}{\partial \boldsymbol{\delta}_{\mathbf{2}} \partial \partial \nu}, \frac{\partial^{2} F}{\partial \sigma \partial \nu}$ were differentiated numerically. For example, suppose that we want calculate $\frac{\partial^{2} F}{\partial \boldsymbol{\delta}_{\mathbf{2}} \partial \sigma}$. Thus,

$$
\frac{\partial^{2} F}{\partial \boldsymbol{\delta}_{\mathbf{2}} \partial \sigma}=\frac{\frac{\partial F\left(y_{2} \mid \eta_{2}, \sigma+\epsilon^{\prime}, \nu\right)}{\partial \boldsymbol{\delta}_{\mathbf{2}}}-\frac{\partial F\left(y_{2} \mid \eta_{2}, \sigma, \nu\right)}{\partial \boldsymbol{\delta}_{\mathbf{2}}}}{\epsilon^{\prime}} \mathbf{X}_{\mathbf{2}}
$$

where $\epsilon^{\prime}$ is equal to $10^{-7}$ for Poisson and negative binomial distribution and $10^{-3}$ for Delaporte, Poisson inverse Gaussian and Sichel distributions. The reason for choosing a different $\epsilon^{\prime}$ for Delaporte, Poisson inverse Gaussian and Sichel distribution is that there is a significant difference between the numerical and analytical derivatives when very small $\epsilon^{\prime}$ values are used. 
Appendix $\mathbf{J}$

Simulation for likelihood function

using trust for analytical Hessian and

specification with and without

instrumental variable 


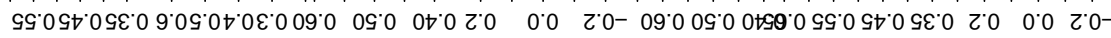
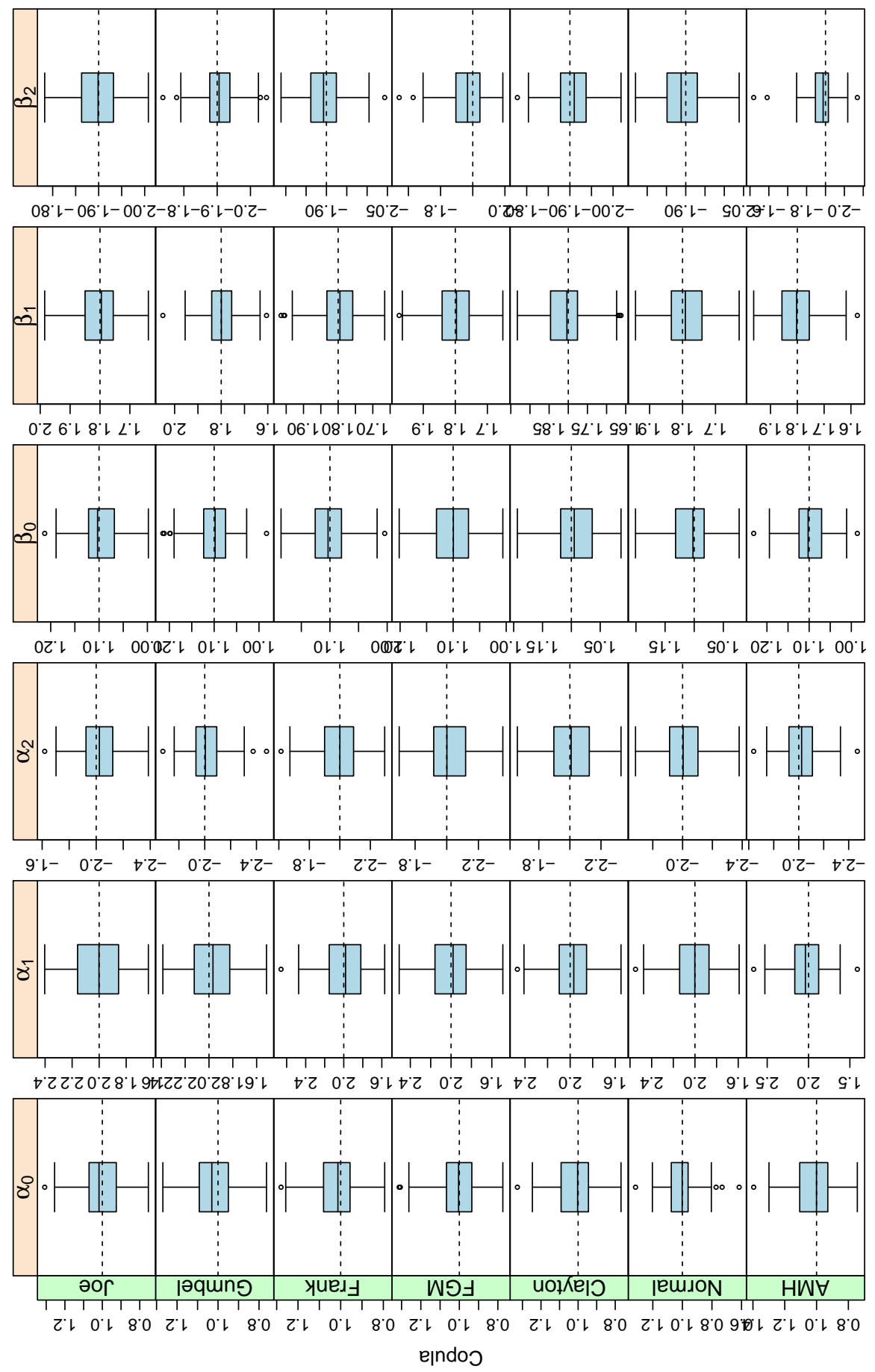


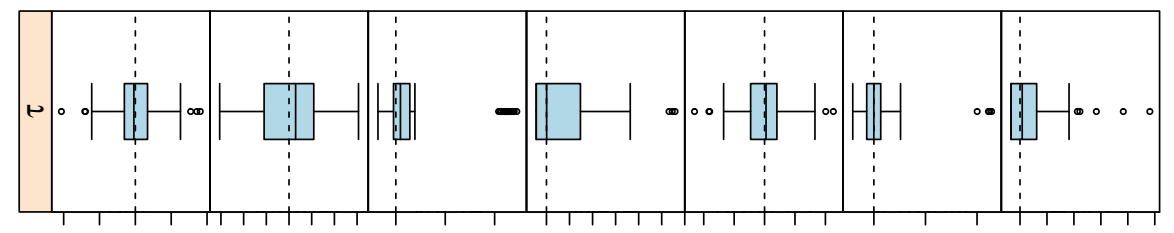

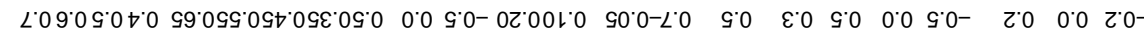

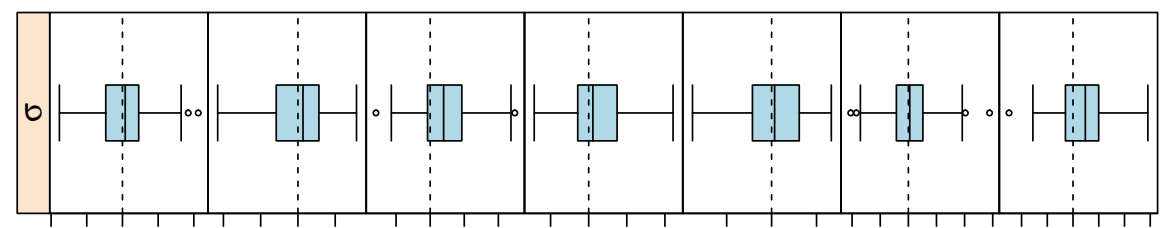

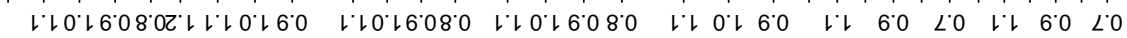
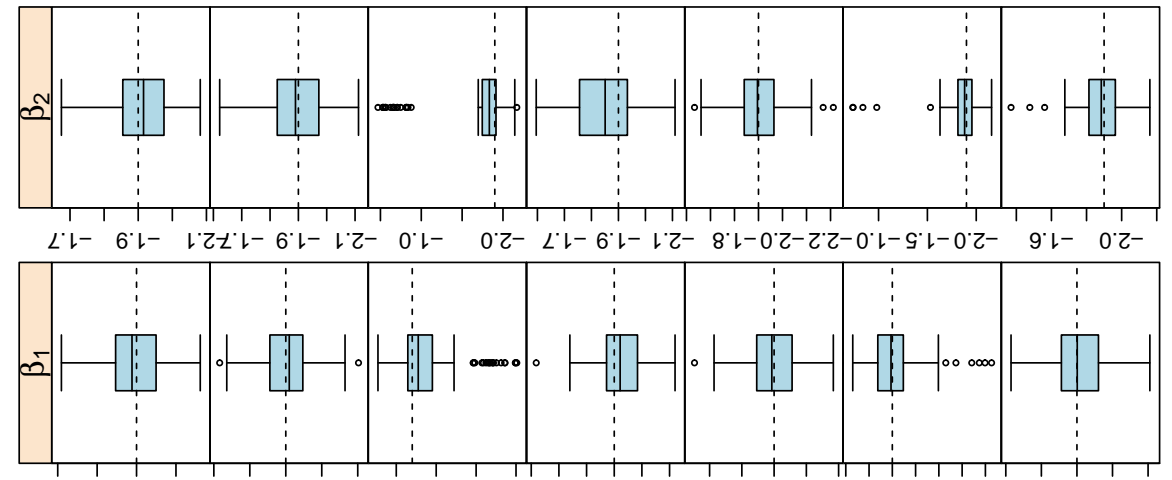

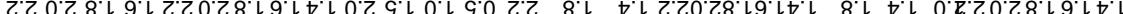

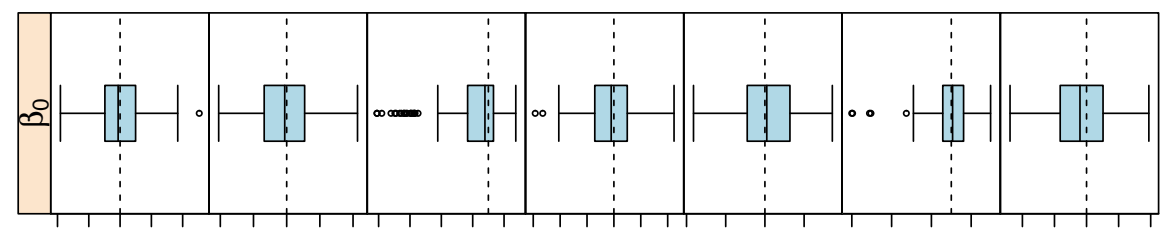

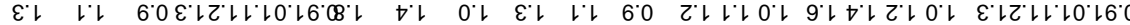
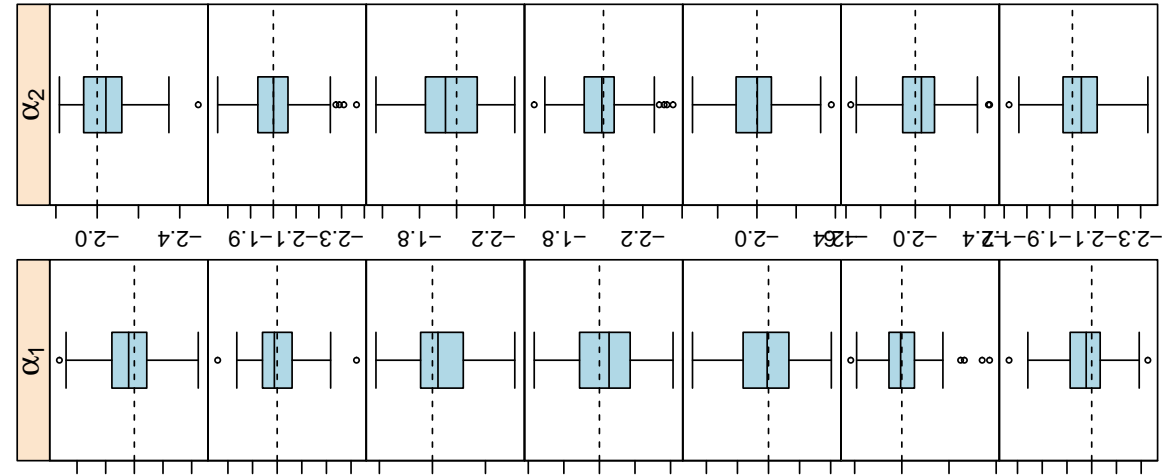

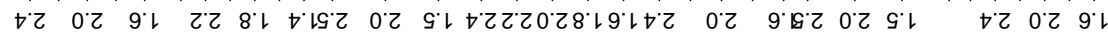

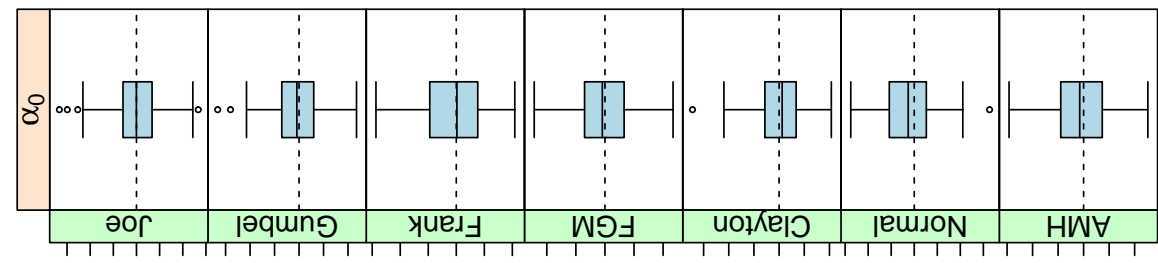

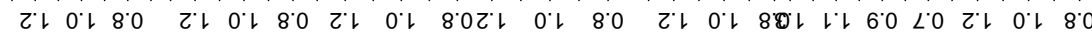

ejndos 

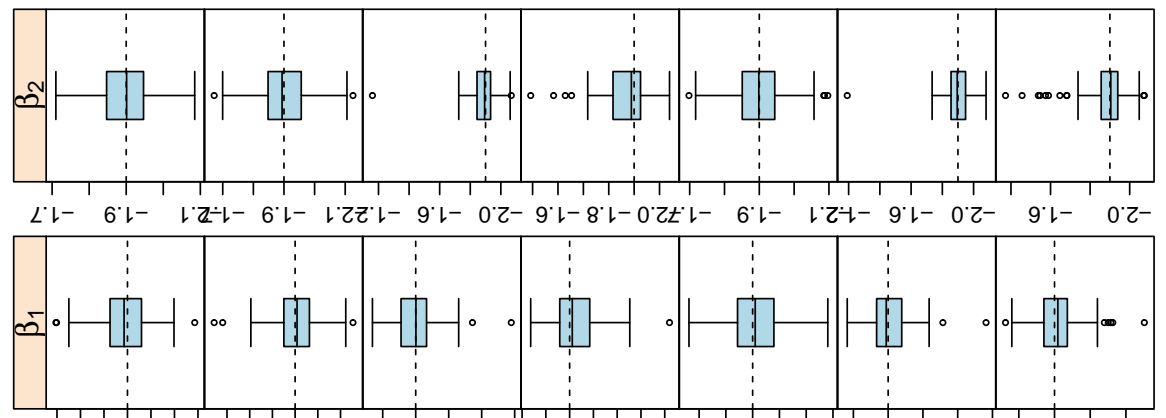

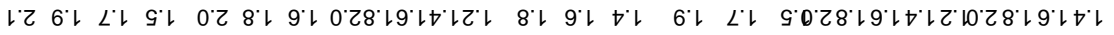

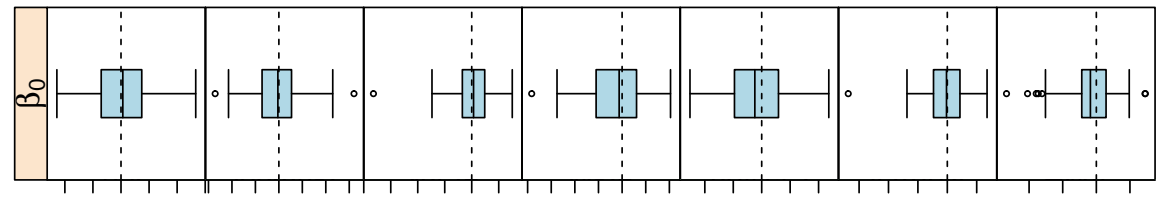

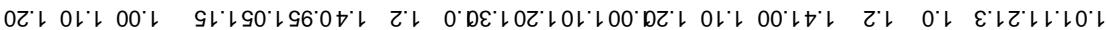
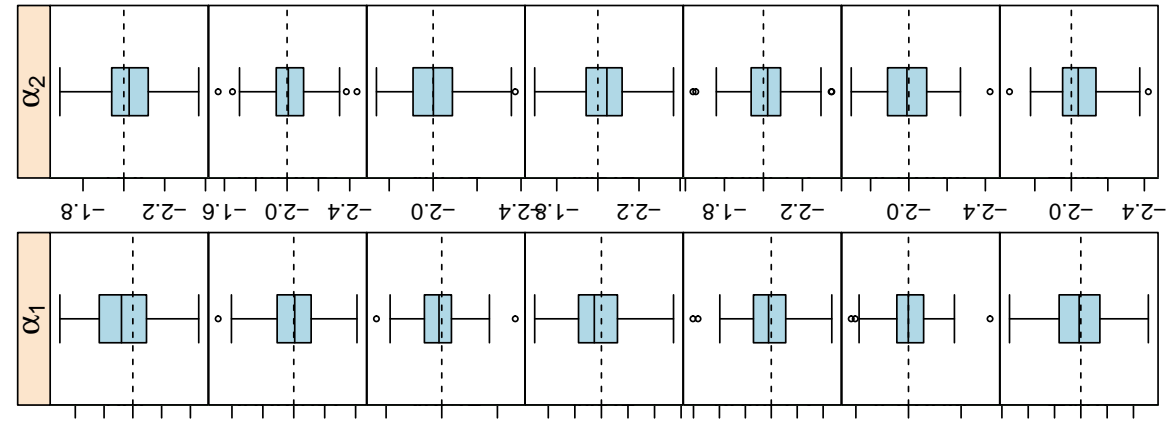

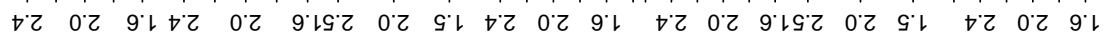

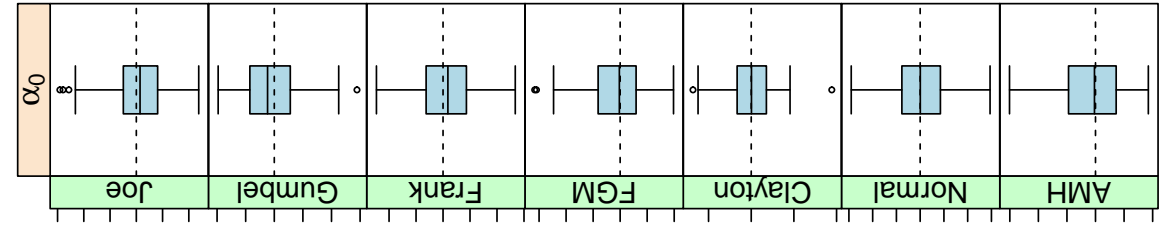

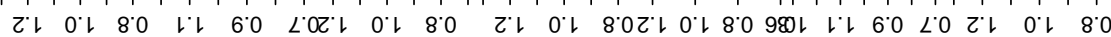
ejndoo 

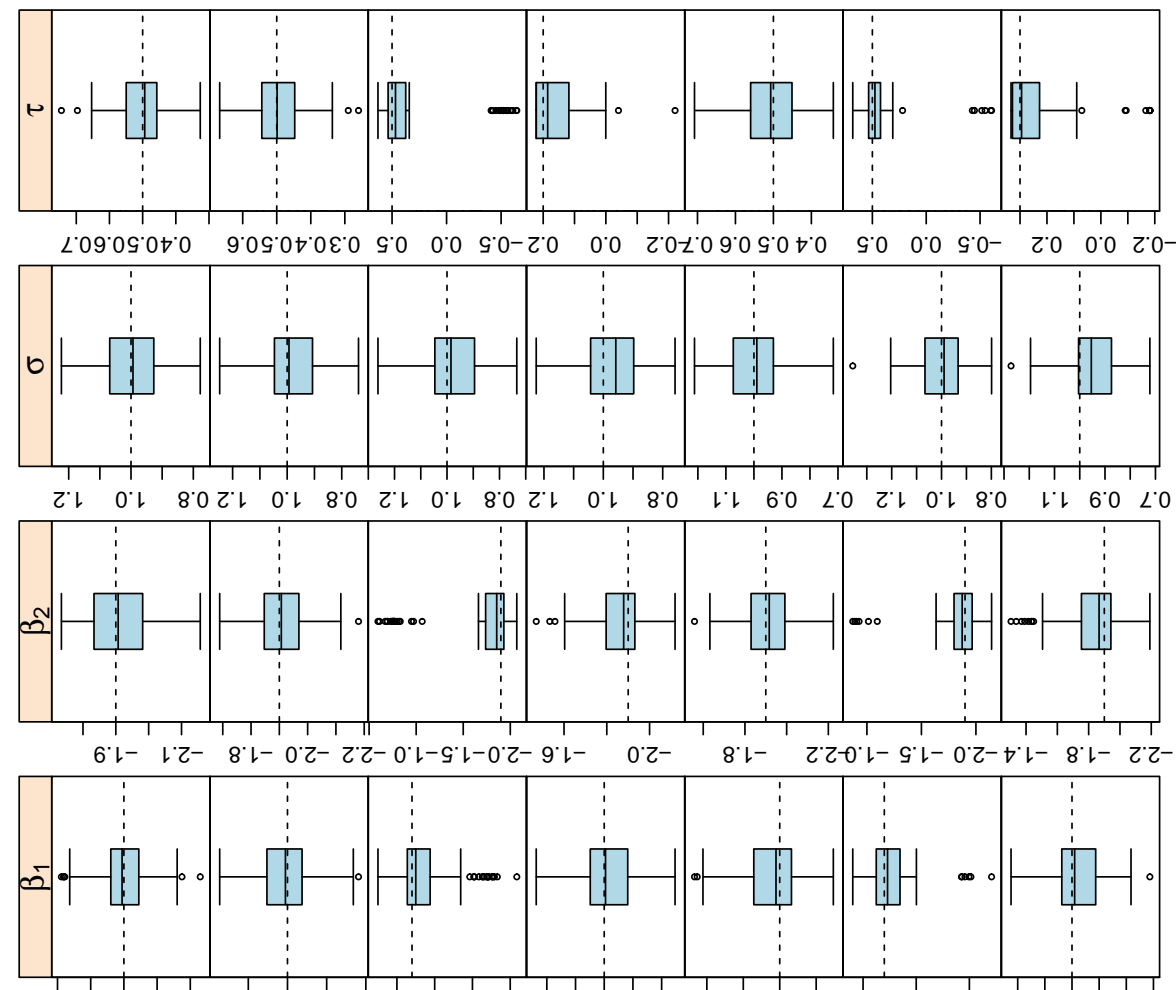

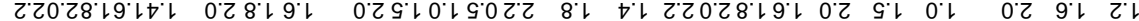
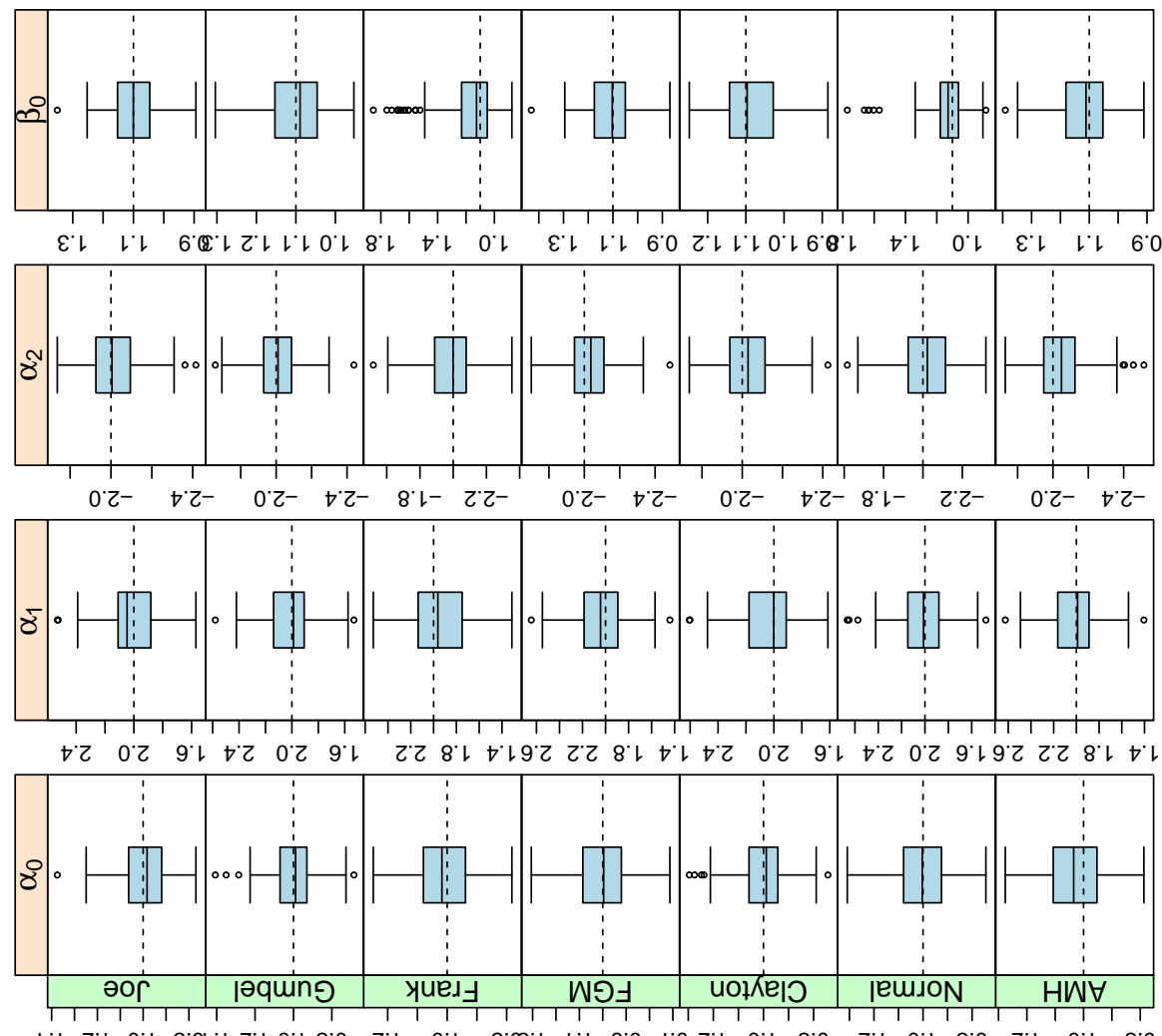

ガレ゙

ejndoo 


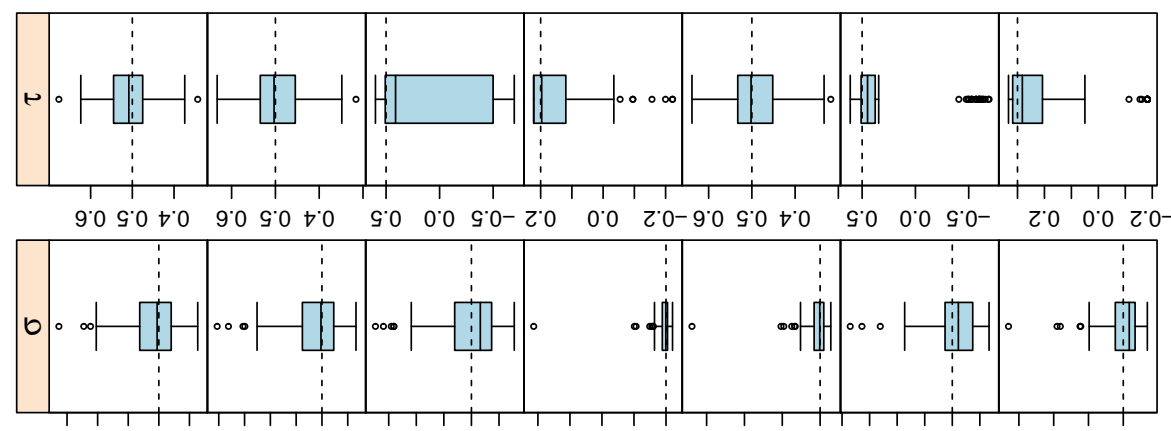

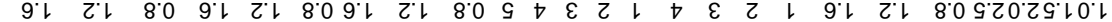
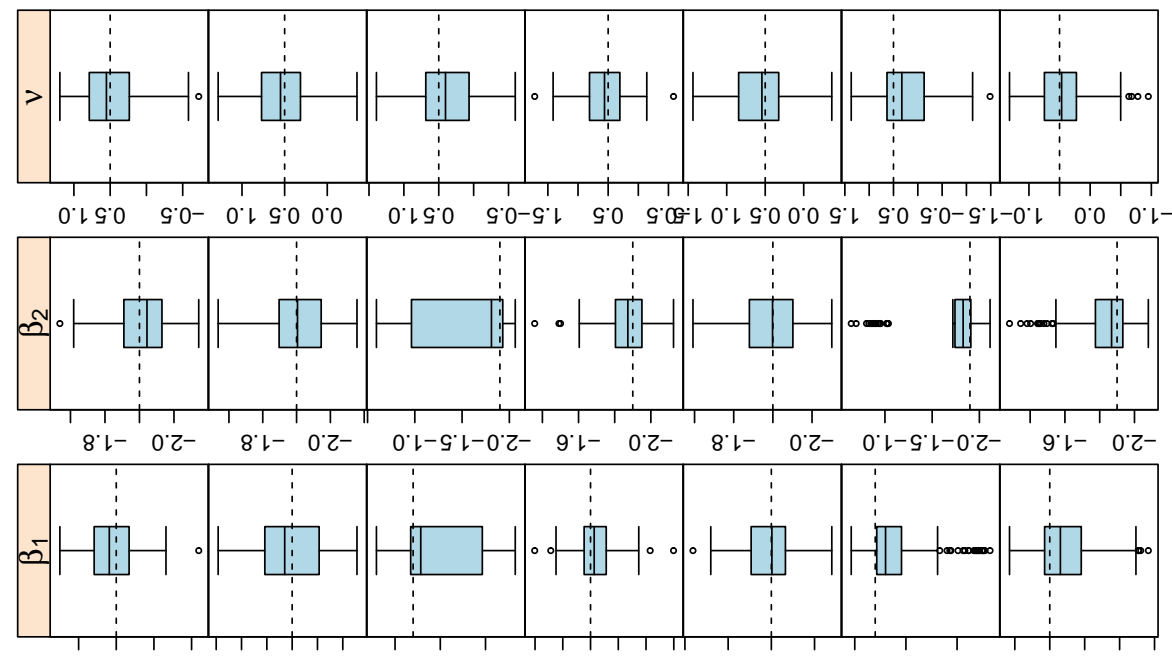

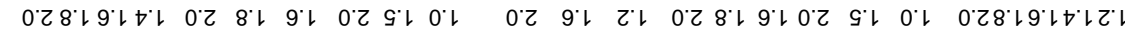
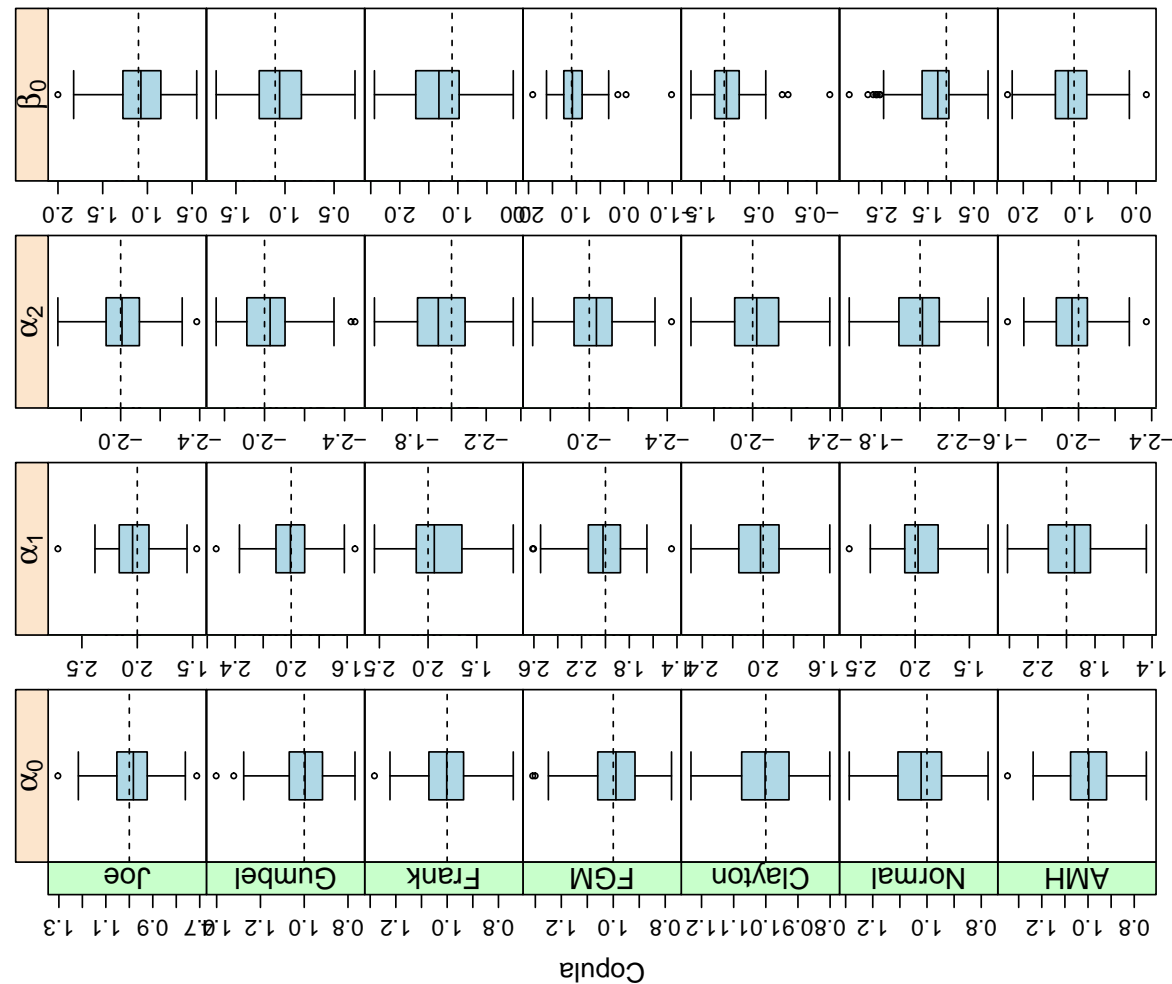


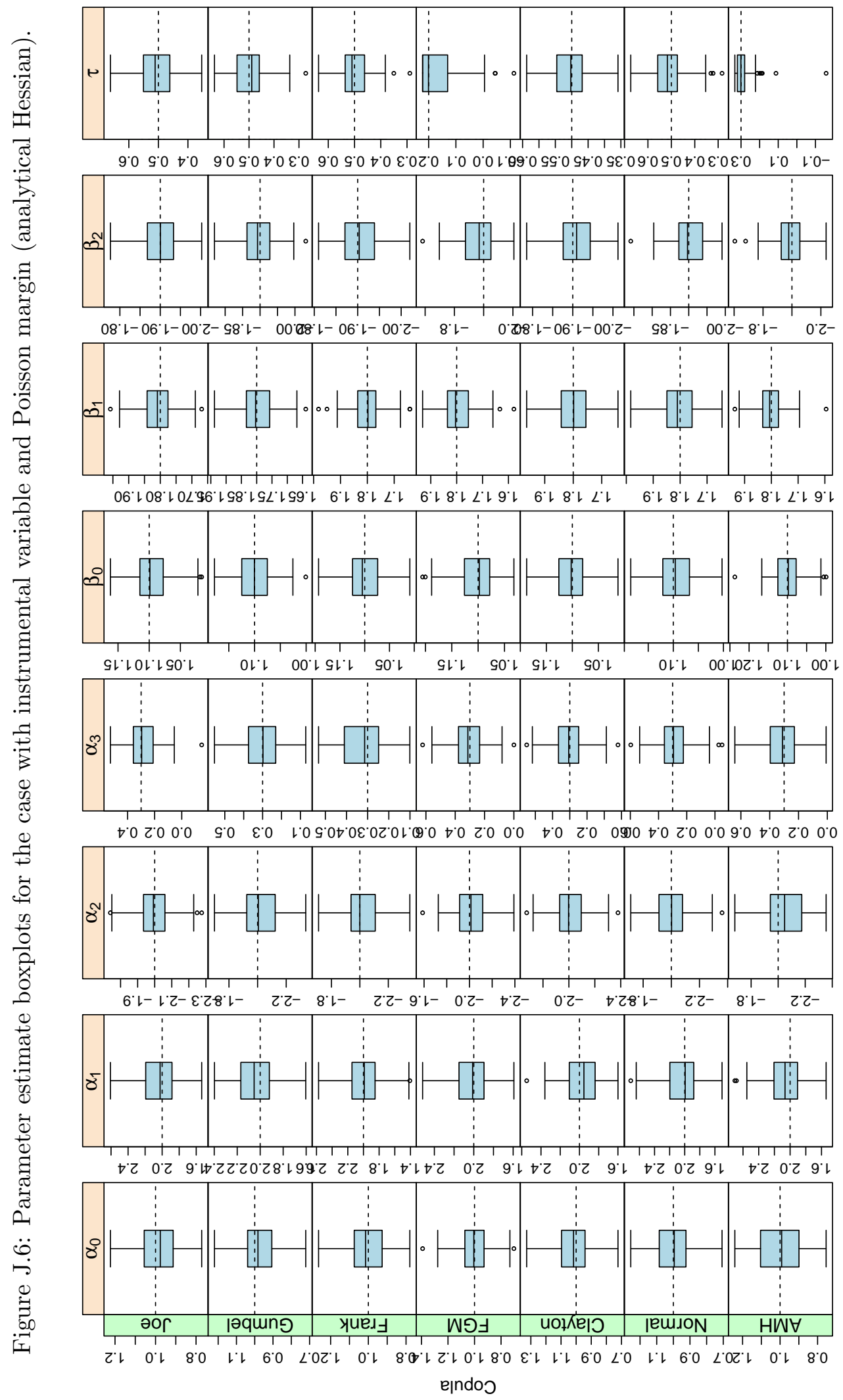




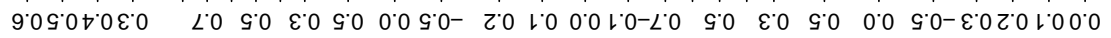

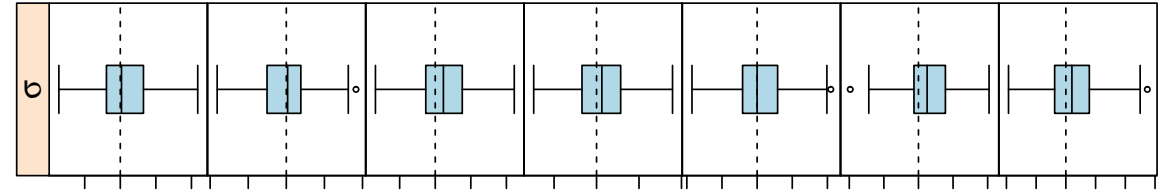

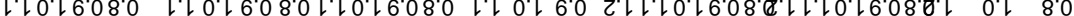
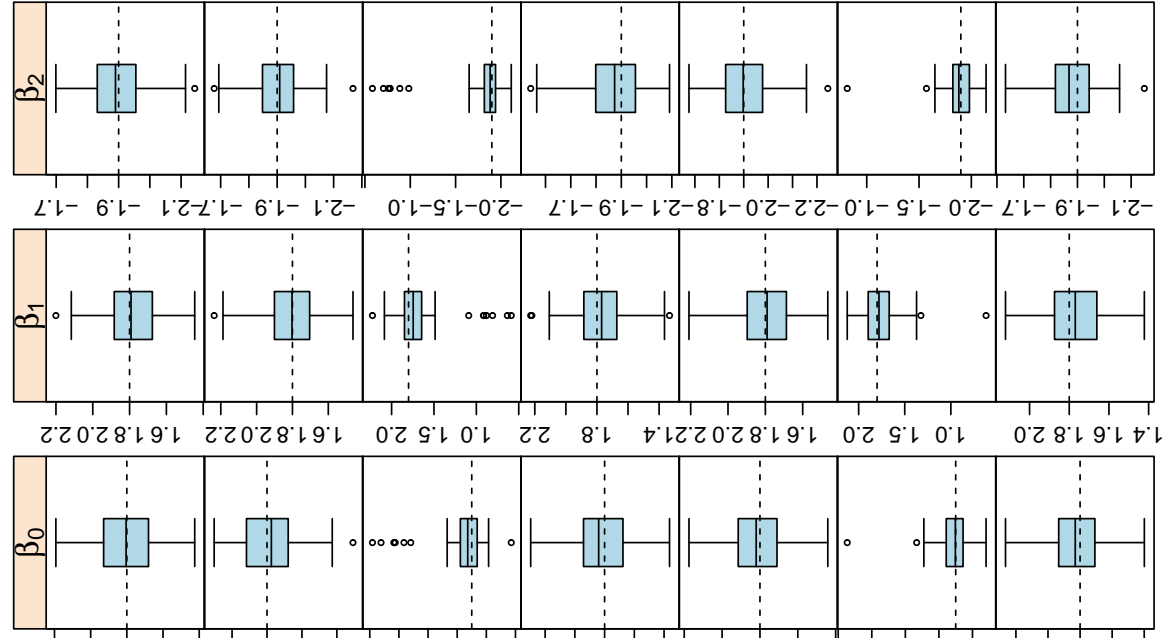

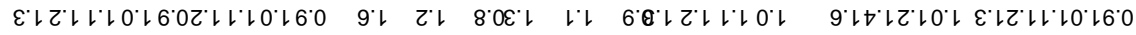

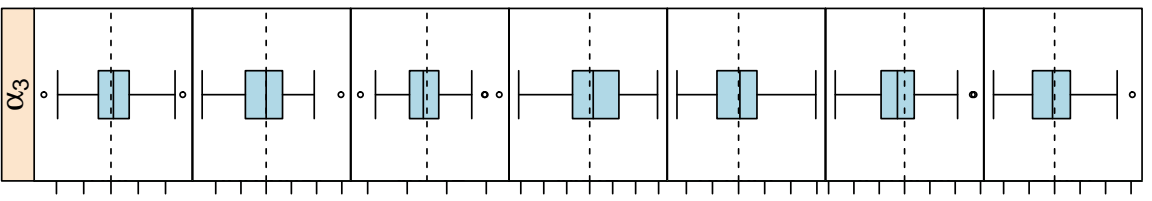

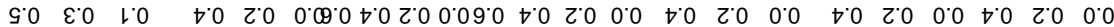
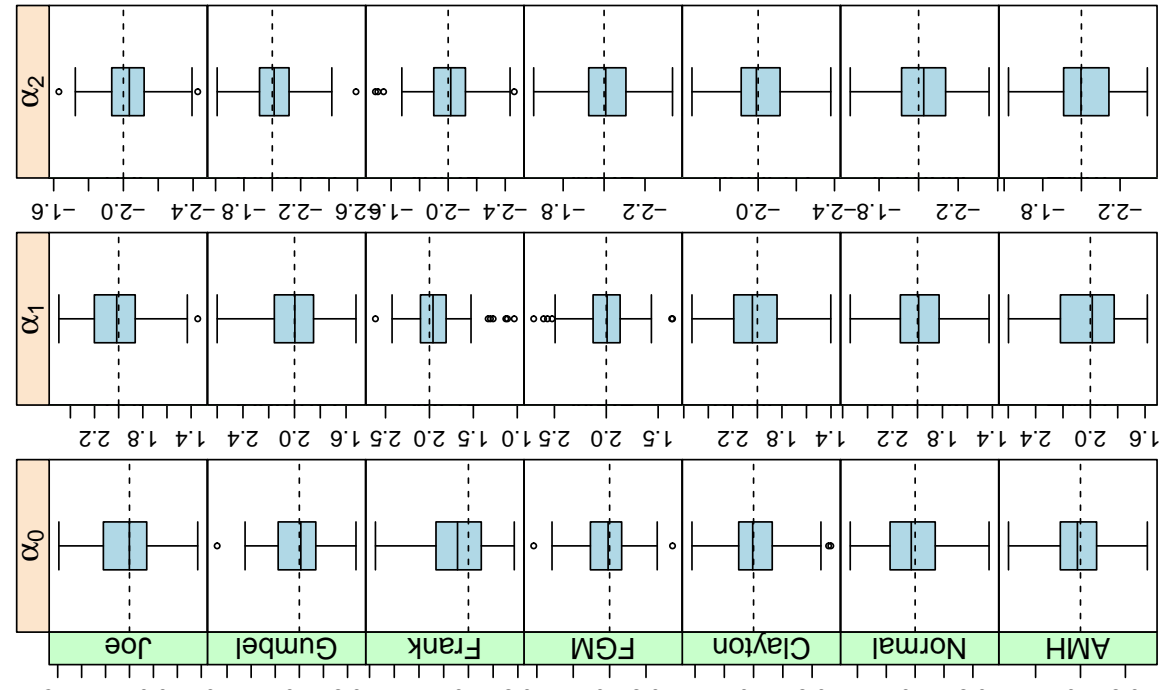

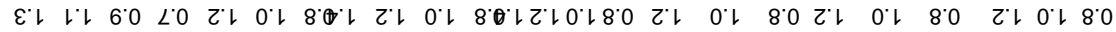
ejndo 


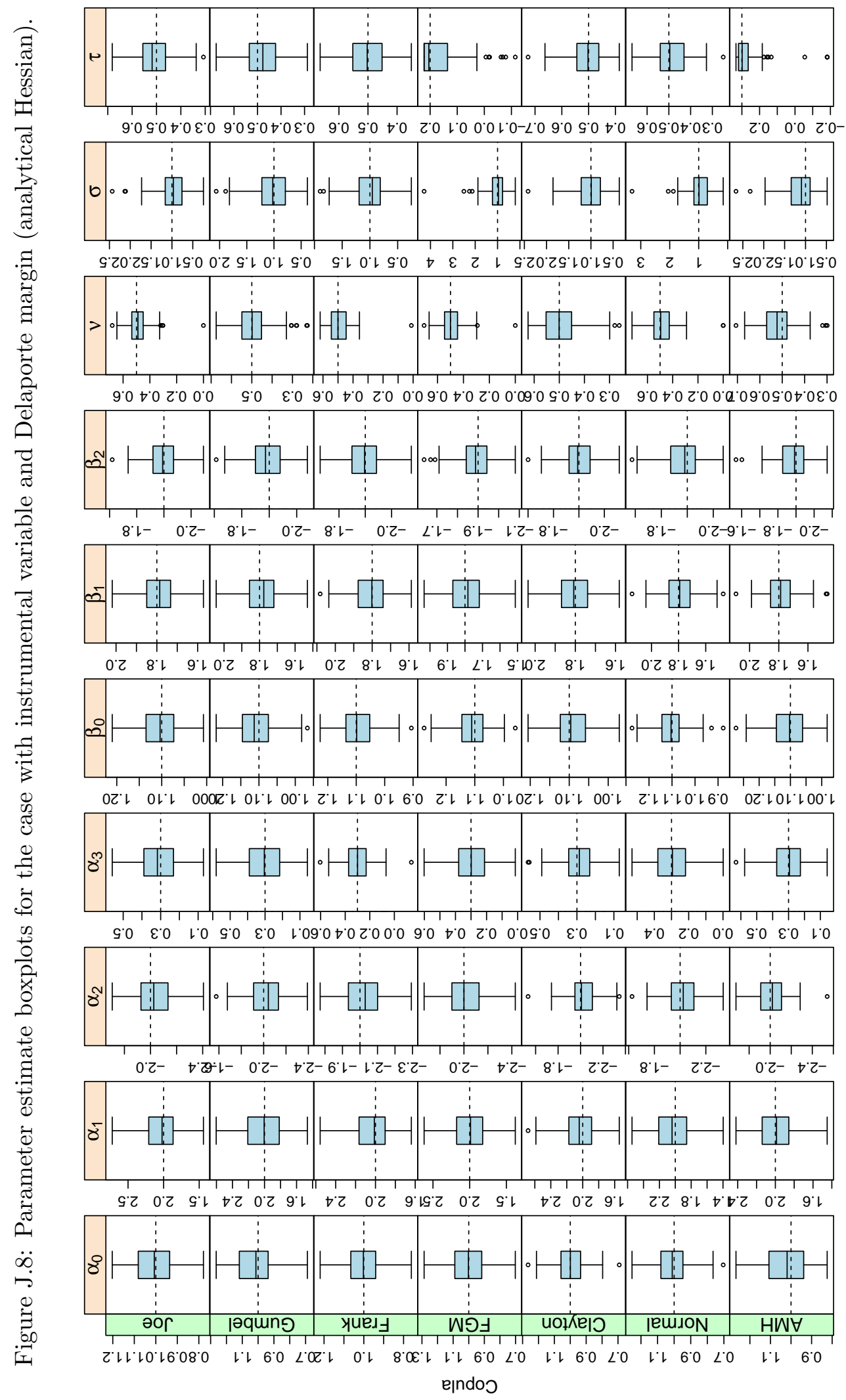




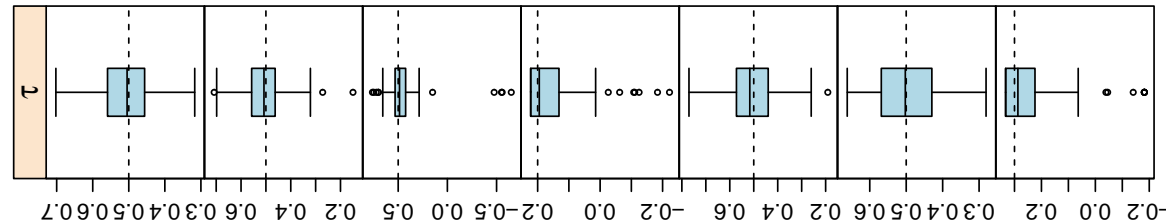

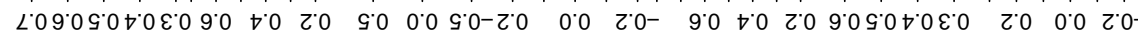

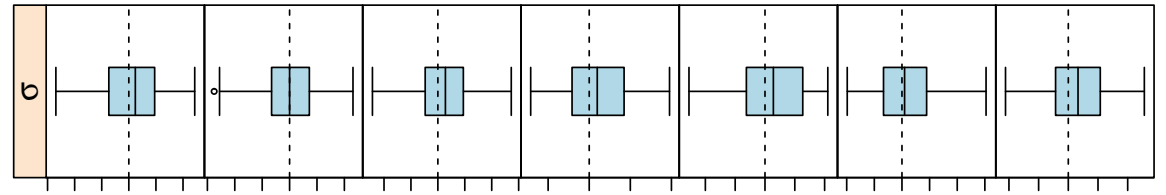

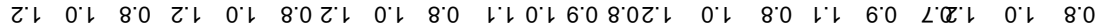
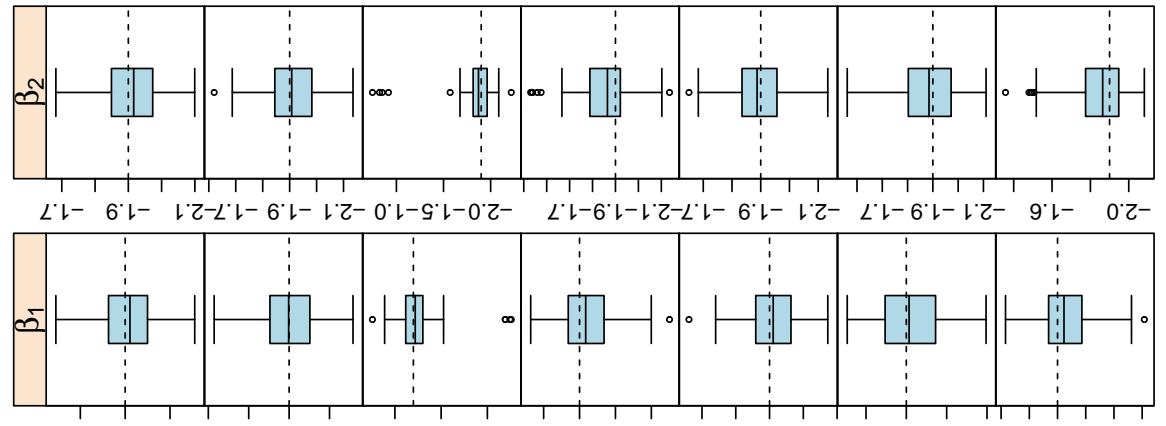

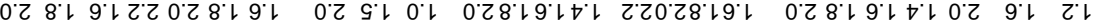

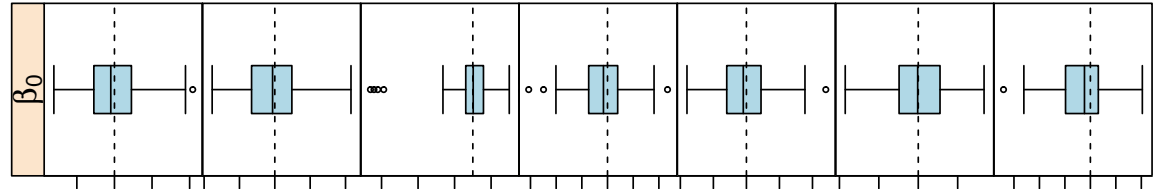

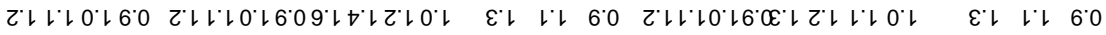

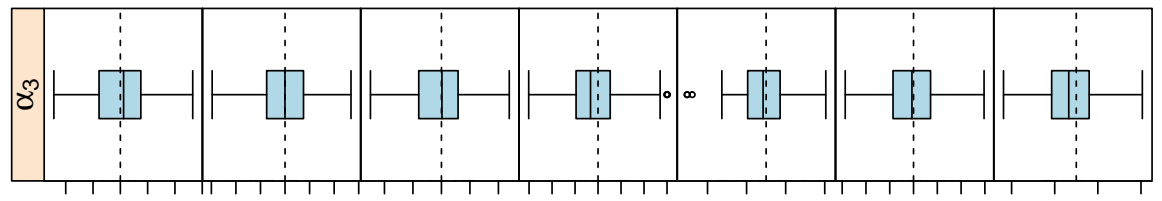

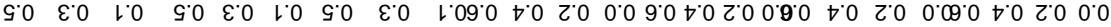
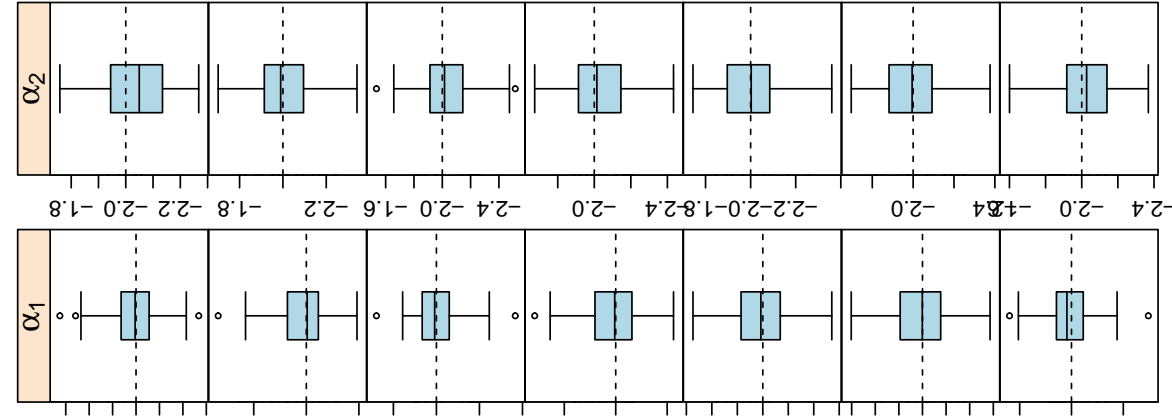

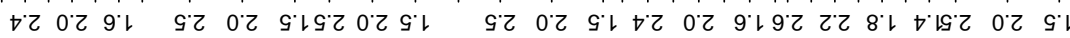

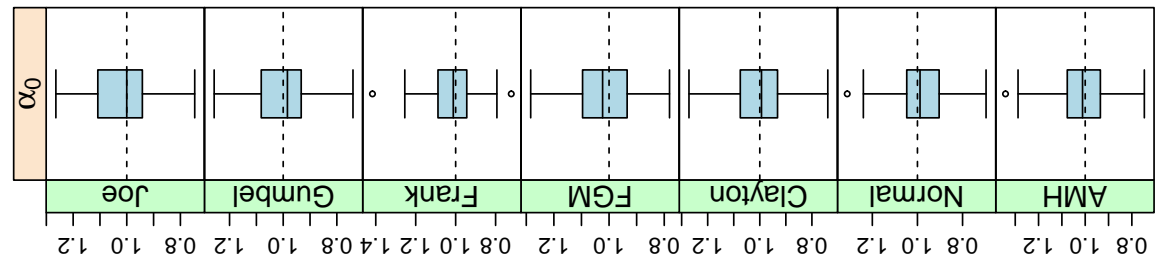

e्pndoo 


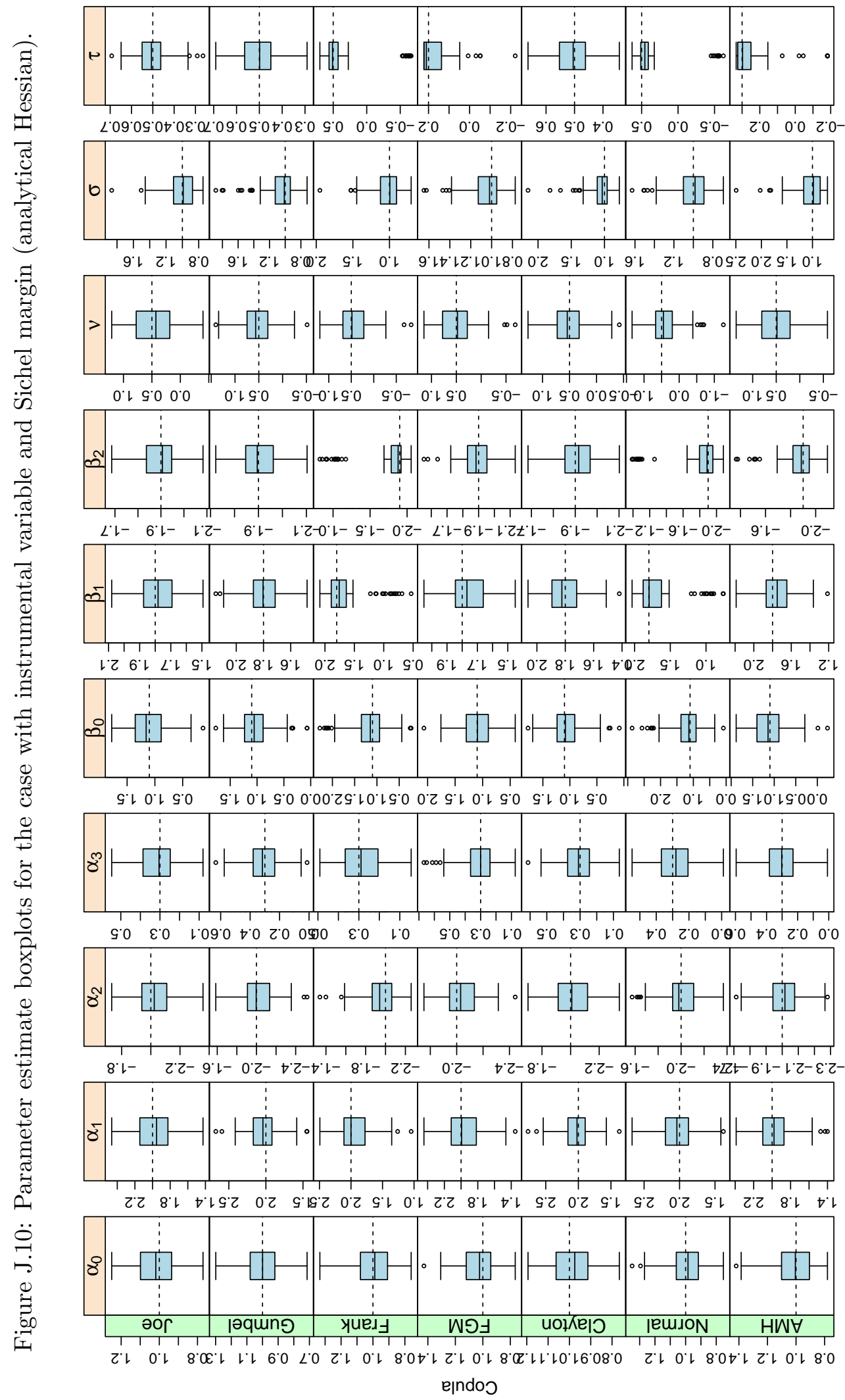




\section{Appendix $\mathrm{K}$}

\section{First and second derivatives of rotated}

\section{copulas}

All derivations below were conducted manually. According to the author's best knowledge, these cannot be found in the literature.

90 degrees copulas

$$
\begin{aligned}
\frac{\partial C_{90}}{\partial u} & =\frac{\partial C(1-u, v)}{\partial u} \\
\frac{\partial C_{90}}{\partial v} & =1-\frac{\partial C(1-u, v)}{\partial v} \\
\frac{\partial C_{90}}{\partial \theta} & =-\left(-\frac{\partial C(1-u, v)}{\partial \theta}\right) \\
\frac{\partial^{2} C_{90}}{\partial u^{2}} & =-\frac{\partial^{2} C(1-u, v)}{\partial u^{2}} \\
\frac{\partial^{2} C_{90}}{\partial v^{2}} & =-\frac{\partial^{2} C(1-u, v)}{\partial v^{2}} \\
\frac{\partial^{2} C_{90}}{\partial u \partial v} & =\frac{\partial^{2} C(1-u, v)}{\partial u \partial v} \\
\frac{\partial^{2} C_{90}}{\partial u \partial \theta} & =-\left(\frac{\partial^{2} C(1-u, v)}{\partial u \partial \theta}\right) \\
\frac{\partial^{2} C_{90}}{\partial v \partial \theta} & =-\left(-\frac{\partial^{2} C(1-u, v)}{\partial v \partial \theta}\right) \\
\frac{\partial^{2} C_{90}}{\partial \theta^{2}} & =-\frac{\partial^{2} C(1-u, v)}{\partial \theta^{2}}
\end{aligned}
$$


180 degrees copulas

$$
\begin{aligned}
\frac{\partial C_{180}}{\partial u} & =1-\frac{\partial C(1-u, 1-v)}{\partial u} \\
\frac{\partial C_{180}}{\partial v} & =1-\frac{\partial C(1-u, 1-v)}{\partial v} \\
\frac{\partial C_{180}}{\partial \theta} & =\left(\frac{\partial C(1-u, 1-v)}{\partial \theta}\right) \\
\frac{\partial^{2} C_{180}}{\partial u^{2}} & =\frac{\partial^{2} C(1-u, 1-v)}{\partial u^{2}} \\
\frac{\partial^{2} C_{180}}{\partial v^{2}} & =\frac{\partial^{2} C(1-u, 1-v)}{\partial v^{2}} \\
\frac{\partial^{2} C_{180}}{\partial u \partial v} & =\frac{\partial^{2} C(1-u, 1-v)}{\partial u \partial v} \\
\frac{\partial^{2} C_{180}}{\partial u \partial \theta} & =-\frac{\partial^{2} C(1-u, 1-v)}{\partial u \partial \theta} \\
\frac{\partial^{2} C_{180}}{\partial v \partial \theta} & =-\frac{\partial^{2} C(1-u, 1-v)}{\partial v \partial \theta} \\
\frac{\partial^{2} C_{180}}{\partial \theta^{2}} & =\frac{\partial^{2} C(1-u, 1-v)}{\partial \theta^{2}}
\end{aligned}
$$

270 degrees copulas

$$
\begin{aligned}
\frac{\partial C_{270}}{\partial u} & =1-\frac{\partial C(u, 1-v)}{\partial u} \\
\frac{\partial C_{270}}{\partial v} & =\frac{\partial C(u, 1-v))}{\partial v} \\
\frac{\partial C_{270}}{\partial \theta} & =-\left(-\frac{\partial C(u, 1-v)}{\partial \theta}\right) \\
\frac{\partial^{2} C_{270}}{\partial u^{2}} & =-\frac{\partial^{2} C(u, 1-v)}{\partial u^{2}} \\
\frac{\partial^{2} C_{270}}{\partial v^{2}} & =-\frac{\partial^{2} C(u, 1-v)}{\partial v^{2}} \\
\frac{\partial^{2} C_{270}}{\partial u \partial v} & =\frac{\partial^{2} C(u, 1-v)}{\partial u \partial v} \\
\frac{\partial^{2} C_{270}}{\partial u \partial \theta} & =-\left(-\frac{\partial^{2} C(u, 1-v)}{\partial u \partial \theta}\right) \\
\frac{\partial^{2} C_{270}}{\partial v \partial \theta} & =-\left(\frac{\partial^{2} C(u, 1-v)}{\partial v \partial \theta}\right) \\
\frac{\partial^{2} C_{270}}{\partial \theta^{2}} & =-\frac{\partial^{2} C(u, 1-v)}{\partial \theta^{2}}
\end{aligned}
$$


Appendix L

Simulation for rotated copulas 


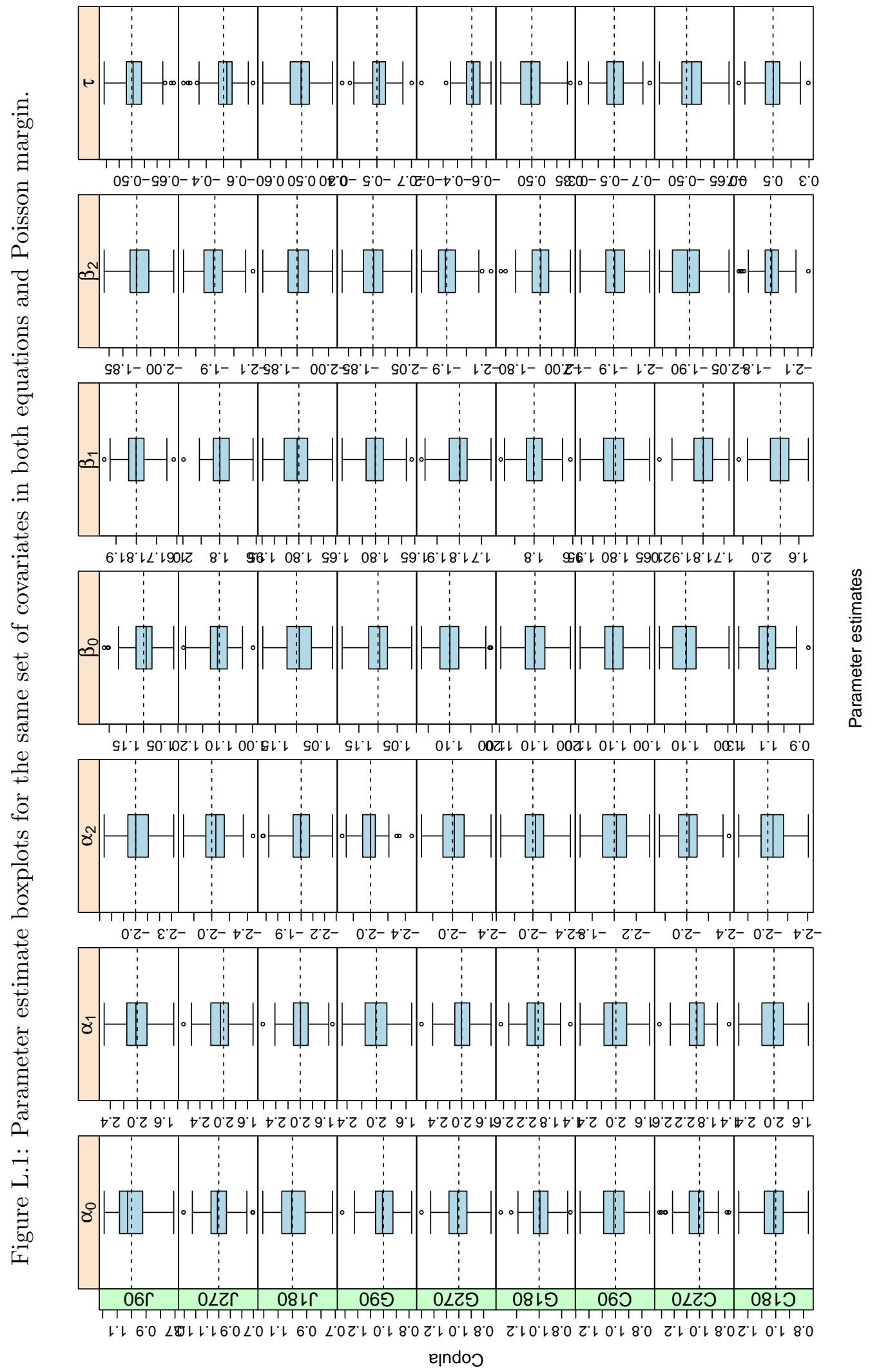




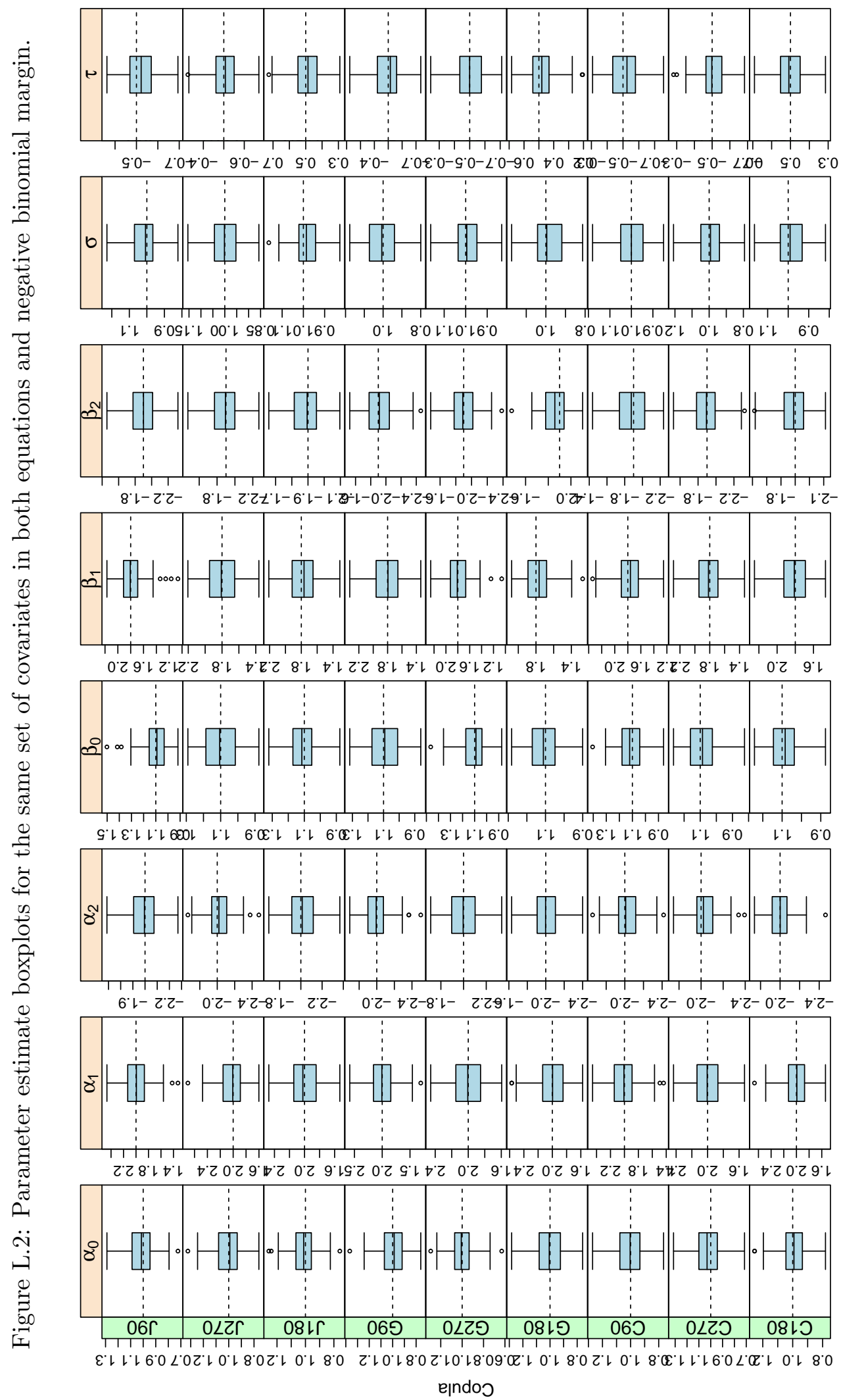




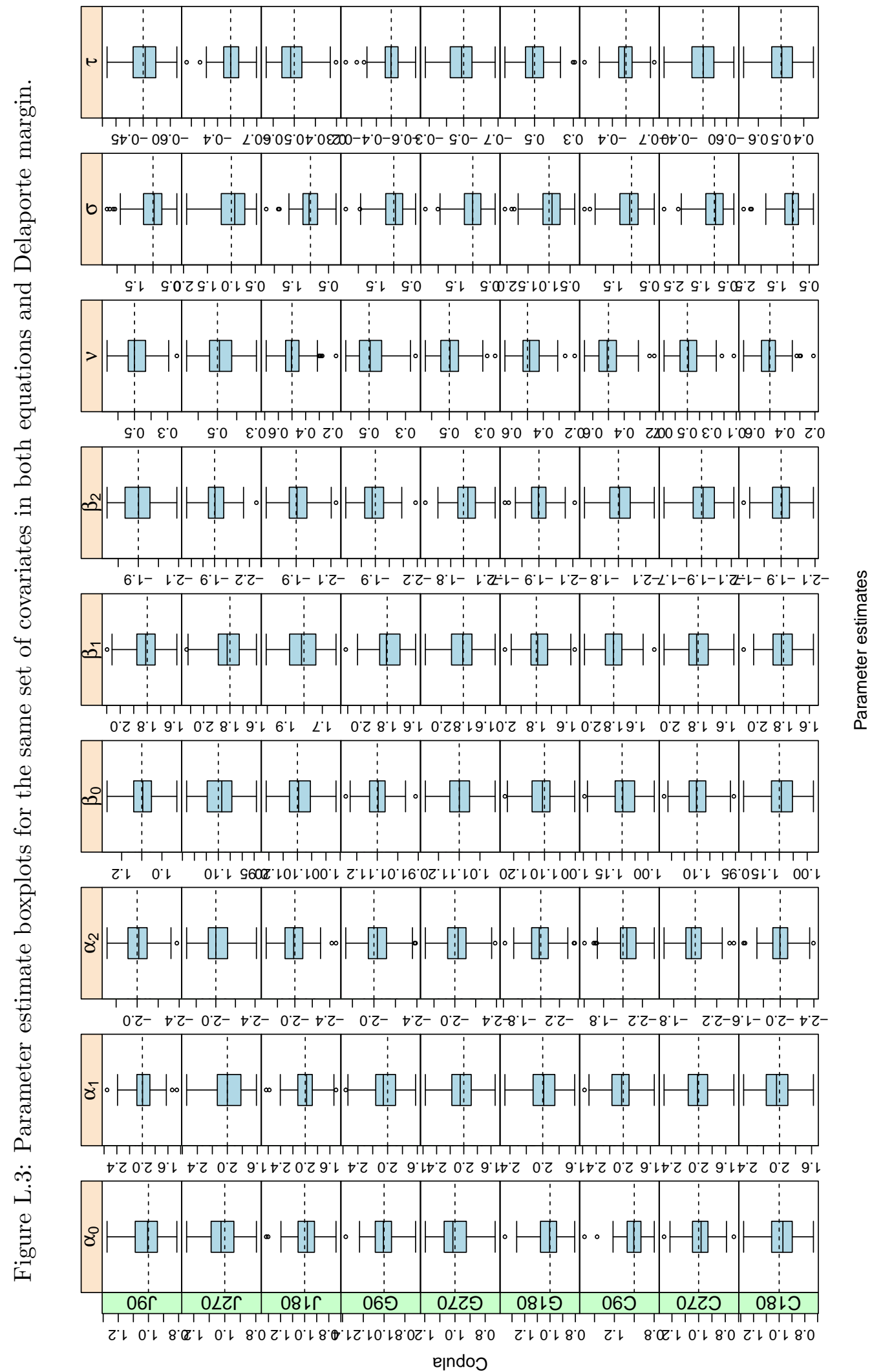




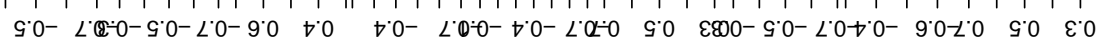

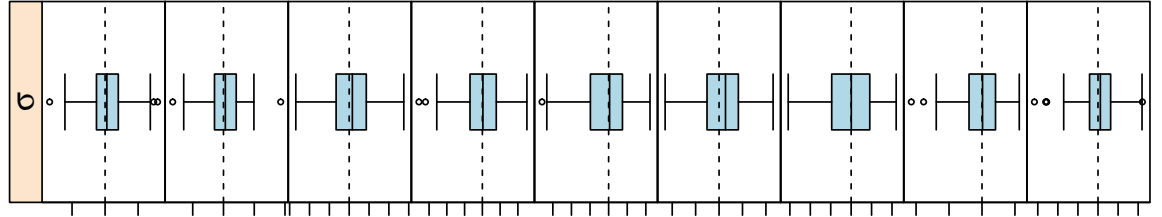

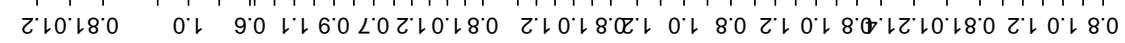

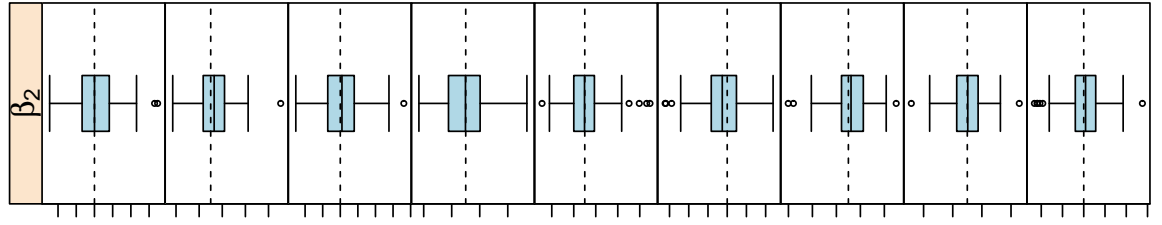

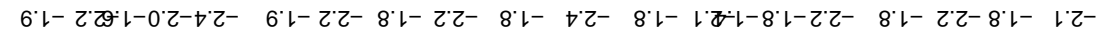
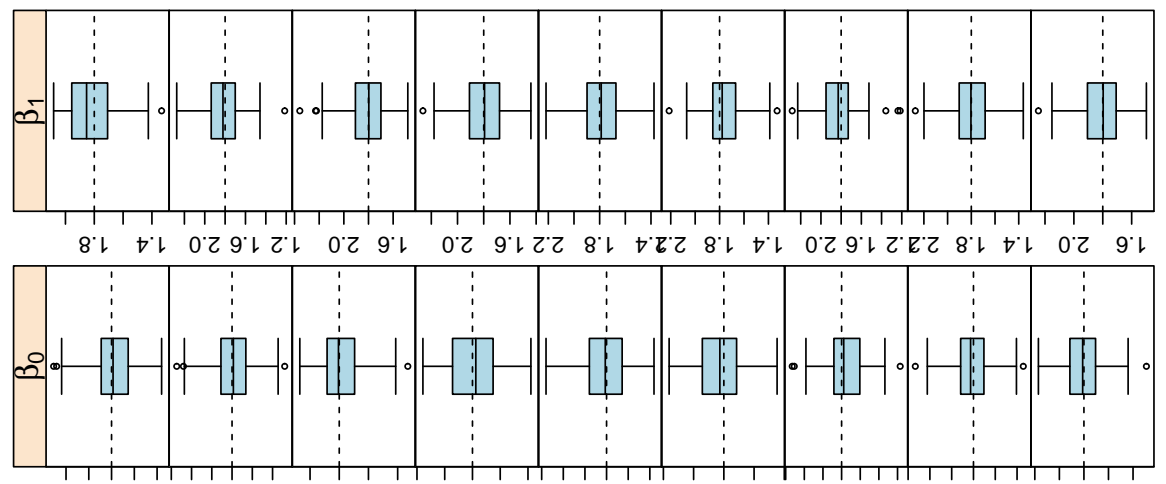

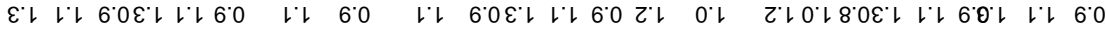
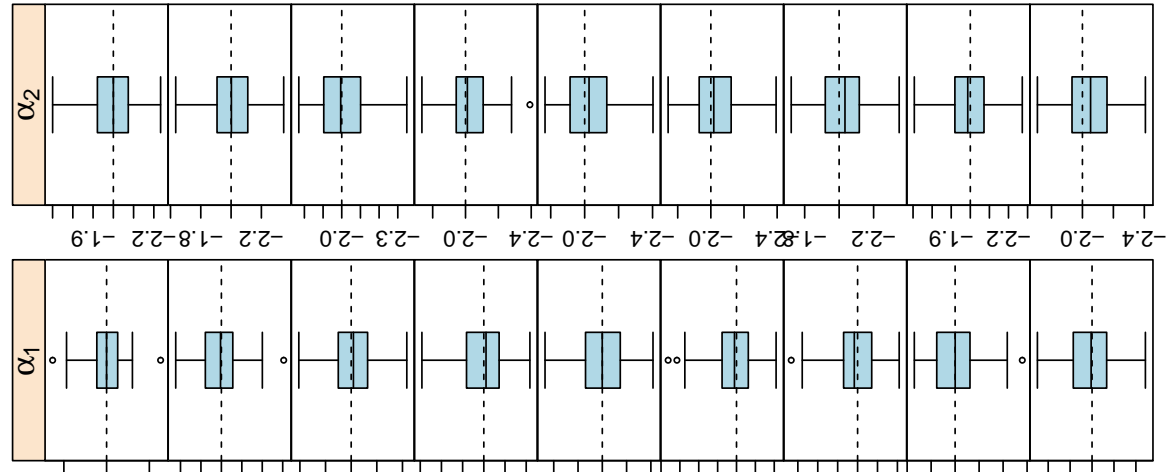
エ゚

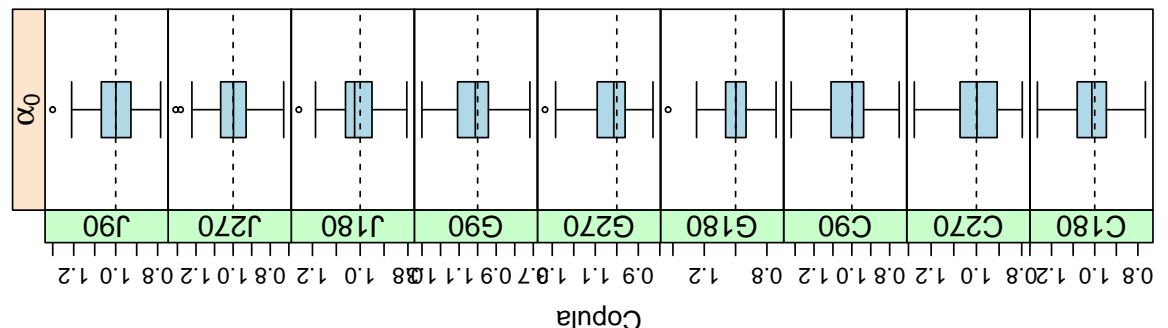




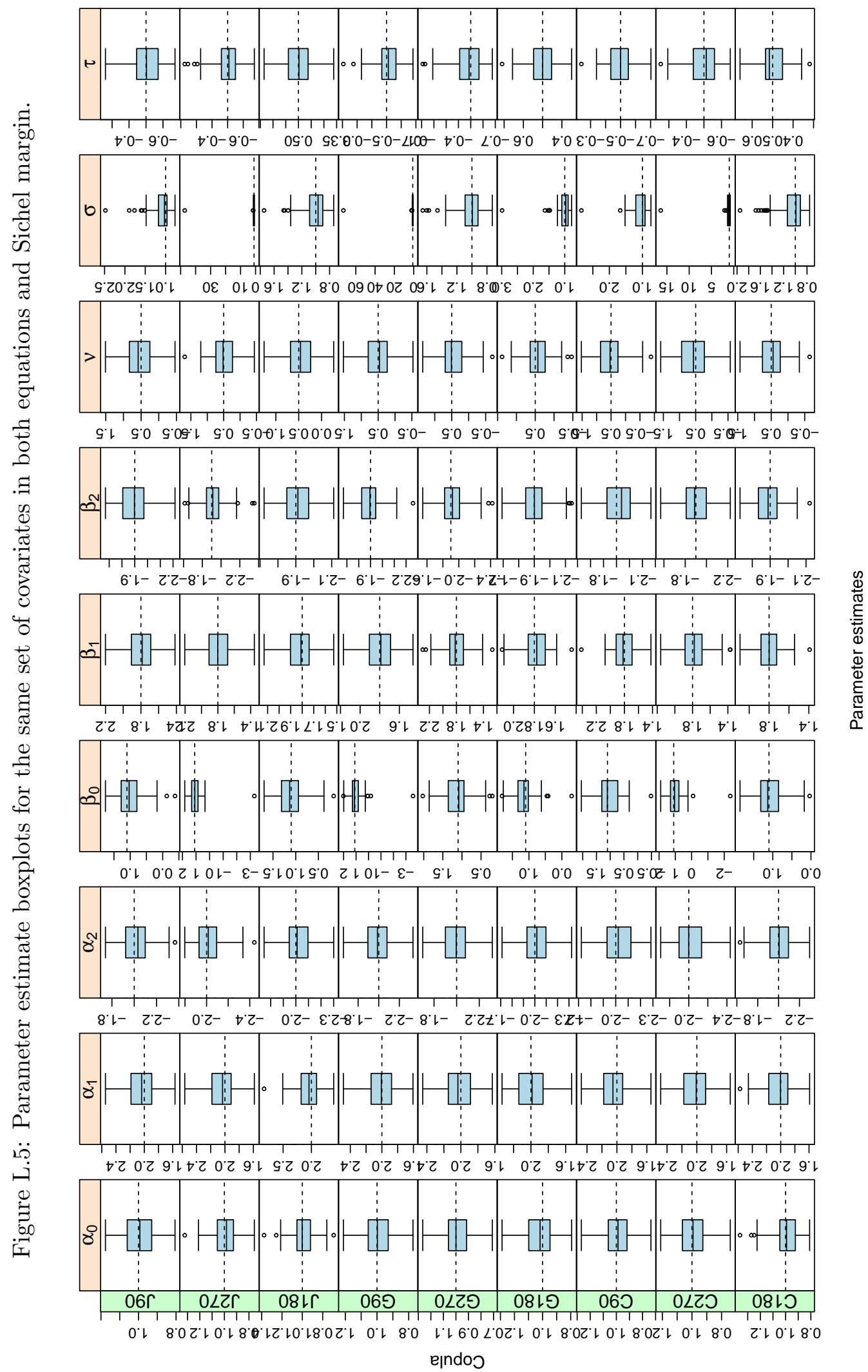




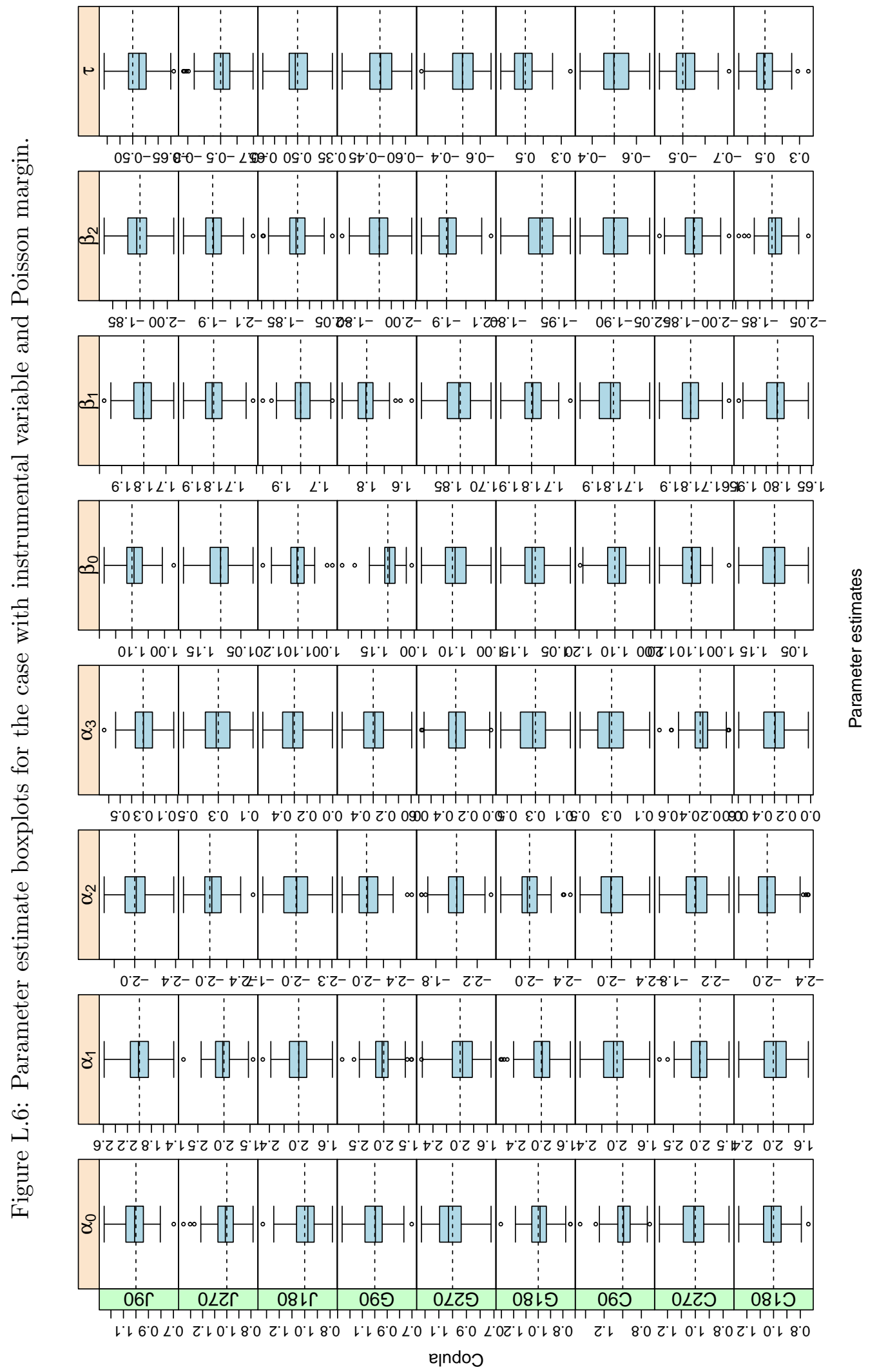




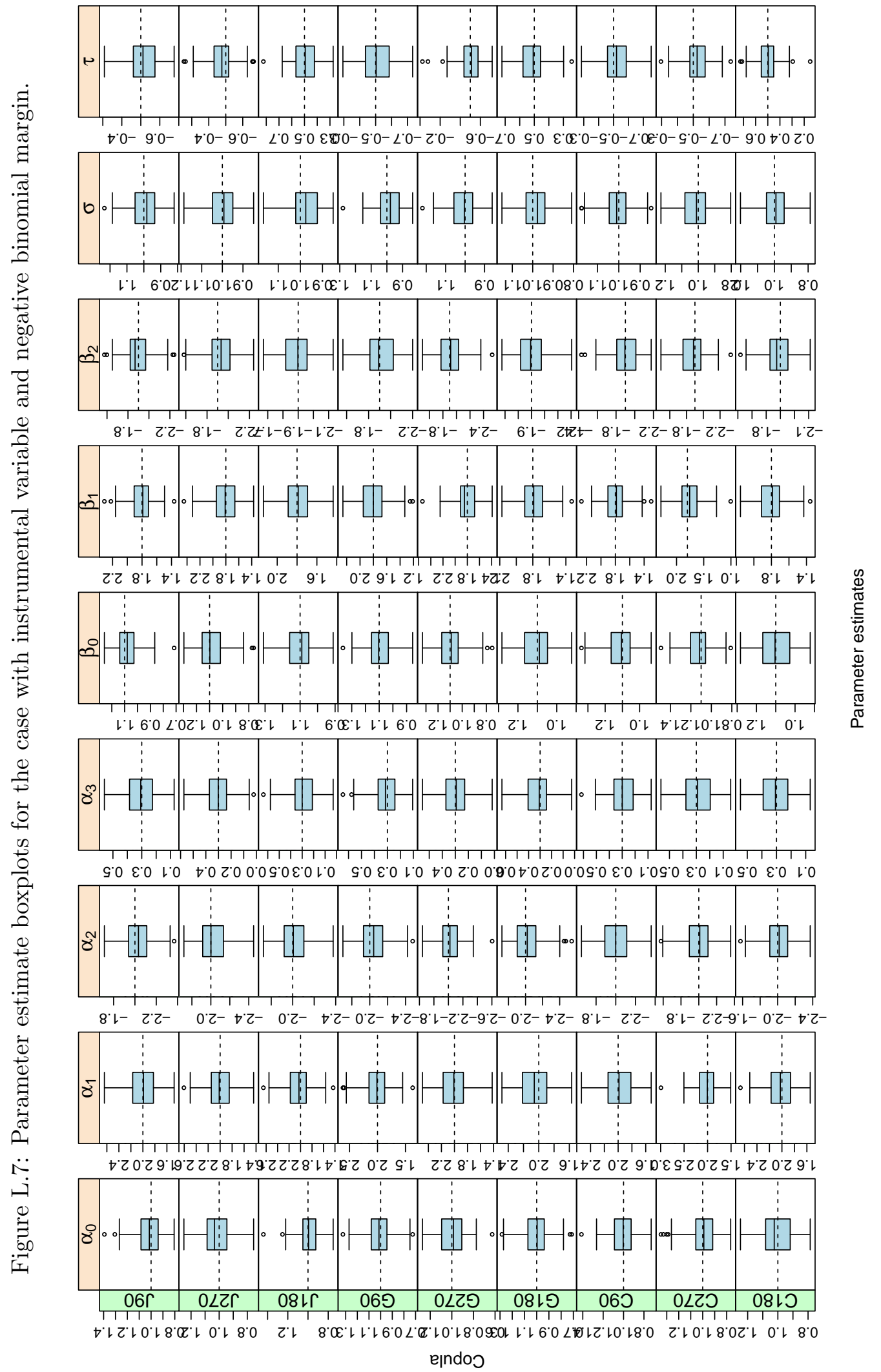




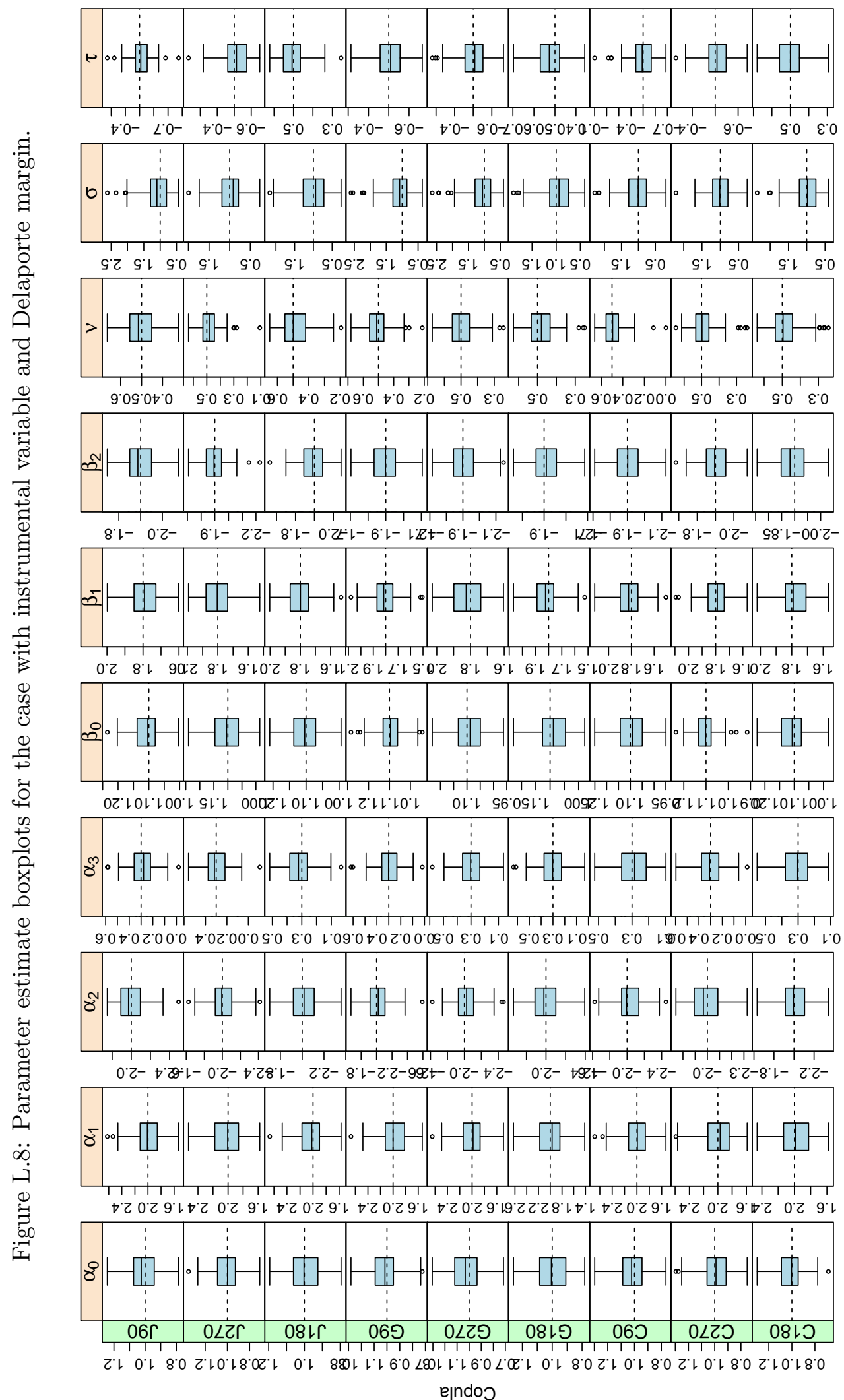

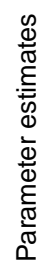




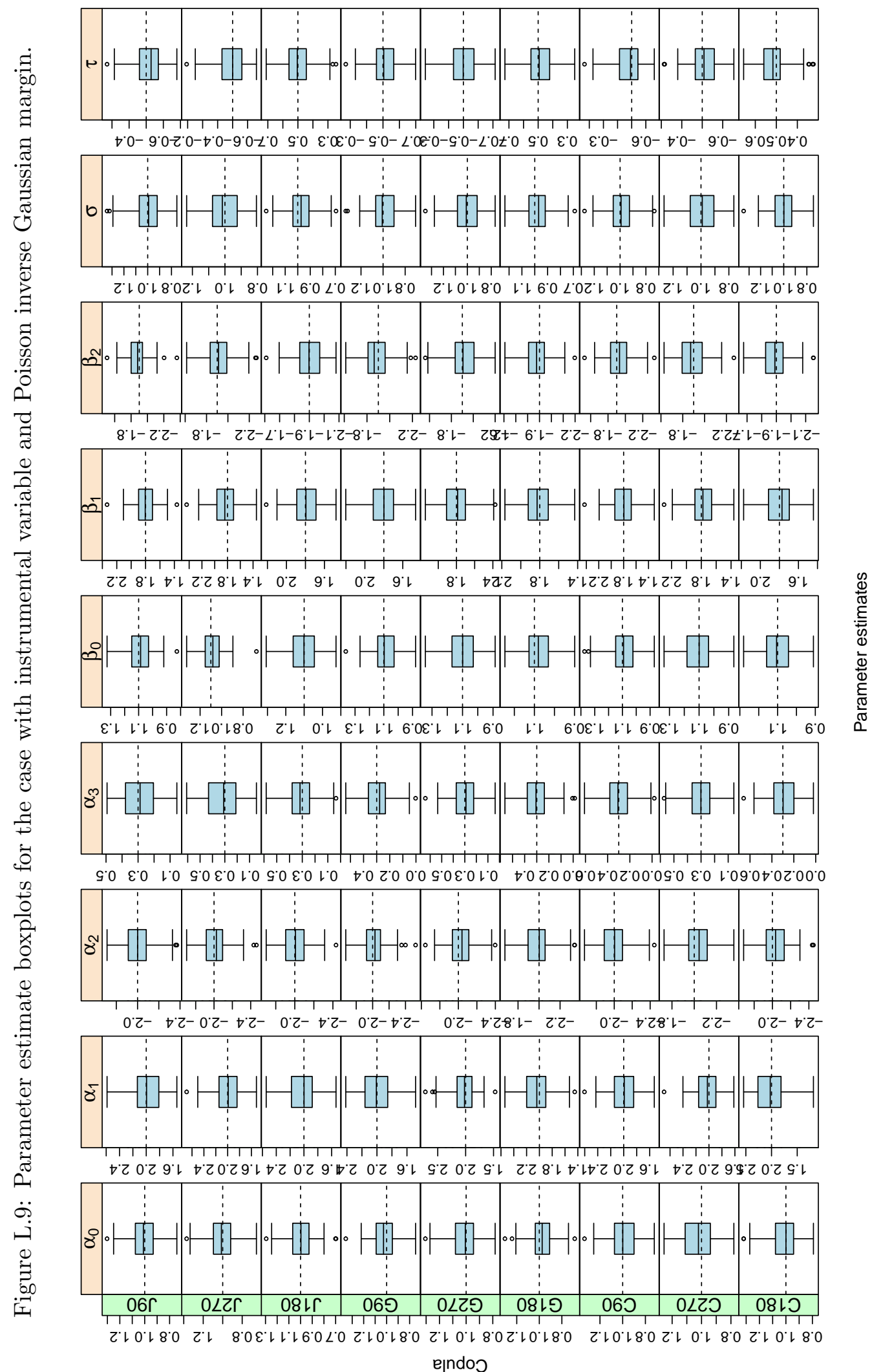




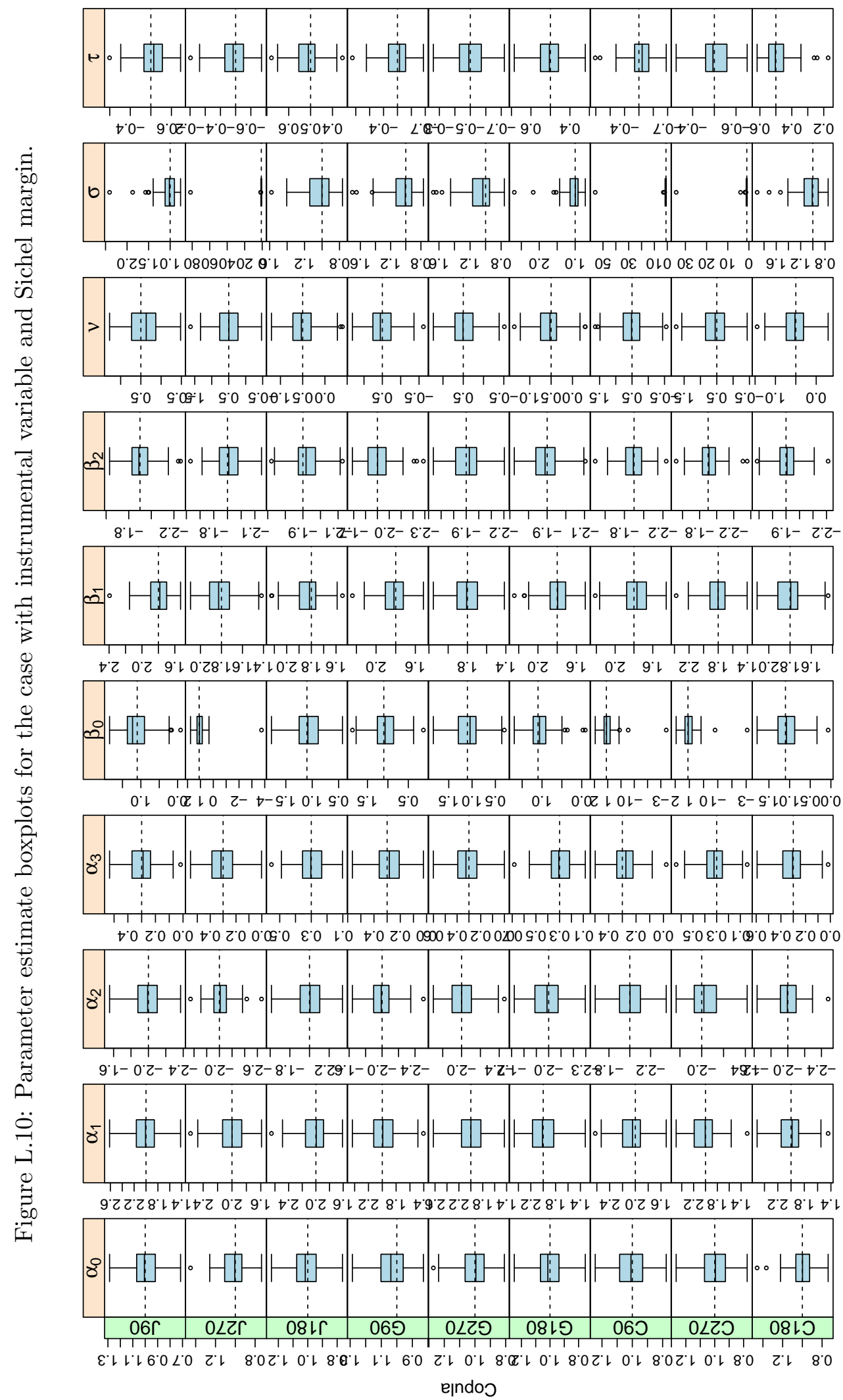




\section{Appendix M}

\section{Data simulation $R$ code (splines)}

The $\mathrm{R}$ code below shows the simulation function used to generate data for the case with splines. The inputs include sample size n, Kendall's $\tau$ (s.tau), $\sigma$ (s.sigma), $\nu$ (s.nu), Pearson's correlation coefficient for the correlation between covariates $(\mathrm{rhC}$ ), outcome margin (outcome.margin) and copula (copula). In summary, we first generate covariates, $x 1$ and $x 2$, from the multivariate normal distribution. Then, we create a copula-specific object Cop that will enter the terminal function. Afterwards, we determine equations for the selection and the outcome (mu_s and mu_o). Continuous functions, which will be modelled using splines, are defined as $f 1$ and $f 2$. We create a marginspecific object, speclist, that will also enter the terminal function. Finally, the function $\operatorname{rMvdc}()$ generates data; missing values are coded as -99. For further comments in the code, see lines starting with a hash.

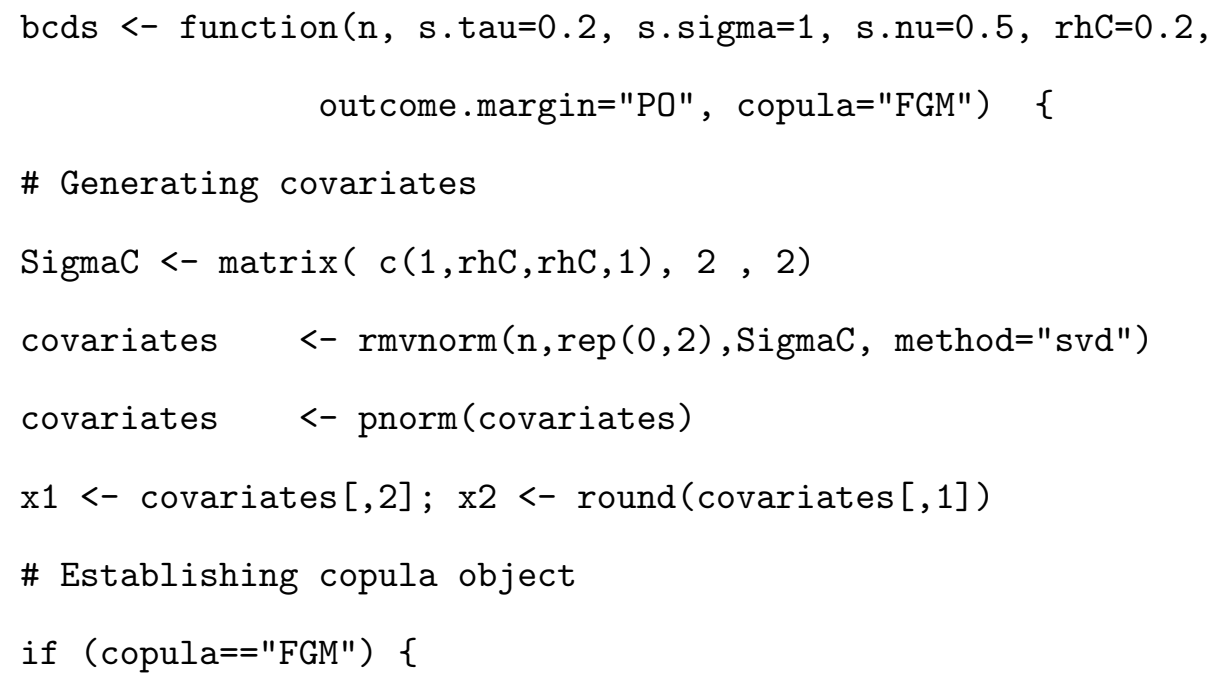




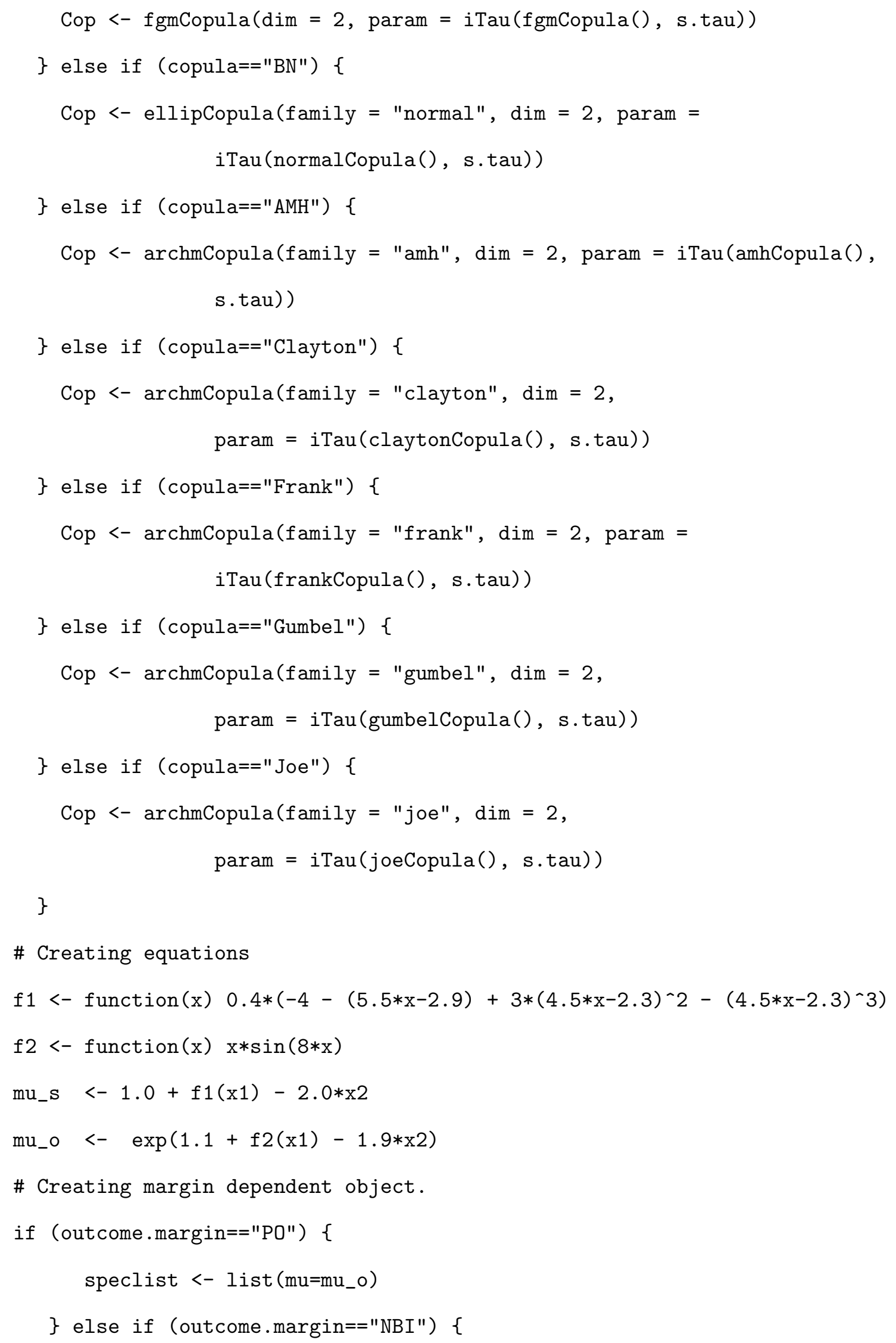




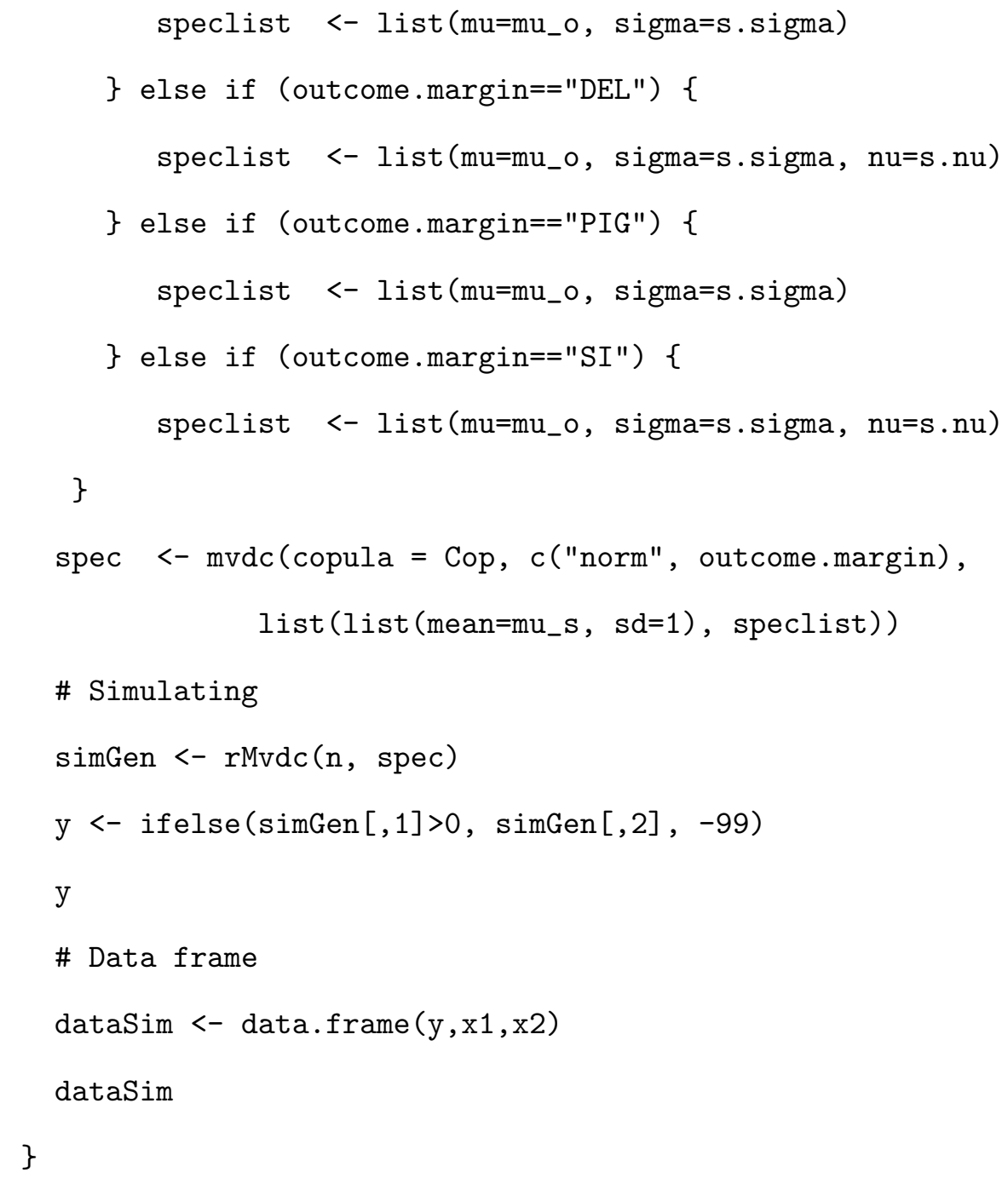


Appendix $\mathbf{N}$

Spline simulation for unrotated

copulas and same covariates 

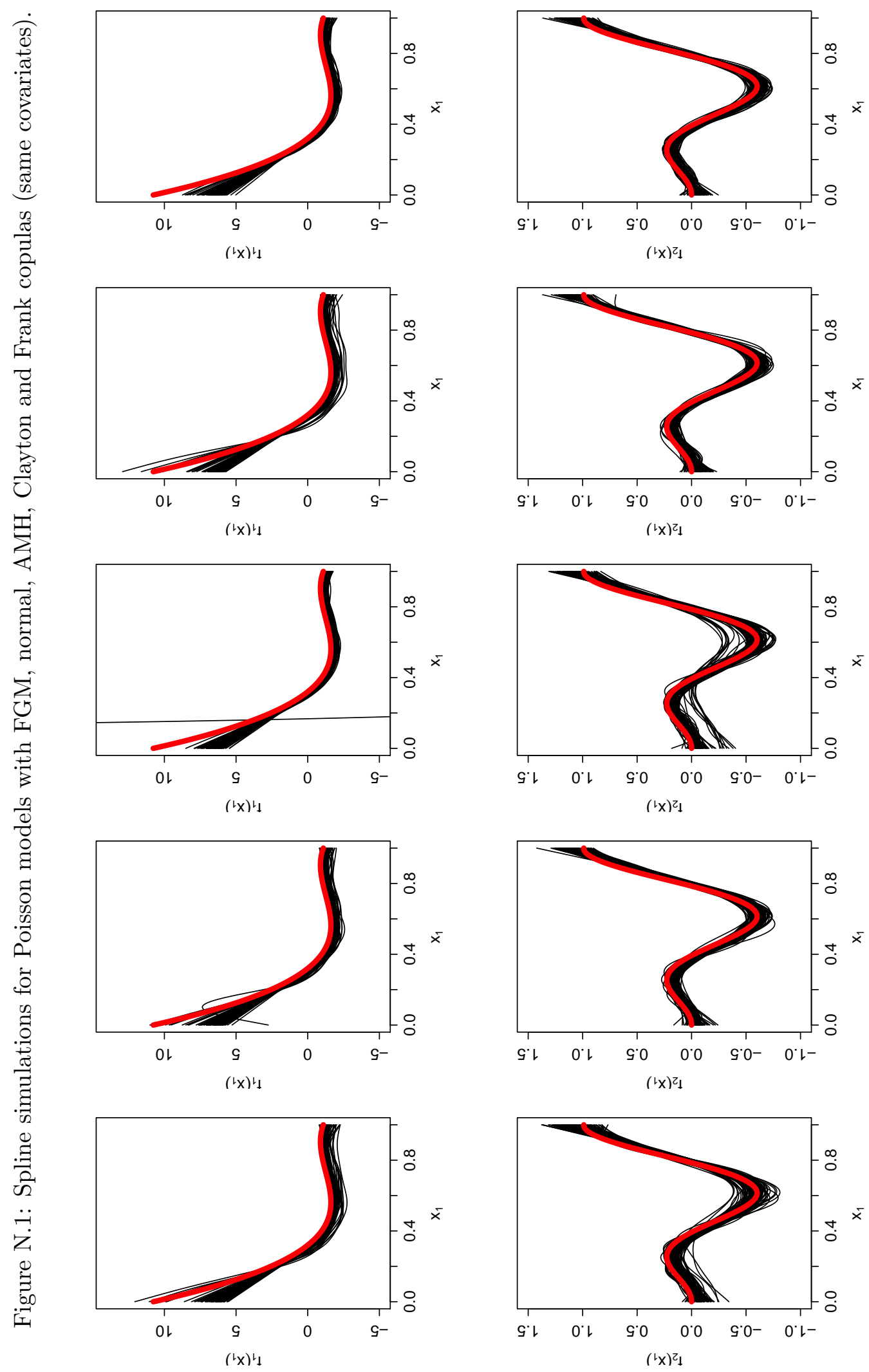

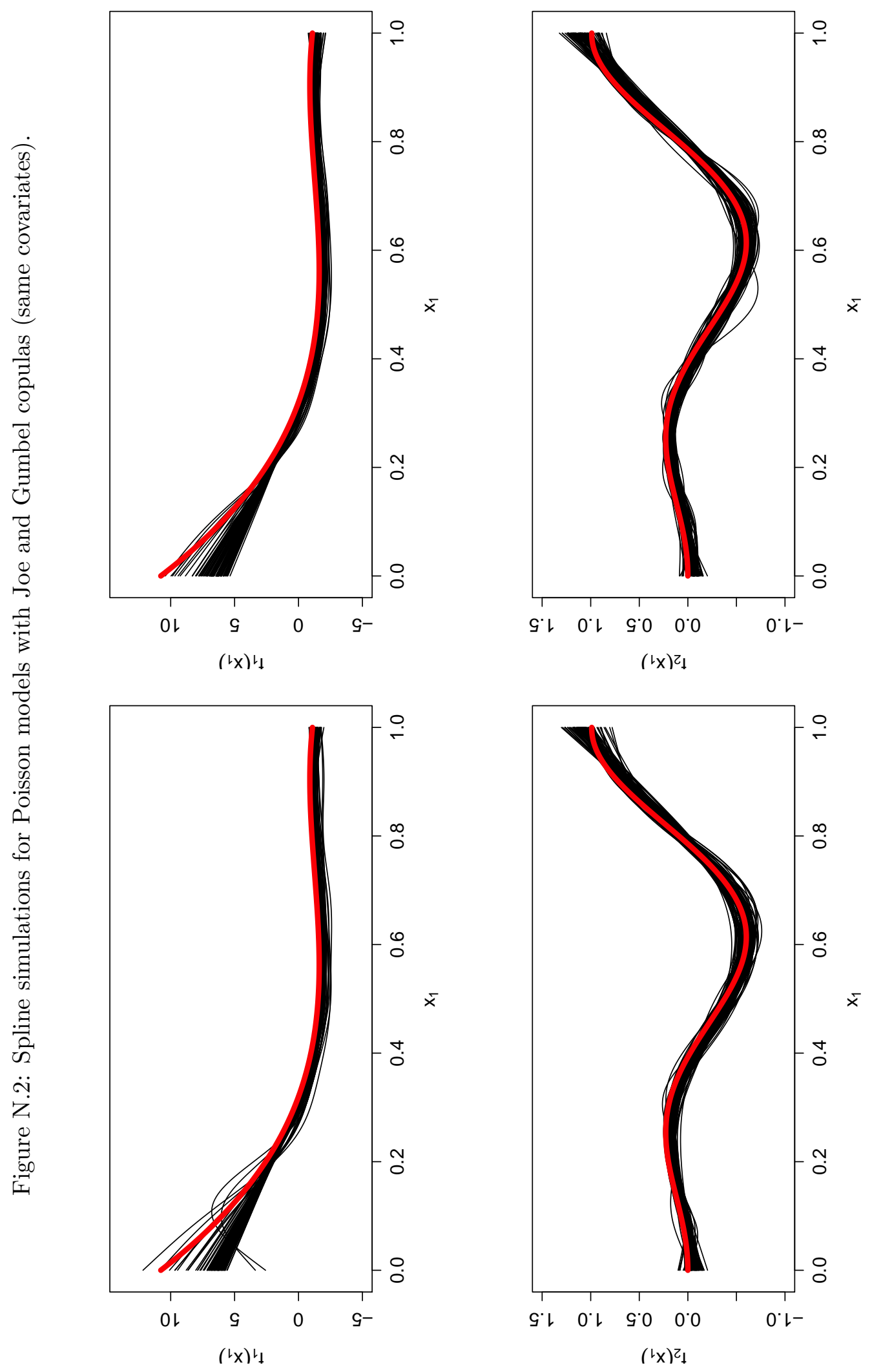

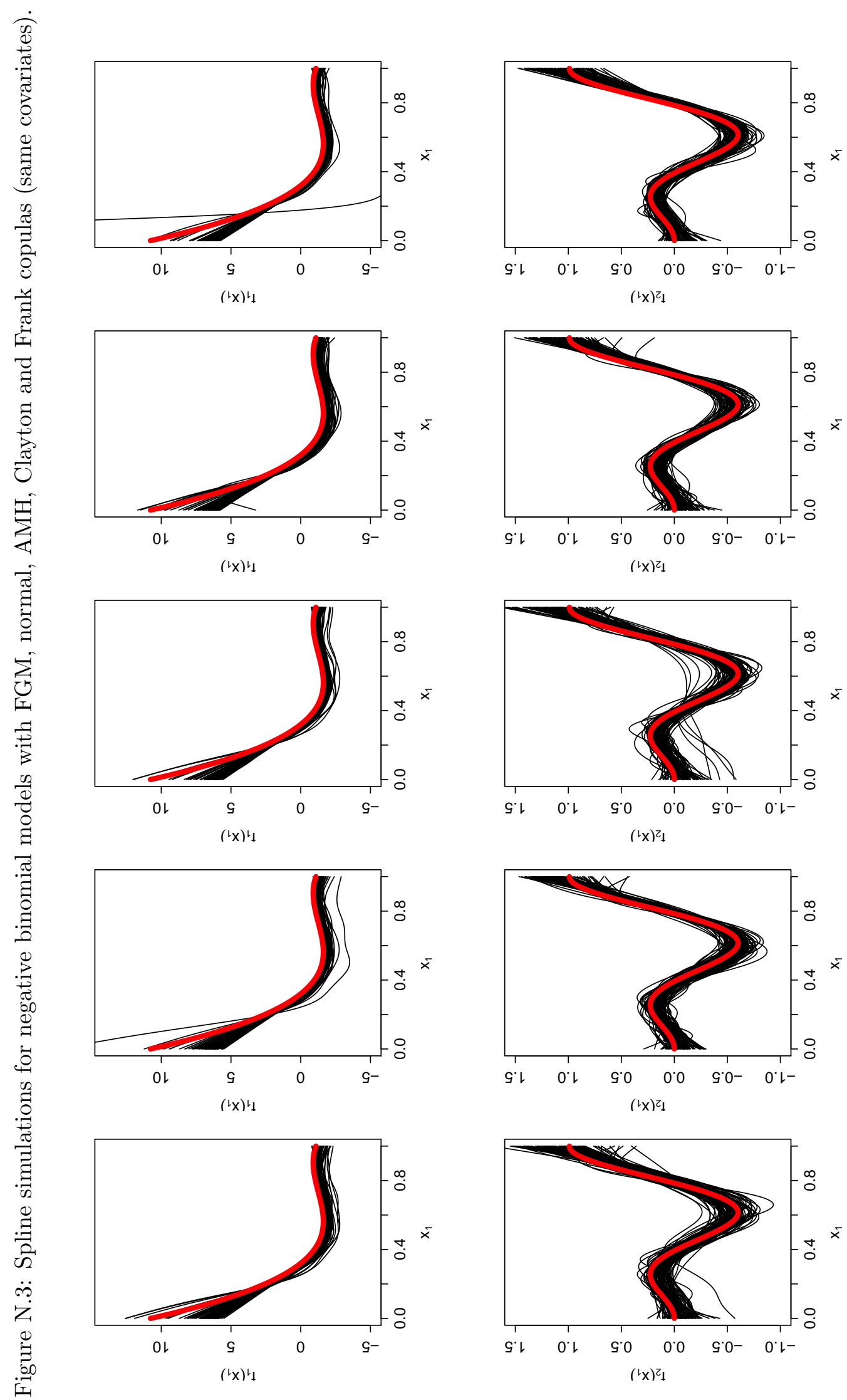

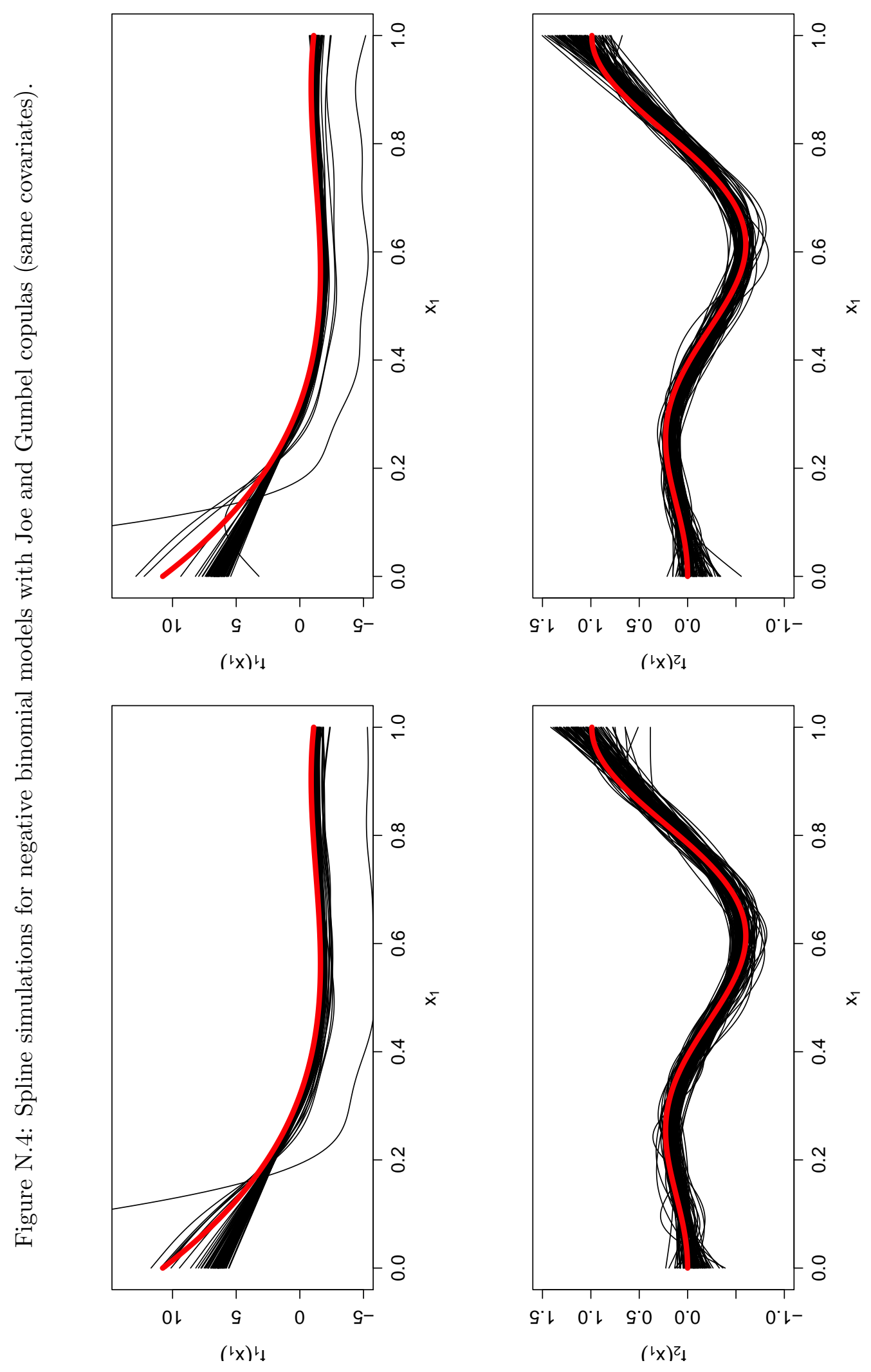

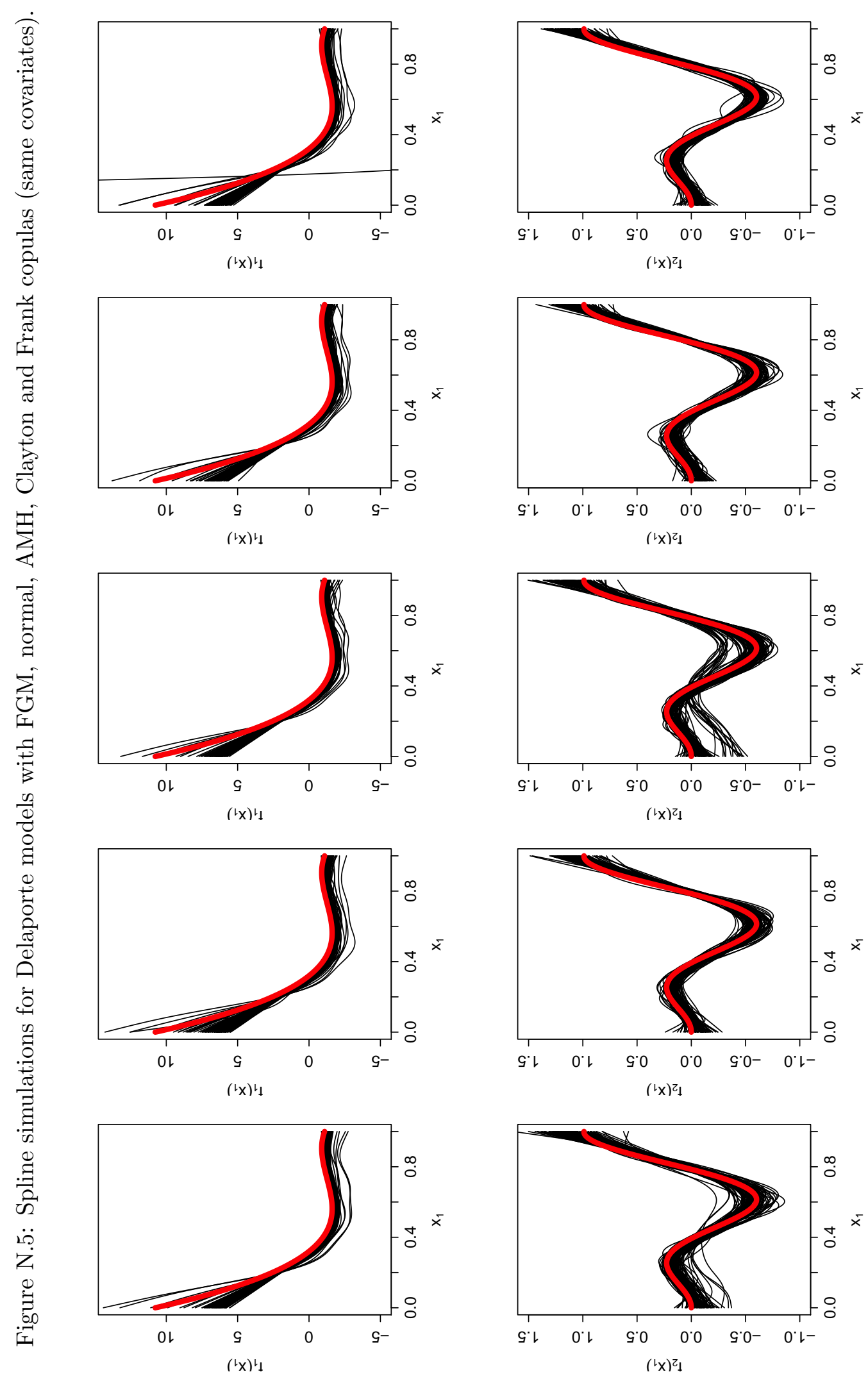

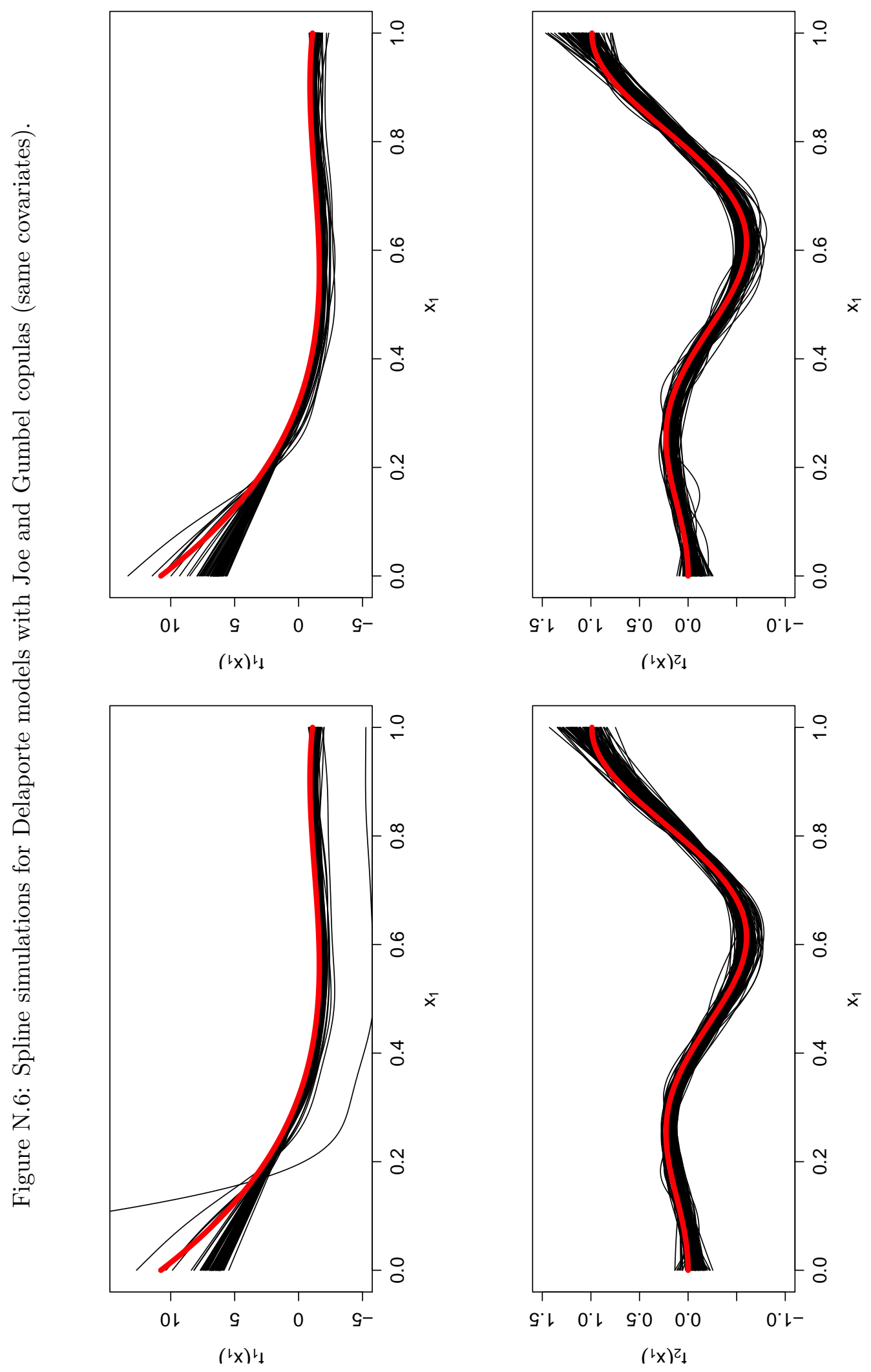

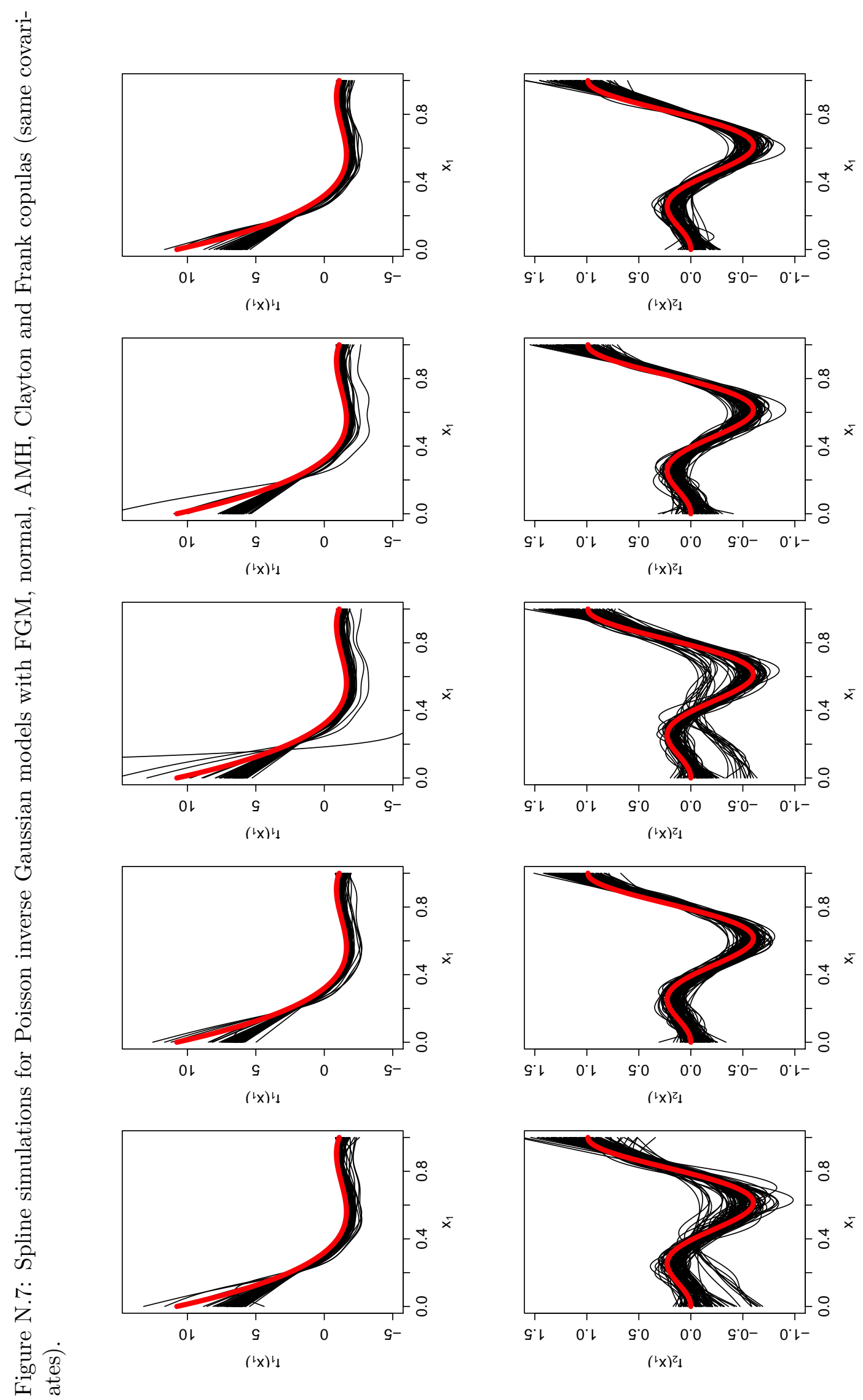

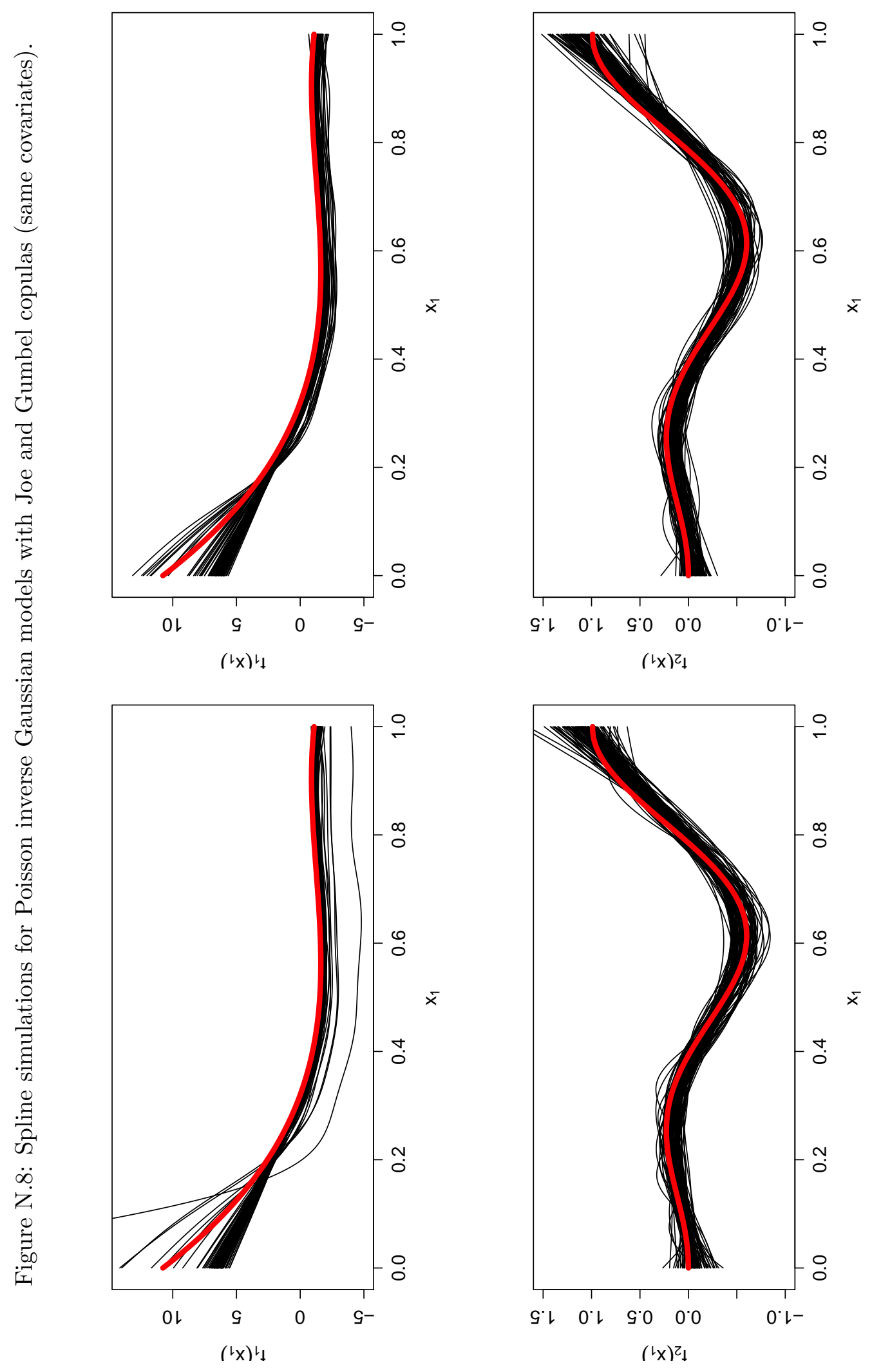

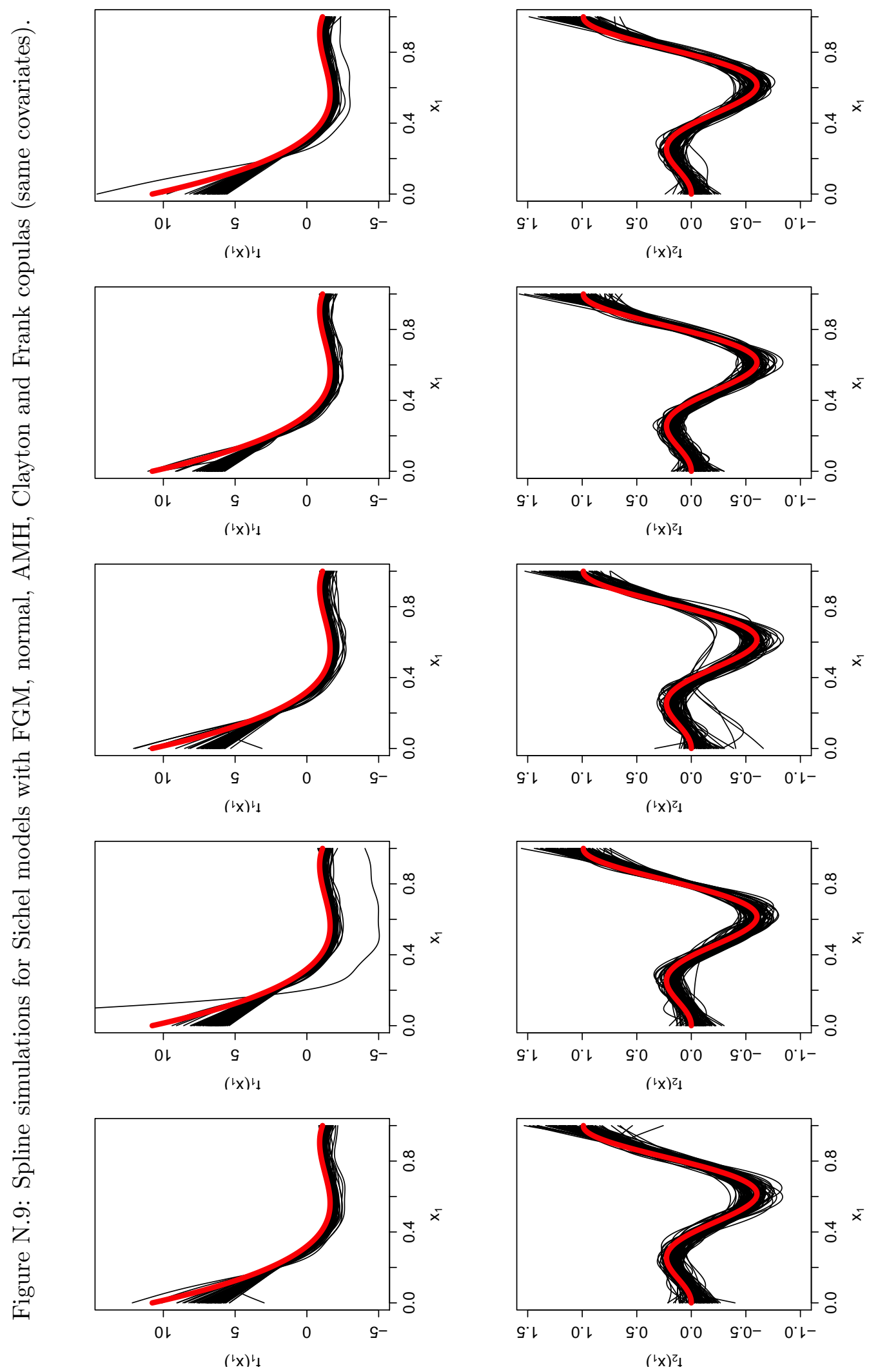

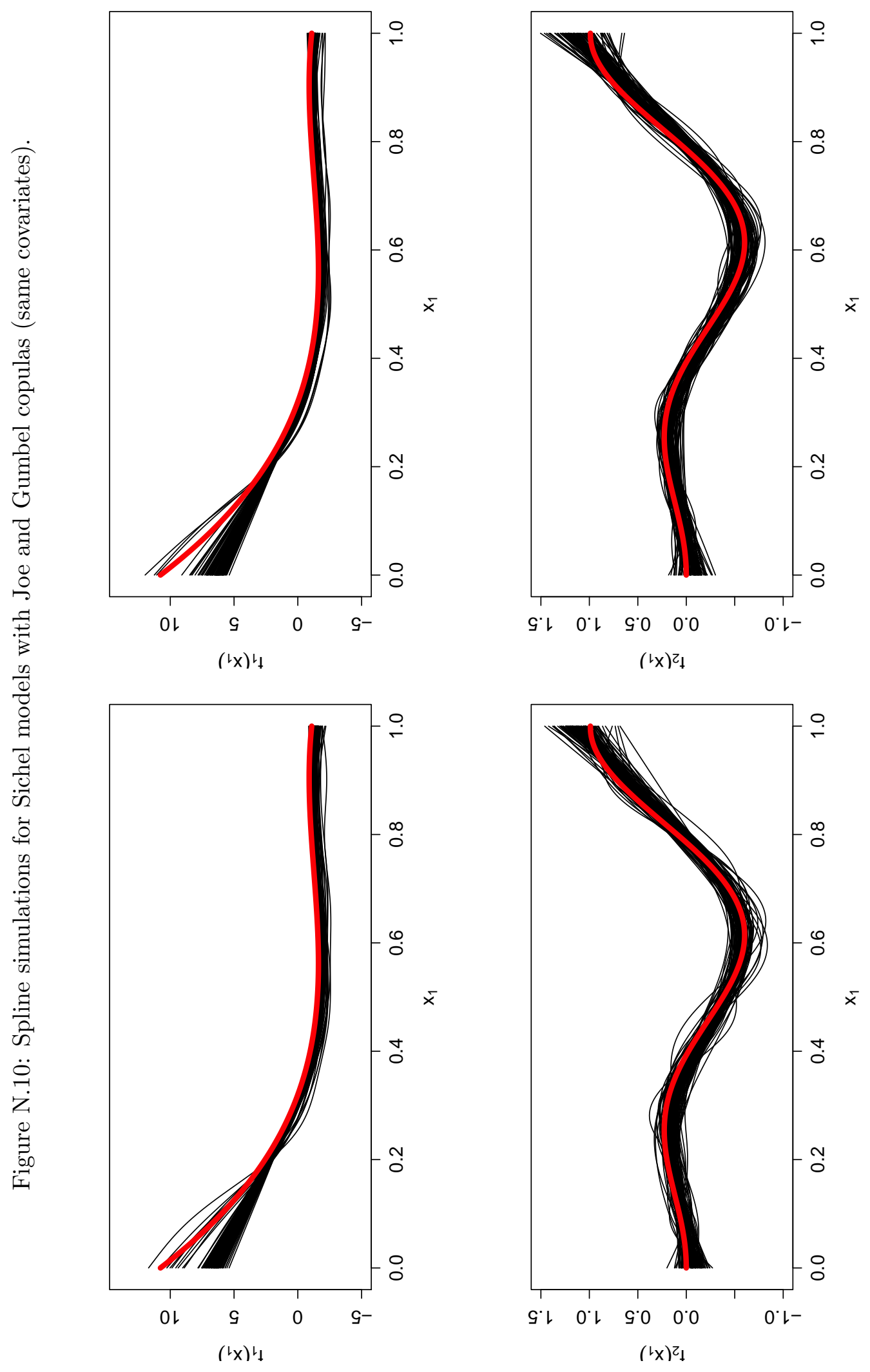
Appendix $\mathrm{O}$

Spline simulation for rotated copulas and same covariates 

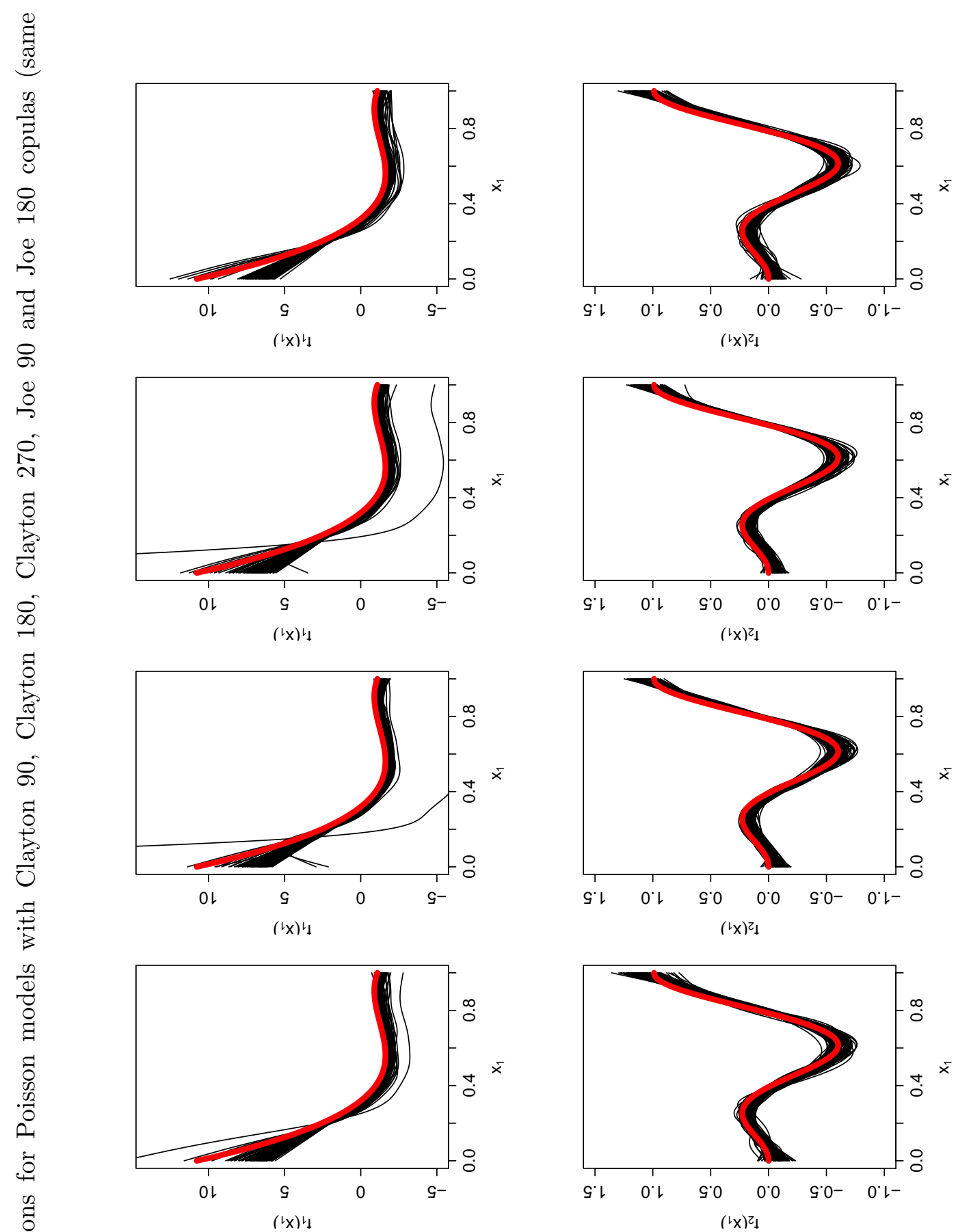

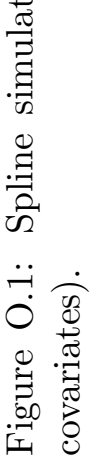
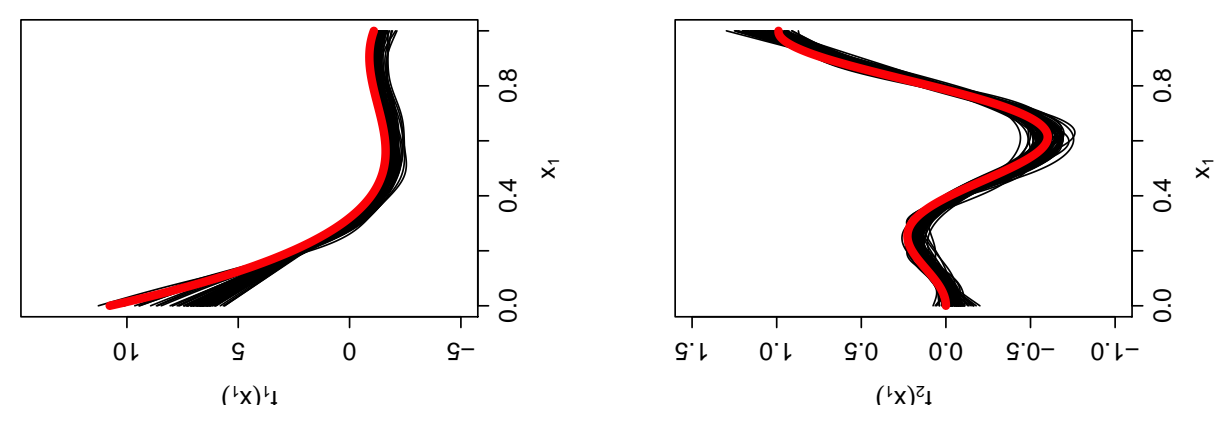

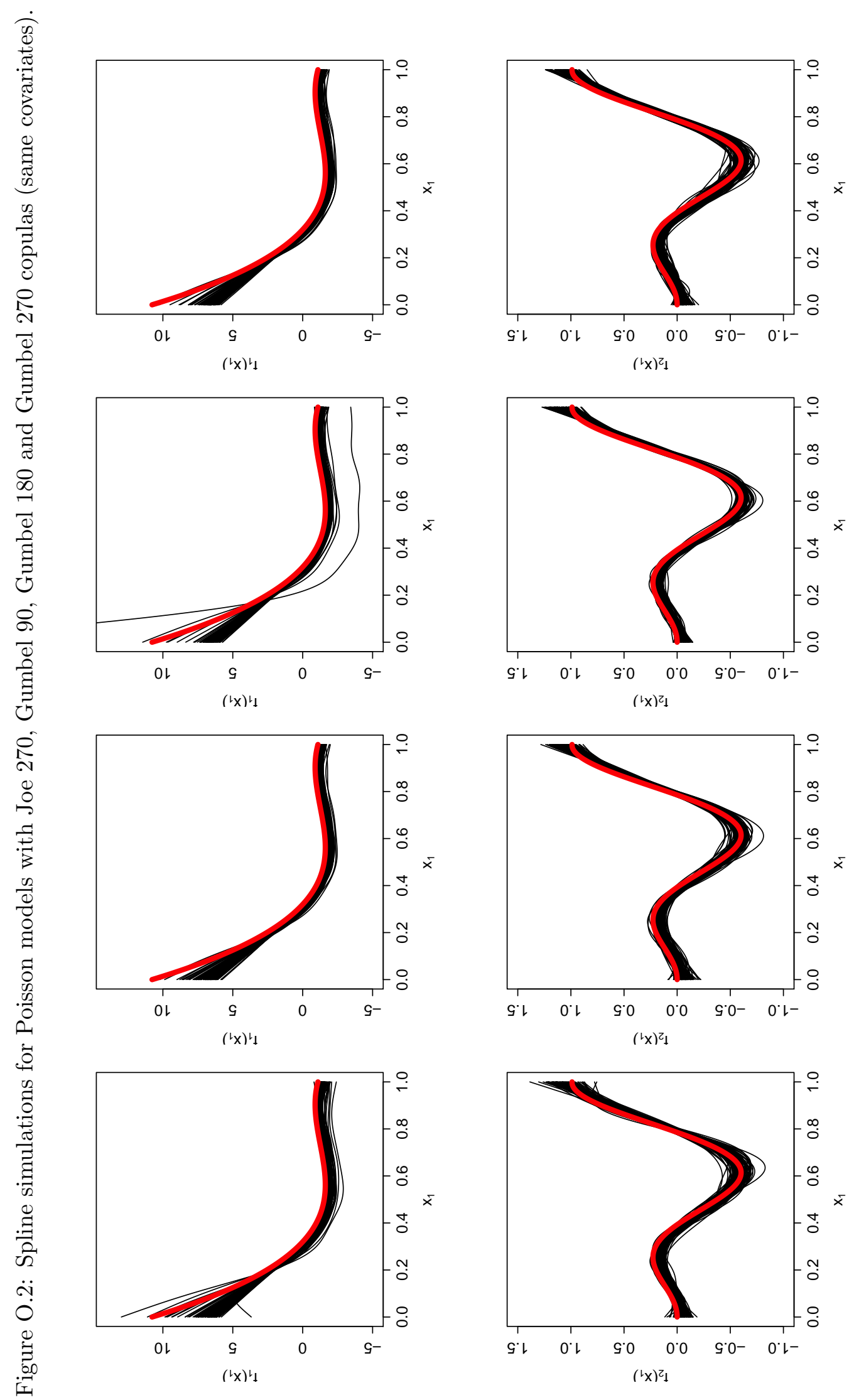

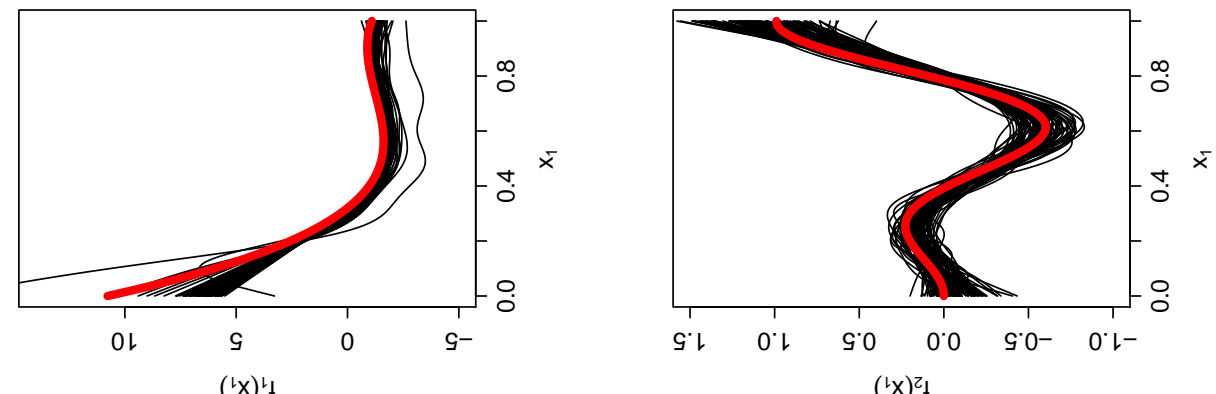

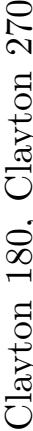
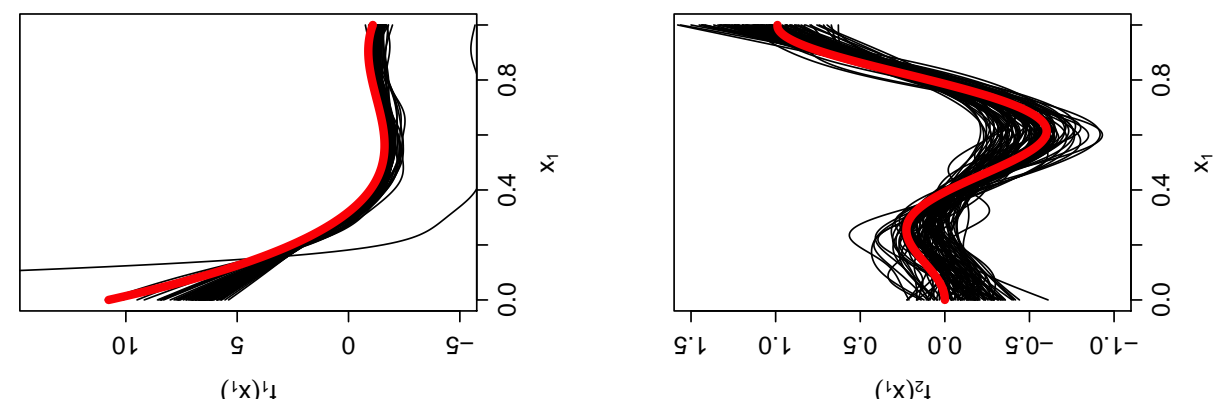

8
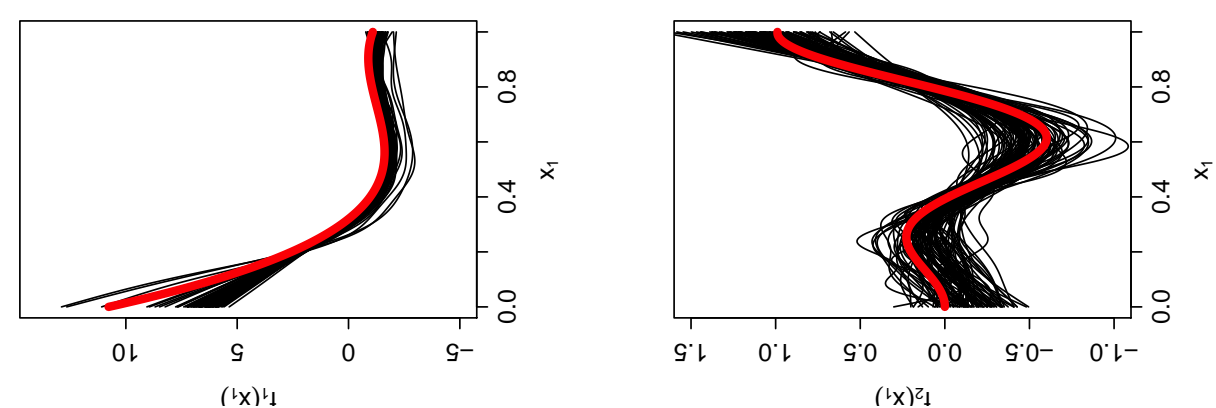

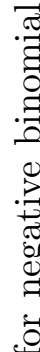

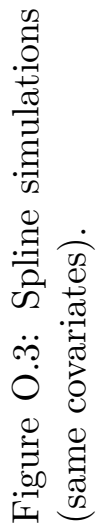
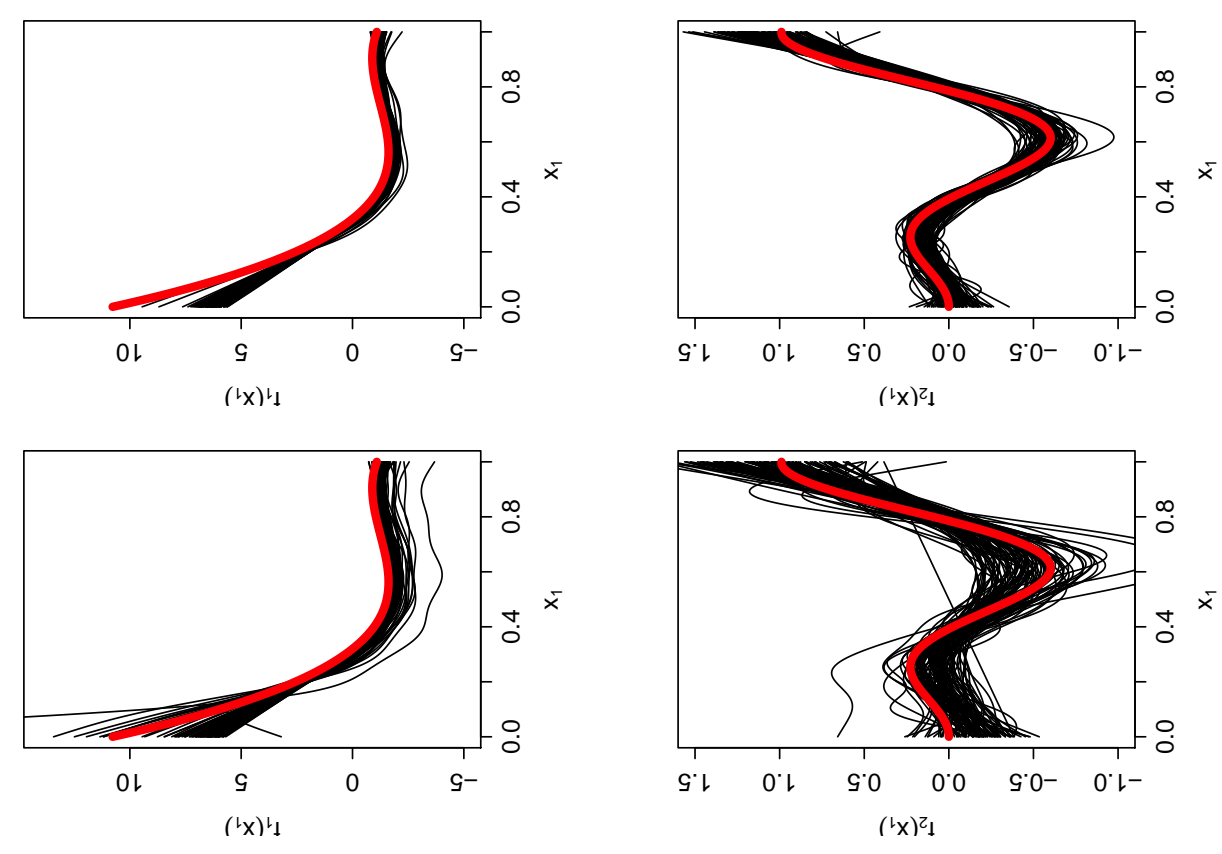

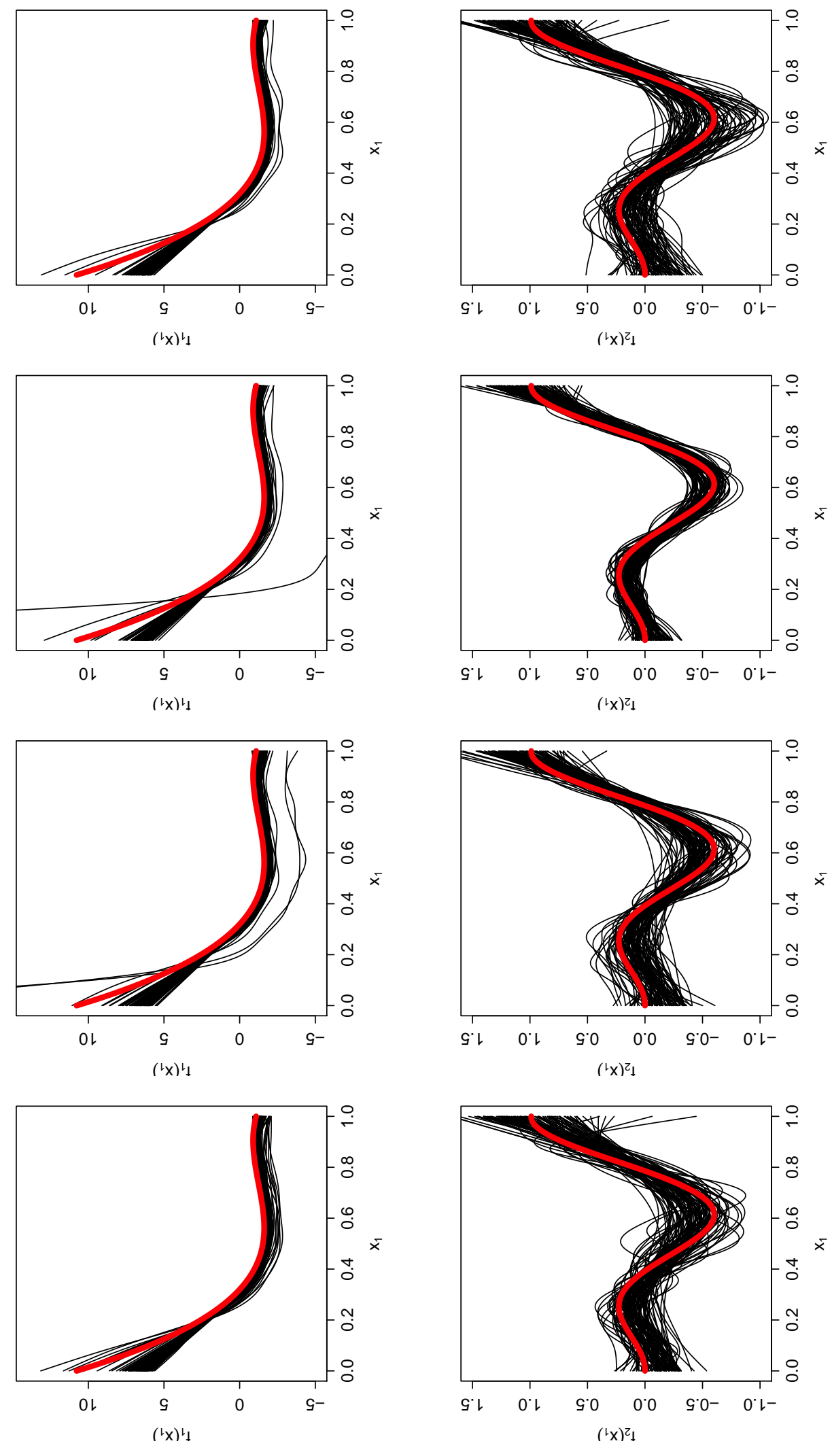

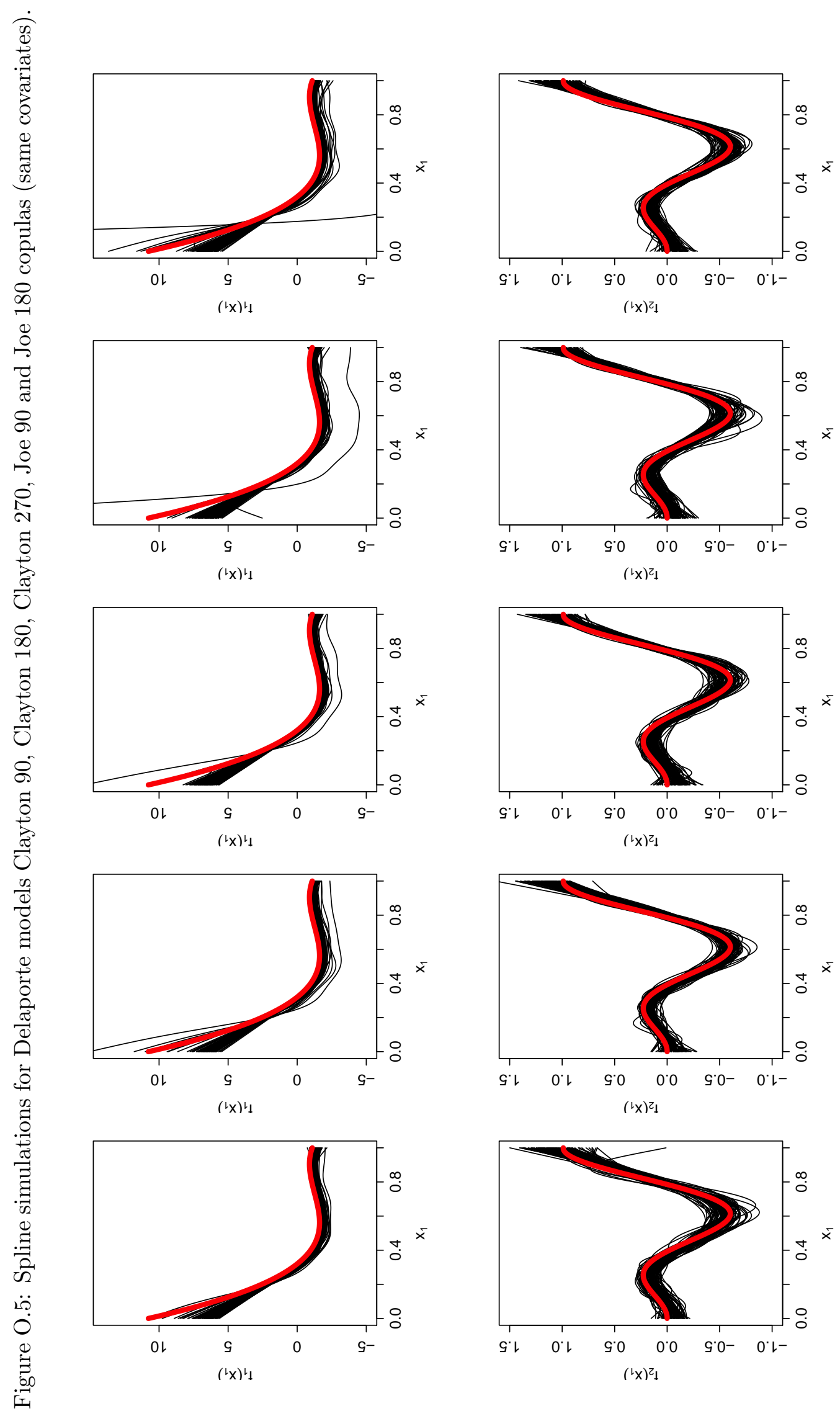

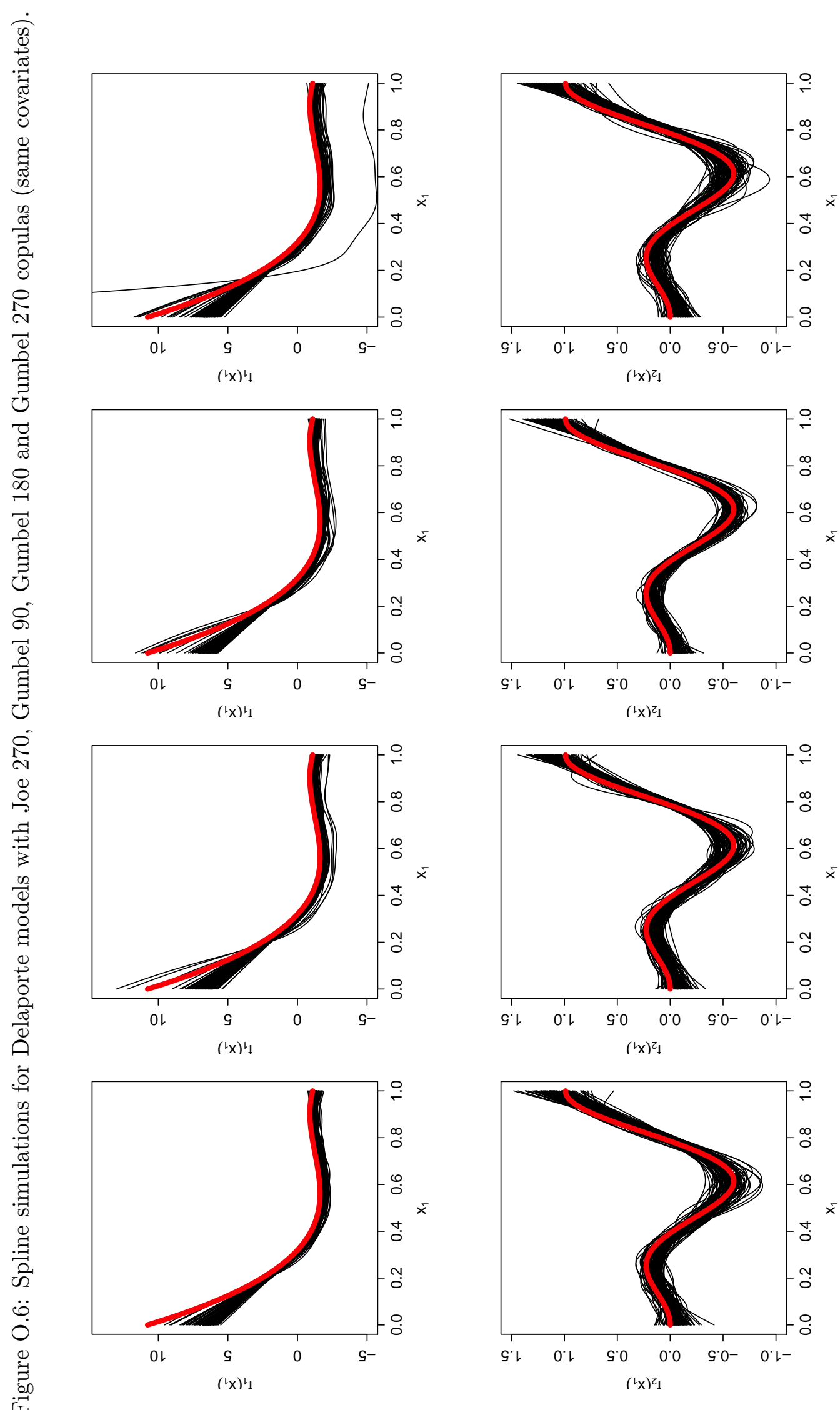

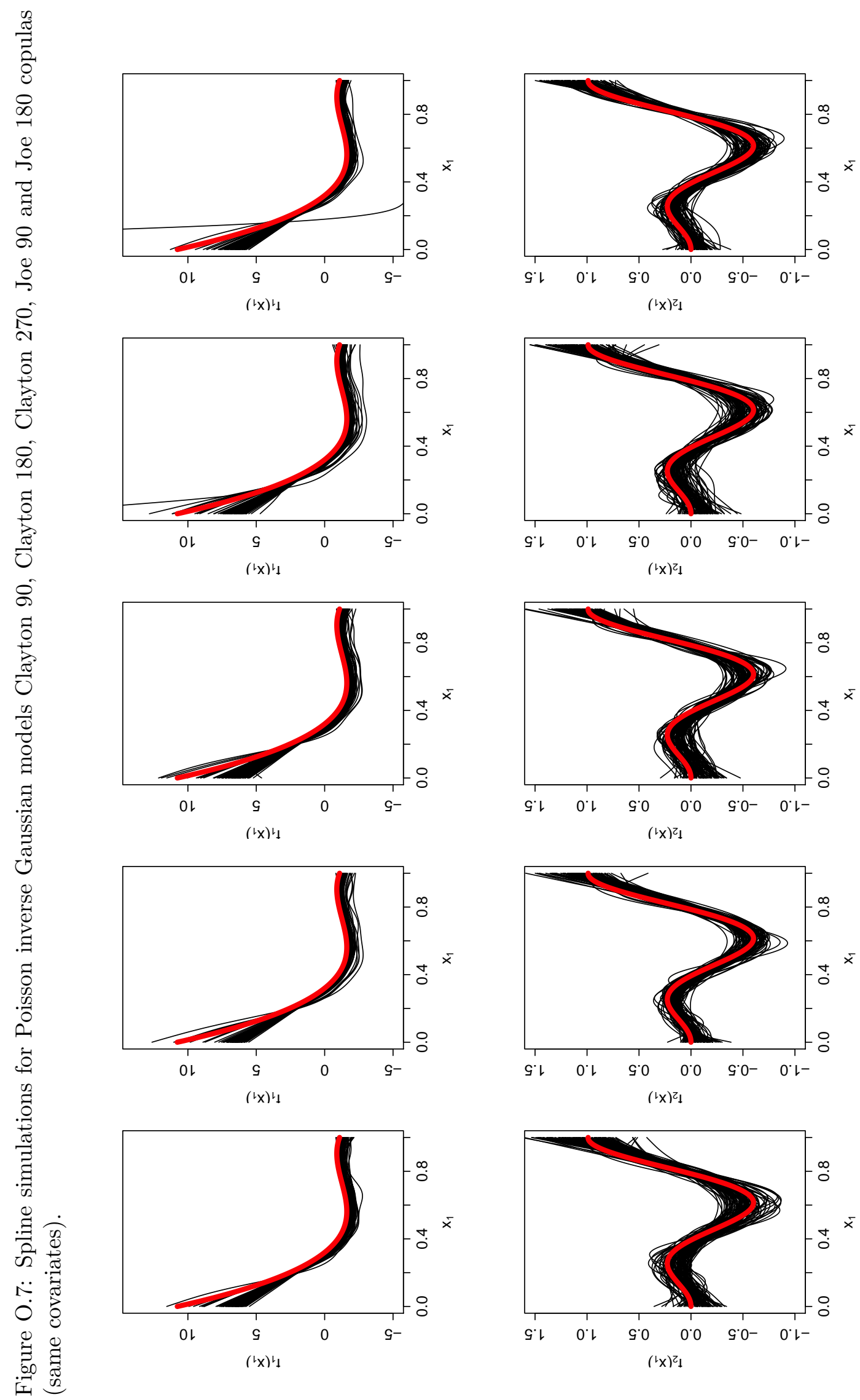

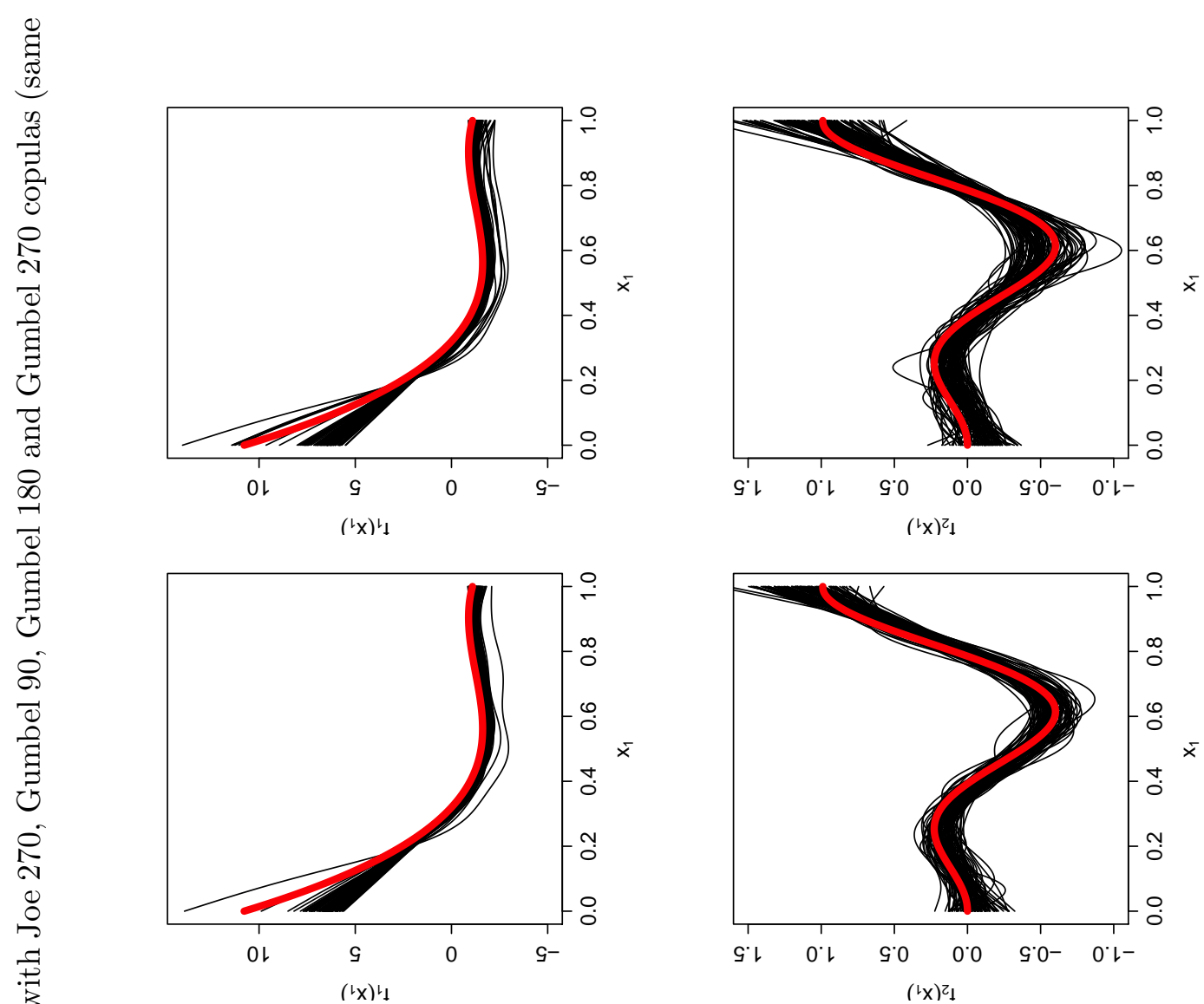

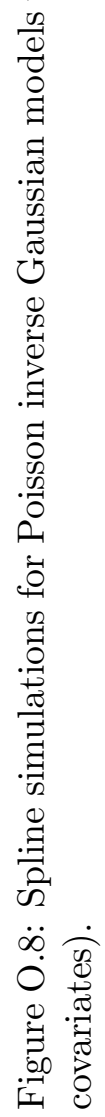
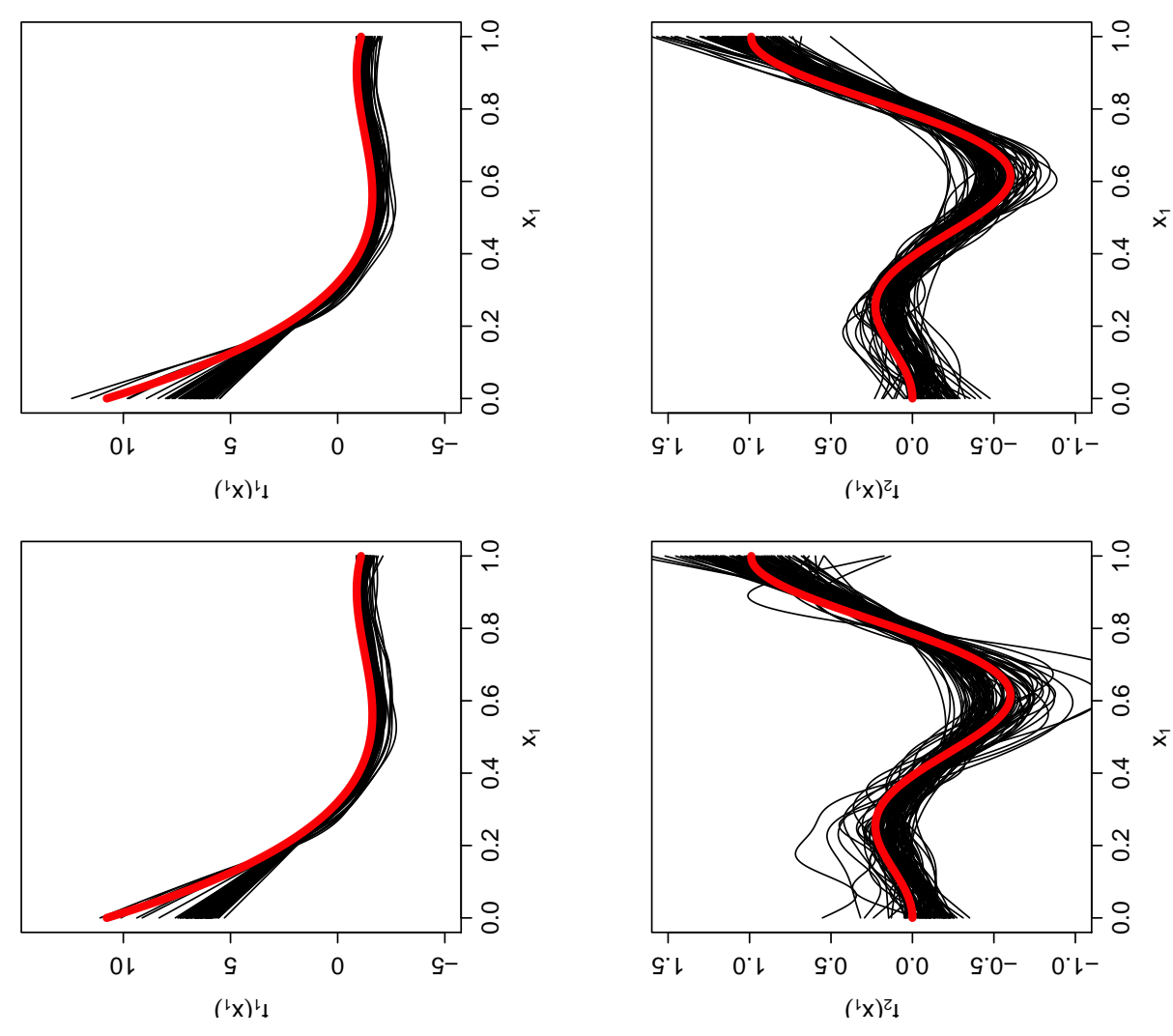

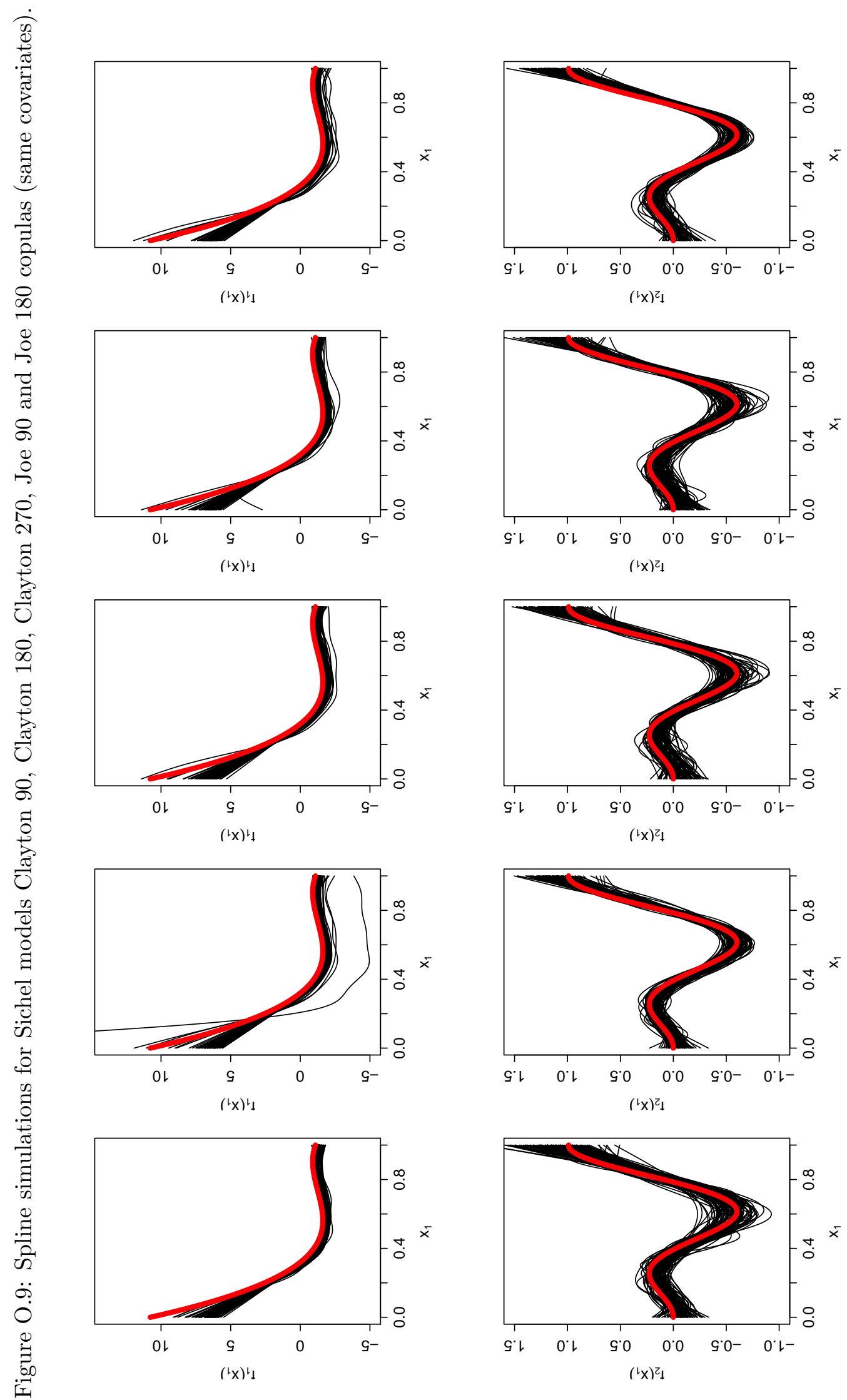

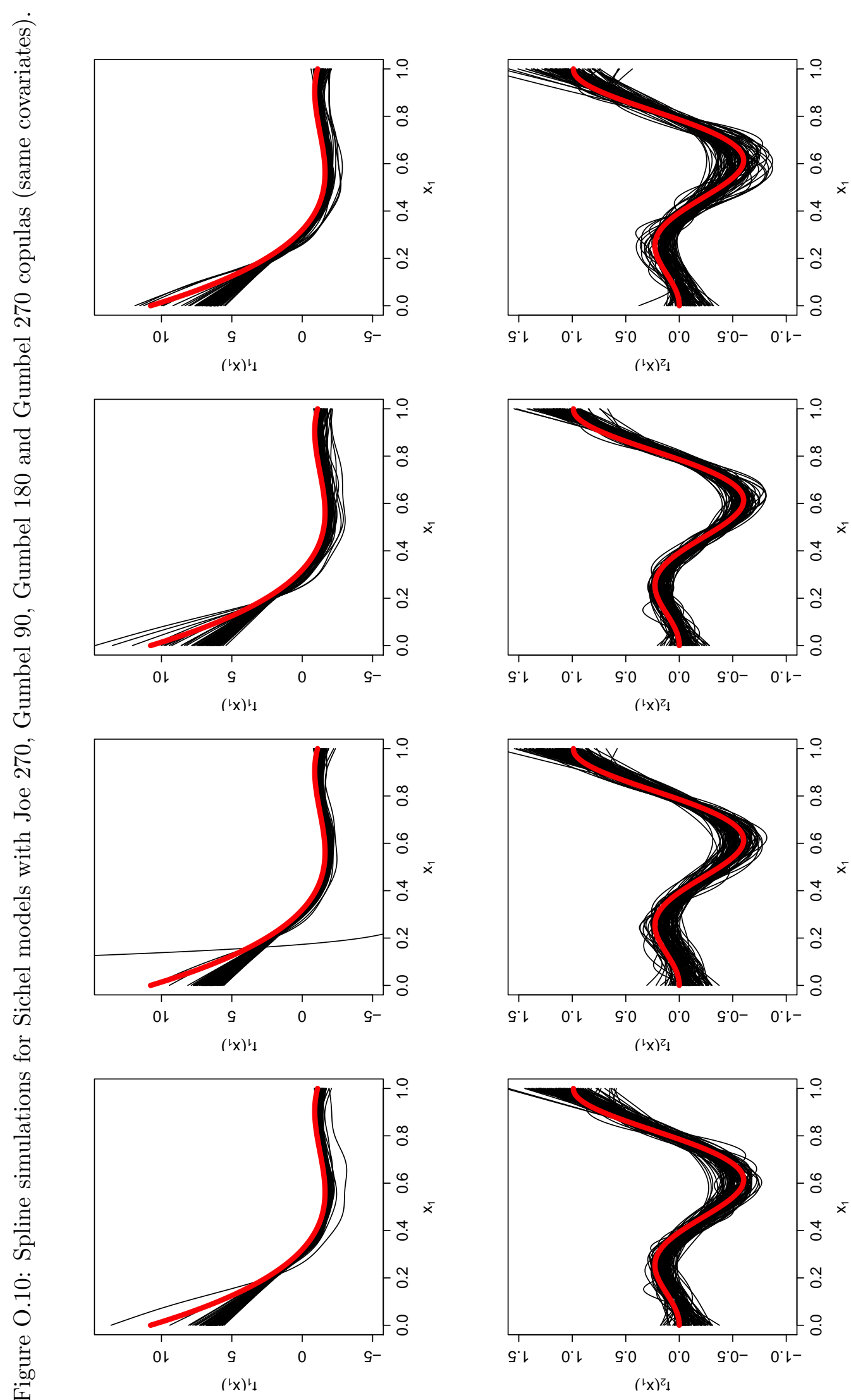
Appendix $\mathbf{P}$

Spline simulation for unrotated copulas and IV 

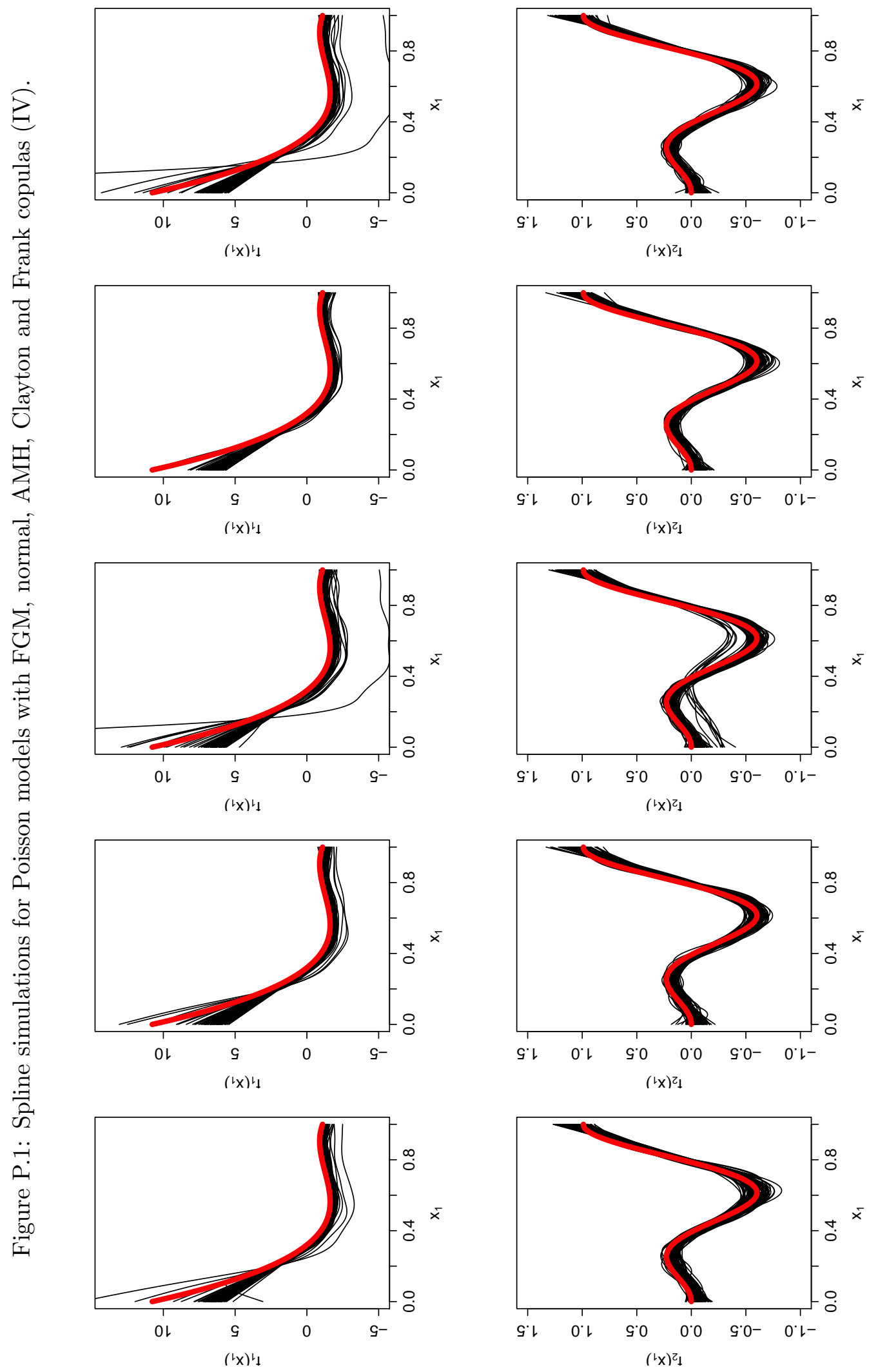

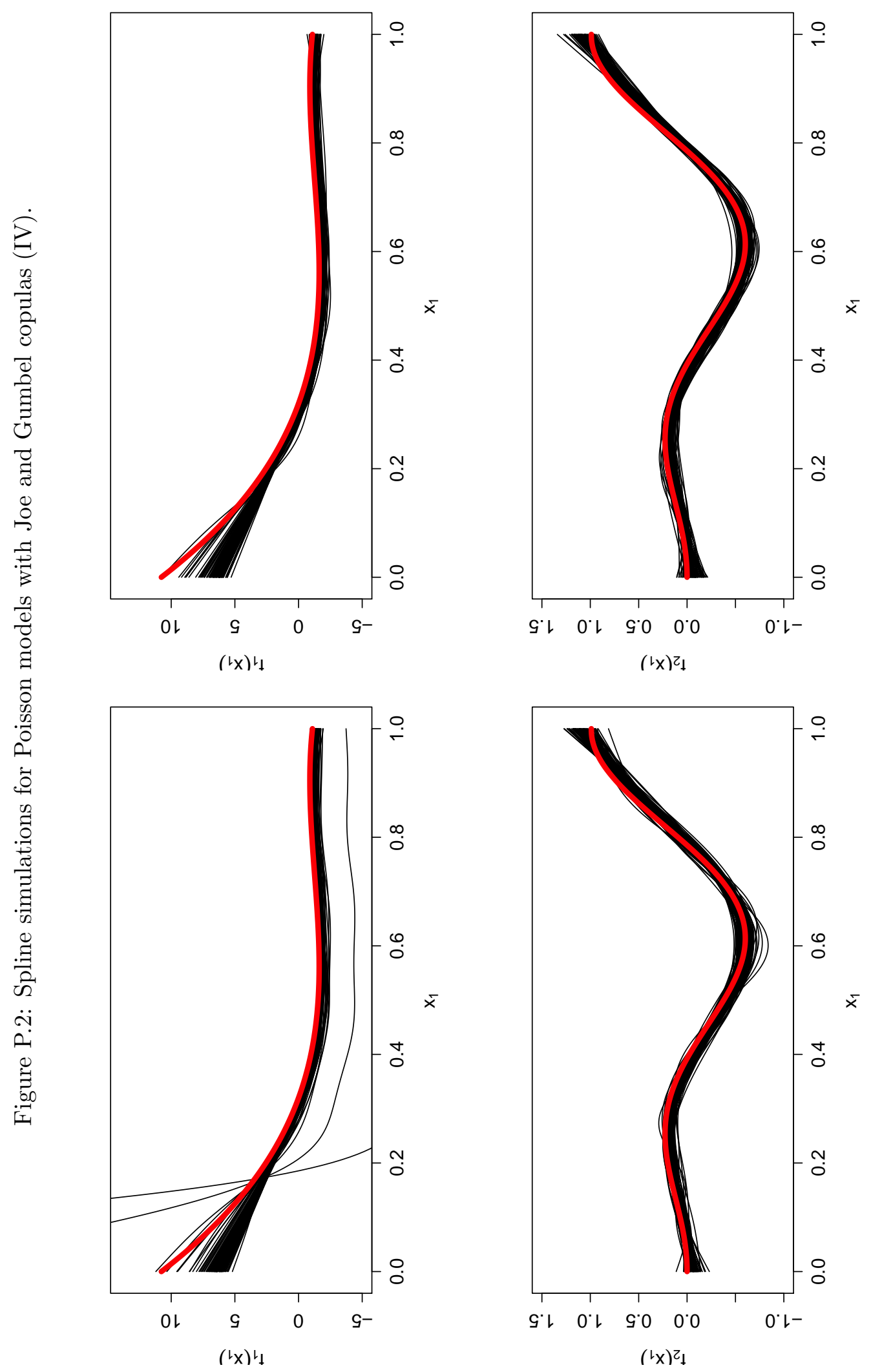

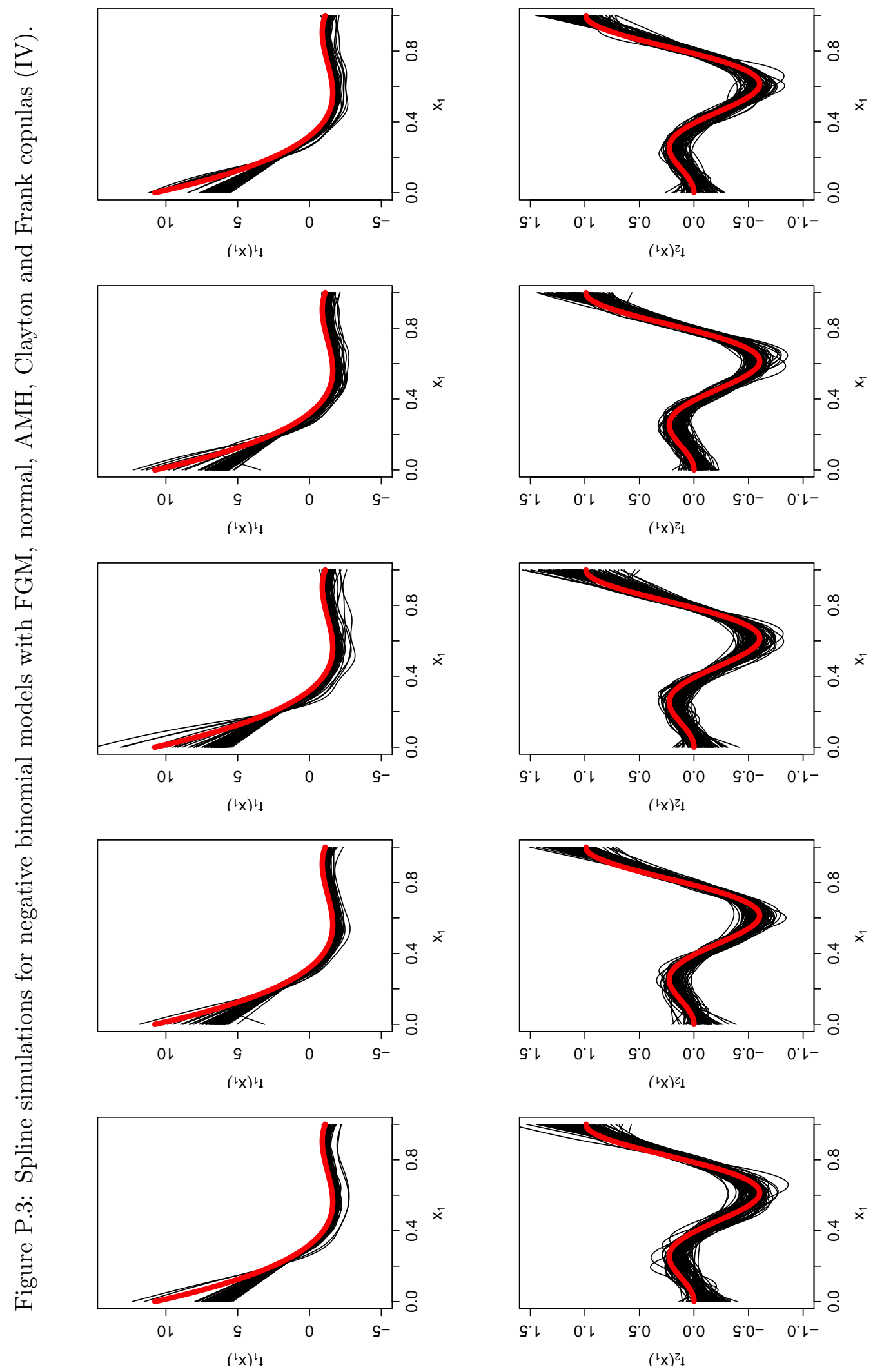

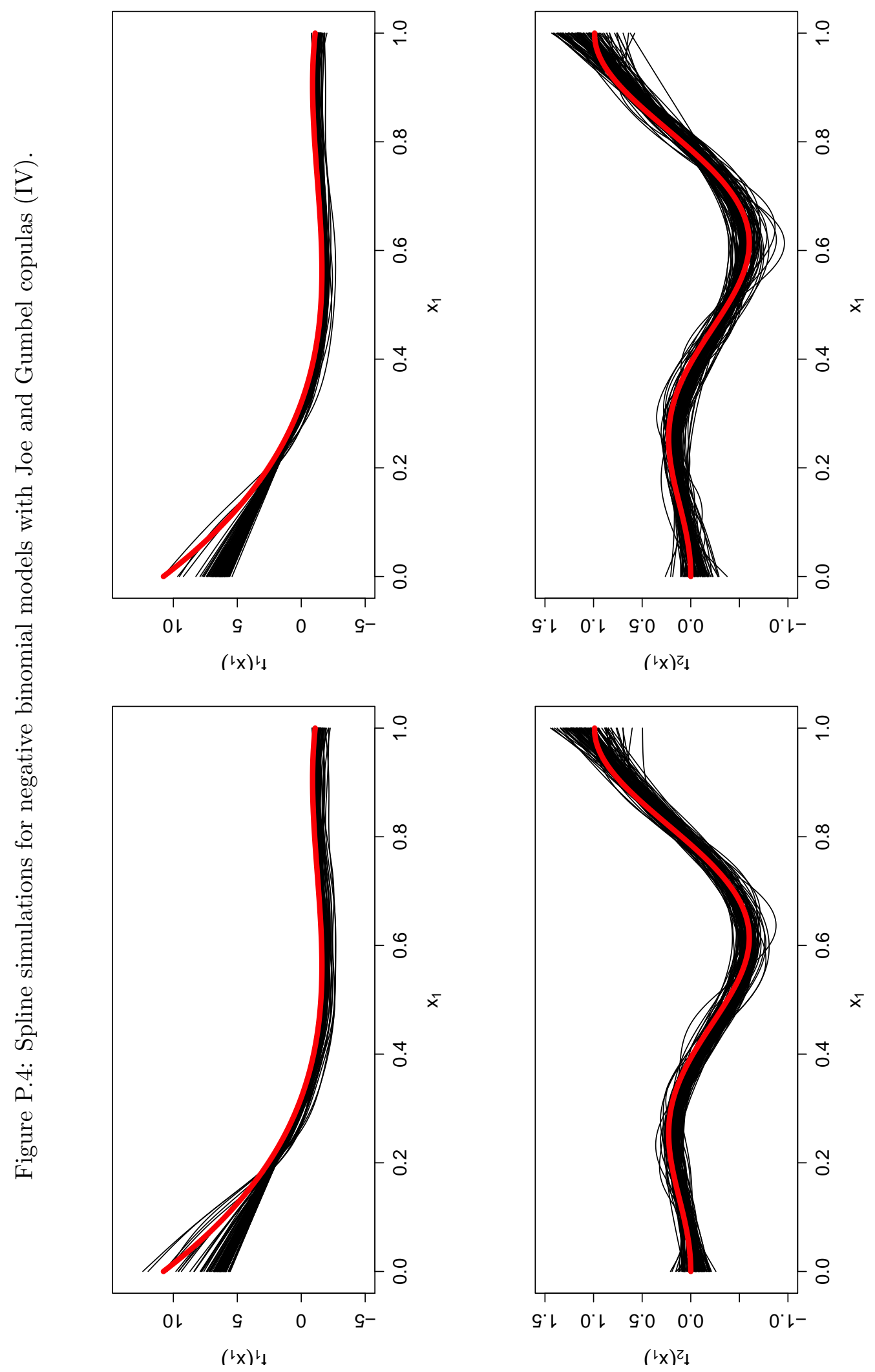

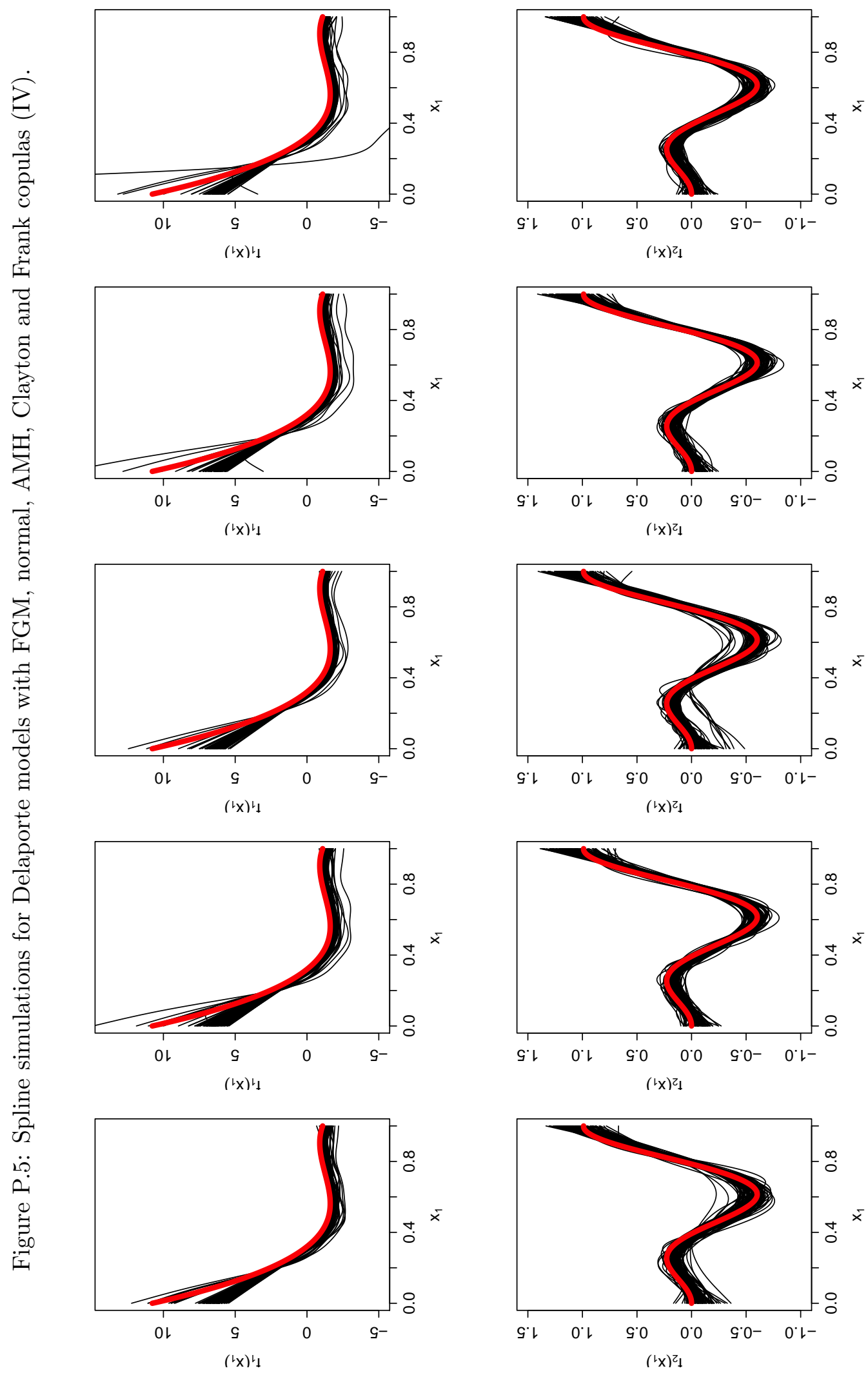

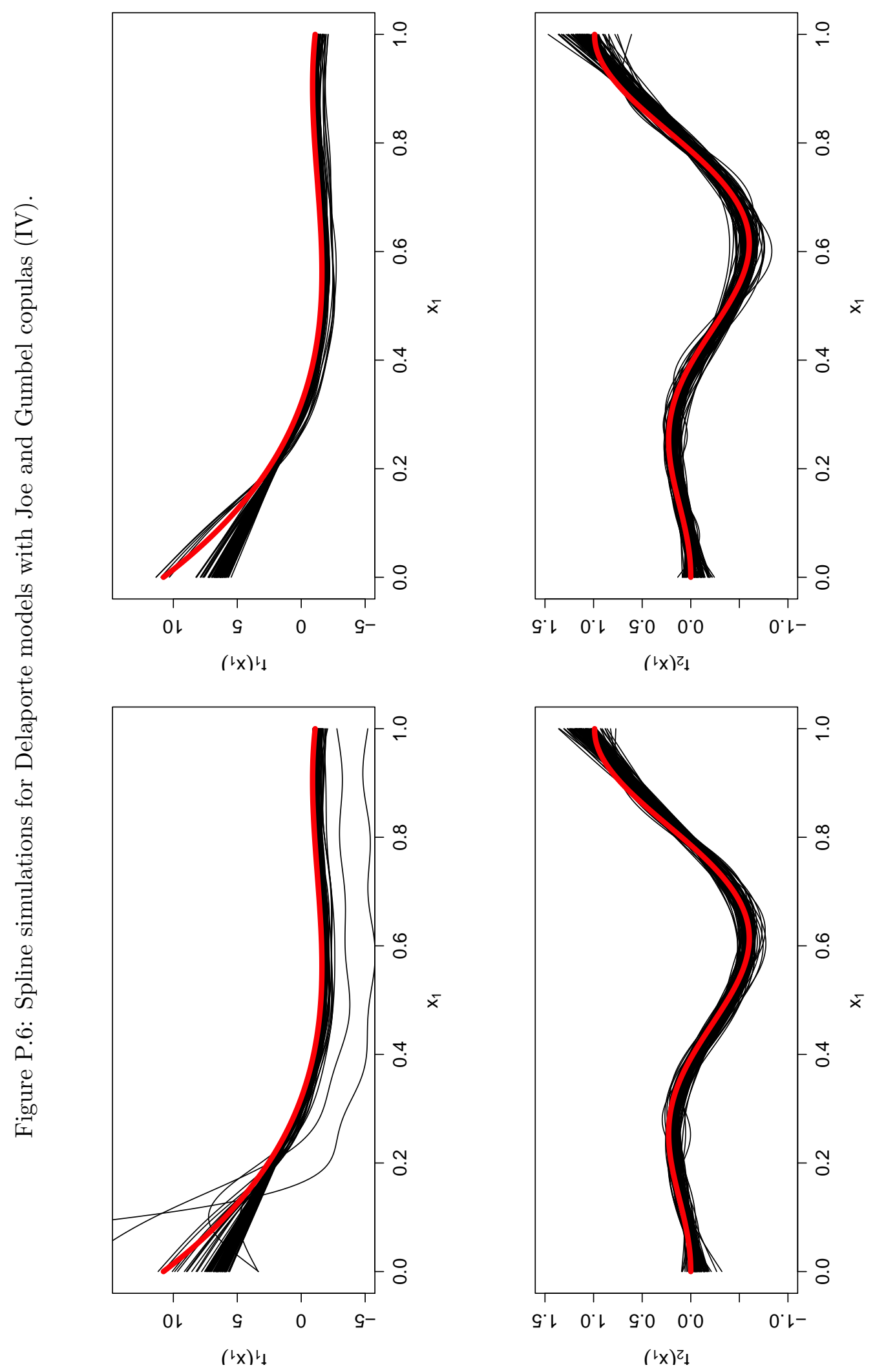

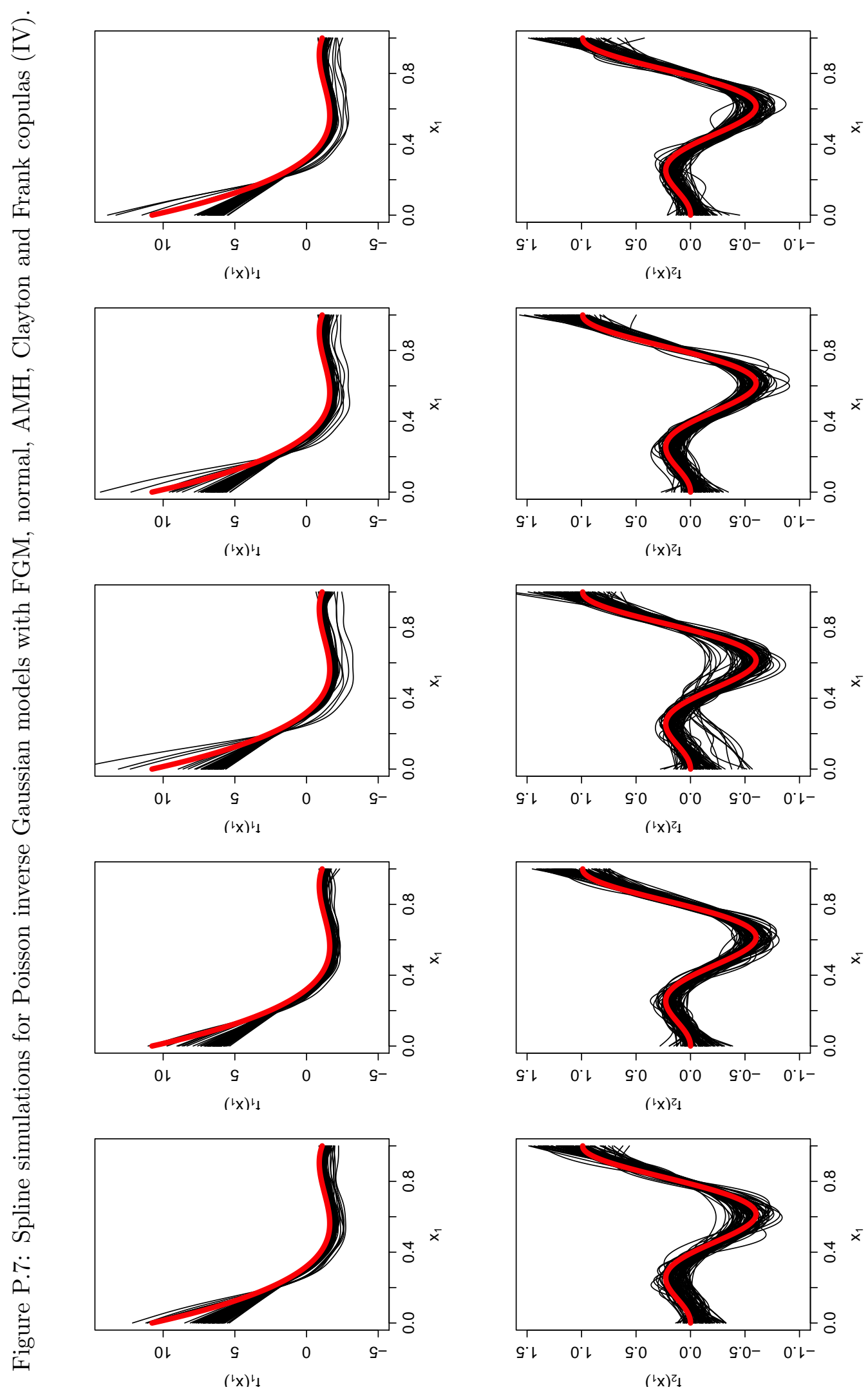

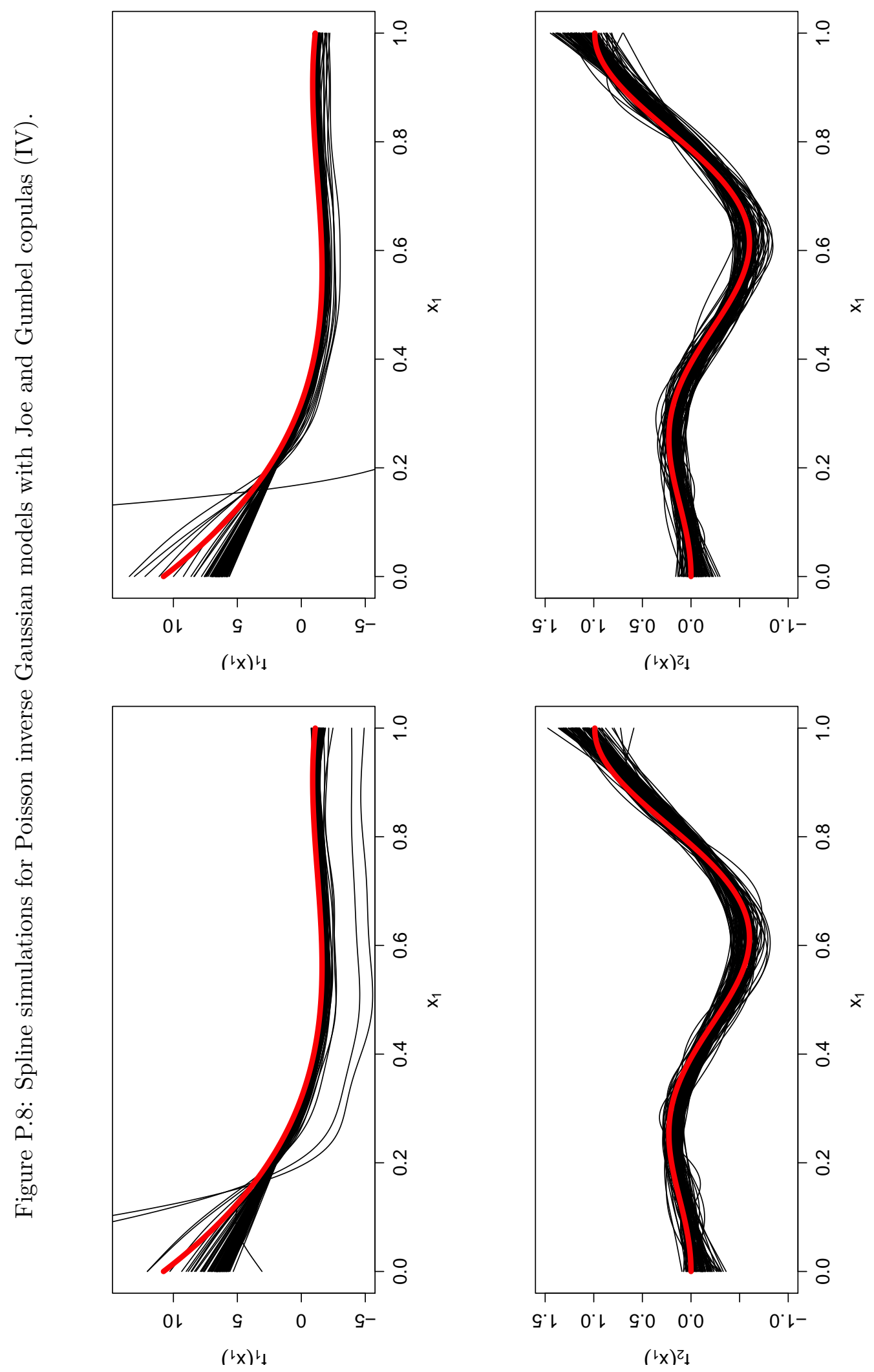

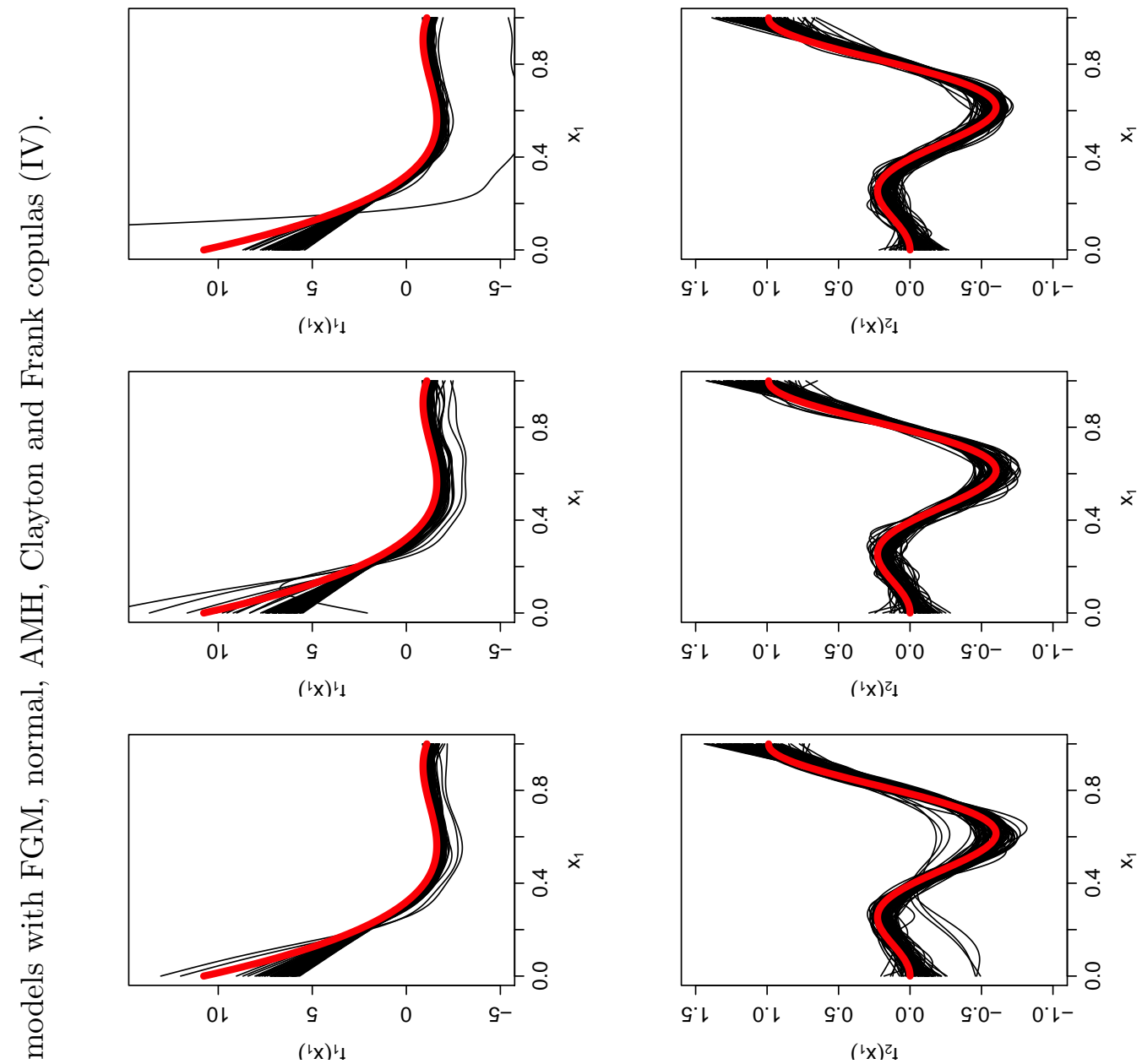

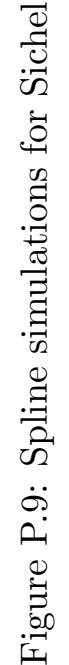
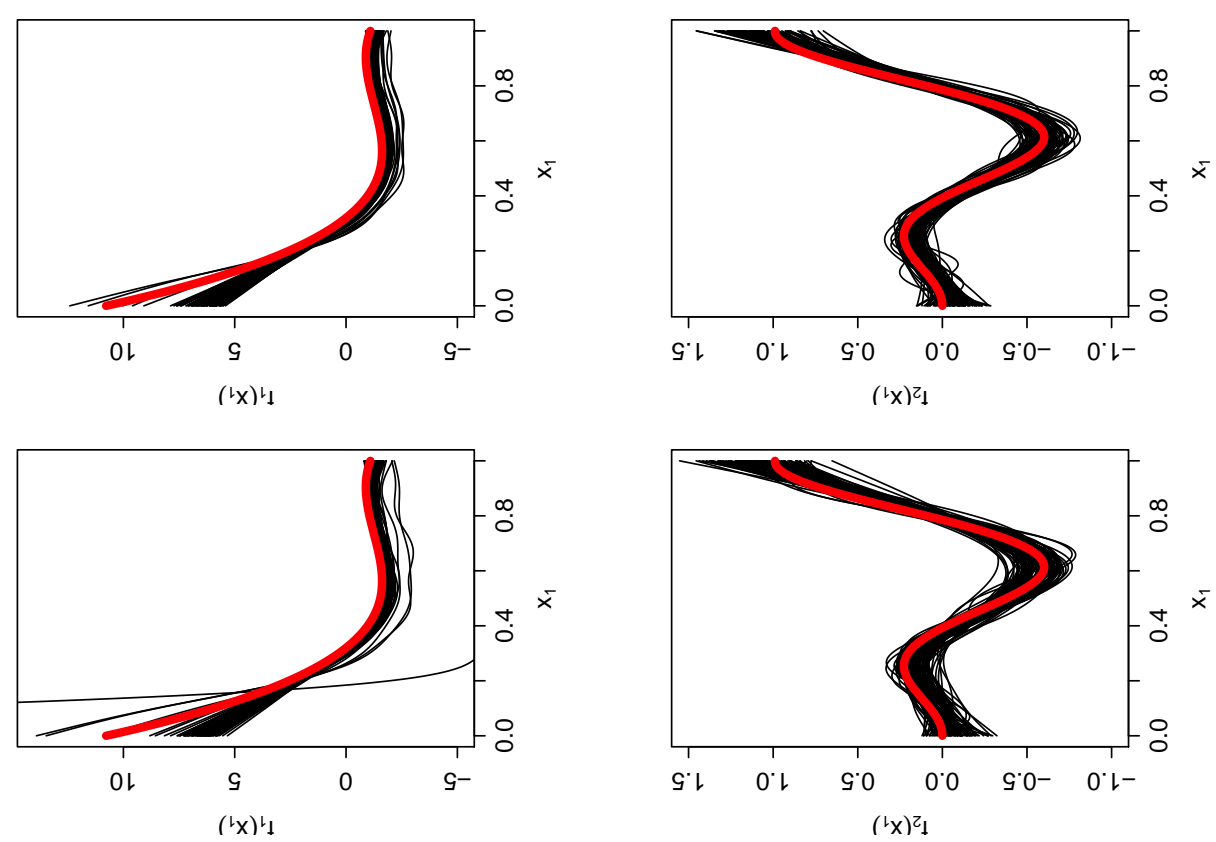

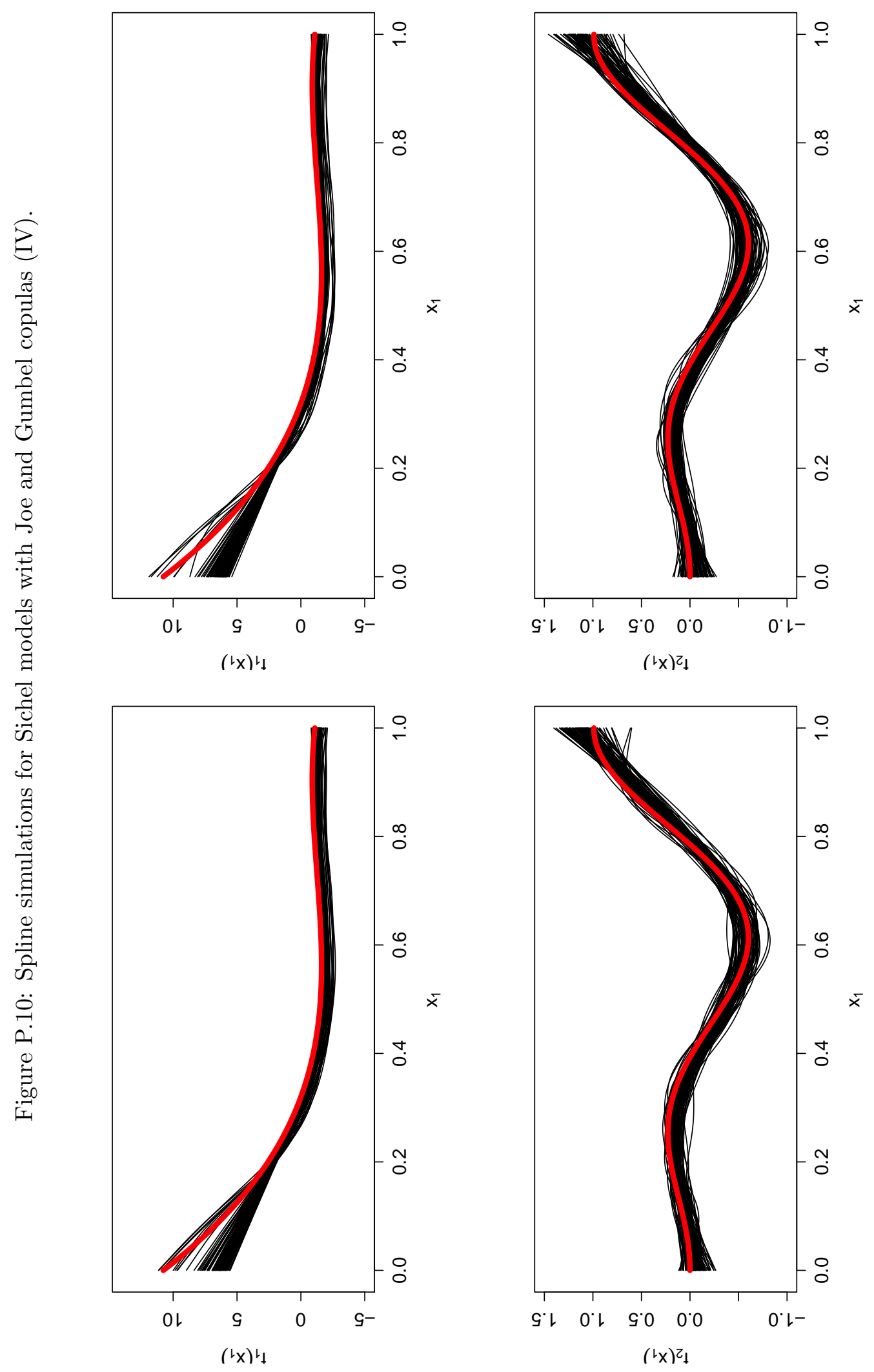
Appendix Q

Spline simulation for rotated copulas and IV 

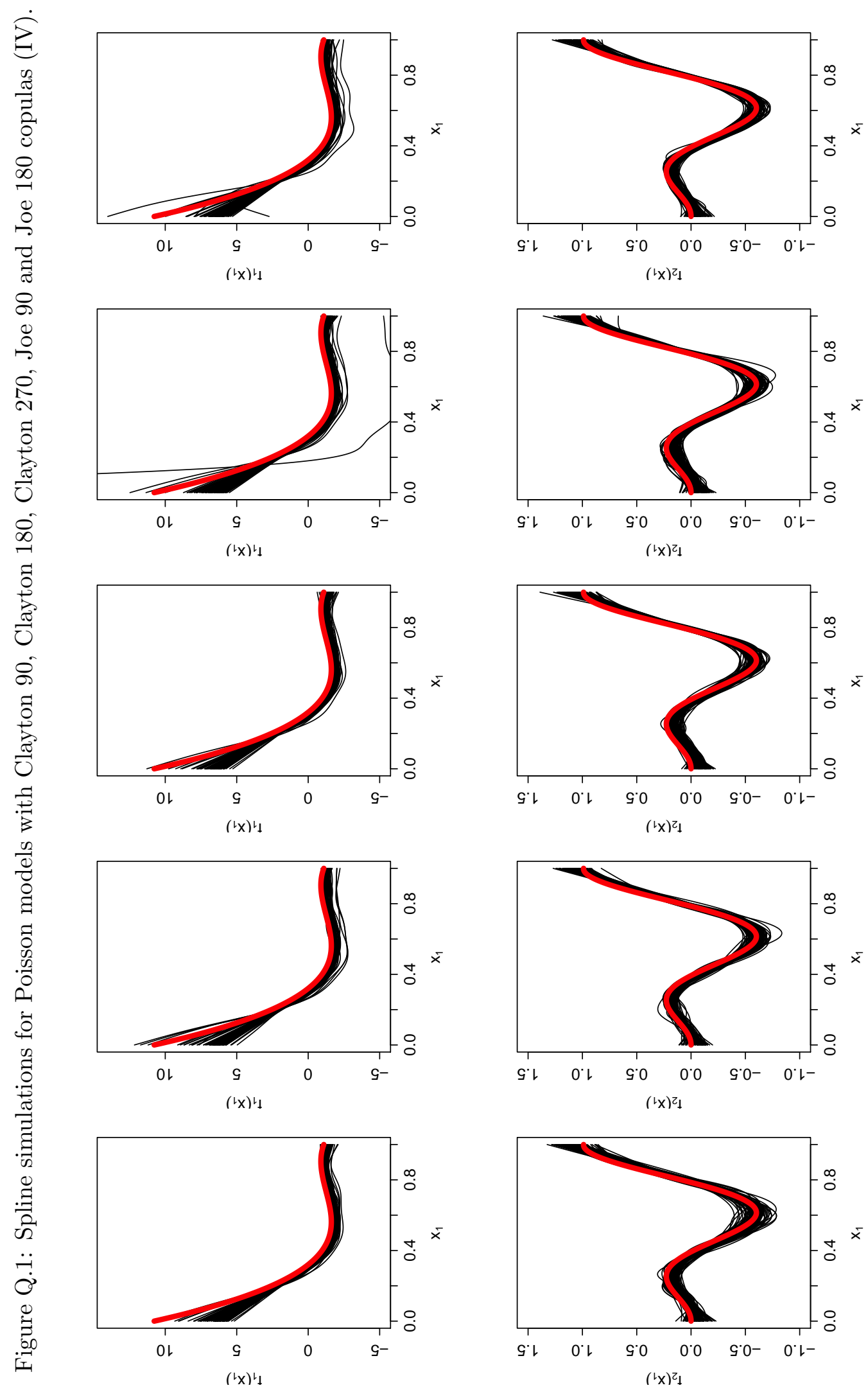

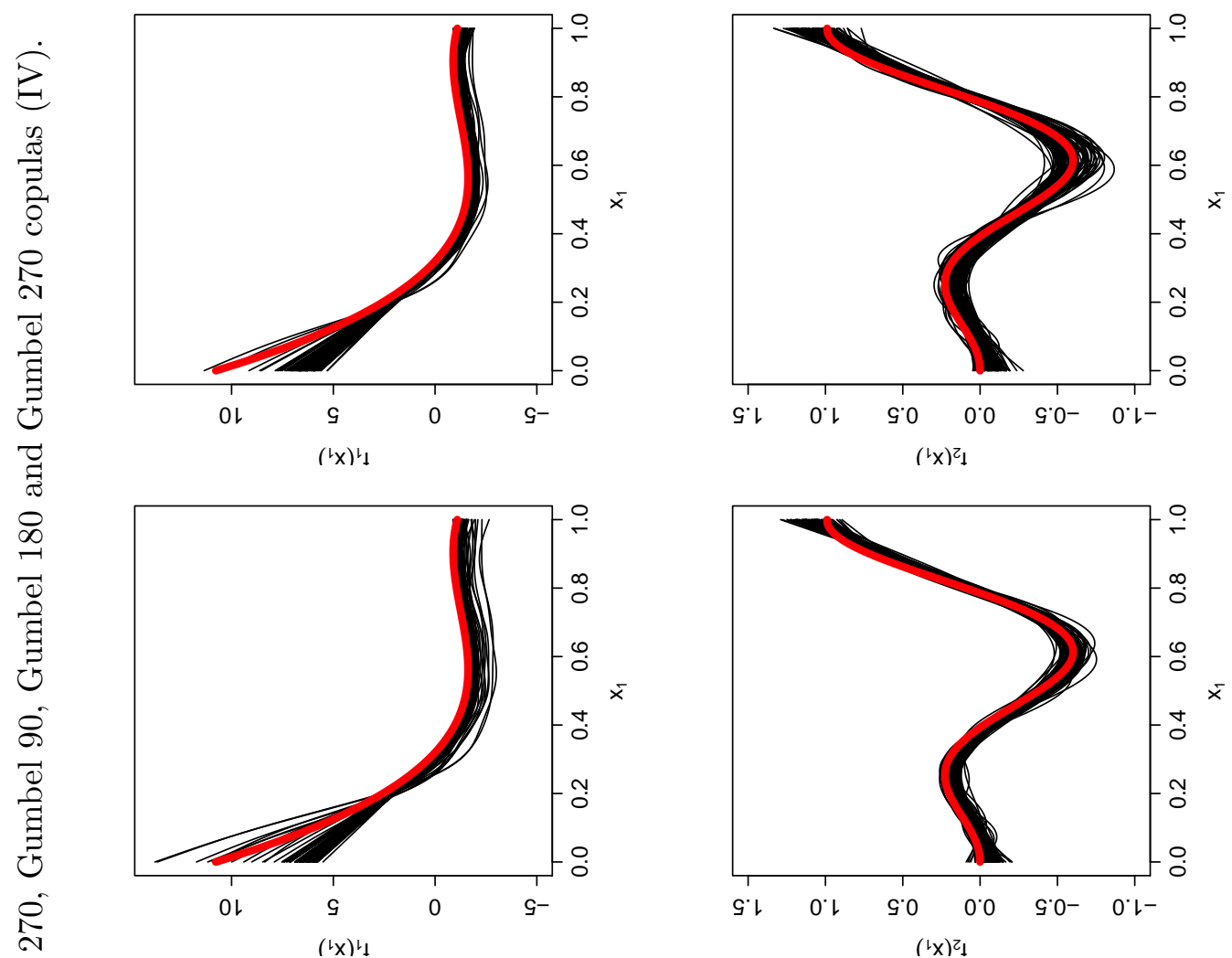

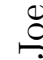

索
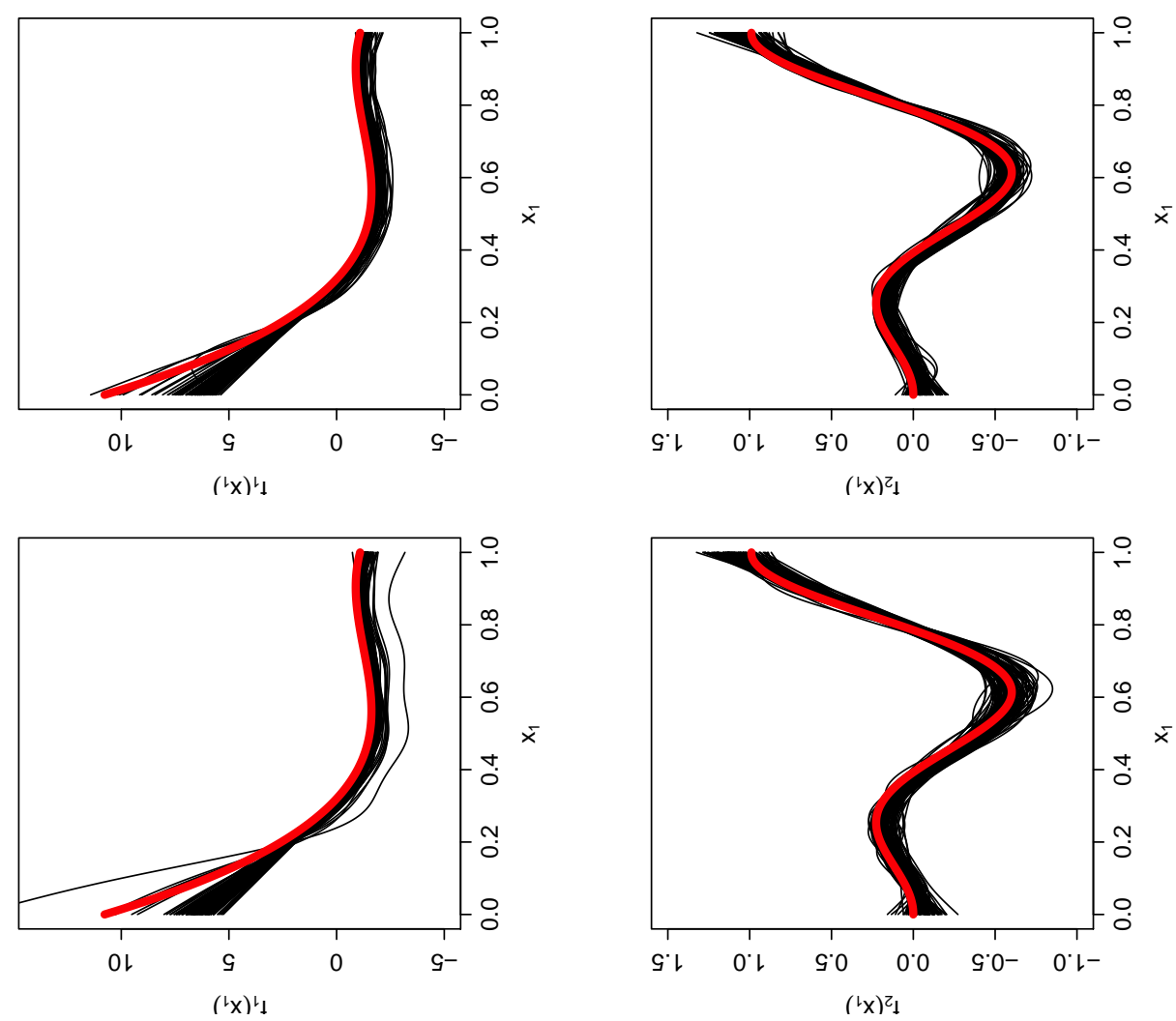

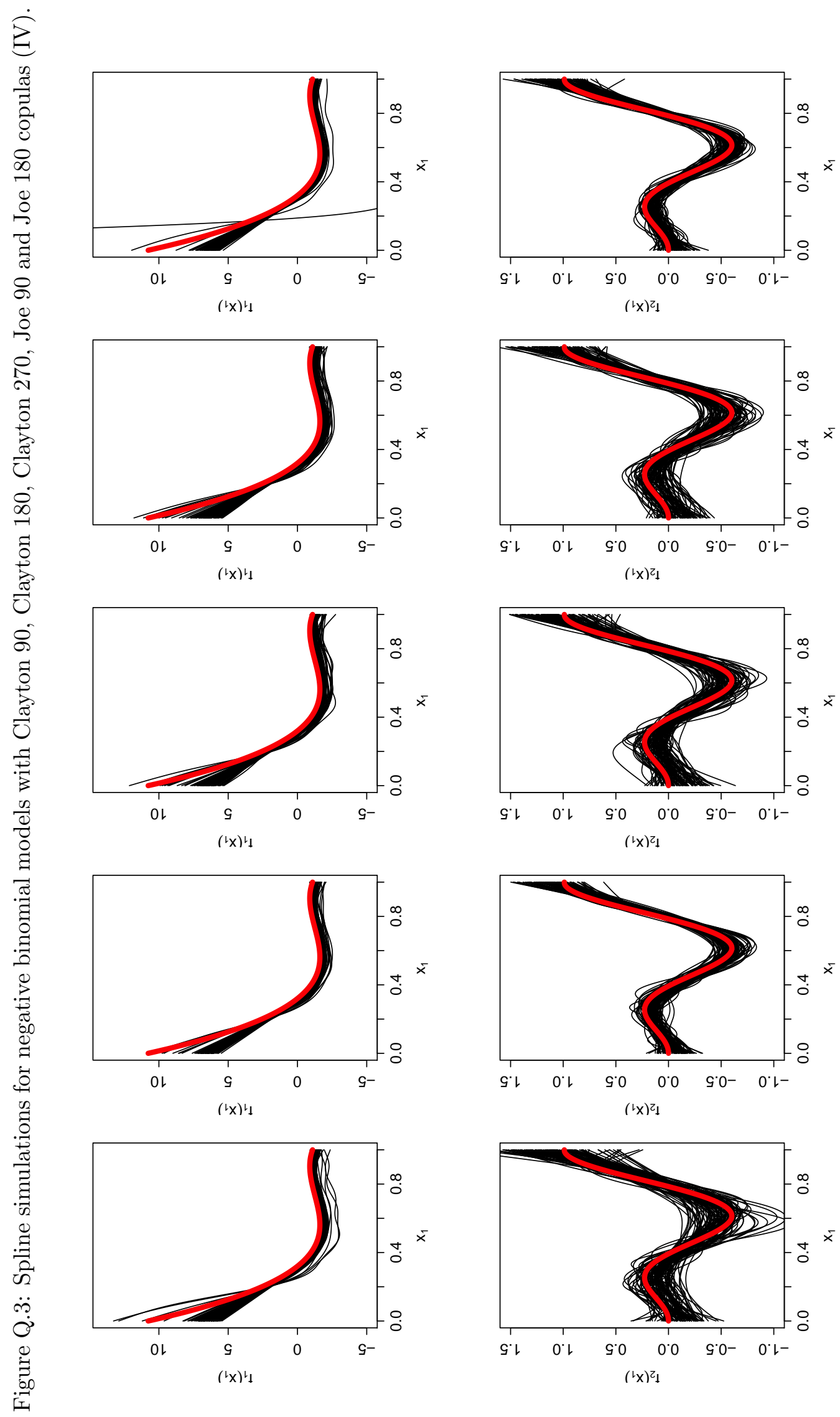

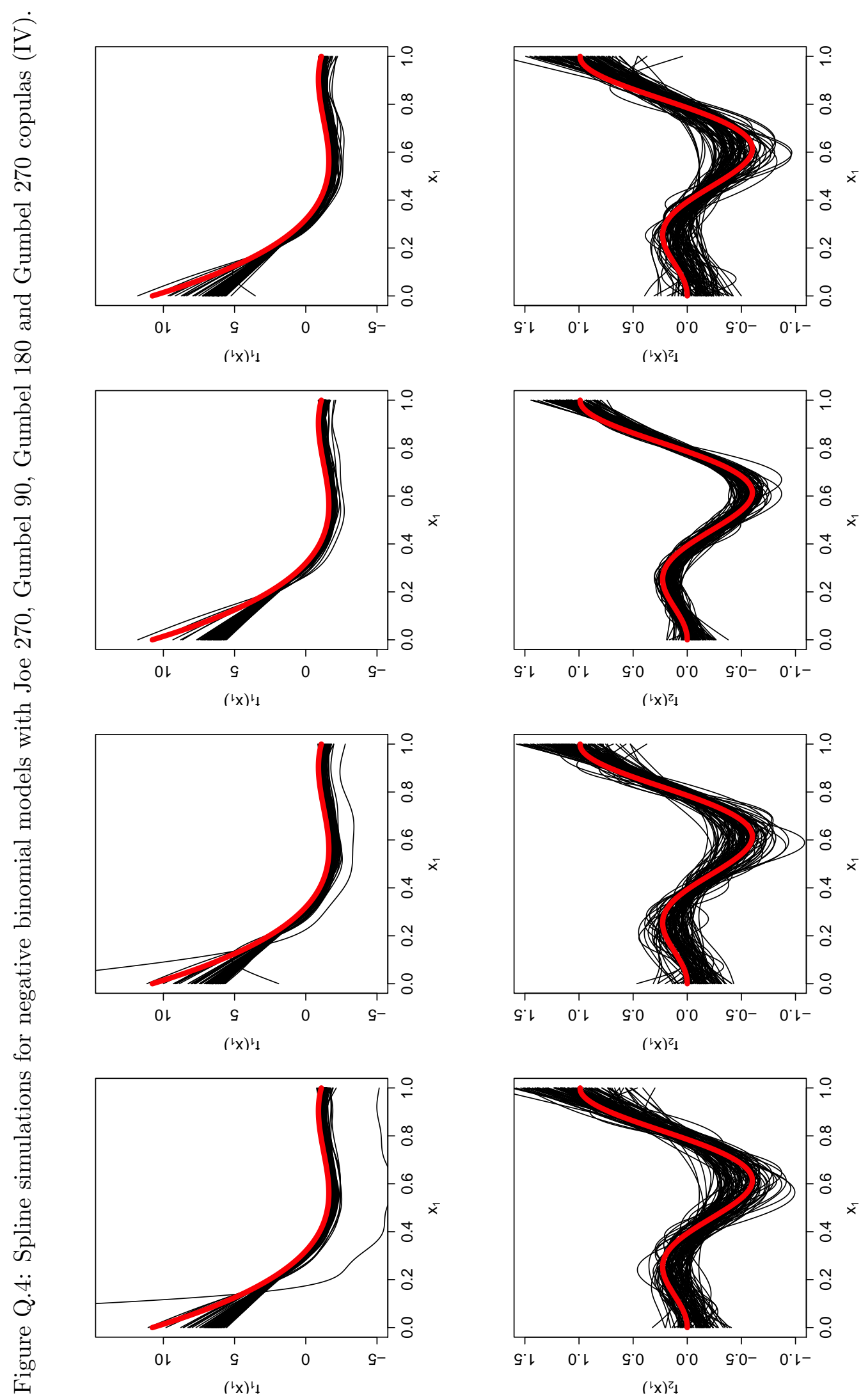

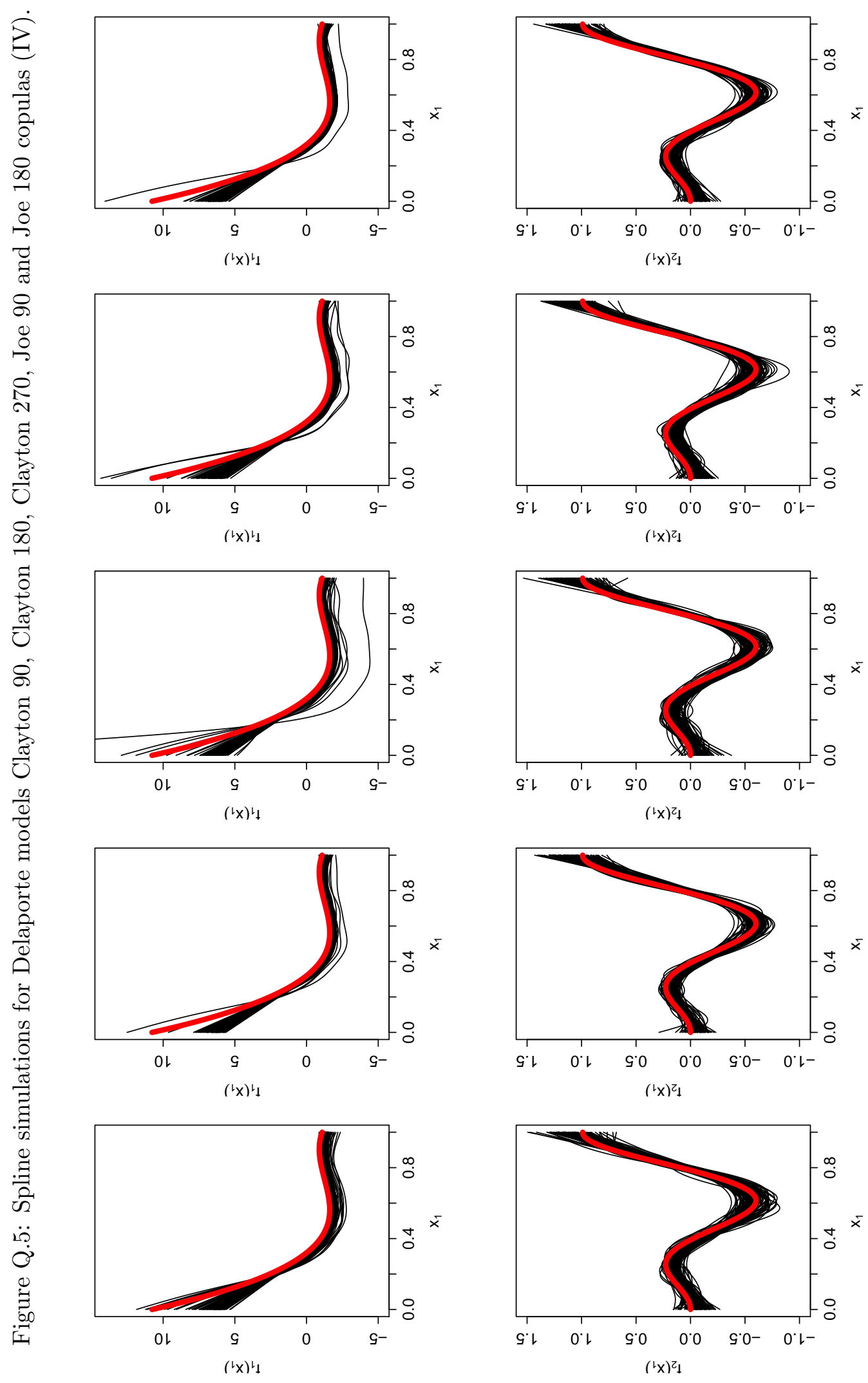

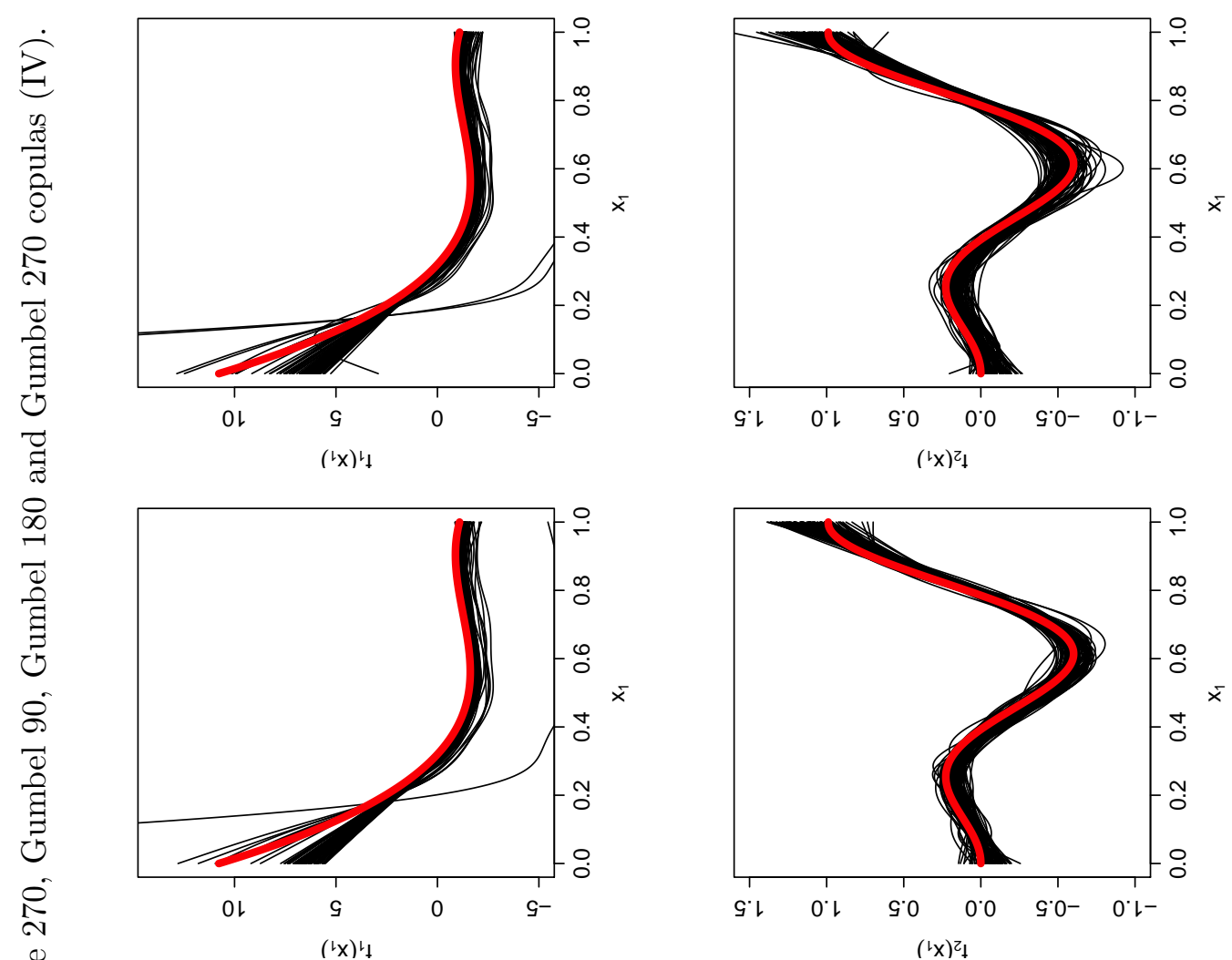

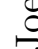

索

造
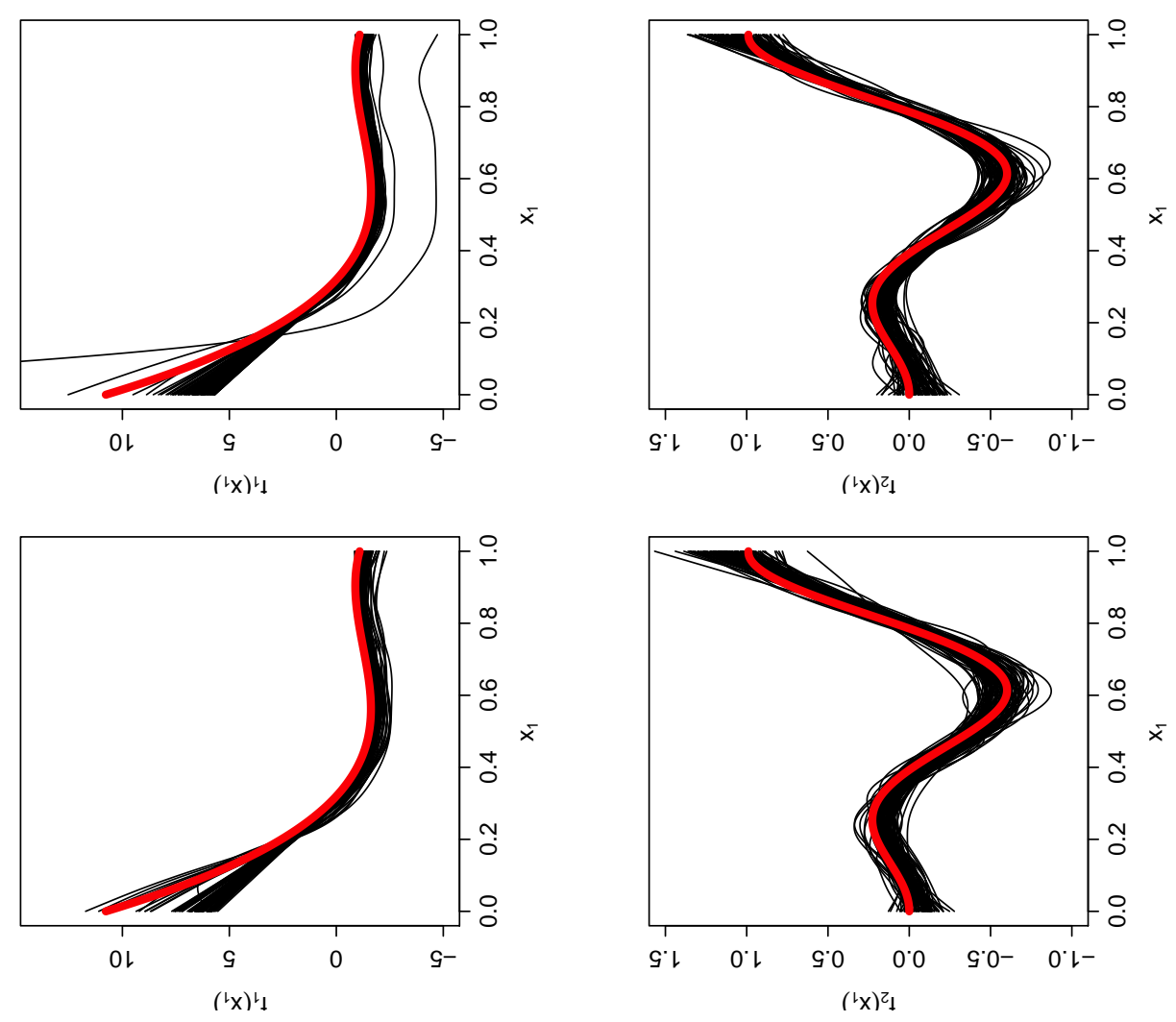

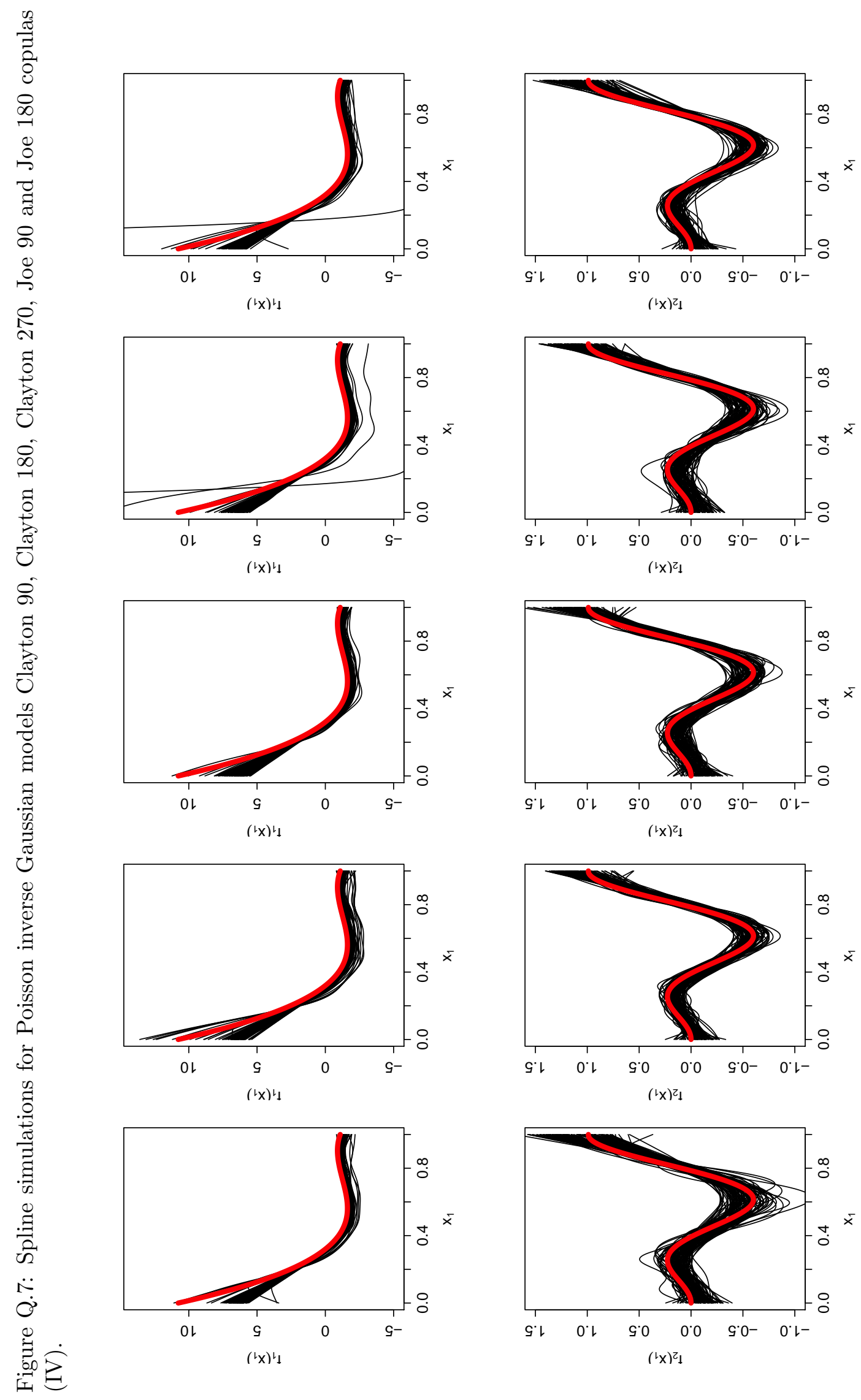

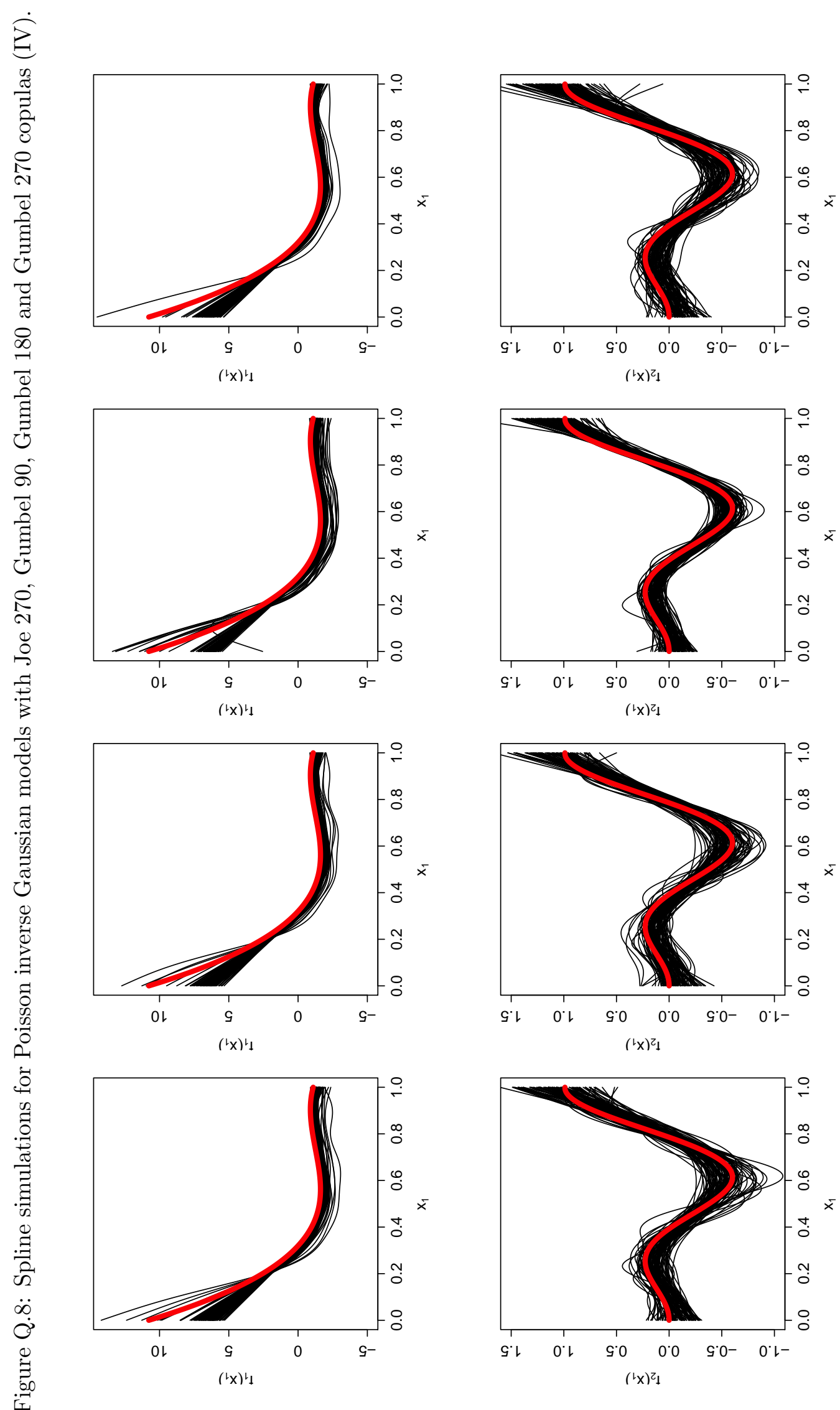

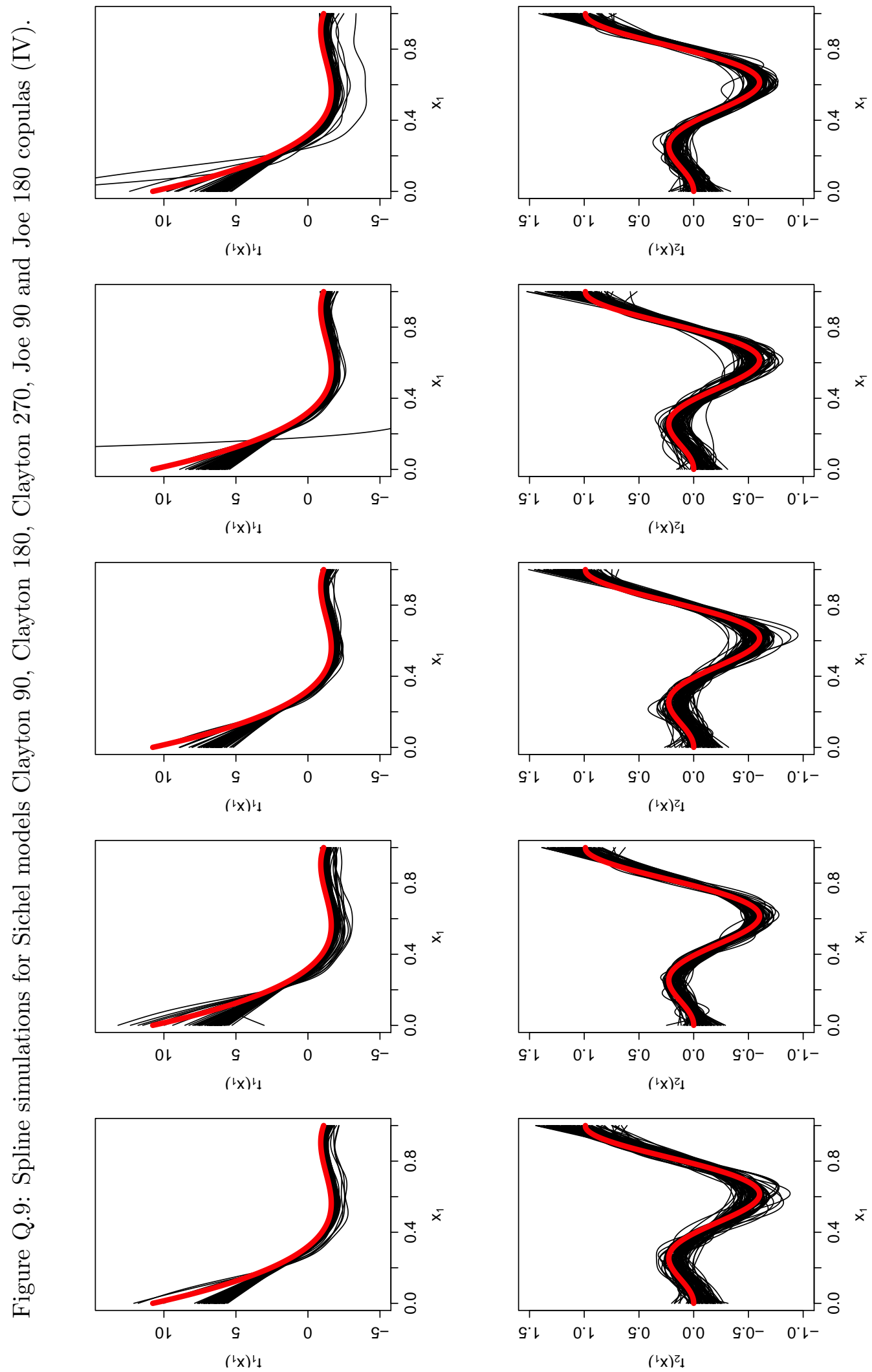

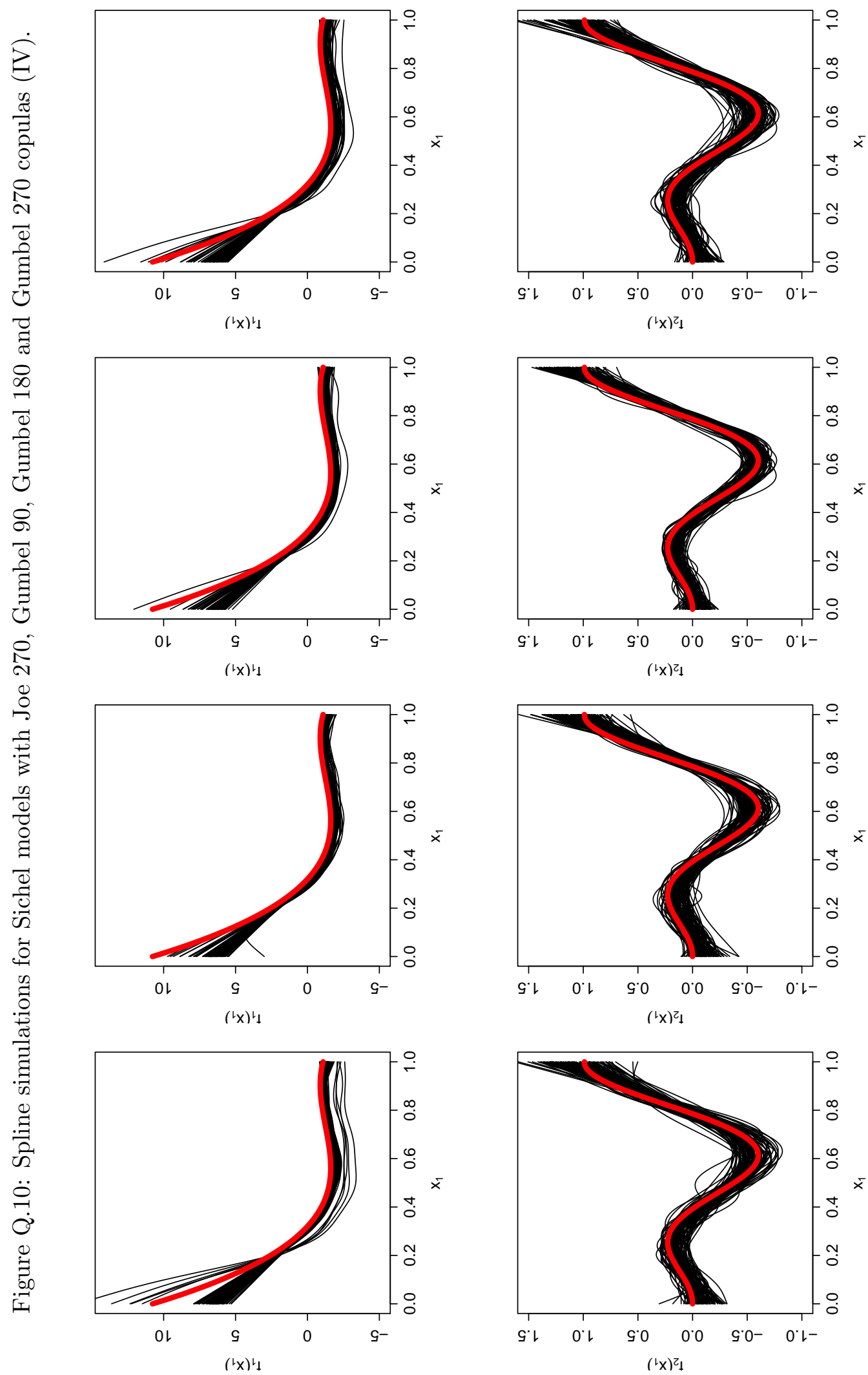
Appendix R

Data illustration results for VA 
Figure R.1: Selection (left) and outcome (right) smooth plots for Sichel Frank model for NUMOTH.
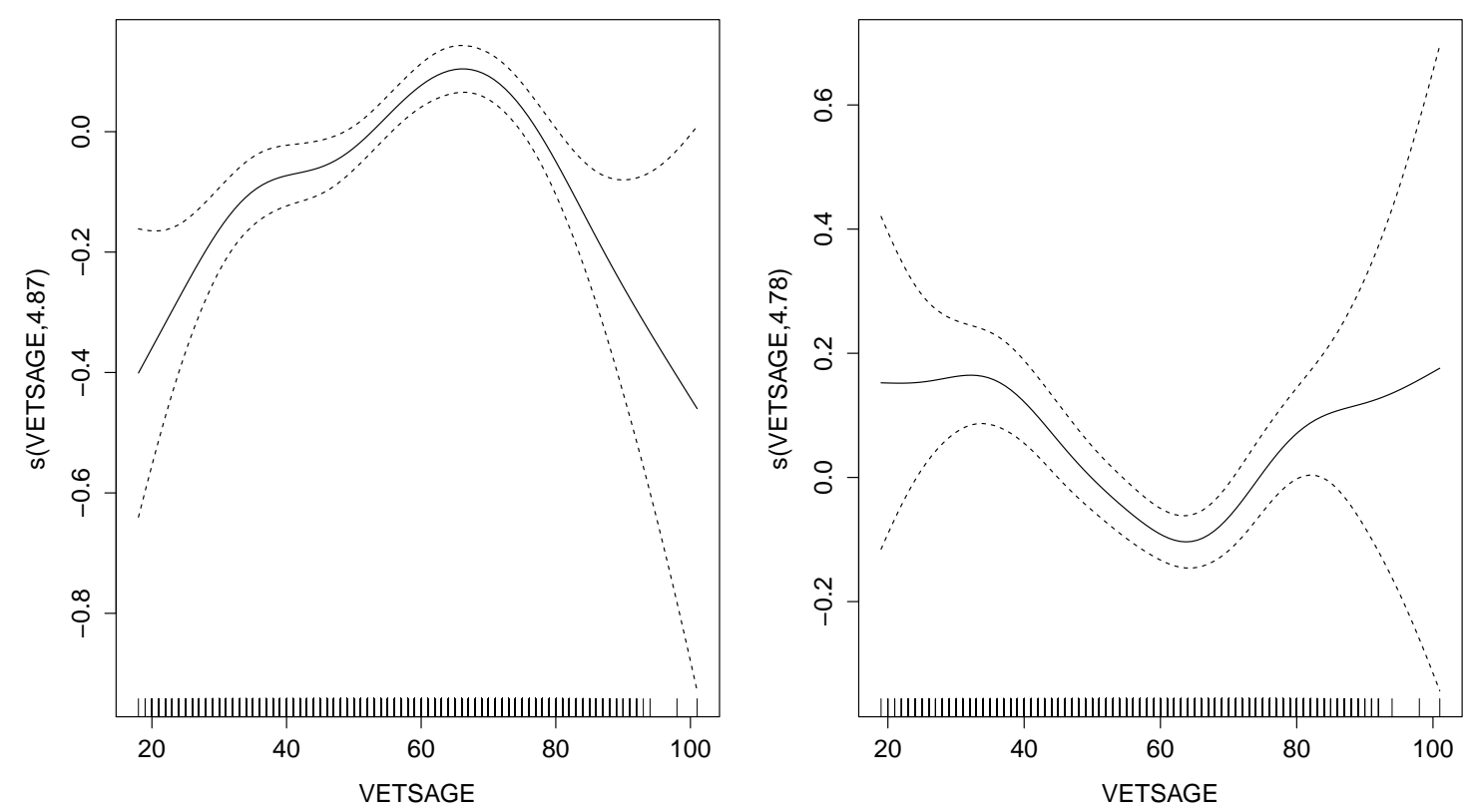

Figure R.2: Selection (left) and outcome (right) smooth plots for Sichel Clayton 90 model for NUMOTH.
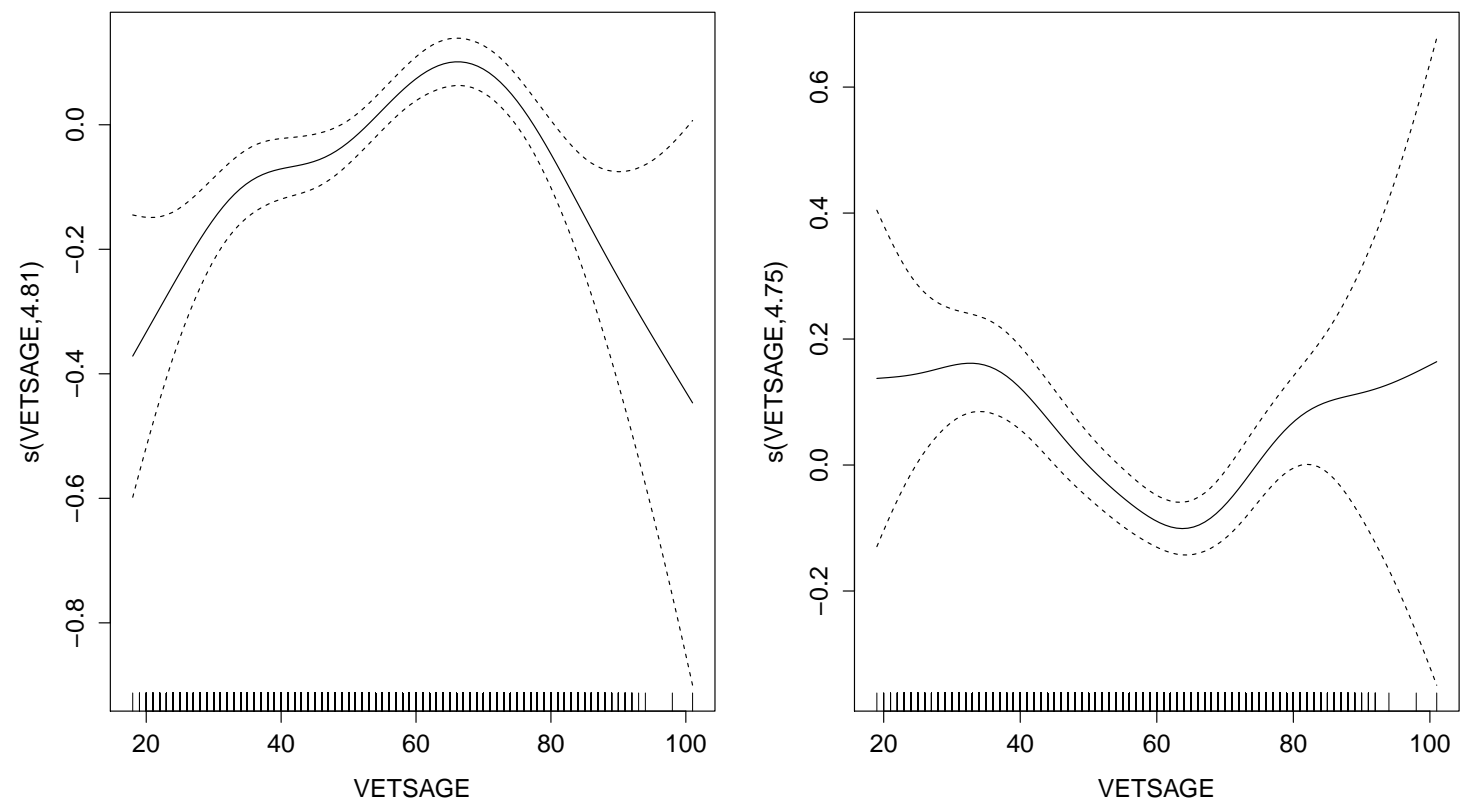
Figure R.3: Selection (left) and outcome (right) smooth plots for Sichel Gumbel 270 model for NUMOTH.
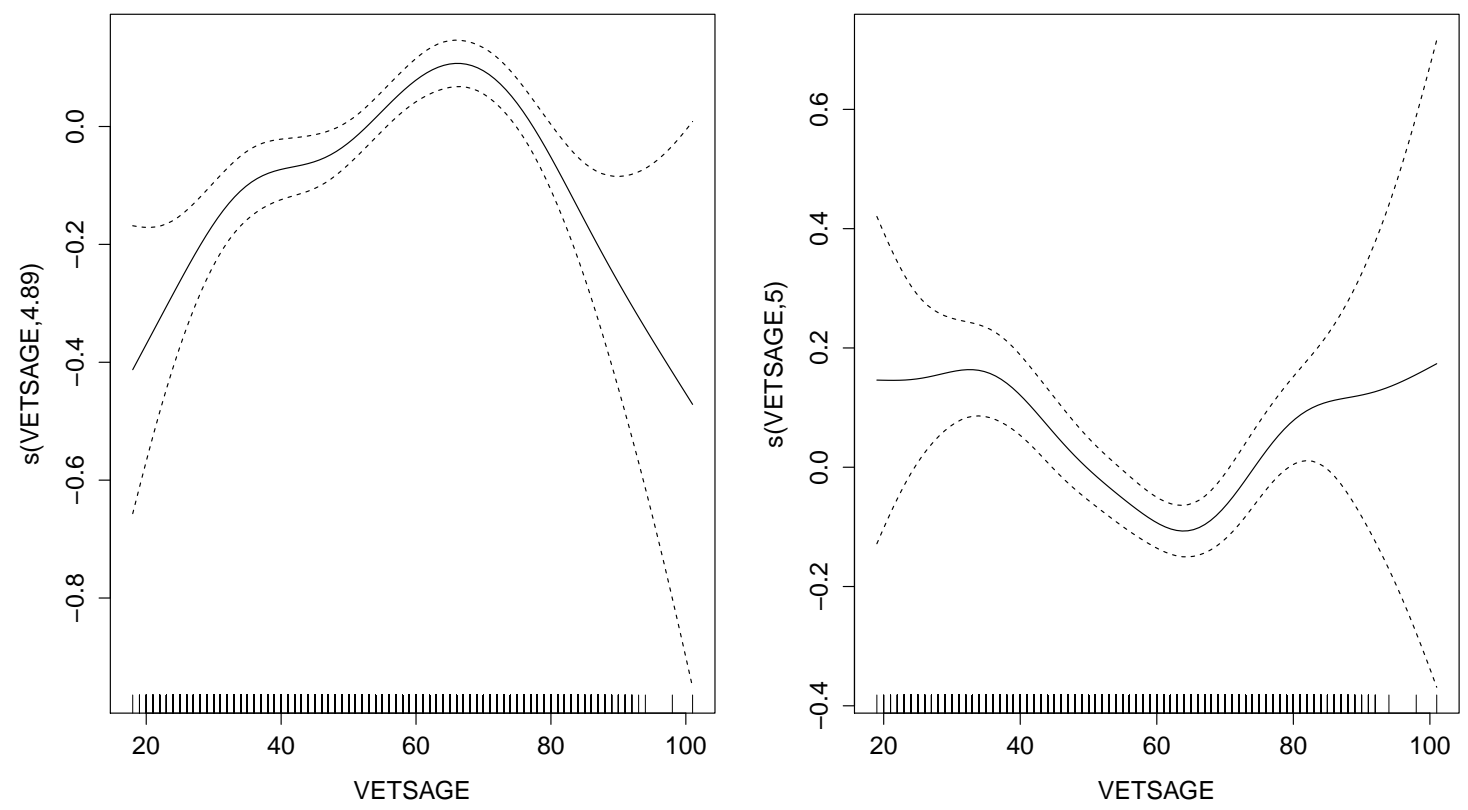

Figure R.4: Selection (left) and outcome (right) smooth plots for Sichel Frank model for VANITES.
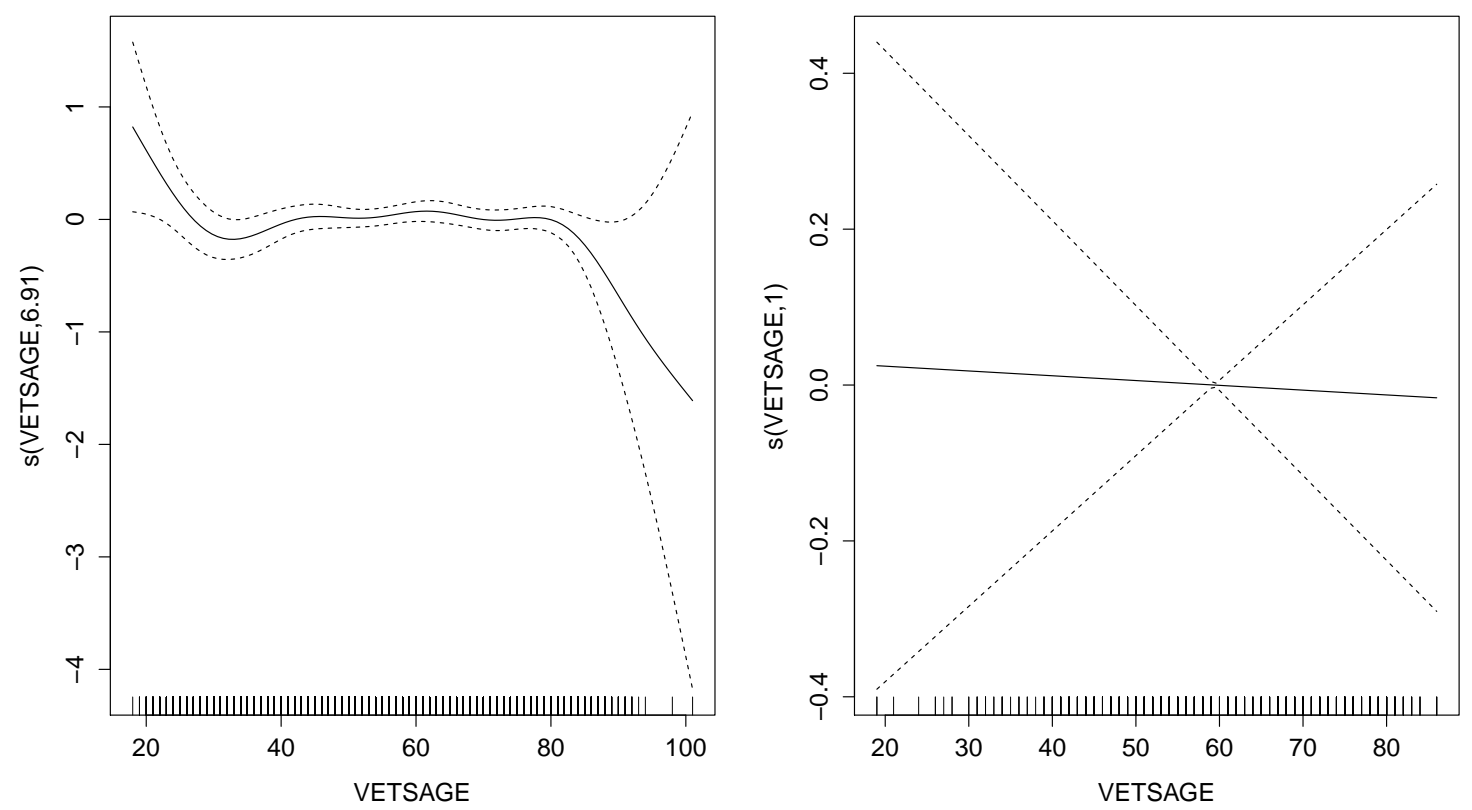
Figure R.5: Selection (left) and outcome (right) smooth plots for Sichel Clayton 90 model for VANITES.
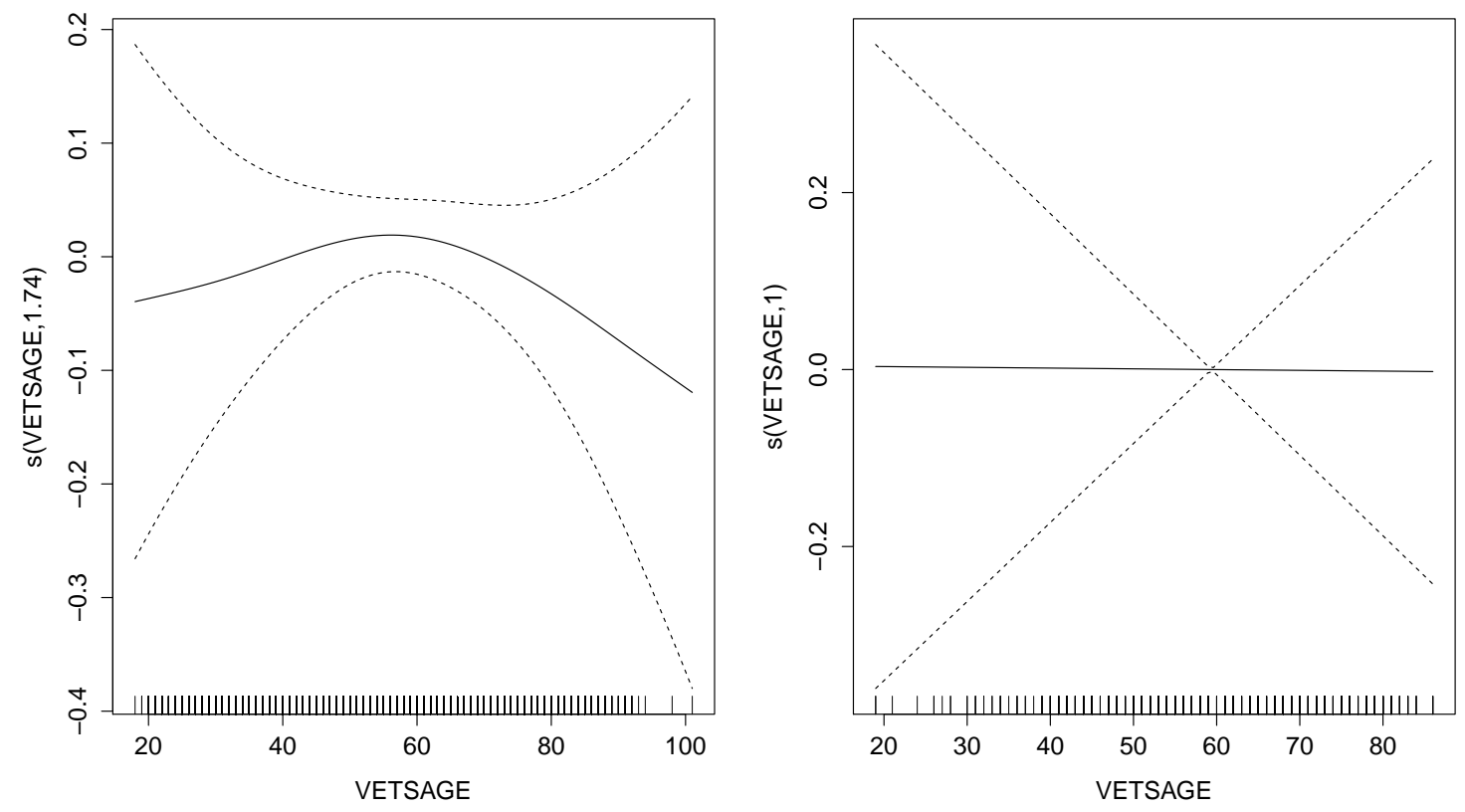

Figure R.6: Selection (left) and outcome (right) smooth plots for Sichel Gumbel 270 model for VANITES.
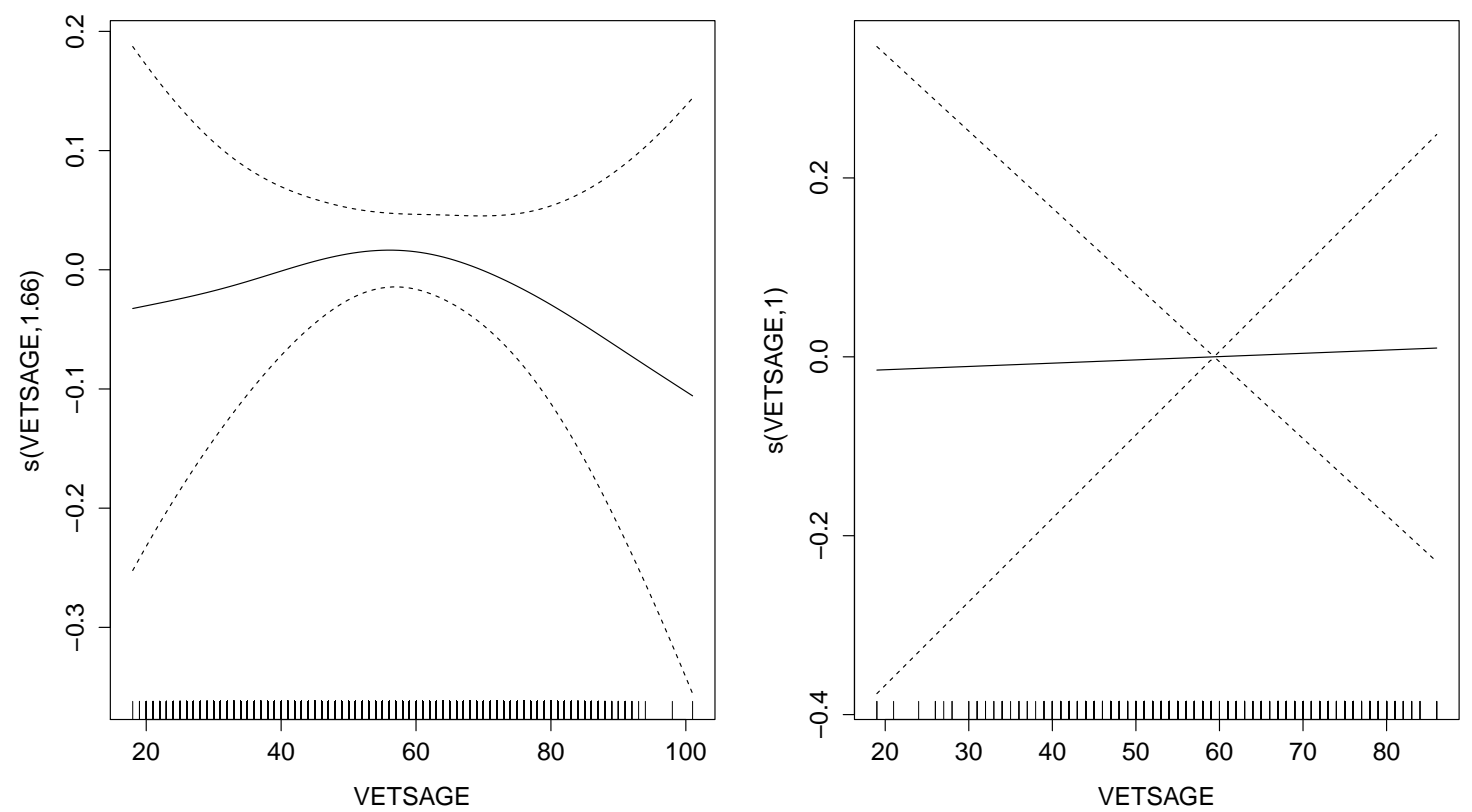
Figure R.7: Selection (left) and outcome (right) smooth plots for Poisson inverse Gaussian Frank model for OTHNITES.
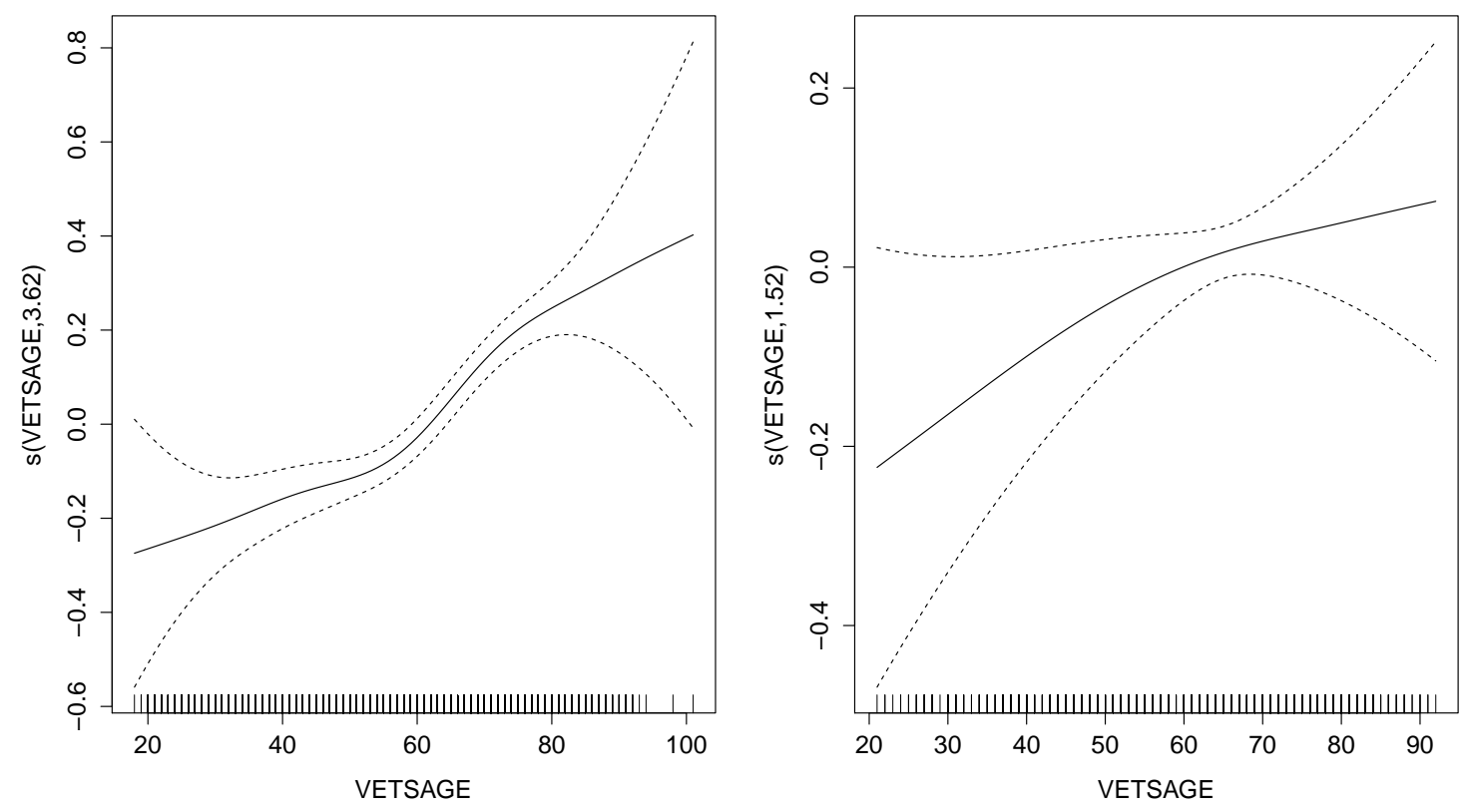

Figure R.8: Selection (left) and outcome (right) smooth plots for Poisson inverse Gaussian Clayton 90 model for OTHNITES.
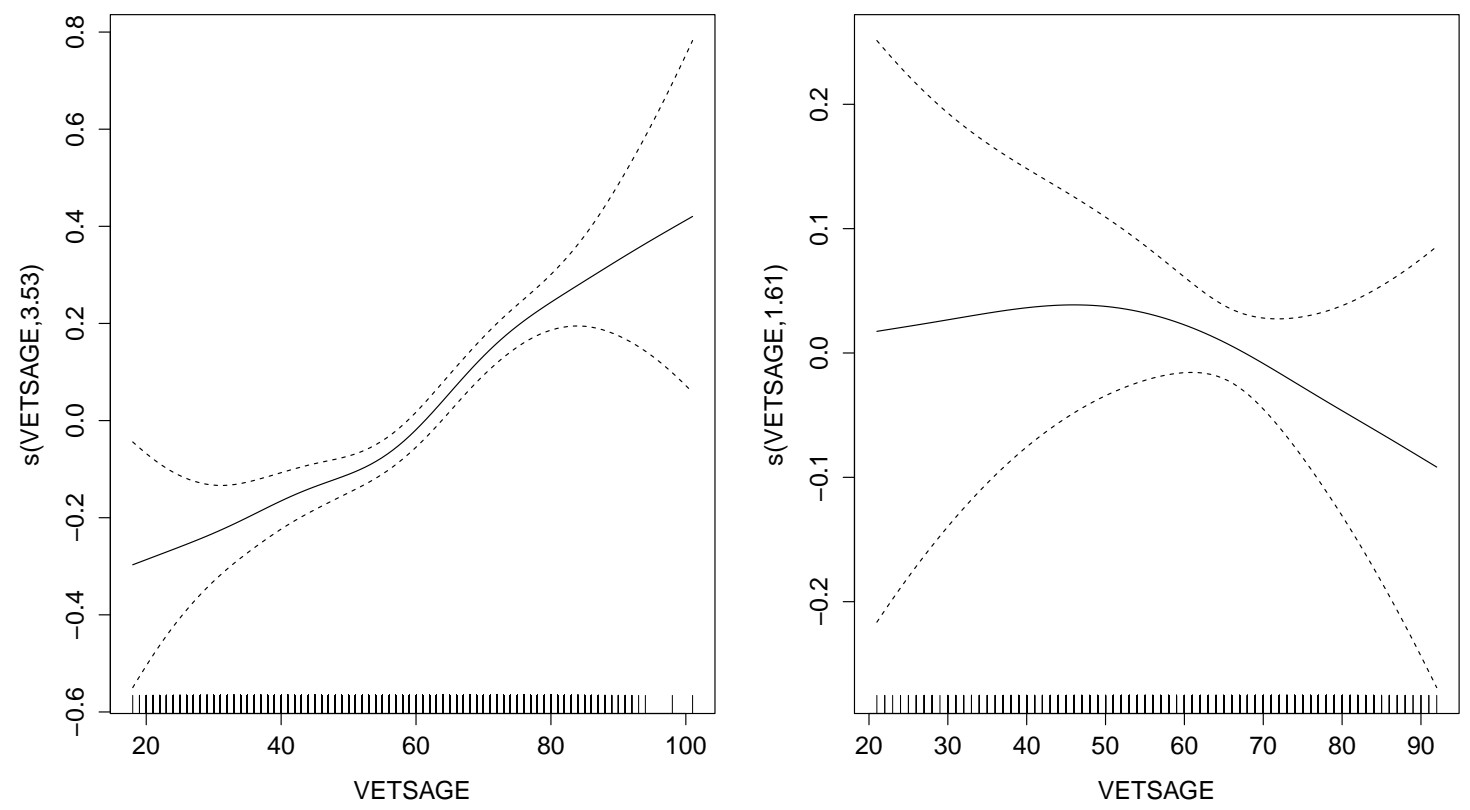
Figure R.9: Selection (left) and outcome (right) smooth plots for Poisson inverse Gaussian Gumbel 270 model for OTHNITES.
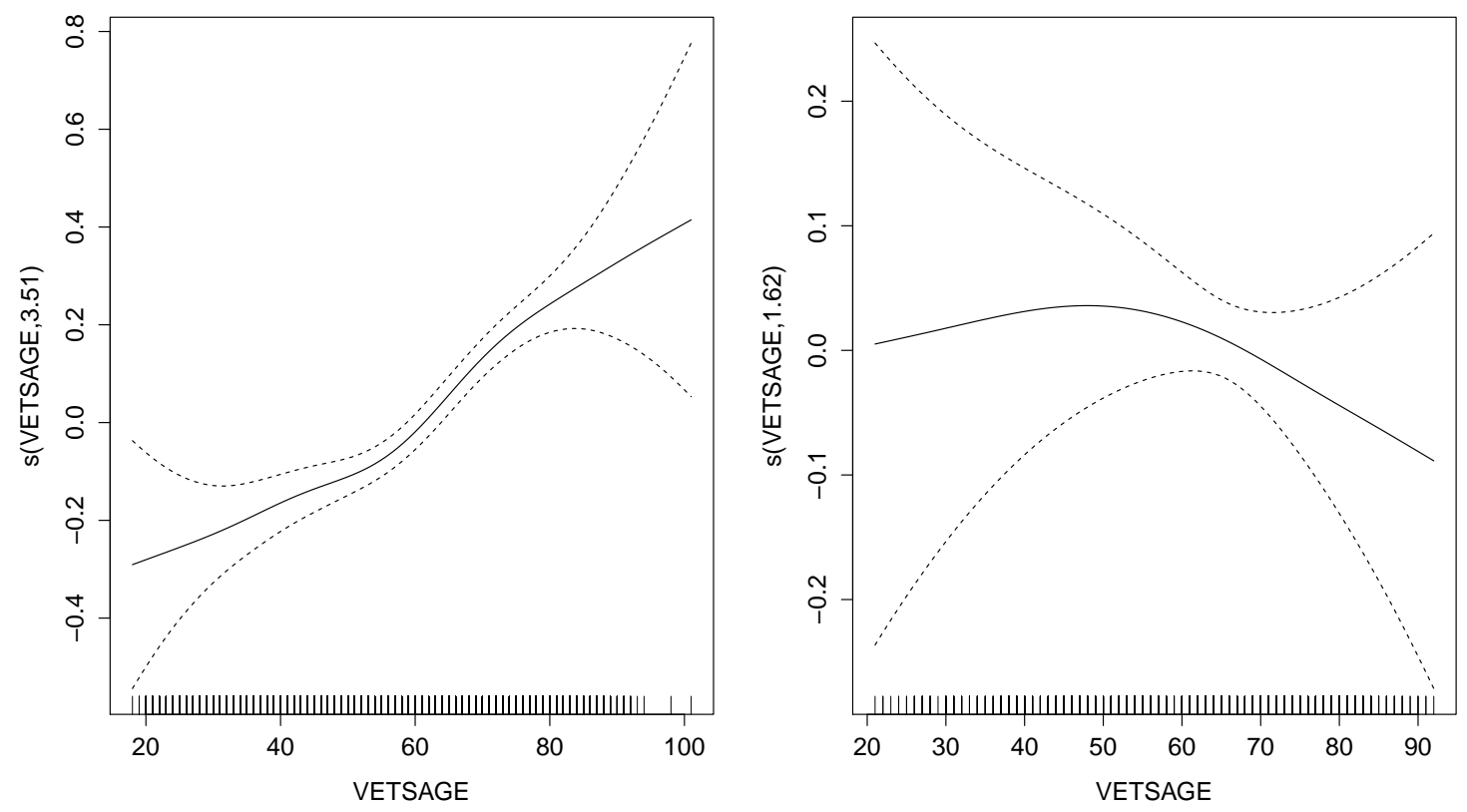


\section{Bibliography}

Abramowitz, M. et al. (1964). Handbook of mathematical functions: with formulas, graphs, and mathematical tables, volume 55. Courier Dover Publications.

Agresti, A. (2002). Random effects: generalized linear mixed models. An Introduction to Categorical Data Analysis.

Akaike, H. (1974). A new look at the statistical model identification. IEEE Transactions on Automatic Control, 19(6):716-723.

Barndorff-Nielsen, O. and Cox, D. R. (1989). Asymptotic techniques for use in statistics. Springer.

Bärnighausen, T., Bor, J., Wandira-Kazibwe, S., and Canninga, D. (2011). Correcting HIV prevalence estimates for survey nonparticipation using heckman-type selection models. Epidemiology, $22: 27-35$.

Boeri, T. and van Ours, J. (2008). The Economics of Imperfect Labour Markets. Princeton University Press.

Braun, M. (2013). trustoptim: An R Package for Trust Region Optimization with Sparse Hessians. Working Paper.

Brechmann, E. and Schepsmeier, U. (2013). Modeling dependence with C-and D-vine copulas: The R-package CDVine. Journal of Statistical Software, 52(3):1-27.

Broyden, C. (1970). The convergence of a class of double-rank minimization algorithms 1. general considerations. IMA Journal of Applied Mathematics, 6(1):76-90.

Cameron, A. and Trivedi, P. (2005). Microeconometrics: theory and applications. Cambridge University Press, New York. 
Chib, S., Greenberg, E., and Jeliazkov, I. (2009). Estimation of semiparametric models in the presence of endogeneity and sample selection. Journal of Computational and Graphical Statistics, $18: 321-348$.

Ciurtin, C., Wyszynski, K., Manson, J., and G., M. (2015). A Beta-Binomial Regression Model to Predict Doppler Score on Ultrasound Examination in Patients with Inflammatory Hand Joint Pain. Rheumatology (Supplement 1), 54. i151.

Clarke, K. (2007). A simple distribution-free test for nonnested model selection. Political Analysis, 15(3):347-363.

Craven, P. and Wahba, G. (1978). Smoothing noisy data with spline functions. Numerische Mathematik, 31(4):377-403.

De Boor, C. (1978). A practical guide to splines. Springer.

Eilers, P. and Marx, B. (1996). Flexible smoothing with B-splines and penalties. Statistical Science, pages 89-102.

Fairclough, D., Peterson, H., Cella, D., and Bonomi, P. (1998). Comparison of several modelbased methods for analysing incomplete quality of life data in cancer clinical trials. Statistics in medicine, 17(5-7):781-796.

Gallant, A. and Nychka, D. (1987). Semi-nonparametric maximum likelihood estimation. Econometrica, pages 363-390.

Genest, C. and Neslehova, J. (2007). A primer on copulas for count data. Astin Bulletin, 37(2):475515.

Genius, M. and Strazzera, E. (2008). Applying the copula approach to sample selection modelling. Applied Economics, 40(11):1443-1455.

Geyer, C. (2011). trust: Trust Region Optimization R package.

Gilbert, P. and Varadhan, R. (2015). numDeriv: accurate numerical derivatives. $R$ package version 2014.2-1. 
Green, P. and Silverman, B. (1994). Nonparametric Regression and Generalized Linear Models: A Roughness Penalty Approach. Chapman\& Hall, London.

Green, P. J. (1984). Iteratively reweighted least squares for maximum likelihood estimation, and some robust and resistant alternatives. Journal of the Royal Statistical Society. Series B, pages $149-192$.

Greene, W. H. (1994). Accounting for excess zeros and sample selection in poisson and negative binomial models. Working Paper, EC-94-10.

Greene, W. H. (1997). FIML Estimation of Sample Selection Models for Count Data. Working Paper, EC-94-10.

Greene, W. H. (1998). Sample selection in credit-scoring models. Japan and the World Economy, 10:299-316.

Greene, W. H. (2002). Econometric Analysis. Prentice Hall, Upper Saddle River, New Jersey, USA, 5th edition.

Gronau, R. (1974). Wage comparisons: A selectivity bias. Journal of Political Economy, 82:11191143.

Gu, C. (1992). Cross-validating non-Gaussian data. Journal of Computational and Graphical Statistics, 1(2):169-179.

Hartenstein, W. and Liepelt, K. (1962). Party Members and Party Voters in W. Germany. Acta Sociologica, 6:43-52.

Hasebe, T. and Vijverberg, W. (2012). A flexible sample selection model: A GTL-copula approach. IZA Discussion Paper.

Hastie, T. and Tibshirani, R. (1986). Generalized additive models. Statistical science, 1(3):297-310.

Hastie, T. and Tibshirani, R. (1990). Generalized additive models, volume 43. CRC Press.

Heckman, J. (1976). The common structure of statistical models of truncation, sample selection and limited dependent variables and a simple estimator for such models. Annals of Economic and Social Measurement, 5(4):475-492. 
Heckman, J. (1979). Sample Selection Bias as a Specification Error. Econometrica, 47:153-161.

Howell, D. (2012). Treatment of missing data: Part 1. David Howell's Homepage at http://www.uvm.edu.

Ilies, R., Hauserman, N., Schwochau, S., and Stibal, J. (2003). Reported incidence rates of workrelated sexual harassment in the United States: using meta-analysis to explain reported rate disparities. Personnel Psychology, 56(3):607-631.

Jung, J. and Liu, J. (2012). Does Health Insurance Decrease Health Expenditure Risk in Developing Countries? The Case of China. Working Paper.

Karlis, D. and Pedeli, X. (2013). Flexible bivariate INAR (1) processes using copulas. Communications in Statistics-Theory and Methods, 42(4):723-740.

Kauermann, G. (2005). Penalized spline smoothing in multivariable survival models with varying coefficients. Computational Statistics \& Data Analysis, 49(1):169-186.

Kenward, M. and Carpenter, J. (2007). Multiple imputation: current perspectives. Statistical Methods in Medical Research, 16(3):199-218.

Klau, F. and Mittelstadt, A. (1986). Labour Market Flexibility. Technical report, OECD.

Kneib, T. (2013). Beyond mean regression. Statistical Modelling, 13(4):275-303.

Kneib, T. and Wiesenfarth, M. (2010). Bayesian geoadditive sample selection models. Royal Statistical Society Series C: Applied Statistics, 59:381-404.

Lahiri, K. and Xing, G. (2004). An econometric analysis of veterans' health care utilization using two-part models. Empirical Economics, 29:431-449.

Lee, L.-F. (1983). Generalized econometric models with selectivity. Econometrica, pages 507-512.

Leung, S. and Yu, S. (1996). On the choice between sample selection and two-part models. Journal of econometrics, 72(1):197-229.

Levit, K., Lazenby, H., Waldo, D., and Davidoff, L. (1985). National Health Expenditures 1985. Health Care Financing Review, 7:1-35. 
Lewis, H. (1974). Comments on selectivity biases in wage comparisons. Journal of Political Economy, $82(6): 1145-55$.

Little, R. (1993). Pattern-mixture models for multivariate incomplete data. Journal of the American Statistical Association, 88(421):125-134.

Little, R. (2008). Selection and pattern-mixture models. Longitudinal data analysis, pages 409-431.

Little, R. and Rubin, D. (2002). Statistical analysis with missing data. Wiley.

Liu, M., P., K., and Yen, S. T. (2012). Who are consuming food away from home and where? Results from the Consumer Expenditure Surveys. European Review of Agricultural Economics, $5: 1-23$.

Manning, W., Newhouse, J., Duan, N., Keeler, E., Benjamin, B., Leibowitz, A., and Zwanziger, J. (1988). Health Insurance and the Demand for Medical Care: Evidence from a Randomized Experiment. Technical report, RAND, Santa Monica, California.

Marchenko, Y. V. and Genton, M. G. (2012). A Heckman selection-t model. Journal of the American Statistical Association, 107(497):304-317.

Marra, G. (2013). On p-values for semiparametric bivariate probit models. Statistical Methodology, 10(1):23-28.

Marra, G. and Radice, R. (2013a). Estimation of a regression spline sample selection model. Computational Statistics and Data Analysis, 61:158-173.

Marra, G. and Radice, R. (2013b). A Penalized Likelihood Estimation Approach to Semiparametric Sample Selection Binary Response Modelling. Electronic Journal of Statistics, 7:1432-1455.

Marra, G., Radice, R., Wojtyś, M., and Wyszynski, K. (2015). SemiParSampleSel: Semiparametric Sample Selection Modelling with Continuous or Discrete Response. R Package Version 1.3.

Marra, G. and Wood, S. (2012). Coverage properties of confidence intervals for generalized additive model components. Scandinavian Journal of Statistics, 39(1):53-74. 
McGovern, M., Bärnighausen, T., Marra, G., and Radice, R. (2015). On the Assumption of Joint Normality in Selection Models: A Copula Based Approach Applied to Estimating HIV Prevalence. Epidemiology.

Meyer, D., Zeileis, A., and Hornik, K. (2005). vcd: R package for visualizing categorical data.

Miranda, A. and Rabe-Hesketh, S. (2006). Maximum likelihood estimation of endogenous switching and sample selection models for binary, ordinal, and count variables. Stata Journal, 6(3):285-308.

Murphy, K. and Topel, R. (1985). Estimation and inference in two-step econometric models. Journal of Business $\&$ Economic Statistics, 3:370-379.

Nelsen, R. B. (1999). An Introduction to Copulas. Springer, New York, 1st edition.

Nelsen, R. B. (2006). An Introduction to Copulas. Springer, New York, 2nd edition.

Nikoloulopoulos, A. and Karlis, D. (2009). Modeling multivariate count data using copulas. Communications in Statistics-Simulation and Computation, 39(1):172-187.

Nikoloulopoulos, A. and Karlis, D. (2010). Regression in a copula model for bivariate count data. Journal of Applied Statistics, 37(9):1555-1568.

Nocedal, J. and Wright, S. (2006). Nonlinear optimization. Springer, New York.

O’Sullivan, F., Yandell, B. S., and Raynor, W. J. J. (1986). Automatic smoothing of regression functions in generalized linear models. Journal of the American Statistical Association, 81(393):96-103.

Pigini, C. (2012). Of Butterflies and Caterpillars: Bivariate normality in the Sample Selection Model. Technical report, Università Politecnica delle Marche.

Prieger, J. (2002). A flexible parametric Selection Model for non-normal data with Application to Health Care Usage. Journal of Applied Econometrics, 17:367-392.

Rabe-Hesketh, S., Skrondal, A., and Pickles, A. (2005). Maximum likelihood estimation of limited and discrete dependent variable models with nested random effects. Journal of Econometrics, $128(2): 301-323$. 
Racine, J. (2014). A primer on regression splines.

Radice, R., Marra, G., and Wojtyś, M. (2015). Copula Regression Spline Models for Binary Outcomes With Application in Health Care Utilization. Research report.

Rubin, D. (1976). Inference and missing data. Biometrika, 63(3):581-592.

Rubin, D. (1987). Multiple Imputation in Nonresponse in Surveys. Wiley, New York.

Ruppert, D., Wand, M., and Carroll, R. (2003). Semiparametric regression. Cambridge University Press.

Sapelli, C. and Vial, B. (2003). Self-selection and moral hazard in Chilean health insurance. Journal of Health Economics, 22:459-476.

SAS Institute (2013). SAS 9.4.

Schwarz, G. (1978). Estimating the dimension of a model. The Annals of Statistics, 6(2):461-464.

Sklar, M. (1959). Fonctions de répartition à $n$ dimensions et leurs marges. Université Paris 8.

Smith, M. (2003). Modelling sample selection using Archimedean copulas. Econometrics Journal, 6:99-123.

Smyth, G. and Dunn, P. (1996). Randomized quantile residuals. Journal of Computational and Graphical Statistics, 5:236-244.

SOEP v28 (2012). Socio-Economic Panel (SOEP). doi: 10.5684/soep.v28.

Sorensen, D. (1982). Newton's method with a model trust region modification. SIAM Journal on Numerical Analysis, 19(2):409-426.

Stasinopoulos, D. and Rigby, R. (2005). Generalized additive models for location, scale and shape. Journal of the Royal Statistical Society: Series C, 54(3):507-554.

Stasinopoulos, D. and Rigby, R. (2007). Generalized additive models for location scale and shape (gamlss) in R. Journal of Statistical Software, 23(7):1-46. 
Stasinopoulos, D., Rigby, R., and Akantziliotou, C. (2008). Instructions how to use gamlss package in R. Technical report, gamlss.

Terza, J. V. (1998). Estimating count data models with endogenous switching: Sample selection and endogenous treatment effects. Journal of Econometrics, 84:129-154.

Trivedi, P. and Zimmer, D. (2007). Copula Modeling: An Introduction for Practitioners, volume 1. Now Pub.

Von Hippel, P. (2007). Regression with missing Ys: An improved strategy for analyzing multiply imputed data. Sociological Methodology, 37(1):83-117.

Vuong, Q. (1989). Likelihood ratio tests for model selection and non-nested hypotheses. Econometrica, pages 307-333.

White, H. (1980). A heteroskedasticity-consistent covariance matrix estimator and a direct test for heteroskedasticity. Econometrica, pages 817-838.

Winkelmann, R. (1998). Count data models with selectivity. Econometric Reviews, 17:339-359.

Wojtyś, M., Marra, G., and Radice, R. (2016). Copula Regression Spline Sample Selection Models: The R Package SemiParSampleSel. Journal of Statistical Software.

Wood, S. (2006). Generalized additive models: An introduction with R. CRC press.

Wood, S. (2012). On p-values for smooth components of an extended generalized additive model. Biometrika.

World Health Organisation (2009). Towards universal access: Scaling up priority HIV/AIDS interventions in the health sector. Technical report, United Nations, Geneva.

Young, R. and Johnson, D. (2010). Imputing the Missing Ys: Implications for Survey Producers and Survey Users. In 64th Annual Conference of the American Association for Public Opinion Research.

Yuan, Y. and Yen, S. T. (2012). Alcohol consumption by individuals in the United States: a sample selection approach. Applied Economics Letters, 19:1353-1358. 
Yuan, Y. C. (2010). Multiple Imputation for Missing Data: Concepts and New Development. SAS Institute. 